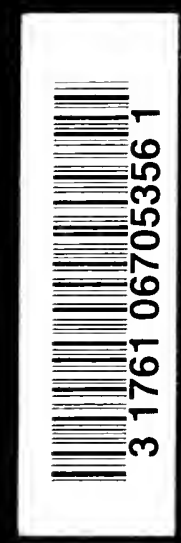




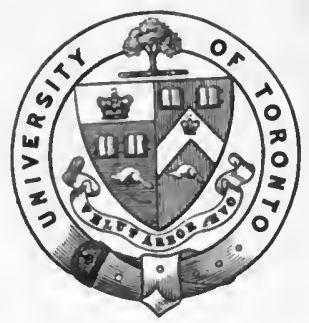

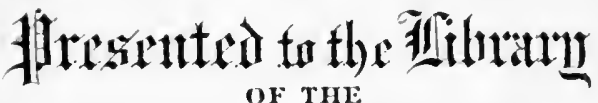
Hltutrexsity of Comontor. 8! 7.h.g. Stan

$$
\text { A.D. } 190
$$




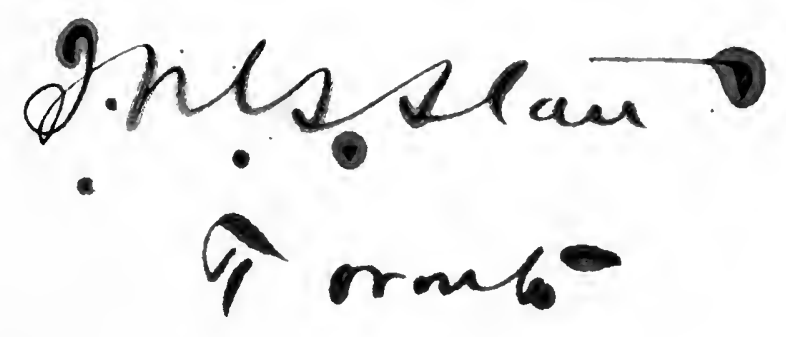




$$
\text { -... }
$$


Digitized by the Internet Archive in 2007 with funding from Microsoft Corporation 


rin.

\section{SURGICAL ANATOMY}

AND

\section{Operative Surgery}

For Students and Practitioners

BY

JOHN J. MCGRATH, M.D.

Protessor or Surgical axatosy axd Operative Surgery at the New Yokk Post-granuate Mepical School, Visiting SUrgeon to the Harlem IIospital, and Assistant Visiting

SuRgeon to the Colcubus Hospital, New York.

\section{(With 227 Illustrations, including Colors and Galf-tones}

Philadelphia

F. A. DAVIS COMPANY, PUBLISHERS 
COPYRIGIIT, 1902,

BT

F. A. DAVIS COMPANY.

[ Hegistered at Stationerg' Hall, London, Eng.]

Philadelphia, Pa., U. S. A.:

The Medical Bulletin Printing-house, 1914-16 Cherry Street. 


\section{PREFACE.}

IN this volume an endeavor has been made to combine, in a practical manner, the subjects of surgical anatomy and operative surgery, because a knowledge of the one is essential to the proper study of the other.

Diagrammatic drawings have been used largely for the purpose of illustration, because these, in my judgment, are the most satisfactory for teaching.

An effort has been made to exclude all those anatomical considerations that are purely technical and not of practical value in the performance of surgical operations.

The arrangement of the subject has been made in accordance with the plan followed in my courses in operative surgery at the Post-graduate Medical School.

John J. MoGrath. 



\section{CONTENTS.}

PART I.

Generat Considerations $\ldots \ldots \ldots \ldots \ldots \ldots \ldots \ldots \ldots \ldots \ldots \ldots \ldots \ldots$

Anæsthesia: Generai anæsthesia, 1; incomplete general anæsthesia, 1; local anæsthesia, Schleich infiltration method, 3 ; analgesia by subarachnoid injection of cocain, 3.- Division of Tissues: Division of soft parts, 4; division of bone, 5.- Hemorrhage: Means to arrest hemorrhage, 6; natural arrest of hemorrhage, 6; artificial arrest of hemorrhage, 6.Suture of the Tissues: Suture of the skin, 13; intracuticular suture, 14; suture of muscle, 14; suture of tendons, 15; suture of nerves, 15; suture of bone and cartilage, 15; suture of serous surfaces, bowel, etc., 16.

\section{PART II.}

Head and Face............................ 19-117

Head: Surgical anatomy of the head, 19; of the scalp, 19; of the skull, 20; of the dura mater, 21; of the pia mater, 23. - Operations upon the Head: Trephining, 23; for depressed fracture of the skuil, 23; for intracranial hemorrhage, 25; craniectomy (linear craniotomy), 29.- - Middle Fossa of the Skuli: Anatomy of the middle fossa, 30; extirpation of Casserian ganglion (Hartley-Krause), 36. - Mastold Region and Ear: Surgical anatomy of mastoid region, 39; anatomy of the ear, 41.-Operations upon the Mastoid, etc.: Wilde's incision, 46; drilling into antrum, 46; to open and drain antrum, 46; for thrombosis sigmoid sinus, 48; for cerebeilar abscess, 50; for extradural abscess in middie fossa, 50; for temporo-sphenoidal abscess, 51.- Face: Surgical anatomy of face, 51; of skeleton of lace, 52; of mouth, 54; side of face, 58; pterygo-maxillary region, 58.-Operations upon Face: Resection of upper jaw, 64; total resection of both superior maxillæ, 69; to drain antrum of Highmore, 69; resection of half of lower jaw, 70; resection of half of body of lower jaw, 73; resection of entire body of lower jaw, 75; resection of part of body of lower jaw (in continuity), 75; resection of part of body of lower jaw (not in continuity), 76; extirpation of Casserian ganglion (Rose-Andrews), 76; division of second and third branches of trifacial nerve (Krönlein-Lücke), 79. - Congenital Deformities of Face: Development of face, 80; formation of palate, 88; teeth, 89; tongue, 89 ; deformities of face, 89 ; deformities in which frontal plate is concerned, 91; lateral clefts of the upper lip, and alveolar process and cleft palate, 91 ; median clefts and notches of the upper lip, 95; oblique facial clefts, 96; deformities in which the first visceral arch is concerned, 98; transverse facial clefts, 98; median clefts of lower lip, lower Jaw, and tongue, 98.-Operations for Harelip, Cleft Palate, etc.: Operations for harelip, 98; operations for incomplete harelip, 100; operations for complete harelip, 102; operation for single complete harelip with cleft of alveoiar process and advancement of the intermaxillary bone, 104; operation for double harelip without a prominent advanced intermaxiliary bone, 104; operation for doubie hareifp with prominent advanced intermaxillary bone, 105; operation for cleft paiate, 
107.- Operations upon the Lips: Excision of whole lower llp, 111; restoration of lower lip, 112; Dieffenbach-Jaesche, 112; Bruns, 113; Langenbeck, 114; Estlander, 115; restoration of upper lip, 116; Estlander, 116; Dieffenbach's Wellenschnitt, 116; Bruns, 117.

\section{PART III.}

Neck and Tongue....................... 118-165

Surgical Anatomy of Neck: Deep cervical fascia, 118; back of the neck, 120; side of the neck, 120; anterior triangle, 122; posterior triangle, 122; sterno-mastoid region, 123; inferior carotid triangle, 123; superior carotid triangle, 125; submaxillary triangle, 126; lingual triangle, 127; occipital triangle, 127; subclavian triangle, 128; front of the neck, 129; hyoid bone, 130; suprahyoid region, 130; infrahyoid region, 130; laryngeal region, 134; thyroid gland, 134; suprasternal region, 135; blood-vessels of the neck, 136; common carotid artery, 136; internal carotid artery, 137; external carotid artery, 138; internal jugular vein, 139; subclavian artery, 140; inferior thyroid artery, 141; vertebral artery, 141.- Operations upon the Neck: Tracheotomy, 142; tampon of trachea, 142; high tracheotomy, 143; low tracheotomy,.144; median tracheotomy, 145; transverse laryngotomy, 146; thyrotomy, 146; laryngectomy, 148; extirpation of half of the larynx, 152; external œsophagotomy, 152; ligation of blood-vessels, 153; common carotid artery, 153; external carotid artery, 155; internai carotid artery, 156; subclavian artery, 156; lingual artery, 158.- Operations upon the Tongue: Amputation of tongue (Kocher), 159; amputation of tongue (Regnoli-Billroth), 161; extirpation of tongue through floor of mouth with division of lower jaw, 162; Sédillot, 162; Langenbeck, 163; Billroth, 164; extirpation of half of the tongue (Whitehead), 164.

\section{PART IV.}

Thorax ............................. 166-204

Surgical Anatomy of Thoracic Wall: Skeleton of thorax, 166; muscles of chest wall, 169; fasciæ of chest, 169; internal mammary artery, 170; diaphragm, 171._-Regions of Chest: Sternal region, 172; upper anterior pectoral region, 172; clavicular region, 173; infraclavicular region, 174; mammary region (breast), 175; lower anterior pectoral region, 177; lateral pectoral region, 177.- Mediastinum and Contents: Pericardium, 178; heart, 179; thymus, 182; arch of aorta, 182; pncumogastric nerves, 183; phrenic nerves, 184; trachea, 184; œsophagus, 185; thoracic aorta, 187; vena azygos, 187; vena hemiazygos, 188; thoracic duct, 188; innominate artery, 188; left common carotid and subclavian arteries, 188.—Pleura: Limits of pleura indicated by lines upon chest wall, 189; anterlor edge of pieura, 189; lower edge of pleura, 190; dome of pleura, 191.-Lungs: Root of lung, 192; lung, 193.- Operations upon the Chest: Incisions for abscess of breast, 194; extirpation of tumors (fibroids) from mammary gland, 195; amputation of breast, 195; amputation of breast (HalstedMeyer), 197; ligation of intercostal artery, 200; ligation of internal mammary artery, 200; thoracentesis, 201; thoracotomy, 201; thoracectomy (Estlander), 202; pleurectomy (Fowler), 203. 
Abdomen: Diaphragm, 205; posterior wall of the abdomen, 206; anterolateral wall of the abdomen, 207; superficial vessels of abdominal wall, 208; muscles of the antero-lateral wall, 208; fascia transversalis, 211; parietal peritoneum, 211; deep vessels of abdominal wall, 212; regions of the abdomen, 213.- The Back:, Muscles of the back, 215; erector spinæ muscle, 216; quadratus lumborum muscle, 217; lumbar fascia, 217; psoas and Hliacus muscles, 218; spinal column, etc., 218.- The Stomach: Surgical anatomy of the stomach, 221.-Operations upon the Stomach: Gastroplication, 226; gastrostomy, 228; Pyloroplasty (Heinecke and Mikulicz), 231; gastrostomy, 232; von Hacker, 232; Ssabanajew and Franck, 233; Witzel, 235; gastrorrhaphy, 237; pylorectomy, 237; Billroth, 237; Murphy button, 241; Kocher, 241; gastrectomy, 244.- The Small Intestine: Surgical anatomy of the small intestine, 247.- Operations upon the Small Intestine: Enterorrhaphy, 252; enterectomy, 254; end-to-end anastomosis, 257; suture, 257; Mounsell's, 259; Murphy button, 261; Laplace forceps, 264; O'Hara forceps, 266; side-to-side, lateral, approximation, 268; suture, 268; Murphy button, 271; McGraw rubber suture, 271; Laplace forceps, 272; O'Hara forceps, 272; gastro-enterostomy, 272; anterior (Woelfier), 272; posterior (von Hacker), 276; Murphy button, 280; McGraw's rubber suture, 281; Laplace forceps, 283; O'Hara forceps, 284.--Large Intestine and Vermiform Appendix: Surgical anatomy of the large intestine, etc., 285; cæcum, 285; vermiform appendix, 285; ascending colon, 287; transverse colon, 287; descending colon, 288; sigmoid flexure, 288; bloodsupply of large intestine, 288.- Operations upon Large Intestine: Colostomy, 289; Maydl, 292; resection of cæcum, 295; end-to-end anastomosis, 296; lateral anastomosis, 297; end-to-side, lateral, implantation, 298; ileo-colostomy, 298; resection of sigmoid flexure, 298.- Operations upon Vermiform Appendix: Appendicectomy, 299; McBurney incision, 300; Battle incision, 301; ligature wlthout inversion, 302; inversion and purse-strlng, 304; inversion (Edebohls), 305; for appendicular abscess, 306; for appendicltis accompanied with general peritoneal infection, 309 . -Liver and Gall-bladder: Surgical anatomy of liver, 310; surgical anatomy of gall-bladder, 312.- Operations upon Liver: Hepatotomy, 314; hepatectomy, 315; injuries of liver, 315.-Operations upon Gall-bladder: Aspiration of gall-bladder, 315; cholecystotomy, 315; cholecystostomy, 318; cholecystostomy in one sittlng, 318; cholecystostomy in two sittings, 320; cholecystostomy with adherent gall-bladder, 322; cholecystostomy when unable to draw gall-bladder up into incision, 322 ; cholecyst-enterostomy, 324; cholecysto-duodenostomy with Murphy button, 325. - Operatlons upon Gall-ducts: Choledochotomy, 326; choledocho-lithectomy, 326; choledocho-lithotripsy, 327; duodenotomy for impacted gall-stone, 327.The Spleen: Surgical anatomy of spleen, 328.-Operations upon Spleen: Splenotomy, 329; splenectomy, 330.-The Pancreas: Surgical anatomy of the pancreas, 331; operations upon the pancreas, 332.-Operations upon the Spinal Column: Laminectomy, 332; lumbar puncture, 334.

\section{PART VI.}

The Rectum.................................. $338-361$

Surgical anatomy of the rectum, 338; sacrum, 338; coccyx, 339; rectum, 339. - Operations upon the Rectum: Dilatation of the sphincter, 342; fistula in ano, 342; for complete fistula, 343; for Incomplete fistula, 344; hemorrhoids, 344; ligation and excislon, 345; clamp and cautery, 346; 
excision of part of rectal wall, 346; Innocent rectal polypi, 348; extirpation of rectum (Volkmann), 348; resection of rectum (Dleffenbach), 351; resection and amputation of rectum through sacral route (Kraske), 353; for resection of rectum, 353; for amputation of rectum, 361 .

\section{PART VII.}

Hernia, Spermatic Cord, Testes, etc.......... 362-402

Surglcal anatomy of groin, 362; superficial layer of superficial fascia, 362; lymphatic glands, 363 ; deep layer of superficial fascia, 363; Inguinal region, 363; descent of the testes, 369; femoral region, 372; study of inguinal and femoral region from within the abdomen, 375 ; Inguinal region, 375; femoral region, 378.- Operations for Hernia: Herniotomy, 380; for inguinal hernla (Bassini), 383; for inguinal hernia (Halsted), 389; for femoral hernia, 392.- Spermatic Cord, Scrotum, etc.: Spermatic cord, 393; scrotum, 394; testes, 394; ejaculatory ducts, 395.- Operations upon Spermatic Cord, Scrotum, etc.: For varicocele, 395; for hydrocele, 397; castration, 401.

\section{PART VIII.}

UrinaRY System...................... 403-436

Kidneys: Surgical anatomy of kidney, 403.—Operations upon the Kldney: Nephropexy, 404; nephropexy (Edebohls), 407; nephrotomy, 410; nephrolithotomy, 411; nephrectomy, 411; decortication of kidney (Edebohls), 412.-Bladder: Surgical anatomy of bladder, 413.-Operations upon Bladder: Suprapubic cystotomy, 415; puncture of bladder, 418. Penis: Surgical anatomy of the penis, 418.- Operations upon the Penis: Forcible dilatation of prepuce, 419; dorsal section, 419; circumcision, 421; circumcision with clamp, 422; amputation of penis, 422.- Perineum and Ischio-rectal Region: Floor of pelvis from without, 424; ischlo-rectal region, 425; perineum, 426; pelvic cavity from withln, 428.-Operations upon Perineum, etc.: Perineal section with a guide, 430; perineal section without a guide, 431; median lithotomy, 432; lateral lithotomy, 433; Prostatectomy (McGill-Fuller), 433; prostatectomy (Alexander), 434; prostatotomy (Bottinl), 435.

PART IX.

The Upper Extremity $\ldots \ldots \ldots \ldots \ldots \ldots \ldots \ldots \ldots \ldots .437-481$

The Axilla: The axilla, 437; the axillary artery, 437.- The Arm: Vessels of the arm, 440; the brachial artery, 440; the radial artery, 442; the ulnar artery, 443; musculo-spiral nerve, 445; median nerve, 445; ulnar nerve, 445. The Hand: Nerve-supply of the hand, 446; ligations, 446; axillary, 446; brachial, 447; radial, 449; ulnar, 449.-Amputations, Resections, etc.: Surgical anatomy of hand, 449; phaiango-phalangeal joints, 449; metacarpo-phalangeal joints, 450; exarticulation of the finger at the phalango-phalangeal joint, 450; exarticulation of finger at the metacarpophalangeal joint, 452; exarticulation of hand at the carpo-metacarpal articulation, 453; surgical anatomy of wrist-joint, 455; exarticulation of hand at wrist-joint, 456; amputation through forearm, 457; surgical anatomy of elbow-joint, 458; exarticulation of forearm at eibow-joint, 460; 
amputation of arm, 461; surglcal anatomy of sboulder-joint, 463; exarticulation at shoulder-joint (Spence), 465; exarticulation at shoulderfolnt (Esmarch), 467; exartlculation at shoulder-joint with deltoid flap, 469.-Resections: Wrist-joint, 472; elbow (Langenbeck), 474; shoulder, 476; tendon suture, 479; nerve suture, 479; intravenous saline infusion, 480 .

\section{PART X.}

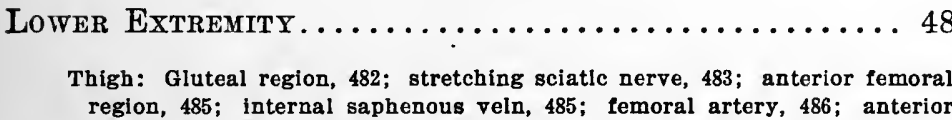
crural nerve, 489; ligation of femoral artery, 489; popliteal space, 491. - Leg: Anterior tiblal artery, 492; anterior tibial nerve, 494; ligation of anterior tibial artery, 494; posterlor tlblal artery, 494; posterlor tibial nerve, 496; ligation of posterior tibial artery, 496; tenotomy, 496; multiple ligature of veins of leg, 497.- Amputations, Resections, etc.: Surglcal anatomy of skeleton of toot, 498; exarticulation of big toe, 500; exarticulation of big toe with removal of first metatarsal, 500; exarticulation of little toe, 500; for Ingrowing toe-nail, 501; amputation through tarsometatsrsal articulation (Lisfranc), 501; smputstion through medio-tarsal articuiation (Chopart), 504; surgical anatomy of ankle-joint, 505; exarticulation of foot at ankle-foint (Syme), 506; exarticuiation of foot, etc. (Pirogoff), 507; amputation of leg, 510; amputation of leg with latersl hooded flaps, 510; surgical anatomy of knee-joint, 513; exarticulation of leg at knee-joint (Stephen Smith), 516; transcondylar amputation (Carden), 518; amputation of knee (Gritti-Stokes), 520; amputation of thigh, 521; surgical anatomy of hip-joint, 523; exarticulation of thigh at hip (Wyeth), 526; exarticulation of thigh with preliminary ligation of common femoral, 529. - Resections: Ankle (Langenbeck-Hueter), 529; ankle (Koenig), 533; ankle (Lauenstein), 535; ankle, osteoplastic (Mikulicz-Wladimirow), 536; knee-joint, 538; hip-jolnt (Langenbeck), 543; osteotomy, 547; suture of patella, 548. 



\section{LIST OF ILLUSTRATIONS.}

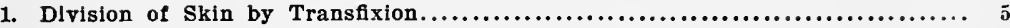

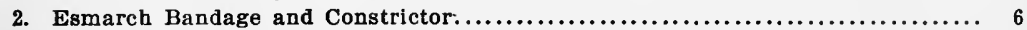

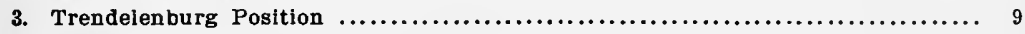

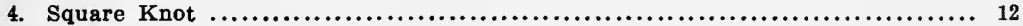

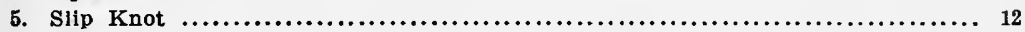

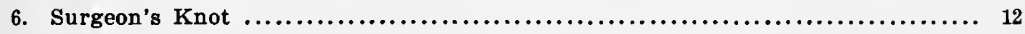

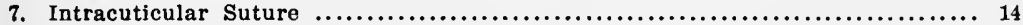

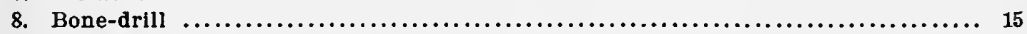

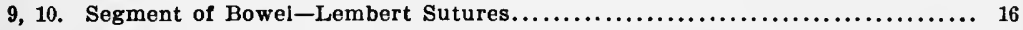

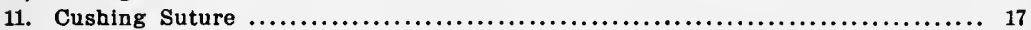

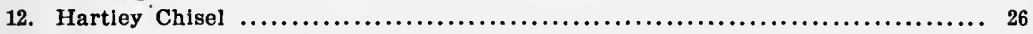

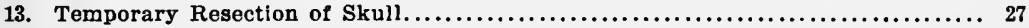

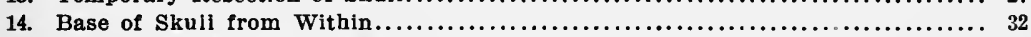

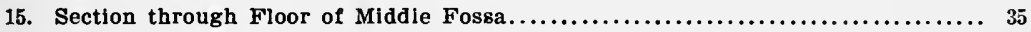

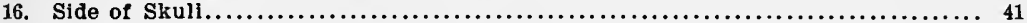

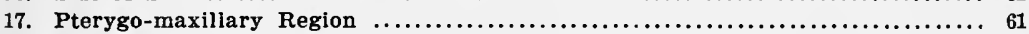

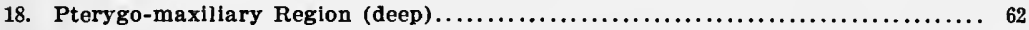

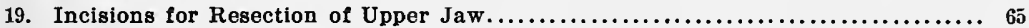

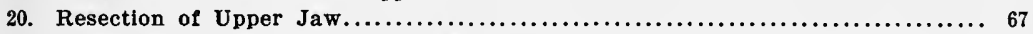

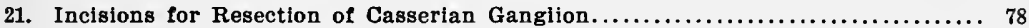

22. Transverse Section of Head End of Embryo Tweive Days Old ................ 81

23. Sagittal Section of Head End of Embryo Tweive Days Oid.................. 81

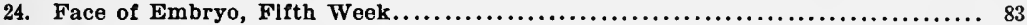

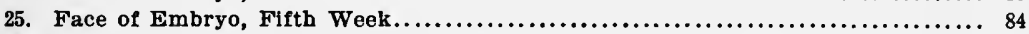

26. Embryo, Fourth Week, Seen from Side.............................. 85

27. Embryo, Eighth Week, Seen from Side............................... 86

28. Face of Embryo about Eighth Week................................... 88

29. Diagram of Congenital Facial Clefts................................... 90

30. Double Complete Harelip .......................................... 92

31. Harelip with Advanced Intermaxiliary Portion............................ 93

32. Double Cleft Palate with Advanced Intermaxillary Portion.................. 94

33. Oblique Facial Cleft..................................................... 96

34. Incomplete Oblique Facial Cleft..................................... 97

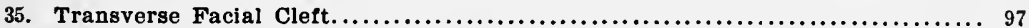

36, 37. Simple Paring for Incomplete Harelip.............................. 100

38, 39. Von Graefe Operation for Incomplete Harelip.......................... 100

40, 41, 42. Nélaton Operation for Incomplete Harelip.......................... 101

43, 44, 45. Malgaigne Operation for Incomplete Harelip....................... 101

46, 47, 48. Mirault Operation for Incomplete Harelip.......................... 102

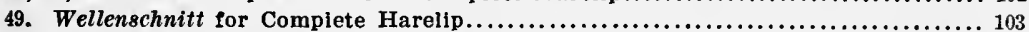

$50,51,52$. Hagedorn Operation for Single Complete Harellp.................... 103

53, 54, 55. Double Malgaigne Operation for Double Complete Harelip............... 105

56, 57, 58. Hagedorn Operation for Complete Double Harelip..................... 105

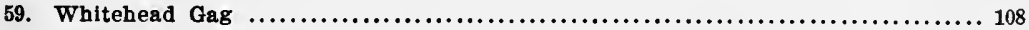

60. Repair of Cleft Palate.................................................. 111

61. Exclsion of Entire Lower Lip............................................. 112

62. Triangular Defect in Lower Lip Closed............................... 112

63. Dleffenbach-Jaesche Operation for Restoring Lower Lip................... 113

64, 65. Bruns Method of Restoring Lower Lip.............................. 114 
66, 67. Langenbeck Method of Restorlng Lower Llp........................... 114

68, 69. Estlander Method of Restorlng Lower Lip............................ 115

70, 71. Dieffenbach Wellenschnitt for Restoration of Upper Llp................ 116

72, 73. Bruns Method of Restoring Upper Llp.............................. 116

74. Section through Neck..................................................... 118

75. Side of Neck to Show Triangles........................................ 124

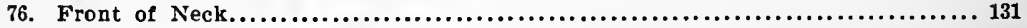

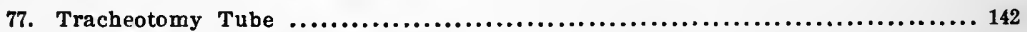

78. Trendelenburg Tampon Cannula........................................... 142

79. Incision for Removal of Lower Jaw, etc................................ 154

80. Transverse Section through Thorax.......................................... 178

81. Outline of Heart, etc................................................ 181

$82,83,84$. Outline of Pleura, etc............................................ 190

85. Section through Seventh, Eighth, and Ninth Ribs....................... 191

86. Amputation of Breast.................................................... 198

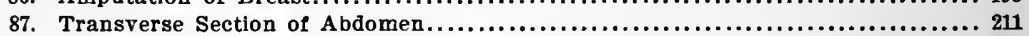

88. Transverse Section of Abdomen......................................... 211

89. Regions of Abdomen.................................................. 213

90. Sagittal Section to Show Arrangement of Greater and Lesser Omenta.......... 223

91. Incisions to Reach Abdominal Viscera.................................... 225

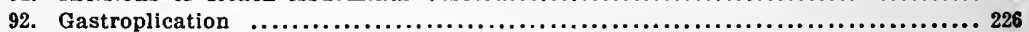

93. Cross Section of Stomach after Gastroplication............................. 227

94. Cross Section of Stomach after Gaștroplication............................. 227

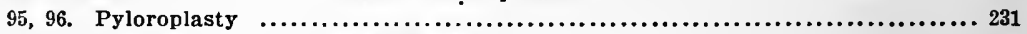

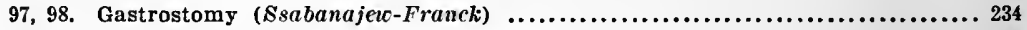

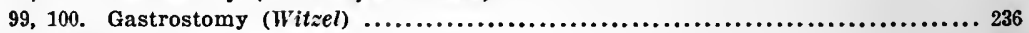

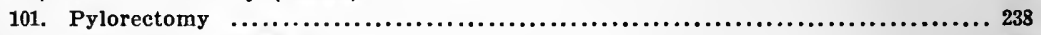

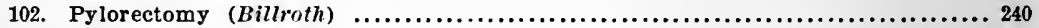

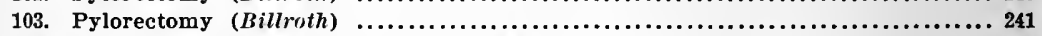

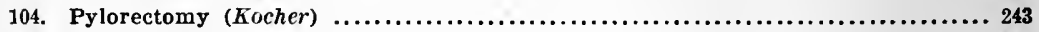

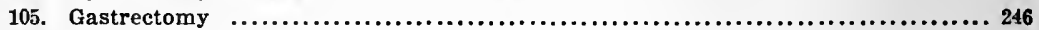

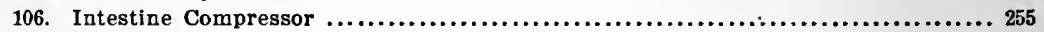

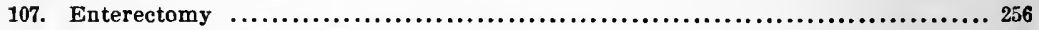

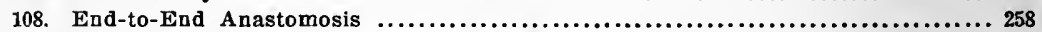

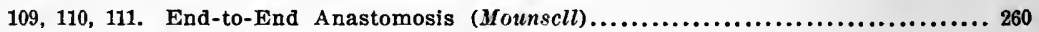

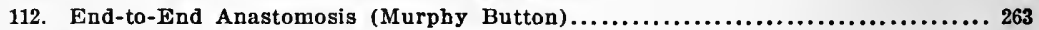

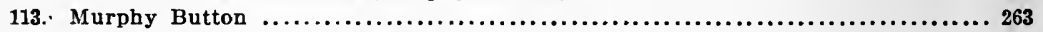

$114,115,116$. Laplace Anastomosis Forceps.................................. 265

117, 118, 119. O'Hara Anastomosis Forceps.................................... 267

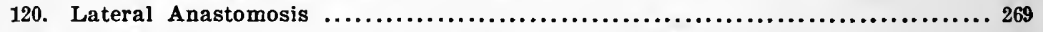

121. Cross Section of the Apposed Coils of Gut (Lateral Anastomosis)............. 270

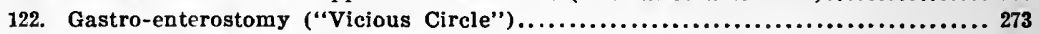

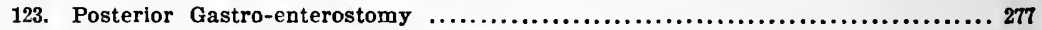

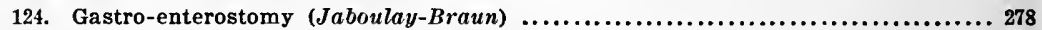

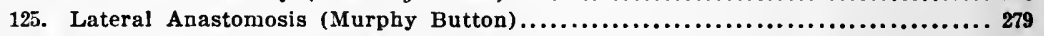

126. Gastro-enterostomy (McGraw Rubber Suture)............................ 282

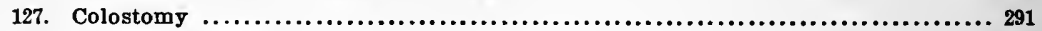

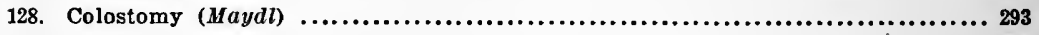

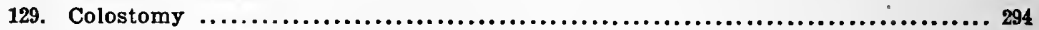

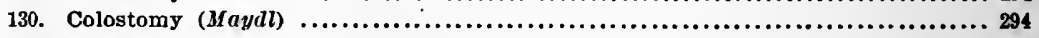

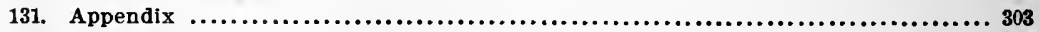

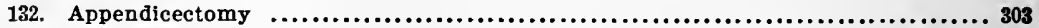

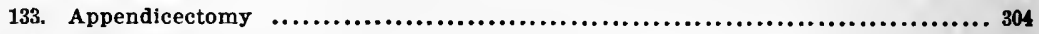

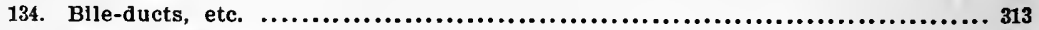

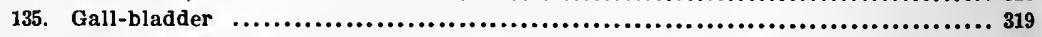

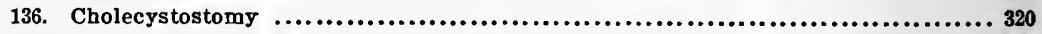

137. Keen Bone Forceps..................................................... 333

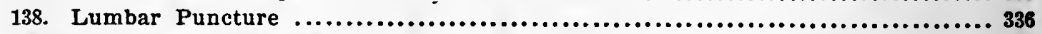

139. Complete Flstula In Ano................................................ 343 


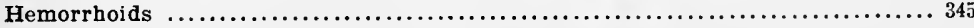

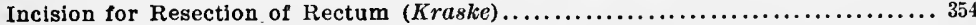

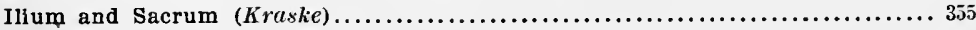

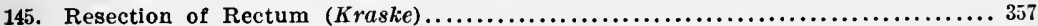

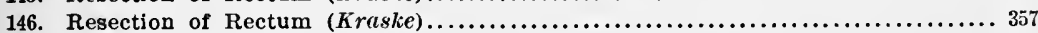

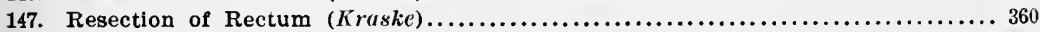

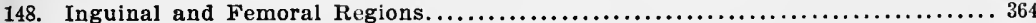

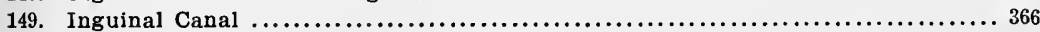

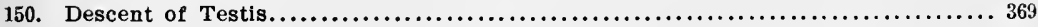

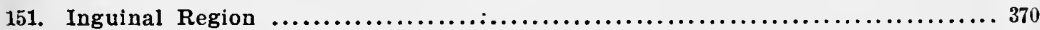

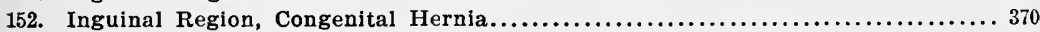

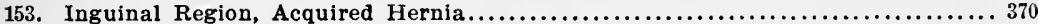

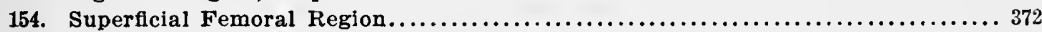

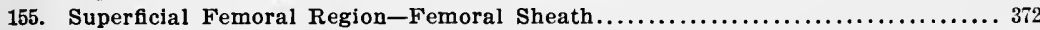

156. Pelvis and Ligaments of Illo-pubic (or Femoral ?) Region...................37

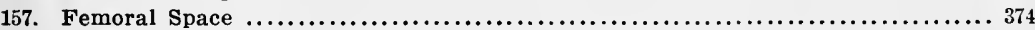

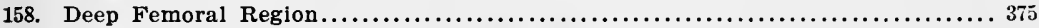

159. Inguinal and Femoral Regions from Within Abdomen ...................

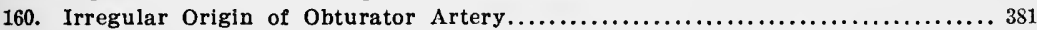

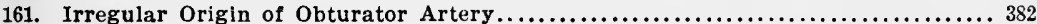

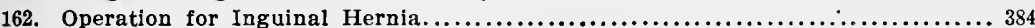

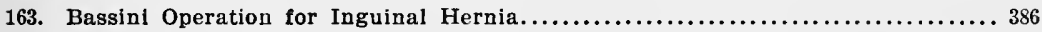

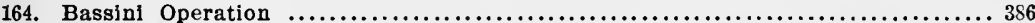

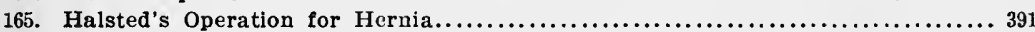

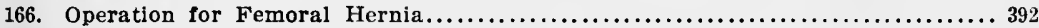

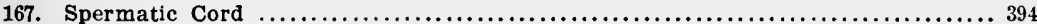

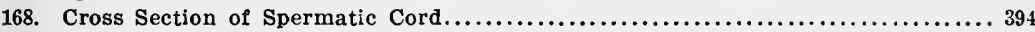

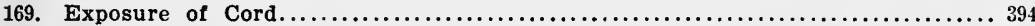

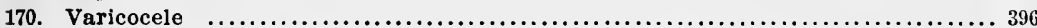

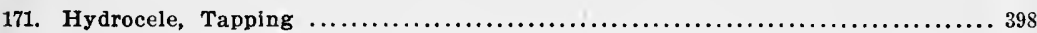

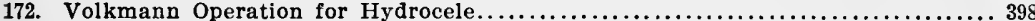

173. Hydrocele, Retroversion of Tunica Vaginalis. .....................

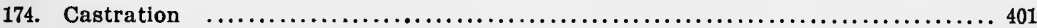

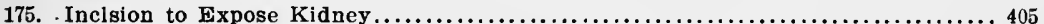

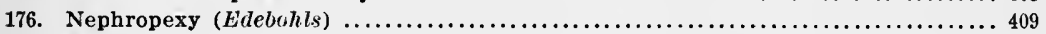

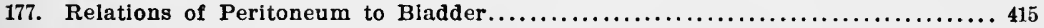

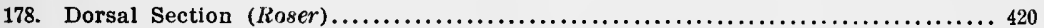

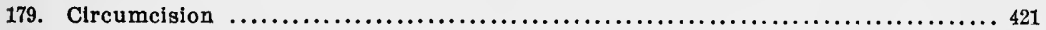

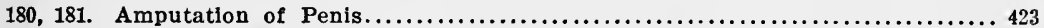

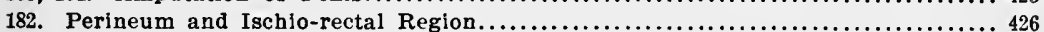

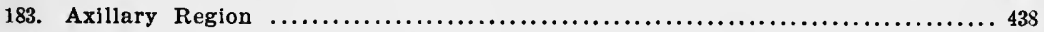

184. Section through Middle of Right Arm $\ldots \ldots \ldots \ldots \ldots \ldots \ldots \ldots \ldots \ldots \ldots \ldots \ldots \ldots \ldots \ldots \ldots$

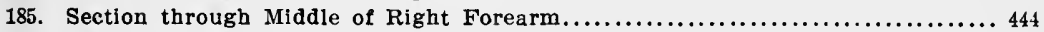

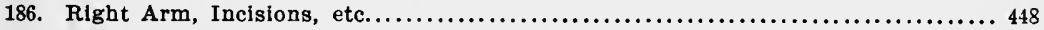

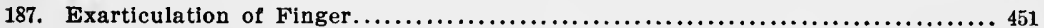

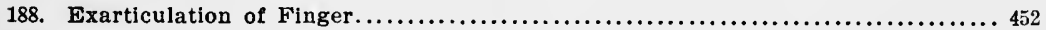

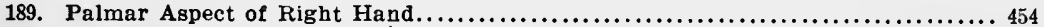

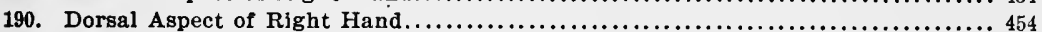

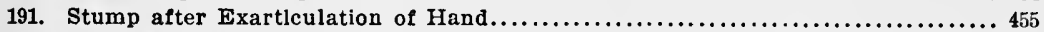

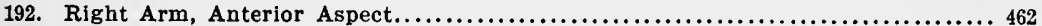

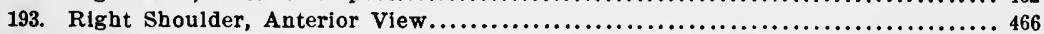

194. Right Shoulder, Posterior Vlew $\ldots \ldots \ldots \ldots \ldots \ldots \ldots \ldots \ldots \ldots \ldots \ldots \ldots \ldots \ldots \ldots \ldots \ldots \ldots \ldots \ldots$

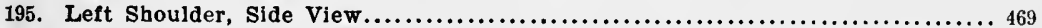

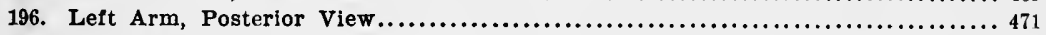

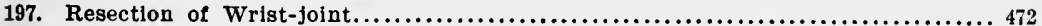

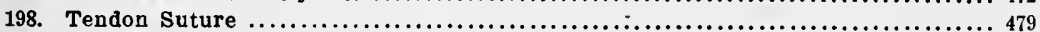

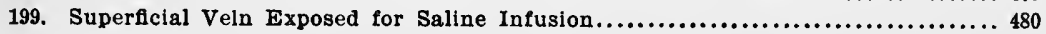




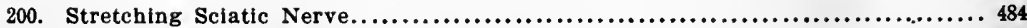

201. Section through the Middle of the Left Thigh................................. 487

202. Ligation of Femoral Artery......................................... 490

203. Section through the Middle of the Left Leg............................... 493

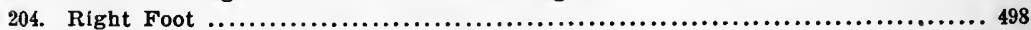

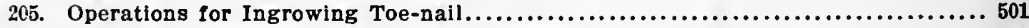

206. Right Foot, Inner Side................................................ 503

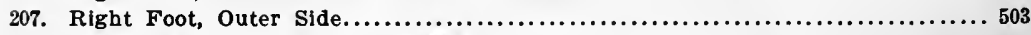

208. Right Foot, Inner Side (Pirogoff's Amputation) $\ldots \ldots \ldots \ldots \ldots \ldots \ldots \ldots \ldots \ldots \ldots \ldots \ldots, 508$

209. Right Foot, Inner Side (Günther's Modiflcation) $\ldots \ldots \ldots \ldots \ldots \ldots \ldots \ldots \ldots \ldots \ldots \ldots \ldots$

210. Right Foot, Inner Side (le Fort's Modification) $\ldots \ldots \ldots \ldots \ldots \ldots \ldots \ldots \ldots \ldots \ldots \ldots \ldots, 508$

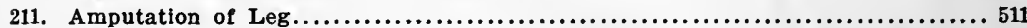

212. Right Leg, Outer Side............................................ 517

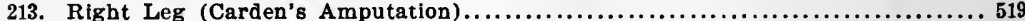

214. Stump after Carden's Amputation.................................... 519

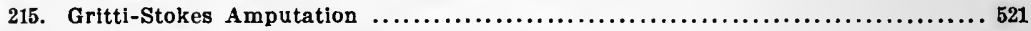

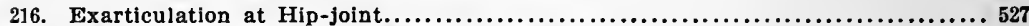

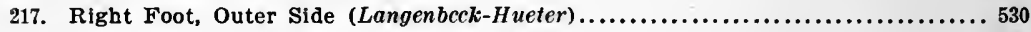

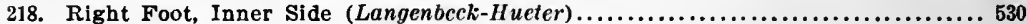

219. Incisions for Resection of Ankle (Koenig) and for Amputation of Big Toe with Removal of the First Metatarsal...................................... 533

220. Resection of Ankle-joint (Lauenstein's Incision) ......................... 536

221. Incision for Mikulicz-Wladimirow Osteopiastic Resection of the Ankle-jolnt..... 537

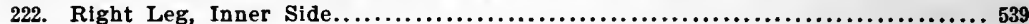

223. Resection of Knee-foint.............................................. 541

224. Resection of Hip (Langenbeck's Incision) $\ldots \ldots \ldots \ldots \ldots \ldots \ldots \ldots \ldots \ldots \ldots \ldots \ldots \ldots, 544$

225. Resection of Hip (Anthony White's Incision) $\ldots \ldots \ldots \ldots \ldots \ldots \ldots \ldots \ldots \ldots \ldots \ldots \ldots \ldots, 545$

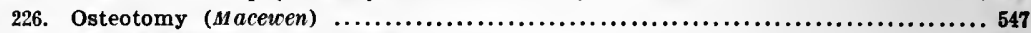

227. Wiring Patella for Fracture.............................................. 549 


\section{PART I.}

\section{GENERAL CONSIDERATIONS}

\section{ANAESTHESIA.}

General Anæsthesia. - Of the general anæsthetics, ether and chloroform are the ones most commonly employed. Ether is used more generally than chloroform, especially in this part of the United States. With ether, the stage of excitement and struggling that precedes the stage of anæsthesia is more prolonged and more pronounced than with chloroform; still, this objection may be partially eliminated by administering nitrous oxide or chloroform until the period of excitement has been passed. The preliminary use of nitrous oxide is much in vogue at present.

Ether stimulates the heart and increases the arterial tension. It has a marked congestive influence upon the kidneys, and acts as an irritant to the respiratory tract.

The first stage of chloroform narcosis is shorter than is that of ether and is not accompanied by as much excitement and struggling. Chloroform does not increase the arterial tension and does not congest the kidneys, but it has a tendency to interfere with the heart-action, especially if the heart-muscle is diseased and in conditions accompanied by chronic anæmia. Therefore, if it become a matter of choice, one should elect ether if the heart-action is unsatisfactory or if the patient is markedly anæmic, and chloroform if the urine shows defective kidneys or if there is a tendency to cerebral apoplexy or pulmonary disease and in cases of empyema. Chloroform is also preferable to ether in young children and in very old people. Alcoholics, as a rule, take chloroform much more satisfactorily than ether.

In operations about the mouth where the mask can only be applied during intervals, and for administration through a tracheotomy tube, chloroform is the preferable anæsthetic.

Incomplete General Anæsthesia.-This plan consists in administering a liberal dose of morphin hypodermically, shortly before commencing the operation, and then giving the chloroform only up 
to the point of deadening the sensation without nullifying the reflexes. In this way the pain is made endurable and at the same time, the reflexes being still active, the patient is able to cough, clear the throat, and expectorate. This plan may be practiced with satisfaction in operations about the upper and lower jaw, nasal passages, larynx, etc., where there is danger of blood entering the respiratory canal and asphyxiating the patient if not coughed out.

Local Anæsthesia.-The skin may be anæsthetized sufficiently for simple incision or puncture by freezing, either by the application of ice, chopped and mixed with salt, in a bag, or by the ethylchloride spray.

Ethyl chloride is a very volatile substance, boiling at the bodytemperature. It is supplied in glass cylinders with a removable brass cap. If the cylinder is held in the hand for a few moments sufficient heat is imparted to volatilize the fluid in the cylinder, which then escapes in the form of a fine spray. The spray is directed against the part to be anæsthetized for a few minutes.

For operations that require a certain amount of dissection cocain in a 2-per-cent. solution, introduced into the skin, hypodermically, is more satisfactory. The cocain is still more effective if it can be confined to the part that is to be operated upon by constricting it with a rubber elastic ligature; for example, in operations upon the fingers and toes and for circumcision, etc., by placing an elastic ligature about the root of the part. The solution should be thrown into the deeper layer of the skin proper so as to raise welts, and not into the loose tissue underneath the skin.

The solution is introduced, a few drops at a time, through several punctures along the line of the proposed incision.

After the first puncture and injection have been made, the needle should be introduced each succeeding time through the skin that has already been anæsthetized; ordinarily from 20 to 30 minims of a 2-per-cent. solution, according to the age, etc., of the patient, may be introduced during the course of a single operation.

Occasionally disagreeable symptoms of cardiac disturbances due to the action of the cocain present themselves, especially if it has been introduced into a part where the circulation cannot be interrupted with a constricting ligature. Mucous surfaces may be anæsthetized by applying a wad of cotton saturated with the cocain solution direct to the part for several minutes.

Eucain is used as a substitute for cocain; it is said to have no 
depressing influence upon the heart. From 20 to 40 minims of a 2-per-cent. solution may be used.

Schleich Infiltration MethoD.-The solution used contains cocain and morphin. It is thrown into the skin with a hypodermic, as described above for cocain, along the course of the intended incision. The solutions vary in strength according to the amount of cocain that they contain, and are known as Nos. 1, 2, and 3.

Solution No. 1.

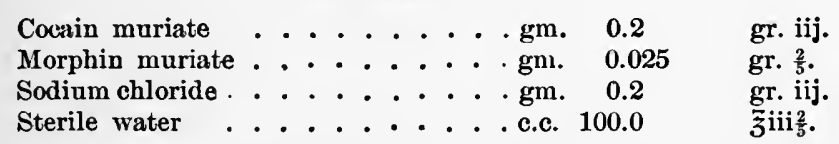

This is the strongest solution. A quantity up to 6 drams may be used.

Solution No. 2.

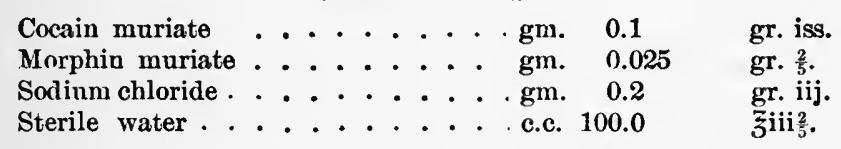

This is the solution that is commonly used, and of this a quantity up to 3 ounces may be injected.

Solution No. 3.

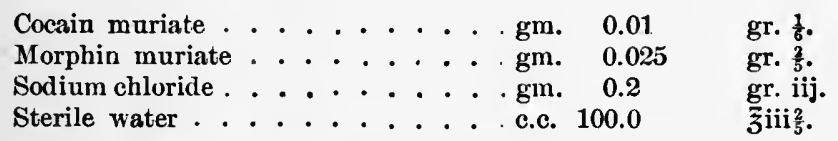

No. 3 is the weakest solution, containing only one-tenth as much cocain as No. 2 .

One may use a pint of this solution.

Analgesia by Subarachnoid Injection of Cocain, etc.-A solution of cocain, eucain, etc., may be thrown into the subarachnoid space with a hypodermic syringe. This method of inducing analgesia was introduced by Bier and has been recently practiced by numerous surgeons with varying degrees of satisfaction, some discarding it after a few trials and others advocating its usefulness. No doubt it will prove of value in certain cases. The method of introducing the fluid is described elsewhere (see "Lumbar Puncture").

A 1- or 2-per-cent. solution of cocain, eucain, or tropacocain may be used. 
If the cocain solution is sterilized by boiling, the potency of the drug is very much impaired. We may thus account for some of the instances where the method has failed to give satisfaction. If cocain, for example, is used, $1 / 2$ grain or less of the crystals of hydrochlorate of cocain is placed in a sterile glass vessel and 1 or 2 drams of ether poured in; this is stirred with a sterile glass rod until the ether evaporates, when the residue is dissolved with sterilized distilled water (Bainbridge). The solution is then ready for injection.

According to the method of Dudley, the cocain may be sterilized by adding a few drops of chloroform to the crystals of the drug. After the chloroform evaporates the residue is dissolved in sterile water.

Three to seven minutes usually elapse before the analgesia reaches the level of the diaphragm. The lower limbs and the lower part of the trunk first show the analgesic effect of the drug, and this gradually extends to the chest and upper extremities.

The analgesic effect lasts from fifteen minutes to several hours.

The method may be applicable in those cases where extreme weakness or cardiac or renal disease renders the use of chloroform or ether especially dangerous.

\section{DIVISION OF THE TISSUES.}

Division of the Soft Parts. Bloody Division of THE SofT Parts.-The division of the integument may be accomplished with the knife or scissors, either by direct incision or by transfixion (Fig. 1). The deeper soft parts may be divided with cutting instruments or by tearing with the fingers or blunt instruments, the handle of the scalpel, thumb forceps, etc. This plan of blunt dissection is especially serviceable in enucleating encapsulated tumors or lymphatic nodes and in separating between different layers of tissue along the normal connective-tissue planes.

The contents of hollow viscera, serous spaces, and cystic tumors may be evacuated or withdrawn in part for the purpose of diagnosis by means of the trocar and cannula or some form of aspirating apparatus. Substances may also be introduced into the body through cannulæ or with some form of syringe.

Bloodless Division of the Soft Parts.-This may be accomplished with the thermocautery, galvanocautery, elastic ligature, écraseur, or wire snare and by the action of corroding chemicals. 
Division of Bone.-Bones may be divided through an incision in the soft parts with the chisel and mallet, bone forceps, or with some form of saw,-circular, chain, or wire, or with the flat saw; with the drill, dental burr, or bone scoop.

The bones are covered with an adherent vascular membrane, the periosteum, which should be incised with the knife and separated from the bone with the elevator before applying the cutting instruments to the bone.

The bone may be divided without an incision in the soft parts -for the purpose of correcting deformities, etc.-either by manual force or by the use of an instrument known as the osteoclast. The osteoclast consists of a solid metal bar with two sliding bracelets

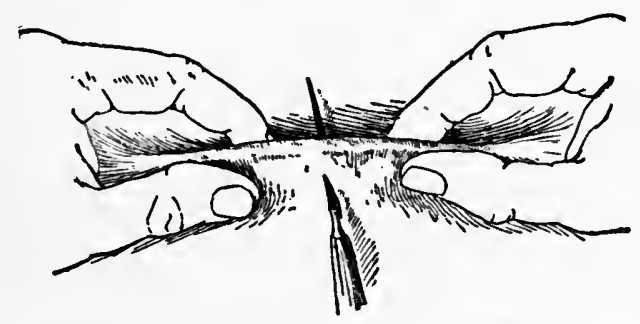

Fig. 1.-Division of the Skin by Transfixion.

one on either end and between these a brace by which the breaking force is applied and which may be raised or lowered by means of a screw.

\section{HEMORRHAGE.}

During the course of an operation the hemorrhage must be controlled in order to minimize the loss to the patient and to keep the field clear for proper work.

Hemorrhage may be described as capillary, venous, and arterial.

Capillary hemorrhage is characterized by a general oozing.

Venous hemorrhage is characterized by a steady welling of blood into the wound, often filling it so as to obscure the bleeding point. Venous blood is rather darker in color than arterial blood. If a large vein is divided close to the trunk,-i.e., in the neck or axilla,-or if one of the intracranial dura mater sinuses is opened, the blood may escape in a remittent stream, synchronous with the respiratory movements, diminishing or ceasing during inspiration and increasing during expiration. During inspiration, under these 
circumstances, air may be sucked into the reins, but, if limited in quantity, this is said to do no harm; nevertheless it should be guarded against.

Arterial hemorrhage is characterized by the brighter color of the blood and by the fact that it escapes in a distinct remittent jet of considerable, though varying, force. The jet is synchronous with the heart's action, increasing during ventricular systole and diminishing during ventricular diastole.

Means to Arrest Hemorrhage. The Natural Arrest or HEMORRHAGE is effected by the clotting of the blood. If the divided vessels are not too large and the blood-pressure not too great, nature will thus be able to bring about a cessation of the hemorrhage. Nature is assisted in her efforts to control hemorrhage from a severed artery by the fact that when an artery is divided its orifice contracts, thus diminishing the size of the opening through
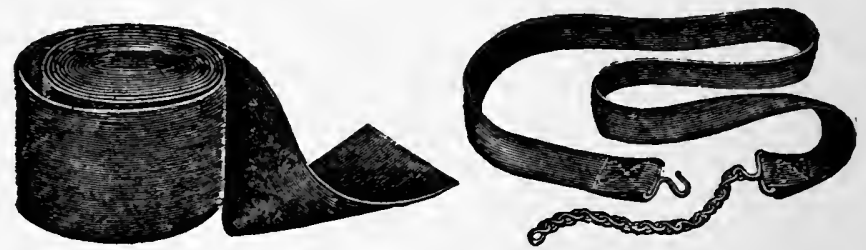

Fig. 2.-Esmarch Bandage and Constrictor. The constrictor is provided with a chain and hook.

which the blood escapes, and further by the fact that the inner elastic coat of the ressel, the intima, retracts, coiling up within the artery, thus blocking the lumen of the vessel and offering a considerable impediment to the flow.

The natural arrest of hemorrhage from a severed vein is facilitated by the low blood-pressure within the ressel and by the collapsibility of its thin, flaccid wall.

Artificial Arrest of Hemorrhage.-Artificial measures are resorted to, as a rule, to control hemorrhage. These may be classified as indirect means, acting outside at a distance from the wound, and direct means, acting locally within the wound.

Indirect Means. The Elastic Bandage and Constrictor (Esmarch).-Operations upon the extremities may be rendered practically bloodless by the use of the Esmarch bandage and constrictor. 
The extremity being elevated, a rubber bandage about three inches broad is applied about the limb, each furn being drawn pretty tight. The bandage is applied spirally about the limb, commencing below and working upward toward the trunk, each turn somewhat overlapping its predecessor; in this way the blood is forced out of the limb. Having reached a point above the site of the proposed operation, a rubber band or thick elastic tube, the constrictor, is passed around the limb several times and then made fast. The rubber spiral bandage may then be removed.

In most cases the application of the rubber spiral bandage may be dispensed with, it being sufficient to elevate the limb to a perpendicular position for a few minutes, at the same time massaging or stripping it from the periphery toward the trunk, in order to force the bulk of the blood out of it. While the limb is thus elevated, the rubber constrictor bandage or tube is applied about the upper part of the limb.

In cases of tuberculous disease, malignant disease, and sepsis one should certainly omit stripping the limb or applying the rubber spiral bandage on account of the likelihood of forcing infectious elements onward into the healthy tissues. Under these circumstances one should be content with elevation of the limb for a few minutes before applying the constrictor.

The rubber constrictor that is placed about the limb may be secured with a band of gauze which is placed underneath the constrictor so that after the first loop of an ordinary knot has been taken in the constrictor the gauze bandage may be tied over this to secure it and prevent it from slipping; the second and final loop is then taken in the rubber constrictor. The constrictor shown in the illustration is provided with a chain and hook.

The constrictor should be applied sufficiently tight to shut off the arterial current, but not tight enough to bruise the nerve-trunks against the underlying bone. The constrictor may be left on for two or three hours without any untoward results.

For operations upon the lower extremity, except at the hip-joint, the constrictor is placed about the thigh, just above the knee-joint or higher up, nearer the hip-joint. For disarticulation at the hipjoint the constrictor is placed about the limb as high up, near the trunk, as possible, and it is then prevented from slipping down by steel pins, or skewers, which are passed through the soft parts (Wyeth). 
For operations upon the upper extremity, except at the shoulderjoint, the ligature is placed about the arm, just above the elbowjoint or higher up nearer the shoulder-joint. For disarticulation at the shoulder-joint the constrictor is applied as high up as possible; it may be passed through the axilla and over the shoulder and prevented from slipping by a steel pin, or skewer, that is thrust through the soft parts, transfixing the upper part of the deltoid muscle mass.

The main arterial and venous trunks, if they have been divided during the course of the operation, may be secured and ligated before the constrictor is removed. Any additional bleeding branches may be secured and ligated after the constrictor has been removed.

By Digital Compression of the Main Arterial Trunk at a Distance from the Site of the Operation.-During amputation of the thigh the common femoral artery, as it emerges from under Poupart's ligament, may be compressed against the underlying pubic bone.

During amputation of the forearm or disarticulation at the elbow-joint the brachial may be compressed against the humerus, and during amputation through the upper arm or at the shoulderjoint the hemorrhage may be controlled by digital compression of the subclavian artery against the first rib. This plan is rather untrustworthy.

Preliminary Ligation in Continuity.-This is a very satisfactory method of controlling hemorrhage in certain bloody operations. For example, in disarticulation at the hip-joint preliminary ligation of the common femoral may be practiced, the vein being tied at the same time through the same incision. In amputation of the tongue one or both linguals may be ligated as a preliminary step to the main procedure. In extirpation of the lower jaw, etc., preliminary ligation of the external carotid may be practiced with great advantage.

Position.-Position of the part has much to do with the severity of the hemorrhage during an operation. Elevation of the part is often sufficient, of itself, to check capillary and venous hemorrhage. The volume of arterial blood sent to the part is diminished and the return-flow through the veins is facilitated. These factors, together, serve to markedly diminish the pressure in all the vessels of the elevated part. This is especially true of the limbs, but also of the pelvis and the head. With the pelvis raised as in the Trendelenburg position, the hemorrhage during the course of operations upon the 
pelvic organs is much diminished. During operations upon the head and face, with the patient in the semi-erect position, the hemorrhage, especially the venous, will be found to be very much less than it would be with the patient in the Rose position, with the head hanging low over the end of the table.

Direct Means of Controling Hemorrhage are applied within the wound itself, and these may be divided into three groups: Agents that act locally through the nervous system; chemical agents that act directly upon the escaping blood, causing it to coagulate; and mechanical agents.

Agents that Act Locally through the Nervous System.-Application of heat or cold, usually in the form of water, hot or cold, or ice,

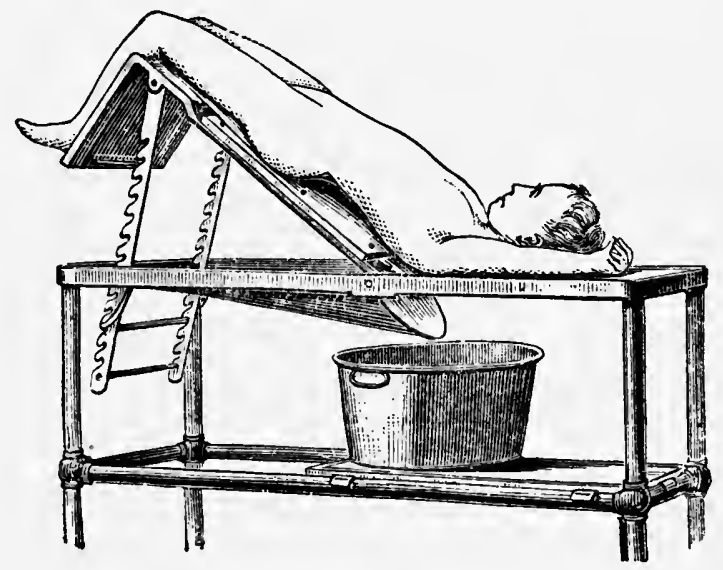

Fig. 3.-Trendelenburg Position.

tends to diminish and check hemorrhage. If hot water is used it should be as hot as the hand can bear, about $120^{\circ} \mathrm{F}$.; if cold, it should be quite cold.

Heat and cold both act by causing the small arterioles to contract and diminish in size. Heat causes albumin to coagulate so that, when heat is applied to a wound, the wound surface becomes glazed with a thin, albuminous film, and in this way heat possesses an additional potency in checking oozing. Heat is a more effective agent in controlling hemorrhage than cold, since the latter acts only by causing a diminution in caliber of the small arteries.

Heat in the form of a hot saline irrigation is a very satisfactory agent to check oozing from capillaries and small arteries and veins. 
Chemical Agents. Styptics.-These agents tend to check hemorrhage by acting directly upon the escaping blood, causing it to coagulate, and thus seal the mouths of the severed vessels. They are but little used except in operations upon the nose, etc., and are of service only to control capillary hemorrhage and oozing from small reins and arteries. The common styptics are the persulphate of iron, tincture of the chloride of iron, powdered alum, tannic acid, extract of suprarenal capsule, etc.

The styptic cotton is ordinary absorbent cotton impregnated with one of these agents.

Mechanical Means. Digital Compression. - With the finger in the wound hemorrhage may be controlled by pressure exerted directly upon a severed vessel, thus closing it until it can be secured with an artery forceps. In operations upon the neck, for example, a large vessel may be divided and then so obscured by the great volume of escaping blood that it cannot be located and secured with the artery forceps. With the finger thrust into the wound the hemorrhage may be checked temporarily by compressing the injured vessel until the wound can be cleared of blood and the vessel located and grasped with an artery clamp. This is especially true of large veins; when cut, the blood may well into the wound in such volume that one is unable to locate the divided vessel.

Digital compression may be applied to the main ressels in the wound before they are divided in order to minimize the loss of blood. For example, in exarticulating at the shoulder-joint, after the incisions have been made, but before the brachial artery and adjoining vessels have been cut, the assistant grasps the mass of soft parts which includes the main vascular trunks and compresses these between the thumb and fingers until after the limb has been amputated and the vessels secured by the operator.

Tamponade.-This is really one way of applying the principle of compression. This method is especially serviceable in controlling oozing and bleeding from veins. For example, hemorrhage from an injured intracranial sinus may be readily controlled by packing a strand of gauze into the wound between the sinus and the skull.

If a wound is tamponed and a good snug dressing applied so as to exert a considerable degree of firm compression, this will usually suffice to check all oozing from capillaries and small veins.

Bleeding from the nutrient artery of a bone may be checked by plugging the orifice of the nutrient canal with a piece of catgut 
or a wooden peg. Oozing from the end of a long bone, from the edges of the bones of the skull in craniectomy, etc., is readily controlled by a few minutes' firm compression with a hot gauze pad.

Suture of the Wound controls hemorrhage from capillaries and small veins by bringing the contiguous surfaces into apposition, and is simply one method of applying the principle of compression.

Forcipressure consists in crushing the coats of the severed vessels with hæmostatic forceps. It is a well-known fact that even large arteries when crushed or torn do not bleed, and it is upon this same principle that forcipressure is applied to control hemorrhage.

The bleeding artery or vein is seized with the forceps, which is then closed down upon the vessel with much force, in this way crushing the coats of the vessel, especially the inner coat and so effectually controlling the hemorrhage. If the vessels are small the forceps may be removed after a few minutes, when it will be found that the hemorrhage has ceased. Forcipressure is a very satisfactory method of dealing with larger vessels when situated deep in a small wound where they are not readily accessible for ligation. Under these circumstances, however, it is wise to allow the forceps to remain in place for twenty-four to forty-eight hours, including them in the dressing, since the hemorrhage might recur if they were removed earlier. By allowing the forceps to remain one gives the blood a chance to form a good firm clot to occlude the vessel.

The angiotribe, a bulky crushing instrument, is applied upon this principle. It is provided with a screw which enables one to apply great pressure to the parts within the grasp of its blades. This instrument has been used for crushing the broad ligament in vaginal hysterectomy, but has not, as yet, won for itself a place in popular favor.

Torsion.-This method of occluding a bleeding vessel consists in seizing the end and twisting it until the inner coat of the vessel is ruptured and the end of the vessel, in the grasp of the forceps, is twisted free. This measure may be applied to small arteries and veins as an adjunct to forcipressure. Torsion may be more effectually applied by grasping the free end of the vessel with one forceps and the vessel itself a short distance beyond, transversely, with a second forceps. While the vessel is steadied with the forceps that grasps it transversely, it is twisted repeatedly upon itself with the forceps that grasps its extremity.

Ligature.-The most commonly employed and safest means of 
securing severed arteries and veins especially if of large caliber. In the day of the non-absorbable, non-aseptic ligature many plans were devised to obviate the use of the ligature, since it had to be cast off before the wound could heal and thus precluded the possibility of union by first intention, and because, as the ligature separated and came away, it was often accompanied by a dangerous secondary hemorrhage.

With the aseptic, absorbable ligature, an ideal method of controlling hemorrhage in the wound was instituted. The aseptic, absorbable ligature permits the immediate closure of the wound and does not in any way interfere with the healing process. Some sur-

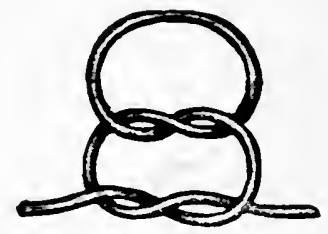

Fig. 4.-Square Knot.

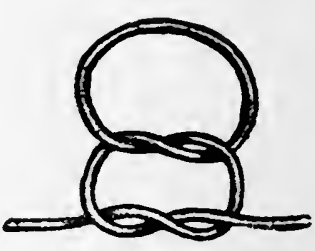

Fig. 5.-Slip-knot.

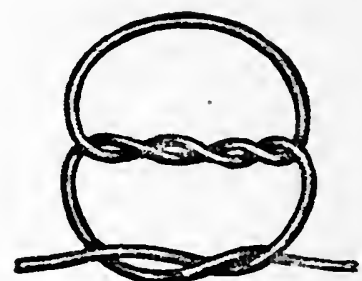

Fig. 6.-Surgeons' Knot. The first loop is made double to prevent slipping while taking the second loop.

geons still use silk for ligature. Although silk may be rendered absolutely aseptic, it has the disadvantage of not being absorbable, and may therefore occasionally act as a foreign body, keeping the wound open until it separates or until it is removed. The ligatures may be applied in the wound before the vessels have been divided or afterward, and may be applied to the isolated vessels or may include the immediately adjoining soft parts as well.

Ligature of blood-vessels before they have been severed is exemplified in the tying of the external jugular in operations upon the neck after the vessel has been exposed in the incision, but before it is cut; the ligature is applied double and the vessel then divided 
between these. Again, in disarticulation through the hip-joint the main vessels may be exposed during the course of the operation, ligated, and then divided. In resecting portions of the alimentary canal the mesentery or omentum that carries the blood-supply to the parts must be tied off. This is usually done in sections, each ligature including from one to one and a half inches of the mesentery or omentum; in this case not only are the blood-vessels included in the ligatures, but all of the tissue from one ligature to the next.

Ordinarily the ligatures are applied to the vessels after they have been severed. The bleeding point is seized with a hæmostatic forceps and the ligature is then slipped over the end of this and tied.

Occasionally, vessels in dense fibrous tissue, in the dura mater and wall of the chest, when cut, retract into the surrounding tissue so that their ends cannot be seized with the forceps. Under these circumstances it may be necessary to carry the ligature around the vessel with a curved needle.

\section{SUTURE OF THE TISSUES.}

The various suture materials may be grouped in two classes: temporary and permanent.

Temporary sutures are made of simple catgut, which softens and becomes absorbed in from five to ten days according to its thickness, and chromicized eatgut, which remains longer, from two to four weeks or even six weeks, according to its thickness and the manner of its preparation.

Permanent sutures consist of silk, silk-worm gut, kangaroo tendon, horse-hair and metal, silver wire, etc. (Kangaroo tendon becomes absorbed after sixty days; so that it is not, in the strict sense, permanent.)

Suture of the Skin.-For this purpose one may use a penetrating stitch, continuous or interrupted, or a non-penetrating intercuticular stitch, which is at present much in favor.

The stitch should not be drawn too tight, as it constricts the parts, and this interferes directly with the blood-supply and the healing process. If the stitch is drawn too tigh' it may cut its way through the tissues, and besides may add much to the pain and discomfort of the patient. The stitch should be drawn just tight 
enough to bring the parts into immediate contact. The knots should be so arranged that they lie to one side or the other of the wound.

The Intracuticular Suture. - For this suture simple or chromicized catgut or some permanent material-silk-worm gut, silk, etc.-may be used. It may be introduced with a straight needle or with a curved needle in a holder.

In introducing this stitch, one should, with the needle, eatch the firm under layer of the skin proper, avoiding the loose, subcutaneous fat and connective tissue, and with each puncture of the needle take a good long bite; after crossing from one edge of the

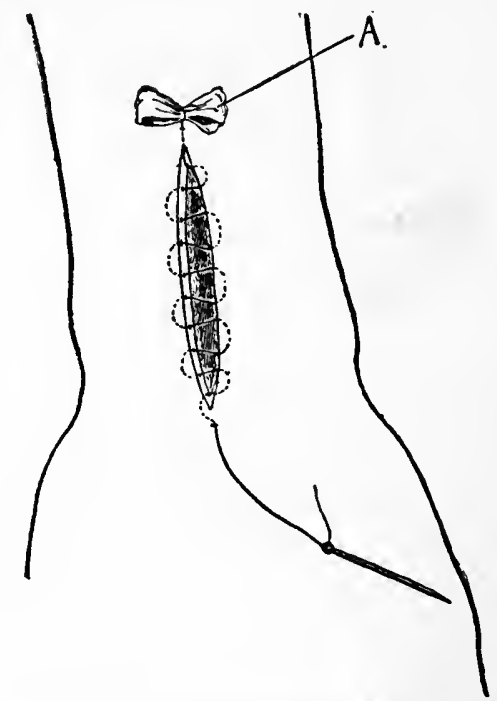

Fig. 7.-Intracuticular Suture. A, end of suture fixed with a pledget of gauze.

incision to the other one should take care to enter the needle directly opposite the point at which it emerged or even a trifle back of this. The suture may be secured at each end with a small pledget of gauze. One pad is fixed to the end of the thread before commencing the suture, and then, after the needle emerges through the last puncture, it is carried through the second pad and the suture secured with oneor two turns about this.

Suture of Muscle.-Divided muscle is usually approximated with absorbable material, simple or chromicized catgut. If the muscle has been cut across, at right angles to the course of its fibers, the 
part should be placed in a position to relax the muscle and special care should be exercised to bring the cut edges securely together. This is accomplished by introducing a sufficient number of interrupted sutures or a continuous suture of moderately thick catgut, each taking a good secure bite in the muscle, or one may use several mattress sutures for this purpose. If the muscle has been divided along the course of its fibers,-i.e., between its fibers,- - several interrupted catgut sutures will usually suffice to retain its edges in. apposition.

If the sheath of a broad muscle has been divided,-for example, the sheath of the rectus, - care should be taken to unite accurately, with catgut, simple or chromicized, the edges of the sheath.

In operations for the cure of hernias the edges of the muscles. are sometimes joined with a non-absorbable suture material,--silk, silk-worm gut, or silver wire,-with the idea of leaving these as. permanent sutures to retain the parts in close apposition.

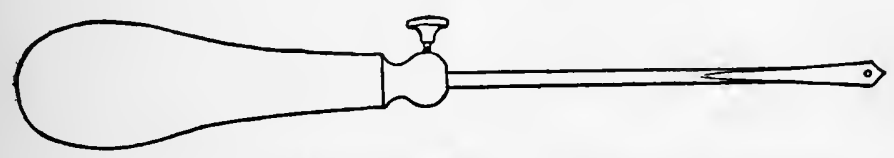

Fig. 8.-Bone Drill with Eye near the Point to carry Suture, etc.

Suture of Tendons.-Severed tendons are sewed end to end with some absorbable suture material. A single mattress suture or one or more ordinary interrupted sutures that pass through the tendon proper are usually employed for this purpose (see Fig. 198). If a part of the tendon has been destroyed so that the ends cannot be approximated, a flap may be turned back from one or both ends in order to meet this deficiency.

Suture of Nerves. - The ends of a divided nerve may be joined with one or two catgut sutures which secure the sheath of the nerve, or, better, these sutures may penetrate the nerve proper.

Bone and Cartilage.-For the purpose of suturing bone and cartilage silver wire is usually employed. Sometimes heavy, chromicized catgut is used. In order to pass the sutures, holes must first be made through the bone. This is done with the drill. Before withdrawing the drill the suture is introduced through the small eye in the point of the drill, and then as the instrument is withdrawn it brings the suture after it. If the suture is too thick to 
enter the eye in the point of the drill, one may pass a loop of silk through the eye of the drill and with this draw the suture through.

Bones are sometimes joined with one or more sutures of chromicized catgut which do not go through the bone, but include the periosteum and the fibrous tissue that cover the bone; this method may be used, for example, to unite a fractured patella so as to avoid entering the knee-joint and the handling that would be necessary in the making of drill-holes.

Bones may also be joined by steel nails, ivory pegs, etc., that are driven from one fragment of bone into the other.

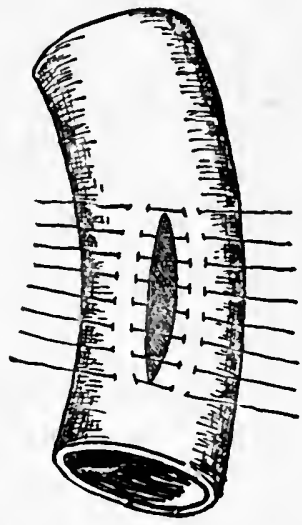

Fíg. 9.-Segment of Bowel. Interrupted Lembert sutures in place.

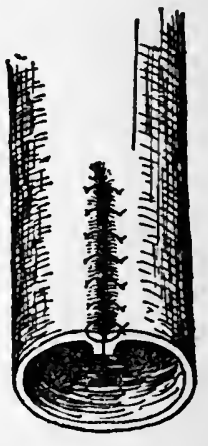

Fig. 10.-Segment of Bowel. Lembert sutures tied. It will be noted that they do not penetrate through the entire thickness of the wall of the gut.

Suture of Serous Surfaces, Bowel, etc.-The essential object is to secure rapid adhesion by approximating serous surface to serous surface, and this is accomplished by means of the Lembert suture.

The Lembert suture catches the serous and muscular coats of the bowel, but does not penetrate into the mucous membrane layer. It should not enter into the cavity of the gut, etc. For this suture silk should be employed. It may be introduced interrupted or continuous, and is applied in such a manner as to invert the edges and join opposite serous surfaces.

A straight round cambric needle is usually employed to carry the Lembert suture, but occasionally, especially in sewing deep within the abdominal cavity, a thin curved surgeon's needle in a holder may be more convenient. 
In applying the Lembert suture the needle is introduced a short distance from the edge of the wound, and after passing through the wall of the gut, catching up the serous and muscular coats, but not entering the mucous membrane coat, it emerges near the edge of the incision; the needle is then carried across the incision and introduced upon the opposite side at a point directly opposite and in a similar manner.

The suture may also be introduced and carried in the wall of the gut along a line parallel with the incision instead of at right angles to the line of the incision.

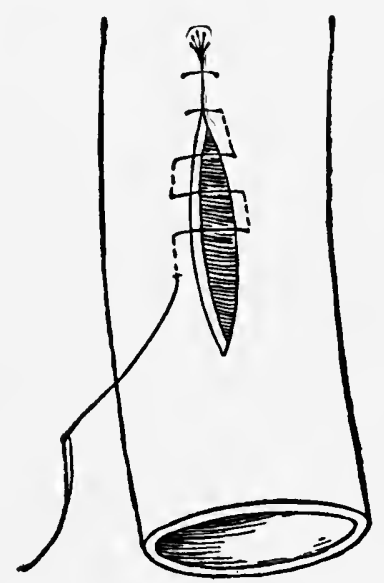

Fig. 11.-Cushing Suture Applled to Close Openlng in the Bowel. It is a continuous stitch and passes through the wall of the gut parallel with the line of the incision instead of at right angles to it.

Small wounds of the bowel may be closed with a single row of Lembert sutures. Larger wounds of the hollow abdominal viscera should be closed, first, with a continuous or interrupted row of silk or catgut sutures that penetrate through all the layers of the organ, joining the parts accurately edge to edge, and then, after the opening has been thus closed, the Lembert stitch, which unites the opposite serous surfaces to each other, is applied. The Lembert stitch buries the penetrating suture and inverts the edges of the wound, so that the serous surfaces become apposed to each other. The outside Lembert suture that buries the deeper penetrating mucous suture is sometimes called the "outside serous" suture. 
Suture of Wounds of the Bladder.-Closure of wounds of the urinary bladder requires special mention. They may involve the serous or the non-serous portion of the organ.

Wounds of the serous portion should be first closed with a continuous catgut stitch, which. should include all the coats except the mucous membrane. Each loop of this suture should be drawn tight. This serves to close the opening. A continuous Lembert stitch of silk is then introduced which unites the opposite serous surfaces, immediately adjacent to the edges of the incision, to each other and buries the first row of sutures.

Wounds of the non-serous portion of the bladder: i.e., its anterior wall. Wounds of this part of the bladder should be closed with a continuous or interrupted row of catgut sutures that include the whole thickness of the bladder wall except its mucous membrane. These sutures should close the opening in the wall of the bladder very accurately; owing to the absence of the serous coat from this part of the bladder, the Lembert suture-"outside serous suture"cannot be applied. Since we cannot look for rapid adhesion in wounds of this part of the bladder, it is well to allow the abdominal incision to remain open, packing with gauze down to the suture line in the wall of the bladder, so that, if there is any leakage, the fluid may find its way out of the wound. 


\section{PART II.}

\section{HEAD AND FACE.}

HEAD.

Surgical Anatomy of the Head. The Scalp.-The head is covered by the scalp, which is a dense layer, composed of the skin, subcutaneous connective tissue, and the aponeurosis of the occipitofrontalis minscle. These three layers together constitute the scalp.

The subcutaneous connective tissue is dense and serves to unite the skin intimately with the underlying aponeurosis of the occipitofrontalis muscle. It is continuous behind, in front, and upon the sides with the superficial fascia (subcutaneous fatty and connective tissue layer) of these parts. In it ramify the blood-vessels and nerves.

The arteries of the scalp are large and numerous. Bleeding from these vessels can often be controlled by pressure applied against the underlying bony surface. Anteriorly are the frontal and supraorbital arteries; on the sides, branches of the temporal; and, behind, the occipital and posterior auricular. These vessels all course from below upward toward the crown of the head, their branches anastomosing freely with each other all around. These arteries are found at times to be very tortuous.

The occipito-frontalis muscle is broad and flat, consisting of an anterior and a posterior muscular portion and an intermediate aponeurotic portion which covers the top of the skull. This aponeurosis is firmly united with the overlying skin, whereas it is but loosely attached to the pericranium beneath. Upon either side the aponeurosis is continued into the temporal fascia. In cases where the scalp is torn off, the aponeurosis of the occipito-frontalis comes away with the skin and subcutaneous connective tissue, thus leaving the pericraninm exposed.

In the temporal region the subcutancous connective tissue layer is looser than upon the top of the head, and in it run the branches of the temporal artery and vein and the auriculo-temporal nerve. Beneath the subcutaneous layer in the temporal region is the temporal fascia. This is a strong, fibrous layer covering in the temporal 
muscle, and is attached above, all around, to the temporal ridge, and, below, to the upper border of the zygomatic arch, where it splits into two layers, between which are included a small arterial and nervous branch. The aponeurosis of the occipito-frontalis muscle thins out upon each side and is continued into this temporal fascia. Beneath the temporal fascia is the temporal muscle. This is a broad, fan-shaped muscle which arises from the whole surface of the temporal fossa and from the under surface of the temporal fascia; it is attached by a strong tendon to the tip, anterior border, and inner surface of the coracoid process of the inferior maxilla.

The pericranium is a shining, fibrous layer of periosteum which is closely attached to the external surface of the bones of the skull: most intimately at the suture lines, through which it is continuous with the dura mater lining the inner surface of the bones.

Collections of pus or blood between the skin and the occipitofrontalis aponeurosis give rise to circumscribed tumors because they cannot become diffused in the dense subcutaneous connective tissue layer. Between the aponeurosis and the pericranium, however, such collections may become widely diffused, owing to the looseness of the tissue which joins the aponeurosis and the pericranium together, and, raising the whole scalp so that it resembles a water-bag, may gravitate and point in the frontal or occipital regions. Beneath the pericranium, between this layer and the surface of the bone, such collections are again limited, owing to the close union between this structure and the underlying bone.

The SKull is a rounded, elastic case made up of a number of bones joined, for the most part, edge to edge. The base of the skull is irregular and is strengthened along certain lines by ribs of bone, the intervening portions being often very thin. It presents many openings for the entrance and exit of important structures. The vault of the skull is arched, rounded, and smooth. The bones entering into the formation of the vault are flat and vary in thickness in different places. These so-called flat bones that enter into the formation of the vault are made up of spongy tissue-diploëinclosed between two plates of hard compact bone: the inner and outer tables. The outer table is twice as thick as the inner. The external surface of the skull is covered by the periosteum (pericranium) already mentioned. The internal surface is lined by the dura mater, which is very closely applied to the surface of the bones, serving the purpose of a periosteum; the large vascular branches 
that ramify upon the inner surface of the skull are lodged in the dura.

The spongy substance-diploë-inclosed between the two layers of compact bone presents an extensive system of venous canals. These communicate with the intracranial venous channels, that are found between the layers of the dura mater, and with the veins of the scalp. The vault of the skull varies in thickness in different places and in different individuals. About the middle it is thin, its average thickness in this situation being from 4 to $5 \mathrm{~mm}$.; it becomes thicker toward the front and still more so toward the occiput. Along the course of the intracranial venous sinuses, and also corresponding to the depressions for the Pacchionian bodies, which are located upon either side along the middle line, the bone is thinner. Where the skull is thin it is at the expense of the diploë, which in certain parts may be entirely absent, the two tables being in direct contact with each other. This is the condition in the temporal region.

Corresponding to the frontal region the skull is marked by the presence of two large air-spaces, one on either side, the frontal sinuses; these are separated from each other by a septum located more or less in the middle line. The anterior wall of these spaces is thick, and consists of two layers of hard, compact bone with intervening diploë. The posterior wall is thin. The frontal sinuses vary in size in different individuals, are lined with mucous membrane, and communicate with the nasal fossa through a large canal, the infundibulum, which opens under the middle turbinated bone, toward the front.

In the mastoid region the bone is prolonged downward in the form of a teat-like process: the mastoid process.

Corresponding to the temporal region, the skull is made up of the squamous portion of the temporal bone, which is very thin, and of part of the parietal bone. Ascending upon the surface of the bone, beneath the temporal muscle, are several deep temporal arterial branches.

The parietal and the occipital bones and the mastoid portion of the temporal bone present openings for the passage of veins from the exterior of the skull which enipty into the intracranial sinuses, and these may be the routes through which infection is carried into the cranial cavity.

The Dura Mater is a strong, non-elastic, fibrous membrane 
which lines the inner surface of the skull and is closely attached to the bones (periosteum), but may be separated without much force. It supports the intracranial arteries and veins (venous sinuses), and when separated from the surface of the bones carries these vessels with it. Anteriorly, ramifying in the dura mater, is the anterior meningeal artery, which is a branch of the ethmoid. Corresponding to the middle fossa of the skull and the temporal region, the middle meningeal artery is found. This is a branch of considerable size, and is of much surgical importance; it is derived from the internal maxillary and enters the skull through the foramen spinosum in the base of the skull. Behind are the posterior meningeal branches which are derived from the occipital and the vertebral.

There are a number of large venous sinuses which are situated between the layers of the dura and which groove the surface of the bones along their course. The largest of these are the longitudinal, the lateral, and the cavernous.

The longitudinal sinus runs from before backward along the line of the sagittal suture from the foramen cæcum in front to the occipital protuberance behind.

The lateral sinus is important surgically. From the center of the occipital bone that of either side passes transversely outward, grooving the internal surface of the occipital bone upon a line corresponding to the attachment of the trapezius and sterno-mastoid muscles and the inner surface of the posterior inferior corner of the parietal; here the sinus curves downward, grooving the inner surface of the mastoid, and from this bone is continued again over on to the occipital, crossing the upper surface of the jugular process of this bone, to join with the inferior petrosal sinus to form the internal jugular vein. The course of the transrerse portion of the lateral sinus corresponds to a line drawn from the external occipital protuberance to the upper margin of the external auditory meatus.

The cavernous sinus is lodged in the groove upon the side of the body of the sphenoid bone. The internal carotid artery passes from behind forward, from the orifice of the carotid canal in the apex of the petrous portion of the temporal bone, where the artery enters the cranium, to the point where it divides into its terminal branches. This part of the internal carotid artery is enveloped by the cavernous sinus, the wall of the sinus being, as it were, wrapped around the artery. The sixth nerve is also inclosed entirely within the sinus, lying below and to the outer side of the artery. The third, 
fourth, and the ophthalmic division of the fifth nerve are located in the outer wall of the cavernous sinus, but are not contained within its lumen as are the internal carotid artery and the sixth nerve.

The blood-pressure within these sinuses is low, and hemorrhage is readily controlled by packing with gauze.

The Pia Mater.-The skull contains the brain inclosed within its own peculiar membrane: the pia mater. This is a connective tissue membrane which serves to support the ressels which supply the brain, and contains within its meshes the cerebro-spinal fluid. The pia mater is not a simple flat membrane, but is really made up of two layers joined together by septa which divide it up into a mesh-work of cellular spaces within which is contained the cerebrospinal fluid. It has been compared to a water-soaked connective tissue. It has no connection with the dura mater; so that between the inner surface of the dura and the external surface of the pia there is a narrow free space, or crevice, which contains a minute quantity of fluid. This is called the subdural space.

Between the layers of the pia mater there is a great system of spaces communicating with each other, and this is sometimes called the subarachnoid space; as already stated, the cerebro-spinal fluid is contained in this space. This membrane, the pia mater, is attached by its deep internal surface directly to the surface of the brain, dipping down between its convolutions and lobes. It acts like a water cushion, preserving the blood-vessels from pressure, and also permits intracranial tumors, etc., to acquire an appreciable thickness before they begin to cause pressure symptoms.

\section{OPERATIONS UPON THE HEAD.}

Trephining.-By trephining we mean making an opening into, or resecting a portion of, the skull. This operation is done to relieve compression either from depressed bone or from extravasated blood, and to treat intracranial conditions, as abscess, tumor, etc.

The patient is placed upon the back with a thin sand bag under the head. The opening in the skull may be made with a trephine, chisel, or rongeur or Keen forceps, or with a circular saw or a rotary drill.

Trephining for Depressed Fracture of the Skull.-If a wound is already present, this should be utilized, and, if necessary, may be enlarged in order to expose the site of fracture. If no wound 
is present and the incision is a matter of choice, a crescentic or crucial incision may be employed, or a U-shaped flap be reflected. In marking out this flap the base should be below, toward the periphery, so as to insure good blood-supply to the flap. The incision should reach through the periosteum down to the surface of the bone, and in reflecting the flap the periosteum should be included.

After the site of the fracture has been exposed and spurting vessels clamped and tied, one may proceed to relieve the compression by elevating depressed bone, clearing out blood-clot, etc. A number of loose pieces of bone, entirely detached from the periosteum (pericranium and dura mater), may be found, and these may be removed with a thumb forceps. We may find other fragments loose, but still attached, at least in part, to the periosteum or dura mater. These may, in some cases, be readily elevated. We may find other depressed fragments so firmly impacted, wedged, that they cannot be elevated, and in order to get at these fragments it may be necessary to remove a portion of the adjoining margin of bone, either with the trephine or chisel. If the trephine is used for this purpose the periosteum is scraped back, laying bare the surface of the bone which is to be removed. When the trephine is first applied the center pin should be lowered beyond the level of the cutting edge of the crown of the trephine, so as to engage in the bone and steady the trephine until the crown has cut a groove within which it may work without slipping, when the pin may be again raised. The trephine should be so placed that its crown will partly overlap the edge of the bone, so that only one-half of a button will be removed from the margin adjoining the impacted fragment. The trephine should be worked with a firm, steady wrist movement, and the groove occasionally probed to ascertain if the bone is cut through at any point. The use of such force as would result in sudden, abrupt penetration of the skull should be avoided. The button may be loosened by gently prying with the elevator. Bleeding from the edge of the bone ceases after a few moments' pressure with a hot gauze pad.

In many cases the liberation of an impacted fragment is best accomplished by using the chisel to cut away the margin of the bone that holds it fast; often, with a few strokes of the mallet, the fragment is freed or a space is made to allow the use of the elevator.

Having removed all loose fragments and elevated those which are still attached to the pericranium and dura mater and rounded off 
the edges of any defect left in the skull, one should search carefully for any loose fragments or spiculæ which may be concealed under the edge of the opening in the bone. The finger or probe should be used for this purpose. Small pieces may be washed out by irrigation with a weak bichloride solution or they may be picked out with a forceps. One should examine carefully as to the condition of the internal table, as this is often more extensively fractured than is indicated by the appearance of the external table. The internal table is at times extensively fractured and depressed when the corresponding part of the external table is apparently uninjured. Extravasated clotted blood, between the dura and the inner surface of the bone, or beneath the dura, between it and the pia mater, should be removed with a scoop and by irrigation and any severed vessels tied with fine catgut. If the dura mater has been torn the edges of the opening may be brought together with a fine catgut suture.

The wound in the scalp may be closed without drainage unless the parts have been exposed to the chance of infection. In this case, for the purpose of drainage, a narrow strip of gauze may be introduced through one corner of the wound and reaching down to the dura mater.

Trephining for Intracranial Hemorrhage (Middle MenINGEAL). - The middle meningeal artery is the usual source of traumatic intracranial hemorrhage.

The middle meningeal is a vessel of considerable size, and is given off from the upper aspect of the first part of the internal maxillary a short distance beyond its origin from the external carotid, as it (the internal maxillary) lies beneath the neck of the condyle of the jaw, between it and the internal lateral ligament. The middle meningeal passes directly upward between the two roots of the auriculo-temporal nerve, which surround the commencement of the artery, toward the base of the skull, and enters the skull through the foramen spinosum. This part of the middle meningeal artery is concealed beneath the external pterygoid muscle, the tendon of which is attached to the front of the neck of the condyle of the jaw. In front and internal to this part of the artery is the inferior maxillary division of the fifth nerve and its motor root, these nerve branches emerging from the skull through the foramen ovale.

After entering the skull the middle meningeal runs a short distance outward in a groove in the floor of the middle fossa and 
then divides into two branches. The anterior, the larger branch, passes forward and outward across the floor of the middle fossa of the skull and across the anterior inferior angle of the parietal bone just behind the outer extremity of the lesser wing of the sphenoid, and may be exposed as it ascends upon the side of the skull at a point which corresponds to the intersection of two lines (Vogt), one vertical, a thumb's breadth behind the external angular process, and, the other, horizontal, placed two fingers' breadth above the zygoma. The posterior branch of the middle meningeal passes outward across the squamous portion of the temporal bone and then ascends upward and backward upon the inner surface of the posterior inferior portion of the parietal bone above and in front of the groove seen here for the lateral sinus. The posterior branch may be exposed by removing a button of bone whose center is one inch above and one-half inch behind the external auditory meatus.

The middle meningeal and its branches ramify in the dura and groove the surface of the bones against which they are applied. The

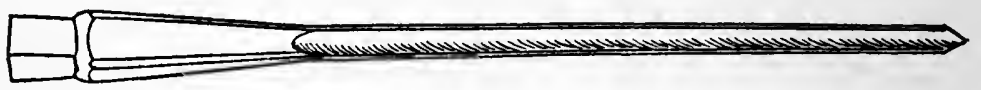

Fig. 12.-Hartley Chisel. This chisel is pointed, $\mathrm{V}$ shape cn section, and is very convenient for cutting the groove in the bone.

anterior branch, as it approaches the anterior inferior angle of the parietal bone, is lodged in a deep groove, which is occasionally converted into a complete bony canal.

Temporary Resection of the Skull. - When the skull is intact, it is preferable, in order to gain access to the cranial cavity, to do a temporary resection of the skull (Wagner), turning back a flap, which consists of the soft parts, periosteum, and corresponding piece of bone, rather than to remove a button of bone, which leaves a permanent defect in the skull. To reach the middle meningeal artery or its divisions this is a most satisfactory method.

A horseshoe-shaped flap is marked out in the temporal region, with its arch above and its base below at the zygoma, the anterior leg being placed a good finger's breadth behind the external angular process and the posterior leg just in front of the tragus. The incision should reach through the soft parts, including the periosteum, down to the bone. The flap thus marked out should measure in its vertical diameter about three inches, and about two and one-half 
inches across its widest part. At its base the flap should be from one and one-half to two inches wide.

The temporal artery and some of its branches are usually divided, and must be clamped and tied.

Retracting the soft parts, but without separating them from the surface of the bone, a groove is cut in the bone all around corresponding to the course of the skin incision. This may be accom-

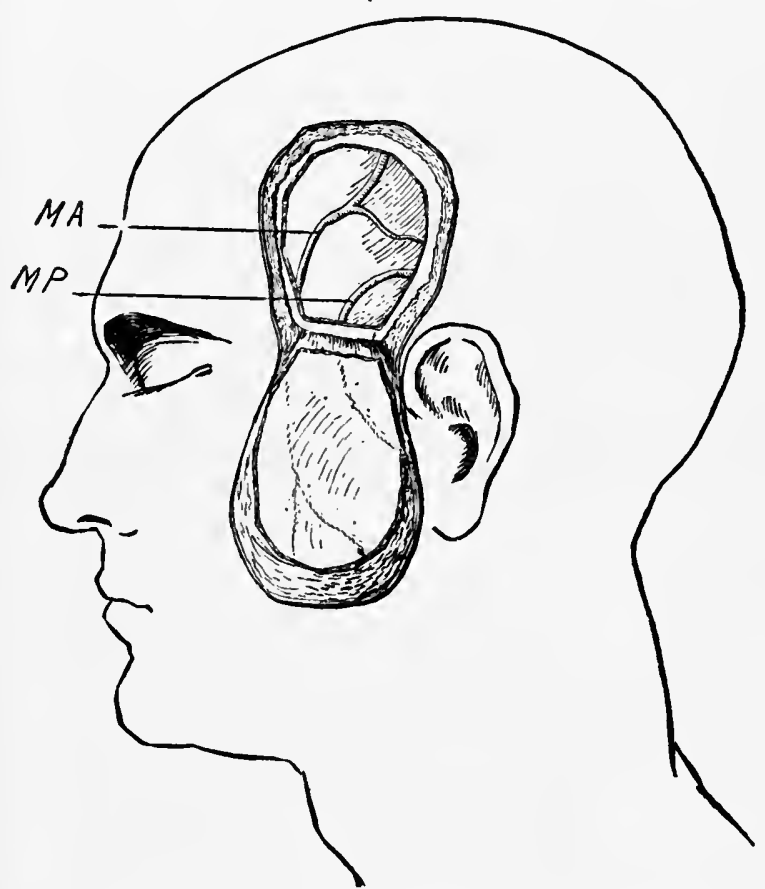

Flg. 13.-Temporary Resection of the Skull. Osteo-tegumentary flap turned down, exposing dura. $M A$, anterior branch of middle meningeal artery. $M P$, posterior branch of mlddle menlngeal artery.

plished with a chisel (Hartley or ordinary chisel), or one may commence by marking it out with a revolving saw and complete it with the chisel, or a small opening may be made in the skull with a trephine and a rotary drill then used. The line of section through the bone should not be direct, but rather somewhat oblique, so that the segment of bone in the flap may have a beveled edge, thus giving, a better fit when it is replaced. After the section through the bone 
has been made, the periosteum elevator is introduced into the upper part of the groove and the piece of bone pried out, breaking it below, through its base, near the zygoma, and then this flap, which consists of all the soft parts with the corresponding segment of bone attached, is turned down over the zygoma, leaving a considerable opening in the skull through which the dura mater and the branches of the middle meningeal artery, which ramify in it, are exposed.

If the opening in the skull is not sufficiently large, it may be further enlarged by cutting away its margins with the bone forceps.

The extravasated blood is usually located between the dura and the bone, so that as soon as the plate of bone has been turned back we expose the blood, which is, as a rule, partly clotted. This may be cleared out with a scoop and irrigation, after which the ends of the divided vessel are sought and tied. Ordinarily they may be seized with a clamp and ligated in the usual manner; there may, however, be some difficulty in securing the ends of the divided vessel, as they may have retracted within the canal in the dura in which they are situated to such an extent that they cannot be readily seized with the artery forceps, and it may then be necessary to carry a ligature around the vessel with a curved needle.

Should the blood have collected beneath the dura mater, between it and the surface of the brain (pia mater), in the subdural space, it would be necessary to make an opening in the dura in order to clear the blood out.

Usually the anterior branch of the middle meningeal is the vessel which is torn, but through the opening made in the skull one can also reach the posterior branch or the main trunk if necessary.

Having entirely removed the blood, tied the ruptured vessel, and sutured the dura, if it has been incised or torn, we replace the osteo-tegumentary flap and without drainage unite the edges of the soft parts all around with interrupted catgut sutures.

Removal of a Button of Bone with the Trephine.-By removing a button of bone with the trephine the anterior and posterior branches of the middle meningeal may be exposed and ligated.

To reach the anterior branch of the middle meningeal, an incision, vertical, is made through the skin, muscle, and periosteum down to the bone, and with the periosteum elevator the surface of the bone, corresponding to the intersection of Vogt's lines, is laid bare (see Fig. 16). Instead of using the vertical incision this area of bone may be exposed by turning down a U-shaped flap with its 
base below near the zygoma. This flap includes all the tissues of the scalp and the periosteum, and is detached from the surface of the bone with an elevator.

The trephine is then used to remove a button of bone, and thus the dura is exposed. If the opening is not sufficiently large it may be enlarged with the rongeur bone forceps. The clot is usually found between the dura and the bone, and is therefore exposed as scon as the button has been removed. It may, however, be situated beneath the dura, in the subdural space, and it may thus become necessary to incise the dura in order to reach it. After clearing out the clot, etc., the ends of the vessels are secured and the incision in the soft parts closed. This operation may be performed more quickly than the temporary resection of the skull, but it does not give as much room, and a further disadvantage is that it usually leaves a permanent defect in the skull.

To expose the posterior branch of the middle meningeal a button of bone may be removed one inch above and one-half inch posterior to the external auditory meatus, as described above. This branch is but seldom injured.

Craniectomy (Linear Craniotomy).-Making linear furrows in the skull for the purpose of providing space to permit of the proper growth of the brain, in cases of microcephalia and idiocy.

This operation was first performed by Lannelongue. It may be done on one or both sides of the skull at one sitting: one side at a time is probably preferable.

A longitudinal incision is made in the scalp in the middle line commencing at a point just above the occipital protuberance and carried forward as far as the hair-line of the scalp; from the anterior end of this a second curved incision may be made reaching downward and outward away from the middle line; this latter incision is also placed within the hair-line of the scalp. The scalp is then raised from the skull with the elevator.

Posteriorly, just above the occipital protuberance, an opening is made in the skull with the trephine, about one-half inch in diameter, and through this opening, with the bone forceps, a furrow is cut which is carried forward to within an inch of the supra-orbital ridge. This channel should be about one-fourth of an inch wide and will vary from five to six and one-half inches in length and should be placed about three-fourths of an inch away from the middle line, in order to avoid the longitudinal sinus. The dura is 
detached from the inner surface of the skull to permit the use of the bone forceps, but should not be opened.

From either end of the longitudinal furrow in the bone an additional channel may be cut, reaching downward and outward for one or two inches away from the middle line.

The periosteum is cut away from the margins of the furrows in the bone to prevent reproduction of the bone. If any of the branches of the meningeal are injured during the course of the operation, they may be surrounded by a ligature carried in a curved surgeon's needle and tied. It is often difficult to secure these branches with the artery forceps, and thus the necessity of carrying the ligature around them in the needle.

The edges of the incision in the scalp are accurately approximated without drainage, to insure primary healing.

The longitudinal furrow in the skull is usually placed to the left of the middle line, but may be placed upon the right side instead, if this appears to be the less developed side.

\section{THE MIDDLE FOSSA OF THE SKULL.}

The Anatomy of the Middle Fossa.-The middle fossa of the skull is narrow in the middle and widens out upon either side. It is limited in front by the posterior border of the lesser wing of the sphenoid and by the optic groove; behind by the dorsum epiphii and the upper border of the petrous portion of the temporal bone. The upper border of the petrous portion is marked by a groove for the superior petrosal sinus and gives attachment to the tentorium cerebelli. The floor of the middle fossa, in the middle line, consists of the upper surface of the body of the sphenoid, presenting in front the optic groove, at either end of which is the optic foramen; behind the optic groove is the sella turcica, a deep depression which lodges the pituitary body and which is bounded behind by the dorsum epiphii; laterally the floor of this fossa consists of the upper surface of the great wing of the sphenoid, the anterior surface of the petrous portion of the temporal, and a part of the squamous portion of the temporal. The body of the sphenoid is marked upon either side by a groove which commences behind at the foramen lacerum medium (carotid foramen) and terminates in front at the optic foramen. This lodges the cavernous sinus, etc.

The foramen lacerum medium is formed at the expense of the 
anterior superior surface of the apex of the petrous portion of the temporal; it is bounded in front by the posterior border of the great wing of the sphenoid and behind by the apex of the petrous portion; through this opening the internal carotid artery enters the cranium. Behind and external to this foramen the anterosuperior surface of the petrous portion presents a depression in which the Casserian ganglion rests. In front of and external to the foramen lacerum medium, in the posterior part of the great wing of the sphenoid, there is a large opening, the foramen ovale. As its name indicates, this opening is oval in shape, its long diameter being directed from without inward and a little forward. This opening is seen externally upon the base of the skull at the root of the pterygoid process, external to the external pterygoid plate. Through this opening the inferior maxillary or third division of the fifth nerve emerges from the cranial cavity. Just external to the foramen ovale and a little behind it, in the apex or angle of the great wing of the sphenoid, is the foramen spinosum, through which the middle meningeal artery enters the skull. From this opening a groove is seen running outward, marking the squamous portion of the temporal near its junction with the petrous portion; this groove lodges the posterior branch of the middle meningeal artery and is continued upward upon the side of the skull across the posterior inferior part of the parietal bone. Commencing at or near the foramen spinosum there is another groove, which runs forward and outward across the squamous portion of the temporal and the great wing of the sphenoid, ascending upon the side of the skull, across. the anterior inferior portion of the parietal bone; in this groove rests the anterior division of the middle meningeal artery. About one-half inch in front of and a little internal to the foramen ovale is the foramen rotundum. This is the commencement of a short canal which passes obliquely forward through the great wing of the sphenoid and opens into the spheno-maxillary fossa through the upper part of its posterior wall; the superior maxillary or second division of the fifth nerre passes through this canal. Toward the front of the middle fossa we have the sphenoidal fissure opening into the orbit; this is a triangular opening between the free border of the great wing and the under surface of the lesser wing of the sphenoid, its base being inward toward the body of the sphenoid. Through this fissure pass the third, fourth, and the ophthalmic or third division of the fifth nerve, the ophthalmic vein, etc. 


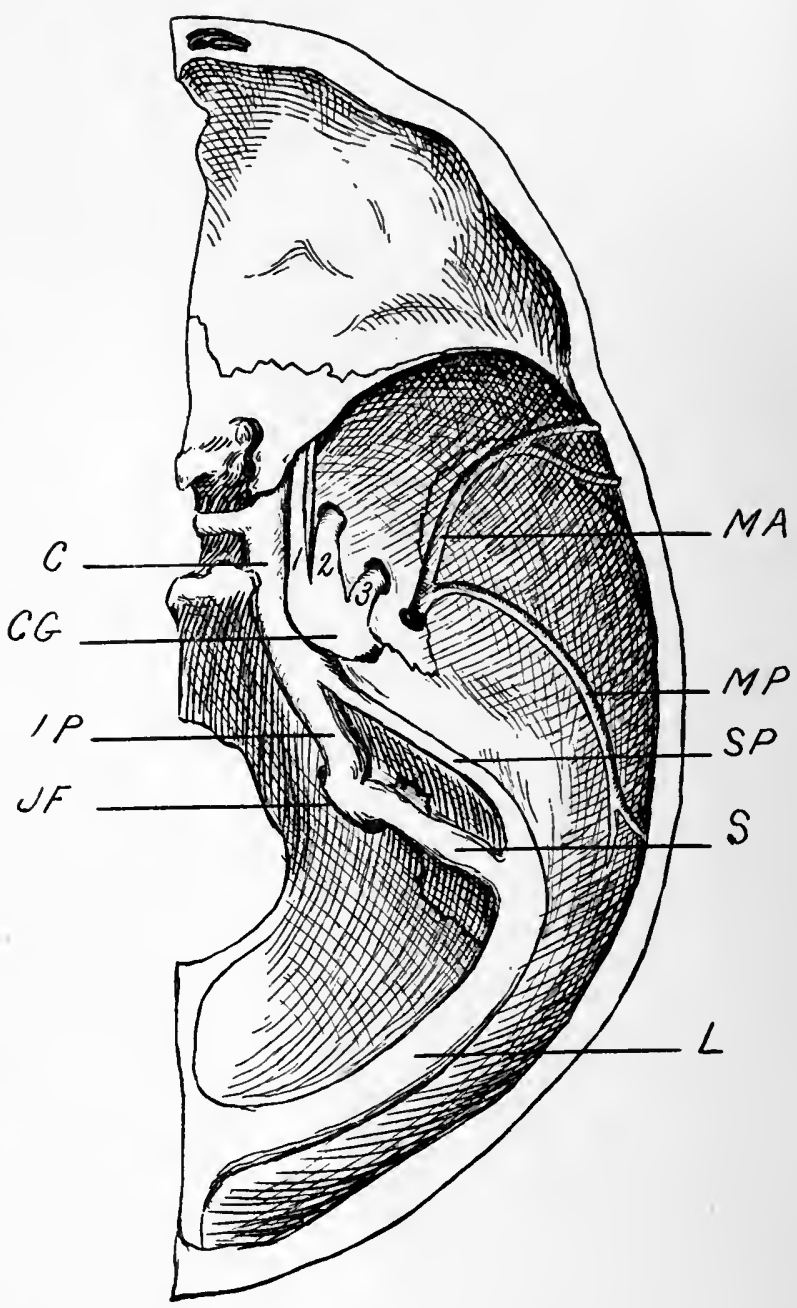

Fig. 14.-Base of Skull from Within. $C$, cavernous sinus; $C G$, Casserian ganglion; $I P$, inferior petrosal sinus; $J F$, juguiar foramen; $L$, lateral sinus; $M A$, anterior branch of middle meningeal; $M P$, posterior branch of middle meningeal; $S P$, superior petrosal sinus; $S$, sigmoid (lateral) sinus; 1 , first (ophthalmic) division of fifth nerve; 2, second (superior maxlliary) division; 3, third (inferior maxillary) division. The first (ophthalmic) division rests upon and is blended with the wall of the cavernous sinus. The second division lies alongside of, but is not connected with, the wall of the cavernous sinus. 
The cavernous sinus is a wide, loose, thin-walled canal, situated between the layers of the dura mater. It reaches from the apex of the petrous portion of the temporal bone behind to the inner end of the sphenoidal fissure in front, being lodged in the cavernous groove upon the side of the body of the sphenoid. The lumen of the cavernous sinus presents a reticular structure, being broken up into numerous cellular spaces by trabeculæ and septa which pass in various directions. Anteriorly, it receives the ophthalmic vein, and, posteriorly, joins both petrosal sinuses and communicates with the pterygoid plexus through veins which enter the skull through the foramina ovale, spinosum, and lacerum medium. The external border of the cavernous sinus corresponds to a line running from before backward, which would skirt the inner margin of the foramen rotundum (see Fig. 14).

The internal carotid artery enters the cranium through the foramen lacerum medium and passes forward, along the side of the body of the sphenoid, enveloped by the cavernous sinus, the sinus being, as it were, wrapped entirely around the artery. (One could not wound the artery in this situation without first cutting into the sinus.) Anteriorly, at the inner side of the anterior clinoid process, the internal carotid, after giving off its ophthalmic branch, turns upward and, passing through an opening in the dura mater, divides into its terminal branches. Along the outer side of the artery, and therefore also inclosed within the cavernous sinus, runs the sixth nerve. In the outer wall of the cavernous sinus and intimately united to it, the third, the fourth, and the ophthalmic or first division of the fifth nerve are lodged; these structures cannot be separated from the wall of the sinus without tearing it, and their relation to each other is in the order given both from within outward and from above downward.

The fifth nerve at its origin appears upon the side of the pons Varolii, and consists of a thick sensory and a small motor root; these pass forward through an oval slit in the dura mater and across the upper border of the petrous portion of the temporal bone, near its apex, into the middle fossa of the skull. As the roots pass over the upper border of the petrous portion, they lie beneath the superior petrosal sinus. In its course the nerve lies outside the dura mater, extradural: i.e., between the dura mater and the base of the skull. Upon reaching the front surface of the petrous portion of the temporal bone the sensory root presents a 
swelling, the Casserian ganglion. The motor root takes no part in the formation of this ganglion, but lies underneath it. The ganglion is reddish gray; crescentic or semilunar in shape; its anterior convex border looks forward, downward, and outward. It is 14 to $22 \mathrm{~mm}$. wide, $4 \mathrm{~mm}$. from before backward, and $1 \frac{1}{2} \mathrm{~mm}$. in thickness.

Given off from the anterior border of the ganglion are the three divisions of the fifth nerve. Of these, the first, or ophthalmic, the longest and thinnest of the three, is the most internal and passes from behind forward and upward along, or rather in, the outer wall of the cavernous sinus, entering the orbit through the sphenoidal fissure. On account of its intimate relation to the wall of the sinus, any attempt to separate it would tear the wall of the sinus; it is in close relation with the third and fourth nerves, the carotid artery, and the sixth nerve. The second, or superior maxillary, division lies external to the preceding, is 8 to $11 \mathrm{~mm}$. long, and passes forward, entering the foramen rotundum, and emerges from this canal in the spheno-maxillary fossa. This branch lies close to the outer edge of the cavernous sinus, but is not joined to it, and may be readily removed without danger to the sinus. The third, or inferior maxillary, division, the most external of the three, is short and thick, and passes forward and outward, leaving the skull through the foramen ovale in company with the motor root. The motor root winds around the third division to get upon its outer side, the two becoming joined just after their exit through the foramen ovale. The ganglion rests in the depression already described upon the front surface of the petrous portion of the temporal bone. The motor root takes no part in the formation of the ganglion, but lies beneath it, between it and the bone. At times the bone is absent in this location and in such cases the ganglion is separated from the carotid artery only by the fibrous tissue which intervenes. The surface of bone upon which the ganglion and its three divisions rest is covered by the periosteum. The ganglion and its divisions, as already mentioned, are placed extradural: i.e., between the dura mater and the base of the skull; the dura roofs them over, and is attached to the margins of the depression in which the ganglion rests and to the floor of the middle fossa of the skull, along the inner margin of the second division and along the outer margin of the third division; so that not only the ganglion, but its second and third divisions as well, are thus roofed in. This space, in which the ganglion and its second and third divisions are thus inclosed, is called the cavum 
Meckelii. Beyond the ganglion and its divisions the dura is, as elsewhere, closely applied to the surface of the bone. The ganglion and its divisions are but loosely attached to the periosteum which covers the surface of the bone upon which they rest (floor of cavum Meckelii) and to the dura mater which covers them and forms the roof of the cavum Meckelii.

The cavum Meckelii is really a space in the floor of the middle fossa of the skull between the bone and the non-attached dura, which lodges the ganglion and its second and third divisions.

The Casserian ganglion is in relation, internally, with the carotid artery and cavernous sinus. Behind the ganglion is the superior petrosal sinus underneath which the roots of the nerve

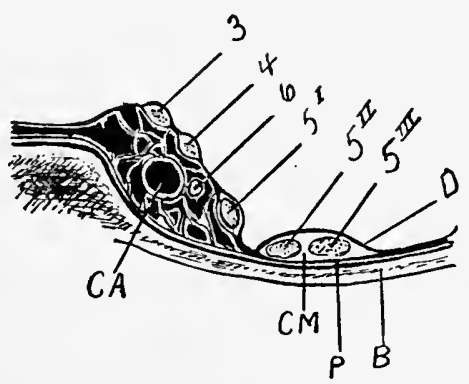

Fig. 15.-Transverse Section through Floor of Middle Fossa. $B$, bone that forms floor of middle fossa; $C A$, internal carotid artery inclosed within the trabeculated cavernous sinus; $C M$, cavum Meckelii; $D$, dura mater lining floor of middle fossa and roofing over cavum Meckelii; $P$, dura lining floor of cavum Meckelii-periosteum; 3, 4, 51, third, fourth, and first (ophthalmic) divisions of the fifth nerve, lodged in the wall of the cavernous sinus; $5^{11}$, 5111, second (superior maxillary) and third (inferior maxillary) divisions of fifth nerve, situated between the dura and base of the skull in the cavum Meckelli; 6, sixth nerve inclosed within cavernous sinus close to the outer side of the Internal carotid.

must pass in order to join the ganglion as it rests upon the front surface of the petrous portion. The superior petrosal sinus is contained in the edge of the tentorium cerebelli, which is attached to the superior border of the petrous portion.

The middle meningeal artery enters the skull through the foramen spinosum just external to and a little behind the foramen ovale (through which the third division passes out of the skull) and would therefore be met with in approaching these structures through an opening in the side of the skull. 
Extirpation of the Casserian Ganglion (Hartley-Krause).-The patient is placed in a semirecumbent position with the head turned partly to one side. A horeshoe-shaped flap, consisting of the integument and the underlying muscle and the corresponding segment of bone, is turned back.

The incision passes through the whole thickness of the soft parts, including the periosteum, down to the bone. This incision commences anteriorly, just above the zygoma, and a good finger's breadth behind the external angular process; it is carried upward upon the temporal region, describing an arc, its posterior limb terminating behind, just in front of the tragus. Hemorrhage should be controlled with clamps. The flap thus marked out should measure in its vertical diameter three inches, about two inches across its widest part, and from one and one-half to two inches at its base, which is just above the zygoma. Corresponding to the skin incision a groove is now chiseled all around in the bone; this groove may be commenced with a circular saw and completed with a chisel. The Hartley chisels are probably the best for this purpose, as they cut a distinct groove; if an ordinary narrow chisel is used, it should be held quite obliquely and only its corner engaged in the bone while cutting. This groove should be deepened to the same extent throughout its whole length, going over it several times before finally penetrating through the entire thickness of the bone. The groove should reach entirely through the bone, except perhaps at its lowest part, down near the zygoma. Care should be taken not to injure the dura with the chisel.

The elevator is now introduced as a lever into the upper part of the groove, and with a prying motion the segment of bone, with the soft parts attached, is broken through at its base and turned well down over the zygoma; if the opening is not sufficiently large, one may cut more bone away from the lower margin of the opening with the bone forceps. It is well if the section through the bone is so made that its edge presents a somewhat beveled margin, so that it may fit better when replaced (see Fig. 13). Through this opening in the skull the dura is exposed, the anterior branch of the middle meningeal ramifying upon it toward the front; at times this branch is torn when the plate of bone is reflected, especially if the groove in which it is lodged is unusually deep; if injured, it should be clamped and tied. Now, with the fingers, the dura is separated from the bone: floor of the middle fossa. This may be done rapidly 
until the middle meningeal artery, as it enters the skull through the foramen spinosum, is met. One should then stop and secure this vessel with a double catgut ligature and divide it; it would probably answer to tie singly or without ligating the vessel, to plug the foramen spinosum with catgut, and then divide the artery-its distal anastomoses are not free. The field of operation should be kept clear of blood with pads on holders. After tying the middle meningeal and still working inward, but rather more cautiously, the dura is separated from the base of the skull with a blunt elevator or with a small gauze pad in a forceps, at the same time lifting the brain away from the base of the skull toward the vault. This is best accomplished with the aid of a narrow, polished, right-angle retractor: serves as a reflector at the same time. A pad of gauze may be interposed between it and the brain; the hemorrhage may be thus somewhat diminished. The hemorrhage caused by separating the dura mater from the bone is sometimes considerable. It may be controlled by a few minutes' pressure or by shifting or withdrawing the retractor for a few minutes and allowing the brain to drop back upon the surface of the bone. Thus gradually working inward we reach the third division of the nerve, which may be seen passing out of the skull through the foramen ovale. This trunk may be seized with a narrow forceps and isolated as far back as the ganglion; it serves as a guide to the ganglion. Without cutting this trunk, we then work a little farther inward, toward the middle line; until we meet the second division. This is likewise isolated from the foramen rotundum back as far as the ganglion. The upper surface of the ganglion is now gradually freed from the dura. While the ganglion is being isolated the brain should be well retracted: lifted away from the base of the skull. The ganglion can be separated from the overlying dura with a blunt periosteum elevator; one may seize and pull upon the third division and use this as a guide to the ganglion. It may be necessary to cut a few connective-tissue bands, between the ganglion and the dura, with the scissors, and in doing this one may accidentally cut into the dura; but this is of no special significance; there may escape some cerebro-spinal fluid, but, according to Tiffany, this is rather an advantage. There may be hemorrhage occasioned in isolating the ganglion, but this may again be controlled by pressure or by shifting the retractor or allowing the brain to drop back in place upon the bone temporarily. The ganglion should be freed as far back as the superior border of the petrous portion, so that 
one may see the white trunk of the nerve beyond the ganglion. Care should be exercised in freeing the inner part of the ganglion, on account of the proximity of this part to the cavernous sinus and the carotid artery. The ganglion may be separated from the surface of the bone, upon which it rests, with the periosteum elevator. At times this surface of bone is absent, and the ganglion is then separated from the artery, as it lies in the carotid canal, by only a thin, cartilaginous or fibrous layer; therefore one should avoid any roughness during this step of the operation.

The ganglion, being finally free all around, is seized with a long artery clamp, and in doing this one should avoid catching the dura, etc., at the same time. The third and second divisions are then cut, either with the scissors or with a tenotome close to their foramina; in cutting the third division, the motor branch of the nerve is usually divided at the same time with it. One should make an effort to avoid cutting the motor branch as the third division is severed, but this is oftentimes difficult and in many cases its division is excusable. When the third division is cut there may be considerable venous hemorrhage from the small meningeal branch which enters the skull through the foramen ovale; this can be controlled by packing or by shifting the retractor and by allowing the brain to drop back for a few minutes upon the base of the skull.

No attempt should be made to isolate or cut the first, the ophthalmic, division on account of the danger of doing damage to the third and fourth nerves and to the cavernous sinus, and, besides, this branch is readily torn away when the ganglion is twisted out.

After the second and third nerves have been divided the ganglion, in the grasp of a long, narrow forceps, is slowly twisted free, tearing it away from the first division and usually bringing away with it a portion of the trunk of the nerve for a greater or less distance beyond the ganglion. Should the cavernous sinus be torn, the hemorrhage is profuse; but this can be controlled by temporarily packing and allowing the brain to drop back into place upon the base of the skull.

The bone is finally replaced and the incision in the soft parts closed with suture. It is well to introduce a strip of gauze through the posterior part of the opening in the skull, especially if there is considerable oozing, for the purpose of drainage.

This operation may be followed by ulcer of the cornea or conjunctivitis, due to infection or the entrance of dirt which is not 
appreciated on account of the loss of sensation in the eye. This may be avoided by bandaging the eye or sealing it with a watch-crystal.

Ptosis, paralysis of the muscles of the eye, etc., may occur as a result of injury to the third, fourth, and sixth nerves. One may avoid these conditions by keeping away from the first division of the fifth nerve and the immediately adjacent third, fourth, and sixth nerves during the course of the operation.

\section{THE MASTOID REGION AND THE EAR.}

The mastoid region and the ear are intimately associated with each other clinically.

The Surgical Anatomy of the Mastoid Region.-The mastoid region is that part of the skull which corresponds to the mastoid portion of the temporal bone.

The integument of this region is thin and contains very little fat; its blood-supply is derived from the posterior auricular artery, which ascends just behind the ear. The occipital artery ascends beneath the sterno-mastoid muscle and becomes superficial midway between the mastoid process and the external occipital protuberance, whence it is continued upward upon the back of the skull.

The surface of the mastoid is uneven and perforated by a number of small vascular openings. At the back part of the mastoid portion, at or just in front of the suture line between it and the occipital bone, there is an opening, the mastoid foramen. Through this a small vein passes into the lateral sinus and a small arterial branch from the occipital artery to the dura mater.

The inner surface of the mastoid portion presents a wide groove, curving from above downward with the convexity forward; this lodges the sigmoid (lateral) sinus. This groove is located about half an inch behind the posterior border of the external auditory meatus, and presents the opening of the mastoid foramen.

The mastoid portion is prolonged below in a teat-like process which varies considerably in size. It is said to be larger in muscular subjects; it is comparatively small in the child. The structure of this process varies. Its cortex may be thin or may be thick and very hard like ivory. The interior may be cut up into a number of cellular recesses lined with mucous membrane and communicating with each other and, through the antrum, with the middle ear, or it may be composed of ordinary spongy bone, or it may be very dense 
and hard, resembling ivory. There is always present, however, even in the newborn, at least one space, the antrum. The mastoid antrum is a space, varying in size from a small pea to a small bean, which is found in the mastoid process just behind the tympanic cavity; these two spaces communicate with each other through an opening in the upper part of the posterior wall of the tympanum. The roof of the antrum is formed by the same plate of bone that forms the roof of the tympanum. The antrum is lined with mucous membrane, which is continuous with that of the tympanum. The antrum is practically a part of the tympanic cavity, and an inflammatory process originating in the tympanum may readily extend and involve the antrum, etc. Externally the antrum may be located upon a level with the upper margin of the external auditory meatus and between 5 and $10 \mathrm{~mm}$. (say, one-fourth inch) behind this opening, and is usually found at a depth of from 12 to $18 \mathrm{~mm}$. beneath the external surface of the bone. In very young children the antrum is comparatively large and very close to the surface of the bone, just behind the upper margin of the external auditory meatus.

The outer margin of the bony portion of the auditory canal is marked above and behind by a spine, the spina supra meatum; this spine is readily recognized after the soft parts have been incised and separated, and may be used as a landmark in locating the antrum. The antrum lies upon the same level as the spine, but about onefourth inch posterior to it.

The mastoid process is usually made up of a number of cellular spaces, the pneumatic mastoid, all lined with mucous membrane and communicating through the antrum with the middle ear (tympanum); these reach to the tip of the process and often penetrate beyond the limits of the mastoid process into the occipital bone or zygomatic process or they may extend backward into the mastoid portion proper, pretty close to the groove which lodges the sigmoid sinus, so that there may be but a very thin shell of bone separating the mastoid cells from the sinus. Mastoids vary in different people and upon opposite sides in the same person as to the extent to which these cells are developed. They begin to develop early in life, but the age differs at which they are found fully developed. From five years on they are fairly well marked, and it is said that at the age of fifteen years they are all developed down to the tip of the process. Some say that they do not reach complete development until a few years later. Occasionally the septa may undergo a process of rare- 
faction, the septa gradually disappearing and the spaces opening into each other until they are all combined in one large space represented by the antrum. Instead of as above described, the structure of the bone may be spongy or it may be excessively dense and without spaces, resembling ivory.

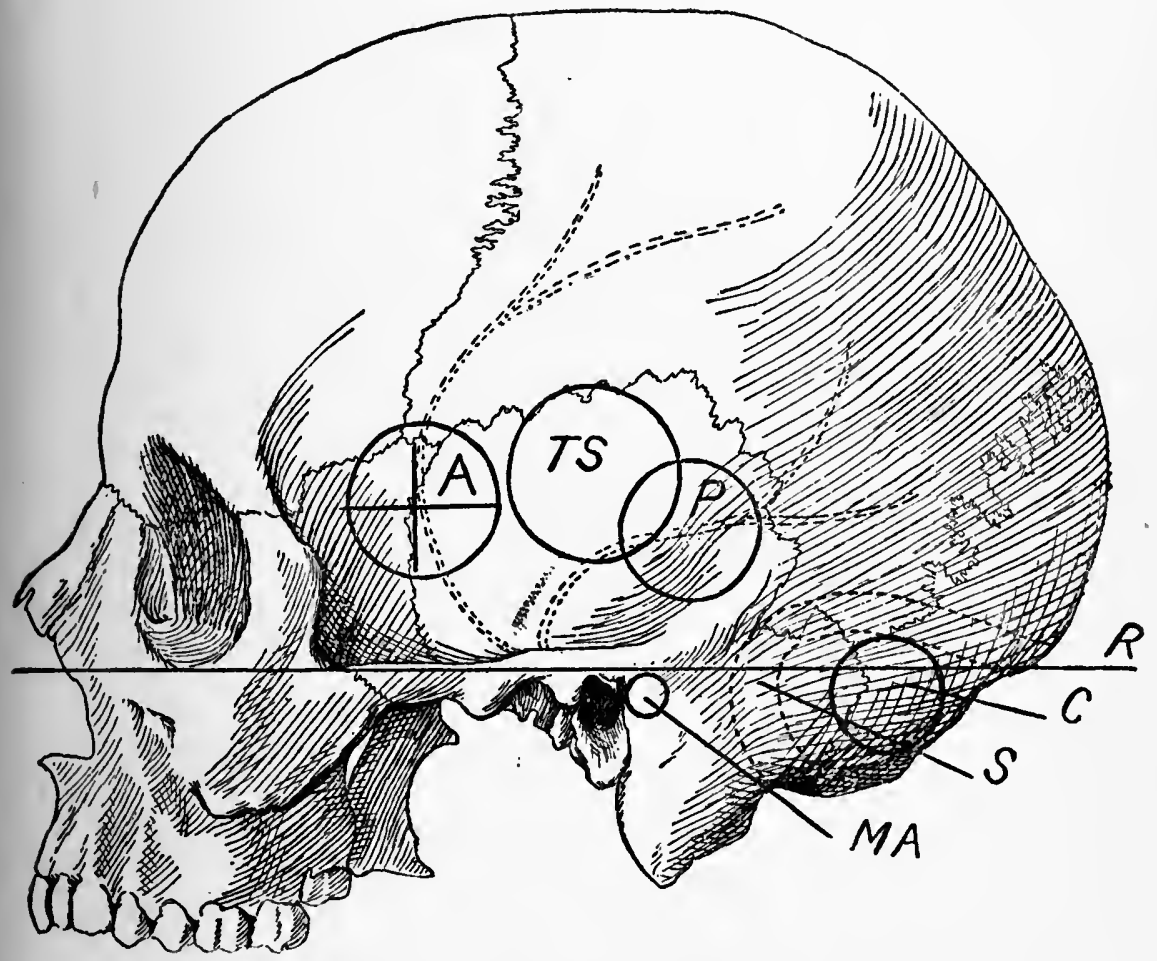

Fig. 16. - Side of Skull. A, position of openlng in skull to expose the anterior branch of the middle meningeal (Vogt's lines); $C$, position of opening for cerebellar abscess; $M A$, location of mastoid antrum (directly in front of circle $M A$ is the spina supra meatum); $P$, opening to expose the posterior branch of middle meningeal; $R$, Reid's base-line continucc backward to external occlpital protuberance; $S$, dotted ines represent course of lateral (sigmoid) sinus; $T S$, opening in the skull for abscess of the temporo-sphenoidal lobe.

The Anatomy of the Ear. - Changes that occur in the first visceral cleft result in the formation of the external and middle ear. The internal ear, labyrinth, etc., are formed within the substance of the petrous portion of the temporal bone. The external fossa, or cleft, develops into the external auditory canal and auricle; 
the internal fossa, or cleft, which opens into the pharynx, becomes the Eustachian tube and tympanum. Where the funduses of these clefts, or fossæ, meet, their walls coalesce and thus form the drum, the partition between the external and the middle ear. The margin of the outer opening of the external cleft, or fossa, becomes thickened and nodulated, and these nodules, coalescing, form the external ear.

The hearing apparatus may be divided into the external ear, which includes the auricle, external auditory canal, and drum; the middle ear, tympanum, which communicates with the pharynx through the Eustachian tube; and the internal ear, labryinth, etc., inclosed within the petrous portion of the temporal bone.

The auricle is made up of a cartilaginous plate considerably folded upon itself and covered with skin; it consists of several parts. It is attached to the side of the head by ligamentous bands; one of these passes forward to the root of the zygoma; the other backward to the mastoid process. Its blood-supply is derived from branches which are given off by the temporal artery in front and the posterior auricular behind. The supply is very abundant, and therefore wounds of the ear heal kindly.

The external auditory canal is about one inch (24 mm., Trölsch) in length; its outer portion, comprising one-third of its length, is cartilaginous and continuous with the auricle; the inner part, comprising two-thirds of its length, is bone. The course of the canal is transverse, but it suffers two curves: one, in its cartilaginous part, with its convexity forward; the second at the junction of the cartilaginous and bony parts, with its convexity backward; this junction is the narrowest part of the canal, and is called the isthmus.

To expose the drum, the auricle is drawn upward, backward, and outward away from the side of the head.

In the newborn child there is no bony portion to the external auditory canal, this part being represented only by a ring of bone into which the drum is fitted. This bony ring, the auditory process, is incomplete, and is applied against the depressed, hollowed-out under surface of the squamous portion of the temporal, which thus completes the ring. At this early age the drum is very near the surface of the body, there being no depth to the bony auditory canal. As the child grows, the bony ring, the auditory process, broadens out, and in the adult is represented by the external auditory process, which corresponds to its outer edge, and by the vaginal process, this 
latter forming the lower and anterior wall of the bony portion of the auditory canal and the back part of the floor of the glenoid cavity. The upper wall of the auditory canal is formed by the grooved under surface of the squamous portion of the temporal bone. The outer edge of the auditory process is rough, and to it is attached, by firm connective tissue, the cartilaginous part of the auditory canal.

The skin which lines the interior of the auditory canal is continuous with that which covers the surface of the drum.

The bony part of the external auditory canal is in relation, above, with the middle fossa of the skull, from which it is separated by a thin, cellular plate of bone, part of the squamous portion of the temporal; behind, it is in relation with the mastoid system of cells, and, in front, with the condyle of the lower jaw and the parotid gland.

Blows upon the chin may be transmitted through the lower jaw to the condyle, and in this way may injure the auditory canal, so that there may be an issue of blood from the external auditory meatus. Purulent processes involving the auditory canal may present cerebral complications, especially in children, without the middle ear being involved, the infection in these cases passing through the roof of the auditory canal directly into the cavity of the skull.

The drum is the septum between the external and the middle ears. It is made up of skin externally, and, internally, of the mucous membrane of the tympanum; interposed between those two is a layer of connective tissue. The drum is set in a bony ring, and forms the greater part of the external wall of the tympanum. It is set obliquely and in such a way that its outer surface looks downward, forward, and outward; the anterior wall of the external auditory canal is thus longer than the upper, posterior wall.

The middle ear consists of the tympanum and adjoining aircells and the Eustachian tube.

The tympanum is a wedge-shaped cavity separated from the external auditory canal by the drum and communicating by an opening in its anterior end, through the Eustachian tube, with the pharynx. In the anterior part is also scen the Glascrian fissure, through which the middle ear communicates with the glenoid cavity and through which the chorda tympani leaves the tympanum.

The carotid artery, surrounded by a venous plexus, traverses a canal, in the temporal bone, which is located just in front of the tympanum and which is separated from this carity by a very thin 
plate of bone that is, at times, perforated. Behind, the tympanum communicates with the mastoid antrum through an opening in the upper part of its posterior wall. The inner wall of the tympanum, that opposite the drum, presents, toward the front, the promontory; behind this, two openings, one above, the foramen ovale, and another below and a little behind, the foramen rotundum. The labyrinth is located beneath this inner wall, in the petrous portion of the temporal bone. This inner wall presents a smooth, curved ridge above the foramen ovale which runs backward and downward toward the back of the tympanum; it corresponds to the position of the Fallopian canal which lodges the facial nerve in its course through the petrous portion of the temporal bone. The layer of bone which separates the nerve from the cavity of the tympanum is sometimes very thin or perforated. The tympanum communicates with the posterior fossa of the skull through the labyrinth and the internal auditory canal, which is traversed by the facial and auditory nerves. The bulb of the jugular vein is lodged in the depression in the temporal bone beneath the floor of the tympanum. The layer of bone which forms the floor of the tympanum is usually comparatively thick, though it may be very thin, perforated, or entirely absent. In the latter case the mucous membrane lining the floor of the tympanum and the wall of the internal jugular vein would be in direct contact with each other. Through small openings in the floor of the tympanum, Jacobson's ncrve, a branch from the glosso-pharyngeal, and some small arterial and venous branches enter the tympanum.

The roof of the tympanum, the most common link between disease of the ear and intracranial complications, is a thin, cellular plate of bone; it may be very thin, perforated, or entirely absent. This plate of bone reaches from the petrous portion of the temporal bone over to the inner surface of the squamous portion, where a suture line, petroso-squamous, exists. In the child this suture line is open and contains a process of dura mater which joins with the mucous membrane lining of the tympanum and carries blood-vessels which take part in the supply of both these membranes. This condition, although not so visible, continues to exist in the adult. This same thin layer of bone, which forms the roof of the tympanum, reaches backward and forms also the roof of the mastoid antrum. The roof of the tympanum and antrum forms part of the floor of the middle fossa of the skull, and is in relation with the dura mater, etc., and with the temporo-sphenoidal lobe of the brain. 
The course of the facial nerve through the temporal bone and its relation to the tympanum and the mastoid antrum are important. The nerve enters the internal auditory canal in company with the auditory nerve, and passes in a direction forward and outward, reaching the inner wall of the middle ear, tympanum, just above the foramen ovale; here it makes a turn and runs backward and downward in the aqueductus Fallopii. The course of this canal is indicated by a prominent linear elevation upon the inner wall of the tympanum just above the foramen ovale; at the back of the tympanum, the nerve, as it curves downward and still contained within the aqueductus Fallopii, is situated but a short distance in front of the antrum. It continues its course through the substance of the petrous portion of the temporal bone, emerging, externally, upon the base of the skull, through the stylo-mastoid foramen. This foramen is located internal to, and a little in front of, the base of the mastoid process. Just before the facial nerve emerges from the stylo-mastoid foramen and while still contained within the canal, it gives off a branch, the chorda tympani, which passes forward and upward through a separate canal in the petrous portion, and enters the tympanum through an opening in its posterior wall, near the drum; it runs forward through the tympanic cavity, being covered by mucous membrane, and escapes through the Glaserian fissure, a slit in the anterior part of the floor of the tympanum, into the glenoid cavity.

The stylo-mastoid artery, derived from the posterior auricular, enters the stylo-mastoid foramen to supply the facial nerve and also the mucous membrane of the tympanum.

The Eustachian tube reaches from the tympanum to the pharynx; its outer one-third is bony; its inner two-thirds, cartilaginous. Where these join, the tube is narrowest: the isthmus. The tube opens into the anterior end of the tympanum, near the drum; its inner end opens into the pharynx above the soft palate and just behind the posterior border of the inferior turbinated bone. The walls of the cartilaginous portion of the tube are usually in contact and the tube is thus closed. To ventilate the tympanum, muscular action, which will open the pharyngeal end of the tube, is required. This is accomplished by the muscles of the soft palate: the tensor and the levator palati. 


\section{OPERATIONS UPON THE MASTOID, ETC.}

Wilde's Incision.-This consists of a simple incision through the soft parts, including the periosteum, down to the bone. It is placed $1 \mathrm{~cm}$. behind and parallel with the auricle, and reaches from the base of the mastoid process to its apex. Usually no vessels are cut and it is not necessary to apply any ligatures. It is often sufficient in very young children.

Drilling into the Antrum.-An incision is made through the soft parts down to the bone, as in the preceding operation, and a channel drilled through the bone down into the antrum. The drill is placed upon the mastoid, upon a level with the upper margin of the external auditory meatus, spina supra meatum, and rather less than one-half inch posterior to it, and a canal is then drilled through the bone in a direction downward, forward, and inward toward the antrum; this canal should not be carried much deeper than one-half inch. This operation is not one to be recommended, as it is uncertain and may be dangerous, especially if one proceeds deeper than one-half inch. It is much more satisfactory to make a free opening into the antrum with the chisel.

To Open into and Drain the Antrum.-The patient is placed with the head upon the side resting upon a thin sand-bag.

Regardless of any condition that may complicate mastoid disease, the first step should always consist in opening into the antrum. (Bacon, Schwartz.)

An incision is made $1 \mathrm{~cm}$. (Schwartz)-one-third inch-behind the attachment of the auricle, through the soft parts, including the periosteum, down to the surface of the bone, and reaching from the base of the mastoid to its tip. In this incision we do not meet the posterior auricular artery, and, as a rule, no vessels that require ligation are divided. With the elevator the soft parts, including the periosteum, are then separated from the surface of the bone, exposing an area as large as a five-cent piece upon a level with and just behind the external auditory meatus. The soft parts are retracted with broad, sharp retractors. The surface of bone, which is thus laid bare, may be soft, discolored, and may further present the orifice of a fistula, or it may be firm and apparently healthy or thickened, sclerosed, and ivory-like. If the first condition exists,- - that is, if the bone is softened, carious, etc.,-one may easily gouge it away with a strong, sharp scoop, continuing thus until the antrum is reached. With the curette one should remove all the bone that 
is apparently diseased, taking away enough of the cortex, especially down toward the tip, to allow good drainage. A rongeur forceps will often be found useful in thus removing the cortex. One should watch for loose pieces of carious bone. In working backward toward the sigmoid sinus one should be careful not, inadvertently, to perforate the dura and enter this channel. If the sinus is simply exposed, this is of no special significance. There may be some hemorrhage from emissary veins that pass through the mastoid foramen into the sigmoid sinus. Fistulæ that are present should be carefully followed, thoroughly curetted, and laid open. They may lead into the auditory canal or into the cranial cavity. During the operation one should take frequent soundings with a blunt probe.

If the surface of bone which is exposed is not softened and apparently healthy and we may select a point at which to commence the excavation in the bone, we choose a point upon a level with the upper border of the external auditory meatus (spina supra meatum) and from 5 to $10 \mathrm{~mm}$. behind it. The antrum is situated about onefourth inch behind the anterior border of the mastoid process upon a level with the upper border of the external auditory canal. In cutting through the bone into the antrum we commence by using a broad chisel,- they vary in width from 2 to $8 \mathrm{~mm}$.,-working rather with the corner of its edge and chipping the bone out in the form of a circle at least three-fourths inch in diameter. This excavation is carried deeper into the substance of the bone, in a direction forward, inward, and downward. As we progress, narrower chisels or gouges may be used and the opening made smaller in diameter. We continue thus, occasionally sounding with the probe, until the antrum is reached. It may contain only a few drops of pus. During this part of the operation the field may be kept cleir of blood and chips of bone by a stream of salt-water or bichloride or by sponging. A funnel-shaped excavation, extending through the substance of the mastoid, is thus made, the base of the opening corresponding to the external surface of the bone and its narrow end to the antrum. The base, or external orifice, of this canal should be sufficiently large to allow of convenient work in its deeper part. The antrum is usually found at a depth of from 12 to $20 \mathrm{~mm}$., but may occasionally be nearer the surface. After having opened into the antrum, if a probe introduced feels firm, healthy bone and if no sinuses are present, one may then proceed to complete the operation by gouging away the cortex down to the tip of the process, in order to expose 
and drain these most dependent cells. One should also see that the communication between the antrum and the tympanum is free. The drum is usually already perforated, and fluid introduced into the antrum may escape in part from the ear. After irrigating, a thin strip of gauze is packed into the opening in the mastoid, reaching into the antrum, and the edges of the soft parts drawn together in part. This would be the procedure in a simple uncomplicated case of mastoid disease.

In order to avoid accidental opening into the sigmoid sinus, the base, the commencement of the cone-shaped canal which is chiseled through the bone into the antrum, is placed anterior to the location of the sinus; and as we proceed deeper into the substance of the bone we work in a direction forward, downward, and inward, so that there is no danger of injuring the sinus, as it lies behind the most posterior part, base, of this excavation in the bone; and as we proceed deeper into the substance of the bone we get farther away from the sinus. It is of but little consequence if the sinus is exposed, but one should avoid accidentally perforating the dura and wounding it. If the sinus is opened, the hemorrhage which results may be controlled by the pressure of an aseptic tampon; air may be sucked into the sinus, if it is opened, but this is not accompanied by any danger (Schwartz). Accidental opening into the middle fossa of the skull is avoided by commencing the channel in the bone below the level of the upper margin of the external auditory meatus, below the spina supra meatum, and, as we proceed, working in a direction rather downward. The floor of the middle fossa will thus lie above the base of the cone-shaped canal which is made in the bone.

If one does not chisel beyond the antrum, there is but little danger of injuring the facial nerve or the inner wall of the tympanum (labyrinth). The facial nerve, contained within the Fallopian tube, lies rather deeper than the antrum and anterior to it, in the inner wall of the tympanum. If one penetrates to a depth of $2 \mathrm{~cm}$. or more, there is then danger of getting beyond the antrum and injuring the facial nerve or the labyrinth.

For Thrombosis of the Sigmoid Sinus.-The sigmoid sinus is encountered about one-half to three-fourths inch posterior to the bony auditory canal (spina supra meatum).

One should always, as a preliminary step, open into the antrum as described above and from here start out to investigate the sinus, 
etc. After the antrum has been opened an incision is carried backward, through the soft parts, for a distance of about two inches, and with the chisel or rongeur the bone is removed in a direction backward until the region of the sinus is reached and the dura exposed. The opening in the skull may be still farther enlarged by cutting away its margin with the rongeur forceps, so that the sinus is freely exposed, and an opening made in the skull which is sufficiently large to work through. This opening in the skull should be at least as large as a silver quarter. Oftentimes pus and granulation tissue are met with just as soon as the dura is exposed,-extradural abscess,- and if the sinus is not diseased it will not be necessary to proceed farther, it being sufficient to curette and drain the parts about the sinus without opening into the latter.

If the sinus is thrombosed, it will appear firm and prominent, and in case of doubt an aseptic aspirating needle may be introduced. If pus is not present in the sinus and the needle withdraws fluid blood it does not necessarily prove that the sinus is unaffected. Tenderness along the course of the internal jugular, etc., is an indication for opening the sinus. If in doubt it is always wise to incise the sinus, as this is not accompanied by any special danger.

If one decides to open the sinus it should be done by making an incision, corresponding to its long diameter, with a sharp, narrowbladed knife. If a clot is found, this should be curetted away first from the jugular end down to the bulb,-if necessary, removing more bone with the rongeur, - until there is a free flow of blood: good, free bleeding tends to wash out any remaining portions of clot. This bleeding may be readily controlled by introducing a small wad of gauze between the sinus and the adjoining bone. This flow of blood does not necessarily prove that there is not a clot in the jugular vein beyond the bulb: blood may flow around from the inferior petrosal sinus.

This procedure is repeated in the other direction-i.e., toward the torcular-until hemorrhage is established; this may then be controlled in a similar manner. It may be well, after the hemorrhage has been controlled, to remove the packing and freely irrigate the sinus with normal salt solution. Before opening the sinus, the internal jugular vein, the facial vein, etc., may be exposed in the neck and tied, or the internal jugular, in its entirety, and including all its branches, may be resected through an incision in the neck after first having tied the vessel below, at the clavicle, and above, 
at the bulb (avoid the pneumogastric nerve). This procedure is indicated especially if tenderness and induration are present along the course of the internal jugular vein: along the anterior border of the sterno-mastoid muscle (McKernon). If the internal jugular vein has not been tied, it may be compressed in the neck, during the operation, to prevent the passage of dislodged clots (Dench).

Besides the condition described, we may find an opening leading through the dura mater to a collection of pus beneath the dura or within the cerebellum; these purulent collections may also be present without thrombosis of the sinus or without a fistulous opening in the dura. All fistulous openings should be thoroughly explored and treated as the condition indicates.

For Cerebellar Abscess.-The opening in the skull may be made with a trephine or chisel. Usually the antrum and sinus have already been explored, and the opening in this case may be simply extended backward with the rongeur. The center of the opening in the skull for cerebellar abscess should be located two inches behind the external auditory meatus, and should be placed below a line drawn from the upper margin of the external auditory meatus to the occipital protuberance (see Fig. 16). The opening in the bone is thus placed below the superior curved line of the occipital bone and we enter therefore below the attachment of the tentorium cerebelli and below the course of the lateral sinus. The bone is here very thin, and the opening may be readily enlarged to any necessary extent with the rongeur. A good free opening should be made in the skull. One may meet pus between the dura mater and the bone or there may be a fistulous opening in the dura leading to a deeper purulent collection. If there is no opening in the dura an aspirating needle may be introduced and search thus made for the pus. When the pus is located, without withdrawing the needle, the dura may be incised and a director or thin artery forceps introduced along the aspirating needle and the opening then enlarged by spreading the forceps so as to permit the introduction of the little finger. The abscess cavity may be irrigated with a double-current tube and then. loosely packed. The opening in the dura may be closed in part.

For Extradural Abscess in the Middle Fossa.-There may be an abscess located between the dura mater and the bone.

If the mastoid antrum has already been explored one may find a fistula leading through the roof of the antrum or tympanum into the middle fossa. The incision, which is already present and through 
which the mastoid antrum has been opened, is prolonged from the base of the mastoid in a direction upward and forward over the ear, dividing the temporal vessels and muscle. With the rongeur or chisel, the bone is cut away so that one may enter the middle fossa just above and in front of the external auditory meatus; here we work in between the tegmen tympani and the dura mater, where the abscess is usually located. The pus is evacuated and the abscess cavity drained as described in the preceding operation.

For Temporo-sphenoidal Abscess. - Associated with the extradural abscess we may find an abscess in the temporo-sphenoidal lobe, and there may be a fistula leading through the dura and communicating with such a collection. In this case the fistula should be followed, enlarging the opening in the dura, evacuating and draining the abscess. A temporo-sphenoidal abscess may be present without an extradural abscess.

If the mastoid has been already explored, one may extend the incision upward and forward over the ear, as described in the preceding operation, and remove sufficient bone with the chisel and rongeur, proceeding from the opening in the mastoid, or, instead of this, a button of bone may be removed with the trephine. This opening in the skull should be at least one inch in diameter with its center located one and one-fourth inches above the bony meatus, and may be farther enlarged with the rongeur forceps to the requisite dimensions (see Fig. 16).

The temporo-sphenoidal lobe may also be exposed by doing a temporary resection of the skull (see "Ligation of the Middle Meningeal Artery").

After the dura has been exposed an aspirator is introduced, and when pus is discovered the dura is incised and, without withdrawing the needle, a director-or, better, an artery forceps-is introduced and the abscess freely opened by spreading the forceps and withdrawing them. The finger may be then introduced and the abscess cavity irrigated with a double-current tube and packed. The opening in the dura may be partly closed.

THE FACE.

Surgical Anatomy of the Face.-The skin of the face is soft, thin, and intimately united to the underlying muscles and connective tissue, and cannot be pinched up without including these deeper 
layers. The subcutaneous tissue of the face is widely meshed, and within these meshes there is contained much fat. Those parts of the face where the fat is absent from the subcutaneous layer are loose and flaccid,- - for example, under the eyes,-and become marked early in life by wrinkles. These parts also readily become swollen and distended in dropsical conditions. In this layer are contained the muscles of expression and the vessels and nerves.

The facial artery is the chief source of supply to the face. It is a large vessel derived from the external carotid. It pursues a tortuous course, upward and forward, across the side of the face, from the anterior border of the masseter to the angle of the mouth, and then, as the angular, continues upward alongside the nose, anastomosing at the inner canthus with a branch of the ophthalmic. Just below the corner of the mouth the facial gives off a branch, the inferior labial, for the supply of the lower lip; those from either side anastomose. At the corner of the mouth the facial gives off the inferior and superior coronary. These branches pass inward, lying a little beyond the edge of either lip and situated beneath the mucous membrane: between it and the muscular structure of the lip. Those from either side anastomose freely with their fellows.

The facial vein, which accompanies the artery, is not tortuous, and lies superficial to the artery.

The facial nerve supplies the muscles of expression, etc., and the buccinator. It emerges from the parotid gland upon the side of the face at a point corresponding to the lower border of the lobe of the ear, and divides into branches which supply the facial muscles and the platysma. The sensory supply to the face and teeth is derived from the fifth nerve.

The Skeleton of the Face.-The upper part consists of the superior maxillary and the adjoining bones with which it articulates and which serve to join it to the skull; it articulates, toward the middle line, with the nasal bones which form the bridge of the nose and laterally with the malar. The malar bone forms the prominent part of the cheek and gives off a process which passes backward and unites with a similar process from the temporal to form the zygomatic arch.

The body of the superior maxillary is pyramidal, its base being directed inward toward the nasal cavity, forming part of its outer wall and presenting the opening into the antrum of Highmore; its apex corresponds to its junction with the malar. The upper surface 
of the superior maxillary is thin and forms the floor of the orbit. Its anterior or facial surface is very thin in places and easily perforated; it is rather concave, and just below the margin of the orbit presents the opening of the infra-orbital canal. A canal descends, as an offshoot from the infra-orbital canal, through the anterior wall of the bone; it transmits a nerve-branch which supplies the upper front teeth. The posterior, or zygomatic, surface of the superior maxilla looks backward and outward toward the zygomatic fossa; it gives origin, in part, to the external pterygoid muscle, and is in close relation with the termination of the internal maxillary artery. This surface presents the commencement of the superior dental canal for the transmission of the superior dental nerve to the upper back teeth.

The body of the bone is hollowed out. The space within, known as the antrum of Highmore, communicates with the nasal cavity through an opening into the middle meatus, and is lined with mucous membrane, which is continuous with that of the nose. The walls inclosing the antrum are thin, but strengthened by columns of bone which ascend from the tooth sockets and converge toward the apex, malar process; in this way the bone is strengthened and the shock of blows distributed. The alveolar process is solid and presents the sockets for the teeth. The palate process, projecting inward, joins with its fellow of the opposite side, and together with the horizontal plates of the palate bones forms the hard palate: the floor of the nasal, and the roof of the buccal, cavity.

The periosteum covering the upper jaw is thin and closely attached to the surface of the bone. It is rather more easily separated from the orbital and facial surfaces.

The lower part of the face is composed of the inferior maxillary, which consists of a body and two rami and which is attached to the skull through the temporo-maxillary articulations. The body of the bone is horseshoe-shaped, presenting an upper border, with sockets for the teeth, and a lower rounded border, which may be felt beneath the integument.

To the inner surface of the body of the inferior maxillary are attached the muscles which form the floor of the mouth, and in front, at the symphysis, are attached the muscles which draw the tongue forward and prevent its dropping back into the pharynx.

The ramus is a perpendicular plate of bone with an upper curved border which presents, in front, a thin, pointed process, the coracoid, to which is attached the tendon of the temporal muscle, 
and, behind, a rather thickened process, the condyle. The upper surface of the condyle is rounded and smooth, for articulation with the glenoid cavity. Below the articular surface there is a rather constricted portion, known as the neck. To the front surface of the neck of the condyle is attached the tendon of the external pterygoid muscle. The lower posterior corner of the ramus is a prominent landmark, and is called the angle of the jaw. The outer surface of the ramus is covered by the masseter and gives attachment to this muscle. The inner surface of the ramus presents, about its middle, the orifice of the inferior dental canal, into which the nerve of the same name passes to supply the teeth of the lower jaw. The anterior margin of this orifice is marked by a small pointed process of bone, to which the long internal lateral ligament is attached. The internal pterygoid muscle is attached to the lower posterior part of the inner surface.

Sixteen teeth are inserted in each jaw, eight on a side: two incisors nearest the middle line, and, following these, one canine, two bicuspids, and three molars.

The Mouth.-The mouth is inclosed by the lips and cheeks.

The lips are composed of fatty connective tissue and muscular tissue, and are covered externally by the skin and internally by the mucous membrane. The muscular fibers are found in the subcutaneous connective-tissue layer, coming from all directions and interlacing with each other, and with much fatty tissue interspersed between them. The mucous membrane, lining the inner surface of the lips, is continued over upon the gums. In the middle line, from the lip to the gum, there is a thin, delicate fold of mucous membrane, the frænum, which is well seen when the lip is drawn away from the gum. The vessels to the lips are the labial and the inferior coronary to the lower lip, and the superior coronary to the upper lip. These branches are derived from the facial.

The cheeks are formed of skin, connective tissue and fat, buccinator muscle, and mucous membrane. The buccinator muscle is attached to the outer surface of the upper and lower jaw-bones just beyond the alreolar processes. This muscle is covered, upon its external surface, by a layer of fascia, bucco-pharyngeal, which is continuous behind with that covering the constrictors of the pharynx. The mucous membrane lining the inner surface of the cheeks is continuous with that of the gums. The buccal cavity may be divided into an outer space, the vestibule, and an inner space, the mouth 
proper. The vestibule is the space between the teeth and the cheeks and lips. When the mouth is closed the mucous membrane lining the cheeks is thrown into folds, which would be caught between the teeth if not prevented by the contraction of the buccinator to which the mucous membrane is firmly attached.

Opposite the second upper molar tooth is the orifice of Stenson's duct. At times this orifice is marked by a papilla, which may assist one in locating it.

The mucous membrane, from the lips and cheeks, is reflected upon the alveolar process of the upper and lower jaw and extends between the teeth. It is intimately united with the periosteum covering the bone, and together with it forms the gums. Behind the last molar tooth the anterior border of the ramus of the jaw may be felt, and upon the outer side of this the masseter muscle may also, when contracted, be distinctly recognized. When the teeth are tightly closed, the vestibule communicates with the cavity of the mouth proper by a small space behind the last molar tooth upon either side.

The cavity of the mouth proper presents a roof and a floor, and is bounded in front and upon the sides by the alveolar processes and the teeth. Behind, the mouth opens into the pharynx. It is separated from the larynx by the epiglottis, and from the posterior nasal space by the soft palate. Where the cavity of the mouth opens into the pharynx it is somewhat narrowed and is called the isthmus of the fauces. The isthmus is bounded above by the free edge of the soft palate; below, by the tongue; and, upon the sides, by the pillars of the fauces.

The roof of the mouth is divided into the hard and soft palate. The hard palate is formed by the junction, in the middle line, of the palatal processes of the superior maxillaries in front, and of the horizontal plates of the palate bones, behind. It is concave, and arched from side to side and from before backward. In front, in the middle line, just. behind the incisor teeth, is a foramen, the orifice of the anterior palatine canal, which transmits the anterior palatine vessels. Extending from this foramen, forward and outward, to a point between the lateral incisors and the canine teeth, on either side, may be seen, occasionally, a line which marks the junction of the intermaxillary bone with the palatal processes of the superior maxillaries.

Near the posterior edge of the hard palate, just to the inner 
side of the last molar tooth, is the orifice of the posterior palatine canal, and passing forward from this is a groove, close to the alveolar process. The posterior palatine vessels descend through the posterior palatine canal and then pass forward, upon the hard palate, lying in the groove just mentioned. Behind the orifice of the posterior palatine canal may be seen the hook-like hamular process: the termination of the internal pterygoid process, around which the tendon of the tensor palati is reflected before it spreads out in the soft palate. The mucous membrane and periosteum, which cover the hard palate, are intimately united with each other and to the surface of the bone. There is little or no anastomosis between the vessels across the middle line; so that in operating for cleft palate it is desirable to retain the arteries in the flaps (Langenbeck).

The soft palate is a curtain-like structure suspended from the posterior border of the hard palate. It is composed of the spreadout aponeuroses of the tensor and levator palati. It marks the boundary line between the mouth and the pharynx. It presents an inferior, or anterior, and a superior, or posterior, surface, each covered with mucous membrane.

The lower, or free, border of the soft palate presents, in the middle line, the uvula and upon either side separates into the anterior and posterior pillars of the fauces. The anterior pillar is continued downward into the side of the base of the tongue at a point just behind the last molar tooth of the lower jaw, and is made up of the palato-glossus muscle. The posterior pillar is continued downward and backward into the side of the pharynx, and is composed of the palato-pharyngeus muscle. Between the two pillars of the fauces there is a triangular space in which the tonsil is lodged. Just above the soft palate, in the side of the pharynx, is the orifice of the Eustachian tube; it is about on a level with the floor of the nose.

In quiet breathing the soft palate hangs passive; but during the act of swallowing it becomes tense, owing to the contraction of its muscles, and its free border then comes into contact with the posterior wall of the pharynx, thus shutting off the posterior nasal space from the cavity of the mouth.

The floor of the mouth is formed of soft parts: chiefly by the mylo-hyoid muscle. This muscle extends from the mylo-hyoid ridge, upon the inner surface of the body of the inferior maxilla, to the body and greater cornu of the hyoid bone, uniting with its fellow 
in the middle line. The upper surface of the muscle, which is directed toward the cavity of the mouth, is covered over by the mucous membrane, beneath which are found, on either side, the sublingual gland, Wharton's duct, the gustatory nerve, etc. The external surface of the mylo-hyoid muscle forms part of the floor of the submaxillary triangle, and is in relation with the submaxillary gland.

The tongue is a muscular organ which projects upward and forward from the floor of the mouth. It is attached by its base and throngh several muscles to the hyoid bone, and is connected with the epiglottis through the glosso-epiglottidean folds of mucous membrane. The tongue is composed of a mass of muscular and connective tissue interspersed with much fat, and is partly divided into two symmetrical halves by a fibrous septum. The tongue is connected with the hyoid bone by the hyo-glossus muscle on each side; with the styloid process by the stylo-glossus; with the soft palate by the palato-glossus, and through the genio-hyo-glossus with the symphysis of the lower jaw-bone-this muscle serves to draw the tongue forward and prevents its dropping back into the pharynx and obstructing breathing.

When the mouth is closed its cavity is almost completely occupied by the tongue. The anterior part of the upper surface of the tongue is in contact with the hard palate; the posterior part, with the soft palate and the epiglottis. The tongue is covered by mucous membrane, that covering the under surface and sides of the organ being similar to that of the rest of the mouth. That covering its upper surface, dorsum, is rough, marked by numerous glands, and composed of a thick layer of flat epithelium, which gives it rather a grayish color. If the tongue is lifted away from the floor of the mouth by its tip, the attachment of its under surface to the floor of the mouth, in the middle line, through a membranous band, the frænum linguæ, is seen.

The sublingual glands consist each of a number of lobules, and are located in the front part of the mouth, upon either side of the frænum, resting upon the mylo-hyoid muscle and corered over by the mucous membrane. The location of the glands is indicated by a slight swelling in the floor of the mouth, which presents the little pin-point orifices of their excretory ducts.

Upon either side of the frænum there is a little papilla showing the orifice of Wharton's duct. This is the excretory duct of the 
submaxillary gland; it passes forward, through the floor of the mouth, lying below and to the inner side of the sublingual gland.

Each half of the tongue is supplied by the corresponding lingual artery; this is a large branch which is given off from the external carotid just above the greater cornu of the hyoid bone. It passes forward beneath the hyo-glossus muscle, and ascends beneath this muscle to the under surface of the tongue, where it is continued forward to its tip. The chief vein of the tongue is the ranine, a large branch, which passes backward upon the outer surface of the hyo-glossus muscle and terminates in the internal jugular.

The nerves to the tongue are the hypoglossal, the gustatory, and the glosso-pharyngeal. The hypoglossal descends in the neck as far as the point where the occipital artery is given off from the external carotid; here it passes forward, above and parallel with the greater cornu of the hyoid bone, resting upon the hyo-glossus muscle. The gustatory is one of the branches derived from the third division of the fifth nerve. From its origin it descends in front of the inferior maxillary nerve, lying between the internal pterygoid muscle and the ramus of the jaw; here it communicates with the chorda tympani, from the facial, and passing forward, beneath the body of the jaw and above the submaxillary gland, gives off its branches to the submaxillary ganglion; continued forward, upon the hyo-glossus muscle, it crosses Wharton's duct, and is continued alongside the tongue to its apex, lying directly beneath the mucous membrane. The glosso-pharyngeal is of but little surgical importance. It descends in the neck, in front of the internal jugular vein and the internal carotid artery, curving forward upon the outer side of the stylo-pharyngeus muscle, to be distributed to the base of the tongue, etc.

The Side of the Face.-Passing transversely from behind forward beneath the integument, the zygomatic arch may be felt. This bony arch is formed by the junction of the zygomatic process of the temporal with that of the malar. It is a prominent landmark, and serves to separate the side of the head, the temporal region, from the side of the face, the pterygo-maxillary region.

The Pterygo-sraxillary Region corresponds to that part of the side of the face which is situated below the level of the zygoma.

The skin of this region is intimately connected with the underlying subcutaneous connective tissue, which is thick and only loosely attached to the fascia covering the masseter muscle. 
The masseter muscle is a strong, thick muscle arising by two portions from the lower border and inner surface of the zygoma. Its fibers pass downward, covering the ramus of the jaw, to the outer surface of which and to the angle of the jaw it is attached. It is covered by an expansion of the cervical fascia, which is attached above to the lower border of the zygoma. The facial artery crosses the lower border of the inferior maxilla just in front of the masseter muscle, grooving the bone in this situation and passing upward and forward across the cheek to the side of the nose. It is accompanied by the facial vein, which joins with a branch from the temporo-maxillary and thus constitutes a big branch, the temporo-facial, which terminates in the internal jugular.

After the skin and subcutaneous fat have been removed in this region the parotid gland is exposed. This gland is situated upon the side of the face, reaching from the zygoma, above, to below the angle of the jaw. It lies in the space bounded by the angle of the jaw and the posterior border of the ramus in front, and the mastoid process behind, and extends forward upon the side of the face, lying upon the back part of the masseter muscle. The parotid gland is covered by a strong layer of fascia, which forms a sort of fibrous envelope and sends prolongations into the gland to support it. This fascia is continued forward on to the masseter and buccinator muscles, and downward upon the side of the neck, where it is continuous with the cervical fascia. It is also attached to the angle of the jaw. The duct of Stenson (duct of the parotid gland) is about two inches long and lies about a finger's breadth below the zygoma, passing forward across the masseter, at the anterior border of which it pierces the cheek to enter the mouth opposite the second molar tooth of the upper jaw.

The facial nerve, after emerging from the styloid foramen, passes forward and downward into the substance of the parotid gland. It crosses the external carotid artery and divides in the substance of the parotid gland into several branches, which form the pes anserinus and which are distributed upon the side of the face to supply the muscles, etc.

The auriculo-temporal nerve emerges upon the face behind the neck of the condyle of the jaw after passing through the upper part of the parotid gland. It ascends across the root of the zygoma, in front of the ear, in company with the temporal artery, to be distributed upon the side of the head (temporal region). 
Beneath the parotid gland or within its substance the external carotid artery divides into its terminal branches: the internal maxillary and the temporal. The temporal ascends through the substance of the gland and across the root of the zygoma, just in front of the cartilage of the ear, the auriculo-temporal nerve lying posterior to it; and about two inches above the zygoma it divides into the anterior and posterior temporal. These branches, lodged in the subcutaneous connective-tissue layer of the temporal region, divide and supply this part of the scalp, anastomosing anteriorly with branches from the frontal and posteriorly with the occipital, etc. The internal maxillary artery is not exposed until after the removal of the ramus of the jaw, etc. (see later). The temporal artery is accompanied by the temporal vein. The temporal vein does not lie within the substance of the parotid gland, but superficial to it; it receives many tributaries, and below the angle of the jaw divides into two branches; the posterior joins with the posterior auricular to form the external jugular vein; the anterior joins with the facial to form a large branch, the temporo-facial, which passes obliquely backward across the upper part of the superior carotid triangle, to enter the internal jugular. This branch is often cut in extirpating glands, etc., in this part of the neck, and may give rise to profuse hemorrhage.

The deeper parts of this region are exposed by dividing the zygomatic arch with the chisel or chain-saw at its anterior and posterior extremities, and then, after cutting the attachment of the temporal fascia from its upper border, turning the detached segment of the arch, with the attached masseter, downward. There is then exposed the upper part of the ramus of the jaw, with its coracoid process, to which the tendon of the temporal is attached. This process is now cut away from the ramus, and, together with the attached tendon of the temporal, turned upward, and we then have exposed to view the pterygo-maxillary region proper. Occupying this space is the external pterygoid muscle. This muscle arises, by its broad anterior end, from the under surface of the great wing of the sphenoid and from the outer surface of the external pterygoid plate; behind, its narrow end is attached to a depression in the anterior surface of the neck of the condyle of the lower jaw and to the anterior margin of the interarticular fibrocartilage of the temporo-maxillary joint. Curving around its lower border and passing forward and upward upon its outer surface may be seen the internal maxillary artery. This vessel gives off branches to the adjoining 
muscles and disappears, anteriorly, by passing into the sphenomaxillary fossa between the two heads of the external pterygoid muscle. This vessel may now be cut away and the muscle cut short at its attachment to the condyle of the jaw and also close to its origin, and in this way the parts which lie beneath the external

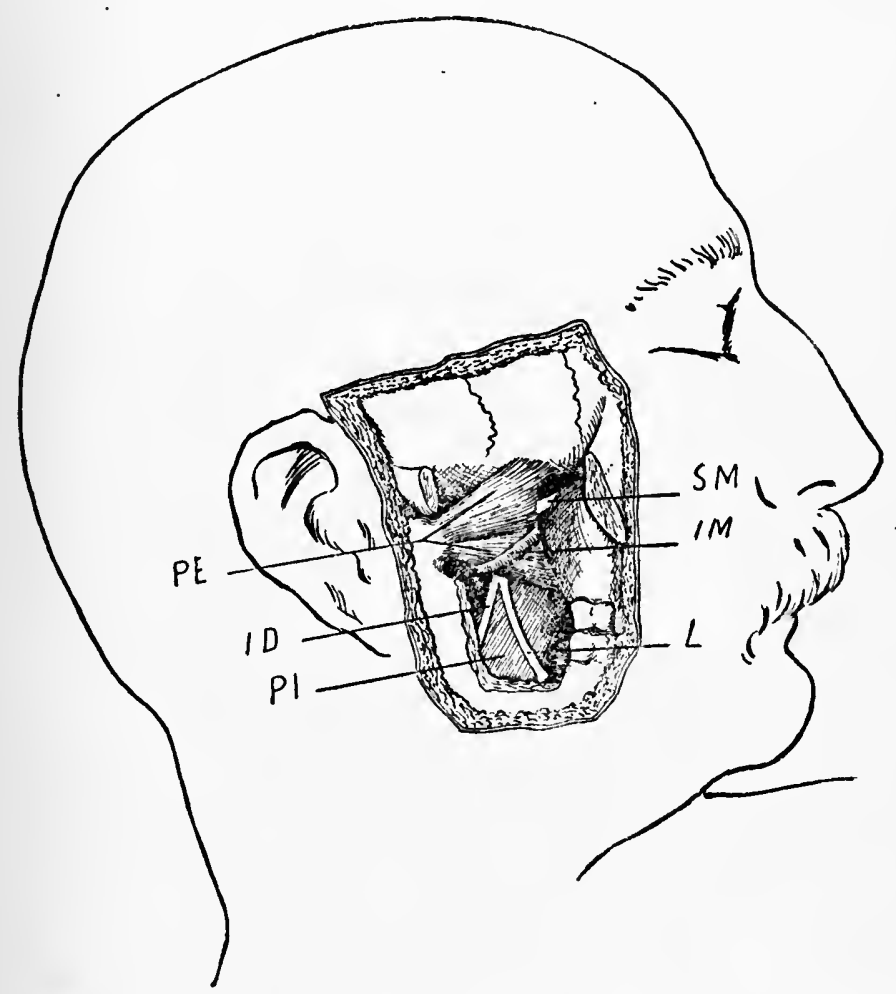

Fig 17.-Pterygo-maxlllary Region. Ramus of the jaw and the zygomatic arch cut away. $I D$, inferior dental nerve; $I M$, internal maxillary artery; $L$, lingual, or gustatory, nerve; $P E$, external pterygoid muscle; $P I$, internal pterygoid muscle; $S M$, superior maxillary (second division of fifth) nerve crossing the spheno-maxillary fossa from behind forward.

pterygoid muscle are exposed,-the zygomatic and spheno-maxillary fossæ, with their important vascular and nervous structures.

The zygomatic fossa is that space which is limited above by the prominent horizontal ridge called the pterygoid ridge which is found upon the under surface of the great wing of the sphenoid 
about opposite the zygoma. The floor of the zygomatic fossa is composed of the under surface of the great wing of the sphenoid (base of the skull) from the pterygoid ridge to the base of the pterygoid process, and also of the surface of the external plate of the pterygoid process. It presents the foramen ovale and the foramen spinosum.

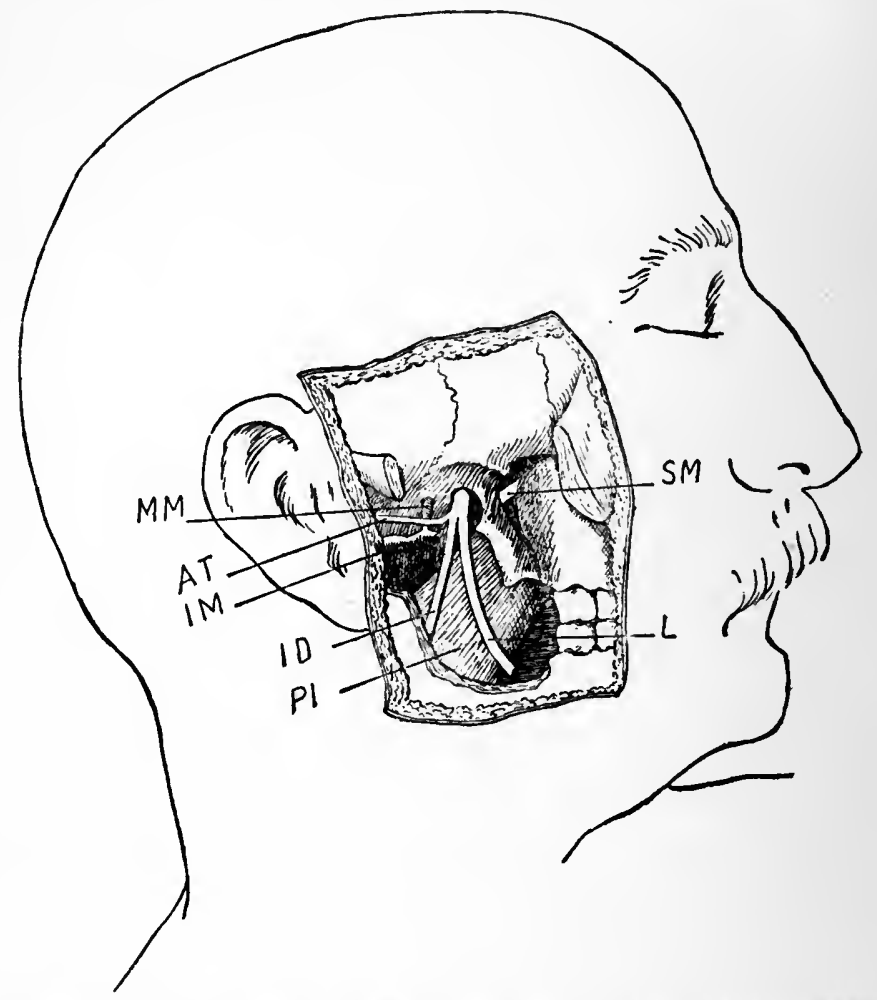

Fig. 18.-Pterygo-maxillary Reglon. External pterygold muscle cut away, exposing external pterygoid plate, etc. $A T$, auriculo-temporal nerve; $I D$, inferior dental nerve; $\boldsymbol{I M}$, internal maxillary artery; $L$, lingual, or gustatory, nerve; $\boldsymbol{M} \boldsymbol{M}$, middle meningeal artery; $\boldsymbol{P I}$, Internal pterygoid muscle; $\boldsymbol{S M}$, superior maxillary (second division of the fifth) nerve passing across the spheno-maxillary fossa.

The spheno-maxillary fossa is the narrow perpendicular space which is bounded in front by the posterior aspect of the superior maxilla and behind by the front of the pterygoid process. Its inner wall is formed by the vertical plate of the palate bone and constitutes a part of the lateral wall of the nasal cavity. Above, this space 
is bounded by the orbital process of the palate bone and the body of the sphenoid. The inner wall presents, above, the spheno-palatine foramen, through which it communicates with the nasal cavity and below the upper opening or commencement of the posterior palatine canal. Into the upper part of this fossa, upon its posterior wall, the foramen rotundum opens; above and internal to this is the opening of the Vidian canal. The anterior wall of this space presents the commencement of the infra-orbital canal.

Located between the inner surface of the condyle of the lower jaw and the internal lateral ligament is the first part of the internal maxillary artery; in this situation the vessel gives off the middle meningeal branch, which passes directly upward and enters the skull through the foramen spinosum. The middle meningeal artery, at its origin, is surrounded by the two roots of the auriculo-temporal nerve; these two roots join posteriorly to form the auriculo-temporal, which passes backward, as far as the temporal artery, and, after emerging from the upper part of the parotid gland, ascends in front of the ear, to be distributed to the integument of the temporal region.

A little in front and to the inner side of the middle meningeal artery may be observed the inferior maxillary division of the fifth nerve. This trunk consists of a large sensory root and a smaller motor root, which emerge from the skull through the foramen ovale and join together outside this opening, just below the base of the skull, to form the inferior maxillary division.

The inferior maxillary division gives off two temporal branches, which pass upward beneath the temporal muscle, and two large branches, which pass downward and forward. One of these, the lingual or gustatory, is joined below by the chorda tympani, a branch of the facial, and the other, the inferior dental, enters the canal on the inner surface of the ramus of the jaw to supply the lower teeth. Attached to the inner posterior aspect of the inferior maxillary division is the otic ganglion; it is located just below the foramen ovale.

In the upper part of the spheno-maxillary fossa is seen the middle, or superior maxillary, division of the fifth nerve. This nerve leaves the skull through the foramen rotundum, passes forward, across the upper part of the spheno-maxillary fossa and, as the infra-orbital, and accompanied by the terminal branch of the internal maxillary artery, enters the infra-orbital canal, and is finally 
distributed to the skin of the front of the face, below the orbit. Suspended from the lower border of the middle division, as it passes across the upper part of the spheno-maxillary space, is Meckel's ganglion, with its descending palatine branches, etc.

We may now remove rather more of the ramus of the jaw in order to expose more completely the internal pterygoid muscle. This is seen to arise from the inner surface of the external pterygoid plate, and, passing downward, backward, and outward, is attached to the inner surface of the angle of the jaw. Between this muscle and the inner surface of the ramus of the jaw are the inferior dental nerve, which enters the canal on the inner surface of the ramus, and the lingual, which is joined by the chorda tympani. The internal lateral ligament of the jaw may also be seen in this dissection.

\section{OPERATIONS UPON THE FACE.}

Resection of the Upper Jaw.-The chief danger in this operation is from the entrance of blood into the larynx. This may be avoided by previously ligating the external carotid or by a preliminary tracheotomy and the use of a Trendelenburg tampon cannula; or an ordinary tracheotomy tube may be used, in this latter case packing the pharynx, through the mouth, with a gauze pad. The operation may be done without a preliminary tracheotomy by operating with the patient in the Rose position, the head hanging over the end of the table, so that the field of operation is upon a lower level than the larynx. It is said that the dependent position of the head, the Rose position, favors venous hemorrhage, which would be a disadvantage. The operation may be done with the patient in a halfsitting position, using incomplete morphin-chloroform narcosis, the patient being but partly anæsthetized, and therefore able to cough and keep the larynx clear of blood.

The incision should be so placed as to aroid Stenson's duct.

Weber's Ircision.-Reaching from the inner angle of the eye, the incision is carried down alongside of the nose and around the ala to the middle line, terminating by splitting the upper lip. To this is added a second incision reaching from the inner angle of the eye, outward, below the lower margin of the orbit. This second incision should pass along the lower edge of the orbicularis palpebrarum in order to avoid cutting into the substance of this muscle. These incisions should penetrate to the bone. Branches of the facial 
nerve are not cut in this incision. The flap which is thus marked out is reflected outward, and should be raised subperiosteally if the character of the disease permits. The infra-orbital vessels and nerve are cut when the flap is separated from the anterior surface of the superior maxilla.

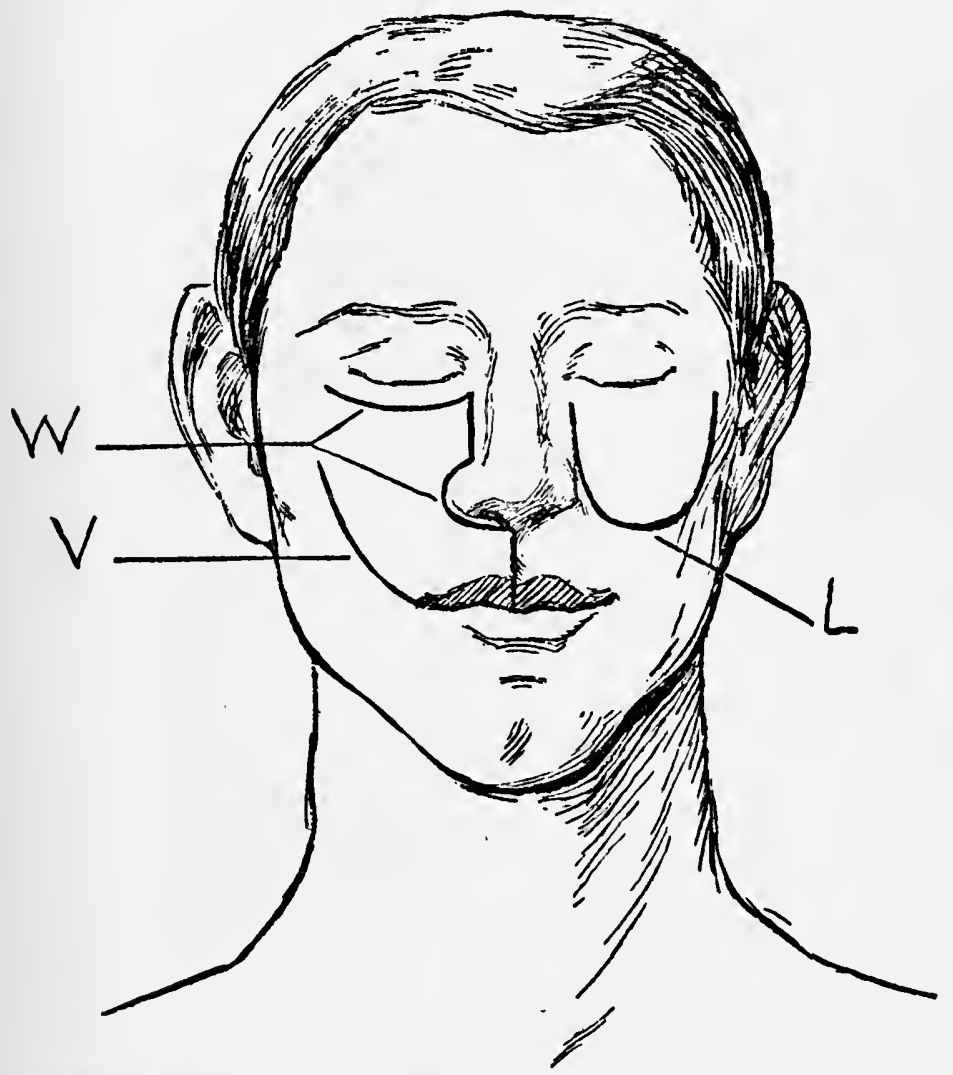

Fig. 19.-Resection of Upper Jaw. $L$, Langenbeck Incision; $\boldsymbol{V}$, Velpeau incision; $W$, Weber incision.

LANGENBECK's INCISION.-A flap, its lower border curved with the convexity downward, is raised. The incision commences at the inner angle of the eye, and passes down alongside of the nose to a point below the level of the ala, as far as the attachment of the upper lip to the alveolar process of the superior maxilla; here it curves outward, corresponding to a line drawn from the ala of the 
nose to the lower border of the lobe of the ear, and is then carried upward to a point over the prominence of the cheek-bone. This incision does not divide the lip, but it will be necessary later to separate the lip from its attachment to the jaw-bone. It divides some branches of the facial nerve, which is a disadvantage. The front surface of the bone is exposed by reflecting this flap upward, subperiosteally, if the conditions permit. In raising the flap from the bone the infra-orbital vessels and nerve are divided.

In making either of these incisions the facial artery is divided and must be clamped and ligated.

After the soft parts have been detached from the bone the cartilage of the nose is separated from the nasal notch, and the soft parts, corresponding to the lower margin of the orbit, raised from the bone, and the tarso-orbital fascia cut along the margin of the orbit. The floor of the orbit is now exposed, and the contents of the orbit raised out of the way with a blunt retractor. We are then ready to cut through the nasal process of the superior maxillary. This division should extend from the margin of the nasal notch, across the nasal process, as far as the lacrymal groove or fossa. One should avoid injury to the lacrymal sac, the upper expanded part of the lacrymal canal, which is lodged in the lacrymal depression upon the lacrymal bone. The division of this process of bone may be accomplished with a chisel, or one may make a hole in the lacrymal bone, which is very thin, just in front of the lacrymal sac, and introduce through the orbit a Gigli saw, bringing its end out through the nasal notch; the Gigli saw is carried around the bone with a loop of silk in a curved needle. Probably a chisel is more convenient for this part of the operation.

We next separate the jaw from its attachment to the malar bone. This may be done with a chisel or with a chain or Gigli saw. The line of division should extend through the maxillary process of the malar bone into the anterior end of the spheno-maxillary fissure. If this section is made with a chain or Gigli saw, the instrument may be carried around the bone with a loop of strong silk in a large, full-curved needle. The contents of the orbit being well retracted, the needle is passed into the orbit, through the sphenomaxillary fissure, and then out through the zygomatic fossa, emerging upon the face below the malar process; the suture is then pulled through, drawing the saw, which thus surrounds the malar bone at its junction with the superior maxillary, after it; the division may 
then be readily made. If it is desired to take the malar bone away in addition to the superior maxillary, the needle, after entering the spheno-maxillary fissure, as above described, should be made to traverse the temporal fossa, appearing above the upper border of the malar bone, thus surrounding its frontal process; after this process has been divided the zygomatic arch may be cut through

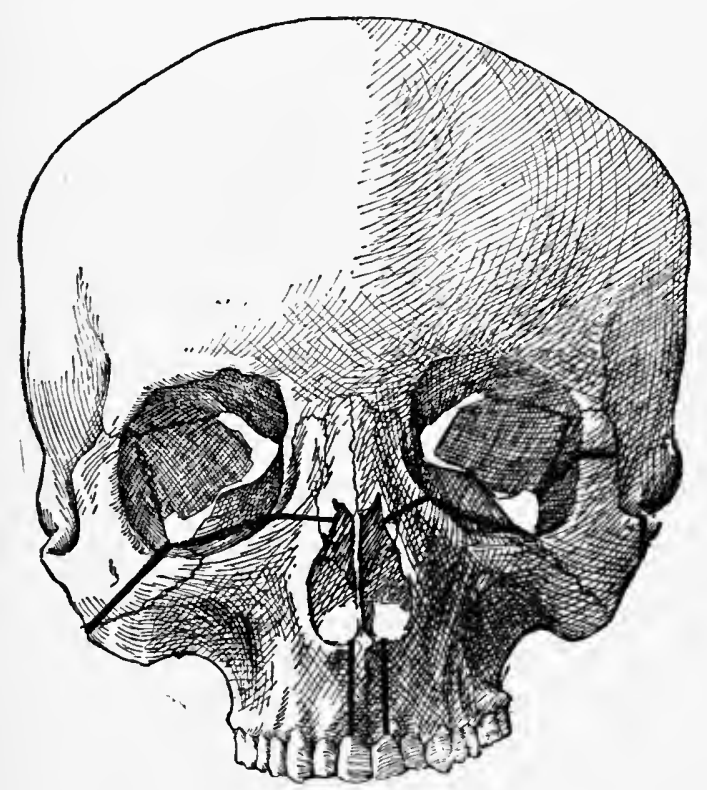

Fig. 20.-Resection of Upper Jaw. When it is desired to leave the major part of the malar bone, the line of section through the bone should be as indicated upon the right side of the skull. If the malar bone is to be removed together with the superior maxillary, the section through the bone should be as is represented upon the left side of the skull, the line of division passing through the frontal process of the malar and the zygoma.

with the chisel, thus separating the malar bone from its connection with the temporal bone.

We are then ready to make the division through the hard palate; this is best done as the last step of the operation, after the other connections have been severed, on account of the hemorrhage into the mouth. Before dividing the hard palate the muco-periosteal layer, which covers it, is detached. An incision is made in the mucoperiosteal covering of the hard palate, commencing anteriorly just 
behind the incisor teeth; this is carried back along the side of the hard palate, close to the alveolar process, as far as the attachment of the soft palate to the posterior border of the hard palate. With a periosteum elevator, this layer is separated from the surface of the hard palate, as far as the middle line; the soft palate is also separated from the corresponding half of the posterior border of the hard palate. A chisel is then placed in the middle line between the two incisor teeth, and the hard palate divided down the middle for its whole length. It is probably better, in some cases, to accomplish this division with a saw. For this purpose we use a narrow saw, which is introduced into the nasal cavity, after the first incisor tooth of the jaw which is to be excised has been extracted, sawing through the floor of the nasal cavity from above downward and from before backward.

The jaw-bone is now free except for its attachment, behind, to the palate bone and to the pterygoid plate of the sphenoid. The floor of the orbit, which is very thin, may be cut through, just behind its anterior margin, with one or two strokes of the chisel, this line of section reaching from the lacrymal fossa across the floor of the orbit into the spheno-maxillary fissure. One should finally see that the soft parts are separated from the facial surface of the bone, well beyond the last molar tooth; this may be done with a few sweeps of the knife, cutting close to the surface of the bone.

The body of the jaw is now seized with a strong bone forceps, and, with a gradually increasing rocking motion, it is forcibly wrenched from its remaining attachment. Usually all of the palate bone, except its orbital process, comes away with the superior maxilla and there is left remaining a part of the orbital surface of the superior maxilla sufficient to support the contents of the orbit. If part of the pterygoid process comes away with the superior maxillary, the bone will still be held by some of the muscles which arise from this process, - the internal and external pterygoids,-and it will be necessary to divide these with a sweep of the knife before the bone can be removed.

There is left a large bloody space, but, as a rule, there is little or no hemorrhage, owing to the tearing of the blood-vessels in wrenching the bone free. The infra-orbital vessels and nerve may be seen hanging free in the wound. The vessels, which may bleed freely, should be seized at once, clamped, and tied, and the nerve cut short. The other branches of the internal maxillary artery also 
are exposed,- the descending palatine and spheno-palatine,-and these should also be clamped and tied.

The wound may now be irrigated and tamponed, the ends of the gauze emerging through the nostril. The incision upon the face is closed with interrupted silk sutures, but, before doing this, the edge of the muco-periosteal flap, which was raised from the surface of the hard palate, should be sutured, with interrupted silk sutures, to the inner side of the cheek, along the line where this was separated from the alveolar process of the superior maxilla. The ends of these sutures should be left rather long and presenting into the mouth, to facilitate their removal later.

During the operation the back of the mouth and the pharynx may be kept clear of blood with gauze pads on long holders.

Total Resection of Both Superior Maxillæ.-This operation is analogous to the preceding.

A curved incision, passing from the angle of the mouth outward and upward to the malar bone on each side, or a double Weber incision, may be used.

The nasal septum, vomer, is divided with bone scissors, and the soft parts, as a whole, including the nose, are then detached and reflected upward, or, if a double Weber incision is used, the lateral flaps are separated from the bone and reflected outward.

The attachments of the superior maxillæ are then divided as in the preceding operation, except that it will not be necessary to split or cut through the hard palate, as this is taken away entirely. If possible, the muco-periosteal covering of the hard palate should be stripped off and preserved; this is done by separating it, with an elevator, through a curved incision which penetrates through this layer down to the bone and which is placed just inside the line of the teeth. The soft palate, at its attachment to the posterior border of the hard palate, is also completely separated. Finally, with lionjaw forceps, the bone is forcibly wrenched free, as in the preceding operation.

The soft parts are then brought together with silk sutures, first uniting the edge of the muco-periosteal flap, which was raised from the hard palate, to the inner side of the cheeks, corresponding to the line where they were separated from the alreolar process.

To Drain the Antrum of Highmore. Throvgh tile Tootir SocKeT.-Empyema is frequently associated with carious teeth. These or their remaining roots may be extracted and an opening 
made into the antrum by gouging out the alveolar cavity, which is often found to be carious. This may be done, as a rule, with a sharp spoon or with a narrow chisel. The chisel should be directed upward toward a point corresponding to the middle of the lower margin of the orbit. Such an opening, if made sufficiently large, provides satisfactory drainage from the antrum. A strip of gauze may be introduced to drain the cavity and to prevent the entrance of particles of food. The opening should be made through the alveolus of the second biscuspid, or, better, the first molar tooth.

Throvgh the Axterior Wall.-Drainage may be established by making an opening through the front wall of the antrum. The upper lip is everted and the nucous membrane cut and the soft parts separated from the front surface of the bone with the periosteum elevator. The front wall of the antrum is perforated through the canine fossa just above and to the outer side of the canine tooth. The socket of this tooth is marked by a prominent ridge.

After the periosteum has been stripped off the bone a goodsized opening is made into the antrum with the chisel or with a strong, sharp-pointed perforator or with a drill. The instrument should be directed upward and somewhat backward toward the floor of the orbit, but care should be taken to avoid entering the antrum abruptly with such force as to endanger the floor of the orbit. A drainage tube may be introduced and left in place for several days until the drainage opening is well established. It is advisable to use a tube with a bulbed end to prevent its slipping out.

This operation may well be combined with drainage through the tooth socket as described above. Both operations may be done with the patient in the Rose position or with partial morphin-chloroform anæsthesia.

Throdgh the Lateral Wall of the Nose.-Mikulicz advises making an opening in the lateral wall of the nose just below the middle of the inferior turbinated. This may be done with a sharppointed perforator somewhat bent upon itself near the end. The bone is thin, and the operation is readily done except when the nasal cavity is narrow or the inferior turbinated much hypertrophied.

Resection of Half of the Lower Jaw.-The incision commences at the middle of the chin and follows along the lower border of the body of the jow as far as the angle, whence it is continued upward along the posterior border of the ramus as high as the lower border of the lobe of the ear (one may cut to this point without-danger of 
injuring the facial nerve; see Fig. 79). This incision for its whole extent should reach to the bone. There may be added in front a vertical incision, splitting the lower lip through the middle line, but this is usually unnecessary. The facial vessels are severed in making the incision along the lower border of the body of the jaw-bone, and these should be clamped and tied.

If the glands, etc., in the submaxillary region are diseased, instead of the above-described incision one may be made which commences anteriorly, in the middle line, at the lower border of the jaw, from which point it passes backward and somewhat downward across the submaxillary triangle, deviating from the lower border of the jaw as it passes backward, as far as the anterior border of the sterno-mastoid muscle, whence it is turned upward toward the apex of the mastoid process. This incision passes through the integument and the platysma. The flap which is thus outlined is turned up over the side of the face, and we are then enabled, as a preliminary step, to clear out the submaxillary triangle, and before doing this we can, if desired, easily expose and ligate the external carotid artery. Some surgeons precede the operation with a preliminary tracheotomy, introducing a tampon cannula; or an ordinary tube may be introduced and the pharynx tamponed. These measures eliminate the danger of blood being inspired into the trachea.

Having cleaned out the submaxillary triangle, or, if this has not been necessary, through the incision along the lower border of the body, the soft parts are separated from the external surface of the body and ramus of the jaw, back as far as the angle, working close to the surface of the bone; the attachment of the masseter is thus separated from the ramus. The separation of the masseter and, in fact, the soft parts from the body of the bone as well, is accomplished with a periosteum elevator, occasionally snipping with the knife. It is desirable, if the nature of the condition present permits, to make this separation subperiosteally. In the mass of soft parts which is raised from the outer surface of the ramus of the jaw are included, besides the masseter muscle, the parotid gland and Stenson's duct, the facial nerve, and the temporal artery. None of these structures are injured if one works close to the surface of the bone. Now, with a clean cut, the cavity of the mouth is entered, incising the mucous membrane close to the anterior border of the ramus and along the dental margin of the body of the jaw as far as the middle line; in this way the outer surface of the lower jaw, 
including the teeth, is laid bare. Anteriorly, where the body of the jaw is to be divided, a tooth is extracted and the floor of the mouth, close to the bone, incised, so that the chain or Gigli saw may be carried around the bone. This is done with a loop of strong silk in a large curved needle, and then the body of the jaw is divided. This division may also be accomplished with a metacarpal saw. The section through the body of the jaw, in front, should, if possible, be made a little external to the middle line, toward the side of the disease, in order to avoid separating the genio-hyoid and genio-hyoglossus muscles from their attachment to the tubercles on the inner aspect of the symphysis mentis. If these muscles are separated from their attachment to the jaw, there is a great tendency, both during and after the operation, for the tongue to drop back into the pharynx, closing down the epiglottis and thus greatly interfere with the patient's breathing.

After the bone has been divided in the middle line its free end is seized with a bone forceps and drawn outward, thus putting the structures attached to its inner surface (floor of the mouth) on the stretch, and they are then divided close to the dental margin (teeth) with a scalpel. If the condition of the periosteum permits, these parts may be separated from the inner surface of the jaw subperiosteally with an elevator. The body of the bone, still firmly grasped with the bone forceps and being now freely movable, is dragged forcibly downward and out of the wound so that the operator can reach the coracoid process to which the tendon of the temporal muscle is attached; this is separated with a knife, cutting close to the bone and aroiding the internal maxillary artery and the bone is then still further luxated. Behind, attached to the inner surface of the ramus of the jaw, at the angle, is the internal pterygoid muscle; this is also cut away close to the surface of the bone.

The inferior dental vessels and nerve enter the jaw-bone through the inferior dental canal on the inner surface of the ramus; these structures may be cut or torn, but before being cut they should be grasped with an artery forceps; later the vessels are tied and the forceps removed, liberating the nerve. If the inferior dental should bleed in the sawn surface of the bone this may be stopped by plugging the orifice of the canal with a strand of catgut. The flap of soft parts is now drawn forcibly upward, and the bone, still held with the bone forceps, dragged downward; so that the outer wall of the capsule of the temporo-maxillary joint may be reached with 
the point of a sharp knife and incised; the tendon of the external pterygoid, which is attached to the front of the neck of the condyle, is likewise divided. In cutting these structures the knife is kept applied close to the surface of the bone in order to avoid the internal maxillary and the temporal arteries. The bone may now be readily twisted out of its socket.

If it should be necessary to separate the muscles of the tongue from their attachment to the symphysis of the jaw, a thick silk ligature should be previously passed through its tip, to be used as a tractor to prevent its being drawn backward into the pharynx and closing the larynx and interfering with respiration. It is probably advisable to introduce such a ligature in all cases.

The cut edge of the mucous membrane, which was separated from the inner aspect of the jaw-bone, is now accurately sutured to the corresponding edge of the mucous membrane, which was separated from the outer aspect, except for a short space behind through which the cavity of the mouth is drained; these sutures should be of silk, knotted on the inside of the mouth, and the ends left sufficiently long to allow of their ready removal later.

The edges of the skin are approximated with interrupted sutures except at the posterior part where the drain emerges.

For the purpose of facilitating drainage, the wound is loosely packed with iodoform gauze, reaching into the cavity of the mouth. This may be removed after a few days, when a sinus is established through which all secretions from the mouth may find exit.

Resection of Half of the Body of the Lower Jaw. - A strong, thick suture is passed through the tongue for use as a tractor, if this becomes necessary. An incision is made along the lower border of the body of the jaw from the middle line in front to the junction of the body and ramus just beyond the last molar, behind; this incision penetrates to the bone. In many cases the facial artery, where it curves over the lower border of the body of the jaw, just in front of the masseter, is divided; but frequently this may be avoided. If the vessel is cut it must be clamped and ligated. There may be added anteriorly a vertical incision which splits the lower lip in the middle line; but, as a rule, this is unnecessary, and should be avoided.

With the elevator or knife, working close to the surface of the bone, the soft parts are separated from the outer surface of the body of the jaw, finally cutting through the mucous membrane close to 
the teeth and thus entering the mouth and exposing the outer surface of the body of the bone and the teeth.

The floor of the mouth is now perforated, anteriorly, near the middle line, close to the inner surface of the bone, and, after extracting a tooth, the Gigli or chain saw is introduced, being carried around the jaw with a loop of silk in a strong curved needle, and the bone is then sawn through; this section should be made to the side of the middle line in order not to disturb the attachment of the muscles of the tongue to the symphysis. If the end of the divided bone bleeds, this may be controlled by plugging the orifice of the canal which contains the nutrient artery. The end of that half of the bone which is to be excised is now seized with the bone forceps and drawn strongly outward, thus putting the soft parts attached to its inner surface (floor of the mouth) upon the stretch. These parts are separated from the inner surface of the bone as far back as the junction of the body with the ramus-beyond the last molar tooth. This may be done bluntly with an elevator, separating subperiosteally, or, if this is contra-indicated on account of the character of the disease, these parts, including the mucous membrane, may be simply cut away from the bone with the knife. After having thus stripped the body of the bone of its soft parts, both upon its outer and its inner or buccal surface, the saw is applied just behind the last molar tooth and the bone cut through. This may be done with the chain or Gigli saw or with a narrow metacarpal saw. While the bone is being divided it should be drawn well downward with the bone forceps.

Hemorrhage from the cut surface of the bone is controlled with a plug of catgut, which is packed into the orifice of the dental canal.

The mucous membrane, which was separated from the outer surface of the segment of bone which has been resected, is sutured to the cut edge of the parts which were separated from the inner surface of the bone. This closes in the cavity of the mouth, and may be done with interrupted silk sutures tied within the mouth, the ends being left moderately long, so that they may be readily removed.

The incision in the skin is closed in part, leaving the posterior end open for drainage. It is probably wise, in most cases, to leave a small opening through the mucous membrane also, so that the cavity of the mouth may be drained; in this case the gauze, which is introduced into the posterior portion of the skin incision, is packed into the mouth. 
Resection of the Entire Body of the Lower Jaw.-This is analogous to the preceding operation, but special care must be exercised to guard against the tongue dropping back into the pharynx after the attachment of the muscles, which pull it forward, have been separated from the inner surface of the symphysis. This accident may be prevented by passing a ligature through the tip of the tongue by which traction may be made.

There is also considerable danger of the tongue dropping back and obstructing the breathing after the operation, and this accident night easily cause the death of the patient; so that the tractor should be allowed to remain in the tongue and fixed outside. The jaw-bone is divided in the middle line, and then each half is resected separately as described in the preceding operation.

Resection of Part of the Body of the Lower Jaw in Continuity. From Wrthix the Mouth.-Precautions must be taken to prevent blood entering the larynx during the operation (see "Resection of the Upper Jaw," etc.). A mouth-gag is introduced and an incision is made through the mucous membrane on either side of the teeth, and the soft parts separated from the inner and outer surfaces and from the lower border of the segment of the jaw-bone that is to be excised, with an elevator. A tooth is then extracted and the Gigli saw passed around the bone with a loop of silk in a large curved needle and the bone divided; this procedure is repeated at the other end of the segment of bone which is to be excised. The hemorrhage from the cut ends of the bone is controlled by a plug of catgut packed into the dental canal. One may separate the soft parts from the surface of the bone subperiosteally, as above described, but in most cases this is not permissible on account of the character of the disease. After removal of the segment of bone the edges of the mucous membrane may be brought together, at least in part, by interrupted silk sutures. A small opening may be made externally through the skin for drainage.

If the anterior portion of the body is resected, necessitating the separation of the tongue muscles from the symphysis, proper measures must be taken to guard against the tongue's dropping back upon the epiglottis and larynx. The operation done from within the mouth is ordinarily rather disadvantageous, as one is unable to properly drain the wound afterward.

From Without.-An incision is made along the lower border of the body of the bone corresponding to that part of the bone 
which is to be resected and reaching down to the surface of the bone. Usually it is not necessary to split the lower lip. The soft parts are separated from the outer surface of the body of the bone with the elevator, if permissible, subperiosteally, and the mucous membrane then incised close to the teeth, thus opening into the mouth. Corresponding to the points at which the bone is to be divided the teeth are extracted and incisions made in the floor of the mouth close to the bone to allow the passage of the Gigli saw; this is carried around the bone with a loop of silk in a full curved needle and the bone then divided from within outward. The segment of bone, which has been thus loosened and to the inner aspect of which the soft parts of the floor of the mouth are still attached, is seized with the bone forceps and the soft parts (mucous membrane and muscles of the floor of the mouth) are then separated with the elevator or cut with the knife close to the surface of the bone and near its alveolar margin. Hemorrhage from the bone may be controlled by plugging its nutrient canal with a piece of catgut.

The mucous membrane, which was separated from the outer surface of the resected segment, is united to that which was separated from the inner surface with several interrupted silk sutures, tied within the mouth, in this way closing in the cavity of the mouth. The external wound is partly closed and drained.

If the part resected corresponds to the anterior portion of the body of the jaw-bone, one should secure the tongue by passing a silk suture through its tip.

Resection of Part of the Body of the Lower Jaw (Not Through. Entire Thickness, Not in Continuity).-Practically as described in the preceding operation, working either from within the mouth or without. The operation consists in resecting the diseased part of the bone and leaving a portion of the body, of greater or less thickness, as a bridge to preserve the continuity of the bone and prevent deformity, and to. facilitate the application of an apparatus. The removal of the bone may be effected with a chisel or with the cutting bone forceps. This operation is but seldom practiced.

Extirpation of the Casserian Ganglion (Rose-Andrews).-The incision commences at a point near the external angular process, curving backward above the zygoma to a point just in front of the ear, whence it extends downward to near the angle of the jaw. This incision penetrates through the skin and fat only, and pains should be taken to avoid injuring the parotid gland, Stenson's duct, and 
the facial nerve. The temporal artery, as it ascends in front of the ear, may be divided, in which case it will be necessary to ligate it. This flap is reflected downward sufficiently to expose the zygomatic arch. The temporal fascia is incised along the upper border of the zygomatic arch.

The next step is the division of the zygomatic arch with the chain or Gigli saw, both in front and behind, and the segment which is thus resected, together with the attached masseter muscle, is then reflected downward. Before dividing the zygomatic arch holes should be drilled through the bone corresponding to the intended line of section, so that it may be wired back in place after the operation has been completed. When this flap, including the detached segment of the zygomatic arch and the masseter muscle, is turned down, the coracoid process of the lower jaw and the temporal tendon, which is attached to it, are exposed. The coracoid process is now divided, first drilling holes for subsequent wiring, and together with the attached tendon of the temporal muscle, this is turned upward. There is now exposed the internal maxillary artery, passing from below, forward, and upward across the outer surface of the external pterygoid muscle. This ressel is tied double and divided. With the periosteum elevator the external pterygoid muscle is separated from its attachment to the under surface of the great wing and from the outer surface of the external pterygoid plate of the sphenoid. All hemorrhage should be controlled by ligature or pressure as the operation progresses step by step. Now, with the finger in the wound, one should feel for and recognize the sharp edge of the external pterygoid plate, and tracing this upward, as a guide, feel or see the foramen ovale at its base (see Fig. 18).

A trephine of small diameter is applied to the base of the skull (under surface of the great wing of the sphenoid, which has been laid bare by detaching the external pterygoid) anterior and a little external to the foramen ovale, and here a small button of bone is removed. After this button of bone has been removed the bridge of bone remaining between the trephine opening and the foramen ovale is cut away with a rongeur bone forceps. The third division of the fifth nerve is now seized with a hook and drawn out through the opening in the skull to serve as a guide to the Casserian ganglion, and then the second division of the nerve is also seized with the hook and pulled out through the opening. These trunks are then both divided and 
used as guides to the ganglion, which lies in a direction backward and inward from the foramen ovale, within the skull, upon the apex of the petrous portion of the temporal bone. The cut ends of the nerves, still attached to the ganglion, are steadied in the grasp of a long, narrow artery forceps, and with a curette, introduced through the opening in the skull, the ganglion is destroyed and scooped out.

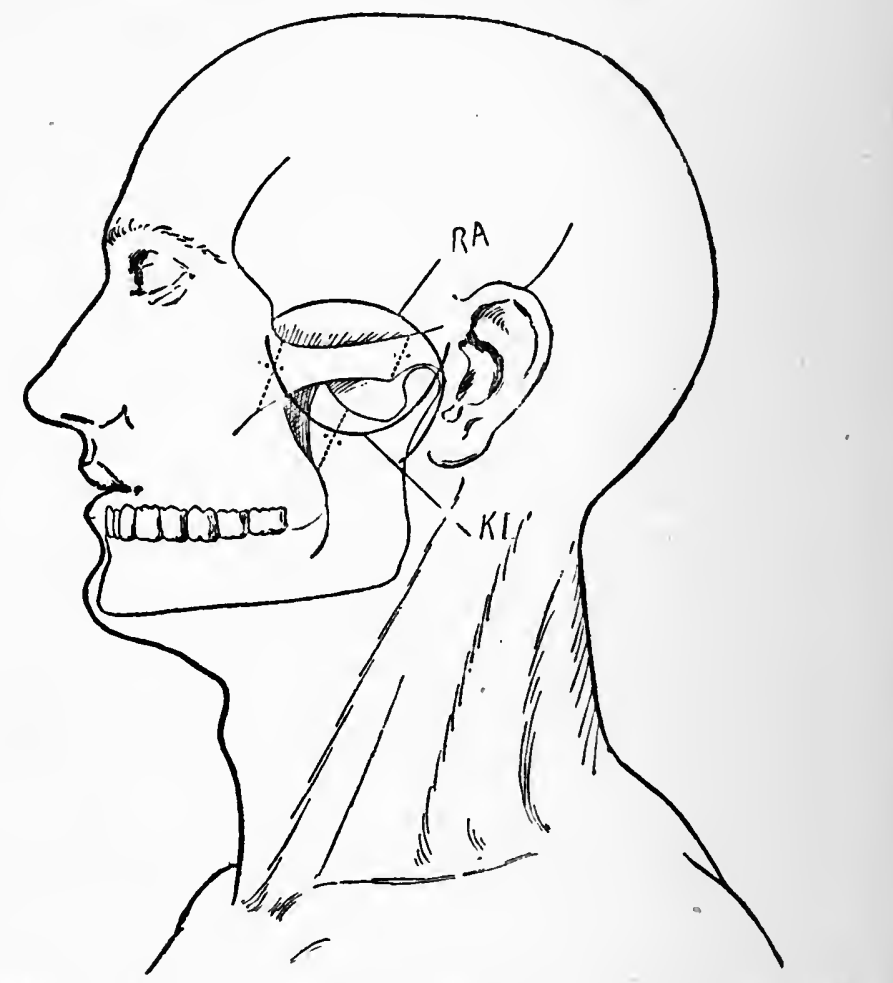

Fig. 21.-Resection of the Casserian Ganglion, etc. $K L$, Krönleln-Lücke Incision; $R A$, Rose-Andrews incision. Dotted lines represent the lines of division through the bones; drill holes for subsequent wiring of the fragments.

The technique of this operation is difficult, as it is almost impossible to reach the ganglion. There is liability to profuse hemorrhage which is difficult to control and also to injury of the Eustachian tube; so that the danger of infection is great. Oozing can be stopped by pressure with a gauze pad. When the operation is finished, the parts are replaced, the coracoid being wired to the ramus of the jaw, 
the segment of the zygomatic arch fixed in place with wire sutures, and the wound in the skin closed.

Division of the Second and Third Branches of the Trifacial Nerve at the Base of the Skull (Kronlein's Modification of Lücke's Operation).-This operation consists in exposing the second and third divisions of the fifth nerve as they emerge from the skull and dividing them or twisting them free from their origin.

An incision marking out a rounded skin-flap, with its convexity downward and its base corresponding to the upper border of the zygomatic arch, is made. It commences anteriorly, one finger's breadth behind the external angular process, and terminates behind, just in front of the tragus. This flap, consisting of the skin and subcutaneous fascia, is raised from the deep fascia covering the parotid gland and masseter muscle, and is reflected upward, thus exposing the arch of the zygoma and the lower portion of the temporal fascia, which is attached to the upper border of the arch. The incision does not reach low enough to injure the facial nerve or Stenson's duct. Bleeding points should be clamped and ligated as the operation progresses.

The temporal fascia, attached to the upper border of the zygomatic arch, is now incised along this border of the arch, and the arch sawn through: first, posteriorly and then anteriorly. Before making this division of the arch holes should be drilled for the purpose of wiring the detached segment in position later. In dividing the arch anteriorly one should take care to get well forward so as to include as much of the length of the arch as possible; the line of division should not be from above directly downward, but from above obliquely downward and forward. This segment of the arch, carrying the attached masseter muscle with it, is reflected downward, exposing the coracoid process of the ramus of the lower jaw and the attached temporal tendon. This process, after making drill holes for subsequent wiring, is then cut away, the line of section extending from the deepest part of the sigmoid notch obliquely downward and forward so as to include practically all that part of the ramus which corresponds to the attachment of the temporal tendon. This segment of bone, carrying the temporal tendon, is reflected upward, and held thus with a retractor. The external pterygoid muscle, and the internal maxillary artery, which passes obliquely across its outer surface, are now exposed. It is well to tie the vessel double and cut it. With the elevator the attachment of the external ptery- 
goid is now separated from the under surface of the great wing of the sphenoid and drawn downward. The finger is then introduced into the space above the upper border of the muscle and is passed inward close to the under surface of the bone (base of the skull), feeling for the posterior sharp edge of the external pterygoid plate and searching for the foramen ovale, which is directly behind and a little external to the root or base of the pterygoid process, external pterygoid plate. We should recognize the thick trunk of the inferior maxillary, or third, division of the fifth nerve as it emerges from the foramen ovale; directly behind this, the middle meningeal artery, surrounded by the two roots of the auriculo-temporal nerve, is seen passing upward to enter the skull through the foramen spinosum (see Fig. 18). The inferior maxillary division is seized with a hook and drawn forward and cut, and then the stump, grasped with a forceps, is twisted free from its origin at the Casserian ganglion. Usually the motor root is grasped at the same time and included with it. We then penetrate into the spheno-maxillary fossa, and in the upper part of this cavity, the superior maxillary, or second, division of the fifth nerve, just before it enters the infra-orbital canal, is seized with the hook and drawn out and cut, and then likewise twisted away from the Casserian ganglion. The Eustachian tube is located close to the inner side of the inferior maxillary, or third, division, and, therefore, just as soon as this trunk of the nerve is accessible, one should not penetrate deeper into the wound for fear of cutting into the Eustachian tube, which would result in certain infection of the wound.

The coracoid process is reunited to the ramus of the jaw with a wire suture and the segment of the zygomatic arch is likewise replaced and wired. The skin incision is then closed.

\section{CONGENITAL DEFORMITIES OF THE FACE.}

The Development of the Face. - About the twelfth day the arrangement of the head end of the embryo is quite simple. A cross section shows it to consist of two tubes, one being situated in front of the other. The anterior is the blind, head end of the alimentary tube: the future pharynx. The posterior is the enlarged neural tube which is later developed into the brain. The anterior wall of this upper, head end of the alimentary tube is called the "oral plate," and marks the location of the future mouth and face. 
A sagittal section will also show this relationship, and further that the neural tube not only lies behind the alimentary tube, but also

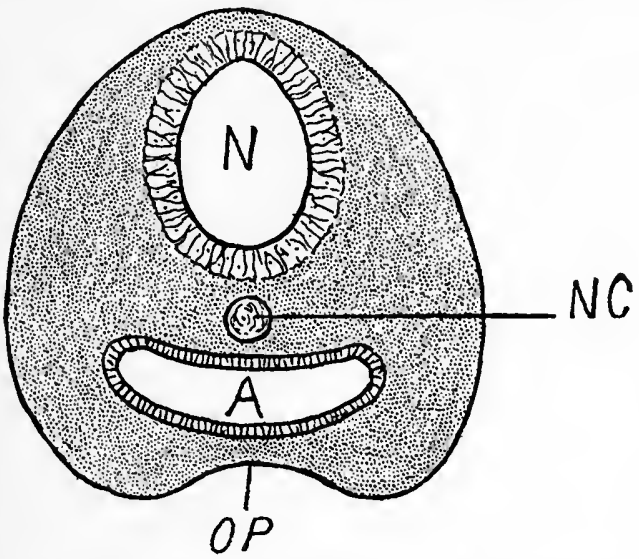

Fig. 22.-Transverse Section of the Head End of an Embryo Twelve Days Old. $A$, allmentary tube; $N$, neural tube; $N C$, notochord; $O P$, oral plate.

arches forward above the upper end of the latter like a hood, overriding it anteriorly. This upper part of the neural tube, which

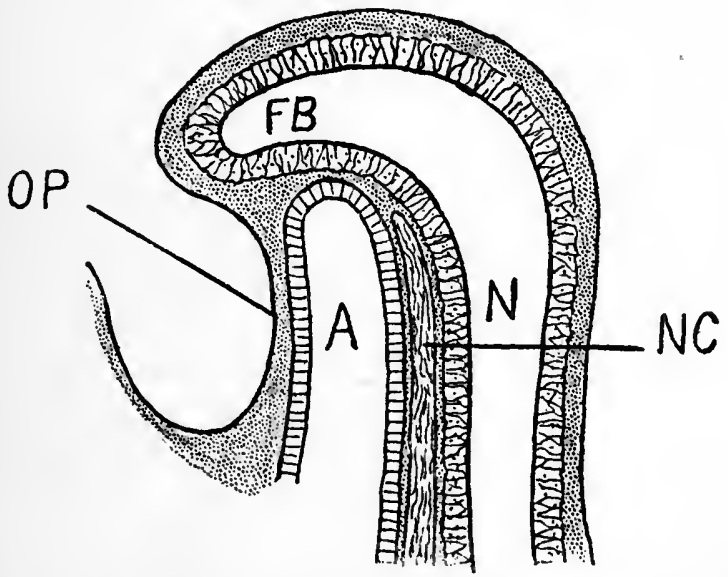

Fig. 23.-Sagittal Section of the Head End of an Embryo Twelve Days old. $A$, alimentary tube; $F B$, vesicle of the forebrain overriding the end of the alimentary tube; $N$, neural tube; $N C$, notochord; $O P$, oral plate (site of future mouth), which ruptures during the fourth week.

projects forward over the end of the alimentary tube, is called the vesicle of the forebrain. 
In the third week there may be seen, upon either side of the head end of the embryo, four transverse plates or ribs of tissue which are separated from one another by deep fissures, or clefts. The thickened plates are called visceral arches, and the intervening spaces, or fissures, visceral clefts. Within the alimentary tube, upon its inner aspect, there may be seen corresponding arches and clefts. These arches are simply thickenings or ribs in the lateral walls of the head end ("schlund," pharynx) of the alimentary tube. Each mass consists of mesoblast, covered upon its outer surface by the epidermic layer, which covers the whole exterior of the body, and upon its inner surface by the endodermic layer, which lines the whole inner surface of the alimentary tube. Between the arches, at the bottom of any two opposed clefts, the wall of tissue is extremely thin; consists practically of the outer (epidermic) and the inner (endodermic) layers. The uppermost of these visceral arches, that concerned in the formation of the face, is the thickest. It extends forward, and in front, where it is narrower, unites in the middle line, with its fellow of the opposite side to form the mandibular arch, which represents the future lower jaw. The second arch is less prominent than the first, and as it passes forward is directed somewhat upward. This second arch does not reach as far as the middle line. The third and fourth arches are still less prominent and still shorter. These lower three arches do not join with their fellows across the middle line in front, but are continued into the plate of tissue which forms the front wall of the (schlund) pharynx. From above downward these arches overlap and partially conceal each other; so that the third and fourth, especially the fourth, are almost entirely concealed by the first and second. The uppermost arch appears earliest. The appearance of these arches is the first indication that marks the commencement of the formation of the face.

Owing to the progressive growth of the visceral arches, which causes a thickening of the parts that immediately adjoin the area already mentioned as the oral plate, and on account of the presence of the prominent overhanging forebrain vesicle (neural tube) above, the oral plate becomes relatively depressed, and we have thus, in its stead, a distinct fossa, which is called the oral pit. The oral pit is bounded above by the overhanging forebrain vesicle and below and upon the sides by the first visceral arches. These are the parts which immediately surround the oral pit and which are finally de- 
veloped into the face; the oral pit represents the future oral and nasal cavities.

The second, third, and fourth visceral arches are not concerned in the formation of the face.

The next change noticed in the parts about the oral pit is the appearance of a thick, rounded mass or process upon the upper back part of the first visceral arch of either side; this is called the superior maxillary process. Above, corresponding to the upper margin

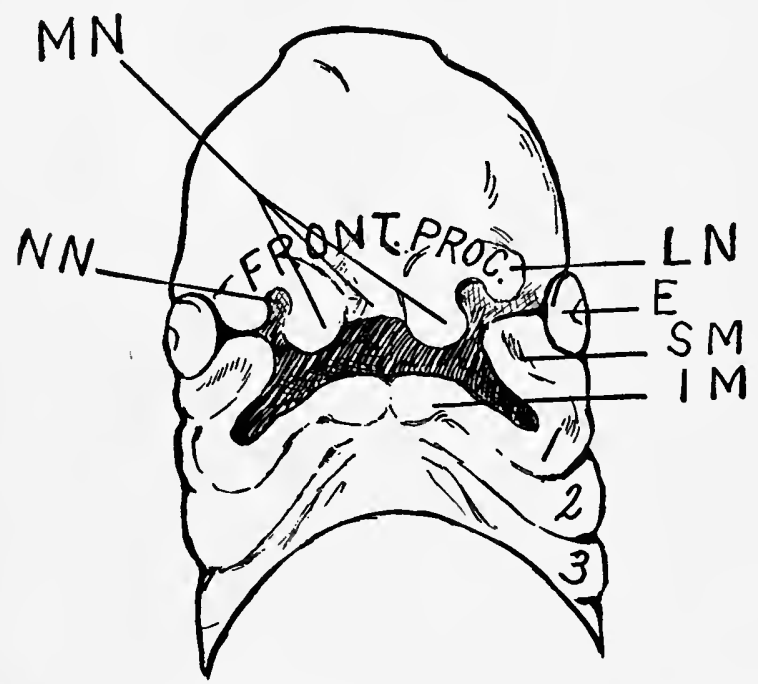

Fig. 24.-Face of Embryo, Fifth Week. Front vlew. $E$, eye; IM, inferior maxillary process (first visceral arch) joins in middle line with its fellow of the opposite side to form the mandibular arch (future lower jaw); $L N$, lateral nasal process (outer extremity of the frontal process); $\boldsymbol{M} N$, middle nasal process (middle portion of frontal process); NN, nasal notch (future nostril); SM, superior maxillary process (upper back part of the first visceral arch); $1,2,3$, first, second, and third visceral arches.

of the oral pit, there appears a single broad process, which is developed by the forward and downward growth of the anterior wall of the vesicle of the forebrain; this is called the frontal process or frontal plate, and is really a prolongation of the front wall of the vesicle of the forebrain; it grows downward and plays a very important rôle in the development of the face. At this stage the oral pit is a five-sided, deep fossa, bounded above by the frontal process or frontal plate, below by the mandibular arch (inferior maxillary processes), and upon each side by the superior maxillary process. 
The eyes are located one upon either side of the head, and are bounded below by the upper back part of the superior maxillary process and internally by the outer border of the frontal process.

The frontal process, frontal plate, is broad, and consists of a middle portion, the middle nasal process, and two lateral portions, the lateral nasal processes.

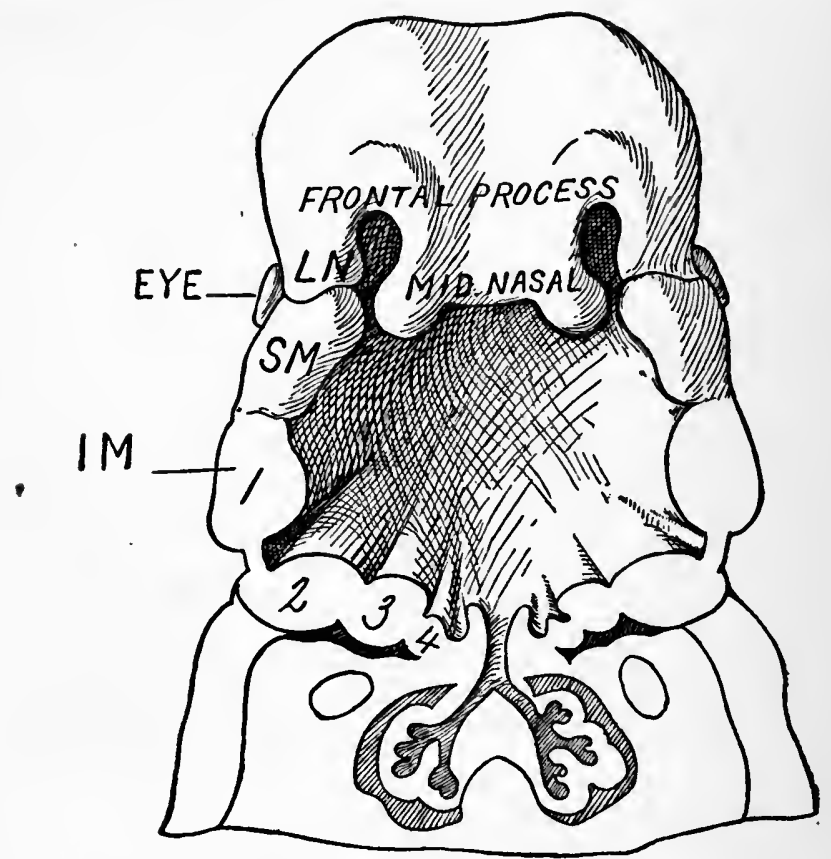

Fig. 25.-Face of Embryo, Fifth Week. Front view. The anterior portion of the visceral arches has been cut away to show the interior of the mouth cavity (pharynx), the wall of which shows the visceral arches with intervening clefts corresponding to those upon the outside. $I M$, cut surface of inferior maxillary process; $L N$, lateral nasal process; $S M$, superior maxillary process; $1,2,3,4$, cut surface of the first, second, third, and fourth visceral arches, showing the corresponding clefts between them. Between $L N$ and middle nasal process is the nasal notch (future nostril).

The middle nasal process is quite broad, and its lower free border is deeply notched in the middle. The lateral nasal process, one on either end of the frontal process, is separated from the middle nasal process by a deep notch, the olfactory groove; the floor of each olfactory groove is intimately related with the base of the cerebral vesicle: organ of smell. 
During the fourth week the plate of tissue which forms the floor of the oral pit becomes very thin, consisting only of the epidermic and endodermic layers. It is called the "rachenhaut of Remak," or the pharyngeal membrane, and during this week ruptures and so establishes a communication from without with the alimentary tube -pharynx.

Somewhat later, about the fifth week, we find that the various processes have approached each other, and the appearance begins

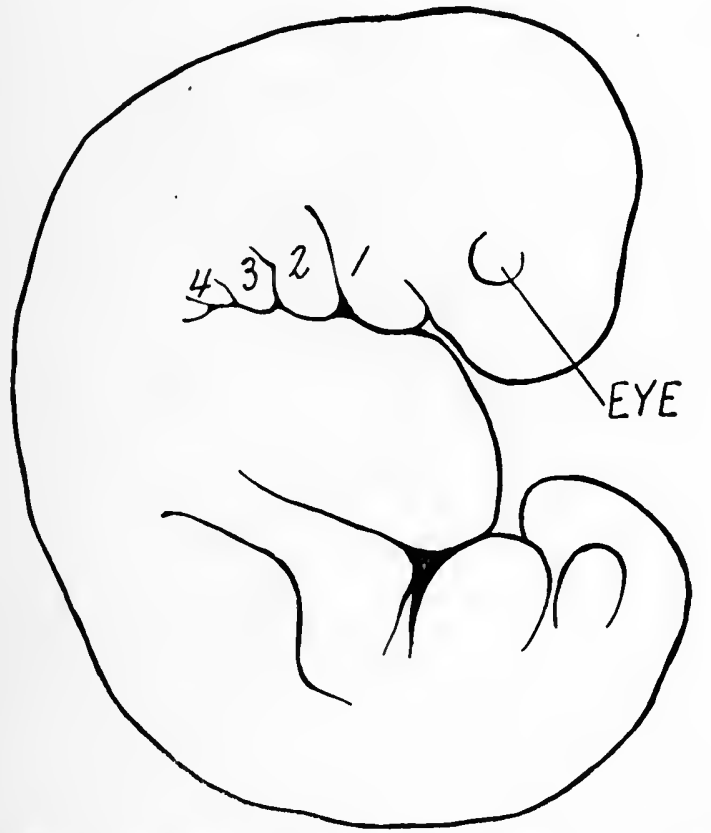

Fig. 26.-Embryo about Fourth Week, seen from Side. 1, 2, 3, 4, visceral arches with clefts between them.

to suggest the ultimate conformation of the face. The superior maxillary processes are nearer the middle line, the whole frontal process is longer, and its separation into a middle and two lateral portions is still more pronounced on account of the increased depth of the olfactory grooves. The eyes are fairly well bounded, but are still located upon the side of the head.

About the seventh week we note that the superior maxillary process, in part, has become fused with the lateral nasal process 
of the frontal plate; this line of fusion corresponds to the position of the tear-duct. If union does not occur along this line, we have a so-called orbito-nasal or oblique facial cleft. The eye is entirely surrounded and is placed more to the front of the face. The middle portion of the frontal plate, the middle nasal process, is still notched in the center and broad; the extremities of this middle nasal process have become fused with the lowest and most internal part of the superior maxillary process, and by this union the upper lip is formed

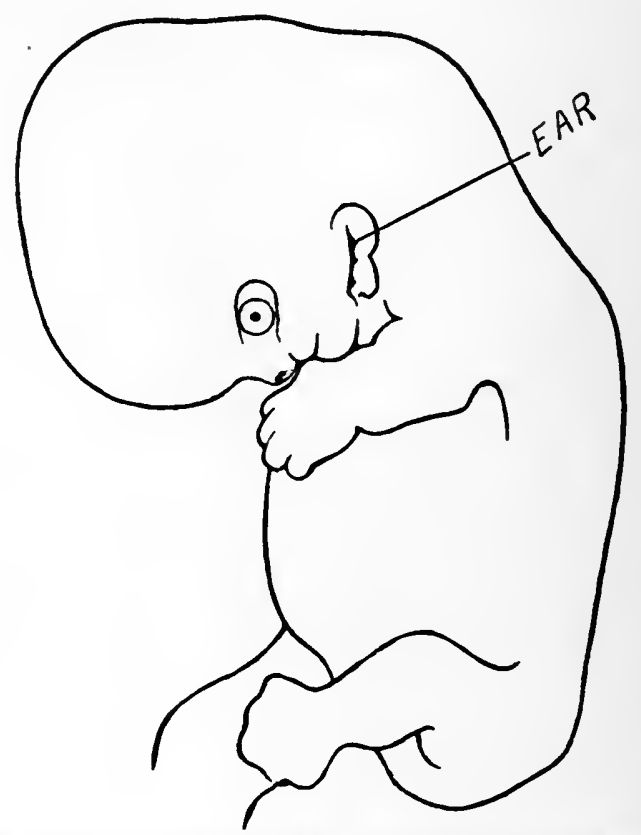

Fig. 27.-Embryo about Eighth Week. Development of face well advanced.

and at the same time the olfactory grooves are bounded below, and are thus converted into round openings: the nostrils. If the superior maxillary process and middle portion of the frontal plate, middle nasal process, fail to unite, we have, as a result, a cleft in the lip,-harelip; this may or may not reach into the opening of the nostril: i.e., may be complete or incomplete according to the extent to which the parts have failed to unite.

The lower edge of the superior maxillary process becomes partially united with the upper border of the mandibular process, the 
inferior maxillary process, which has also become thickened, and in this way the size of the mouth is much diminished. If this union falls short of normal we have a characteristic deformity: macrostoma or transverse facial cleft. The face, as a whole, is, therefore, at this period closed in, but the nostrils are still far apart, the nose broad, and perfectly flat and directed forward, and the upper lip is still notched in the middle line. This type of face often persists, and we then have a peculiar "pug face."

The openings for the external auditory meatus are seen low down upon either side of the head.

The external auditory canal is the remains of the posterior part of the first visceral cleft: i.e., that between the first and second arches. The margins of the orifice of the auditory canal later become nodulated; these nodules coalesce, and in this way the auricle is formed. The Eustachian tube and the tympanum are the remains of the corresponding first internal cleft (from pharynx). The eardrum represents the point where the epiderm, at the bottom of the outer cleft, and the endoderm, at the bottom of the inner cleft, have coalesced with each other.

At the end of the second month the eyes are located toward the front of the face. The nose is still broad and flat, although the nostrils are rather closer together. The upper lip, representing the middle portion, middle nasal process, of the frontal plate, is still notched in the middle line. The cavity of the mouth is fairly well closed in by the upper and lower lips.

To recapitulate: The first visceral arch is eventually developed into the inferior maxillary bone and the adjoining soft parts, including the lower lip and the floor of the mouth, and assists in the formation of the tongue. The superior maxillary process of the first visceral arch is developed into the superior maxillary bone and the adjoining soft parts, including the hard and soft palate. The frontal plate, its lateral portion, the lateral nasal process, forms the side of the nose, including the nasal bones; its middle portion, the middle nasal process, forms the bridge of integument between the nostrils, reaching from the tip of the nose to the upper lip, and the cartilaginous and bony portions of the nasal septum (vomer and perpendicular plate of the ethmoid); also the middle portion of the upper lip and intermaxillary bone.

The intermaxillary bone was first described by the poet Goethe. It is a small, wedge-shaped, bony process which is attached to the 
anterior end of the vomer and fits into a corresponding triangular space in the anterior part of the hard palate, and carries the four incisor teeth. The line of union between this bone and the palatal processes of the superior maxillary may often be plainly seen in the adult upper jaw-bone. The anterior palatine canal marks the junction of these parts. A non-united, abnormally placed intermaxillary bone often complicates harelip.

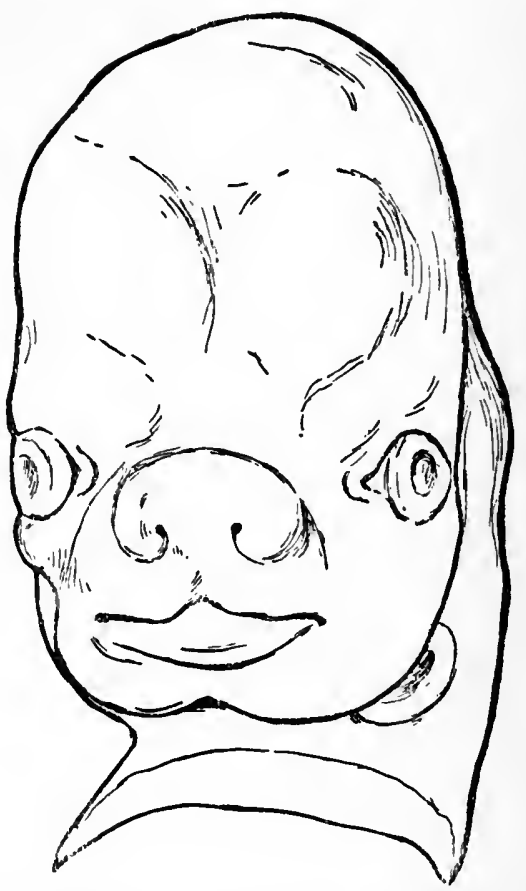

Fig. 28. - Face of Embryo about Eighth Week. The various processes that go to make up the face have coalesced, but the embryonal type of the face is still well marked. Eyes located upon the side of face. Ears low down. Nose flat and projecting forward, with nostrils far apart. Upper lip still notched in the middle.

Formation of the Palate.-The superior maxillary process of either side gives off, upon its inner aspect, a shelf-like process: the palate process. These processes gradually grow toward the middle line and unite with each other, and thus form the hard and soft palate, the union taking place from before backward, the uvula being the last part to unite. Union between the palatal processes is com- 
plete at about the eleventh week. With the formation of the hard and soft palate, the nasal cavity is separated from the oral, or mouth, cavity. Failure of union between the palatal processes gives rise to the various degrees of cleft palate. In front, where the two halves of the hard palate join with the intermaxillary bone, there are a suture line and the anterior palatine canal.

The vomer and the perpendicular plate of the ethmoid are developed from the middle portion-the middle nasal process-of the frontal plate, and divide the nasal cavity into two parts. The junction between the lower border of the vomer and the hard palate occurs after the two palatal processes have united with each other in the middle line. The nasal cavity opens in front upon the face through the nostrils and behind into the pharynx through the posterior nares.

The Teeth.-The margins of the upper and lower jaw become prominent, and in this way form the alveolar processes; the epithelium covering these processes becomes invaginated,-dips down into the substance of the processes, - and from this the teeth are formed.

The floor of the mouth is developed from the first visceral arch.

The Tongue.-The tongue is developed, its anterior portion from the first arch and its posterior portion from the second and third arches. The anterior part-the body and tip-is developed from a tubercle which appears in the front part of the mouth at the junction of the two halves of the first arch. The back part, the root, is developed in the back part of the mouth from the wall of the pharynx, from two tubercles at the junction of the second and third arches. These two parts of the tongue, the anterior and the posterior, become joined, the line of union being indicated by the V-shaped row of papillæ upon the dorsum of the adult tongue. At the apex of the $\mathrm{V}$ there is a dimple, the foramen cæcum, which indicates the point of junction of the parts of which the tongue is formed. As the tongue is developed, it increases rapidly in size, occupying the mouth cavity and projecting up into the future nasal cavity. As the palatal processes grow inward to meet each other in the middle line, however, the tongue is gradually forced down into the mouth cavity proper, where it belongs.

Deformities of the Face.-These consist of abnormal clefts and atresias, which may be partial or complete.

Clefts are due to the entire or partial absence of normal union between the original embryonal processes by whose coalescence the 
face is formed. Atresias are caused, on the other hand, by excessive union, beyond the normal, between these processes, and as a result we get a partial or complete closure of the facial orifices: mouth, nostrils, and eyes. Still further, the union between the processes may occur to its normal extent, but the lines of union may remain permanently marked by cicatricial seams or irregular tags and nodules.

The failure of the embryonal processes properly to coalesce,

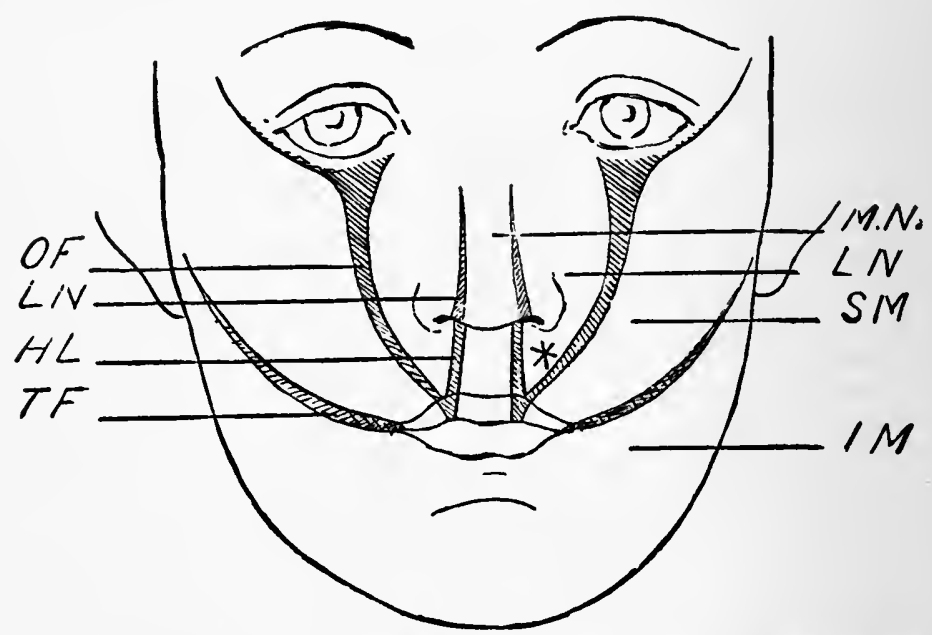

Fig. 29.-Diagram of Congenital Facial Clefts. Shaded portions Indicate the location of the different congenital fissures. $H L$, harelip; $I M$, inferior maxillary process; $L N$, *, lateral nasal process of frontal plate; $L N$, lateral nasal cleft; $M . N .$, middle nasal process of frontal plate; $O F$, oblique facial cleft; $S M$, superior maxillary process; $T F$, transverse facial cleft; *, lower part of lateral nasal process which takes part in the formation of the upper lip, but not of its red border; the free red margin of the lip is formed by the union of the lower part of the middle nasal process $(M N)$ and the lower part of the superior maxillary process ( $S M)$.

with the resulting clefts, is really due to the incomplete development of the processes themselves; they are deficient: i.e., too small to meet each other, and hence the clefts. The clefts vary in degree from narrow, incomplete fissures to widely gaping spaces. The margins of the clefts may be smooth or they may be irregular and marked by nodular processes, tags, etc.

The congenital deformities of the face may be divided into two general groups:- 
(A) Those in which the frontal plate or process is concerned. Under this heading we have:-

1. Lateral clefts of the upper lip and the alveolar process; clefts of the palate may also be conveniently included in this group.

2. Median clefts or notches of the upper lip and deformities of the nose.

3. Notching of the wing of the nose.

4. Oblique facial fissures, etc.

(B) Those in which the first visceral arch is involved. In this group we have:-

1. Transverse facial fissures.

2. Median fissures of the lower lip, lower jaw, and tongue.

3. Deformities of the lower jaw.

Deformities in Which the Frontal Plate is Concerned. LATERAL Clefts of the Upper Lip axd of the Alveolar Process axd Cleft Palate.-Clefts of the upper lip and alveolar process depend upon imperfect union of the middle portion, middle nasal process, of the frontal plate with the corresponding lower portion of the superior maxillary processes: to failure of the intermaxillary bone and its accompanying soft parts to unite with the adjoining portion of the face. These clefts are always lateral and may be present on one or both sides. Clefts of the palate (hard and soft) depend upon non-union, partial or complete, of the palatal process of the superior maxillary process of either side with each other. These clefts are median when the processes of both sides are at fault. If the palatal process of one side only is involved, the fissure will be present upon the corresponding side of the middle line, the palatal process of the other side being joined with the lower border of the vomer, thus shutting off the nasal cavity, on that side, from the mouth.

If union has failed, on both sides, between the middle process of the frontal plate, the middle nasal process, and the corresponding part of the superior maxillary process of either side (double harelip and fissure of the alveolar process) and between the palatal processes of the superior maxillary processes of either side (cleft of the hard and soft palate), we have the most extreme variety of this group of deformities. There are found all degrees of this variety of deformity from this exaggerated form down to a mere notching of the upper lip (incomplete harelip) or bifurcation of the urula.

Harelip.-This condition may be incomplete or complete.

Incomplete harelip consists in a vertical notch in the free mar- 
gin of the upper lip. It is located to one side of the middle line between the middle segment and the lateral segment of the lip. It varies in depth from a barely noticeable notch to a deep fissure which may extend almost through the entire lip, leaving but a narrow bridge of integument separating the angle of the notch from the nostril.

In complete harelip the fissure extends all the way through the upper lip into the nostril. It may be associated with cleft of the alveolar process and with cleft palate. The nose is apt to be unusually broad and flattened, the wing of the nose, on the side corresponding to the cleft, being carried outward away from the middle line. These deformities may involve one or both sides. If double,

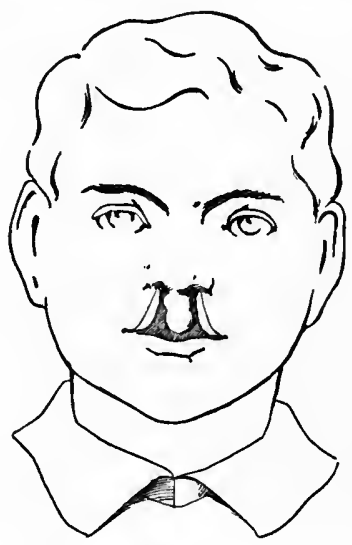

Fig. 30.-Double Complete Harelip.

those of the two sides may differ from each other, the fissure on one side may be complete, that of the other side incomplete, or those of both sides may be complete. They may be associated with cleft of the alveolar process and with cleft palate, the intermaxillary bone often being small and misplaced forward. The entire middle segment of the lip may be absent, together with the intermaxillary bone and the vomer. In this case the upper lip shows a broad, median space, which opens into the nasal cavity.

Cleft of the Alveolar Process. - With harelip, as already mentioned, there may also be present a cleft of the alveolar process, and this may vary from a narrow, incomplete fissure to a broad, open space; it may be unilateral or double, and is usually associated with 
cleft palate. If there is no cleft of the hard palate, the cleft of the alveolar process terminates at the anterior palatine foramen: the meeting point of the palatal process of either side and the intermaxillary bone. If the cleft in the alveolar process involves both sides, the intermaxillary bone, which is continuous with the front of the vomer, may be placed forward in advance of the rest of the alveolar process, especially if cleft palate is also present; so that it and the corresponding portion of the upper lip seem to be suspended from the point of the nose. In this case the lower tegumentary part of the septum of the nose is absent, the soft parts which represent the middle part of the lip being continued directly with the tip of the nose. This advancement of the intermaxillary bone is due to the

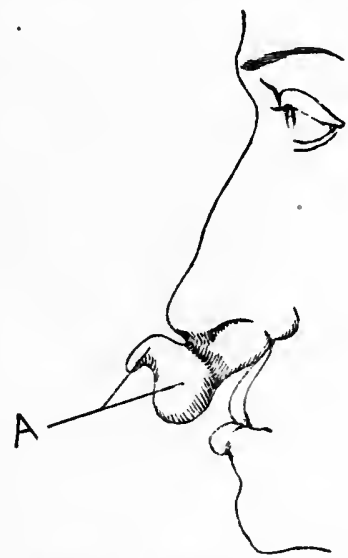

Fig. 31.-Harelip with (A) Advanced Intermaxillary Portion.

unrestricted forward growth of the vomer, which is not inhibited as is normally the case when it is joined to the palatal processes. If the cleft is confined to one side of the alveolar process and the hard palate, the intermaxillary bone, as it is carried forward by the growth of the vomer, is apt to become markedly twisted upon its long axis, so that its anterior surface, instead of being directed forward, looks almost directly toward the normal side of the face, presenting its prominent sharp lateral edge anteriorly. The intermaxillary segment may be entirely absent, as already mentioned.

Cleft Palate.-The presence of a longitudinal fissure which may involve the hard or soft palate or both. It is caused by a failure of the palatal processes of the superior maxillary processes to meet 
in the middle line and coalesce. In these cases the base of the skull may be unusually broad and the pterygoid processes unusually far apart.

Cleft of the Hard Palate.-This may be unilateral or double. If one-sided, the palatal process of the normal side is seen to be united with the lower border of the vomer, shutting off that side of the nasal cavity from the mouth, while upon the affected side the palatal process is deficient and falls short of meeting its fellow of the opposite side, and there is thus left an opening which leads into the corresponding half of the nasal cavity. In double cleft palate both palatal processes are deficient, and the lower free edge of the vomer may be seen between the separated edges of the cleft.

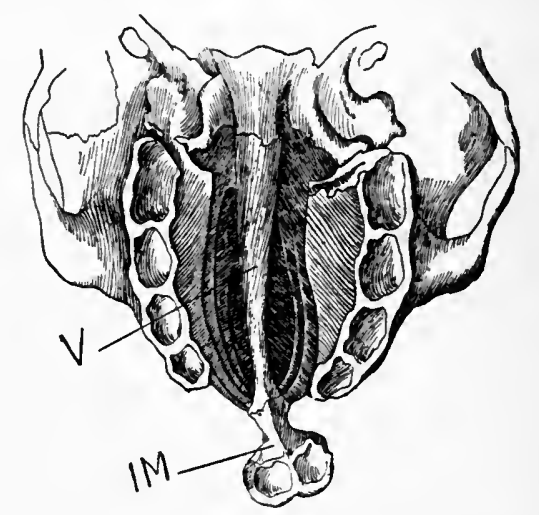

Fig. 32,-Double Cleft Palate with Advanced Intermaxillary Portion (IM) Carrying the Sockets of Two Incisor Teeth. $\nabla$, vomer (septum of the nose).

Usually the lower border of the vomer does not reach low enough to present itself in the fissure between the edges of the cleft, and the cleft thus has the appearance of a median cleft when it is, in reality, a bilateral, or double, cleft.

At times we may find the palatal processes of either side properly united with each other, but the vomer fails to grow down suffciently far to articulate with them, and there is thus left a space below the lower border of the vomer through which the two sides of the nasal cavity communicate with each other. It should be remembered that the vomer does not play any part in the formation of the hard palate.

Cleft of the hard palate ends anteriorly, either at the anterior 
palatine foramen, which marks the point of junction between the intermaxillary bone and the palatal processes of the superior maxillaries, or else it is combined with a single or double cleft of the alveolar process and harelip. It usually ends, posteriorly, in cleft of the soft palate.

In cleft palate, especially if double, the forward growth of the vomer is unrestricted on account of its not being joined to the palatal processes, and by this forward growth the intermaxillary bone and its corresponding soft parts may be carried forward beyond the line of the alveolar processes, the intermaxillary bone often being bent upward or twisted upon its long axis (see Fig. 32). This advancement of these parts adds very much to the difficulty of correcting the deformity.

Cleft of the Soft Palate.-The fissure extends from the tip of the uvula for a varying distance into the soft palate. It may be simply a bifurcation of the uvula, but, as a rule, it extends all the way through the soft palate as far as the posterior border of the hard palate or for some distance into the hard palate. It may be combined with a lateral or double cleft of the hard palate. As is the case with cleft of the hard palate, there is not only a simple lack of union between the two halves of the palate, but an actual deficiency of tissue which prevents the parts from meeting and coalescing in the middle line, and this fact is important in considering the operative treatment of this condition.

With the exaggerated forms of cleft palate there is frequently associated imperfect development of the middle nasal process of the frontal plate or it may be entirely absent: the intermaxillary bone may be absent, with or without absence of the vomer. If the intermaxillary bone, etc., are absent, we have a median cleft of the upper lip, or, better, a double harelip with absence of its middle segment; and this condition is usually associated with a broad cleft in the hard and soft palate, and the nose may be soft and flattened, on account of the absence of the nasal septum, etc. This condition is apt to be accompanied with defective cerebral development.

Median Clefts and Notches of the Upper Lip.-These deformities depend upon exaggeration and persistence of the embryonal notch of the middle portion, the middle nasal process, of the frontal plate and failure of the nostrils to approach each other. These defects are much less frequent than the preceding. There may be simply a notch or fissure in the middle of the upper lip reaching part 
way through, or this may be combined with a grooving or furrow upon the point and dorsum of the nose and a wide separation between the nostrils. This condition may be so pronounced that the nose appears to consist of two halves completely separated from each other and each containing one nostril. Instead of this extreme degree of deformity the nose may be simply flattened, the bridge depressed, the nostrils far apart and looking directly forward: "dog nose." The fissure in the upper lip instead of simply notching the lip may extend completely through the whole lip and into the intermaxillary bone. This variety of deformity may also be represented by a fistula of the tip or dorsum of the nose.

Lateral Nasal Clefrs.-These occur with or without harelip and cleft palate; the notch or fissure involves the wing of the nose. If they extend upward for a considerable distance through the side

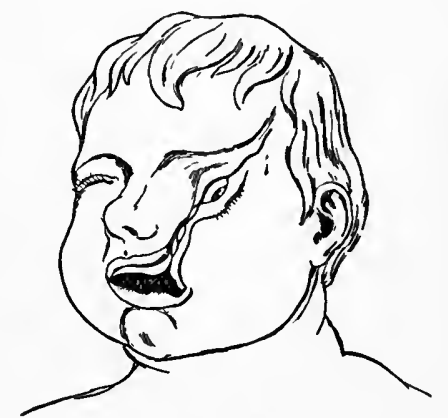

Fig. 33.-Oblique Facial Cleft Extending into the Temporo-frontal Region.

of the nose, they terminate above, not in the inner canthus, but to the inner side of the inner corner of the eye; they represent the embryonal notch between the middle and lateral nasal processes of the frontal plate. Fissures of the side of the nose, that resemble these, but terminate above in the inner canthus of the eye, are varieties of oblique facial clefts.

Oblique Facial Clefts.-Failure of normal union between the lateral process of the frontal plate and the superior maxillary process of the first visceral arch. They correspond to the embryonal orbitonasal line of coalescence. These deformities may be very extensive or slight: one-sided or double. They commence below at the edge of the upper lip, and, after splitting this at the usual harelip site, extend upward through the cheek, alongside of the wing of the nose, 
not into the nostril, like harelip, and terminate above, at the lower margin of the eye (lower lid) or inner canthus. They may extend

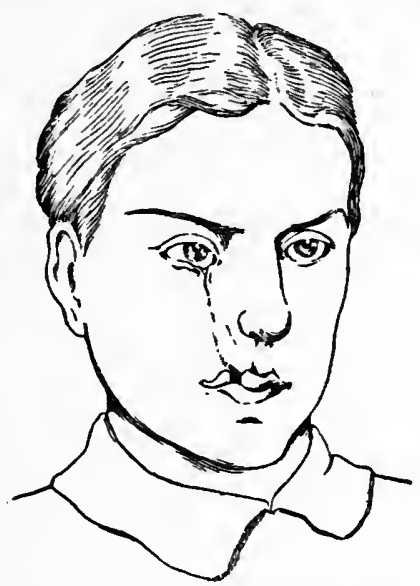

Fig. 34.-Incomplete Oblique Facial Cleft. The edge of the upper lip is notched and a cicatricial line extends across the cheek to the lower eyelfd, which is everted.

beyond the orbit, from its outer corner, upward and outward into the fronto-temporal region of the skull. They vary from a narrow

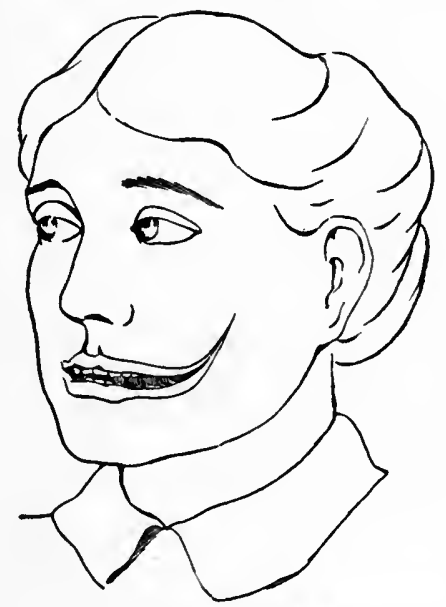

Fig. 35.-Transverse Facial Cieft.

fissure or incomplete notch to a wide, gaping fissure, between the edges of which is the eyeball. This class of deformity is frequently 
represented in its simplest form by a notch or coloboma of the lower or upper eyelid. Instead of a fissure, this deformity may be represented by a cicatricial, nodulated seam, indicating the orbito-nasal junction.

Deformities in Which the First Visceral Arch is Concerned. Transterse Facial Clefts, ETc.-These are due to a failure of the inferior maxillary process of the first visceral arch and its superior maxillary process to coalesce to the normal extent. This deformity may be unilateral or double. The cleft extends from the corner of the mouth outward through the cheek and exposes the teeth: macrostoma. If the reverse of this process occurs, we may have a mouth so small as to require surgical interference: microstoma.

Median Clefts of the Lower Lip, Lower Jaw, and Tongue. -These conditions are very rare. They are due to failure of the two halves of the first visceral arch (mandibular processes) to unite with each other in the middle line. They vary from a slight notching of the lower lip, in the middle line, to a complete separation through the lower lip, the lower jaw at the symphysis, and the tongue. The tongue, by itself, may be split or absent or bound down to the floor of the mouth or adherent to the side of the cheek, etc.

The lower jaw may be imperfectly developed, rudimentary, etc. It may be split in the middle line or there may be absence of the condyles, etc. As the formation of the face advances the jaw is gradually protruded forward, and, if arrested, we have, as a result, the receding chin, etc.

\section{OPERATIONS FOR HARELIP AND CLEFT PALATE, ETC.}

Operations for Harelip.-In speaking of harelip-if single-the flap corresponding to the angle of the mouth is called the lateral flap, or segment, and the other, the middle; if the harelip is double, one speaks of the middle segment and two lateral segments, the right and the left.

Koenig advocates early operation, within a few days after birth. Trendelenburg advises delaying the operation until later, operating between the third and sixth months, and, if very complicated, waiting still longer. Trendelenburg claims that the difficulty in nourishing the children is not a good ground for early operation; that children, even with a cleft palate in addition to the harelip, can feed from 
a bottle if it is provided with a nipple attached to the neck of the bottle itself, and especially if the child is assisted by the nurse holding the bottle. If the child is able to take the breast it will not be necessary to change to the bottle after operation and the breast nursing may still be continued. At the time of operation the child should be free from intestinal trouble, and there should be little or no nasal catarrh.

For children under one year no anæsthetic is necessary; for older children one may use incomplete chloroform anæsthesia.

The child should be wrapped in a blanket in such a way that the arms and legs are confined and then held upright in the arms of a nurse who sits opposite the operator. The child's head is steadied by an assistant, who thrusts the head a little forward to prevent the blood entering the mouth during the operation.

The instruments that are required consist of a sharp, narrowbladed knife with a sharp point, several tenacula, mouse-tooth forceps, and narrow-bladed sharp-edged scissors. The steps of the operation consist in freshening the edges of the cleft and suturing. In freshening the edges one should cut with a view to providing broad, raw surfaces for apposition; they should be cut somewhat obliquely, and more taken away from the skin than from the mucous surface. During this step of the operation the hemorrhage may be controlled by an assistant, who compresses either segment of the lip between the finger and thumb, or a clamp may be applied on either side of the defect in the lip (Trendelenburg). With the mouse-tooth forceps the edge of the defect is seized and transfixed with a knife, and the incision made with a sawing motion and with deliberation. In order to bring the raw surfaces into apposition it is occasionally necessary to liberate the flaps by cutting them free from their attachment to the deeper adjoining parts: the alveolar process and anterior surface of the superior maxilla.

As suture, several harelippins may be used, each with a figure-ofeight coil of silk floss. Without these pins, or in addition to them, one may unite the raw surfaces with one to three heavier silk sutures carried in a straight or curved needle. These should penetrate deep into the substance of the lip, down to, but not through, the mucous membrane, and should take a good hold. Between these the skin and mucous membrane are brought accurately together, edge to edge, with a number of superficial sutures of rather finer silk. 
Operations for Incomplete Harelip. Sisiple Freshening of the Opposing Edges aND Suture.-This plan would not answer even for incomplete harelip, since a notch would remain which would increase with time as the scar contracts, especially if the cleft is deep.

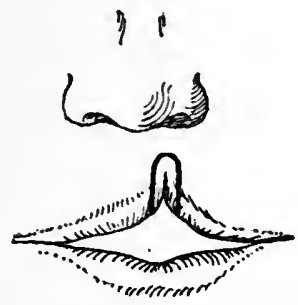

Fig. 36.-Simple Paring of the Edges of the Notch for Incomplete Harelip.

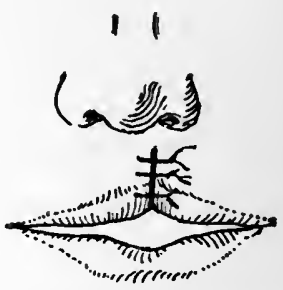

Fig. 37.-Imperfect Result After Simple Paring and Suture, Showing the Notch still Present.

VoN Graefe proposed a very simple method to increase the length of the apposed edges of the freshened surfaces. This method will answer, however, only for the very incomplete defects, and not for wide or complete splits. It consists in paring the edges of the notch by making a circular incision, which arches over the corner of the notch.

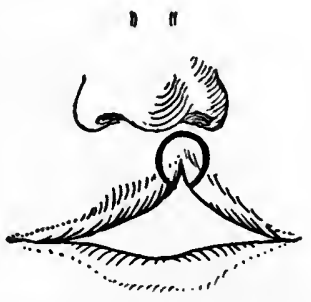

Fig. 38.-Von Graefe Method of Paring an Incomplete Harelip so as to Increase the Length of the Raw Apposed Edges.

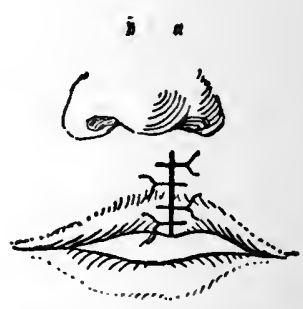

Fig. 39.-Result After Suturing.

NÉLATON MethoD.-Without removing any tissue, an incision is made through the substance of the lip, around the corner of the cleft and parallel with its edges, and after converting this incision into a vertical one its edges are united with several interrupted stitches. 


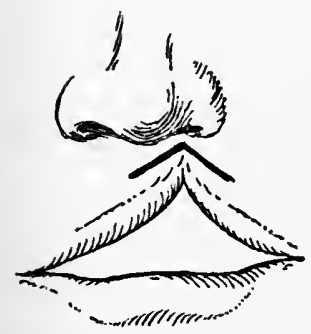

Fig. 40.-Nélaton Operation for Incompiete Harelip. Line of incision.

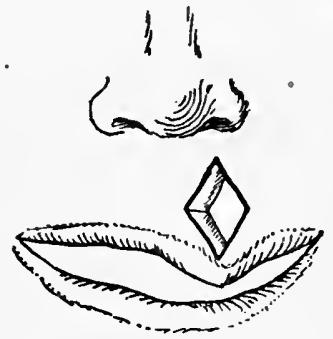

Fig. 41. - Incision Converted into a Perpendicuiar, ready for Suture.

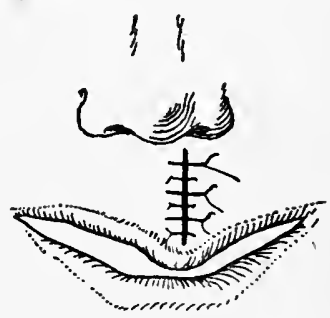

Fig. 42. - Resuit After Suture.

Malgaigne proposed to close the defect, especially where the defect is considerable, by making use of flaps in addition to freshening the edges. In his operation the tissue is removed from the angle of the defect only, the second part of the operation consisting in the formation of flaps by simply cutting into the substance of the lip along either side of the defect, commencing near the angle and cutting toward the red border of the lip. The base of the flap

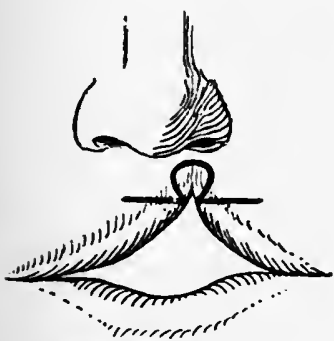

Fig. 43.-Maigaigne Operation for Incomplete Harelip. Paring and formation of flaps.

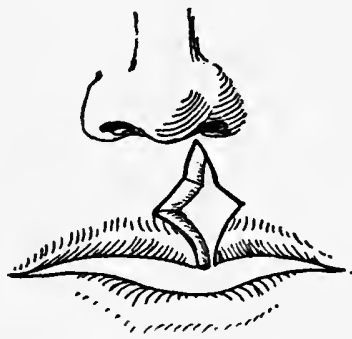

Fig. 44. - Fiaps Turned Down, ready for Suture.

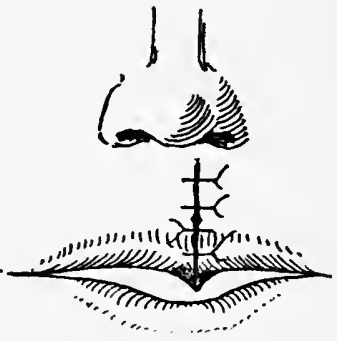

Fig. 45. - Resuit After Parts have been Sutured.

should be no thicker than the red of the lip; otherwise it is very difficult to turn it down. The tongues of tissue thus marked out are turned down and sutured together, with the result that the cleft is not only filled in, but a little tongue of tissue is left projecting beyond the free line of the lip to allow for future retraction.

The objection to this operation is that, on account of the considerable torsion to which the flaps are subjected, their nourishment is uncertain and they may become gangrenous, especially in very young children. 
Miravit's operation is an improvement on Malgaigne's. Only one flap is made, and that is taken from the edge of the lateral segment. The flap which is thus formed is sutured to the freshened edge of the middle segment. This single flap is not likely to become gangrenous as is the Malgaigne, because it is not necessary to turn it down so far, and, secondly, because its base may be made suffieiently broad to inelude the coronary vessels. In forming the flap a single eut is made into the substance of the lip proper, striking well above the red margin so that the base of the flap eorresponds to the lower third of the breadth of the lip. This is a very satisfactory operation. It has been modified by Simon, Koenig, Esmarch, Hagedorn, and others.

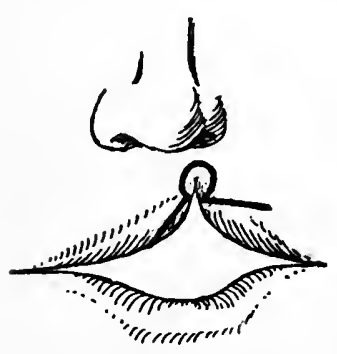

Fig. 46.-Mirault Operaation for Incomplete Harelip. Paring and formation of one flap.

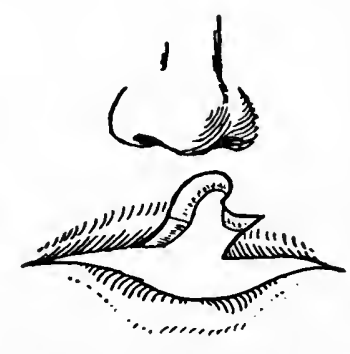

Fig. 47. - Parts ready for Suture.

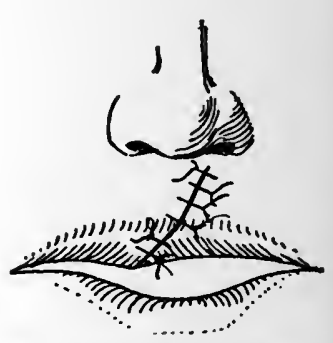

Fig. 48.-Result After Suture. .

Operations for Complete Harelip. - Cases in which the split extends through the entire width of the lip.

In these cases it is not only neeessary to freshen and prepare the edges for suture, but one must, in addition, detach the soft parts, in order that the raw surfaces may be brought together and sutured; one must unite the whole width of the lip from the nasal opening down to its free border, and further strive to correct the accompanying nasal deformity. It usually suffices to separate the outer or lateral segment, that nearer the corner of the mouth, from its attachment to the superior maxillary bone. Only in extreme cases does it become necessary to detach the other flap as well. To separate the flap from the underlying bone one seizes its edge with a mouse-tooth forceps, and draws it inward toward the middle line, and forward, away from its attachment to the bone. In this way the fold of the mucous membrane which attaches the lip to the gum is 
put upon the stretch, and may be incised with the edge of the knife, cutting toward the bone (superior maxillary). This incision is carried sufficiently far and deep to liberate the lateral flap and the

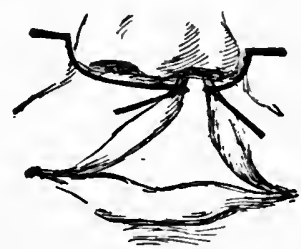

Fig. 49.-Wellenschnitt for Complete Harelip. Incision, carried around the alæ of the nose in order to fiberate the segments. Formation of flaps by incision into each segment.

corresponding side of the nose and to allow of the parts being readily apposed without tension. Hemorrhage from this incision is often considerable, especially if it is necessary to cut deep, and this is given as one of the reasons for waiting in these cases, at any rate, until the third or fourth month (Trendelenburg). This hemorrhage, however, usually ceases when the sutures are inserted and compression applied; still, any spurting vessels that are to be seen should be clamped and ligated with fine catgut.

Occasionally, in order to free the flap sufficiently it may be necessary to make an incision around the wing of the nose; this, however, is but seldom necessary (Dieffenbach's Wellenschnitt). The Mirault or the Hagedorn operation is usually done for this condition of complete harelip.

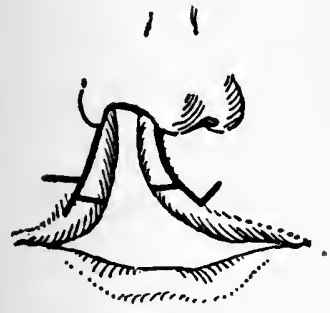

Fig. 50.-Hagedorn Operation for Single Complete Harelip. Lines of incision.

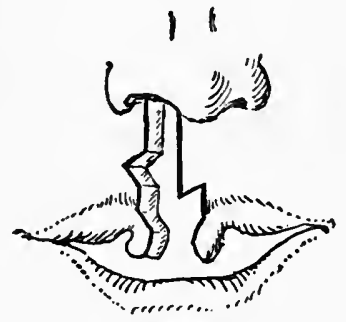

Flg. 51. - Parts Freshened and Ready for Suture.
10

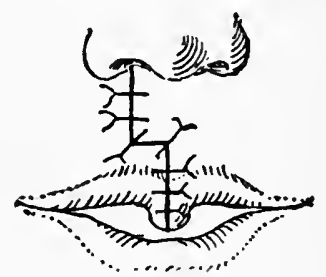

Fig. 52.-Result After Suture.

HAGEDORN's operation consists in paring away the edges of each flap, first from the margin of the lateral flap,- that nearer the angle of the mouth, -and then from the margin of the other flap. A 
horizontal incision is then made into the substance of the lateral flap and an oblique one into the median flap. Then, with a scissors, the long strips of vermilion border which have been pared away from the edges of the flaps are snipped off. When the parts are sutured there is left a little process hanging from the edge of the lip; this retracts in time.

Operation for Single, Complete Harelip Associated with Cleft of the Alveolar Process and Advancement of the Intermaxillary Bone. -In these cases the intermaxillary bone, besides being misplaced, may be rotated upon its long axis in such a way that it presents, anteriorly, a prominent, sharp edge, which would greatly interfere with the healing process.

Under these circumstances it becomes necessary to place the bone in its natural position. An effort should be made, by twisting it upon its long axis, to set it square so that its sharp, lateral edge will not project under the suture line. If necessary, with the bone forceps or the chisel the process may be separated forcibly from its attachment to the alveolus and brought into position by rotating it partly upon its long axis. The vomer, to the front of which the intermaxillary is joined, may prevent this replacement, and then it may be wise to resect the prominent edge of the intermaxillary with a chisel or rongeur, but if we do this we lose an incisor tooth. These measures complicate the operation and occasion considerable hemorrhage, and therefore it is often well, with this condition, to defer the operation in very young children. After the intermaxillary bone has been reduced or resected, closure of the split in the lip may be accomplished by any of the methods described above.

Operation for Double Harelip without a Prominent Advanced Intermaxillary Bone.-The middle segment is always found to be too short to take part in the formation of the free border of the lip, but it may be used to form the middle portion of the lip. From the whole of the middle segment and from each lateral segment in part, the mucous membrane edge is trimmed away, and a Malgaigne flap then made from the edge of each lateral segment. One side may be done at a sitting, or one may, by freshening the lateral margins of the middle segment and the corresponding margins of the lateral segments, transform the condition into a double; incomplete harelip and later do a second operation to correct this. If the nose is flattened and the alæ spread out, one should try to correct this deformity at the same time by separating the lateral segments of 
the lips and the sides of the nose from their deep attachments. Instead of the Malgaigne, a double Hagedorn may be done for this condition.

Operation for Double Harelip with Prominent Advanced Intermaxillary Bone.-This may be remedied by resecting the bony part of the prominent intermaxillary portion, leaving the soft parts to assist in making the lip. The middle segment may be placed very far forward upon or near the point of the nose, in which case the cuta-

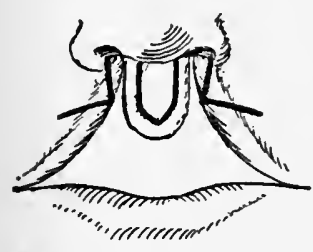

Fig. 53. - Double Malgaigne Operation for a Double Complete Harelip. Paring of edges of defects and formation of flaps.

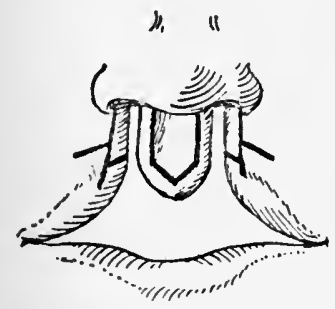

Fig. 56.-Hagedorn Operation for Complete Double Harelip. Paring and formatlon of flaps.

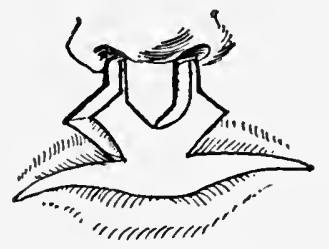

Fig. 54. - Flaps Turned down ready for suture.

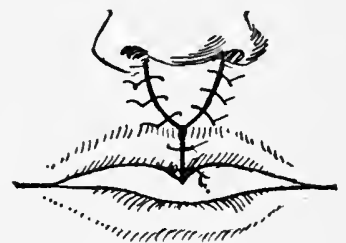

Fig. 55.-Result After Suture.

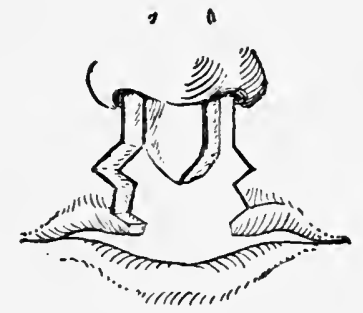

Fig. 57.-Parts Ready for Suture.

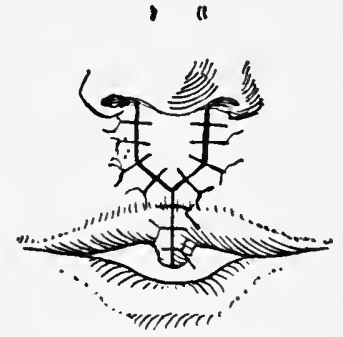

Fig. 58.-Result After Suture.

neous part of the septum of the nose is absent (see Fig. 31). Under these circumstances the soft parts of the middle segment of the lip must be used to make the tegumentary part of the nasal septum, and then the whole lip must be formed from the two lateral segments without the assistance of the middle portion. It may be necessary to liberate the flaps by separating them from the alveolar process of the superior maxillary or, in addition to this, by making an incision, upon either side, around the ala of the nose (Wellenschnitt of Dieffenbach). 
An attempt may be made to gradually force the intermaxillary segment into place by long-continued pressure. If this method is to be tried, the double cleft is closed after having first liberated the side flaps by an incision, on either side, around the wing of the nose (Dieffenbach's Wellenschnitt), and then an elastic band is applied which exerts pressure, continuously, upon the middle segment. This bandage must we worn for a long time.

Immediate forcible replacement of the intermaxillary portion may be done. It is seized with the forceps and broken away from the vomer, or the line of fracture may extend upward and backward through the vomer proper. The segment is then forced back into proper position and the edges of the flaps freshened and sutured.

Blandin recommends the resection of a triangular-shaped portion from the nasal septum posterior to the intermaxillary segment. The base of the triangular piece of bone which is thus resected corresponds in width to the space that intervenes between the middle segment and the intermaxillary notch, its apex running upward into the septum of the nose. In young children this resection may be made with a pair of ordinary strong scissors, but in children over ten years of age it will be necessary to use the bone scissors. The apex of the resected triangular piece should be directed upward and forward, toward the bridge of the nose, in order to avoid the anterior palatine ressels. The intermaxillary segment may then be readily forced back into proper position and the cleft closed. If the anterior naso-palatine artery is cut in removing the triangular piece of bone, the hemorrhage will be severe.

Bardeleben has modified the above procedure in that he first separates the periosteum, upon either side of the septum, behind the middle segment, and then, with the ordinary strong, straight scissors, simply cuts through the septum without attempting to resect a triangular piece. The middle segment is then pushed back into place, the edges of the divided septum sliding past and overlapping.

As a rule, the attempt to replace the middle segment should be made during the first, second, or third year of the patient's life, because later the segment becomes too large and the corresponding intermaxillary space too small.

Many surgeons make it a rule to excise the intermaxillary bone entirely, and indeed it is very questionable if anything is gained by leaving or replacing a deformed, misplaced middle portion. If it is removed, the four incisor teeth are lost, but a plate can be fitted to sub- 
stitute for these. If the intermaxillary bone is allowed to remain and is replaced, it is very likely to remain rudimentary and wabbly, and the corresponding teeth are apt to be crooked and imperfect. If a considerable part of the septum of the nose has been removed, in order to place the intermaxillary portion in its normal position, the point of the nose will be drawn down so close to the front of the face as to give it a peculiar flattened, "bird-like" appearance.

Operation for Cleft Palate.-The cleft may be limited to the soft or hard palate or may extend through both.

The operation upon the soft palate is called staphylorrhaphy; that upon the hard palate, uranoplasty. At times cleft palate is combined with harelip. This latter condition may be remedied during the first few months of life, leaving the cleft in the palate until later: seventh to eighth year. Julius Wolff operates upon cleft palate earlier, during the second or third year, and this seems advisable. The operation for closure of a complete cleft may be done in two sittings: closure of the hard palate first and the soft palate subsequently at a second sitting. As a rule, however, it is preferable to close the entire cleft at one sitting.

The operation is probably best done with the head in the Rose position, the patient lying upon the back, with the head hanging over the end of the table, and under complete anæsthesia (chloroform). Some operators advise a preliminary tracheotomy with the introduction of a tampon cannula, or an ordinary tracheotomy tube may be introduced, in this latter case, packing the pharynx, in addition, with a pad of gauze. Blood is thus prevented from entering the larynx, and the anæsthetic is administered through the tracheotomy tube. Even whell these measures are resorted to, the Rose position is still preferable. The mouth, teeth, and nasal passages should be thoroughly cleansed and disinfected, and during the operation the mouth and nose may be frequently irrigated with a hot, saline solution, which cleanses the parts and checks hemorrhage. The corners of the mouth are retracted with curved retractors held by an elastic band fastened around the patient's neck. The jaw's are held apart with a Smith or Whitehead gag, which not only holds the jaws open, but at the same time depresses the tongue. If this gag is used, the retractors for the side of the mouth may be dispensed with. Bleeding is controlled by pressure with hot pads on long sponge holders, and one should, at short intervals, interrupt the operation for this purpose; usually the hemorrhage is simply an 
oozing from the cut edges. By carefully minimizing the loss of blood we are enabled, with safety, to operate upon quite young children: less than two years of age (Julius Wolff).

Staphylorrhaphy.-Closure of a split in the soft palate. The first step of the operation consists in freshening the edges of the cleft. The free extremity of one side of the split uvula is seized with a long mouse-tooth forceps, and, while the uvula is thus held taut, it is transfixed, near its tip, which is steadied in the grasp of the forceps, with a narrow-bladed, sharp-pointed, double-edged knife, and with a sawing motion a thin strip is eut away from its margin; the edge is pared along the entire extent of the split toward the posterior

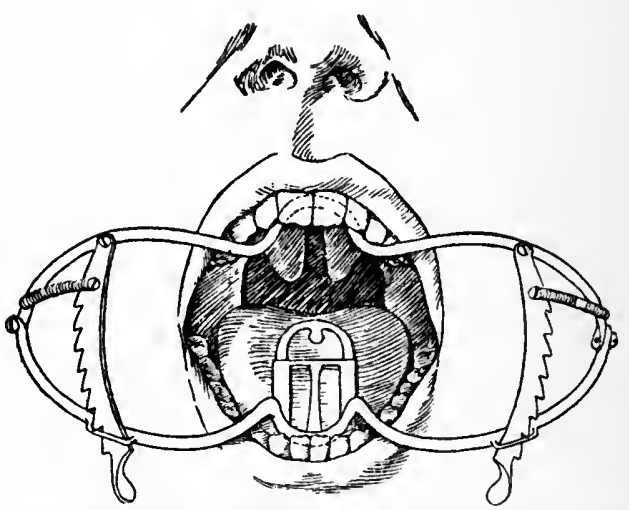

Fig. 59.-Whitehead Gag and Tongue Depressor in Place. For operations upon the hard and soft palate.

border of the hard palate. The opposite edge is then freshened in a like manner and the strips finally cut away from the tip of the uvula. Care should be taken to freshen the angle of the split. The strips should be so cut that the freshened margins present a beveled edge, more tissue being taken away from the buccal than from the nasal aspect of the soft palate, as this gives us broader surfaces for suture. The freshening of the margin of the split may be done with long, narrow-bladed scissors instead of with the knife. After the edges have been freshened, one should, with sharp tenacula, attempt to appose the raw edges in order to estimate what degree of tension, if any, exists. It is absolutely necessary that there be no tension whatever. In order to overcome tension of the soft palate a liberat- 
ing incision may be made upon either side. These incisions are made with a narrow-bladed, double-edged knife, which is introduced just to the inner side of the hamular process. This process, which is located behind and internal to the last molar tooth, is very readily felt. These incisions pass through the entire thickness of soft palate, from behind forward, and divide the tendons of the levator and tensor palati as they turn around the hamular process to spread out into the soft palate. One may wait with these incisions until after the soft palate has been sutured, since they may not be necessary, especially if care has been taken to thoroughly detach the soft palate from the posterior border of the hard palate and also from the adjoining portion of the pterygoid process, which corresponds to the most external portion of the posterior border of the hard palate. If this separation is thorough, the two halves of the soft palate may be readily approximated without tension, and the liberating incisions can then be dispensed with (Julius Wolff). Even when the cleft is limited to the soft palate, one may with advantage raise a mucoperiosteal flap, as when closing clefts of the hard palate; so that, working underneath this flap, close to the surface of the bone, the soft palate may be completely separated from the posterior border of the hard palate. This may be done with a periosteum elevator bent upon itself near the end to almost a right angle.

To unite the freshened edges of the soft palate a small, short, surgeon's needle with a moderate curve or a short, straight needle may be used. The needle is carried in a long needle holder, and as it pierces the tissues its end may be seized with an artery forceps for the purpose of withdrawing it: A combination needle and holder in one piece is preferred by some surgeons.

The stitches, which may be of silk, silk-worm gut, or silver wire, are introduced from before backward, and are not tied until they are all placed. Instead of tying the sutures they may be fixed with perforated shot. To prevent the suture ends becoming confused one may confine them temporarily, until ready to tie them, in incisions cut in a piece of cardboard. From four to five sutures are required, and they should be placed abont one-fourth inch apart. The edges of the soft palate should be accurately apposed without tension and free from hemorrhage.

Uranoplasty.-Closure of clefts of the hard palate. The operation of Langenbeck as described by him in 1862 . This condition is usually associated with cleft of the soft palate, in which case both 
should be closed at the same time. The tip of one side of the uvula is seized with a long, mouse-tooth forceps and transfixed as described above. The paring process is carried forward as far as the posterior border of the hard palate and then continued along the margin of the cleft in the hard palate, close to its edge, cutting through the muco-periosteal covering down to the surface of the bone, as far as the anterior limit of the cleft. Upon the other side, beginning, again, behind, near the tip of the soft palate, the margin of the cleft in the soft palate and in the hard palate is freshened in a similar manner. During this step of the operation one should pause occasionally for a few minutes and apply steady, firm pressure with a hot pad in order to control the bleeding.

The next step of the operation is the raising of a muco-periosteal flap from the surface of the hard palate upon either side of the cleft. An incision, corresponding to the length of the cleft, is made upon the surface of the hard palate and close along the inner margin of the alveolar process. This incision usually extends from a point. anteriorly, behind the incisor teeth, to a point posteriorly, beyond the last molar tooth. In making this incision we should remember the point where the posterior palatine artery emerges from the canal in the back part of the palate, and place the incision fairly close to the alveolar process so that this vessel may be left in the flap to nourish it and also in order that we may avoid the hemorrhage that would follow its division. Many surgeons claim that it is a matter of indifference whether this vessel is cut or not, as the flap is nourished just the same in either case and that the resulting hemorrhage is readily controlled by pressure; nevertheless one should try to avoid dividing it. Into this incision a sharp periosteum elevator is introduced,it may be narrow and rather bent near the end,-and with this the muco-periosteal layer is lifted away from the surface of the bone and thus made freely movable so that it can be brought over to meet the edges of the flap on the opposite side. Care should be exercised to separate thoroughly the soft palate from the whole posterior border of the hard palate. This is accomplished by working close to the surface of the bone with a periosteum elevator bent upon itself. If this detachment of the soft palate is thorough, it will, in nearly all instances, do away with the necessity for liberating incisions, etc.

The apposed edges of the cleft are now sutured together, commencing in front, behind the incisor teeth, and working backward, completing the operation by uniting the edges of the soft palate. As 
already mentioned, the sutures are not tied until after they hare all been placed. The raw space left on either side of the hard palate after raising the muco-periosteal flaps is packed.

Ordinarily the sutures may be removed after six days. The mouth and nose should be irrigated and washed out frequently both during and subsequent to the operation. If a preliminary tracheotomy has been performed the mouth may be packed, the patient breathing freely through the tracheotomy tube. The original defect of the hard palate is closed ultimately by bone produced from the periosteal surface of the flaps.

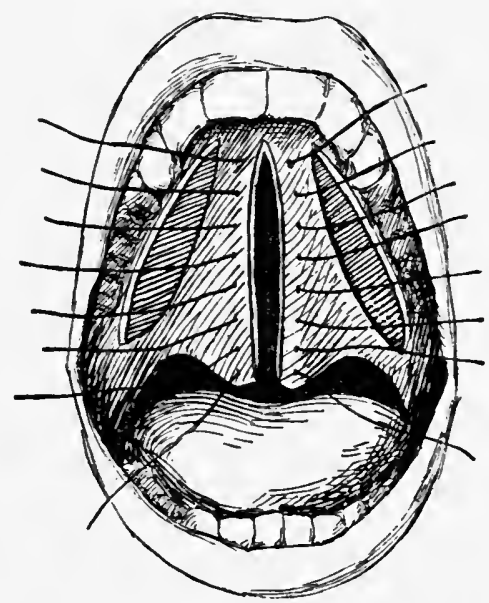

Fig. 60.-Repair of cleft Palate. Muco-periosteal flaps raised and edges of cleft in hard and soft palate pared. Sutures all introduced and ready for tying.

\section{OPERATIONS UPON THE LIPS.}

Excision of the Whole Lower Lip.-This operation is done for malignant disease. At times the angle of the mouth is involved and the upper lip is also encroached upon, so that it becomes necessary, in addition to excising the lower lip, to excise a triangular portion of the upper lip. The cutting is done with a scissors, and during the operation the bleeding is controlled by compression with the fingers. If the jaw-bone is involved in the disease one may resect the diseased portion with the chisel or saw, but should leave, if possible, a bridge of bone sufficient to preserve the continuity of the jaw. 
Restoration of the Lower Lip After Excision of a Wedge-shaped Portion.-After the whole lower lip has been removed, the triangularshaped defect that remains may, in many cases, be remedied by simply drawing the edges of the wound together. The edges of the wound may be united with several sutures of rather heavier silk which go through the entire thickness of the lip down to, but not including, the mucous membrane, and these may be placed so as to control the hemorrhage at the same time. There are then applied additional sutures of finer silk that bring the edges of the wound accurately together. As a result, we have a small, rounded, puckered opening, representing the mouth, which is formed entirely from

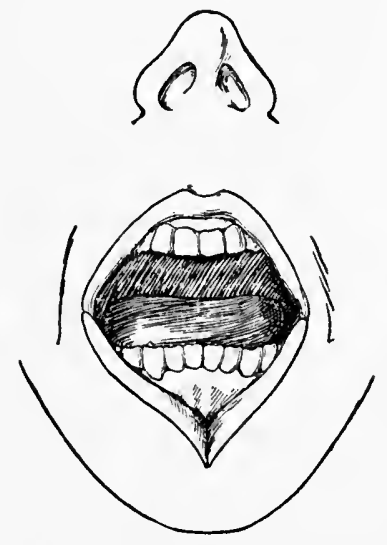

Fig. 61.-Excision of Entire Lower Lip, with Resulting Triangular Defect.

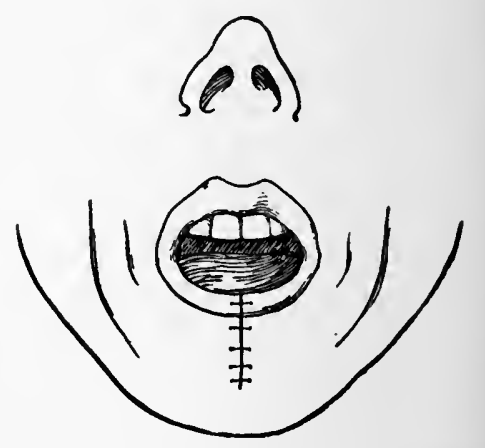

Fig. 62.-Triangular Defect in Lower Lip Closed by Suture.

the upper lip, but this regains an appearance very much like normal, after six to eight months.

Formation of the Lower Lip After Complete Excision. DrefrenBACH-JAESCHE METHOD.-To remedy a triangular defect in the lower lip. In estimating the area of the flaps required one should allow one-third for shrinkage.

From each corner of the mouth an incision is carried outward and somewhat upward into the cheek for a sufficient distance to close the defect in the lip, allowing one-third for shrinkage. From the end of each of these incisions a second curved incision is then carried downward and inward toward the chin so as to terminate near the lower border of the jaw and under the angle of the mouth. 
Stenson's duct should be avoided in making these flaps. This second incision, being curved, makes the flaps more movable. The mucous membrane, corresponding to that part of the incision that reaches outward from the corner of the mouth, should be cut upon a higher level than the skin in order thus to obtain a mucous membrane flap which may be sutured to the edge of the skin to form the free border of the new lower lip. For the rest of its extent the incision goes through the skin and mucous membrane upon the same level. The two flaps are now separated from the lower jaw, avoiding, as far as possible, cutting the fold of mucous membrane that is reflected from the inner surface of the lips to the gums. If the flaps are not sufficiently movable to bring them together, the incisions may be pro-

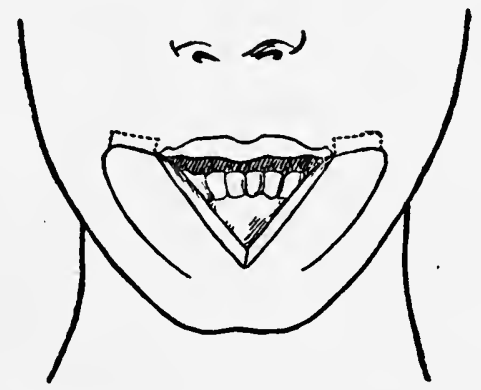

Fig. 63.-Dieffenbach-Jaesche Operation for Restoring Lower Lip. Dotted lines represent the edges of the mucous membrane, which is cut long in order to cover over the free margin of the new lip. The edges of the flaps are drawn together and the mucous membrane, which was cut long, is sewed over the free edge of the new lip. The defect upon each side caused by the sliding of the flaps is closed by suture.

longed downward beyond the lower border of the jaw into the neck and the flaps loosened still farther from the lower jaw. The edges of the flaps are then united with interrupted silk sutures which include the whole thickness of the lip down to, but not including, the mucous membrane. A second set of intermediate silk sutures brings the edges of the skin and mucous membrane into accurate apposition. Corresponding to the free border of the new lip, the edges of the mucous membrane flaps, which were intentionally cut long, are sutured to the skin. Finally the semilunar defects upon either side are closed with sutures. In the male the scar is hidden by the beard.

BRuns MethoD.-For a quadrangular defect of the lower lip. A square cornered flap is taken from either side of the face, includ- 
ing the whole thickness of the cheek, and these are turned down into the defect through an angle of ninety degrees. These flaps have a good blood-supply. Avoid Stenson's duct. The apposed

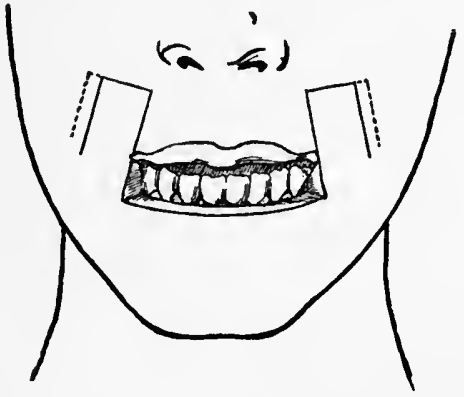

Fig. 64.-Bruns Method of Restoring the Lower Lip. Dotted lines indicate that the mucous membrane is cut longer than the skin in order to provide a mucous membrane border to the new lip.

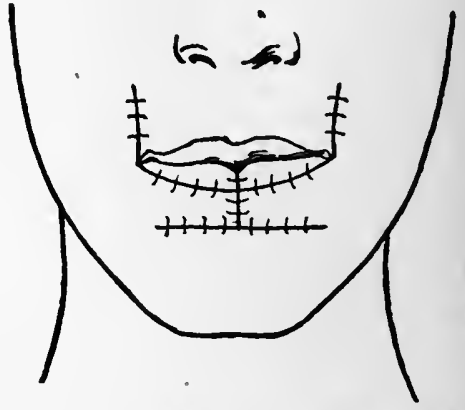

Fig. 65.-Flaps Turned down and Joined to Form New Lip. Mucous membrane is sutured over the free margin of the new lip. The defect upon each side of the cheek is closed by suture.

edges of the flaps are united and the mucous membrane sutured to the edge of the skin to form the free margin of the new lip. The lateral defect on either side is then closed. The scars that result are upon the cheek.

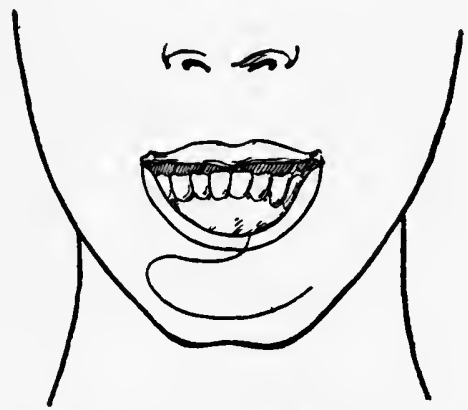

Fig. 66.-Langenbeck Method of Restoring the Lower Lip. An oval flap is taken from the region of the chin.

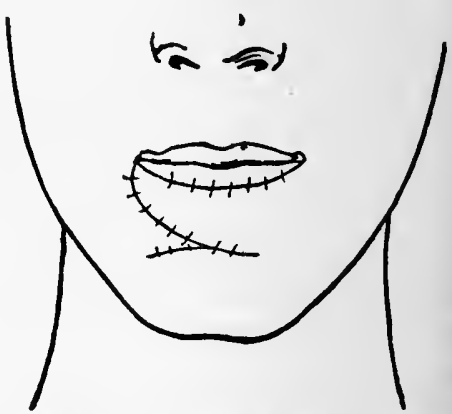

Fig. 67.-Oval Flap is Raised and Sutured into Place and the Defect thus Closed.

LANGENBECK's MeThoD.-Formation of the lower lip for oval defect. A long, rounded flap is taken from the region of the chin with its base directed upward and outward. Between the upper 
border of the flap which is thus marked out and the lower margin of the defect there is a triangular tongue of tissue. This tongue of tissue is partly loosened from its attachment to the underlying tissues. The long flap is raised from the underlying parts and shoved upward, filling in the defect in the lip, and the triangular tongue of tissue is brought under it. These flaps are fixed in their new position with sutures. The whole defect may be closed over if the flaps are sufficiently detached. The great disadvantage of this method is that the new lip, upon its free edge and posterior surface, is not covered by mucous membrane, and shrinks and contracts as it cicatrizes.

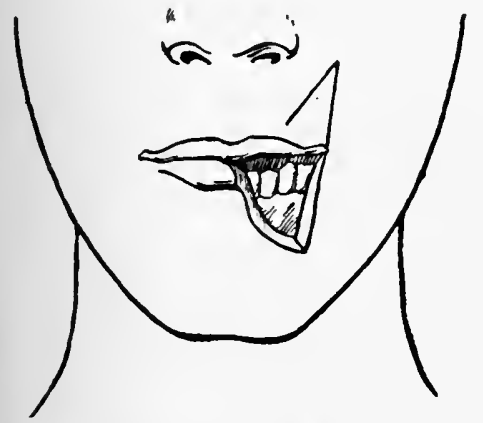

Fig. 68.-Estlander's Method of Restoring the Lower Lip After Partial Excision. A triangular flap is taken from the upper lip and cheek.

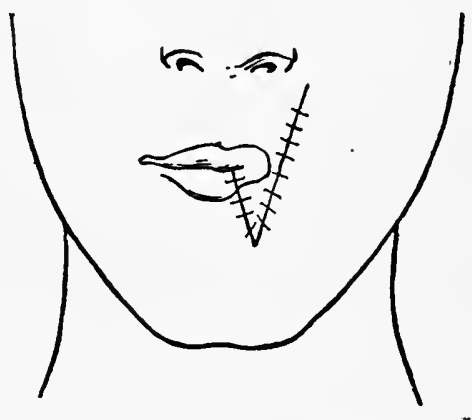

Flg. 69. - The Triangular Flap is Turned down and sutured in Place, thus Closing the Defect.

Estlander's MethoD.-As large a defect as that left after excision of three-fourths of the lower lip may be covered by this method. An incision is made reaching from the corner of the mouth upward, through the whole thickness of the cheek, to the level of the infra-orbital foramen and then downward, past the wing of the nose, toward the philtrum, to a point close to the carmine border of the upper lip. If the coronary branch of the facial artery is not divided, the flap will be well nourished. The flap is then turned down into the defect in the lower lip through an angle of one hundred and serenty degrees. One may feel the pulsating coronary artery before cutting the flap and should positively avoid severing it.

The resulting deformity is bad, the mouth one-sided, the corner of the mouth corresponding to the philtrum. In order to correct this feature one might, subsequently, do another operation, extending the 
corner of the mouth outward, but it would be necessary to wait at least six weeks, in order to insure a good blood-supply, before undertaking this second operation; otherwise there would be danger of gangrene. Without doubt this deformity will, in time, correct itself to a considerable degree, so that the secondary operation may not be necessary.

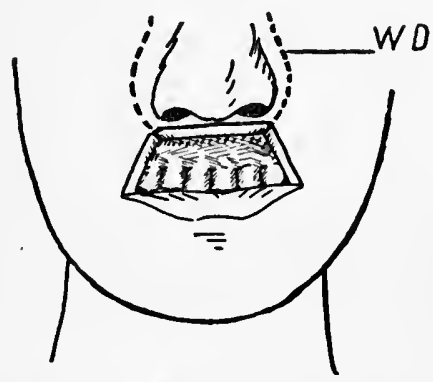

Fig. 70.-Dieffenbach Wellenschnitt for Restoration of the Upper Lip. An incision $(W D)$ is carried around each side of the nose, extending through the cheek.

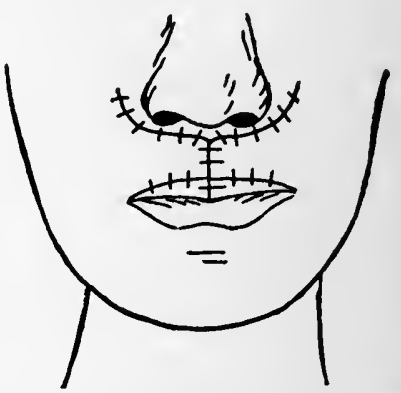

Fig. 71.-The Flaps are Liberated trom the Upper Jaw-bone and are Drawn Down into Place and Sutured. The raw space npon either side of the nose is closed with suture.

Restoration of the Upper Lip.-Restoration of the upper lip is not often required, as this part is but rarely the seat of disease that calls for its excision.

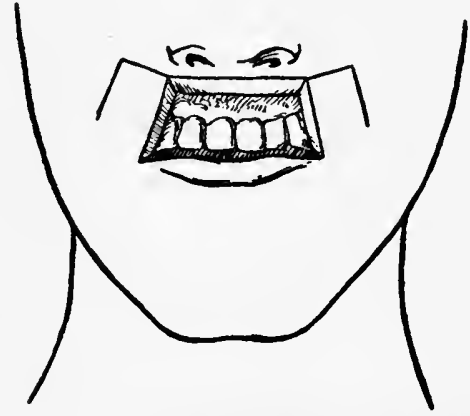

Fig. 72.-Bruns Method of Restoring Upper Lip. A square flap taken from either cheek.

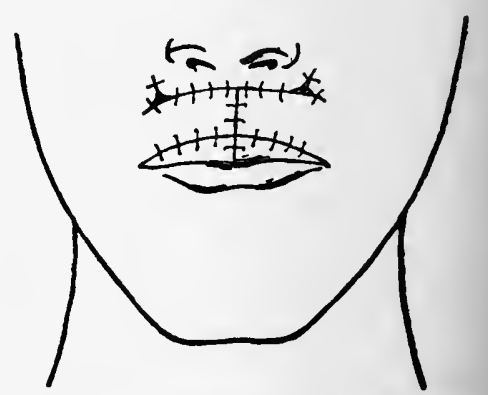

Fig. 73.-Flaps are Turned down into Place and Sutured. Defect in either cheek is closed with sutures.

Estlander's Method may be used to close a wedge-shaped defect in the upper lip, the flap being taken from the lower lip.

DiefFenbach's WeLlenschnitT.-A curved incision is made through the whole thickness of the cheek around the corner of the 
nose. The flaps which are thus marked out are separated from the maxillæ and then drawn toward the middle line and turned down, so that the raw edges of the original defect become the free border of the new lip. The two flaps are then united and the edges of the mucous membrane and skin sutured together along the free margin of the new lip. The mucous membrane corresponding to this margin may be cut a little longer than the skin, in order to facilitate the union of these edges. After uniting the flaps in the middle line the edges of the defect around the side of the nose may be brought together with sutures.

Small, wedge-shaped defects may be closed by simple suture, if necessary, combining this with detachment of the cheek by Dieffenbach's Wellenschnitt.

BRons Method may also be used to restore the upper lip after its complete excision. 


\section{PART III. \\ NECK AND TONGUE.}

\section{SURGICAL ANATOMY OF THE NECK.}

THE neck is the constricted part of the body that joins the head to the trunk. The spinal column passes through the posterior part of the neck, inclosing within its canal the spinal cord. The anterior part of the neck is made up of important organs and of channels that pass between the head and the trunk.

The Deep Cervical Fascia.-This is an aponeurotic layer that serves to bind the structures that comprise the neck, into a compact, cylindrical mass. This fascia offers a strong barrier to the extension of superficial suppurative processes into the deeper parts of the neck, and at the same time hinders, to a considerable degree, the spontaneous evacuation, externally, of pus which is located deep in the neck.

Anteriorly, between the edges of the sterno-mastoid muscle, the deep cervical fascia covers the depressor muscles of the hyoid bonethe sterno-hyoid, sterno-thyroid, and omo-hyoid. Upon the side of the neck it is found beneath the sterno-mastoid, and may be traced from the posterior border of this muscle backward across the posterior triangle of the neck and beneath the trapezius muscle, where it serves to bind the long muscles of the neck to the vertebral column.

Above, the deep cervical fascia is attached to the lower border of the jaw and to the back of the skull, and, below, to the upper border of the sternum, the clavicle, the spine of the scapula, and the spinous process of the seventh cervical vertebra: vertebra prominens. In the middle line of the neck, behind, the deep cervical fascia is blended with the ligamentum nuchæ, which is prolonged deep into the neck to be attached to the tips of the spinous processes of the cervical vertebræ. The deep cervical fascia is firmly attached to the body and horns of the hyoid bone.

Anteriorly, between the edges of the sterno-mastoid muscles, the deep cervical fascia covers the depressor muscles of the hyoid bone, and consists of two layers, the anterior of which is attached 


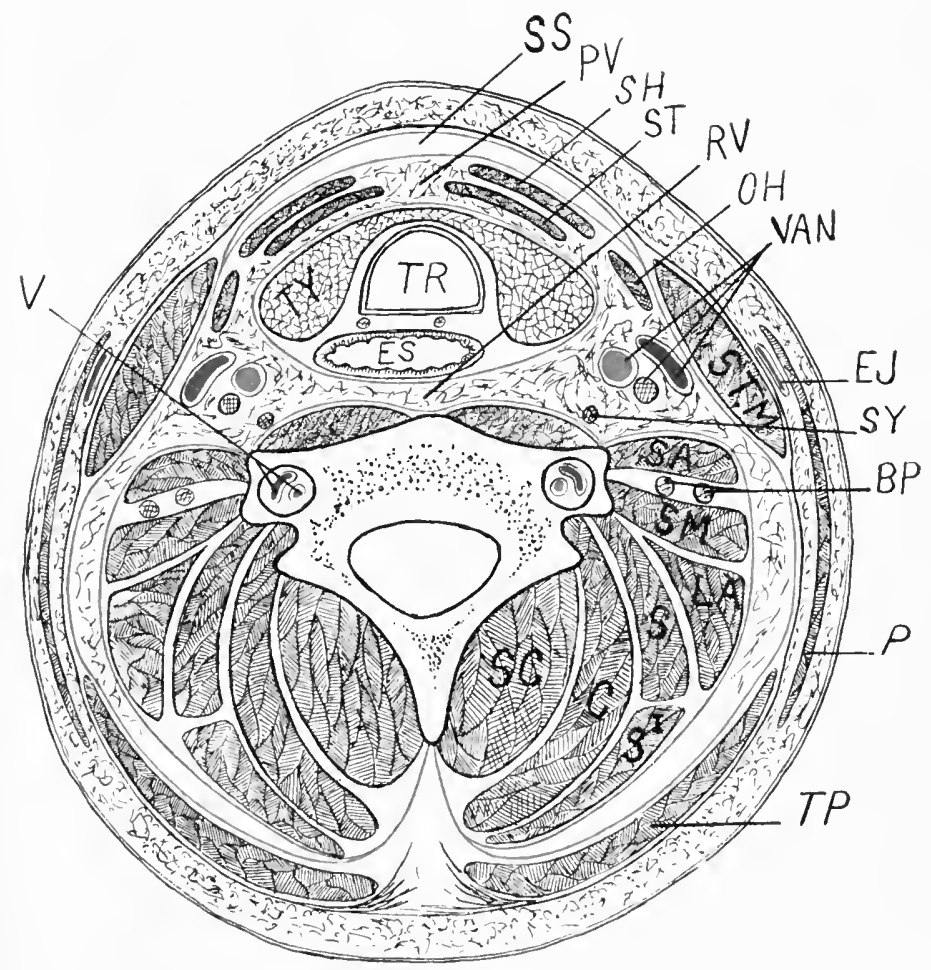

Fig. 71.-Section through the Neck, Level of Sixth Cervical Vertebra, to Show Arrangement of the Deep Cervical Fascia (Indicated in Red). $B P$, trunks of brachial plexus; $C$, complexus muscle; EJ, external jugular vein: $E S$, cesophagus; $L A$, levator anguli scapula muscle ; $O H$, omo-hyoid muscle; $P$, platysma muscle; $P V$, previsceral space; $R V$, retrovisceral space: $S$, $S^{I}$, splenius capitis et colli muscle; $S A$, scalenus anticus muscle; $S C$, semispinalis colli muscle ; $S H$, sterno-hyoid muscle; $S M$, scalenus medius muscle; $S S$, suprasternal space; $S T$, sternothyroid muscle ; $S T . M$., sterno-mastoid muscle ; $S Y$, sympathetic nerve; $T P$, trapezius muscle ; $T R$, trachea; $T Y$, thyroid gland; $V$, vertebral artery and vein ; $V A N$, internal jugular vein. carotid artery, and pneumogastric nerve inclosed in a mass of loose connective tissue. 

to the anterior and the posterior to the posterior margin of the upper border of the sternum. Between the two layers there is a space known as the suprasternal space, which contains some fat, lymphatic tissue, and a venous branch, the anterior jugular, that enters the external jugular beneath the attachment of the sternomastoid.

The suprasternal space extends upward almost as far as the hyoid bone and laterally as far as the anterior edge of the sternomastoid muscle.

A suppurative process in this space is pretty effectively shut off from the deep parts of the neck by the posterior layer of the deep cervical fascia.

In the front part of the neck, below the level of the hyoid bone, the pharynx and œsophagus and the larynx and trachea are bound together in a single bundle by a layer of fascia that completely envelops them; the thyroid gland is also included within this sheath of fascia and is fixed by it to the trachea. Another layer of fascia forms a sheath for the muscles that are contiguous to the vertebral column: anteriorly, the recti and longus colli; laterally, the scaleni, cords of the brachial plexus, and the levator anguli scapulæ; posteriorly, the splenius, complexus, etc.

Above the hyoid bone the deep cervical fascia reaches from the body of the jaw-bone to the hyoid bone. The submaxillary gland, surrounded by a mass of loose connective tissue, is lodged in the submaxillary triangle, beneath the deep cervical fascia.

Connective-'Tissue Spaces Beneath the Deep Cervical Fascia. Pravisceral Space.-This space corresponds to a mass of loose connective tissue that is situated in front of the trachea and thyroid gland and beneath the deep cervical fascia and depressor muscles of the hyoid bone.

If an opening is made in the deep fascia and a probe introduced into this space, it may be readily forced down into the mediastinum, and a collection of pus in this space may readily gravitate along the same route into the mediastinum with fatal results.

Retrovisceral Space.-This is the recess between the pharynx and œsophagus in front and the vertebral column behind; it reaches from the base of the skull down into the chest. Pus in this space may readily find its way down along this path into the chest.

Vascular Space.-Upon either side of the pharynx and œsophagus and the larynx and trachea the carotid artery and its adjoining 
structures are found. These structures, beside the carotid artery, consist of the internal jugular vein and pneumogastric nerve, sympathetic nerve, and loop formed by the descendens and communicans noni. These structures are not provided with a distinct sheath, but are lodged in a mass of loose connective tissue, which may be traced all the way down into the thoracic cavity.

Suppuration may spread along the course of these structures,for example, the internal jugular vein,- and thus invade the chest cavity.

The Back of the Neck.-This region of the neck corresponds to the cervical portion of the trapezius muscle. It is limited above by the occipital protuberance and superior curved line of the occipital bone, below by the vertebra prominens, and upon the sides by the edges of the trapezius muscle.

The skin of this region is intimately united with the subcutaneous connective tissue, which is very dense and is marked by hairfollicles and sebaceous glands. Inflammatory processes which attack the structures of the skin in this region show but little tendency to spread and are excessively painful (carbuncles).

This region presents two longitudinal, rounded swellings-one on either side of the middle line-which correspond to the trapezius muscle. Between these, in the middle line, is a depression marked by the spinous processes of the cervical vertebræ. The spinous processes of the cervical vertebræ are short and not distinctly felt, except the lower ones; that of the seventh, the vertebra prominens, is especially prominent. They are joined together by a dense, ligamentous band,- the ligamentum nuchæ,-which is continued upward as far as the external occipital protuberance. The cervical portion of the vertebral canal is roomy and contains the spinal cord. This part of the vertebral column lies at a considerable depth from the surface, and is well protected by the overlying muscles.

The Side of the Neck.-This region is quadrilateral; bounded above by the lower border of the jaw-bone and an imaginary line drawn from the angle of the jaw to the mastoid process; below, by the clavicle; in front, by the middle line of the neck; and, behind, by the anterior border of the trapezius. It is divided into two triangles-an anterior and a posterior-by the sterno-mastoid muscle.

The sterno-mastoid muscle is a most important surgical landmark. It is attached above to the mastoid process and the adjacent part of the occipital bone; below, to the inner end of the clavicle 
and the upper end of the sternum. This muscle not only divides the side of the neck into an anterior and a posterior triangle, but, being a broad muscle itself, covers important structures not seen in either of the triangles; therefore in addition to the triangles one might well describe a sterno-mastoid region.

The side of the neck is covered by the skin, beneath which the subcutaneous fat and superficial fascia are found, and, beneath these, there is a broad, thin, muscular layer: the platysma. This muscle, which is spread out in a thin sheet, extends from the lower border of the inferior maxilla downward and backward, being continued downward beyond the clavicle, where it is blended with the subcutaneous tissue of the upper part of the chest. The platysma is intimately united with the skin, and together with it is freely movable upon the parts which lie beneath it and with which it and the skin are united by loose connective tissue. It will be observed that the platysma does not cover the anterior portion of the neck in the laryngeal and tracheal regions.

Beneath the superficial fascia and the platysma-i.e., between these and the deep cervical fascia-are found the external and anterior jugular veins together with some nervous branches which are derived from the cervical plexus and from the facial.

The External Jugular Vein, during efforts of straining and in conditions of obstructed venous return, may become distended and sufficiently prominent to be recognized beneath the skin. This vessel is formed above, behind the angle of the jaw, by the junction of the posterior auricular vein and the posterior branch of the temporo-maxillary vein; it passes straight down the side of the neck, crossing the sterno-mastoid muscle from its anterior to its posterior border, and, below, pierces the deep cervical fascia, just above the clavicle and behind the attachment of the sterno-mastoid to this bone, to empty into the subclavian. After it pierces the deep cervical fascia and before it terminates in the subclavian, which it does just external to the tendon of the scalenus anticus, it receives the suprascapular, transverse cervical, and anterior jugular veins.

The Anterior Jugular Vein.-This is formed in the hyoid region by the junction of several veins from the upper anterior part of the neck, and passes downward, anterior to the edge of the sternomastoid muscle, between the superficial fascia and platysma and the deep cervical fascia; in the lower part of the neck it pierces the anterior layer of the deep cervical fascia in front of the sterno- 
mastoid and then passes backward, beneath this muscle, through the suprasternal space, to join the external jugular just before this vessel enters the subclavian. The external and anterior jugular veins are often cut in making incisions in the neck, but may be readily clamped and ligated or they may be recognized and ligated before they are cut.

The Nerves that are found in this part of the neck beneath the superficial fascia and platysma are some superficial ascending and descending branches of the cervical plexus and descending branches from the facial; these, however, are of no special surgical importance.

The Anterior Triangle.-The base of this triangle is above, and corresponds to the lower border of the jaw and an imaginary line drawn from its angle to the mastoid process. Its apex is below at the sterno-clavicular articulation; its posterior border is formed by the anterior edge of the sterno-mastoid muscle, and its anterior boundary is indicated by the middle line of the neck.

The anterior triangle is subdivided into an upper and a lower triangle by the anterior belly of the omo-hyoid; this is a thin, doublebellied muscle that swings obliquely across the side of the neck, being attached above to the hyoid bone and below and behind to the upper border of the scapula. The lower triangle is called the inferior carotid, and the upper, the superior carotid triangle. The anterior triangle presents, in its upper part, a third triangular space: the submaxillary triangle.

The Posterior Triangle.-This is the reverse of the anterior triangle. Its apex is above at the mastoid process; its base, below, is formed by the clavicle; its anterior border corresponds to the posterior edge of the sterno-mastoid muscle and its posterior border to the anterior edge of the trapezius. The posterior triangle is subdivided by the posterior belly of the omo-hyoid into two: an upper or occipital triangle, and a lower or subclavian triangle. In order to demonstrate these triangles it is necessary to draw the posterior belly of the omo-hyoid a little upward, as it usually lies pretty near the clavicle, being fixed in this position, to the first rib, by a slip of the deep cervical fascia.

Since the sterno-mastoid, as already mentioned, is not a line, but a muscle of considerable breadth and covers structures of importance, one might describe, besides these triangular spaces lying in front of and behind the sterno-mastoid muscle, a "sterno-mastoid" 
region, and we will proceed to do this at once and thus dispose of it, and then consider the triangles more in detail.

The Sterno-mastoid Region.-The sterno-mastoid region is covered by the skin and fat (superficial fascia) and to a considerable extent by the platysma. After removing these layers we come down upon the surface of the muscle covered by the deep portion of the superficial cervical fascia. The fibers of the muscle have an oblique direction from above downward and forward, and it is crossed from above downward by the external jugular vein.

To examine the structures that lie beneath the sterno-mastoid, we may divide the muscle through its middle and reflect either end. Then, after cutting through the deep cervical fascia, there are exposed the deep muscles which lie beneath the sterno-mastoid and which are connected with the vertebral column, the longus colli, scaleni, levator anguli scapulæ, etc., the cervical plexus of nerves, the carotid vessels, internal jugular vein, etc., and numerous lymphatic glands.

The Inferior Carotid Triangle.-This triangle is bounded in front by the middle line of the neck, above and behind by the anterior belly of the omo-hyoid, below and behind by the anterior border of the sterno-mastoid.

This triangle contains the larynx, trachea, thyroid gland, and esophagus. These structures are partly covered over and concealed by the sterno-hyoid, sterno-thyroid, and thyro-hyoid ${ }^{1}$ muscles.

The œsophagus, which projects well beyond the left border of the trachea, is more accessible in the left triangle than in the right. Ascending in the recess between the trachea and the œsophagus is the recurrent laryngeal nerve; this nerve enters the larynx between the thyroid and cricoid cartilages, behind the articulation of these two cartilages. Lying to the outer side of these structures (larynx, trachea, and osophagus) are the common carotid artery, with the internal jugular vein upon its outer side, and the pneumogastric nerve between them, but on a plane posterior. The middle thyroid vein passes outward across this space to enter the internal jugular vein, passing across the front of the common carotid artery to reach its destination.

In this triangle the common carotid artery and the internal jugular vein lie beneath the anterior border of the sterno-mastoid

1 The thyro-hyoid is realiy the continuation of the sterno-thyroid. 


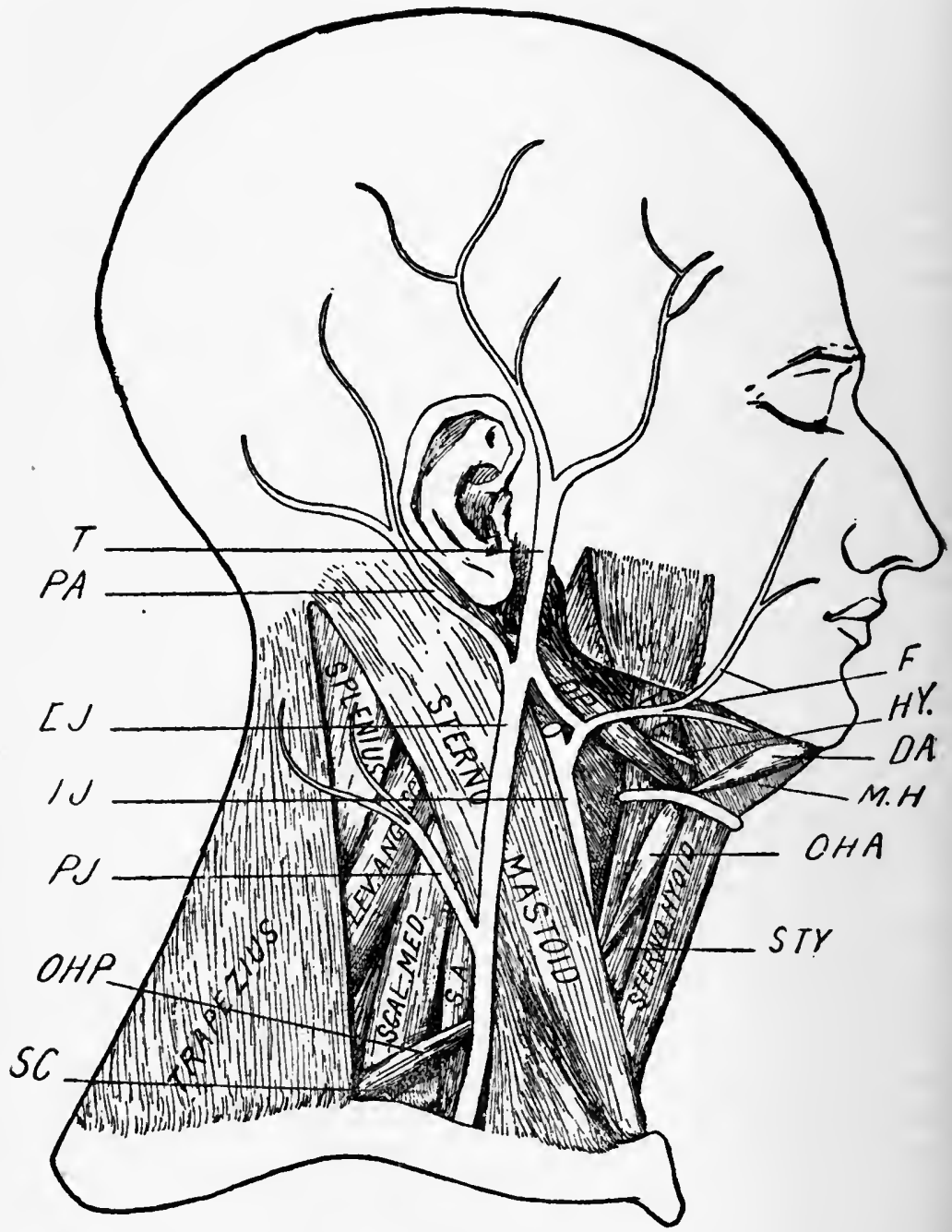

Fig. 75.-Side of Neck to Show Triangles. DA, anterior belly of the digastric; $D P$, posterior belly of the digastric; $E J$, external jugular veln; $F$, facial vein; $H G$, hyo-glossus muscle; $H Y$, hypoglossal nerve; $I J$, internal jugular vein; $M . H$, mylo-hyold muscle; OHA, anterior belly of the omohyold; $O H P$, posterior belly of the omo-hyoid; $P A$, post-auricular vein; $P J$, posterior jugular vein; $S A$, scalenus anticus muscle; $S C$, subclavlan artery: $S . T Y$, sterno-thyroid muscle; $T$, temporal vein. 
muscle, which is the guide to them and which must be drawn outward (backward) in order to expose them. Lying still deeper in this part of the neck, beneath the carotid artery and the internal jugular vein, are the inferior thyroid artery, which passes inward and upward behind these vessels to reach the lower part of the thyroid gland, and the vertebral artery, which enters the foramen in the root of the transverse process of the sixth cervical vertebra. The sympathetic nerve is also found deep in this space behind the carotid vessels, resting upon the muscles which cover the front of the vertebral column, and in this situation it presents its middle cervical ganglion.

The Superior Carotid Triangle. - This space is bounded behind by the anterior border of the sterno-mastoid, above and in front by the posterior belly of the digastric and the stylo-hyoid, and below and in front by the anterior belly of the omo-hyoid. The floor of this space is formed by the constrictor muscles of the pharynx and the thyro-hyoid and a part of the hyo-glossus muscles. It contains the upper part of the common carotid artery and its bifurcation into the internal and external carotids, which division occurs upon a level with the upper border of the thyroid cartilage. The internal jugular vein lies in close contact with the outer side of the common carotid artery and its continuation, the internal carotid; and the pneumogastric nerve still holds its place between the artery and vein, but on a plane posterior to both.

The vessels in this triangle are superficial, not being covered by the anterior edge of the sterno-mastoid, but lying anterior to it. The edge of the muscle is here also the guide to the vessels. A chain of lymphatic nodes is located along the front border of the sterno-mastoid muscle, and some of them are in very close proximity to the internal jugular vein.

In this triangle, the external carotid, as it ascends to a point behind the angle of the jaw, describes a slight curve with the convexity forward, and lies rather beneath the posterior belly of the digastric and stylo-hyoid and upon a plane anterior to the internal carotid, giving off several important branches: among them the superior thyroid, which passes to the upper part of the thyroid gland; the lingual, which passes forward beneath the hyo-glossus muscle to supply the tongue; and the facial, which passes upward and outward over the lower border of the jaw. The occipital and the posterior auricular are derived from the posterior aspect of the 
external carotid artery and ascend in a direction upward and backward.

The hypoglossal nerve arches forward across the external carotid artery upon a level with the origin of the occipital artery.

In this space the facial vein is joined by a large branch from the temporo-maxillary, and then passes downward and outward across the external carotid and internal carotid arteries to enter the internal jugular vein. This vein is often cut during extirpation of glands in this triangle and gives rise to a copious hemorrhage, which is readily controlled by pressure with the finger in the wound and artery forceps. It may often be recognized and tied double before it is cut.

The Submaxillary Triangle.-The submaxillary triangle is bounded above by the lower border of the jaw and an imaginary line drawn from the angle of the jaw to the tip of the mastoid process, below and in front by the anterior belly of the digastric muscle, and below and behind by the posterior belly of the digastric and the stylo-hyoid muscle. The apex of the triangle corresponds to the attachment of these muscles to the hyoid bone. When the coverings of this triangle-consisting of the skin, subcutaneous fat, platysma, and deep fascia-are reflected, we find it fairly well occupied by the submaxillary gland, which rests in a bed of loose connective tissue, and various lymph-nodes. The back part of this triangle is crossed by the facial artery, which passes upward and forward over the upper border of the submaxillary gland to reach the lower border of the jaw, over which it curves on to the side of the face, grooving the bone just in front of the attachment of the masseter muscle. The facial vein, which lies superficial to the facial artery, after receiving the submental vein, also crosses the posterior part of the submaxillary triangle, passing downward and backward across (superficial to) the posterior belly of the digastric and stylo-hyoid muscles and, after uniting with a large branch from the temporo-maxillary vein in the upper part of the superior carotid triangle, enters the internal jugular.

After the submaxillary gland has been raised out of its bed, its duct, Wharton's, may be seen passing forward beneath the posterior edge of the mylo-hyoid muscle to open anteriorly in the floor of the mouth. The gland may be isolated and cut away from its duct, and then the floor of the triangle is exposed to view. The floor of the triangle is formed, for the most part, by the mylo-hyoid muscle, 
whose fibers have an oblique direction, and the hyo-glossus, which lies upon a deeper plane than the mylo-hyoid and forms the posterior part of the floor of the triangle; the fibers of the hyo-glossus muscle run straight up and down from the hyoid bone to the under surface of the tongue. The lingual artery lies beneath the hyo-glossus muscle. The submental branch of the facial artery passes forward parallel with and close to the inner surface of the body of the jaw, resting upon the mylo-hyoid muscle. The hypoglossal nerve may be seen passing forward, entering the submaxillary triangle from beneath the posterior belly of the digastric muscle. In the triangle this nerve rests upon the hyo-glossus muscle, disappearing anteriorly beneath the posterior border of the mylo-hyoid muscle. Accompanying the hypoglossal nerve is the lingual vein, which passes backward and enters the facial.

The hypoglossal nerve forms the base of a second smaller triangle, which corresponds to the apex of the submaxillary triangle and which is called the lingual triangle.

The Lingual Triangle.-The base of the lingual triangle, which is above, is formed by the hypoglossal nerve; its borders, anterior and posterior, by the respective bellies of the digastric. The apex of the triangle is located below where this muscle is attached to the hyoid bone. The floor of the triangle is formed by the fibers of the hyo-glossus muscle. Directly beneath this muscle, in the space marked out as the lingual triangle, the lingual artery is located, and in this situation it is very readily found and ligated. The hyo-glossus muscle is picked up with mouse-tooth forceps and snipped through, when the lingual artery comes into plain view and may be easily surrounded with a ligature in a carrier.

The Occipital Triangle.-This space is bounded in front by the posterior border of the sterno-mastoid, behind by the anterior border of the trapezius, and below by the posterior belly of the omohyoid. This triangle is of but little surgical importance. It is covered by the skin, superficial fascia (fat), by the platysma in part, and by the deep cervical fascia. Beneath the deep cervical fascia there is a mass of loose fat. Lying upon the deep fascia (superficial to it) is the posterior jugular vein, which, below, at the posterior border of the sterno-mastoid muscle, joins the external jugular. A chain of lymphatic nodes, which lie along the posterior border of the sterno-mastoid in this triangle, are frequently diseased and require removal. The space is crossed by the superficial descending 
branches of the cervical plexus. The spinal accessory nerve emerges from the posterior border of the sterno-mastoid, at the junction of its upper and middle thirds, and passes obliquely downward and backward across this space, beneath the deep cervical fascia, and disappears under the anterior border of the trapezius muscle, which it supplies. The floor of this space is formed, from above downward, by the splenius, the levator anguli scapulæ, and the middle and posterior scaleni.

The Subclariax Triangle.-This triangle corresponds to the lower part of the posterior triangle. It is covered by the skin, fat, and superficial fascia, the platysma, and deep cervical fascia, and is crossed by the superficial descending branches of the cervical plexus. In the front part of this space, just behind the posterior border of the sterno-mastoid muscle, the external jugular vein pierces the deep cervical fascia. After the integument, etc., including the deep cervical fascia, have been incised, the boundaries of the subclavian triangle may be sought for. These are, below, the clavicle; in front, the posterior border of the sterno-mastoid muscle; and, above, the posterior belly of the omo-hyoid; this latter muscle lies low in the neck, close to the clavicle, and in order to demonstrate the triangle it may be necessary to draw it somewhat upward.

Crossing the space from without inward, just above the clavicle, are the transversalis colli and suprascapular reins; these form a plexus beneath the deep cervical fascia and terminate in the external jugular; the external jugular vein enters the subclavian just external to the tendon of the scalenus anticus. The external jugular vein, after piercing the deep cerrical fascia and immediately before it terminates in the subclavian, also, as a rule, receives the anterior jugular vein. This latter drains the front of the neck, originating above in the hyoid and suprahyoid regions. In the subclavian triangle there is also found (beneath the deep cervical fascia) a mass of lymphatic nodes, fat, and loose connective tissue which communicates with the lymphatics of the breast and axilla and which may become involved in disease of the breast. The floor of the subclavian triangle is formed by the scalenus anticus and scalenus medius muscles. In order to expose the scalenus anticus muscle, the sterno-mastoid, which conceals it, must be drawn forward (inward). When the scalenus anticus is thus exposed the phrenic nerve may be seen passing obliquely downward and inward across its anterior surface, descending into the chest across the front of 
the first part of the subclavian artery. Beneath the venous plexus above mentioned, and lying close upon the muscles that form the floor of the triangle, are the transversalis colli and suprascapular arteries: branches from the first part of the subclavian. Emerging from between the scalenus anticus and the scalenus medius and passing obliquely downward and outward are the three cords of the brachial plexus. They disappear beneath the clavicle into the axillary space. The third part of the subclavian artery is found below the cords of the brachial plexus, deep in the subclavian triangle, below the level of the clavicle, resting in the groove upon the upper surface of the first rib, external to the attachment of the tendon of the scalenus anticus. The tendon of the scalenus anticus is the guide to the artery, and is readily recognized in the inner or forward part of the subclavian triangle as a tense cord and may be followed downward with the finger as far as its attachment to the first rib. The subclavian vein lies some distance away from the artery in front of, and internal to it, the artery and vein being separated from each other by the tendon of the scalenus anticus.

As the subclavian artery emerges from the chest it arches outward and forward to reach the first rib. That portion of the subclavian which lies behind the tendon of the scalenus anticus is the second part of the artery; the part which lies to the inner side of this tendon is the first part; and that which lies external to the tendon of the scalenus anticus, resting upon the upper surface of the first rib, is the third part of the artery: the part that is usually ligated. The second and first parts of the subclavian artery, the parts behind and internal to the tendon of the scalenus anticus, are in direct relation with the dome of the pleura and the apex of the lung, which projects upward into the root of the neck, beneath the scaleni muscles, for a distance of 3 to $3 \frac{1}{2} \mathrm{~cm}$. above the level of the clavicle. In tying the third part of the subclavian artery one should not mistake for it one of the cords of the brachial plexus, which lie above. The artery is deep, and rests directly upon the first rib. The subclavian vein is pretty well separated from the artery, lying in front of and internal to it and upon a rather lower level than the artery. By drawing the shoulder down we depress the clavicle, and may thus make the artery more accessible.

The Front of the Neck.-This part of the neck may be divided into the suprahyoid region, the part above the hyoid bone, and the infrahyoid region, the part below the hyoid bone. The infrahyoid 
region presents for consideration the larynx, trachea, and thyroid gland, and the œsophagus, which lies behind these.

The Hroid Bove.-This is a horseshoe- or U- shaped bone, with a body and two lateral horns, which are prolonged backward, one on either side, and two lesser horns, directed upward.

In the natural position of the head the hyoid bone is on a level with the lower border of the inferior maxillary bone, and is not distinctly recognized until the head is thrown back. It is not stationary, but may be said to be about opposite the fourth cervical vertebra. To it are attached numerous muscles, coming from different directions. To the upper surface of its body is attached the base or root of the tongue; from its lower border is suspended the larynx. The epiglottis is placed behind the body of the bone, and is attached to its posterior surface. To the upper surface of its lateral horn is attached the middle constrictor of the pharynx, and it thus serves to support the wall of the pharynx and provide a fixed point for the action of the muscles in deglutition.

SUPRahyoId Region.-This is the space between the hyoid bone and the lower border of the jaw. This region is covered with skin, superficial fascia (fat), platysma, and deep fascia; the deep fascia is attached to the body and cormua of the hyoid bone. Beneath the platysma, between it and the deep fascia, are several venous branches which go to form the anterior jugular. Upon removal of the deep fascia a triangular space is exposed: the submental triangle. The apex of this triangle corresponds to the symphysis of the lower jaw, its sides to the anterior belly of either digastric, and its base to the hyoid bone. Its floor consists of the mylo-hyoid muscle, with its raphe in the middle line. This space contains, beneath the deep fascia, several lymphatic nodes, which are occasionally the seat of disease and may demand extirpation. Beneath the mylo-hyoid, upon either side, in the floor of the mouth, the sublingual glands are lodged. The floor of this space is, at times, cut through in operations upon the lower jaw and in order to reach the tongue.

INFRAHYOID REgION.-This is the region below the hyoid bone. The skin is but loosely attached to the underlying structures; beneath the skin are fat and the deep cervical fascia. The platysma is not met with in this part of the neck. Below the hyoid bone may be felt the thyroid cartilage, that of either side uniting in the middle line to form the prominence "Adam's apple." The Adam's apple is not prominent in the female or child, and is not, therefore, a good 
surgical guide. Below the thyroid the cricoid cartilage may be felt. This is located opposite the sixth cervical vertebra, and marks the point where the omo-hyoid muscle crosses the common carotid

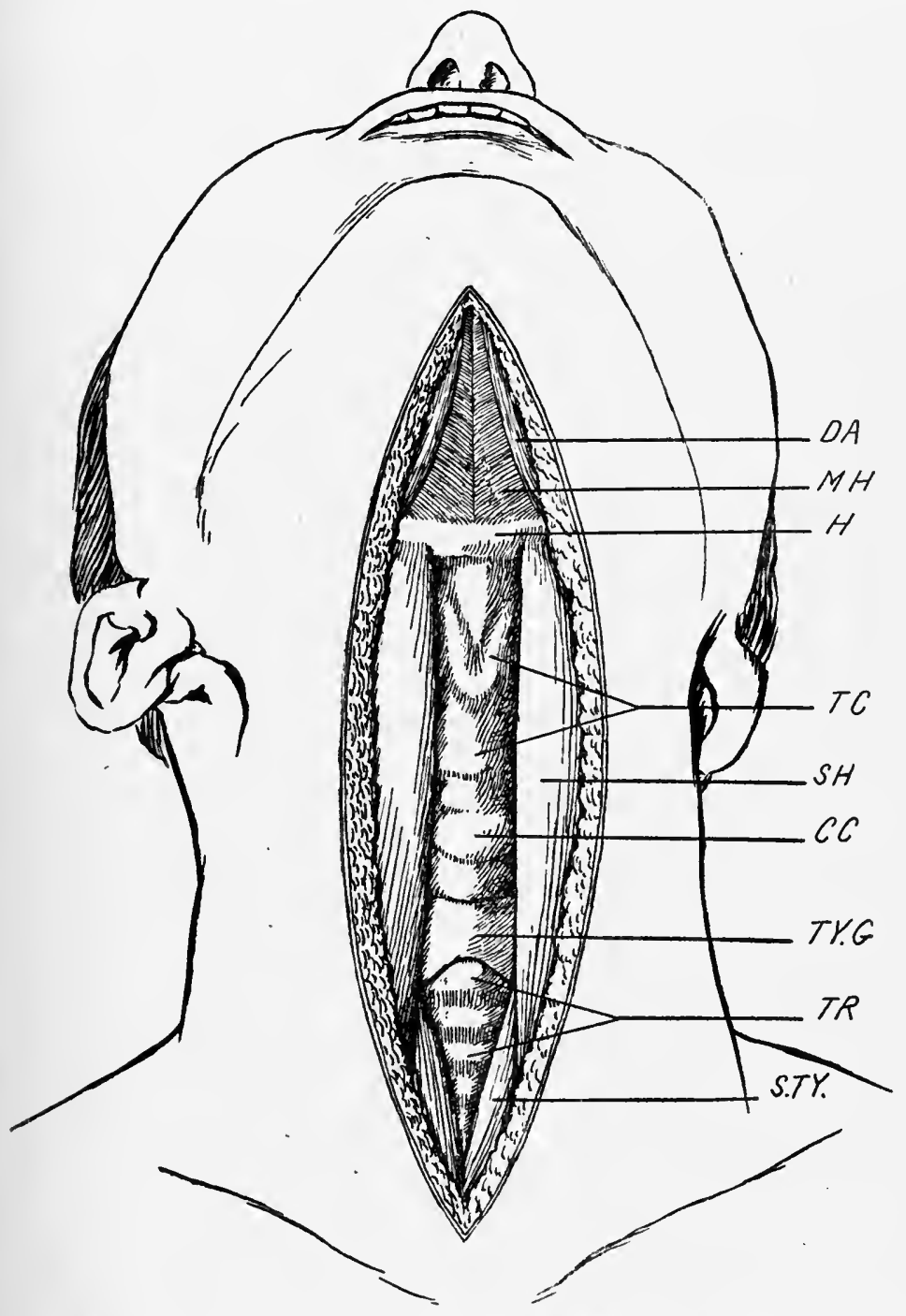

Fig. 76.-Front of the Neck. $C O$, cricoid cartilage; $D A$, anterior belly of digastric; $H$, hyoid bone; $M H$, mylo-hyoid muscle; $S H$, sterno-hyoid muscle; $S . T P$, sterno-thyroid muscle; $T C$, thyroid cartilage; $T R$, trachea; TY.G, isthmus of thyrold gland. 
artery. The cricoid is a ring of cartilage which is rather narrow anteriorly, but of considerable breadth posteriorly; it is always very readily felt, and is therefore a good guide. From the cricoid down to the upper border of the sternum the space is occupied by the trachea. Above, at its commencement at the cricoid cartilage, the trachea is quite superficial, lying just beneath the integument; but lower down it gets to lie deeper, farther away from the surface, and is less accessible. Just below the cricoid cartilage the isthmus of the thyroid gland lies transversely across the front of the trachea, each lobe of the gland extending outward and upward beneath the sterno-hyoid and sterno-thyroid muscles, reaching upward upon the side of the thyroid cartilage and getting into close proximity to the common carotid artery and its adjoining structures. Between the cricoid cartilage and the isthmus of the thyroid gland there is usually a space about one-half inch wide. On either side of the middle line, passing from the hyoid bone and thyroid cartilage down to the sternum, are two long, flat, ribbon-like muscles, one superimposed upon the other: the sterno-hyoid and sternothyroid. The sterno-thyroid lies beneath the sterno-hyoid, being partly concealed by the latter. The sterno-thyroid is attached to the side of the thyroid cartilage and does not reach the hyoid bone, but is continuous with the short thyro-hyoid muscle, which is attached to the hyoid bone. The attachments of these muscles are indicated by their names. The inner edges of these muscles do not meet in the middle line of the neck, but are connected with each other through the intervening deep cervical fascia. They partly cover the trachea and sides of the larynx and the lateral lobes of the thyroid gland. Between the edges of the muscles, in the middle line, from above downward, and covered only by the interposed deep fascia, are the thyroid and cricoid cartilages, the isthmus of the thyroid gland, and the trachea.

Between the hyoid bone and the upper border of the thyroid cartilage there is a space which is filled in by the thyro-hyoid membrane. This membrane is pierced on either side by the superior laryngeal vessels and the internal laryngeal branches of the superior laryngeal nerve. This membrane may be cut in attempts at suicide: cut throat. Between the lower border of the thyroid cartilage and the upper border of the cricoid there is also a space which is filled in by a membrane: the crico-thyroid. This may also be divided in cut throat. Above the hyoid bone, running transversely 
inward and anastomosing with the branch of the opposite side, is the hyoid branch of the lingual artery. Below the hyoid bone there is a similar transverse branch, the hyoid, which is derived from the superior thyroid and which passes likewise inward, anastomosing across the middle line with its fellow of the opposite side. A third transverse branch passes inward, above the cricoid cartilage, upon the membrane between the lower border of the thyroid cartilage and upper border of the cricoid cartilage. This is the crico-thyroid branch of the superior thyroid artery. It also anastomoses with its fellow of the opposite side. Below the level of the cricoid cartilage no arterial branches cross the middle line except through the isthmus of the thyroid gland.

The œsophagus lies behind the trachea, closely applied to its posterior wall, and when empty is flattened out against the vertebræ. It projects a considerable distance to the left of the trachea, and is therefore easier to reach through an incision upon the left side of the neck than upon the right. Above, the œsophagus is continuous with the pharynx, into the commencement of which the larynx opens, the orifice of the larynx being protected by the overhanging epiglottis, which is situated below and behind the root of the tongue. The posterior wall of the larynx, which is formed by the broad posterior portion of the cricoid cartilage, is in close relation with the front wall of the pharynx. Only a thin layer of connective tissue intervenes between the anterior wall of the pharynx, which consists merely of a layer of mucous membrane, and the posterior part of the larynx, which is made up chiefly of the broad posterior part of the cricoid cartilage. When the pharynx is empty it is flattened out against the rertebral column, and the larynx, under these circumstances, also lies close to the vertebral column.

From the cricoid cartilage down, the esophagus and trachea, although in close proximity to each other, form two distinct tubes, which may be readily separated, one from the other. The posterior wall of the trachea, which is in direct relation with the cesophagus, is devoid of cartilaginous bands, and therefore a foreign body, lodged in the esophagus, might press upon this contiguous, non-cartilaginous portion of the wall of the trachea and causc symptoms of strangulation. In the recess between the trachea and œesophagus, on either side, the recurrent laryngeal nerve ascends to enter the lower back part of the larynx bchind the articulation which exists between the cricoid and thyroid cartilages. 
The Laryngeal Region is covered in front by skin and deep fascia, but laterally by the muscles, the sterno-hyoid and sternothyroid and thyro-hyoid, and by the lobes of the thyroid gland.

The interior of the larynx may be examined after splitting the thyroid cartilage, taking care to make this section in the midle line, between the anterior attachments of the vocal cords. The true and false vocal cords are then exposed to view. The true cords are the lower, and are attached anteriorly, upon either side of the middle line, to the thyroid cartilage, midway between the lowest part of the incisura in its upper border and the lower border; posteriorly the true vocal cords are attached to the arytenoid cartilages, which rest, swivel-like, upon the upper surface of the cricoid cartilage.

The false vocal cords are the loose folds of mucous membrane which are situated above the true cords, inclosing much loose connective tissue; these may readily become œdematous-œdema glottis -and act as a dangerous obstruction to respiration.

The Thyroid Gland.-The isthmus is the narrowest part of the thyroid gland. It joins the two lobes of the gland across the middle line, resting transversely upon the upper part of the trachea. At times there projects from the upper border of the isthmus a process of glandular tissue, which is located in front of the larynx and which may be encountered in operations in this locality. The thyroid gland is fixed to the cricoid and thyroid cartilages by bands of connective tissue. These bands connect the isthmus of the gland to the cricoid cartilage and the lateral lobes, adjacent to the isthmus, to the sides of the thyroid cartilage. It is necessary to divide those bands that connect the isthmus to the cricoid cartilage before the isthmus can be dislocated downward in order to expose the upper rings of the trachea in performing the operation of high tracheotomy. The two lobes of the thyroid gland, one on each side, are prolonged backward and upward upon the sides of the trachea and larynx, reaching as far back as the cosophagus and thus getting into close relationship with the common carotid artery and its adjacent structures. As the recurrent laryngeal nerve of each side ascends between the trachea and œsophagus to enter the lower, posterior part of the larynx it lies beneath the corresponding lateral lobe of the thyroid gland. The isthmus of the thyroid lies just beneath the skin and deep fascia, whereas the lateral lobes extend upward and backward underneath the sterno-hyoid and sterno-thyroid muscles.

On account of the intimate relationship that exists between the 
thyroid gland and the trachea, tumors involving the gland may press upon the trachea and push it to one side; so that if tracheotomy becomes necessary in these cases it may be difficult to locate the trachea. When the thyroid is enlarged by tumors, etc., it may be seen to rise and fall with the larynx in movements of swallowing. The thyroid is supplied by the superior and inferior thyroid arteries of each side, and drained by the superior, middle, and inferior thyroid veins. At times an arterial branch from the transverse portion of the arch of the aorta ascends upon the front of the trachea to reach the lower part of the gland: the arteria thyroidea ima.

The Suprasternal Region is the space in the lower front part of the neck above the upper border of the sternum and limited on either side by the anterior border of the sterno-mastoid. The surface shows a depression here known as the suprasternal fossa, or fossa jugularis. This region is covered by the skin, beneath which lies the deep cervical fascia, which splits into two layers, an anterior and a posterior; these layers are attached below to the anterior and posterior edges of the upper border of the sternum, inclosing a space - the suprasternal-between them which is occupied by some connective tissue and lymphatic glands. A communicating venous branch which connects the anterior jugulars of either side is also included between these two layers. The suprasternal space is shut off from the mediastinum by the posterior layer of the deep cervical fascia, and pus in this space is thus hindered from breaking into the mediastinum and is more apt to open externally through the skin. Beneath the deep fascia lies the trachea, its anterior surface being readily accessible for operation. This part of the trachea may be lengthened by throwing the head back:. partly by drawing the trachea out of the chest and partly by stretching it.

If the trachea is incised transversely the wound gapes, and, if completely severed, it retracts into the chest to such an extent that it may be difficult or impossible to reunite it. At times the arteria thyroidea ima ascends in front of this lower part of the trachea and might complicate an operation upon this part of the tube.

Descending obliquely downward and outward, from the lower part of the thyroid gland, are the inferior thyroid veins. These enter the right and left innominate veins or both may enter the left innominate, within the chest, behind the first piece of the sternum. The inferior thyroid veins are large and lie one on either side of the middle line. As they descend they get farther away from the 
middle line, so that they are not likely to be encountered in the operation of low tracheotomy if the incision is kept strictly in the median line.

The Blood-vessels of the Neck. The Common Carotid Artery. -This vessel ascends in the neck from behind the sterno-clavicular articulation to the level of the upper border of the thyroid cartilage, where it divides into the external and internal carotid. The course of the artery is indicated by a line drawn from the sterno-clavicular articulation to a point midway between the angle of the jaw and the mastoid process. The muscular guide to the artery is the anterior border of the sterno-mastoid.

The common carotid is crossed about the level of the cricoid cartilage by the omo-hyoid muscle; so that the lower part of the artery lies in the inferior carotid triangle and the upper part in the superior carotid triangle. The artery is more accessible for ligation in the upper triangle. In the lower part of its course, below the omo-hyoid, the artery lies beneath the anterior edge of the sterno-mastoid, whereas above, in the superior carotid triangle, it does not lie beneath the edge of the sterno-mastoid, but rather in front of it, and is here quite superficial, being covered only by the integument, platysma, and deep cervical fascia. Opposite the thyroid cartilage the lateral lobe of the thyroid gland comes into close relation with the artery, the latter grooving the gland. In its course up the neck the artery is accompanied by the internal jugular vein, which lies close upon its outer side, and by the pneumogastric nerve, which lies between the vein and the artery, but on a plane posterior to both. These structures are lodged in a loose, connective-tissue bed, which is continuous below with the connective tissue of the mediastinum.

Upon the front of the artery, opposite the middle of the thyroid cartilage, the descendens and communicans noni form a loop from which some branches are given off to supply the depressor muscles of the hyoid bone. Posteriorly the artery rests upon the transverse processes of the lower cervical vertebræ and the attachments of the vertebral muscles. The sympathetic nerve lies behind the artery and is united rather closely to the fascia that covers the prevertebral muscles. Below, opposite the sixth cervical vertebra, the inferior thyroid artery, which arises from the first part of the subclavian, curves inward, behind the carotid, etc., to reach the lower part of the thyroid gland. To the inner side of the artery are the 
trachea and œsophagus, and, higher up, the larynx and the lower part of the pharynx. The larynx projects forward between the arteries of either side. Ascending between the trachea and the œsophagus is the inferior, recurrent, laryngeal nerve. Opposite the thyroid cartilage the artery, as mentioned above, is in close relation with the lateral lobe of the thyroid gland. Upon the outer side of the artery the internal jugular vein is situated, and in close proximity to the vein a chain of lymphatic nodes. The common carotid artery is crossed above the omo-hyoid muscle by the superior thyroid vein and about its middle-i.e., below the omo-hyoid—by the middle thyroid vein. Both these veins terminate in the internal jugular. Lower in the neck the artery is crossed by the anterior jugular vein, which, as a rule, terminates in the external jugular.

The artery is covered by the integument, superficial fascia, platysma, and deep fascia. The lower part of the artery lies beneath the sterno-mastoid, and this muscle must therefore be drawn aside in order to expose the vessel. Above, upon a level with the thyroid cartilage, the artery lies quite superficial, not being overlapped by the sterno-mastoid, but in front of it and here its pulsation may be both felt and seen.

The Intersal Carotid is continued upward in the same course as the common carotid, lying alongside of the pharynx. The internal jugular vein lies along its outer side, and the pneumogastric nerve lies between both, but on a plane posterior. At the base of the skull the artery enters the carotid canal in the petrous portion of the temporal bone, and after traversing this canal enters the cranium through the middle lacerated foramen. In the neck the internal carotid lies in the superior carotid triangle, covered by the anterior edge of the sterno-mastoid; it is situated deeper than the external carotid and upon a plane posterior to it. The stylo-glossus and stylopharyngeus muscles, as they pass forward to the tongue and to the side of the pharynx, are interposed between the internal and external carotids. Behind, the artery rests upon the transverse processes of the upper cervical vertebrr and upon the rectus capitis anticus major muscle. The sympathetic nerve, with its superior ganglion, lies behind the artery, between it and the anterior vertebral muscles. Internally the artery is in relation with the side of the pharynx. The superior laryngeal nerve descends between it and the pharynx. At its origin the artery lies quite superficial, being covered by the integument, platysma, and deep fascia and over- 
lapped by the anterior margin of the sterno-mastoid muscle. In the upper part of its course it lies deep in the neck beneath the posterior belly of the digastric and stylo-hyoid muscles and the parotid gland and the stylo-pharyngeus and stylo-glossus muscles, these two latter muscles separating it from the external carotid.

At the base of the skull the internal jugular vein leaves the internal carotid artery and enters the skull through the jugular foramen. This foramen is located external and posterior to the opening which marks the commencement of the carotid canal. Just below the base of the skull the glosso-pharyngeal nerve passes forward between the internal jugnlar vein and the internal carotid artery and then continues forward, below the stylo-glossus muscle, to reach the side of the base of the tongue. Just above the level of the hyoid bone the hypoglossal nerve curves forward between the artery and the rein. The spinal accessory, at the base of the skull, is situated between the internal carotid artery and the internal jugular vein, but passes backward and outward to reach the deep surface of the sterno-mastoid muscle.

The External Carotid Artery, at its origin, is located in the superior carotid triangle in front of the internal carotid artery. It passes upward to a point between the posterior border of the ramus of the jaw and the mastoid process, and here, within the substance of the parotid gland, divides into the temporal and internal maxillary. As it ascends upon the side of the neck it describes a gentle curve with the convexity forward and is placed upon a plane anterior to the internal carotid, giving off many branches to the muscles and structures in the neck and to the tongue. It lies in front of the anterior border of the sterno-mastoid, being covered only by the skin, platysma, and deep fascia; higher up, on a level with the angle of the lower jaw, it is covered by the posterior belly of the digastric and stylo-hyoid, and at its bifurcation into its terminal branches it lies deep within the substance of the parotid gland.

The external carotid artery does not lie as deep in the neck as the internal carotid; upon a level with the angle of the lower jaw these two vessels are separated from each other by the stylo-glossus and stylo-pharyngeus muscles (together with the glosso-pharyngeal nerve). Both these muscles arise from the styloid process and pass forward, between the external and internal carotid arteries, in their course to reach the side of the tongue and the pharynx.

As the external carotid artery lies within the parotid gland it 
is crossed, upon a level with the lower border of the lobe of the ear, by the divisions of the facial nerve. The temporo-maxillary vein, which is formed by the junction of the temporal and internal maxillary veins, also lies superficial to it. Below the angle of the jaw the artery is crossed by the temporo-facial vein; this vessel is formed by the facial and a large branch from the temporo-maxillary, and after receiving the lingual and sometimes the superior thyroid, terminates in the internal jugular.

Below the level of the hyoid bone the external carotid gives off the superior thyroid. This branch passes forward and downward to the lateral lobe of the thyroid gland and gives branches to the larynx. The next branch given off above the superior thyroid is the lingual. This vessel passes forward, beneath the digastric and stylohyoid muscles and beneath the hyo-glossus, to supply the tongue. The next branch above is the facial. The facial is directed forward and upward and curving over the inferior border of the lower jaw, in the groove just in front of the masseter muscle, ascends upon the side of the face, nose, etc. At its origin the facial artery lies beneath the posterior belly of the digastric and stylo-hyoid muscles close to the posterior border of the submaxillary gland, which it grooves and supplies; here it gives off its submental branch, which runs forward upon the under surface of the mylo-hyoid muscle close to the body of the lower jaw. From its posterior aspect, upon a level with the origin of the facial, the external carotid artery gives off its occipital branch. This vessel passes upward and backward across the internal jugular vein and ascends beneath the anterior border of the sterno-mastoid muscle to reach the occipital region of the head. Above the origin of the occipital, also from its posterior aspect, the external carotid gives off the posterior auricular. This ressel courses upward and backward, running close behind the ear and supplying this and the mastoid region. The hypoglossal nerve swings forward across the outer side of the external carotid artery upon a level with the origin of the occipital.

The Irternal Jugular Vein lies close to the outer side of the common carotid artery and its continuation, the internal carotid. This vessel is large, as big around as the little finger, very thin walled, and lies in the same connective-tissue bed with the artery and the pneumogastric nerve. It is formed above, at the base of the skull, by the union of the lateral (sigmoid) and inferior petrosal sinuses. These ressels emerge from the interior of the 
skull through the jugular foramen, which is situated behind and external to the commencement of the carotid canal; the pneumogastric, spinal accessory, and glosso-pharyngeal nerves also emerge from the cranium through the jugular foramen. Just outside the skull the lateral and the inferior petrosal sinuses join and form a bulbous dilatation, which marks the commencement of the internal jugular vein. At the root of the neck the internal jugular terminates by joining with the subclavian to form the innominate. In its course through the neck the vein receives a number of large branches: the temporo-facial, lingual, and superior and middle thyroids. A chain of lymphatic nodes is situated along the outer side of the rein, close to its wall, and these may be diseased and require extirpation. On the right side, in the root of the neck, where the internal jugular unites with the subclavian, the right lymphatic duct is seen to enter the vessel. Upon the left side of the neck the thoracic duct enters the vein at its junction with the subclavian; the thoracic duct arches over the third part of the subclavian artery and across the front of the tendon of the scalenus anticus in order to reach the vein.

The Subclavian Artery.-This vessel upon the right side is derived from the innominate, which bifurcates behind the right sterno-clavicular articulation into the common carotid and subclavian. The left subclavian is given off from the left end of the transverse part of the arch of the aorta and ascends in the upper part of the chest as far as the left sterno-clavicular articulation.

From the sterno-clavicular articulation, upon either side, the subclavian artery arches outward across the root of the neck and passes into the axilla to become the axillary. In the root of the neck the artery is found in the subclavian triangle resting directly upon the first rib.

The tendon of the scalenus anticus, at its attachment, is situated in front of the subclavian artery, and thus, for purposes of description, serves to divide the vessel into three parts. The first part of the artery corresponds to that portion which is included between its origin and the inner margin of the tendon of the scalenus anticus; the second part of the artery corresponds to the portion immediately behind the tendon of the scalenus, and the third part of the artery reaches from the outer border of the tendon of the scalenus anticus to the point where it enters the axilla to become the axillary. The first and second parts of the artery are in intimate relation with the 
apex of the lung and dome of the pleura; the third portion rests upon the upper surface of the first rib. The trunks of the brachial plexus in their course through the subclavian triangle are situated above the subclavian artery. The subclavian artery gives off several large branches; from its first part the vertebral, internal mammary, and thyroid axis (inferior thyroid, suprascapular, transversalis colli); from the second part, the superior intercostal. The origin of these branches varies in different individuals and in the same individual upon either side.

The subclavian vein is the continuation of the axillary. It passes inward across the root of the neck, beneath the clavicle and in front of the scalenus anticus tendon, resting upon the upper surface of the first rib and lying in front and to the inner side of the artery. It is situated a considerable distance away from the artery, from which it is separated by the tendon of the scalenus anticus. The subclavian vein joins with the internal jugular to form the innominate. Upon the right side where these two veins join they receive the right lymphatic duct, and upon the left side, at their junction, they receive the thoracic duct.

The Inferion Thyroid Artery is seen deep in the lower part of the inferier carotid triangle. It is a branch of the thyroid axis which arises from the first part of the subclavian, and curves upward and inward, passing inward, behind the common carotid artery, about the level of the transverse process of the sixth cervical vertebra in order to reach the lower part of the thyroid gland. As this vessel passes behind the common carotid artery, etc., it is crossed from above downward by the sympathetic nerre. This nerve usually descends in front of the inferior thyroid artery, but sometimes behind it. Just before the artery reaches the thyroid gland it is crossed by the recurrent laryngeal nerve, which ascends in the space between the trachea and the osophagus to reach the larynx.

The Vertebral Artery lies deep in the lower part of the neck. It arises from the first part of the subclavian between the scalenus anticus muscle in front and the longus colli behind and enters the foramen in the base of the transverse process of the sixth cervical vertebra. The prominent tubercle on the transverse process of this vertebra is a good guide to the artery. The artery may be reached through the subclavian triangle by drawing the sternomastoid forward toward the middle line or by nicking or incising its posterior border. 


\section{OPERATIONS UPON THE NECK.}

Tracheotomy means opening into the air-passage either for relief when obstruction exists or as a preliminary step to other operations; for example, extirpation of the larynx, amputation of the tongue, etc.

In 1869 , as a preliminary to excision of the jaw, Nussbaum performed a tracheotomy and tamponed the pharynx with a compress to prevent blood from entering the larynx during the operation, the anæsthetic being administered through the tracheotomy tube.

TaMpon of THE Trachea.-Trendelenburg uses a tracheotomy tube which is surrounded by a thin, balloon-like structure provided with a cannula so that it may be inflated after it has been introduced

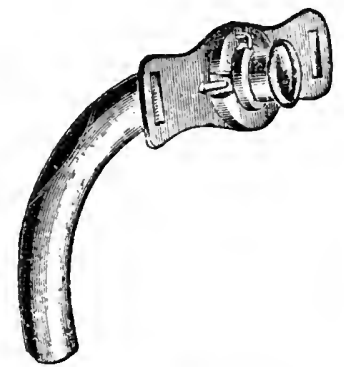

Fig. 77.-Tracheotomy Tube.

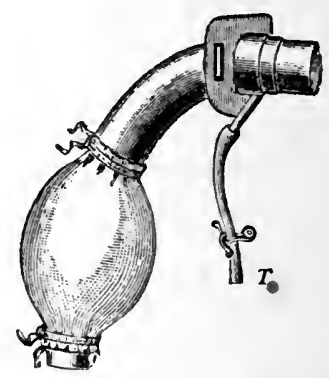

Fig. 78.-Trendelenburg Tampon Cannula. $T$, tube to inflate balloon. Anæsthetic is given through a long tube and funnel attached to tracheotomy tube.

into the trachea, in this way plugging the trachea and preventing the entrance of blood, etc. The anæsthetic is administered through the tracheotomy tube, to which a long rubber tube provided with a funnel is attached; in the bottom of the funnel there is a wad of cotton upon which the anæsthetic is dropped. The tracheotomy tube and tampon may be allowed to remain in the trachea for seven or eight days after the operation.

The Site of Operation.-The opening into the air-passage may be made:-

1. Through the trachea above the isthmus of the thyroid gland (high tracheotomy). This is the preferable operation and usually includes, in addition, division of the cricoid cartilage (crico-tracheotomy). 
2. Through that part of the trachea which is covered by the isthmus of the thyroid gland (median tracheotomy).

3. Through the trachea below the isthmus of the thyroid gland (low tracheotomy). This operation is rather less preferable, because at this level the trachea lies deeper-farther away from the surface, and, besides, one may meet the inferior thyroid veins or some of their branches or there may be an arteria thyroidea ima present. This is the site usually selected for a preliminary tracheotomy in conjunction with operations upon the larynx; for example, extirpation of the larynx.

4. Through the crico-thyroid membrane. This is really a laryngotomy, but it is well to include it with the tracheotomies.

High Tracheotomy (Crico-tracheotomi).-This is the operation usually performed, and has the advantage that no vessels of moment are met with; and that this part of the air-tube is located quite superficially, near the surface.

The patient lies upon the back with the shoulders raised and the head thrown back. If the symptoms of suffocation are urgent, one may dispense with an anæsthetic or may give simply a few whiffs. of chloroform. The operation may be done under cocain anæsthesia.

By palpation, the ring-like cricoid cartilage, which is the best landmark, is readily located. In men the prominent thyroid cartilage may be felt and seen as Adam's apple, but in women and children this is not prominent and is not, therefore, a good guide.

An incision is made through the skin and subcutaneous fat from the lower border of the thyroid cartilage-just above the cricoiddownward, in the middle line of the neck, for a distance of one and one-half inches. In making this skin incision some small tributaries of the anterior jugular vein may be encountered; to these clamps. are applied and the skin retracted, exposing thus the deep cervical fascia, which unites the edges of the sterno-hyoid muscles of either side with each other. This layer of fascia is incised along the middle line, corresponding to the incision in the integument. The edges of the wound being now retracted, there are exposed, above, the cricoid cartilage and just below the cricoid, lying transversely across the front of the trachea, the isthmus of the thyroid gland. The isthmus of the thyroid gland is located about one-half inch below the cricoid cartilage, to which it is connected by a process of the deep cervical fascia. This slip of fascia covers or conceals the upper two rings of the trachea; so that, in order to expose these, it is necessary to 
pick up this band and snip it transversely, after which the isthmus may be drawn downward and the upper rings of the trachea exposed to view.

The next step is to enter the air-passage, but before doing this all bleeding points should be clamped. At times, during the operation, the larynx moves violently up and down in forced efforts at respiration, and in order to steady it a tenaculum must be employed. This is introduced into the larynx above the cricoid cartilage, piercing the crico-thyroid membrane, and hooks the cricoid cartilage firmly upon its posterior aspect a little to the right of the middle line. The operator holds this tenaculum with the left hand, thus steadying the larynx and trachea, and, with a sharp-pointed knife held short in the right hand, the cricoid and one or two upper rings of the trachea are cut deliberately from above downward. One guards the knife blade in order to avoid injuring or perforating the posterior wall of the trachea. Having made an opening in the airtube about one-half inch long and still retaining the tenaculum which was hooked into the cricoid to the right of the middle line, a second tenaculum is now hooked into the other side of the cricoid, to the left of the middle line, and the incision in the air-passage thus held open while the tube is being introduced.

Occasionally the thyroid gland has a well-marked middle lobe occupying the site of the isthmus and ascending upon the front of the cricoid. This extra lobe is seldom present, but, when it is, it must be dislocated downward in order to expose the cricoid and the upper part of the trachea. Usually it is not necessary to apply any ligatures, as the cut vessels cease bleeding after a few minutes' application of the artery forceps; still, if any spurting vessels are met, they should be ligated. The edges of the skin may be brought together with two interrupted catgut sutures, one above and the other below the tube.

The tube is held in place by a tape tied around the neck and the wound dressed with gauze packed loosely about the wound and the tube.

Low Tracheotoms. - The opening is made into the trachea below the isthmus of the thyroid gland. This is not usually the site of choice, although it is at times indicated. This part of the trachea lies farther away from the surface, deeper, and one may meet the inferior thyroid veins, which descend in front of the trachea, although they usually lie well to either side of the middle line, thus 
leaving the line of incision free. At times there is an arteria thyroidea ima ascending in front of this part of the trachea: a rather unusual condition.

The incision, in the middle line of the neck, commences above at a point just below the cricoid cartilage, and is continued downward toward the sternum, for a distance of one and one-half to two inches. The incision penetrates first through the skin and fat, and is then continued deeper through the deep cervical fascia, exposing the front of the trachea. After the trachea has been exposed all bleeding points must be clamped; usually the hemorrhage is only venous and ceases after the artery forceps have been applied for a few minutes. The operator is now ready to make the opening in the trachea, which should be placed below the level of the isthmus of the thyroid gland; the isthmus may be drawn upward toward the cricoid cartilage in order to give more room. All bleeding should be, controlled before the trachea is opened.

Before making the incision in the trachea a tenaculum is introduced into the trachea, just below the isthmus of the thyroid gland and a little to one side of the middle line, to steady the trachea, and with a sharp-pointed knife, held short by the blade, an incision is made into the trachea from below upward, cutting two or three rings. Still steadying the trachea with the first tenaculum, a second tenaculum is introduced into the incision in the trachea, and while it is thus held open the tube is introduced. The tenacula are not withdrawn until the tube is in the trachea.

Any spurting vessels or large veins may be ligated, and one or two stitches may be taken in the skin wound. The left innominate vein is not in danger if, in incising the trachea, the knife is not carried below the level of the sternum.

Median Tracheotomy.-The opening into the trachea is made beneath the isthmus of the thyroid gland, which is divided in order to expose this part of the trachea. This operation can be done rapidly.

The incision passes through the skin and fat and reaches from the cricoid cartilage downward, in the middle line of the neck, for a distance of one and one-half to two inches. The incision is then carried deeper through the deep fascia, between the edges of the sterno-hyoid muscles, when the isthmus of the thyroid gland is exposed. The isthmus is divided and the trachea recognized. In dividing the isthmus we cut several venous branches, which bleed 
and must be clamped. The bleeding should be controlled before the trachea is opened. Bleeding points may be clamped and their ligation postponed until after the tube has been introduced into the trachea if time is limited. The trachea is steadied with a tenaculum and incised, and the tube introduced, as in the foregoing operation.

Transverse Laryngotomy.-This is an emergency operation and may be rapidly performed. One may open the windpipe, after locating the cricoid cartilage and using this as a guide, by cutting transversely through the skin and crico-thyroid membrane: i.e., between the upper border of the cricoid and the lower border of the thyroid cartilage. There is some probability of wounding the cricothyroid artery, a small branch, yet this is not very likely as the incision is made transversely: parallel with the course of the artery.

Thyrotomy.-Division of the thyroid cartilage may be either incomplete or complete.

INCOMPLETE ThYRotomy.-The incision is placed in the middle line of the neck and commences, above, at a point just below the upper border of the thyroid cartilage, and is continued downward to a point just below the cricoid cartilage; it is about one and onehalf to two inches long and reaches through the skin and deep fascia, exposing the cricoid and thyroid cartilages. The edges of the wound are retracted and the crico-thyroid membrane incised, thus entering the larynx. In incising the crico-thyroid membrane the crico-thyroid branches may be cut; these are small branches, but they should be clamped if they bleed, as even a small quantity of blood sucked into the wind-pipe may seriously embarrass respiration. We then proceed to enlarge the opening into the larynx by dividing the cricoid cartilage and the lower part of the thyroid cartilage, to an extent sufficient to permit the extraction of foreign bodies, etc. One should avoid, if possible, incising the thyroid cartilage beyond the level at which the true vocal cords are attached.

If this operation is done for the removal of a foreign body, one may close the opening in the larynx and omit the introduction of a tube; still it is probably not unwise to insert the tube and leave it for a few days in all cases, because, as a result of the operation, there may be some œdema of the glottis caused.

Complete Thynotomy consists of a median section through the thyroid cartilage. This operation is done for the purpose of exploring the interior of the larynx and for the removal of foreign bodies, growths, etc. 
During the operation the trachea must be kept clear of blood. The operation should be performed with the patient in the Rose position unless a tampon cannula is used, when the patient may be placed in the usual tracheotomy position with the shoulders raised and the head thrown back. The tampon cannula may be introduced through a preliminary high tracheotomy, done at the same sitting, previous to opening the larynx, or else the cannula may be inserted through the incision that is made in the larynx and which may be prolonged downward, through the cricoid and upper rings of the trachea for this purpose. Instead of a high tracheotomy, a preliminary, low tracheotomy may be performed and the tampon cannula introduced at this point.

The incision is placed in the middle line of the neck, reaching from the hyoid bone, above, to a point below the level of the cricoid cartilage. The incision extends through the skin, and deep fascia and exposes the thyroid cartilage.

The next step is to open the larynx. The point of the knife is introduced through the crico-thyroid membrane between the cricoid and the lower border of the thyroid cartilage. In doing this the crico-thyroid branch may be cut and should be clamped and tied. Now, with a curved probe-pointed knife, which is introduced into the larynx and passed upward between and beyond the vocal cords, the thyroid cartilage is split into its two halves from within outward, in the middle line, throughout its entire length up to or into the thyro-hyoid membrane. The thyroid cartilage may also be divided from without inward. At times the thyroid cartilage is ossified, and a strong scissors or thin saw is necessary in order to accomplish its division.

After the thyroid cartilage has been split its edges are held apart with sharp retractors or tenacula, and the interior of the larynx may then be freely explored. We may, in addition, divide the cricoid cartilage and the upper rings of the trachea if this has not already been done or if more room is required or in order to introduce a tampon cannula.

In cutting into the thyro-hyoid membrane one should avoid the superior laryngeal vessels and nerve, which pierce this membrane upon either side to enter and supply the larynx.

It may not be necessary to suture the two halves of the thyroid cartilages, as these often adapt themselves very well without suture, especially if the cricoid cartilage has not been divided. It is probably 
wise, however, in all cases, to introduce two or three chromicized catgut sutures through the perichondrium to hold the edges of the two halves of the thyroid cartilage in contact or one silver wire suture may be passed through each edge of the cartilage. The incision in the skin may be partly closed with catgut sutures.

The tampon cannula, if used, may be left in place for a few days if it is well borne, as it prevents the entrance of blood and discharge into the trachea and lungs.

Laryngectomy (Extirpation of the Larynx). - This operation should be preceded by a low tracheotomy, which may be done a week or more in advance of the major operation in order to accustom the patient to the presence of the tube and to bring about fixation of the trachea to the skin, etc., of the neck.

If the preliminary tracheotomy has not been done, one should operate with the patient in the Rose position, or, if the operation is done with the patient in the customary tracheotomy position, it will be necessary, as soon as the larynx has been isolated and all the vessels that supply it ligated, to cut the larynx away from the trachea below and then, at once, introduce the tampon cannula into the upper end of the trachea. The preliminary tracheotomy, with the introduction of the tampon cannula, is probably the most preferable plan. The incision is made in the middle line from the hyoid bone to a point below the cricoid cartilage; to this incision a second transverse incision may be added which extends outward, parallel with the hyoid bone, between the hyoid bone and upper border of the thyroid cartilage, as far as the anterior border of the sterno-mastoid muscle, thus making a T-shaped incision. This latter supplementary incision is especially advantageous if the lymphatic glands, etc., are involved in the pathological process.

The incision extends through the skin and subcutaneous fat and deep cervical fascia, and exposes the thyroid cartilage.

The edges of the sterno-hyoid muscles are next recognized and the muscle of either side divided transversely either partially or completely. The parts being now retracted, we expose the sternothyroid and thyro-hyoid muscles, which are attached upon either side of the thyroid cartilage, the lateral lobes of the thyroid gland being situated beneath the sterno-thyroid muscles.

We now begin the isolation of the larynx, separating all the soft parts either with an elevator or with the knife, the edge of the instrument working close to the surface of the thyroid cartilage. If 
we use the elevator, this is pushed under the thyro-hyoid muscle, between it and the thyroid cartilage, and the attachment of this muscle separated from the side of the thyroid cartilage, separating the attachment of the sterno-thyroid at the same time; this separation may be accomplished in part with the knife. These two muscles are really one and the same continuous muscle; so that, after they have been detached from the thyroid cartilage, they hang together as one continuous flat band. Instead of detaching these muscles as described they may be simply eut away from the sides of the thyroid eartilages with the knife. The soft parts are now retracted and a tenaculum is hooked into the side of the thyroid cartilage, and with this the larynx is drawn forward and to one side, so that we are enabled to reach the superior laryngeal artery and its accompanying nervous branch, as they pierce the side of the thyrohyoid membrane to enter the larynx; the vessel is tied double and cut. The lateral lobe of the thyroid gland, which lies upon the side of the larynx (in the natural relation of the parts being covered by the sterno-thyroid musele), is readily separated from the side of the larynx with the elevator or the finger. At this stage of the operation the superior thyroid artery, which ramifies upon the upper front surface of the thyroid gland, is usually met with. This vessel need not be cut. The thyroid isthmus is also liberated from its attachment to the cricoid cartilage and pushed downward out of the way.

The crico-thyroid branch of the superior thyroid, which runs forward and inward transversely across the erico-thyroid membrane, may be cut and should be clamped and tied. There is also an inferior laryngeal branch, from the inferior thyroid, which accompanies the inferior laryngeal nerve into the larynx; it enters the lower back part of the larynx, behind the articulation between the cricoid and thyroid eartilages, beneath the lateral lobe of the thyroid gland; this branch may be eut and should be tied. The small transverse branch, from the superior thyroid, which runs transversely inward across the thyro-hyoid membrane, below the hyoid bone, to anastomose with its fellow of the opposite side, is also cut and tied.

The larynx is now drawn to the other side, and while the soft parts are retracted the above described procedures are repeated upon this side.

The isolation of the larynx is continued. The soft parts are strongly retracted to one side and with a sharp hook or vulsella the larynx is drawn to the opposite side; then, with the knife, the in- 
ferior constrictor of the pharynx is separated from the side of the thyroid cartilage. This muscle is attached upon the side of the thyroid cartilage close to its posterior border, which may be readily felt by the fingers in the wound. This muscle is separated from the cricoid cartilage also. Care should be exercised to work close to the surface of the cartilage in separating this muscle so as to avoid opening into the pharynx, and also to avoid division again of the vessels that have already been divided and tied. The parts are then separated in a similar manner upon the other side of the larynx.

We are now ready for the final step of this part of the operation: the separation of the larynx from the hyoid bone above, from the anterior wall of the pharynx behind, and from the trachea below.

The knife is introduced through the thyro-hyoid membrane between the thyroid cartilage and the hyoid bone, and this membrane is cut in a direction outward and backward, at the same time drawing the side of the larynx forward with a sharp hook or vulsella. In performing this step of the operation one should avoid again cutting the superior laryngeal artery upon the proximal side of its ligature if it has already been divided and tied. The other half of the thyro-hyoid membrane is then cut in a similar manner. If it is desired to excise the epiglottis also, and this is usually wise, a probe-pointed knife may be introduced through the incision in the thyro-hyoid membrane, between the upper border of thyroid cartilage and the hyoid bone, in a direction upward and backward; so that, as the cut is made, the blade of the knife passes between the base of the tongue and the epiglottis. The finger in the mouth may serve to guide the knife. If the epiglottis is to be left, we cut directly backward between the upper border of the thyroid cartilage and the hyoid bone, thus leaving the epiglottis attached to the posterior aspect of the hyoid bone and to the root of the tongue. The front of the larynx is now seized with a sharp hook or vulsella forceps and drawn directly forward; so that its posterior wall, composed of the broad posterior part of the cricoid cartilage, may be separated from the anterior wall of the pharynx; the anterior wall of the pharynx is very thin, consisting practically only of a layer of mucous membrane. If the growth involves the anterior wall of the pharynx, this part may be excised together with the larynx. If the pharynx is not involved in the disease, the separation of the larynx from the pharynx is not difficult.

After the separation of the larynx from the pharynx has been 
completed to a point below the level of the cricoid cartilage, the larynx is cut away from the trachea, from behind forward, below the level of the cricoid cartilage. In thus severing the larynx from the trachea the inferior laryngeal arteries and nerves are cut, and, if the ressels have not already been tied, they should be secured as they spurt. Thus the extirpation is complete.

Instead 'of operating as described above, we may, after freeing the larynx upon the sides, etc., complete the operation by cutting the larynx away from the trachea below the level of the cricoid cartilage, packing the stump of the trachea at once with a pad to prevent the entrance of blood (a preliminary tracheotomy having been done); and then, drawing the larynx forward with a sharp hook or vulsella, this is separated from the anterior wall of the pharynx from below upward; and, as the final step of the operation, the larynx is cut away from its attachment to the hyoid bone by carrying the knife through the thyro-hyoid membrane.

The superior laryngeal arteries, that enter the larynx upon the sides, are best secured before beginning the actual isolation of the larynx, but they may be again divided accidentally during the final ${ }^{\circ}$ steps of the operation, and in this case should be again clamped and tied; other vessels may be secured as they are encountered during the course of the operation. The wound is best left open. If the accessory lateral skin incisions have been made, and the sternomastoids have been divided, these parts may be brought together with sutures. The opening in the pharynx especially, if it has been necessary to remove a considerable part of its wall, may be closed in part by interrupted silk sutures with their ends left long to facilitate their removal later.

One should arrange good drainage, with the head low, so as to avoid the entrance of wound secretions into the trachea. It is well to leave the tampon cannula in the trachea for a few days if it has been used during the operation. The wound should be properly packed and the dressings changed at rather frequent intervals.

Feeding is carried on through a tube introduced into the stomach either through the mouth or through the wound in the pharynx. If a tracheotomy has not preceded the laryngectomy by a week or more, the stump of the trachea should be sutured to the skin in order to prevent too great retraction of the trachea. In cases where the disease has not spread beyond the larynx, the operation is comparatively easy and not accompanied by much hemorrhage. 
Before proceeding with the radical operation the larynx may be split in the middle line for the purpose of exploration. It may be that in some early cases the removal of one-half of the larynx will suffice.

Extirpation of Half of the Larynx.-This operation is analogous to the preceding. The larynx is first split in the middle line without injuring the vocal cords.

It is a less difficult and dangerous operation, and recurrence is no more frequent after this less radical operation, if the disease is limited to one side of the larynx, than after complete extirpation. The function is good after the partial operation.

External CEsophagotomy.-This operation is usually done for the removal of a foreign body impacted in the osophagus.

The patient lies upon the back, with the shoulders raised and the head thrown back and over toward the right side.

A soft rubber tube is introduced into the œsophagus as far as it will go to serve as a guide. The œsophagus is approached through an incision in the left side of the neck.

The incision is made about three inches long, corresponding to the anterior border of the sterno-mastoid muscle, the midpoint of the incision being upon a level with the cricoid cartilage; it is carried through the skin and subcutaneous fatty layer, including the platysma, and exposes the anterior edge of the sterno-mastoid muscle. The sterno-mastoid is then drawn aside and the underlying layer of deep cervical fascia is incised, when the internal jugular vein and the common carotid artery, lying in their connective-tissue sheath and crossed by the anterior belly of the omo-hyoid muscle, are exposed. These vessels are drawn outward with a blunt retractor. The lateral lobe of the thyroid gland, partly covered by the sterno-hyoid and sterno-thyroid muscles, is then recognized. These structures are drawn toward the middle line with a blunt retractor. The trachea, which may now be readily felt with the fingers, is a guide to the œsophagus, the œsophagus being located posterior to the trachea and protruding well beyond its left border. The tube in the œsophagus assists in locating it, and the foreign body, if present, may also be felt. The middle thyroid vein, as it passes outward from the thyroid gland to enter the internal jugular, may be met with, and, if it is in the way, may be cut and tied. The inferior thyroid vein may also be seen.

The esophagus is entered in the inferior carotid triangle,--i.e., 
below the omo-hyoid,-and, if necessary, this muscle may be drawn to one side or divided. The recurrent laryngeal nerve, as it ascends to enter the larynx, lies in front of the osophagus, in the space between the trachea in front and the œsophagus behind, and should be avoided in incising the œesophagus. The nerve, during the operation, is not encountered, and may be avoided by making the opening in the œesophagus well upon the side and thus keeping away from the front of the tube.

The wall of the csophagus is picked up with two mouse-toothed forceps, and an incision made corresponding to its long axis and of sufficient length to permit the extraction of the foreign body or any other necessary manipulation.

In incising the œsophagus one should make a clean cut in order to avoid getting between the layers of the wall of the tube, which may readily happen owing to the looseness of the tissue between its muscular and mucous coats. Some œsophageal branches of the inferior thyroid may be divided in making the opening in the wall of the œsophagus and these must be clamped and ligated.

The wound in the wall of the cesophagus may be closed with several interrupted sutures of silk or chromicized catgut, but the external wound in the neck, leading down to the incision in the œsophagus, should be packed and left unsutured.

If the object of the operation is to establish a permanent fistula (œsophagostomy), the edges of the incision in the œsophagus, including its mucous and muscular coats, may be fixed to the edges of the skin incision with several interrupted silk stitches.

Ligation of Blood-vessels. The Commor Caro'tid ARTery.The common carotid may be tied either above or below the point where the omo-hyoid crosses it, which is upon a level with the cricoid cartilage. It is ligated preferably and more readily in the so-called superior carotid triangle: above the crossing of the omo-hyoid.

The linear guide to the common carotid is a line drawn from a point midway between the angle of the jaw and the mastoid process to the sterno-clavicular articulation. The muscular guide is the anterior border of the sterno-mastoid muscle.

The incision is made about two inches long, corresponding to the anterior border of the sterno-mastoid, its midpoint upon a level with the cricoid cartilage. This incision penetrates through the skin and subcutaneous fatty layer, including the platysma, and should expose the anterior border of the sterno-mastoid muscle. 
The edge of the sterno-mastoid should be recognized and drawn outward, and then, after carefully incising the underlying layer of deep cervical fascia,- the fascia that separates the ressels from the sterno-mastoid muscle,- the vessels, surrounded by some loose connective tissue, are exposed-first, the internal jugular vein, big and thin-walled, lying to the outer side of the artery, and then the common carotid, whose pulsation is readily felt and seen and which lies to the inner side of the vein. The pneumogastric nerve, which is

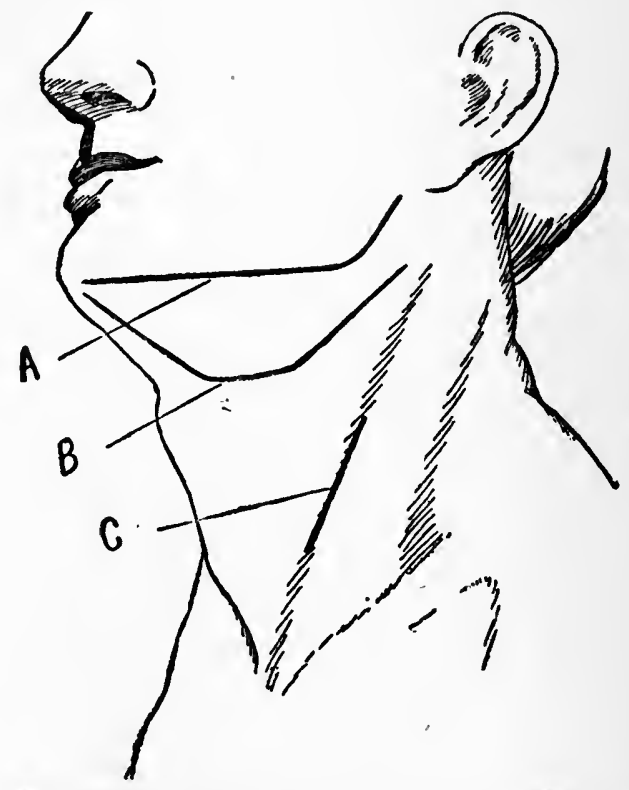

Fig. 79. $-A$, incision for removal of lower jaw; $B$, incision for ligation of lingual artery and Kocher's amputation of tongue; $C$, incision for ligation of common carotid and for csophagotomy.

located between the artery and vein, but behind them, is not seen. The anterior belly of the omo-hyoid is seen as it crosses the vessels opposite the cricoid cartilage. The loop formed by the descendens and communicans noni may also be recognized upon the front of the vessels. The superior thyroid vein crosses the artery from within outward above the omo-hyoid muscle, and the middle thyroid vein below this muscle. If these vessels are cut, they should be clamped and tied.

The connective-tissue sheath which incloses the artery should 
be picked up with mouse-tooth forceps, and nicked with the point of the knife in the direction of the long axis of the vessel; into the opening thus made, a director is introduced, and, working close to its wall, the vessel is separated all around, taking care to avoid the pneumogastric nerve, which lies posteriorly. A blunt-pointed aneurism needle is then introduced into the opening and carried around the artery from without inward, entering between the artery and the vein. The ligature is then drawn around the vessel, and we are ready to tie. The ligature should be of ordinary catgut and tied with a square knot. After the ligature is in place and before it is tied the parts should be again inspected in order to make sure that the nerve is not included. Some surgeons tie the artery double and divide it between the ligatures, but this is probably unnecessary. The incision is closed with a catgut suture.

The External Carotid.-The ligation of the external carotid is practiced as a preliminary to many bloody operations about the mouth, jaws, etc., and to control hemorrhage from parts supplied by its branches when the branches themselves are not accessible. The linear guide to the artery is the same as that for the common carotid; the muscular guide is the anterior edge of the sterno-mastoid. At the upper border of the thyroid cartilage the common carotid artery bifurcates into the external and internal carotids, and it is close to its origin, near the upper border of the thyroid cartilage, that the external carotid is ligated. The incision commences at the level of the hyoid bone and is carried downward, for a distance of about two inches, along the anterior border of the sterno-mastoid. The incision penetrates through the skin, fat, and platysma muscle down to the deep cervical fascia, exposing the edge of the sterno-mastoid muscle, which should be recognized. The edges of the incision are drawn apart with blunt-pronged retractors and the deep cervical fascia is then incised.

The pulsation of the artery, within its connective-tissue sheath, may now be both seen and felt. The external carotid artery lies a little in front of the anterior edge of the sterno-mastoid. The internal carotid, together with the internal jugular vein and pneumogastric nerve, lies posterior to the external carotid, beneath the anterior edge of the sterno-mastoid. Corresponding to the upper border of the thyroid cartilage, the loose connective tissue that invests the artery is picked up with a thumb forceps and snipped with the point of the knife, cutting in a direction corresponding to the long 
axis of the vessel; into the opening which is thus made a blunt director is introduced and worked around the vessel, sticking close to its wall. Through the path thus made by the director a ligature is carried around the vessel in the eye of an aneurism needle. The ligature is then tied and the incision closed. After the ligature has been carried around the artery it may be left untied, with its ends hanging out of the incision, to be tied only in case an emergency arises calling for its use.

The Internal Carotid.-The ligation of the internal carotid is but seldom called for. The internal carotid may be tied through an incision similar to that for ligation of the external carotid. The vessel is found underneath the anterior edge of the sterno-mastoid, which is the muscular guide to it. The internal carotid has the same relations to the internal jugular vein and pneumogastric nerve that the common carotid has, the internal carotid being really the continuation of the common; and these structures must be avoided in isolating the vessel and passing the ligature.

The Subclavian Artery.-The third part of the subclavian artery is tied after it is exposed in the subclavian triangle.

The patient is placed with the shoulders somewhat raised and the head thrown back and turned toward the opposite side, the arm being drawn down to depress the shoulder. The incision corresponds to the middle third of the clavicle. It is placed just above the clavicle, and extends from the anterior border of the trapezius forward and inward almost as far as the outer border of the sternomastoid muscle; the incision falls a little short of the edge of the sterno-mastoid muscle in order to avoid the external jugular vein. The incision in the skin may be made by drawing the integument of the neck downward over the surface of the clavicle and then cutting through it, down upon the surface of the clavicle; when the skin is released, the incision is found to lie just above and parallel with the clavicle. This incision reaches through the skin, fat, and platysma down to the deep fascia. The deep fascia, which reaches from the edge of the trapezius muscle behind to the sterno-mastoid in front, is now incised, avoiding the external jugular vein, which pierces the deep cervical fascia behind the outer edge of the sternomastoid muscle. Beneath the deep fascia the venous plexus, formed by the transversalis colli and suprascapular, is encountered. These veins may be wounded, but are readily clamped; often, however, they can be avoided as the knife may be discarded after the deep 
fascia has been incised. Beneath the deep fascia there is also a considerable quantity of loose fat and connective and lymphatic tissue.

The posterior belly of the omo-hyoid muscle, which lies pretty low down near the clavicle, is now sought and must be drawn upward to show the subclavian triangle, of which it forms the upper boundary, the anterior boundary being formed by the sterno-mastoid and the inferior boundary by the clavicle.

Within the triangle, passing transversely outward, are the transversalis colli and suprascapular arteries. These vessels should be avoided. The tendon of the scalenus anticus, which is the guide to the subclavian artery, may be felt as a tense cord passing straight up and down beneath the posterior or outer border of the sternomastoid and attached below to the first rib. If this tendon is followed downward as far as its attachment to the first rib, one may locate the subclavian artery as it passes outward and forward from behind the tendon of the scalenus anticus muscle, resting directly upon the upper surface of the first rib. That part of the subclavian artery which lies upon the first rib is the part which is ligated. The subclavian vein lies a considerable distance to the inner side of and anterior to the artery, the tendon of the scalenus anticus intervening between them, and is not apt to be encountered during the operation. Within the triangle, above the subclavian artery, may be seen the three cords of the brachial plexus. These pass obliquely downward and outward from behind the scalenus anticus muscle, and should not be mistaken for the artery, which is the lowest structure in this triangle and rests directly upon the upper surface of the first rib. These structures may all be exposed by blunt dissection, separating with the finger or handle of the knife, after the deep fascia has been incised.

With blunt retractors the wound is held open and the connective-tissue sheath, which envelops the artery, picked up and snipped with the scissors and the artery then separated from the adjoining structures with a blunt director, working around the artery close to its wall. The aneurism needle is passed around the artery from without inward, avoiding the cords of the brachial plexus. The subclavian vein, which lies below and internal to the artery, is not apt to be in the way.

It should also be remembered that the dome of the pleura reaches above the clavicle into the subclavian triangle, and that the subclarian artery (second part), as it lies behind the tendon of 
the scalenus anticus, rests upon the pleura, and care should be taken to avoid injuring this structure, especially in making way for the passage of the ligature.

The ligature is tied with a square knot, deep in the wound, without lifting the artery too much out of its bed.

The Lingual Artery.-This operation is usually performed in combination with Kocher's amputation of the tongue. The lingual is a vessel of considerable size, that of each side supplying the corresponding half of the tongue. In order to prevent the entrance of blood into the larynx during the amputation of the tongue, the patient is placed in the Rose position, or, if a preliminary tracheotomy has been done and a Trendelenburg tampon cannula introduced, or if an ordinary tracheotomy tube has been introduced and the pharynx tamponed, one may operate with the patient in the ordinary position, the shoulders somewhat raised, and the head thrown back and over toward the opposite side.

An incision is made which corresponds to the boundaries of the submaxillary triangle. It commences in front, at the symphysis mentis, and is carried down to the hyoid bone, thence backward above and parallel with the greater horn of the hyoid bone and then in a direction upward and backward toward the mastoid process as far as the angle of the lower jaw (see Fig. 79).

This incision penetrates through the skin, fat, and platysma, down to the deep fascia. The apex of the flap, which is thus marked out, is seized with the fingers and reflected upward upon the side of the face as far as the lower border of the jaw-bone. In reflecting this flap we may, toward the back, cut the external jugular vein, and this should be clamped and tied. The deep fascia is then incised and the submaxillary gland exposed. This gland, which is lodged in a bed of loose connective tissue, is seized with toothed forceps and enucleated, together with the adjoining lymphatic nodes. This is accomplished by cutting with the knife close to the gland or by blunt dissection with the handle of the knife or with the finger, the gland being finally cut away from its duct, which disappears anteriorly beneath the posterior border of the mylo-hyoid muscle on its way to open into the anterior part of the floor of the mouth. The facial artery, if not previously cut, is usually divided in enucleating this gland, and should be tied when cut, or, still better, it may be tied, before it is cut, close to its origin and before it reaches the submaxillary gland. 
The facial vein is also usually divided during this part of the operation; this vessel bleeds freely, but may be clamped and ligated. After the submaxillary gland has been removed, the boundaries of the submaxillary triangle are readily made out; above, the lower border of the jaw, and, below, in front, and behind, the anterior and posterior bellies of the digastric muscle. The floor of the submaxillary triangle is formed in front by the oblique fibers of the mylohyoid and behind by the perpendicular fibers of the hyo-glossus, which muscle lies on a deeper plane than the mylo-hyoid, being partly overlapped by the posterior margin of the latter. Passing from behind, horizontally forward, above and parallel with the hyoid bone and lying directly upon the hyo-glossus muscle is the hypoglossal nerve; this nerve disappears anteriorly beneath the posterior edge of the mylo-hyoid muscle. This nerve marks the upper boundary of the lingual triangle, which is really the apex of the submaxillary triangle. The base of the lingual triangle is formed by the hypoglossal nerve, and its lower borders, in front and behind, by the anterior and posterior bellies of the digastric. The floor of the lingual triangle is formed by the hyo-glossus, and beneath this muscle the lingual artery, accompanied by a vein, is located; so that, if this muscle is picked up with tooth forceps and snipped through with the knife or scissors, the lingual artery is readily found and may be hooked up with an aneurism needle and tied. Locating and tying the lingual artery in this triangle is very simple. We are then ready to proceed with the amputation of the tongue.

Should it be desirable to tie the lingual artery without removing the submaxillary gland, one may, after cutting through the deep fascia, draw the gland up out of the way and then proceed as above. In this case it is not necessary to make such an extensive incision.

\section{OPERATIONS UPON THE TONGUE.}

Amputation of the Tongue (Kocher), with Preliminary Ligation of the Lingual Artery.-Amputation of the tongue according to the method of Kocher has many advantages: the hemorrhage is easily controlled, diseased glands are readily removed, and the incision is well placed for drainage.

The position of the patient, etc., has been described in connection with the ligation of the lingual artery. 
An incision, as described above for the ligation of the lingual artery, is made upon the side of the neck, laying bare the boundaries of the submaxillary triangle. The lymphatic nodes and submaxillary gland are then excised and the lingual artery sought for and tied; it is not necessary to ligate the lingual of each side, yet this may be done with advantage, especially if the lymphatics of both sides are involved, as they can then be extirpated at the same time that the vessel is ligated.

After having excised the submaxillary lymphatic nodes and gland and tied the lingual artery and secured all bleeding points, an incision is made with the knife through the floor of the submaxillary triangle,-i.e., through the mylo-hyoid muscle and the mucous membrane of the mouth,-close to the inner surface of the body of the lower jaw. This opening may be farther enlarged with the scissors or fingers. The tip of the tongue is then seized with a forceps and drawn out into the wound in the neck, through the opening in the floor of the mouth, and making considerable traction, first to one side and then to the other, the tongue is separated from its attachment to the floor of the mouth, as far back toward the base as possible. This is done with the blunt-pointed curved scissors, snipping through the septum of the tongue and working close to its under surface. During this step of the operation, and while traction is being made upon the tongue, one should examine occasionally with the finger for bands, etc., which tend to bind the tongue within the mouth. The anterior pillars of the fauces, which are attached to the sides of the tongue, near its base, should be cut close to the surface of the tongue, and then it will be observed that the organ can be drawn out of the mouth for a considerable distance, when it may be amputated quite close to its root. This is done with the scissors.

The half of the tongue, corresponding to the side upon which the lingual has been tied, may be cut through without occasioning any bleeding; but, if the lingual artery of the other side has not been previously tied, the hemorrhage, when this second half of the tongue is cut through, may be embarrassing, as there may be some difficulty in catching the cut end of the artery. This, however, may be provided against by seizing the base of the tongue with a toothed clamp behind the point where it is intended to amputate it before cutting through; so that, when we divide this half of the tongue, we may puli the stump forward, and seize the divided ressel, when it spurts, with an artery clamp. 
The wound in the side of the neck may be closed with interrupted silk-worm gut sutures, except its posterior part, which is left open and packed to carry off the secretions, etc., from the mouth. The packing should be introduced well into the cavity of the mouth. The patient is fed through a stomach tube, which is passed through the mouth or through the nose. This tube may be passed before the patient recovers from the anæsthetic.

Amputation of the Tongue (Regnoli-Billroth).-This method is applicable to those cases where the floor of the mouth is considerably involved in the disease.

The patient is placed in the Rose position, or if a preliminary tracheotomy has been done and a Trendelenburg tampon cannula introduced into the trachea, or if an ordinary tracheotomy tube has been introduced and the pharynx has been tamponed, the patient may lie in the usual position with the shoulders raised and the head thrown back.

An incision is made along the lower border of the body of the jaw about $6 \mathrm{~cm}$. long, the midpoint of the incision corresponding to the symphysis mentis. This incision penetrates through all the soft parts down to the bone and extends backward, upon either side, nearly as far as the anterior edge of the masseter muscle. In making this incision, the facial artery, as it turns up over the lower border of the jaw-bone, just in front of the masseter, may be avoided.

From either end of this incision additional ones are made which reach straight downward as far as the hyoid bone, passing through the integument and the platysma. Through the lateral incisions, on either side, the lingual artery may be sought and tied, at the same time extirpating any diseased glands, etc.

The cavity of the mouth is now entered by severing the muscles attached to the inner surface of the body of the lower jaw with a knife. They should be cut fairly close to the bone, and the point of the knife may be guided with the finger in the mouth. Those muscles that are attached to the inner aspect of the symphysis in the middle line are divided first. A suture should be passed through the tip of the tongue or it may be seized with a toothed clamp in order to exercise traction and prevent its falling back into the pharynx and obstructing the breathing during the course of the operation.

After a sufficiently large opening has been made in the floor of the mouth, the tongue is drawn through the wound, under the 
jaw, and may then be removed together with the floor of the mouth as far back as the epiglottis.

If the lingual arteries have not been previously ligated, the base of the tongue should be seized with a vulsella forceps before it is amputated, in order to facilitate the clamping of these vessels in the stump of the tongue.

The flap of skin and soft parts is replaced and the wound closed except posteriorly, on one or both sides, where the incision is left open and packed in order to drain the cavity of the mouth.

Extirpation of the Tongue through the Floor of the Mouth, with Division of the Lower Jaw.-The operation is preceded by a tracheotomy and the introduction of a Trendelenburg tampon cannula, or an ordinary tracheotomy tube may be used and the pharynx tamponed. A soft rubber tube for feeding purposes may be passed into the stomach, before the patient recovers from the anæsthetic, either through the mouth or the nose.

Sedillot's Method, with Division of the Lower Jaw in the Middle Line.-The first incisor tooth of the lower jaw is extracted. An incision is made, as in the Regnoli-Billroth operation, along the lower border of the jaw and reaching as far as the masseter on either side. The lower lip is then split in the middle line, the incision being carried down to the bone through the gum and periosteum. The lower jaw is then sawn through with a metacarpal or a chain or a Gigli saw, and the muscles and the mucous membrane composing the floor of the mouth incised close to the inner surface of the body of the lower jaw-bone.

Each half of the jaw is now drawn well outward, away from the middle line, thus giving very free access to the tongue and to the floor of the mouth. The tongue and that part of the floor of the mouth which is involved in the disease may then be extirpated.

If the linguals have not been previously tied, they may be clamped after the tongue has been amputated, drawing the stump of the tongue forward with a vulsella in order to facilitate this.

The tonsils and the pillars of the fauces may also be reached in this operation, and, if the lower jaw-bone is involved, it can be resected in part. Diseased lymphatic glands in the neck may also be excised through this incision, which may be made as extensive as necessary.

One should attempt to bring the raw surfaces in the mouth together, at least in part, with interrupted chromicized catgut or 
silk sutures, their ends being left long to facilitate their removal later.

The two halves of the jaw are brought together and carefully wired, and the incision closed except at its posterior part on one or both sides, where it is left open for packing and drainage.

Langenbeck's Method, with Division of the Lower JaW on oNe Side.-Upon the side corresponding to the disease an incision is carried from the corner of the mouth through the lower lip as far as the lower border of the jaw, whence it is continued downward through the integument of the neck as far as the side of the hyoid bone. The upper part of this incision splits the lip and gum, passing through the periosteum down to the bone; the lower part of the incision passes through the skin, fat, and platysma. All bleeding points are clamped.

Through the lower part of the incision, after cutting through the deep fascia, the submaxillary gland and the neighboring diseased lymphatic nodes of this side may be removed, and the lingual artery tied as it lies in the lingual triangle, above the hyoid bone and beneath the hyo-glossus muscle.

The canine tooth of the lower jaw is now extracted and an opening made in the floor of the mouth so as to allow the use of the chain or wire saw with which the jaw-bone is divided. The section through the jaw should be, not straight up and down, but obliquely from above downward and inward toward the symphysis, so that the tendency to dislocation caused by the pull of the masseter muscle may thus be counteracted. The jaw-bone may be divided with a narrow, flat saw or with a chain or wire saw.

The segments of the divided jaw-bone, especially the shorter piece, are now drawn well apart with sharp retractors, and the soft parts, muscles and mucous membrane, which form the floor of the mouth, separated from their attachment to the inner surface of the bone, as far back, if need be, as the anterior pillars of the fauces. The tongue is then seized with the toothed forceps and drawn well forward and over toward the well side and removed. One may excise the floor of the mouth, the pillars of the fauces, and the tonsils, if they are diseased, and also resect a part of the jaw-bone if this is involved.

If the linguals have not been previously ligated, we may clamp them in the stump after the tongue has been amputated. The segments of the jaw-bone are brought into apposition and wired, and 
the wound in the soft parts, except its lower part, which is left open and packed to carry off the secretions from the mouth, is closed with interrupted silk-worm gut sutures.

One should try to diminish the raw surface left in the buccal cavity as much as possible by drawing the parts together with separate chromicized catgut sutures.

Billroth's Method, with Bilateral Division of the Lower $\mathrm{J}_{\mathrm{AW}}$.-This is probably not so satisfactory as the preceding operations, owing to the difficulty of getting union of the loose segment of the jaw.

The canine tooth upon either side of the lower jaw is extracted, and an incision made from each corner of the mouth, through the lower lip, gum, and periosteum, down to the bone, and continued downward, in the neck, through the skin, fat, and platysma as far as the hyoid bone.

Corresponding to the place upon either side where the canine tooth has been extracted the lower jaw is sawn through, from its upper border downward to its lower border; this may be done with the chain, wire, or flat saw.

The soft parts, which correspond to the floor of the mouth and which are attached to the middle, loose segment of the jaw-bone, are separated upon the inner aspect of the bone, and the flap of soft parts, which includes the free middle segment of the bone, is reflected downward.

The lingual arteries may be ligated and diseased glands removed through the incisions in the neck previous to amputating the tongue, or the arteries may be clamped and ligated in the stump after the tongue has been cut away. We gain free access to the floor of the mouth, tonsils, etc., in this operation.

The segments of the jaw are finally wired together and the incisions closed except the lower part, upon one or both sides, which may be left open and packed for drainage.

Extirpation of Half of the Tongue (Whitehead).-The patient may be placed in a half-sitting posture. Anæsthesia is not complete. A liberal dose of morphin may be administered hypodermically shortly before the operation, and only sufficient chloroform used to keep the patient fairly quiet. In this way sufficient reflex is retained to enable the patient to keep the larynx clear of blood by coughing and expectorating.

This operation is advisable when only half of the tongue is to 
be removed, or, if the whole tongue is to be extirpated, where the disease is limited and has not involved the floor of the mouth.

One or both lingual arteries may be previously tied through a small incision upon either side of the neck.

The jaws are separated with a gag and the mouth held wide open with flat retractors placed in either corner. A strong silk suture is passed through the tip of the tongue, and with this as a tractor the tongue is drawn well forward and split down the middle with sharp scissors. The diseased half of the tongue is then separated from the floor of the mouth and amputated as far back toward the root of the organ as desired. If the lingual artery has not been tied as a preliminary step to the operation, the bleeding vessel must be seized with the artery forceps in the stump of the tongue and ligated. In excising a portion of the tongue one should cut wide of the apparent diseased area. If the disease has approached near the middle line it is probably better to sacrifice the whole tongue, in which case the second half of the tongue may be amputated in a similar manner.

This operation will probably suffice for early cases where the floor of the mouth and the lymphatics are not yet involved. 


\section{PART IV.}

THE THORAX.

\section{THE SURGICAL ANATOMY OF THE THORACIC WALL.}

The Skeleton of the Thorax.-The thorax consists of a conical cage of bone and cartilage. Entering into its construction are the dorsal vertebræ, ribs, sternum, and interposed costal cartilages. The spaces between the ribs and costal cartilages are filled in, and the walls of the chest thus completed, by the intercostal muscles.

The thoracic cavity is rather cone-shaped, with its base below and its small end above, and is somewhat flattened from before backward.

The upper orifice of the thorax is kidney-shaped, narrow from before backward, and broader from side to side. It is bounded in front by the upper border of the sternum, behind by first dorsal vertebra, and laterally, on each side, by the first rib. The first rib is set very obliquely; so that its anterior end strikes a much lower level than its posterior end. The upper border of the sternum is opposite the intervertebral cartilage between the second and third dorsal vertebræ.

The lower opening of the thorax is large. It is bounded by the lower border and tip of the twelfth rib, the tip of the eleventh and the costal cartilages of the tenth, ninth, eighth, and seventh ribs. Anteriorly, in the middle line, is the ensiform cartilage; posteriorly is the body of the last dorsal vertebra.

A transverse section through the middle of the thoracic cavity shows it to be rather heart-shaped, owing to the projection forward of the bodies of the vertebræ. On either side of the vertebral column there is a longitudinal recess, which serves to deepen the space for the accommodation of the lungs; this is called the fossa pulmonis. The cartilages of the lower ribs, the seventh to the tenth, meet at the lower end of the sternum and form an angle the apex of which corresponds to the ensiform cartilage. This is known as the costal angle.

The thoracic carity is closed in, below, by the diaphragm, which projects upward, dome-like, into the carity of the chest, forming its 
floor and at the same time the roof of the abdominal cavity. By the projection of the diaphragm upward into the chest the capacity of the chest cavity is diminished and that of the abdomen correspondingly increased. In the living body the chest appears to be broader above, at the shoulders, than below at the waist; this appearance is due to the broad shoulder girdle, which partially encircles the chest above and which is made up of the clavicle and the scapula of either side.

The space within the chest consists of an air-tight compartment on either side, each containing one of the lungs, and a middle space called the mediastinum, in which are lodged the heart and the great vessels at its base, the trachea, œsophagus, thoracic duct, and the thymus gland or its remains.

The Dorsal Vertebra. - These are twelve in number and form the back part of the skeleton of the chest. They give stability to the thorax and at the same time, on account of the presence of the elastic intervertebral pads, free motion is allowed in all directions.

This part of the vertebral column shows a sagittal curve with its concavity forward and a slight lateral curve with its concavity toward the left (aorta).

ThE RiBs are twelve in number (may be eleven or thirteen) on each side. They are flat bones articulated behind to the vertebræ and directed obliquely downward and forward. They form the bony frame-work of the back, sides, and part of the front of the chest.

The lower the rib is situated, the greater is its inclination downward. They increase in length from the first to the eighth.

The first to the seventh are true ribs: i.e., they are each connected individually, through their cartilages, with the sternum.

The eighth to the twelfth are false ribs: their cartilages do not articulate with the sternum. The eighth, ninth, and tenth ribs are indirectly connected with the sternum through the junction of their respective costal cartilages with those of the ribs which immediately adjoin them above.

The eleventh and twelfth are floating ribs; they are short and their cartilages are free.

The lower border of each rib, upon its inner aspect, is grooved for the lodgment of the corresponding intercostal vein, artery, and nerve, that being their order from above downward.

The first rib is important surgically. It is very short, and its 
surfaces look almost directly upward and downward. It is set so obliquely that its posterior end, head, articulates with the upper part of the body of the first dorsal vertebra, whereas its anterior end, at its attachment to the sternum, is upon a level with the intervertebral pad between the second and third dorsal vertebræ. The inner border of this rib presents a tubercle for the attachment of the scalenus anticus muscle; external to this tubercle, upon the upper surface of the rib, there is a groove for the subclavian artery. The subclavian vein also passes across the upper surface of the first rib, but internally to the artery, the tendon of the scalenus anticus being interposed between the two vessels.

The inner border of the first rib is in direct relation with the dome of the pleura and the apex of the lung.

The Costal Cartilages.-These are the elastic bands which join the ribs to the sternum (except the eleventh and twelfth). The cartilage of the first rib is very short. The first and second costal cartilages, as they pass to the sternum, are directed somewhat downward like their ribs. The cartilage of the second rib articulates with the sternum at the junction of the manubrium with the gladiolus. The cartilage of the third rib is directed horizontally; the cartilages of the fourth, fifth, sixth, and seventh ribs are directed upward with increasing obliquity as they pass to the sternum. The cartilages of the eighth, ninth, and tenth make quite a sharp. turn upward toward the sternum at the angle of junction with their ribs, and do not reach the sternum directly, but are fixed each to the cartilage immediately above, and finally, through the junction of the cartilage of the eighth rib with that of the seventh, to the sternum. The cartilages of the eleventh and twelfth ribs are short and free.

The Sternum.-This bone is rarely fractured, owing to the elasticity of the parts with which it articulates. It consists of a manubrium, or handle; a gladiolus, or body; and a cartilaginous tip, the ensiform or xiphoid cartilage. The junction between the manubrium and the body is marked by a prominent transverse line, and presents an angle directed forward: angulus Ludovici. This transverse ridge, which is readily felt under the skin, is an important landmark in counting the ribs: it corresponds to the articulation of the costal cartilage of the second rib with the sternum.

The ensiform cartilage varies in length and shape; its lower extremity is usually on a level with the tenth dorsal vertebra; it. may be bifurcated or deflected to one side. The junction of the 
ensiform cartilage with the body of the sternum corresponds with the line that marks the lower border of the heart as it lies within the chest behind the sternum.

The Muscles of the Chest Wall. The Intercostal Muscles are placed between the ribs and costal cartilages, and consist of two sets: external and internal.

The External Intercostals. - The fibers of the external intercostals have a direction similar to those of the external oblique muscle of the abdomen: that is, from above downward and forward. In front, between the costal cartilages, the muscular fibers are absent, their place being taken by aponeurotic bands, the ligamenta intercostalia anterior, which represent the muscles.

The Internal Intercostals.-The direction of the fibers of the internal intercostal muscles is the reverse of those of the external. They correspond to the internal oblique muscle of the abdomen, and their fibers have a similar direction: upward and forward. Behind, the internal intercostals are deficient, their place being occupied by aponeurotic sheaths: the ligamenta intercostalia posterior.

The Triangularis Sterni is situated anteriorly within the chest. It is a thin sheet of muscle which is attached along the lateral border of the posterior aspect of the sternum. It spreads upward and outward in four or five processes, which are attached separately to the inner surfaces of the cartilages of the second to the sixth ribs. The internal mammary artery is located between this muscle and the costal cartilages. The triangularis sterni is the transversus thoracis anterior of Henle.

The Musculi Subcostales are a few sets of muscular fibers that are found upon the internal surfaces of the posterior ends of the ribs near the vertebral column; the direction of the fibers of these muscles is similar to that of the internal intercostals: they reach from the inner surface of one rib to the first or second rib above. These muscles correspond to the musculus transversus thoracis posterior of Henle, and together with the triangularis sterni are the analogues of the transversus abdominis, the most internal, deepest, of the flat muscles of the abdomen.

The Fasciæ of the Chest.-A thin fascia covers the outer surface of the ribs and the external intercostals. A similar fascia is spread over the inner surface of the ribs and the internal intercostals, triangularis sterni, and subcostales. This fascia corresponds to the fascia transversalis of the abdomen, and is known as the fascia endo- 
thoracica. The fascia endothoracica is also spread over the thoracic surface of the diaphragm. It lines the whole inner surface of the thoracic cavity, and is everywhere interposed between the parietal layer of the pleura and the inner surface of the chest, serving thus to bind the pleura to the chest wall and at the same time to strengthen it. Upon the posterior surface of the sternum this fascia forms a strong fibrous layer. Above it projects into the root of the neck together with the dome of the pleura, which it strengthens and fixes to the vertebræ and to the deep surface of scaleni muscles, etc.

The Internal Mammary Artery supplies the front part of the intercostal spaces and the diaphragm and gives perforating branches to the muscles of the chest and to the mammary gland. At its origin from the first part of the subclavian artery it lies behind the subclavian vein, resting upon the pleura, and is crossed by the phrenic nerve. It passes down into the thoracic cavity and descends alongside of the sternum, a distance of from 5 to $10 \mathrm{~mm}$. intervening between it and the lateral border of this bone. Behind the seventh costal cartilage the internal mammary artery divides into the musculo-phrenic and the superior epigastric. The musculo-phrenic continues downward parallel with the free border of the ribs, supplying branches to the intercostal spaces. The superior epigastric enters the posterior sheath of the rectus, anastomosing with the deep epigastric, which is derived from the external iliac, and in this way forms an important communication between this trunk and the subclavian. The internal mammary artery is accompanied by two veins, one upon either side, but above these two unite to form a single vein, which lies to the inner side of the artery. The artery is also accompanied by a chain of lymphatic glands.

Within the chest the artery rests upon the costal cartilages and the internal intercostal muscles, alongside the sternum, and is separated from the parietal pleura by the fascia endothoracica and the triangularis sterni muscle. Opposite each intercostal space the internal mammary gives off an intercostal branch, which, passing outward, divides into two, and these, anastomosing with the intercostal branches from the aorta, serve to establish a communication between the subclavian and the aorta. These intercostal branches are located between the internal and the external intercostal muscles close to the upper and lower borders of the contiguous ribs. The internal mammary gives off perforating branches, which pass forward through 
the intercostal spaces to supply the muscles of the breast and the manmary glands. Those which pass through the second, third, and fourth intercostal spaces are large, and are distributed to the mammary gland.

The Diaphragm.-The lower orifice of the thorax is closed in by the diaphragm. This is a musculo-tendinous partition which separates the thorax from the abdominal cavity. It forms the floor of the thoracic cavity and the roof of the abdomen. The thoracic surface of the diaphragm is covered by the fascia endothoracica and the diaphragmatic portion of the parietal pleura. Its middle part from before backward forms the floor of the mediastinum, and upon either side of this it forms the bottom of each pleural cavity.

The position of the diaphragm, immediately after death, corresponds with that found at the end of quiet expiration during life, but after a short time, owing to the further collapse of the lungs, it reaches to a still higher level.

Luschka places the highest point reached by the diaphragm at the end of forced expiration upon the right side at the level of the fourth rib. Most authors say that this is too high and give, instead, the fourth intercostal space. Upon the left side the diaphragm does not reach as high as upon the right by the breadth of one rib.

The upper orifice of the thoracic cavity is shut in on either side by the arching subclavian artery, scalenus anticus and medius muscles, and the fascia endothoracica. This fascia is intimately blended with the dome of the pleura, and attaches the same to the adjacent fixed points.

\section{THE REGIONS OF THE CHEST.}

The following imaginary lines serve to facilitate the location of points upon the chest:-

1. The midsternal, which passes through the middle of the sternum.

2. The lateral sternal, which corresponds to the lateral border of the sternum.

3. The mammary, which is drawn through the nipple.

4. The parasternal, which is drawn midway between the lateral border of the sternum and the mammary line.

5. The axillary, which is located midway between the anterior and the posterior borders of the axilla. 
6. The scapular passes through the lower angle of the scapula.

The chest is divided into a number of regions as follows:-

1. The sternal.

2. The upper anterior pectoral, which is subdivided into a clavicular, an infraclavicular, and a mammary.

3 . The lower anterior pectoral.

4. The lateral pectoral.

The Sternal Region.-This region corresponds to the sternum. It is depressed below the level of the rest of the chest, especially in muscular subjects and in females.

The skin of this region, in the male, is usually covered with hair and is rich in sweat-glands. The subcutaneous tissue is poor in fat and allows ready palpation of the sternum beneath. The skin and periosteum covering the sternum are so intimately blended with each other that separation between these two layers is somewhat difficult, and, therefore, collections of blood or pus beneath the skin in this region remain circumscribed, as is the case in the subcutaneous tissue of the scalp. Above, we observe the upper notched border of the sternum with the sterno-clavicular articulation upon either side and the attachment of the tendon of the sterno-mastoid. Below is the ensiform cartilage, to which is attached the linea alba. The junction of the manubrium with the body of the sternum is marked by a prominent transverse ridge and presents an angle directed forward: the angle of Ludovici. The sternum forms the anterior wall of the mediastinal space, and its posterior surface is in close relation with the pleura and the edges of the lungs. Below, the heart, inclosed in the pericardial sac, lies close behind the sternum.

The Upper Anterior Pectoral Region.-This area corresponds to the region of the pectoralis major muscle, and shows the prominence of the breast surmounted by the nipple and the areola. The skin is soft, especially in women, and during lactation is marked by blue lines, which correspond to large superficial veins. The skin is freely movable, owing to the looseness of the subcutaneous tissue, which is rich in fat and within which the mammary gland is contained. The mammary gland is freely movable upon the underlying pectoralis major muscle. The anterior surface of the pectoralis major is covered by a thin, cellular fascia, which also lines the posterior aspect of this muscle. Beneath the pectoralis major are the pectoralis minor and the subclavius muscle. The pectoralis major and minor form the front wall of the axilla. 
The Pectoralis Major is a broad, flat muscle which occupies all of this region. It takes its origin from the cartilages of the six or seven upper ribs and from the edge of the sternum: the sternal portion of the muscle. It also arises from the inner half of the anterior surface of the clavicle: the clavicular portion of the muscle. From these points of origin the fibers converge to form a flat tendon, about two inches broad, which is attached to the outer edge or lip of the bicipital groove: a depression which marks the upper part of the front of the humerus. The pectoralis major muscle is covered by a thin fascia, which dips down between its fasciculi and from which the overlying fat and mammary gland are readily separated. This fascia is rich in lymphatics, which may become involved in disease of the mammary gland. Below, this fascia is continuous with the superficial fascia which covers the abdominal muscles and laterally with that which covers the serratus magnus. It dips down into the space between the deltoid and the pectoralis major, and is there continuous with the loose fascia that invests. the pectoralis minor and the posterior surface of the pectoralis major.

The Pectoralis Minor.-This muscle is exposed by dividing the tendon of the pectoralis major close to its insertion and reflecting the muscle downward. The pectoralis minor arises from the tip of the coracoid process; passing downward and inward and becoming broader, it is attached to the third, fourth, and fifth ribs. The pectoralis minor is invested by a fascia which is continued upward and inward heyond the upper border of the muscle, covering in the first part of the axillary artery and adjoining structures and the subclavius muscle. This layer of fascia is called the costo-coracoid membrane and is attached to the under surface of the clavicle and to the first rib. It is somewhat thickened, and perforated by various vascular and nervous branches, which pass to and from the axillary vessels and adjacent nerves.

The Subclavius Muscle.-This muscle is exposed after the costo-coracoid membrane has been removed. It arises from the under surface of the clavicle and passing downward and inward is attached to the cartilage of the first rib.

This upper anterior pectoral region may be considered as the clavicular, the infraclavicular, and the mammary regions.

The Clavicular Region. - The clavicle can be readily palpated beneath the freely movable integument which covers it from its inner end, where it articulates with the sternum, to its outer end, 
where it articulates with the acromion process of the scapula. The acromion process of the scapula forms the most external and prominent point of the shoulder.

Beneath the skin in the clavicular region are found the platysma and the deep fascia.

To the upper surface and posterior border of the clavicle are attached, internally, the sterno-mastoid muscle, and externally the trapezius. To the inner half of the front surface of the clavicle is attached the pectoralis major muscle (clavicular portion), and, to its outer half, the deltoid muscle.

The under surface of the clavicle shows, at its inner end, the attachment of the rhomboid ligament. This ligament extends between the under surface of the clavicle and the cartilage of the first rib. External to this the subclavius muscle arises from the under surface of the clavicle.

The inferior surface of the outer end of the clavicle is connected with the coracoid process of the scapula by strong ligamentous bands.

Beneath the clavicle, between it and the first rib, the bloodvessels and nerves pass from the root of the neck into the axilla.

The Infraclavicular Region.-This is the region below the clavicle. Between the pectoralis major and the deltoid muscle, close to the clavicle, there is a triangular depression, the fossa of Mohrenheim: the infraclavicular fossa.

In the space, or groove, between the pectoralis major and the deltoid are lodged the cephalic vein and the descending branch of the acromio-thoracic artery, which is given off from the axillary. If the two muscles are widely separated, we expose the upper part of the pectoralis minor, covered by its fascia, some loose connective tissue and fat, and the coracoid process. This process is readily felt underneath the skin, and in thin persons can be seen.

If the pectoralis major is cut away from its attachment to the clavicle and from the upper part of the sternum and reflected downward, the infraclavicular region proper is uncovered. The pectoralis minor muscle is now more freely exposed. The cephalic vein may be seen passing from without inward across the pectoralis minor into a mass of fat and connective tissue on the inner side of the muscle, where it disappears through an opening in the costo-coracoid membrane to reach the first part of the axillary vein, which lies underneath this membrane. 
The acromio-thoracic and branches of the superior thoracic which are derived from the axillary artery are seen to emerge through openings in the costo-coracoid membrane, as is also the external anterior thoracic nerve, which supplies the pectoralis major.

The costo-coracoid membrane is a sheet of fascia which is continued from the inner or upper border of the pectoralis minor muscle upward and inward, and is attached to the under surface of the clavicle and to the first rib; it covers in the first part of the axillary artery and the structures that accompany it and the subclavius muscle. When the costo-coracoid membrane is removed, we expose the first part of the axillary artery and its acromio-thoracic and superior thoracic branches, the cords of the brachial plexus, which lie above the artery, and the axillary vein, which lies below and internal to the artery. The cephalic vein may be seen passing across the axillary artery to enter the axillary vein. All these structures are gathered together into a single bundle, and are accompanied by a mass of fat, connective tissue, and lymphatics (see Fig. 183).

The Mammary Region (Breast). - The mammary gland is rudimentary in the male and naturally well developed in the female. It rests upon the pectoralis major muscle from the third to the sixth rib. In unmarried and in young females it is hemispheroidal, firm, and projects forward; but after child-bearing, and especially in some races more than others, it is pendulous, and hangs down over the lower part of the thorax.

The skin of this region is thin and fine and is freely movable upon the underlying tissue. The superficial veins may show through the skin as irregular blue streaks. The skin of the nipple is especially thin and pigmented, and may be fissured and split, and shows the orifices of the milk-ducts, fifteen to twenty in number, as very fine, needle-point openings; through these infection may reach the mammary gland tissue proper.

In the unpregnant the nipple is depressed and pinkish, but is prominent and dark colored during pregnancy. The nipple is surrounded by a pigmented area, areola, which is fixed to the underlying tissue and marked by little nodules which correspond to sebaceous and sweat-glands.

In the unmarried the mammary gland proper is small, the prominence of the breast being due chiefly to the abundance of the fatty tissue in which the gland is imbedded. It does not reach its full development until after pregnancy. The mammary gland is a teg- 
mentary organ inclosed within its own proper fibrous capsule and lodged in the subcutaneous fat. It consists of a number of lobules, which are separate and distinct from each other; so that the secretion of milk and nursing may be continued even after one or more lobules have become the seat of a suppurative process. Between the mammary gland and the anterior surface of the pectoralis major muscle there is a layer of loose fatty tissue, which permits the gland to be freely moved about upon the surface of the muscle.

Occasionally a process of gland tissue almost entirely disconnected from the main gland may be found lying under the border of the pectoralis major, dipping beneath the muscle into the axilla. This process of gland tissue is often difficult to recognize. All the ducts of the gland converge from the periphery toward the nipple; they may become occluded and distended, giving rise to cystic tumors whose contents consist of milk or of a buttery material: galactocele.

The arteries of the breast consist of perforating branches from the internal mammary, especially the second and third and branches of the long thoracic from the axillary. Of the veins, the superficial ramify beneath the skin and the deep ones accompany the arteries.

The lymphatics are important and of these there are two sets: those of the integument and those which drain the gland proper. The lymphatics of the integument are very superficial and numerous, especially upon the nipple and in the areola; corresponding to the region of the areola, they form a fine capillary net-work which spreads outward toward the periphery, some branches dipping inward to enter a plexus which surrounds the milk-ducts beneath the skin of the areolar region. The lymphatics from the gland proper, from the acini and substance of the gland, are abundant. According to Sappay, they all tend toward the surface and end as goodsized vessels in the plexus already mentioned which surrounds the milk-ducts beneath the skin of the areola. The lymph from this subareolar plexus is collected into two main channels: one above and one below the nipple. These lymphatic vessels pass outward toward the outer border of the gland, and, after being joined by one or two vessels from the periphery of the gland, terminate in the nearest lymphatic nodes, which are found near the anterior wall of the axilla in the neighborhood of the third and fourth ribs, being covered usually by the edge of the pectoralis major. These are, as a rule, the first lymphatic nodes to become involved in disease of the mammary gland. The lymphatic nodes in the root of the neck 
also receive tributaries from the breast, and may be found involved when the mammary gland is diseased.

The Lower Anterior Pectoral Region.-This is the area which lies between the lower limits of the pectoralis major muscle and the free border of the ribs. This region is important surgically only on account of the structures which lie beneath it, within the chest and abdomen.

The Lateral Pectoral Region:-This space is included between the border of the pectoralis major in front and that of the latissimus dorsi behind. It presents the ribs covered by serrations of the serratus magnus and by the latissimus dorsi and obliquus abdominis externus.

The arteries of this region are derived from the axillary (long thoracic) and intercostals. The posterior thoracic nerve is found in this region descending upon the serratus magnus, which it supplies.

\section{THE MEDIASTINUM AND CONTENTS.}

The mediastinum is a space within the chest, between the two pleural cavities, which is occupied by the heart and pericardium, the thymus or its remains, the trachea, œsophagus, aorta, and several nerves, and a mass of loose connective tissue and lymphatics.

Rather more of the space lies to the left of the middle line than to the right. It is limited in front by the sternum, behind by the vertebral column, and its floor is formed by the diaphragm. Above, the loose connective tissue of this space is continuous into the root of the neck with that which surrounds the œsophagus and trachea and the great vessels in the neck. Laterally the mediastinum is walled off on either side from the pleural cavity by the parietal pleura (mediastinal portion of the parietal pleura).

The mediastinum, as mentioned above, is not an empty space, but is fairly closely occupied by various organs. In the lower part of this space, in front, is the heart, inclosed within its pericardial sac; behind the heart, between it and the vertebral column, the space is not large, and is occupied by the esophagus, thoracic duct, thoracic aorta, vena azygos, vena hemiazygos, and various nerves. In the upper part of the mediastinum, in front, is the thymus or its remains, and behind this the trachea and osophagus, the latter lying just in front of the vertebral column. Immediately above the base of the heart are the great vessels connected with the heart-the arch 
of the aorta, vena cava superior, pulmonary artery and its branches -and the bifurcation of the trachea. A number of lymphatic glands which communicate with the lymphatics of the neck and axilla are packed in between these structures.

The Pericardium.-The heart, occupying the lower anterior part of the mediastinum, lies close to the anterior wall of the chest (sternum) inclosed within its own serous sac, the pericardium. The pericardium, as a thin serous layer, is closely applied to the whole surface of the heart and to the great ressels at its base for a part of

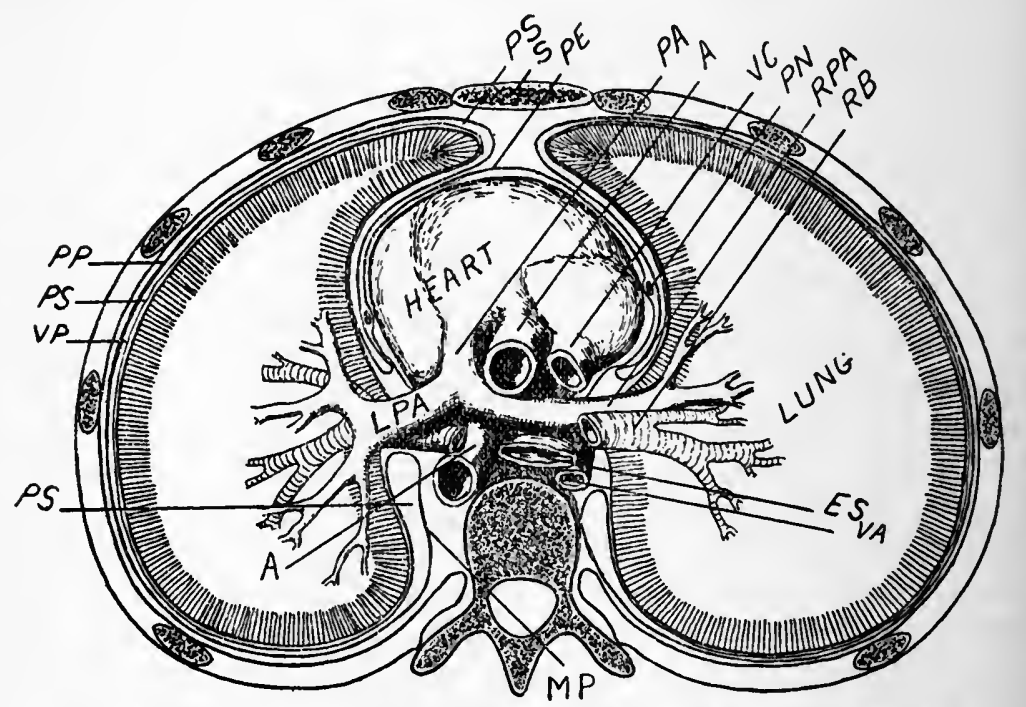

Fig. 80.-Transverse Section through Thorax just Above the Heart and Root of the Lungs. $A, A$, aorta; $E S$, œsophagus; $L P A$, left pulmonary artery; $M P$, mediastinal pleura passing forward to the posterior aspect of the root of the lung; $P A$, pulmonary artery; $P E$, pericardium; $P N$, phrenic nerve; $P P$, parietal layer of pleura; $P S$, space between parletal and visceral layers of the pleura; $R B$, right bronchus; $R P A$, right pulmonary artery; $\delta$, sternum; $V A$, vena azygos; $V C$, vena cava superior; $\nabla P$, visceral layer pleura.

their extent; above, after inclosing the first or ascending part of the arch of the aorta, it is reflected as a thin, loose, membranous sac, which completely envelops the heart and is attached below by its broad base to the dome of the diaphragm. The highest limit, or the apex, of the pericardial sac is that portion which incloses the first part of the arch of the aorta. Its broad base, which is below, corresponds to its attachment to the diaphragm. The pulmonary artery is also 
included within the pericardial sac as far as its bifurcation, but its two divisions are not included. The vena cava superior is also partially invested.

In front, the pericardial sac is in relation with the sternum and the costal cartilages, from which it is separated by the interposed pleura and the edges of the lungs. Behind the lower part of the sternum there is a triangular space-with its apex above upon a level with the fourth costal cartilage, a little to the left of the middle line, and its base below, corresponding to the junction of the body of the sternum with the ensiform cartilage: i.e., on a level with the articulation of the sixth costal cartilage-where the pericardium lies in direct relation with the posterior surface of the sternum. Corresponding to this area the pleura and the edge of the lung are not interposed between the sternum and the pericardial sac. Occasionally, according to some descriptions, the edge of the left pleura fails to reach the left border of the sternum behind the fifth costal cartilage and fifth intercostal space, and under these circumstances one could puncture through the fifth space close to the left border of the sternum and enter the pericardial sac without meeting the pleura. In all cases the edge of the left lung is notched in this region, incisura cardiaca; so that, although one might encounter the pleura in puncturing in this situation, he would not, in any case, meet the lung. Corresponding to the incisura cardiaca is the region of the "cardiac impulse," and here the heart is most exposed. Behind, that part of the pericardial sac which covers the left auricle is in close relation with the œsophagus. The trachea bifurcates just above and close to that part of the pericardial sac that covers the left auricle. On each side the pericardium is firmly adherent to the mediastinal portion of the parietal pleura, and between the apposed layers of both these structures, upon either side, the phrenic nerve descends in its course to reach and supply the diaphragm.

The Heart.-The heart, inclosed within the pericardial sac, is located in the lower anterior part of the mediastinum, almost completely surrounded by the lungs, which show a hollowed-out cavity on their internal surface corresponding to the size and shape of the heart. The impression upon the left lung is deeper than that upon the right.

Behind the heart is the vertebral column, and in the space between the heart and the spinal column, in the lower back part of 
the mediastinum, are the œsophagus, accompanied by the pneumogastric nerves; the thoracic aorta and thoracic duct; the vena azygos, which lies to the right of the vertebral column; and the vena hemiazygos, which lies to the left of the column.

The heart, with its long axis directed downward, forward, and to the left, rests with its posterior surface, which is composed chiefly of the left ventricle, upon the central tendon of the diaphragm. Here the diaphragm is somewhat flattened, and to the right of the middle line is perforated for the passage of the vena cava inferior. This vessel, after passing through the diaphragm, enters almost immediately the lower contiguous part of the right auricle.

The anterior surface of the heart, composed mainly of the right ventricle and auricle, lies close to the posterior surface of the sternum and costal cartilages, from which it is separated, for the most part, by the pleura and the lungs, these being interposed between the heart and the sternum and costal cartilages.

The base of the heart, which is directed upward and backward toward the spinal column, is made up of the auricles; the right auricle is placed anteriorly, and receives above the vena cava superior and below the vena cava inferior; the left auricle forms the posterior part of the base, lying close to the œsophagus, and receives the pulmonary veins from either lung.

The apex of the heart, the lowest part of the left ventricle, is found in the fifth left intercostal space midway between the parasternal and mammary lines.

Above the heart are the arch of the aorta, with the superior vena cava placed close upon the right side of its first or ascending part, the pulmonary artery and its bifurcation, the bifurcation of the trachea, and a mass of lymphatic glands and fat.

The Odthines of the Heart upon the Chest Wall.-The lower border of the heart corresponds to the line of junction between the body of the sternum and its ensiform cartilage. The upper border of the heart corresponds to the upper border of the third costal cartilage. To the right of the sternum lies the right auricle, its boundary corresponding to a curved line which is drawn from the articular end of the third costal cartilage downward and through the fifth costal cartilage close to its articulation with the sternum. The right ventricle reaches over for a considerable distance to the left of the sternum, with a portion of the left ventricle adjoining and forming the left border of the heart. The apex, the extreme end 
of the left ventricle, is situated in the fifth intercostal space midway between the parasternal and the mammary lines.

One-third of the heart lies to the right and two-thirds to the left of the middle line.

The pulmonary orifice, valve, corresponds to a line which is
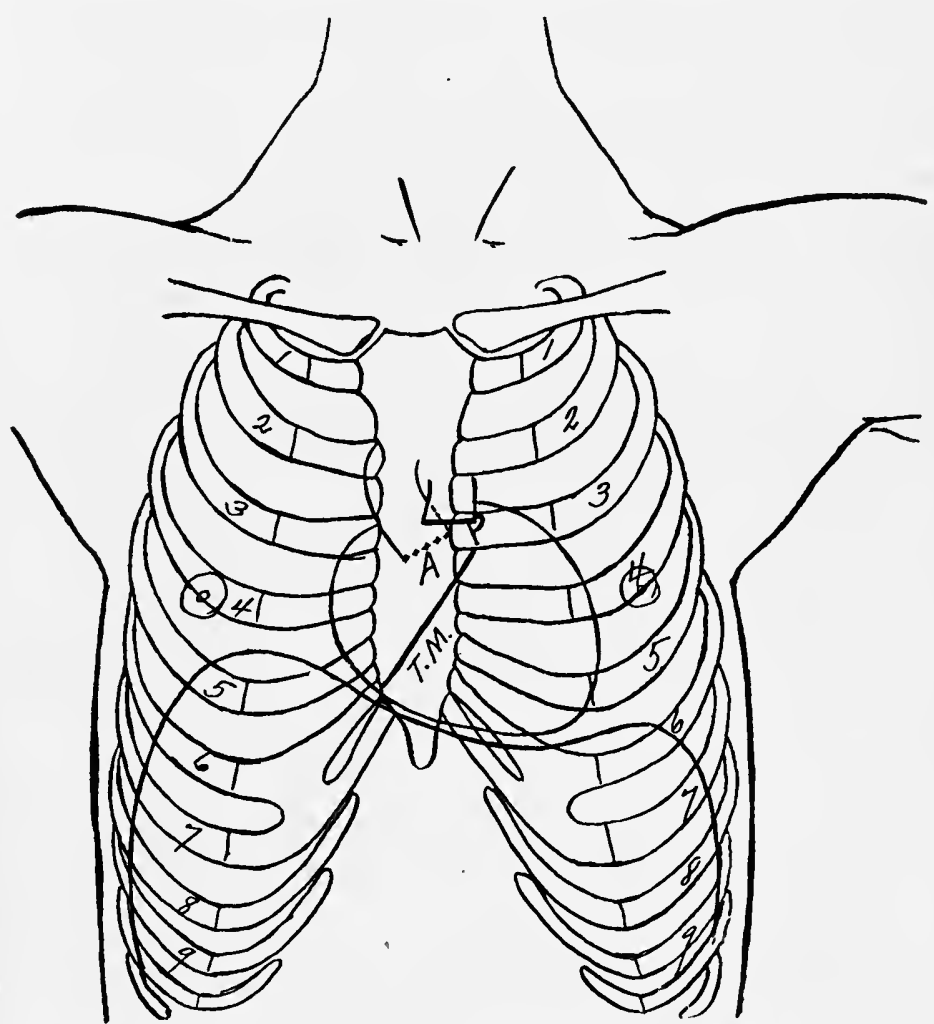

Fig. 81.-Outline of Heart and Location of Valves. $A$, aortic orifice, left semilunar valve (dotted line); $P$, oriflce of pulmonary artery, right semllunar valve; T.M., line of right and left auriculo-ventricular orifice. Upper part of line corresponds to left auriculo-ventricular oriflce, mitral valve. Lower part of line corresponds to right auriculo-ventricular opening, tricuspid valve. Position of the diaphragm is indicated by the curved line that passes below the inferior border of the heart.

placed upon the junction of the third costal cartilage with the left border of the sternum, half of the line upon the cartilage and half upon, the sternum.

The aortic orifice, valve, may be indicated by a line drawn from 
the junction of the third costal cartilage,with the left border of the sternum, just below the line indicating the pulmonary valve and diverging from this, as far as the middle line, to a level with the third space.

The auriculo-ventricular openings are represented by a line extending from the lower border of the third left costal cartilage, one finger's breadth beyond the left border of the sternum, downward and toward the right, across the body of the sternum, as far as the junction of the sixth right costal cartilage with the right border of the sternum. The lower part of this line represents the tricuspid (right auriculo-ventricular) orifice and the upper part represents the mitral (left auriculo-ventricular) orifice.

The Thymus.-The thymus body in the newborn is located in the upper front part of the mediastinum behind the sternum and in front of the upper part of the pericardial sac. Its upper portion reaches well upward, in front of the trachea, into the root of the neck. In the upper part of the mediastinal space the thymus lies directly in front of the trachea, the left innominate vein, which passes from left to right, across the front of the trachea, being interposed between them. In the root of the neck the thymus lies upon the front of the trachea, and is in relation, on either side, with the common carotid artery and the internal jugular vein.

The lower part of the thymus lies behind the body of the sternum and in front of the great vessels at the base of the heart, dipping down between the pericardial sac and the edges of the lungs and pleura.

The thymus increases in size from birth until the second year, and then remains stationary or atrophies slowly until puberty. After puberty it atrophies rapidly, undergoing fatty changes.

The Arch of the Aorta. - The arch of the aorta is well surrounded by the lungs, the edges of which nearly meet behind the sternum.

It arises from the left ventricle, and at its origin lies behind the root of the pulmonary artery. It first passes upward, forward, and toward the right as far as the right border of the sternum; it then turns backward and toward the left, arching over the left bronchus; and near the upper border of the body of the fourth dorsal vertebra, upon its left side, it turns downward and is continued as the thoracic aorta.

The arch, as it passes backward and to the left over the left 
bronchus, reaches its highest point, which is upon a level with the upper border of the first costal cartilage.

The Ascending Part of the Arch.-Upon the right side and close to the ascending or first part of the arch lies the superior vena cava, which enters the upper part of the right auricle; this part of the arch and the superior vena cava are situated in front of the root of the right lung. The vena azygos, passing forward from the right side of the vertebral column, crosses the root of the right lung and empties into the vena cava superior through its posterior wall.

The Transverse Part of the Arch.-The transverse part of the arch passes from right to left and from before backward, from the right border of the sternum to the left side of the body of the fourth dorsal vertebra, arching over the root of the left lung. Its upper border is upon a leve. : th the upper border of the first costal cartilage. From the upper aspect of the transverse part of the arch are given off the innominate and the left common carotid and subclavian arteries.

Below the transverse part of the arch is the pulmonary artery and its bifurcation, the branches passing transversely-one to the hilum of each lung-and lying in front of the bronchi. Behind the transverse part of the arch, in the back part of the mediastinum, the trachea and the œsophagus are located.

In front of the transverse part of the arch are the sternum, the thymus or its remains, and the edges of the pleura and the edges of the lungs, which nearly meet directly behind the sternum. A little above and in front of the transverse part of the arch, passing from left to right across the middle line, is the left innominate vein. The left superior intercostal vein passes forward from the third left intercostal space near the spinal column and enters the left innominate in front of this part of the arch. To the left of the middle line, the left pneumogastric nerve descends in front of and close to the transverse part of the arch, and gives off its recurrent laryngeal branch, which curves around the arch and ascends into the neck. Also descending in front of the transverse part of the arch, but nearer the middle line than the left pneumogastric, is the left phrenic nerve.

The Pneumogastric Nerves.-These pass through the thoracic cavity, in close relation with the cesophagus, on their way to the stomach.

The right pneumogastric, at the root of the neck, lies between 
the common carotid artery and the internal jugular vein. It descends into the chest, across the front of the first part of the subclavian artery, between it and the subclavian vein. Within the chest it passes obliquely backward, close to the right side of the trachea and across the posterior aspect of the root of the right lung, where it takes part in the formation of the posterior pulmonary plexus. The nerve then approaches the middle line and descends upon the posterior surface of the cesophagus and through the csophageal opening in the diaphragm, to be distributed to the posterior surface of the stomach.

The left pneumogastric dips into the chest between the left carotid and subclavian arteries, behind the left innominate vein, and, descending across the front of the left end of the transverse part of the arch of the aorta, is continued downward, behind the root of the left lung and thence upon the front surface of the œsophagus and through the diaphragm, to be distributed to the anterior surface of the stomach.

The Inferior Recurrent Branches. - Upon the right side the inferior recurrent is given off from the pneumogastric as it passes across the front of the first part of the subclavian artery. Curving around this vessel, it ascends in the neck, in the recess between the osophagus and the trachea, to enter the lower part of the larynx.

Upon the left side the recurrent is given off as the pneumogastric passes across the front of the transverse part of the arch of the aorta. It winds around the lower border of this part of the arch and ascends in the neck, having a similar relation to the cesophagus and trachea as that of the right side.

The Phrenic Nerves.-In the root of the neck the phrenic nerve of either side may be seen crossing the front of the scalenus anticus tendon in a direction from above downward and inward. After entering the chest they pass down in front of the root of either lung; the left, in its course, passes across the front of the transverse part of the arch of the aorta parallel with the left pneumogastric, but more internally, nearer the middle line; the right passes down upon the right side of the superior vena cava. They then descend between the pericardium and the mediastinal portion of the pleura as far as the diaphragm, which they supply.

The Trachea.-This is an elastic membranous tube which is put upon the stretch when the head is extended. Set into its wall are 
a number of cartilaginous plates, each forming part of a circle. These cartilaginous plates are absent in the posterior part of the trachea.

The trachea is the continuation of the larynx. It begins in the neck below the cricoid cartilage at the sixth cervical vertebra, and in this part of its course lies quite superficial.. As it passes downward it gets to lie deeper, farther away from the surface. In the chest, opposite the fifth dorsal vertebra, just above the base of the heart, the trachea divides into the two bronchi.

In front of the trachea, in the upper part of the mediastinum, are the sternum, the thymus or its remains, connective tissue, and fat. It is crossed from left to right and obliquely from above downward by the left innominate vein; into this vein in front of the trachea, one on each side of the middle line, empty the inferior thyroid veins. ${ }^{1}$ Occasionally a large arterial branch, the thyroidea ima, arises from the upper aspect of the transverse part of the arch of the aorta and ascends upon the front of the trachea. Lower down, the trachea is crossed by the transverse part of the arch of the aorta and the vessels arising from the superior aspect of this vessel. The innominate and left carotid arteries, at their origin, are placed in front of the trachea. The right pneumogastric, in the upper part of the chest, lies close to the right side of the trachea. The œsophagus is situated behind the trachea. It is intimately related to the posterior, non-cartilaginous wall of the trachea; so that foreign bodies lodged in the œsophagus may, by pressure upon the posterior wall of the trachea, seriously narrow its lumen and produce symptoms of strangulation. In the immediate neighborhood of the bifurcation of the trachea are twenty to thirty lymphatic nodes.

The Esophagus. - The csophagus is the continuation of the pharynx, and consists of a thick muscular coat with a mucous membrane lining. The mucous membrane is connected with the muscular coat by a very loose submucous connective tissue.

When collapsed, the œsophagus appears as a flat, transverse band, with the mucous membrane thrown into longitudinal folds, and upon cross section it shows a stellate figure.

The œsophagus commences behind the cricoid cartilage on a level with the sixth cervical vertebra; it descends through the neck and thorax, piercing the diaphragm upon a level with the tenth

1 The right inferior thyroid often empties into the right innominate. 
dorsal vertebra, and terminates at the cardiac end of the stomach upon a level with the eleventh dorsal vertebra.

In the neck and upper part of the thorax, as far as the fourth dorsal vertebra, the osophagus lies close to the front of the vertebral column, but from this point downward it gets to lie farther away, and as it passes through the diaphragm it is located quite some distance in front of and to the left of the tenth dorsal vertebra.

'The œsophagus, throughout its course, is surrounded by loose, cellular tissue by which it is connected with adjoining structures. The average length of the esophagus is about $35 \mathrm{~cm}$., and the distance from the teeth to the cardiac orifice of the stomach is about $50 \mathrm{~cm}$. To get the distance from the mouth to the cardiac orifice of the stomach, in any individual case, one may measure from the spinous process of the eleventh dorsal vertebra to that of the vertebra prominens, and thence across the shoulder to the mouth.

The lumen of the œsophagus is narrowest at its commencement behind the cricoid cartilage, again narrow opposite the third or fourth dorsal vertebra and again as it passes through the diaphragm. At its narrowest part the caliber of the csophagus has a diameter of $14 \mathrm{~mm}$., but it is capable of much distension beyond this.

Reuations of the CEsophagus. In the Neck the osophagus lies upon the front of the spinal column and immediately behind the trachea, to the posterior non-cartilaginous wall of which it is united by loose connective tissue. The œsophagus, situated behind the trachea, protrudes a considerable distance beyond the left border of the latter, and is therefore in closer relation with the common carotid artery, internal jugular vein, etc., upon the left side of the neck than upon the right side. In the recess between the trachea in front and the osophagus behind, upon either side, the recurrent laryngeal nerve ascends to enter the lower part of the larynx. Above, where the lateral lobes of the thyroid gland rest upon the sides of the trachea, they reach backward so as to get into close proximity with the œsophagus.

Within the Chest.-In the upper part of the chest the œsophagus is still situated in front of the spinal column close behind the trachea, protruding somewhat beyond the left border of the latter. Opposite the third dorsal vertebra it is placed, together with the trachea, behind the transverse part of the arch of the aorta. Opposite the fourth dorsal vertebra the descending part of the arch of the aorta lies to the left side of the œsophagus, pushing it (the œesophagus) a 
little over toward the right; but immediately below this the azygos vein, appearing upon the right side of the œsophagus, forces it again to the left, and here at this level the œsophagus is found behind the root of the left lung, to which it is loosely attached by connective tissue. As the œsophagus descends it remains in close relation with the aorta, which vessel gradually passes behind it in order to reach the middle line in front of the vertebral column. Opposite the eighth dorsal vertebra the œsophagus lies in front of the aorta, and opposite the tenth, as it pierces the diaphragm to terminate in the stomach, it lies in front and to the left of the aorta and spinal column.

In the space behind the heart, between it and the vertebral column, in the lower back part of the mediastinum, the œsophagus lies in close proximity, anteriorly, with the left auricle, which is enveloped in the pericardial sac. In this space, upon the right side of the vertebral column, is the azygos vein; upon the left, the hemiazygos; and in front of the vertebral column, the thoracic duct; the aorta is situated behind the œsophagus. The mediastinal portion of the pleura, as it passes forward to the root of the lung, is reflected upon either side of the œsophagus. Descending upon the anterior wall of the œsophagus is the left pneumogastric, and, upon its posterior wall, the right pneumogastric nerve. These nerves accompany the œsophagus through the œsophageal opening in the diaphragm and are distributed to the stomach.

The Thoracic Aorta.-This is the continuation of the arch. It lies at first upon the left side of the bodies of vertebræ, but as it descends it approaches the middle line, and finally, as it passes into the abdomen behind the diaphragm, it lies in front of the body of the last dorsal vertebra. Throughout its course the thoracic aorta is closely related to the esophagus; at first it lies to the left side of the œsophagus, but as it descends it gets behind it, between it and the vertebral column; below, the esophagus is placed in front of and to the left of the aorta, the latter (aorta) as it passes into the abdomen being situated upon the front of the spinal column. The thoracic aorta gives off the intercostal branches: one for each intercostal space from the third downward.

The Vena Azygos. - This vein ascends upon the right side of the spinal column; it is made up of branches from the lumbar region and receives the intercostals in its course. About the level of the fourth dorsal vertebra it passes forward over the root of the right lung, and enters the vena cava superior through its posterior wall. 
The Vena Hemiazygos.-The origin and course of this vessel are analogous to those of the azygos. It ascends upon the left side of the vertebral column. Opposite the eighth dorsal vertebra it passes across the front of the spinal column behind the aorta and thoracic duct, and upon the right side of the vertebral column joins the vena azygos.

The Thoracic Duct passes into the thorax behind the diaphragm in company with the aorta, between this vessel and the front of the spinal column. As it ascends through the thorax it lies upon the bodies of the dorsal vertebræ. In the upper part of the chest it arches forward and outward toward the left, and, passing over the subclavian artery and across the front of the tendon of the scalenus anticus, it enters the left subclavian vein where this vessel joins the left internal jugular.

The Innominate Artery has a caliber corresponding to the thickness of the little finger. It springs from the right end of the upper border of the transverse part of the arch of the aorta, and is about $5 \mathrm{~cm}$. long. At its origin it lies in front of the trachea; it terminates by dividing into the subclavian and common carotid behind the right sterno-clavicular joint.

Situated in front of this vessel are the sternal attachments of the sterno-hyoid and sterno-thyroid muscles, the manubrium of the sternum, and the remains of the thymus gland. The left innominate vein passes across the front of the root of the innominate artery, and upon its outer (right) side joins with the right innominate vein to form the vena cava superior. The right inferior thyroid vein, as it descends from the lower part of the thyroid gland to enter the right

- innominate vein, also passes across the front of the innominate artery. To the outer side of the innominate artery lie the right pneumogastric and the right phrenic nerves and the pleura and apex of the right lung. To the inner side of the innominate is the left common carotid, the distance between the two vessels varying.

The Left Common Carotid and Left Subclavian Arteries arise from the upper border of the transverse part of the arch. They lie deep within the chest, and are, in this region, not subject to surgical interference.

\section{THE PLEURA.}

The pleura of each side is a completely closed fibro-serous sac. It lines the entire inner surface of the cavity, within which the lung 
is contained, and, besides, as a thin, serous layer, invests the whole surface of the lung.

That portion of the pleura which is applied to the surface of the lung is called the visceral layer, and that which lines the whole inner surface of the cavity in which the lung is contained is called the parietal layer. That part of the parietal pleura which lines the inner surface of the wall of the chest, sternum, costal cartilage, ribs, etc., is spoken of as the pleura sterno-costalis; that portion which is spread out upon the surface of the diaphragm, the pleura diaphragmatica; and that which limits the mediastinum on each side, passing from before backward like a partition and separating the mediastinal space from the space which contains the lung, is called the pleura mediastinalis.

The parietal layer, after lining the inner surface of the ribs, intercostal muscles, etc.,- that is, the whole inner aspect of the wall of the thorax,-is found, behind, upon either side of the vertebral column, to leave the posterior wall of the thorax and pass forward, forming the posterior part of the mediastinal pleura; that of the left side, as it passes forward, covers the adjacent wall of the aorta and, lower down, the œsophagus; that of the right side, as it passes forward, covers, below, the side of the rena azygos and, higher up, the side of the œsophagus. Upon reaching the posterior aspect of the root of the lung the pleura is reflected on to the surface of the lung and as the visceral layer completely invests it, being also continued in between the lobes and intimately united with its surface; after thus entirely enveloping the lung it reaches the anterior aspect of the root of the lung, whence it is reflected forward toward the sternum as the anterior portion of the mediastinal pleura; upon reaching the posterior surface of the sternum it becomes continuous with that part of the parietal pleura which lines the inner surface of the wall of the chest: the pleura sterno-costalis. Above and below the level of the root of the lung the mediastinal pleura passes all the way as an uninterrupted layer from behind forward, from either side of the spinal column to the posterior surface of the sternum.

Limits of the Pleura as Indicated by Lines upon the Chest Wall. The Anterior Edge of the Pleura.-The line which indicates the anterior edge of the right pleural sac commences, above, behind the right sterno-clavicular articulation; from this point it passes downward and inward behind the sternum, and at the junction of 
the manubrium with the body of the sternum it lies close to the middle line; it is then continued downward behind the middle of the body of the sternum, and opposite the articulation of the fourth costal cartilage it curves outward, as it descends, to reach a point corresponding to the lower border of the sternal end of the sixth costal cartilage, whence it may be traced farther downward and backward as the lower edge of the pleura.

The line which marks the anterior edge of the left pleural sac is somewhat different. It commences above, behind the left sternoclavicular articulation, from which point it curves downward and inward toward the middle line and may then be traced downward behind the body of the sternum parallel with the anterior edge of the right pleural sac to a point upon a level with the junction of the fourth costal cartilage with the sternum; here it curves outward, but more obliquely than upon the right side, and reaches the sternal end of the sixth costal cartilage at its upper border, whence it is continued obliquely downward and backward as the lower edge of the pleura.

According to Merkel, the anterior edge of the left pleural sac, upon a level with the fourth costal cartilage, passes still more obliquely outward than has been described above so as to strike the sixth costal cartilage, not at its junction with the sternum, but some little distance beyond this articulation, thus leaving a space between the anterior edge of the left pleural sac and the left border of the sternum, corresponding to the fifth costal cartilage, fifth intercostal space, and sixth costal cartilage, which is not covered by the pleura. If this condition were present, one might introduce an aspirating needle into the pericardial sac through the fifth intercostal space, close to the left border of the sternum, without encountering the pleura.

Without doubt the anterior edge of the left pleural sac is subject to considerable variation. I have found the first description to hold for most cases.

The Lower Edge of the Pleura corresponds to a line that commences, in front, behind the junction of the sixth costal cartilage with the sternum; it passes downward and backward, crossing obliquely the eartilage of the seventh rib in the parasternal line and passing into the seventh intercostal space in the mammary line; still continued downward and backward it reaches its deepest point, corresponding to the tenth rib or tenth intercostal space, a little behind 


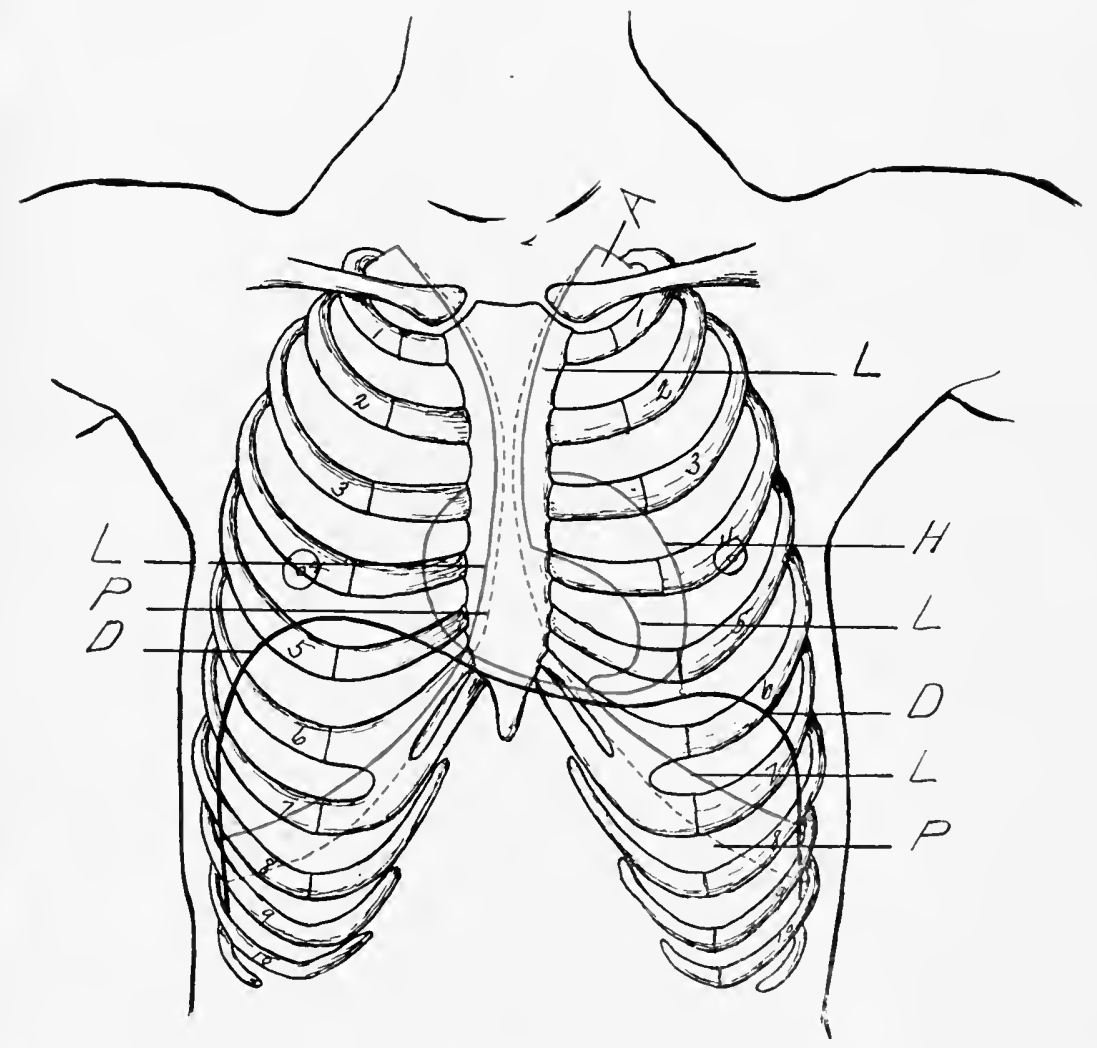

Fig. 82.-Outline of Pleura, etc. Front view. A, apex of lung and dome of pleura; $D$, line of diaphragm; $H$, outline of heart; $L$, solid lines show the edges of the lungs; $P$, dotted lines correspond to the edges of the pleura. 


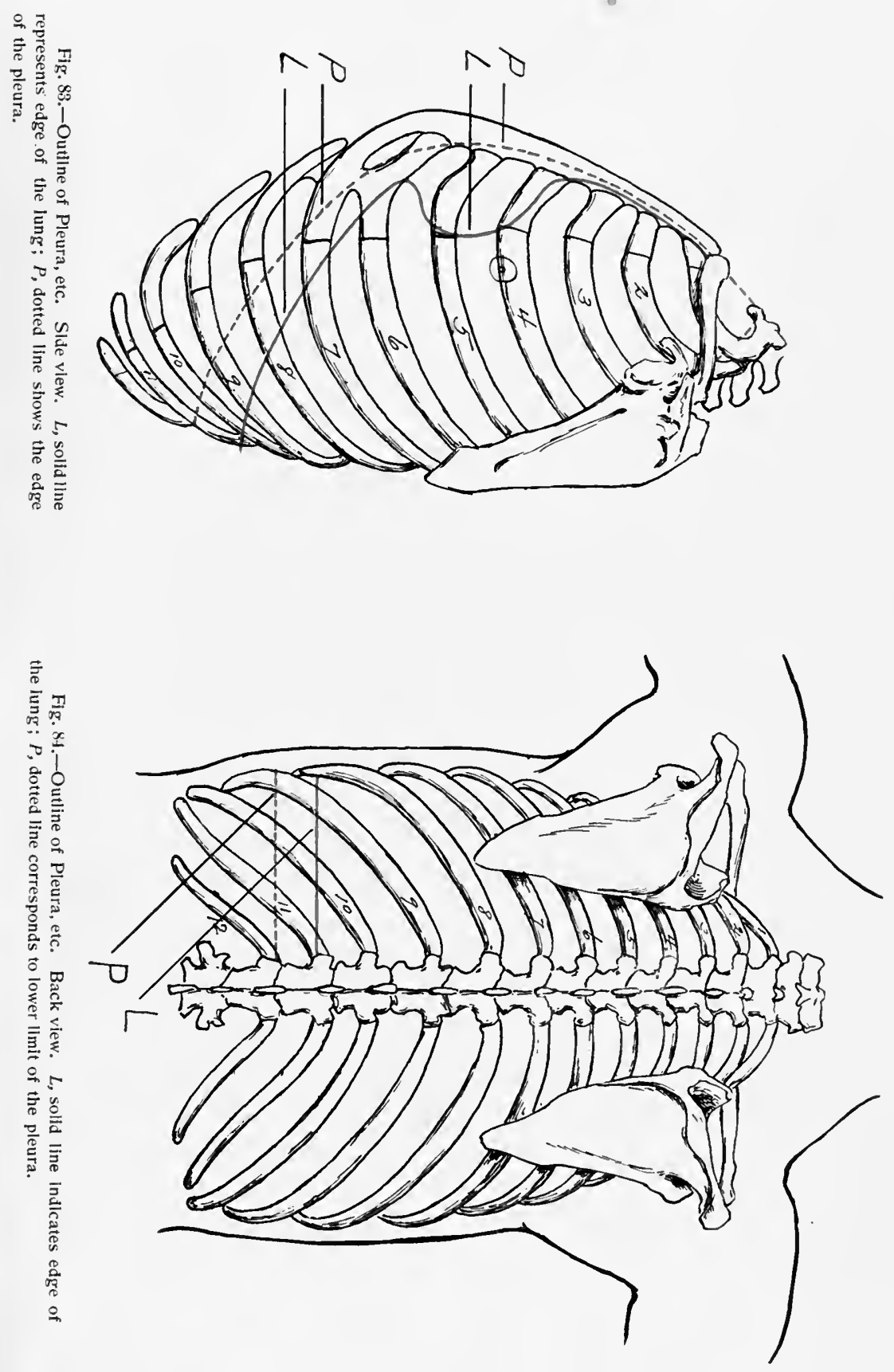

the axillary line, whence it may be traced almost horizontally backward and inward to the articulation of the twelfth rib with the spinal column. Behind, in the scapular line, the lower edge of the pleura corresponds to the tenth intercostal space.

It will be observed that the lower edge of the pleura, as it is reflected from the inner surface of the chest wall over on to the surface of the diaphragm, does not dip down into the bottom of the recess between the costal portion of the diaphragm and the ribs. This space varies in depth at different parts. Occasionaily the lower

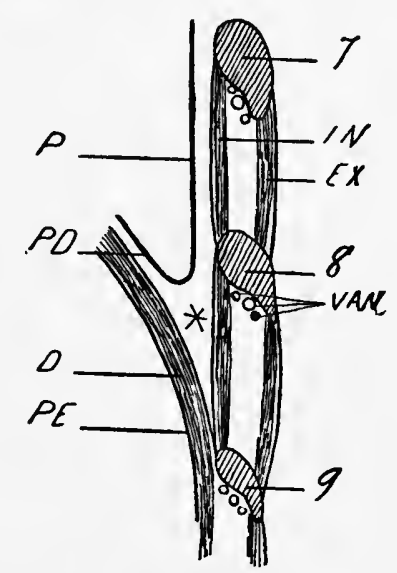

Fig. 85.-Section through Seventh, Eighth, and Ninth Ribs Anterior to the Axillary Line. $D$, diaphragm; $E X$, external intercostal muscle; $I N$, Internal intercostal muscle; $P$, pleura covering inner aspect of the chest wall; $P D$, pleura that covers the diaphragm; $P E$, peritoneum that is reflected upon the under surface of the dlaphragm; $\nabla A N$, intercostal veln, artery, and nerve situated under lower border of the ribs; $7,8,9$, cut surface of ribs; * represents the space between the dlaphragm and chest wall into which the pleura does not descend, as it is reflected from the chest wall on to the upper surface of the diaphragm.

edge of the pleura, behind, reaches down between the twelfth rib and the diaphragm as far as the lower border of the twelfth rib, or, even beyond this, down to the level of the transverse process of the first lumbar vertebra.

The Dome of the pleura is that part of the pleural sac which projects upward into the root of the neck above the level of the first rib; it reaches to a distance of $5 \mathrm{~cm}$. above the level of the anterior part of the first rib, but does not reach above the level of the back part of the first rib; the first rib is set very obliquely, its anterior portion being upon a much lower level than its posterior part. 
The dome of the pleura reaches from 2 to $4 \mathrm{~cm}$. above the level of the clavicle; so that a knife introduced above this bone and passed directly backward would pierce both the pleura and the lung. In front of the dome is the first rib and the posterior surface of the scalenus anticus muscle and the clavicle. Internal to the dome are the trachea and the œsophagus.

The subclavian vessels pass forward and outward across the dome, grooving it and the apex of the lung, which lies beneath. Care is necessary in ligating the subclavian or innominate arteries not to wound the pleura.

As the internal mammary artery dips down into the chest it is crossed by the phrenic nerve and lies in close relation with the dome of the pleura.

The dome of the pleura is re-enforced by the fascia endothoracica, and connected behind, through ligamentous bands, with the first rib and the last cervical and the first dorsal vertebræ and in front with the deep surface of the scaleni muscles.

The mediastinal portion of the pleura and the pericardium are adherent to each other, and between these two serous layers the phrenic nerves descend to the diaphragm.

\section{THE LUNGS.}

The Root, or Pedicle, of the Lung.-The root of the lung is located in the back part of the mediastinum behind the ascending part of the arch of the aorta and above the base of the heart. That of each lung is composed of the bronchus, the pulmonary artery, and the pulmonary veins, together with lymphatics (also blood-vessels for the supply of lung tissue proper and plexuses of nerves).

The trachea bifurcates opposite the fifth dorsal vertebra, and its divisions, the bronchi, are directed outward and downward toward the hilum of either lung. The right bronchus is more horizontal, shorter, and of wider caliber than the left, and its lumen is more directly continuous with that of the trachea; so that foreign bodies dropped into the trachea are more apt to enter the right than the left bronchus.

The pulmonary artery springs from the upper part of the right ventricle, and at its origin lies in front of the root of the aorta. It is a short trunk, directed upward and backward, and under the trans- 
verse part of the arch of the aorta divides into the right and left pulmonary. These pass outward, in front of the bronchi, to the hilum of either lung. At the hilum the pulmonary arteries are located upon a higher level than the bronchi, and may get to lie partly behind these as they enter the lung.

The pulmonary veins are short trunks which, upon leaving the hilum of the lung, pass transversely inward and enter the corresponding side of the left auricle; they lie some little distance below the level of the bronchi and the pulmonary arteries.

There are numerous lymph nodes irregularly arranged about the root of the lung, but there is always a well-marked group below the bifurcation of the trachea.

Over the root of the left lung, arching from before backward, is the arch of the aorta. The vena azygos passes over the root of the right lung, from behind forward, and enters the vena cava superior, which lies just in front of the root of the right lung, upon its posterior aspect.

The Lung, suspended by its root, occupies the pleural cavity and is entirely enveloped by the visceral layer of the pleura. At the root of the lung this visceral layer of the pleura is continuous with the mediastinal part of the parietal pleura. The base of the lung rests upon the diaphragm; its apex projects into the root of the neck for a distance of 4 or $5 \mathrm{~cm}$. above the front end of the first rib. In the natural sitting position the apex of the lung reaches to a point about $3 \mathrm{~cm}$. above the clavicle.

The right lung consists of three lobes, the left of two. Each lung upon its inner surface shows a depression corresponding to the heart, that upon the left lung being deeper than that upon the right lung.

The lung does not entirely fill the pleural cavity except above, where the apex occupies all the space corresponding to the dome of the pleura.

LIMITS of THE LUNGS.-The posterior border of each lung is found alongside the vertebral column. The anterior border of the lung corresponds to the line of the pleura from the sterno-clavicular articulation to the level of the fourth costal cartilage. The anterior border of the right lung continues to be the same as that of the pleura down to the level of the sixth costal cartilage. The anterior border of the left lung, at the junction of the fourth costal cartilage with the sternum, passes almost transversely outward behind the cartilage 
of the fourth rib, forming the upper border of the incisura cardiaca, ard then, midway between the border of the sternum and the nipple, it turns downward behind the fourth intercostal space and fifth costal cartilage, and in the fifth space passes sharply inward, forming the lower border of the incisura cardiaca.

The lower border of either lung is represented by a line which commences at the junction of the sixth costal cartilage with the sternum; it passes downward and backward, behind the sixth costal cartilage, and crosses the seventh rib in the mammary line; from this point the line passes backward, almost transversely, crossing the eighth and ninth ribs in the axillary line, the tenth rib in the scapular line, and reaches the vertebral column upon a level with the articulation of the eleventh rib. Although the line, after crossing the seventh rib in the mammary line, is continued almost transversely backward, it cuts the eighth, ninth, and succeeding lower ribs, owing to the obliquity of the ribs.

The lower edge of the lung does not reach to the bottom of the pleural cavity; so that a space is left which is called the sinus phrenico-costalis. This space commences in front, and gradually becomes deeper; upon the sides it is deepest, and may measure up to two inches. In more forcible inspiration this space is partly obliterated by the increased expansion of the lung.

A similar pleural space, unoccupied by the lung (incisura cardiaca), is found in front of the pericardium and heart, corresponding to the fourth intercostal space and fifth costal cartilage, to the left of the sternum.

In the child the distance between the lower border of the lung and the bottom of the pleural cavity is one-half to one space deeper than described above. In old age the distance between the lower border of the lung and the bottom of the pleural cavity becomes one-half to one space shorter.

Luschka gives the depth of the sinus phrenico-costalis as follows: In the sternal, parasternal, and mammary lines, $2 \mathrm{~cm}$; in the axillary line, $6 \mathrm{~cm}$; and near the vertebra, $2.5 \mathrm{~cm}$.

\section{OPERATIONS UPON THE CHEST.}

Incisions for Abscess of the Breast.-These should radiate from the region of the nipple toward the periphery of the breast in order to avoid, as far as possible, cutting across the milk-ducts, which all 
converge toward the nipple. The incisions should be liberal, and should be so placed as to allow the discharge to drain through the lower, dependent part of the breast, and, if necessary in order to accomplish this, one or more counter-openings may be made. Liberal incisions should be made through the skin and fat, and the abscess cavity penetrated with closed artery forceps, which are spread apart as they are withdrawn.

Extirpation of Tumors Out of the Substance of the Mammary Gland (Fibroids, for Example).-An incision is made corresponding in length to the size of the tumor and radiating from the areola toward the periphery of the breast.

These tumors are usually encapsulated and well defined, and can be dissected out with blunt-pointed scissors or may at times be enucleated by blunt dissection with the finger.

Amputation of the Breast.-The patient lies upon the back with the arm abducted. The incision depends upon the size of the tumor and the condition of the skin. If the skin is involved in the pathological process, the diseased portion should be sterilized and packed or covered with gauze, and the incision placed at least two inches outside of the affected area of the skin.

The incision should be so arranged that the edges of the wound may be brought together with sutures, after the breast has been removed, for the purpose of obtaining primary union; yet one should not hesitate to sacrifice all suspicious integument, since any defect that remains may be covered by skin grafting.

The usual incision is one which includes an elliptical area of the skin and the nipple, set obliquely so as to run parallel with the fibers of the pectoralis major, the upper end of the ellipse being continued along the border of the pectoralis major into the upper arm in order to empty the axilla. The edge of the skin, on the inner side of the ellipse, is seized with the fingers or a thumb forceps, and, including little or none of the subcutaneous fat, is separated from the underlying tumor and breast beyond its farthest limits and down to and exposing the surface of the pectoralis major muscle.

If the breast (tumor) is not adherent to the pectoralis major it may be readily detached from the surface of this muscle with the fingers, and then, after separating the skin which covers the outer part of the breast it may be turned out of the wound ; as the fascia, however, which covers the pectoralis major is often involved in the disease even when apparently healthy (Volkmann), it is better, in 
all cases, to remove this fascia, together with the superficial portion of the muscle, along with the breast, all in one mass.

At times, portions of the mammary gland, partially disconnected from the main mass of the gland and lying in the adjacent fat, are difficult to recognize, or the gland itself may be flattened out and difficult to identify, or a portion of the gland, almost completely detached from the main gland, may be found externally under the outer border of the pectoralis major. Care must be exercised to include all these parts, and this can only be accomplished by excising the entire gland and the fat in which it is imbedded, together with the fascia which covers the pectoralis major and the superficial portion of this muscle and the contents of the axilla, all in one mass.

If the muscle is deeply involved, the whole muscle down to the ribs should be sacrificed, and it may even be necessary, at times, to remove the surface of the ribs; but these are rather hopeless cases at best.

After the breast (tumor) has been entirely freed from the skin and pectoralis muscle, but not yet detached, cut away, at its axillary end, the axilla is opened and its entire contents-glands, connective tissue, fat, etc.-excised in one mass, which still remains continuous with the breast (tumor).

In this way the whole axillary space is completely cleaned out, working close along the course of the axillary vessels and adjoining nerves and ligating all vascular branches as they are encountered. The glands that are involved often extend high up into the axilla under the clavicle, and there may be some difficulty in removing these.

The axillary vein should be exposed early during this part of the operation. It rests upon the tendon of the latissimus dorsi, which is the guide to the vessel, below and to the inner side of the axillary artery. The axillary vessels are accompanied by large nerve trunks and, these, together with the vessels, are located, all in a bunch, close to the humerus, resting upon the tendon of the latissimus dorsi, beneath the edge of the coraco-brachialis and the short head of the biceps. If the vessels are thus sought for and exposed early in the operation they are less liable to be accidentally injured. It is also necessary to clean out the space between the pectoralis major and the pectoralis minor muscles.

If the mass already involves the axillary vessels and nerves, and this is usually indicated by shooting pains in the arm and codema 
of the arm, the case is, at best, rather hopeless, and it is questionable whether the operation had not better be left undone.

In clearing out the axilla some large arteries and veins may be severed, but these may be clamped and ligated; they may be often seen before they are cut, and can then be tied before they are divided, or they may be avoided.

The long thoracic nerve, which supplies the serratus magnus, lies upon the side of the chest, in the posterior part of the axillary space; it is usually seen and may be avoided, although it is of but little consequence if it is accidentally divided. One should also, if possible, avoid the long subscapular nerve which runs in eompany with the subscapular vessels upon the posterior wall of the axilla.

If the clearing out of the axilla is commenced below, close to the under border of the pectoralis major, the long thoracic artery and vein and long thoracic nerve are encountered early, and they may be avoided or used as guides in seeking the axillary vein.

-During the operation the parts must be well retracted, and, in order to minimize the loss of blood as much as possible, each vessel should be clamped as it is cut, and that part of the wound which is not under immediate consideration compressed with hot pads to prevent oozing. The bleeding should be entirely controlled before the wound is finally closed.

With interrupted sutures the edges of the wound are coapted as far as possible, and the area which is then still left uncorered may be provided with skin grafts. It is wise to place a tube in the axilla for the purpose of drainage.

Amputation of the Breast (Halsted-Meyer). - The breast, together with the pectoralis major and minor muscles and the glands and connective tissue of the axilla, must all be removed in one single mass and without cutting into the diseased tissue.

An incision is made through the healthy skin and fat, elliptical and circumscribing the tumor; from the upper end of the ellipse the incision should be continued along the edge of the pectoralis major to a point upon the upper part of the arm a little beyond (below) the attachment of the tendon of this muscle to the humerus. Although it is desirable to bring the edges of the wound together with sutures at the end of the operation, yet one should not, on this account, take any chance in leaving suspicious looking integument, because if we are unable to close the wound with sutures we can cover any remaining raw space with skin grafts. 
To this first incision a second is added which runs obliquely from the junction of the middle and outer thirds of the clavicle down into the upper border of the elliptical incision. The skin flaps which are thus marked out, and including little or no fat, are then dissected away from the breast (tumor) and well beyond its periphery. In this way we expose the tendon of the pectoralis major externally, the border of the latissimus dorsi below and externally, and above the space or groove between the edge of the deltoid and the upper border of the pectoralis major; in this space the cephalic

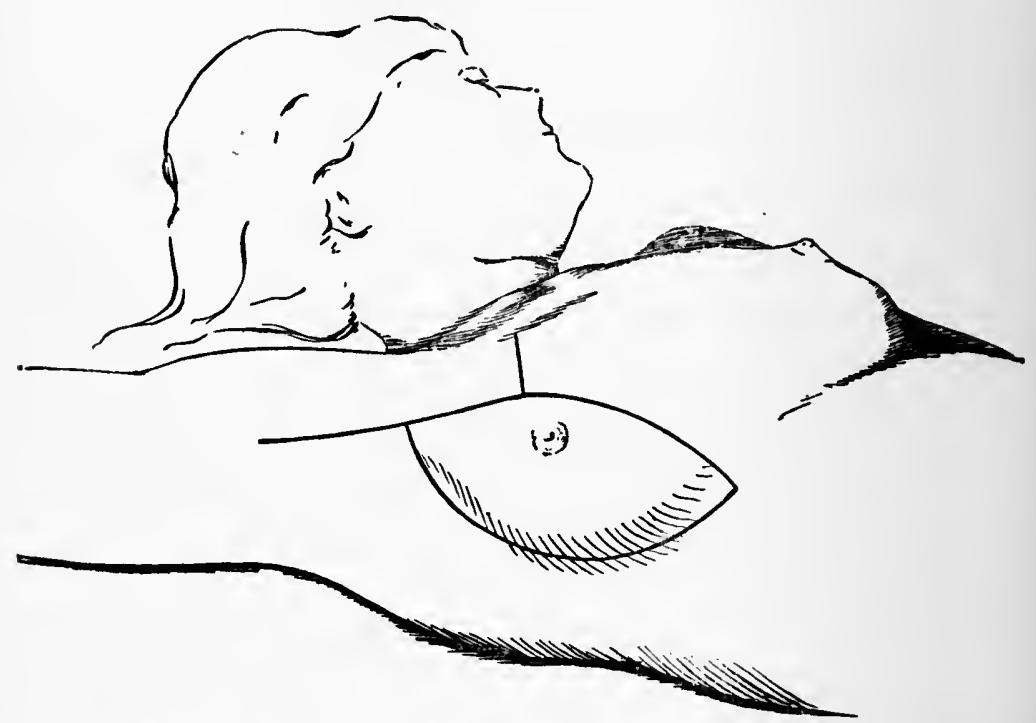

Flg. 86.-Amputation of the Breast. Halsted-Meyer incision for amputation of the breast and to clean out the axilla.

vein and the descending branch of the acromio-thoracic artery are found.

The tendon of the pectoralis major is next divided close to its attachment to the humerus, and then, following along the upper border of this muscle, between it and the edge of the deltoid as far as the clavicle, this muscle (pectoralis major) is cut away from its attachment to this bone (clavicle) and reflected downward, thus exposing the next underlying layer, or "étage," which consists of the pectoralis minor covered by its fascia and some loose connective tissue. The fascia that covers the pectoralis minor is continued 
upward from the inner border of the muscle as the costo-coracoid membrane, and is attached to the first rib and under surface of the clavicle, thus covering in the structures of the infraclavicular region. This fascia, costo-coracoid membrane, which is perforated by the cephalic vein and other structures, is now cut away from its attachment to the clavicle, and we thus uncover the structures of this region, namely: the first part of the axillary artery; the axillary vein, which lies along the inner, lower side of the artery; and close to the vein a chain of lymphatic vessels and nodes, connective tissue, and fat. The nerve trunks, which are derived from the brachial plexus, run parallel with the vessels, but above them. All these structures pass upward and inward, under the clavicle and beyond the first rib, into the root of the neck.

Commencing as high up as possible, the space beneath the clavicle being made more accessible by elevating the shoulder, all the fat and connective tissue are cleaned away from the vessels, ligating all branches as they are met with and working outward and downward along the course of the vessels. After the space beneath and above the clavicle has been thoroughly cleared of all fat and connective tissue, the pectoralis minor is cut close to its origin from the coracoid process and reflected downward, together with the connective tissue and fat that lie upon it and also the fat and connective tissue that are found underneath it adjacent to the vessels and nerves in this part of the axilla. This dissection is continued down along the course of the vessels and nerves as far as the attachment of the tendon of the pectoralis major to the humerus, and should be thorough. The tissue which is thus removed should not be taken away piecemeal, but dissected free from the vessels, etc., in one continuous mass, and allowed to remain connected with the general tumor mass.

Now, from the posterior wall of the axilla and from the side of the chest, all the fat and connective tissue and lymphatic tissue are cleared, working from behind forward and laying bare, behind, the anterior surface of the latissimus dorsi, subscapularis, and teres major muscles (posterior wall of the axillary space) and, upon the side of the thorax, the ribs and serratus magnus muscle. Upon the posterior wall of the axilla the subscapular nerve, in company with the subscapular vessels, is encountered. This nerve should be saved, if possible, and likewise the vessels, if they have not already been cut.

Upon the side of the chest we meet the long thoracic vessels 
and the long thoracic nerve; if the nerve is recognized it may be possible to avoid cutting it.

The whole mass-which consists of the breast (tumor), pectoral muscles (major and minor), axillary contents, etc.-is now grasped by an assistant and lifted away from the chest wall when the attachments of the pectoral muscles to the ribs and sternum are cut, and then, the mass being gradually turned out of the wound, the extirpation is completed and the bare wall of the chest, together with the axillary vessels and the nerves which accompany them, is exposed to view. When the mass is lifted away from the chest wall, the perforating vessels-branches of the intercostals and the internal mammary-may be seen as they enter the posterior surface of the pectoralis major and care should be taken not to tear these or cut them too close to the surface of the chest wall, as it might then be difficult to clamp and tie them. They may often be secured with clamps before they are cut.

The edges of the wound are brought together by suture, and if too much integument has not been removed the wound may be thus closed entirely. The little triangular flap, corresponding to the outer edge of the elliptical incision, is turned up into the axilla and fixed there. If there is any raw space remaining, it may be covered with rubber tissue and skin grafted later, or the grafts may be applied at once. A tube may be placed in the axilla for drainage, and this may be removed on the sixth or seventh day, when the dressing is changed.

One should minimize the loss of blood as much as possible, clamping vessels before or immediately after they are cut.

Ligation of the Intercostal Artery.-Each intercostal artery is situated, together with the intercostal vein and nerve, beneath the lower border of the corresponding rib. These vessels may be injured in stab wounds, etc.

At times it becomes necessary to resect a part of the rib subperiosteally in order to get at the bleeding points. It is necessary to tie both ends of the vessel.

Ligation of the Internal Mammary Artery. - To secure this vessel one must resect the costal cartilage of the second or third rib close to the sternum or the vessel may be ligated through a transverse incision placed midway between the contiguous cartilages and close to the sternum in the third intercostal space. The vessel descends upon the posterior surface of the anterior chest wall, its 
vein alongside of it; it is accompanied also by a chain of lymphatic nodes.

Thoracentesis.-Puncture through the chest wall into the pleural cavity.

This operation may be performed to show the presence and to determine the nature of fluid in the pleural cavity or to evacuate such fluid. If for diagnosis only, an ordinary hypodermic syringe may be used. If necessary to evacuate a considerable quantity of fluid, one may use a rather good sized aspirating needle attached to a Dieulafoy syringe. The patient should be semirccumbent or lying down. The puncture should be made at the point where the physical signs locate the fluid. To anæsthetize the skin a spray of ethyl chloride may be used. Before the needle is introduced, the skin is drawn upward or downward so that the track of the needle through the muscles may not be upon the same level as the puncture in the skin. The needle is thrust into the chest between the two ribs nearer the lower than the upper one.

If one may choose the point at which the needle is to be introduced, either the eighth space, just below the angle of the scapular, or the sixth space, in the middle of axilla, just in front of the border of the latissimus dorsi, is usually selected.

The fluid should be evacuated slowly, and, if the quantity is great, care should be taken not to remove too much. One should stop if persistent cough occurs or if the pulse ehanges. At times, the needle becomes plugged with pieces of fibrin, which may be dislodged by introducing a stylet or by pumping some of the fluid back into the pleural cavity. After the fluid has been withdrawn the needle is removed and the small wound in the skin covered with collodion, etc.

One should remember that the lower limits of the pleural cavity fall short of the free border of the ribs, and, further, that if the needle is inserted straight inward for a considerable distance it may pass through the pleura and diaphragm into the abdominal cavity.

If we find pus in the pleural cavity, in the adult, it is necessary to establish drainage, resecting part of a rib. In the child it often suffices to simply evacuate the pus with the needle without providing drainage.

Thoracotomy. - This means cutting through the wall of the chest, usually with the resection of part of a rib, for the purpose of establishing drainage. 
The patient lies upon his well side, and should be anæsthetized. The seventh rib, that portion of it which lies anterior to the latissimus dorsi, is usually resected, as this is not covered by muscle and is sufficiently low for proper drainage.

Immediately before proceeding with the operation one should insert the exploring needle in order to ascertain positively the location of the pus, and there, where the pus is located, should the entrance into the pleural cavity be made. As already mentioned, if we have the choice, the seventh rib is the one selected for resection.

The incision, usually about two inches long, corresponds to the course and direction of the rib to be excised; it is carried down through the soft parts, including the periosteum, upon the surface of the rib. With the elevator the periosteum and all the soft parts are peeled off the bone, which is thus laid bare. Care must be exercised, in working around the upper and lower borders of the ribs to reach its internal surface, not to perforate the pleura nor wound the vessels that are lodged in the groove along the lower border of the rib. When the length of bone that is to be excised has been denuded of its periosteum it is cut through at either end with the sharp bone forceps. The opening into the chest cavity is made by incising the pleura with the knife. The opening which is thus made may be enlarged by introducing an artery forceps, the blades of which are spread apart as they are withdrawn so as to make a hole large enough to permit exploration of the interior of the chest with the finger and the introduction of one or two good-sized tubes.

If it is discovered, with the finger in the chest, that the opening is a considerable distance above the bottom of the pus cavity, one may, in order to facilitate the drainage, make a second counteropening at a lower level: through the eighth space, for instance, or even lower, depending upon the part of the chest which is involved (see limits of lower edge of pleura). The drainage tubes should be secured to the edge of the incision in the skin with a silk stitch. If the skin wound is unnecessarily large, it may be partially closed with one or two silk sutures. The administration of 20 or 30 minims of aromatic spirits of ammonia hypodermically, immediately before the opening is made into the pleural cavity, will often ward off the condition of collapse which sometimes occurs at this time.

Thoracectomy, Resection of the Chest Wall (Estlander).-An oval or U-shaped flap, consisting of the skin and subcutaneous fat, with its base behind, at the axillary line, is raised from the side of the 
chest, exposing three or four ribs; or a vertical incision, six inches long, may be made in the axillary line over the fifth, sixth, seventh, and eighth ribs, with two additional incisions along the course of the ribs, the middle of each of these accessory incisions corresponding to the upper and lower ends of the vertical incision. The two flaps which are thus marked out are reflected, one backward and the other forward, exposing four to six inches of three or four ribs.

Each rib is denuded of its periosteum all around, as described in the preceding operation, and resected with the bone pliers. A long incision is then made in the pleura and the thickened pleura excised. The bleeding is controlled by clamps and ligatures. The cavity of the pleura may be curetted if thought necessary. The edges of the skin flap are brought together with several silk sutures and the pleural cavity packed.

Pleurectomy (Fowler).-Detachment and excision of the thickened, diseased pleura, visceral and parietal, entire or in part, in old, intractable cases of empyema.

An incision is made along the course of the ribs corresponding to the site of the fistula, which is always present (since this operation is usually practiced in cases which have already been operated upon unsuccessfully), and the location of the disease; to either end of this incision there may be added accessory incisions, an anterior and a posterior. The flaps that are thus marked out, including all the soft parts, are raised so as to expose two or three ribs for four or five inches of their length. Instead of the incision as described above one may employ an elliptical or the double flap incision, as described in the Estlander operation.

The periosteum is stripped off two or three ribs with the elevator, and then from three to five inches of the two or three ribs that have been thus denuded are resected with the bone forceps. All bleeding points should be clamped and ligated. Cutting from the fistula, the parietal (costal) pleura, which is now exposed, is opened up with a free incision, and entrance thus gained into the suppurating pleural cavity. The pleura which invests the lung (visceral) is incised, and by blunt dissection with the finger or bluntpointed scissors this is peeled off the lung; here and there it will be necessary to cut a band with the scissors. In many cases the pleura may be separated from the lung with comparative ease, and this should be done with care, so as not to tear into the lung tissue proper. 
As the decortication of the lung progresses there may be considerable oozing from the denuded lung surface, but this may be controlled by compression with gauze pads, which are applied to the bleeding surface following up the fingers of the operator, or the blunt scissors, according as the pleura is detached.

After the pleura has been peeled off the lung the parietal pleura is stripped off the chest wall and then off the diaphragm. In separating the pleura from the contiguous portion of the pericardial sac care should be exercised to avoid any undue pulling or tearing. As a rule, the pleura is fairly easily separated and removed.

At times it is convenient to commence the detachment of the pleura by stripping it away from the chest wall; it is then peeled off the diaphragm and finally from the surface of the lung.

Occasionally the conditions that exist preclude the possibility of excising all of the diseased pleura, and under these circumstances the operator must content himself with the excision of the visceral or parietal (costal and diaphragmatic) pleura in part, or else simply incise the visceral pleura and strip it away from the surface of the lung without removing it.

After the pleura has been removed, either entire or in part, the cavity in the chest is loosely tamponed with gauze and the edges of the skin approximated with silk-worm gut sutures, except for a part of its extent, where the tampon emerges.

As a rule, as the detachment of the pleura from the lung progresses, the lung gradually expands more and more.

This operation has the advantage of removing the pathological suppurating membrane, and besides eliminates an obstacle to the expansion of the lung. The operation is not advisable in cases of diagnosible pulmonary tuberculosis. The discovery, during the course of the operation, of tuberculous deposits in the lung would warrant the surgeon in discontinuing the operation. 


\section{PART V. \\ THE ABDOMEN AND BACK.}

\section{THE ABDOMEN.}

THE abdomen corresponds to the lower part of the trunk, and consists of a eavity with elastic museular walls.

Within the eavity are contained the ehief part of the alimentary canal and the organs of digestion and the kidneys, ete. These organs are all more or less movable, and are provided with a more or less complete investment of peritoneum.

Externally the abdomen is limited above by the free border of the costal eartilages and below by the erest of the iliac bone of either side and Poupart's ligaments. The walls consist almost entirely of soft parts, and may be eonveniently considered as the posterior and the antero-lateral. The eapacity of the abdominal eavity is greater than is indicated by its external limitations.

The roof of the abdominal eavity is formed by the diaphragm; below, the abdominal cavity includes, on either side, the iliac fossa and communicates through a wide, heart-shaped opening with the cavity of the true pelvis. The margin of the inlet into the pelvie cavity is called the pelvie brim.

The interior of the cavity of the abdomen is lined by the parietal layer of the peritoneum, and is entirely shut off from communication with the exterior of the body except in the female, where a communication exists through the vagina, uterus, and Fallopian tubes, and this is frequently the channel through which infection is carried to the peritoneum from without.

The Diaphragm, which forms the roof of the abdominal eavity, is a musculo-aponeurotic structure that separates the cavity of the chest from that of the abdomen. It is dome-shaped, bulging into the eavity of the thorax and presenting its lower coneave surface to the abdominal eavity.

It arises by muscular fibers, which vary in length, from the inner surface of the ensiform process of the sternum and from the inner surfaces of the eartilages of the lower ribs. Behind, it arises from the ligamentum areuatum externum and ligamentum arenatum in- 
ternum and by its two crura from the anterior surface of the bodies of the three upper lumbar vertebræ. From these points of origin the muscular fibers converge and are continued into a three-leafed aponeurotic structure: the central tendon of the diaphragm. Behind the diaphragm there is an opening, the aortic, through which the aorta passes from the thorax into the abdomen; the posterior boundary of this opening corresponds to the body of the twelfth dorsal vertebra. In the back part of the diaphragm, to the left of the middle line, there is an opening which is surrounded by muscular fibers and through which the œesophagus passes to the cardiac end of the stomach. To the right of the middle line, toward the front, at the junction of the right and middle segments of the central tendon, there is an opening for the passage of the inferior vena cava; the edges of this opening are formed of aponeurotic fibers. The heart, wrapped in its pericardial sac, rests upon the upper surface of the central tendon of the diaphragm.

In front, close to the sternum, on either side of the bundle of fibers which arises from the ensiform process, there is a space where the muscular fibers of the diaphragm are absent; so that, in this situation, an opening exists through which the contents of one cavity may be forced into the other, giving rise to a so-called diaphragmatic hernia.

On the right side, owing to the presence of the liver, the diaphragm reaches higher into the chest than on the left. The thoracic surface of the diaphragm is covered by a thin fascia, the fascia endothoracica; the abdominal surface is likewise covered by a fascia which is very thin, the fascia transversalis.

The Posterior Wall of the Abdomen, the lumbar region of the back, corresponds to the five lumbar vertebræ and to the several muscles which fill in the space between the last rib and the crest of the ilium on either side of the spinal column. Externally we find the skin and beneath this the subcutaneous fatty layer. Between the muscles of the lumbar region are interposed strong layers of fascia-which serve to strengthen this region very much. The internal or abdominal aspect of the posterior wall of the abdomen is lined by that part of the transversalis fascia which covers the psoas and quadratus lumborum muscles.

The kidney, inclosed within its fatty capsule, is located in the lumbar region between the transversalis fascia-i.e., the anterior layer of the lumbar fascia-and the parietal peritoneum, its ante- 
rior surface only being covered by the peritoneum; so that the organ is thus excluded from the peritoneal cavity.

The Antero-Lateral Wall of the Abdomen is made up of several layers of soft parts. It consists of the skin with its underlying fatty layer; several broad, flat muscles, the oblique, the transversalis, and the recti; and the aponeuroses which correspond to these muscles; the fascia transversalis is found beneath these muscles, and, beneath the fascia transversalis, the subperitoneal fat is encountered, and, finally, deepest, most internal of all, is the parietal peritoneum.

In the female the abdomen is more rounded and contains a considerable pad of fat; in the male, especially in athletes, the fatty layer is less marked or almost entirely absent; so that the markings of the muscles show through the skin and give the characteristic appearance to the abdomen.

In the middle line, about midway between the ensiform process and the symphysis pubis, there is a well-marked depression, the navel; this is an important landmark, although its position may vary somewhat in different individuals, and marks the place where the fotal umbilical vessels and fotal channels enter and pass out of the abdomen. Above, in the middle line, is the ensiform process of the sternum, and passing downward from this there is a furrow which corresponds to the space between the rectus muscles, but which does not reach downward as far as the symphysis. On either side of the middle line, corresponding to the outer border of the rectus, is the location of the linea semilunaris. Below, on either side, the anterior superior iliac spines-important surgical landmarks-may be seen, and upon the pubic bones, on either side of and close to the symphysis, the spinous processes of the pubes may be felt.

Corresponding to Poupart's ligament, which reaches from the anterior superior spine to the spine of the pubes, there is a linear crease in the skin which separates the abdomen from the front of the thigh.

The whole abdomen is covered by the skin, underneath which is the subcutaneous fat; the abdomen is a favorite site for the accumulation of fat in the obese, and this layer varies much in thickness in different individuals; it is continuous with the corresponding fatty layer upon the breast and below with the fat of the thighs. At the navel the fat is absent, the skin being depressed and fixed to the aponeurosis beneath, so that the depth of the navel corresponds to the thickness of the abdominal pad of fat. The subcuta- 
neous fatty layer is readily separated from the underlying muscle and aponeurosis, leaving these structures covered by a thin, loose, cellular fascia, the so-called deep layer of the superficial fascia, but which is really a part of the subcutaneous connective-tissue layer. This fascia is more intimately attached to the linea alba and Poupart's ligament and to the pillars of the external inguinal ring than elsewhere. From the pillars of the ring it is prolonged downward around the spermatic cord and into the scrotum, where it is continuous with the dartos.

The Superficial Vessels of the Abdominal Wall.-In the subcutaneous fatty layer the superficial arteries and veins ramify.

Above, branches of the superior epigastric, which perforate the rectus and the anterior layer of its sheath, are distributed to the integument and subcutaneous tissue. Below, the superficial epigastric, which is derived from the femoral, curves obliquely upward across Poupart's ligament toward the umbilicus and supplies the skin and fat in this region.

Upon the sides of the abdomen branches from the lumbar arteries pierce the muscles and ramify in the subcutaneous tissue.

These vessels are all accompanied by their corresponding veins. Underneath the skin of the abdomen are seen many large veins which communicate with those within the abdomen, and therefore when the blood-current is obstructed in the portal vein or the vena cava these superficial abdominal veins become swollen and prominent and are readily recognized beneath the skin.

After the skin and subcutaneous fatty layer, including the thin deep layer of the superficial fascia, have been removed from the front and sides of the abdomen, the broad, strong aponeurosis of the external oblique upon the front of the abdomen and the fleshy portion of this same muscle upon the side of the abdomen are exposed.

The Muscles of the Antero-Lateral Wall. The External Oblique is a broad, flat muscle, the most superficial of the abdominal muscles, and occupies the side of the abdomen. The muscle arises by fleshy slips from the external surface of the eight lower ribs, interdigitating with the processes of origin of the pectoralis major and the latissimus dorsi. The fibers of this muscle have a general oblique direction, downward, forward, and inward, terminating in the broad, strong aponeurosis which occupies the front of the abdomen. Those fibers which arise from the lowest ribs pass almost directly downward and are attached to the anterior half of the outer 
lip of the crest of the ilium. The posterior free border of the external oblique muscle forms the anterior border of the triangle of Pettit. The posterior border of this triangle is formed by the outer free edge of the latissimus dorsi, its base by the crest of the iliac bone, its floor by the internal oblique muscle.

The aponeurosis of the external oblique is a broad, strong, pearly white, glistening, fibrous structure which occupies the front of the abdomen and is exposed after the integument and underlying fatty layer (superficial fascia) have been removed. The fibers of the aponeurosis are, for the most part, directed downward and inward, covering in the recti and joining in the middle line, between these muscles, to form the linea alba.

The linea alba is a strong, fibrous band which reaches from the ensiform cartilage above to the symphysis pubis below; it marks the union of the aponeuroses of either side and separates the recti from each other. The linea alba is interrupted by the navel. Above the navel the linea alba is broad: in the epigastric region it is 1 to 2 $\mathrm{cm}$. wide, and below, toward the navel, becomes still broader. Below the navel, however, it is not so broad, owing to the closer approximation of the edges of the recti. Above, where it is broad, it is thin from before backward, and below, where it is narrow, it is thick from before backward. Below, at its attachment to the symphysis pubis, it spreads out and is known as the adminiculum lineæ albæ.

Those fibers of the aponeurosis of the external oblique, that pass from the anterior superior spine of the ilium downward and inward to the spine of the pubes, form Poupart's ligament; where this ligament is attached to the pubic spine, the aponeurosis of the external oblique splits and leaves a triangular opening which is called the external inguinal ring, and through this the spermatic cord in the male, and the round ligament in the female, emerge. Below Poupart's, the aponeurosis is continuous with the fascia lata of the front of the thigh.

Along the outer edge of the rectus, at the linea semilunaris, the aponeurosis of the external oblique is blended with the aponeuroses of the underlying muscles; from the linea semilunaris the aponeurosis is continued inward, forming the anterior layer of the sheath of the rectus, and in the middle line joins with that of the opposite side to form the linea alba.

The Internal Oblique Muscle lies beneath the external oblique upon the side of the abdomen, a thin, loose, cellular con- 
nective tissue being interposed between them. The fibers of this muscle have a direction the opposite to those of the external oblique.

This muscle arises below from the anterior two-thirds of the middle lip of the crest of the ilium and from the outer half of Poupart's ligament. From this origin the fibers pass in a general direction upward and forward, some being attached to the lower border of the cartilages of the four lower ribs, the others terminating in the anterior aponeurosis, at the outer border of the rectus, the linea semilunaris. The lowermost fibers, which arise from Poupart's ligament, pass inward and then, curving downward, join with a similar process from the transversalis to form the conjoined tendon, which is attached to the crest of the os pubis.

The Transversalis is the deepest of the three broad abdominal muscles. It occupies the side of the abdomen lying next beneath the internal oblique, a thin, loose, cellular connective tissue intervening between them. Its fibers have a transverse direction. This muscle arises behind, through the lumbar fascia, from the transverse processes and spines of the lumbar vertebræ; above, from the inner surface of the six lower ribs; below, from the crest of the ilium and the outer one-third of Poupart's ligament. The fibers pass forward and inward, and, at the outer border of the rectus, terminate in the anterior aponeurosis. Those fibers of the transversalis which arise from Poupart's ligament pass inward, and curving downward join with a similar process from the internal oblique to form the conjoined tendon, which is attached to the crest of the pubes behind the external inguinal ring. Beneath the transversalis muscle, the transversalis fascia, which lines the whole inner surface of the abdomen, is found.

The Rectus is a long, flat muscle occupying the front of the abdomen, one on either side of the middle line, the linea alba being interposed between them.

Above, the rectus muscles are broad and attached to the cartilages of the fifth, sixth, seventh, and eighth ribs and to the sides of the ensiform cartilage. Below, they become narrow and are attached to the symphysis and crest of the pubes. The recti are marked by several transverse fibrous intersections, which are united to the anterior layer of the sheath of this muscle, but not to the posterior; they are usually three in number, two above the umbilicus and one below.

The Aponeuroses of the external and internal oblique and transversalis are blended with each other along the outer border of 


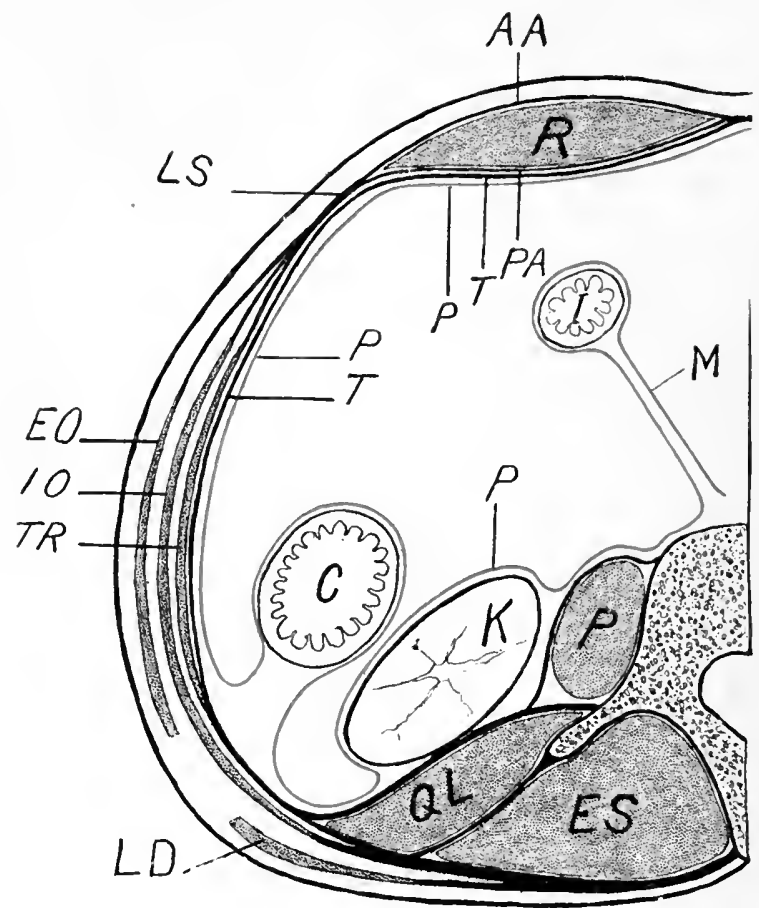

Fig. $47 .-$ Transverse Section of the Abdomen Above the Semilunar Fold of Douglas. $A A$, anterior layer of the split aponeurosis of the oblique and transversalis musclesanterior layer of sheath of the rectus; $C$, descending colon; $E O$, external oblique muscle; $E S$, erector spinxe muscle; $I$, intestine suspended by the mesentery; $I O$, internal oblique muscle; $K$, kidney ; $L D$, latissimus dorsi muscle; $L S$, linea semilunaris ; $M$, mesentery (suspends small intestine to vertebral column); $P$, psoas muscle; $P, P, P$, peritoneum lining inner aspect of abdominal wall; $P A$, posterior layer of split aponeurosis of the oblique and transverse muscles-posterior layer of sheath of rectus: $Q L$, quadratus lumborum muscle; $R$, rectus muscle; $T, T$, transversalis fascia; $T R$, transversalis muscle.

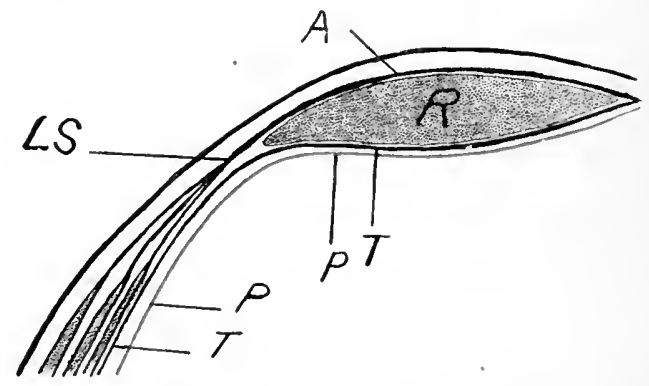

Fig. 88.- Transverse Section of the Abdomen Below the Semilunar Fold of Douglas, Showing the Entire Aponeurosis Passing in Front of the Rectus Muscle. A, aponeurosis of the abdominal muscles (oblique and transversalis), passing undivided in front of the rectus. For explanation of letters see Fig. $s_{i}$. 
the rectus muscle. Here, corresponding to the linea semilunaris, they form one aponeurotic layer. At the outer border of the rectus the conjoined aponeurosis splits into two layers, - an anterior and a posterior,-and these include the rectus between them, one passing in front of the muscle and the other behind it and both joining again with each other, between the recti, in the middle line, to form the linea alba. This disposition of the aponeurosis and sheath of the rectus is very simple and holds for the upper three-fourths of the muscle. Corresponding to the lower fourth of the rectus, the whole aponeurotic layer, without splitting, passes in front of the muscle; so that this lower fourth of the rectus, upon its posterior aspect, is without a proper sheath and is covered only by the general fascia transversalis.

Upon the posterior aspect of the rectus, where the posterior layer of the sheath terminates, it presents a sharp, curved edge: the semilunar fold of Douglas.

The Fascia Transversalis. - Lining the inner surface of the transversalis muscle and continued over the whole internal surface of the abdomen is a strong fascia, the fascia transversalis. Above, this fascia is thin and lines the abdominal surface of the diaphragm; below, and in front, especially in the inguinal region, it is thicker. Behind, upon the posterior wall of the abdomen, it covers the psoas and quadratus lumborum muscles, forming here the anterior layer of the lumbar fascia; it is also attached to the crest of the ilium and to Poupart's ligament except where the femoral vessels pass under Poupart's ligament into the thigh. It covers the psoas and iliacus muscles in the iliac fossa, where it is known as the fascia iliaca, and dips into the true pelvis, which it lines and is here called the pelvic fascia. All these fasciæ, although having different names, are simply parts of the general transversalis fascia.

The Parietal Peritoneum.-The whole interior of the abdominal cavity is lined by the parietal layer of the peritoneum. Between this parietal layer of the peritoneum and the transversalis fascia there is a layer of loose connective tissue which contains a considerable quantity of fat. This is the so-called subperitoneal connective tissue.

Through an incision in the anterior abdominal wall placed just to the left of the middle line, we may study the round ligament of the liver. This structure is the remains of the fœtal umbilical vein and reaches from the posterior aspect of the navel upward and to 
the right as far as the under surface of the liver. A fold of the parietal peritoneum, which is reflected from the anterior abdominal wall around the round ligament, is called the falciform ligament.

The presence of these structures would complicate somewhat entrance into the abdomen through an incision placed near the middle line upon the right side of the navel, and therefore when it is necessary to prolong an incision in the middle line either upward or downward beyond the navel one should pass to its left side.

Accompanying the round ligament of the liver from the region of the umbilicus are several veins (one, the largest, enters the portal system, and thus establishes a communication between the veins of the skin of the abdomen and the portal circulation); in the newborn infection may be carried from the region of the navel to the liver through this channel.

Reaching downward, in the middle line from the umbilicus to the summit of the bladder, is the urachus. This is a musculo-fibrous cord,- - the remains of the fotal allantois, - and may be found more or less pervious in the child or adult; so that a communication may thus exist between the umbilicus and the bladder. As the parietal peritoneum, which lines the posterior surface of the anterior abdominal wall, passes over the urachus, it is raised in the form of a distinct longitudinal fold: the plica vesico-umbilicalis media.

The Deep Vessels of the Abdominal Wall.-Between the layers of the muscles of the abdomen the deep vessels of the abdominal wall ramify. Above are found the terminal branches of the internal mammary, the superior epigastric, and the musculo-phrenic. The superior epigastric is continued from the thorax, through the opening in the diaphragm, between its costal and sternal portions, and then, piercing the posterior layer of the sheath of the rectus, it supplies this muscle and gives off branches which perforate the muscle and the anterior layer of its sheath to supply the subcutaneous tissue and skin of the abdomen. It anastomoses with branches of the superficial epigastric and deep (inferior) epigastric.

Below, the deep epigastric and the deep circumflex iliac, which are derived from the external iliac, are encountered; these are given off just before this vessel passes under Poupart's ligament into the thigh to become the femoral.

The deep epigastric is directed upward and inward toward the umbilicus, resting upon the posterior surface of the rectus, between the transversalis fascia and the parietal peritoneum, and 


$$
\text { . }
$$




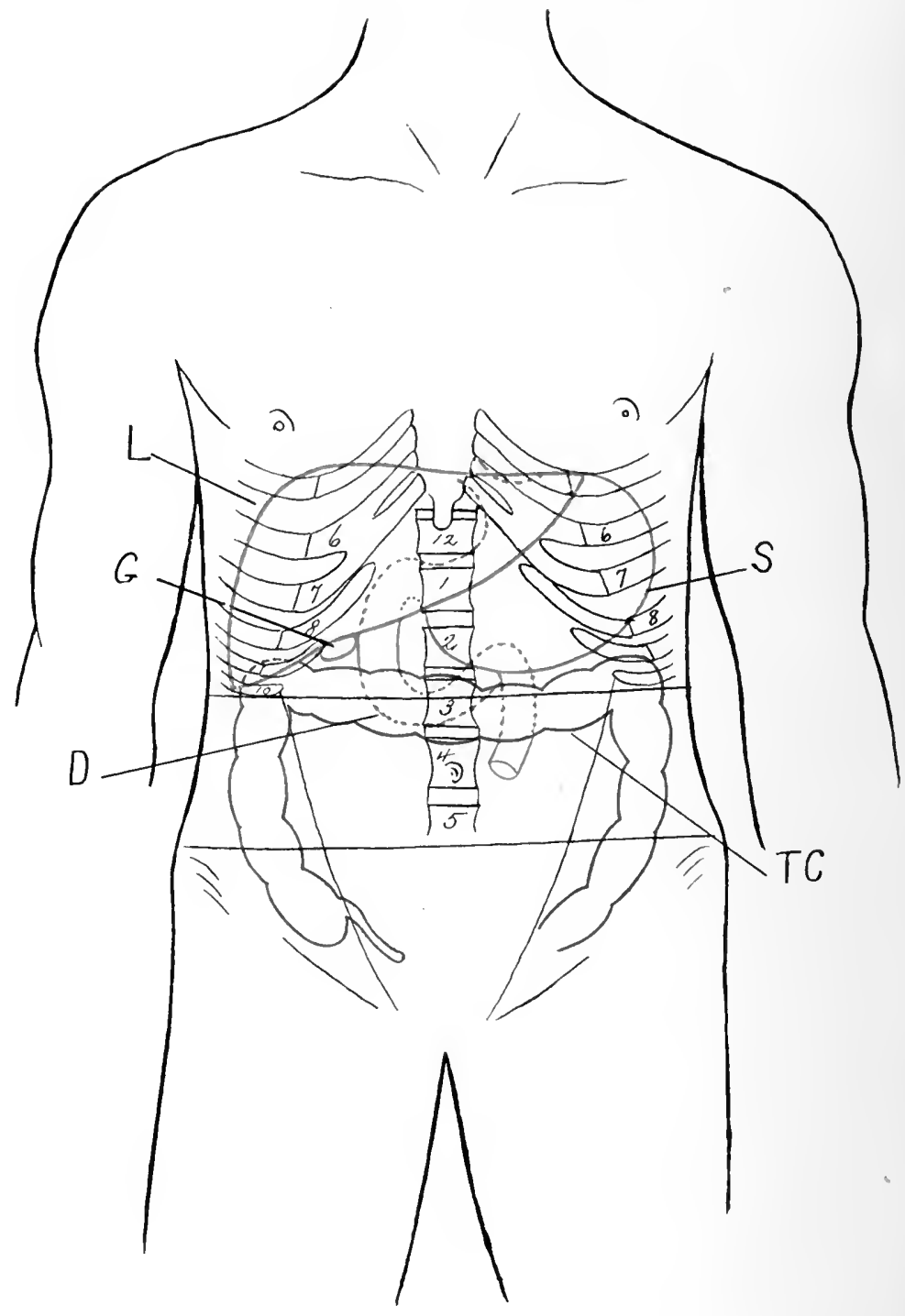

Fig. 89.-The Regions of the Abdomen as Indicated by Two Transverse Lines Drawn through the Tips of the Tenth Costal Cartilages and the Anterior Superior iliac Spines and Two Oblique Lines Drawn from the Tips of the Tenth Costal Cartilages down to the Pubic Spines. D, duodenum indicated in red (the dotted portion represents that portion of the duodenum which lies beneath the liver, transverse colon, and stomach); $G$, gall-bladder; $L$, liver; $S$, stomach ; $T C$, transverse colon. 
enters the substance of this muscle below the semilunar fold of Douglas, supplying it and anastomosing with the end branches of the superior epigastric. Some branches from this vessel pierce the anterior layer of the sheath of the rectus muscle and ramify in the fatty layer beneath the skin.

The deep circumflex iliac passes upward and outward beneath and parallel with Poupart's ligament toward the anterior superior iliac spine; it then runs along the crest of the ilium and after piercing the transversalis fascia is distributed to the muscles of the abdomen.

From behind come the abdominal branches of the lumbar arteries: usually four. They pass forward between the muscles and anastomose with the branches of the musculo-phrenic, superior epigastric, the deep epigastric, and the deep circumflex iliac. These arteries are all accompanied by their corresponding vein.

The Regions of the Abdomen.-The surface of the abdomen is marked off into areas by several lines which intersect each other. Two of these are transverse, the upper passing through the tips of the tenth ribs, the lower through the highest points of the iliac crests. These are crossed by two lines which pass from the tip of the tenth rib of either side downward and inward to the pubic spine.

Above the upper transverse line is the:-

(a) Regio epigastrica;

between the two transverse lines is the

(b) Regio mesogastrica;

and below the lower transverse line is the

(c) Regio hypogastrica.

The regio epigastrica is subdivided into three portions:-

1. Regio epigastrica proper.

2. Regio hypochondriaca dextra.

3. Regio hypochondriaca sinistra.

The regio mesogastrica is also subdivided into three portions:-

1. Regio umbilicalis.

2. Regio abdominis lateralis dextra.

3. Regio abdominis lateralis sinistra.

The regio hypogastrica is subdivided into three portions:-

1. Regio pubica.

2. Regio inguinalis dextra.

3. Regio inguinalis sinistra. 
THE BACK.

When we speak of the back we mean the whole posterior part of the trunk. The back may be divided into three regions: the dorsal, the lumbar, and the sacral.

It is better to consider the back as a whole, since these regions merge directly into each other without any definite dividing line.

Above the back is limited by the vertebra prominens and below by the tip of the coccyx. The dorsal portion corresponds to the chest, and includes the dorsal vertebræ and the ribs, the scapulæ and the muscles of this region. The lumbar portion forms the posterior wall of the abdominal cavity, and includes the five lumbar vertebræ and the thick mass of muscle on either side which fills in the space between the last rib and crest of the ilium.

The sacral region corresponds to the posterior wall of the true pelvic cavity and includes the sacrum and the coccyx.

In the middle of the back there is a longitudinal furrow in which the spinous processes of the vertebræ, from the seventh cervical, vertebra prominens, above, to the sacrum below, may be distinctly felt; those which correspond to the sacrum are less prominent.

To either side of this median furrow there is a prominent mass formed by the longitudinal muscles of the back. These masses extend from the sacrum to the occiput, and, the more pronounced they are, the deeper is the median groove.

In the dorsal region, on either side, are the scapulæ-shoulderblades. These bones are triangular in shape and are located between the first and eighth ribs toward the outer part of the thorax. The inner or vertebral border of the scapula is nearly parallel with the spinous processes of the vertebræ when the arm hangs by the side. This bone is freely movable and its position varies according to the position of the upper extremity. The spine of the scapula is felt beneath the skin and may be traced. outward and upward; its outer end, which is prolonged outward and flattened from above downward, is called the acromion process and overhangs the shoulderjoint, articulating with the outer end of the clavicle. The lower extremity of the scapula, the angle, corresponds to the eighth rib, and is a surgical landmark of some value.

The skin and subcutaneous connective tissue of the back is continuous with the corresponding layers of the adjoining parts of the trunk. The subcutaneous tissue is rather firm and fibrous and contains a varying amount of fatty tissue. The deep fascia of the 
back is a strong, dense, fibrous layer which covers in the superficial muscles; it is attached in the middle line to the spinous processes of the vertebræ and may be traced upward, upon the trapezius muscle, as far as the occipital bone, to which it is attached. In the dorsal region it is attached to the subcutaneous surface of the spine of the scapula. Below it is attached to the crest of the ilium and to the sacrum.

The Muscles of the Back are numerous and may be divided into several layers.

First Layer of Muscles.-Trapezius and latissimus dorsi.

The Trapezius is a broad, flat muscle, one on either side; together they are lozenge-shaped and occupy the dorsal and cervical regions. Each muscle arises from the superior curved line of the occipital bone, from the ligamentum nuchæ, which corresponds to the spinous processes of the cervical vertebræ, and from the spinous processes of all the dorsal vertebræ. From this extensive origin the muscle of each side is attached as follows: Those fibers which arise from the occipital bone pass downward, outward, and forward, and are attached to the upper surface of the outer one-third of the clavicle; those from the dorsal and cervical vertebræ converge and are attached to the whole length of the upper border of the spine of the scapula. That portion of the muscle which corresponds to the lower cervical and upper dorsal vertebræ shows an aponeurotic origin, which, together with that of the opposite side, is oval in shape.

The Latissimus Dorsi is broad, triangle-shaped, and flat, and occupies the lumbar and lower dorsal regions, being partly overlapped by the trapezius.

It arises by aponeurotic fibers from the spinous processes of the five or six lower dorsal and the lumbar vertebræ. Below the aponeurotic origin of the latissimus dorsi is intimately blended with the aponeurosis that covers the erector spinæ; the muscle also arises from the back part of the outer lip of the crest of the ilium and by three or four slips from the external surface of the lower ribs. From this extensive origin the fibers all converge, and at the angle of the scapula they form a thick, flat, fleshy muscle, which, making a halfturn upon itself, passes upward, in front of the teres major, and is attached by a narrow, flat, aponeurotic tendon to the inner lip of the bicipital groove of the humerus. The tendon of the latissimus dorsi and the teres major form the lower border of the posterior wall of the axilla. 
Second Layer of Muscles:

Levator anguli scapulæ.

Rhomboideus $\left\{\begin{array}{l}\text { Major. } \\ \text { Minor. }\end{array}\right.$

The Levator Anguli Scapulce is located in the side of the neck and the upper dorsal region. It arises by tendinous slips from the tubercles on the transverse processes of the four upper cervical vertebræ; passing down the side of the neck, it is attached to the upper part of the inner, or vertebral, border of the scapulæ.

The Rhomboids are two flat muscles placed one above the other, both lying upon the same plane and really forming one broad, flat muscle. Internally they are attached to the spinous processes of' the last cervical and four or five upper dorsal vertebræ, and externally to the vertebral border of the scapula.

Third LaYer of Muscles.-Splenius; serratus posticus, superior and inferior.

The Splenius is located in the back of the neck and upper dorsal region, reaching from the occiput downward as far as the sixth dorsal vertebræ below.

The Serratus Posticus.-The superior and inferior are two thin, flat muscles, the superior being located in the upper dorsal region, the inferior in the lower dorsal and lumbar regions.

The Muscles of the Fourth Layer are numerous and have a longitudinal direction, reaching upward, alongside of the spinal column, from the sacrum as far as the occiput. The muscles of this group, except the erector spinæ, are of but little importance surgically.

The Erector Spinæ below, in the lumbar region, forms a large musculo-tendinous mass, which fills in the space on either side of the lumbar part of the spinal column, being superimposed upon the quadratus lumborum in this region. From the lumbar region the erector spinæ is continued upward into the dorsal region. In the dorsal region this muscle divides into a number of processes, each of which receives a different name and is described as a separate muscle. The erector spinæ below, in the lumbar region, is covered by a dense aponeurotic structure: the posterior layer of the lumbar fascia. The muscle arises from the back part of the iliac crest and, through its aponeurosis, from the posterior surface of the sacrum and from the spinous processes of the lumbar and two or three lower 
dorsal vertebræ. The erector spinæ is included between the posterior and middle layers of the lumbar fascia. The quadratus lumborum lies beneath the erector spinæ.

In the lumbar region the erector spinæ forms a well-marked muscular mass, and its outer edge is an important guide in cutting down upon the kidney.

The Muscles of the Fifth Layer are numerous, and are made up, for the most part, of longitudinal strips that connect adjoining vertebræ to each other. They are all more or less continuous with each other, but receive different names in different regions. They are lodged in the groove upon either side of the spinous processes, and extend from the sacrum to the occiput.

The Quadratus Lumborum is really a muscle of the abdomen, forming part of its posterior wall; it is quadrilateral in shape, broad, and thick. It fills in the space on either side of the spinal column from the last rib to the crest of the ilium. It is broader below at its attachment to the crest of the ilium than above at its insertion into the last rib. Its outer border is free and lies more external than that of the erector spinæ, and forms an important surgical guide.

The muscle arises by aponeurotic fibers from the upper part of the ilio-lumbar ligament and from the adjacent part of the crest of the ilium for a distance of about two inches. From this origin the muscle passes upward and is inserted into the inner half of the lower border of the last rib and, by fleshy slips, to the transverse processes of the four upper lumbar vertebræ.

The muscle is inclosed between the middle and anterior layers of the lumbar fascia, and lies directly beneath the erector spinæ, from which it is separated by the middle layer of the lumbar fascia.

The Lumbar Fascia.- In the lumbar region there is a strong aponeurotic structure called the lumbar fascia; it is through this fascia that the transversalis muscle is connected with the spine. The lumbar fascia is usually described as consisting of three layers (see Fig. 87). The anterior layer is rather thin, covers the front surface of the quadratus lumborum muscle, and is attached internally to the anterior aspect of the transverse processes of the lumbar vertebræ; above, this layer of the fascia is attached to the lower border of the last rib, where it constitutes the ligamentum arcuatum externum. The middle layer of the lumbar fascia is strong, is attached to the apices of the transverse processes of the lumbar vertebræ, and is placed between the quadratus lumborum and erector 
spinæ muscles. The posterior layer of the lumbar fascia is attached to the apices of the spinous processes of the lumbar vertebræ; it forms the posterior aponeurotic covering of the erector spinæ, and is blended with the aponeurosis of origin of the latissimus dorsi. At the outer border of the quadratus lumborum the three layers of the lumbar fascia unite to form a single aponeurotic layer, through which the transversalis muscle is connected with the spinal column.

The Psoas and Iliacus Muscles.-In the back of the abdomen, lying one upon either side of the spinal column, is the psoas muscle. It arises by slips from the transverse processes and bodies of the last dorsal and the lumbar vertebræ, and passing downward joins with the iliacus.

The iliacus muscle occupies the iliac fossa, taking its origin there, and, together with the psoas, passes out of the abdomen under Poupart's ligament to be attached to the lesser trochanter of the femur and to the surface of the bone immediately below this.

The psoas and iliacus are covered by a fascia, the iliac fascia. This is simply a part of the general transversalis fascia of the abdomen. That part which covers the psoas muscle is thickened above, where it is known as the ligamentum arcuatum internum; laterally, beyond the edge of the psoas muscle, this fascia is continuous with that which covers the quadratus lumborum: the anterior layer of the lumbar fascia. The iliac fascia covers the iliacus muscle also, and is attached to the erest of the ilium and the brim of the pelvis, and to Poupart's ligament except where the femoral vessels pass down into the thigh. In this situation the fascia is continued downward, under Poupart's ligament, behind the vessels into the thigh, covering the anterior surface of the psoas-iliacus muscle.

The parietal peritoneum is spread out over the inner surface of the posterior wall of the abdomen. The kidney, incased in its capsule of fat, lies between this layer of the peritoneum and the faseia which covers the quadratus lumborum muscle.

The Spinal Column, etc.-The spinal column is made up of the vertebræ and intervertebral pads, the sacrum, and the coccyx; it is located at a considerable depth from the surface of the body. The spinal column gives solidity, combined with flexibility, to the trunk, and furnishes a canal to contain and protect the spinal cord.

We may palpate the body of the first cervical vertebra, the atlas, through the mouth, its anterior tubercle lying just behind the soft palate; those vertebræ which lie below this down as far as the fifth 
cervical may also be palpated through the mouth. Lower in the neck and in the dorsal region palpation of the bodies of the vertebræ is impossible. The bodies of the lumbar vertebræ can be felt through the abdomen, especially in thin persons. The sacrum and coccyx may be palpated through the rectum.

The laminæ meet behind, in the middle line, to form the spinous processes and inclose the canal which contains the spinal cord.

In the cervical and lumber regions the spaces between the laminæ are broad, and a knife-blade might easily be introduced through these into the spinal canal. This could not be so readily done in the dorsal region, however, where the laminæ and spines overlap each other like the shingles on a roof.

The spaces between the laminæ are occupied by the ligamenta subflava, which serve to complete the canal and even it out upon its inner aspect.

The bodies of the vertebræ are joined to each other by the anterior and posterior common ligaments; the posterior common ligament, besides connecting the bodies of the vertebræ with each other, also serves to even out the irregularities upon the internal aspect of the canal. The spines of the vertebræ are connected with each other by ligaments: the interspinous and the supraspinous.

The spinal column presents three curves in the sagittal direction, antero-posterior, and one lateral with the concavity toward the left (aorta).

Fractures of the spine usually involve the fifth and sixth cervical, last dorsal, and first lumbar vertebræ, and are usually caused by indirect violence, the curved parts of the spine being bent beyond the limit of their elasticity.

The spinal canal is widest in the neck and triangular upon section; narrower in the dorsal region and circular upon section. It is narrowest at the level of the ninth dorsal. From the eleventh dorsal it becomes wider again. In the sacrum it is flattened from before backward and terminates upon the posterior surface of this bone.

The spinal canal shows a series of openings-intervertebralupon either side, just behind the bodies, for the passage of nerves and vessels to and from the canal. The contents of the canal are well protected. It is an uncommon accident for an instrument to penetrate into the canal, and unusual force is required to injure the cord inclosed within these bony walls.

Contained within the canal is the spinal cord, which is much 
smaller and shorter than the canal; the spinal cord commences at the upper border of the posterior arch of the atlas, where it is continuous with the medulla, and terminates below in the conus terminalis on a level with the lower border of the first lumbar vertebra. From the conus terminalis the cord is prolonged still farther downward as the filum terminale.

The spinal cord, as it lies within the canal, is inclosed by the dura and pia mater. The dura mater is continuous with the dura mater, periosteum, of the skull, and is adherent to the margin of the foramen magnum. Here it splits into two layers, the external of which is applied to the inner aspect of the spinal canal as a lining membrane, periosteum, whereas the other, the inner layer, forms a loose, sack-like envelope for the cord, the dura mater proper, and is continued all the way down to the coccyx, where it is blended with the periosteum of that bone. Between these two layers there is a space in which veins and arteries ramify and into which hemorrhage may take place and spread up and down the canal. Each nerve, at its exit from the spinal canal, has prolonged upon it a tubular process, which is derived from the dura and pia mater.

Beneath this dura mater sheath is the pia mater, a reticular structure like that which invests the brain; the outer surface of the pia is known as the arachnoid, and the inner, which is applied directly to the surface of the cord, is known as the pia mater proper and carries the vessels which penetrate into the substance of the cord to supply it.

Between the two surfaces of the pia there is a space, which is called the subarachnoid space, and which is subdivided, cut up, by numerous trabeculæ into a net-work of small spaces. In the subarachnoid space, between the two layers of the pia, the cerebrospinal fluid is found. From the pia mater laterally, between the roots of the nerves, there arises a longitudinal septum which is attached to the inner surface of the dura mater by a number of processes. The line of origin from the pia is continuous. The line of attachment to the dura mater is interrupted. This is known as the ligamentum dentatum.

The surfaces of the dura and the pia mater (arachnoid) are not joined to each other except for occasional strands of connective tissue that unite them here and there. The space between the dura and pia mater is known as the subdural space. 
Each nerve-root is provided with an envelope consisting of a process of the pia and dura.

Arteries that supply the cord consist of branches from the vertebral, intercostals, lumbar, and lateral sacral; from above downward these vessels pass through the intervertebral foramina to supply the coverings and the cord.

Veins, in the form of plexuses, are found on the front and back of the cord, within the canal, between the two layers of the dura, or, better, between the dura proper and the periosteum.

\section{THE STOMACH.}

The Surgical Anatomy of the Stomach.-The stomach is a pearshaped, pouched portion of the alimentary canal with a capacity of from five to eight pints. It is suspended obliquely in the upper part of the abdomen, upon the left side, extending from the œsophagus to the duodenum. Its walls are thick, and consist of a serous, a muscular, a submucous, and a mucous membrane coat.

The larger end of the stomach, the cardiac, is above and toward the left side; the smaller end, the pyloric, is below and toward the right side.

The œsophageal opening is called the cardiac, and the opening into the duodenum, the pyloric, orifice. The dilated left end of the stomach-i.e., that part to the left of the osophageal opening-is called the fundus; the middle part, the body; and the right, rather constricted portion, the pylorus.

The stomach presents an upper or right border, the lesser curvature, and a lower or left border, the greater curvature. It has an anterior wall directed forward and upward and a posterior wall which is directed backward and downward.

The adult stomach, moderately distended, measures in its longest diameter from ten to twelve inches; from the greater to the lesser curvature, four to five inches; and from the anterior to the posterior wall about three and one-half inches. When the stomach is empty the first and second diameters are diminished and the third disappears, as the walls come into contact with each other. In this condition the mucous membrane lining is thrown into numerous folds and rugæ.

The opening between the pylorus and the duodenum is indicated by a well-marked thickening of the wall of the stomach, which may be felt from without; it is made up of circular muscular fibers, 
which act as a sphincter and which serve to shut off the cavity of the stomach from that of the duodenum.

The stomach lies in the left hypochondriac and the epigastric regions; about five-sixths part of the organ lies to the left of the middle line, the pyloric end lying to the right of the middle line. The cardiac orifice is located one inch below the diaphragm, to the left of the eleventh dorsal vertebra, and at a depth of $11 \mathrm{~cm}$. from the front wall of the abdomen, on a line directly behind the articulation of the seventh left costal cartilage with the sternum. The pyloric orifice lies to the right and a little below the ensiform cartilage and nearer the anterior wall of the abdomen. The direction of a line drawn from the cardiac orifice to the pyloric orifice of the stomach would be downward and to the right. The fundus of the stomach reaches upward as high as the level of the sixth costal cartilage, and is separated from the base of the left lung by the diaphragm.

The anterior surface of the stomach, toward the left, is in relation with the seventh, eighth, and ninth ribs, the diaphragm being interposed; toward the right, the pyloric end of the stomach is covered by the left lobe of the liver.

Below the stomach, along its great curvature and attached to it by the so-called gastro-colic ligament, is the transverse colon.

A triangular area of the anterior wall of the stomach-near the left free border of the ribs-is in direct relation with the anterior abdominal wall, and is here accessible for operation. The base of this triangular space is indicated by a transverse line, which corresponds to the transverse colon and greater curvature of the stomach and which is drawn through the tip of the ninth rib (costal cartilages) of either side. The other lines of the triangle are, upon the left, the free border of the ribs, and, upon the right side, a line corresponding to the anterior thin edge of the left lobe of the liver, which is drawn from the tip of the tenth right costal cartilage to the tip of the eighth left costal cartilage.

Behind the stomach lie the pancreas, with the splenic vessels passing along its upper border; the upper part of the left kidney and suprarenal capsule; and, toward the left, the spleen.

Behind the pyloric end of the stomach are the duodenum, portal vein and common bile-duct, the head of the pancreas and the first lumbar vertebra, the crura of the diaphragm, the aorta with the cœliac axis, the solar sympathetic plexus, the thoracic duct, vena cava inferior, etc. 
The spleen lies to the left of the stomach and rather behind it. The gall-bladder is in relation with the pyloric end of the stomach.

The stomach is entirely enveloped by the peritoneum, which forms its serous coat; above, extending between the transverse fis-

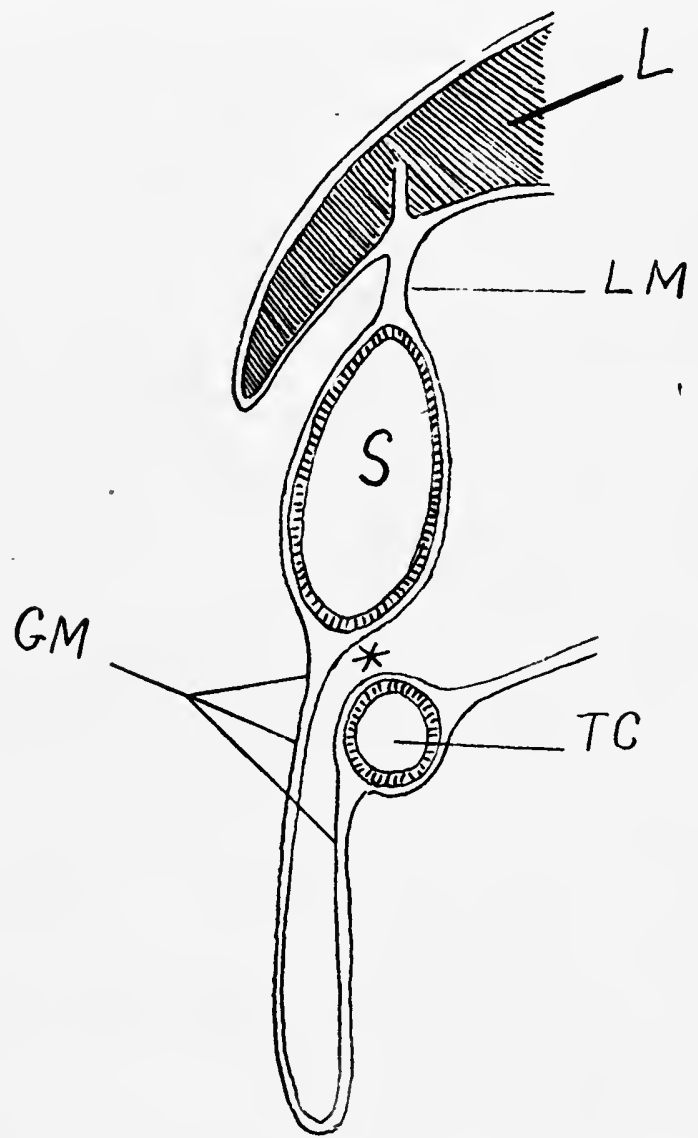

Fig. 90.-Sagittal Section to Show the Arrangement of the Great and Lesser Omenta, etc. $G M$, great omentum; $L$, liver; $L M$, lesser omentum; S, stomach; TC, transverse colon; *, situation where the layers of the great omentum become fused to that portion of the peritoneum which invests the transverse colon, thus joining the latter to the lower border of the stomach.

sure of the liver and the lesser curvature of the stomach, the two layers of the peritoneum join to form the lesser omentum, gastrohepatic ligament, between the layers of which, toward its right edge, the hepatic artery, portal vein, and common bile-duct are located. 
Below, at the greater curvature, the two layers of peritoneum, after enveloping the stomach, again join to form the great omentum through which the transverse colon is attached to the greater curvature of the stomach. That portion of the great omentum which joins the stomach and transverse colon is called the gastrocolic ligament. Toward the left, the two layers of peritoneum which cover the anterior and posterior surfaces of the stomach are also joined-gastro-splenic omentum-and are reflected over upon the spleen, inclosing this organ and connecting it with the fundus of the stomach. Between the layers of the gastro-splenic omentum the vasa brevia pass to the fundus of the stomach.

The arteries which supply the stomach are derived from the cœliac axis, and consist of large branches which course along the lesser and greater curvatures; these vessels give off large branches, which ramify upon the anterior and posterior walls of the stomach, coursing from the periphery toward the middle of each surface; along the lesser curvature, the pyloric artery, a branch of the hepatic, and the gastric artery anastomose; along the greater curvature, anastomosing with each other, are the gastro-epiploica dextra, from the hepatic, and the gastro-epiploica sinistra, from the splenic. The vasa brevia, from the splenic, ramify upon the left end, fundus, of the stomach.

The stomach may be reached through several incisions:-

1. In the linea alba, commencing one inch below the ensiform cartilage and reaching down to the umbilicus. After cutting through the skin, subcutaneous fat, and the strong fibrous layer, the linea alba, the parietal peritoneum is reached and incised. If necessary to prolong this incision beyond the navel, it should be carried to the left of that structure.

2. Von Hacker's incision: Through the left rectus muscle, one and one-fourth inches to the left of the linea alba, penetrating bluntly between the fleshy fibers of the rectus muscle with the handle of the knife.

3. Just to the left of the linea alba, through the inner portion of the rectus muscle, thus avoiding an incision through the linea alba.

4. Fenger's incision: One finger's breadth distant from and parallel with the free border of the ribs (left side). This incision may be two to three inches in length or longer, its upper end being placed a short distance from the tip of the ensiform cartilage. 


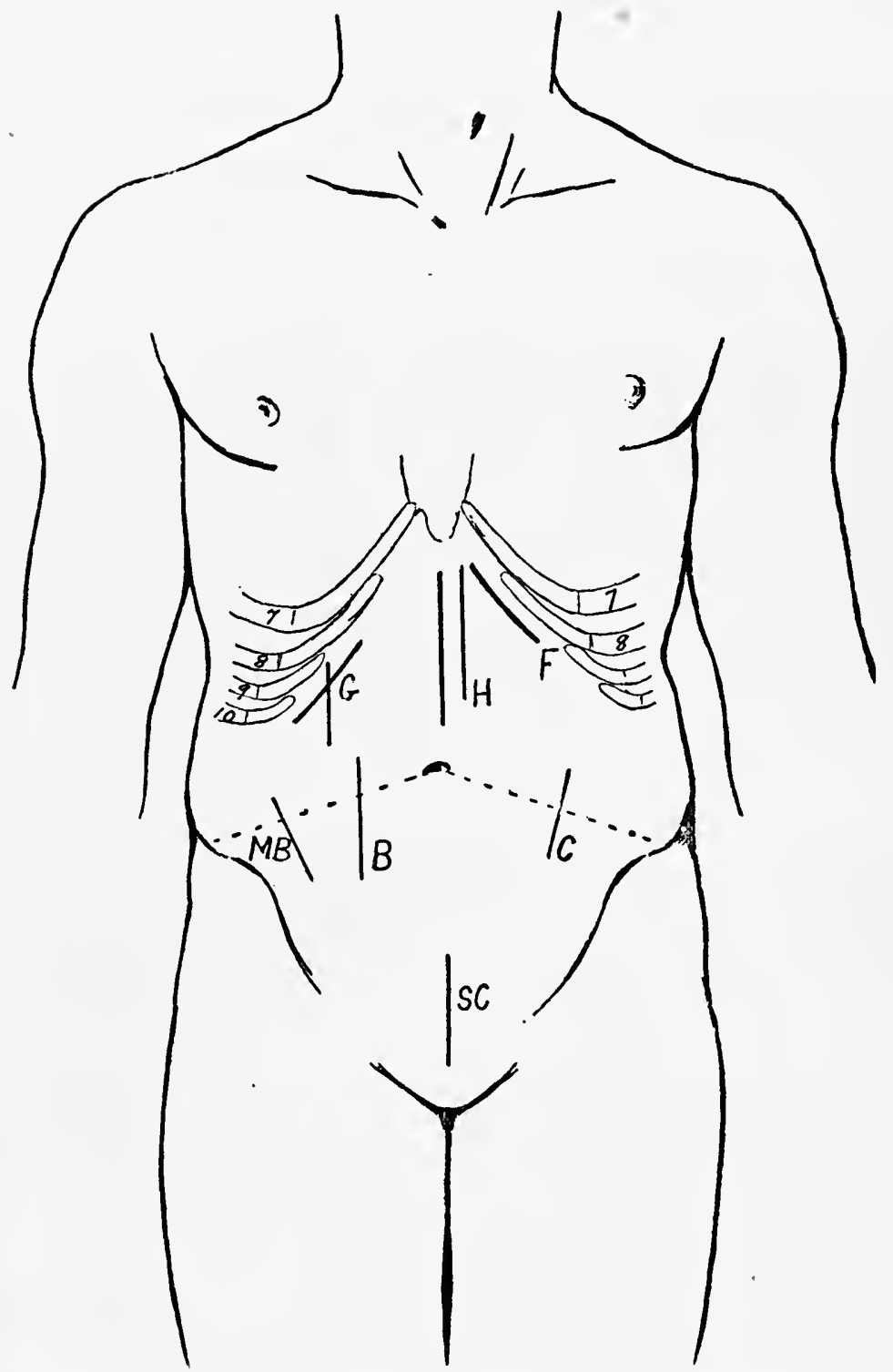

Fig. 91.-Incisions to Reach Abdominal Visccra, etc. $B$, Battle incision; $C$, left colostomy; $F$, Fenger incision for stomach; $G$, incisions for operations upon gall-biadder (perpendicular and oblique); $H$, von Hacker's incision for gastrostomy (in the middie line, aiongside of $H$, is the linea alba incision for operations upon stomach); $H B$, McBurney incision for appendicectomy; $S C$, incision for suprapubic cystotomy. 
Hemorrhage may be controlled by clamping vessels as they are met and cut, afterward ligating any that may require it.

\section{OPERATIONS UPON THE STOMACH.}

Gastroplication.-The folding in of a portion of the wall of the stomach in order to diminish the size of the organ. This operation was first performed by Bircher, and is especially applicable to cases of dilatation without stenosis of the pyloric orifice.

The abdominal incision, five to six inches in length, may be

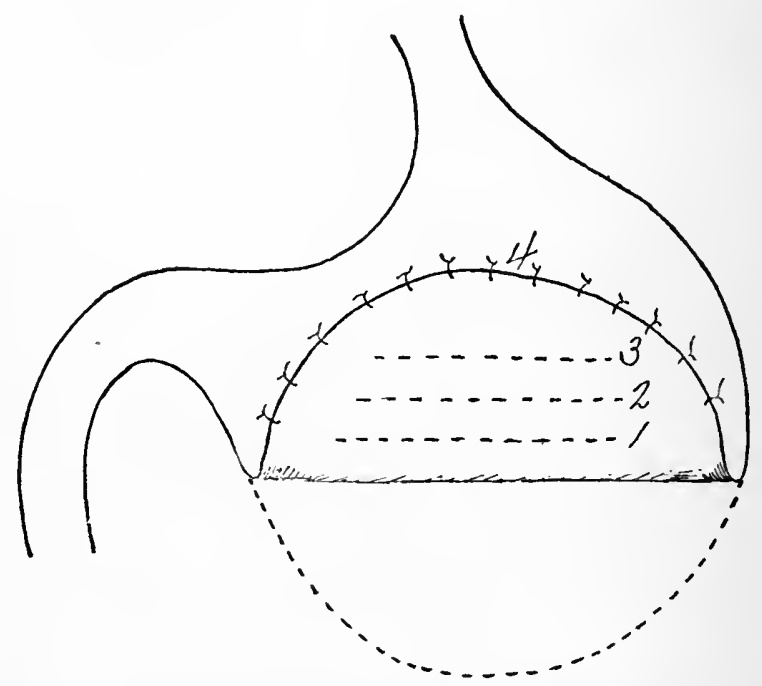

Fig. 92.-Gastroplication. Lower border of the stomach is turned up and stitched near the lesser curvature with a single row of sutures (4), method of Bircher; with four rows of sutures $(1,2,3,4)$, method of Weir.

placed a finger's breadth distant from and parallel with the left free border of the ribs, commencing above near the tip of the ensiform process, or it may be located in the linea alba, reaching from a point one inch below the tip of the ensiform process downward as far as the umbilicus.

Through either of these incisions the stomach may be brought out upon the abdominal wall.

According to Bircher, the anterior wall of the stomach is folded upon itself; so that the greater curvature may be brought up close to the lesser curvature and fixed in this position with a row of inter- 
rupted silk sutures; these should take a good, broad bite in the wall of the stomach, including its serous and muscular coats. Care should be exercised that the sutures do not penetrate through the entire thickness of the wall of the stomach. Twelve to fourteen sutures are usually required.

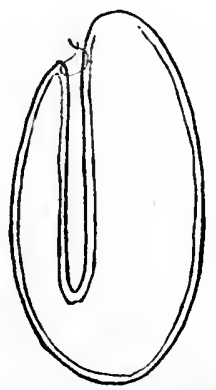

Fig. 93.-Cross Section of the Stomach After Gastroplication according to the Method of Bircher.

According to Weir, the fixation may be made with three or four separate tiers of sutures, one superimposed upon the other. After the stomach has been brought out through the abdominal incision, its anterior wall, corresponding to the long diameter of the organ,

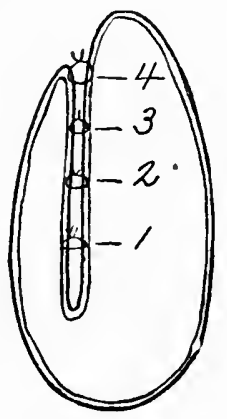

Fig. 94.-Cross Section of Stomach After Gastroplication; the Turned-Up Portion Fixed by Four Rows of Sutures. (Weir.)

is inverted, and the edges of the furrow thus made in the wall of the stomach united with a row of continuous or interrupted silk sutures. A second row of sutures is then introduced parallel with and about one inch distant from the first. A third and finally a 
fourth row may be introduced, the last row joining the greater curvature to the upper part of the anterior wall of the stomach near its lesser curvature. In this way six or eight inches of the stomach wall may be reefed in and the organ materially reduced in size. No doubt the folding of the stomach wall is made more secure when several rows of sutures are used.

Gastrotomy.-This operation consists in making an incision into the stomach for the purpose of extracting a foreign body lodged in the stomach or impacted low down in the osophagus; for exploration of the interior of the stomach, ulcer, hemorrhage, etc., and to treat strictures in the lower part of the œsophagus.

Immediately preceding any operation upon the stomach the organ should be emptied and irrigated, if the conditions permit, with the stomach tube. This is best done after the patient has been anæsthetized.

The incision may be made in the middle line through the linea alba, three inches long, commencing above about one inch below the ensiform process, and extending downward toward the umbilicus; or an incision may be made just to the left of the linea alba, passing through the inner margin of the left rectus muscle; or the Fenger incision, parallel with the free border of the left ribs, may be employed. This last incision (Fenger) is probably the best if the ultimate object is to reach the œsophagus.

Having carried the incision down to the parietal layer of the peritoneum, this is picked up with two toothed forceps and a small incision made between them with the knife; through this the finger is introduced, and upon the finger, with a blunt-pointed scissors, the opening in the peritoneum is enlarged so as to correspond in length with the incision in the abdominal wall. Two fingers are then introduced into the abdomen and the stomach searched for. If there is a foreign body in the stomach, this may oftentimes be felt and serves as a guide to the stomach. The thin anterior edge of the left lobe of the liver may be always readily recognized, and this is a good guide to the stomach, as the stomach lies directly underneath this organ, being partly covered by it; that part of the anterior surface of the stomach which is not covered by the liver is accessible for operation; it is seized with two fingers and drawn out of the abdominal incision. Pads of dry, sterile gauze are then placed about the stomach and tucked into the abdominal incision and the rest of the operation done extraperitoneally. If the stomach is dimin- 
ished in size there may be some difficulty in bringing it out through the incision upon the abdomen.

One should not mistake the transverse colon for the stomach. The transverse colon lies below and close to the greater curvature, being connected with the greater curvature by the great omentum (gastro-colic ligament); the great omentum is suspended free, apronlike, from the transverse colon, and when this part of the intestine is drawn out upon the abdomen the great omentum is drawn out with it; the colon can be further recognized by its sacculation, by the little fatty appendices attached to it, and by the striæ which run along its length.

The wall of the stomach is smooth, and the blood-vessels ramifying upon its surface have a characteristic course, converging from the periphery toward the center; the gastro-epiploica dextra and sinistra run along the greater curvature from either end of the stomach, anastomosing with each other.

A portion of the stomach wall which has been drawn out through the abdominal incision is now incised. When opened, care should be taken to catch any escaping contents, especially if the stomach has not been previously emptied, washed out, in order to prevent these entering the abdominal cavity.

The stomach is best incised in its long diameter, and the incision may vary from one to three inches. Bleeding vessels may be caught with artery forceps. Venous hemorrhage stops after the artery foreeps have been applied for a short time, but spurting arterial branches should be elamped and tied with either fine silk or fine catgut.

After the removal of the foreign body or examination of the interior of the stomach, either by the finger introduced or by inspection, bringing, piece by piece, different areas of its inner surface into the incision, treatment of ulcer by curette or Paquelin, etc., the opening may be closed.

The closure of the incision in the stomach is best effected by a continuous Lembert suture of fine silk, which is applied with a fine curved surgeon's needle. This suture includes the serous and muscular coats and takes a good bite, each loop being drawn fairly tight. This line of suture may be reinforeed by a second similar row of Lembert sutures which bury the first. Before closing the opening in the stomach wall its edges should be wiped with a wet bichloride pad. After the closure has been accomplished the parts should be 
again mopped off with the bichloride pad, followed by salt solution, and the stomach then returned into the abdomen.

The wound in the abdomen is closed first by a continuous catgut stitch which approximates the edges of the parietal peritoneum, and then a sufficient number of interrupted silk-worm sutures-each including the skin, aponeurosis, and muscle-are introduced.

If the stomach is opened for the purpose of treating a cicatricial stricture of the lower end of the œesophagus, the finger may be introduced through the incision in the stomach into the osophageal opening; at times it is necessary to make a little steady pressure with the finger before this opening yields so as to allow the finger to enter. Conical gum bougies of increasing caliber may then be introduced into the œsophagus beyond the stricture. If the stricture is dense and unyielding, one may, according to the method of Abbe, pass a thin bougie, carrying a strand of braided silk, up into the csophagus, through and beyond the stricture, so that the end of the bougie with the silk cord may be felt in the pharynx. The piece of silk is then grasped in the back of the pharynx through the mouth or through an opening which may be made for that purpose in the side of the neck and œsophagus, and the bougie again withdrawn through the opening in the stomach. The silk string having been thus carried through the œsophagus, a conical bougie is now again introduced into the osophagus from below through the opening in the stomach; this bougie should be large enough to become tightly engaged in the stricture; the ends of the silk string are then seized, and after this has been drawn back and forth several times it will be observed that the bougie can be passed farther and farther into the stricture; and one may thus use bougies of increasing caliber until the stricture is sufficiently relieved. The incision of the stricture which is made by the silk string is accomplished with but little hemorrhage. The bougie and string are withdrawn and a tube permitted to remain in the œsophagus, with its end projecting through the opening in the stomach and out through the abdominal incision.

Besides this a second tube is left in the stomach for the purpose of feeding. The abdominal incision is left partly open.

One may again repeat the procedures if necessary, after an interval of a few days.

The gastric fistula which results either closes spontaneously or may be closed by a secondary plastic operation. 
Pyloroplasty (Heinecke and Mikulicz).-For cicatricial stricture of the pylorus causing obstruction to the emptying of the stomach.

The stomach is exposed through a median incision and its pyloric end drawn out through the incision. Pads are then properly placed to protect the peritoneal cavity during the rest of the operation.

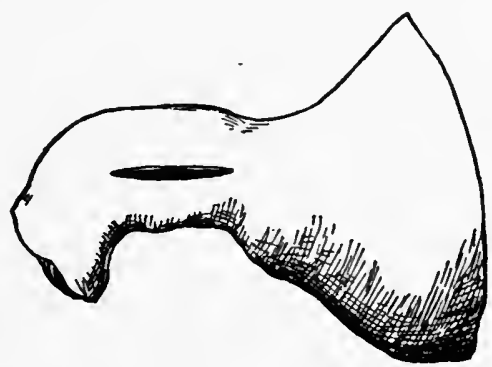

Flg. 95.-Pyloroplasty. Horizontal incision into the pylorus.

The pylorus is incised in its long axis, a clean cut being made through all its coats; this incision should be from 4 to $6 \mathrm{~cm}$. long; the edges of the incision are then drawn widely apart by tenacula in the middle of each edge, and in this way the transverse incision becomes converted into a vertical one. In this position, after sponging

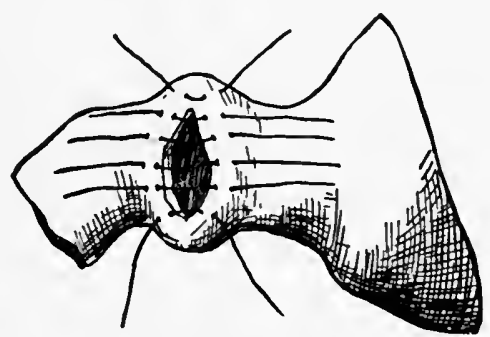

Fig. 96.-Pyloroplasty. Horizontal converted into a vertical incision and sutures placed.

its margins with a bichloride pad, the opening is closed by a row of interrupted Lembert sutures which take a good, deep, and broad bite, these being reinforced and buried by a second row of Lembert sutures, which may be continuous. All the sutures are of silk. Care should be taken to close the opening accurately, especially in the middle: the points which correspond to the extremities of the original incision. 
The result is a marked widening of the pyloric orifice. The wound in the abdomen is closed in the usual way.

Gastrostomy.-The formation of a permanent gastric fistula for the purpose of feeding in cases of simple or malignant stricture of the œsophagus. The fistula should permit the introduction of nutriment and at the same time prevent the escape of stomach contents.

Von HaCker's MethoD.-The operation may be done, if necessary, under cocain anæsthesia. This method is used only in advanced cases, where time presses.

The incision, three inches long, is made through the left rectus muscle; it should be placed about one and one-fourth inches to the left of the middle line, commencing above, about one inch below the free border of the ribs. After passing through the integument the anterior layer of the sheath of the rectus is reached and incised, and then, separating between the fibers of this muscle bluntly with the handle of the knife, the posterior layer of the sheath of the rectus is exposed; after this layer has been incised the parietal peritoneum is exposed; this is picked up with two toothed forceps and between these a small incision is made with the knife. Through this small opening the finger is introduced into the abdomen and the incision further enlarged with blunt-pointed scissors. Now, corresponding to the middle of the abdominal incision, upon each side, the parietal peritoneum is fixed to the edge of the muscle with a single catgut suture. Two fingers are then introduced into the abdomen and the anterior wall of the stomach is seized and drawn out of the wound. Two silk sling sutures are introduced into the wall of the stomach; these should take a good, broad bite in the wall of the stomach, but should not penetrate into its cavity, and should be placed about one and one-half inches apart and one above the other; they are simply to serve as tractors to steady the stomach in the wound, and should not be withdrawn until after the stomach has been opened. They are useful guides when the time comes later to incise the stomach.

Now, with a fairly large curved surgeon's needle a silk suture is passed through the edges of the upper part of the abdominal incision; this should include the whole thickness of the abdominal wall, care being taken not to omit the parietal peritoneum. This suture is not tied. A similar stitch is then passed through the lower end of the wound, and this is also left untied. Now, just below the upper suture, the first one introduced, another similar suture is 
passed, but this includes, in addition, the wall of the stomach: one should take a good, broad bite in the wall of the stomach, but without entering its cavity; this suture is placed just above the upper of the two sling tractor stitches; again, in the lower part of the wound, a stitch is taken similar to the preceding which likewise includes the wall of the stomach and lies just below the lower sling stitch. These four sutures are then tied and cut short, and the wound is thus partly closed above and below, and the stomach fixed at the same time to the edges of the incision by two good, firm sutures. The wall of the stomach is now further fixed to the edges of the incision by three or four additional silk stitches on each side; these may be of fine chromicized catgut or of fine silk.

The wound is then packed and dressed, and after the lapse of two days the stomach is opened between the two sling sutures which were left in situ. It is better to make this opening in the stomach with a sharp knife, clamping any bleeding points, rather than with a Paquelin, which causes a sloughy wound which, when it cicatrizes, may be larger than desired. The opening in the stomach should be one-half inch long, just large enough to adnit a tight-fitting tube. The sling stitches may then be withdrawn.

If one should desire to open the stomach immediately, which should be avoided if possible, the union of the stomach to the edges of the abdominal incision must be made more accurate in order to prevent possible leakage and peritoneal infection.

Method of Ssabanajew and Franck. - A very satisfactory operation. The incision (Fenger) should be placed parallel with the left free border of the ribs and should be not more than two inches long, commencing above to the side of the ensiform process. The middle of the incision should be opposite the tip of the cartilage of the eighth rib. The incision is continued down through the muscles and parietal peritoneum. The margins of the peritoneum are fixed to the edges of the muscles in the abdominal incision with one or two silk or catgut stitches on either side, near the middle. The anterior wall of the stomach, near the fundus, is then seized with two fingers, and drawn out of the wound in a cone-shaped process about one and one-half inches long and a silk sling suture passed through its apex to serve as a tractor. The base of this process of the stomach wall is fixed all around to the edges of the incision in the abdomen with a continuous silk stitch. This stitch should include the serous and muscular coats of the stomach and the edges of the parietal peritoneum 
and transversalis fascia and deep muscular layer in the abdominal ineision. 'They do not pass through the skin nor should they pass through the entire thickness of the stomach wall. After this step of the operation has been completed a second short incision about threefourths inch long is made through the integument, about one inch above and parallel with the first incision and just beyond the free border of the ribs. The bridge of integument that intervenes between this and the first incision is then raised bluntly with the handle of the

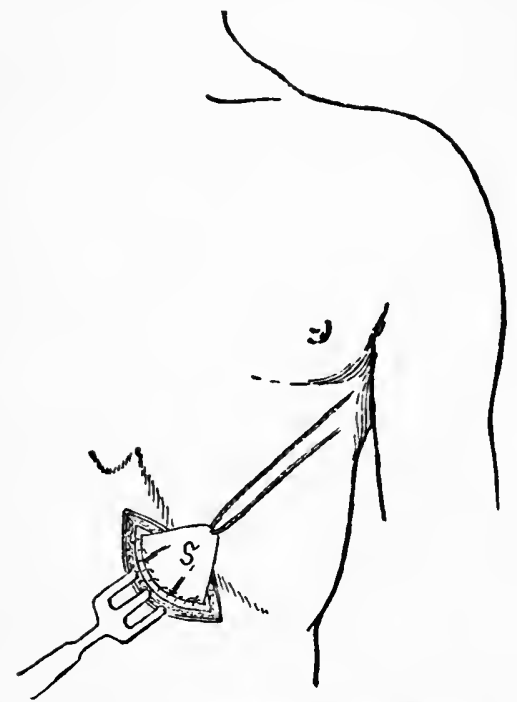

Fig. 97. - Gastrostomy (Ssabanajew$F^{\prime}(r, k)$. Cone-shaped process of the anterior wall of the stomach $(S)$ drawn out through abdominal incision with a silk tractor passed through Its apex, its base sutured all around to the edges of the parietal peritoneum and transversalis fascla, etc.

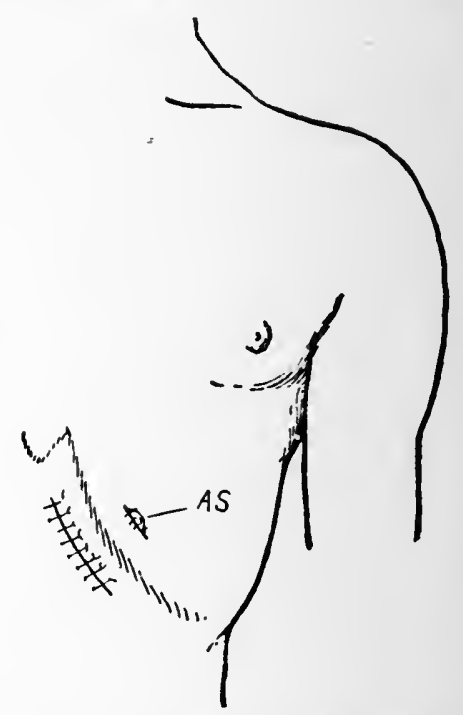

Fig. 98. - Gastrostomy (SsabanajevFranck). Apex of cone-shaped process (AS) sutured to the edges of second incision over the ribs.

knife, and, with the silk sling as a tractor, the apex of the cone-shaped process of the stomach wall is drawn through into the second small incision, where it is fixed with about four interupted silk sutures. The edges of the skin corresponding to the first incision are finally approximated with several interrupted silk-worm gut sutures, the conical process of the stomach wall being thus buried underneath the bridge of tissue between the two incisions. After the apex of the cone-shaped process of the stomach has been sutured to the second 
small incision, it may be opened and a tube introduced for the purpose of feeding. A fistulous tract about two inches long, leading into the stomach, is the result.

Witzel's Method, also a very satisfactory operation. An incision (Fenger) about two inches long is made through the integument, aponeurosis, and muscle down to the parietal peritoneum, which layer is then picked up with toothed forceps and incised.

Instead of cutting through the muscles of the abdominal wall one may, after the aponeurotic layer (sheath of the rectus) has been exposed and divided, separate bluntly between the fleshy fibers of the several museles, between those of the rectus in a vertical direction and those of the transversalis in a transverse direction; the muscles, having been thus separated, are drawn apart with broad retractors, and the peritoneum incised for a length corresponding to the length of the incision in the integument.

It is probably just as well in most cases to cut the muscles, as the separation between the fibers adds to the difficulty of the operation and consumes time, all to little or no purpose.

Instead of the Fenger incision as described above one may make a vertical incision (Mikulicz), 4 to $5 \mathrm{~cm}$. long, just to the left of the middle line, passing through the inner edge of the rectus muscle and located midway between the ensiform process and the umbilicus.

Whichever incision is employed, after the abdomen has been opened the parietal peritoneum is fixed to the edges of the incision with one or two catgut stitches on each side to prevent its retraction. These stitches fix the parietal peritoneum to the transversalis fascia and the deeper layer of muscles, but do not include the skin.

Two fingers are now introduced into the abdomen, and the anterior wall of the stomach seized and drawn out of the wound and surrounded with aseptic gauze pads, so that the succeding steps of the operation may be done extraperitoneally.

A No. $25 \mathrm{~F}$. soft rubber catheter is placed upon the surface of the stomach so that it is directed obliquely downward and toward the left, and in this position it is fixed with four interrupted silk sutures, which pick up the wall of the stomach on either side of the catheter, each taking a good, broad bite, but not penetrating through the entire thickness of the wall of the stomach. In this way the stomach wall is raised in a fold, or plait, upon each side of the tube; so that when the sutures are tied these two folds meet and completely bury the tube. Corresponding to the end of the catheter a very small 
opening is now made in the stomach wall with the point of the knife, and through this the end of the catheter is pushed so that about three inches of its length is within the stomach. The opening in the stomach should be so small that the tube will be a tight fit. The free end of the tube is closed with a ligature or forceps to prevent the escape of stomach contents. The four sutures which have been introduced across the tube into the stomach wall are now tied, and thus the tube is imbedded between the two folds, which form a canal about two inches long containing the tube. A sufficient number of additional silk sutures should be introduced to secure the accurate coaptation

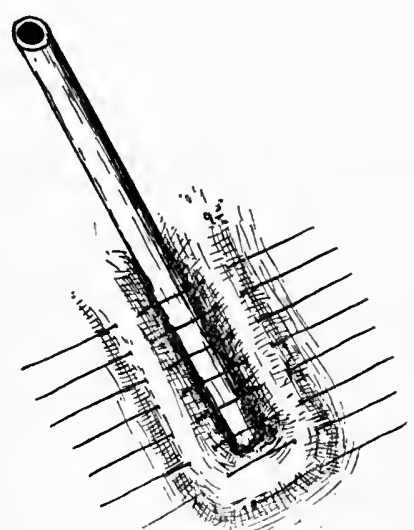

Fig. 99. - Gastrostomy (Witzel). The end of the tube is passed through a smali incision into the stomach, the wall of the stomach being raised up upon each side of the tube and sutures introduced.

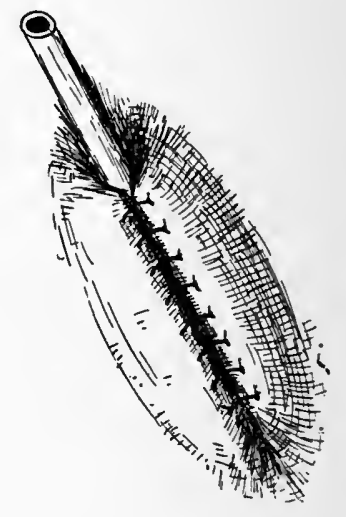

Fig. 100.-Gastrostomy (Witzel). The sutures tied, thus uniting the folds of the stomach to each othi $r$ and burying the tube.

of the two folds of the stomach wall over the tube, and at the point where the end of the tube enters the stomach the sutures should be extended a sufficient distance beyond to insure against leakage from the stomach around the tube. That part of the stomach wall underneath which the tube is buried and that immediately adjacent to the catheter as it emerges from the canal formed by the plaiting of the wall of the stomach should now be joined with interrupted silk sutures to the edges of the parietal peritoneum and transversalis fascia upon either side of the abdominal incision; these sutures should take a good, broad bite in the wall of the stomach, but should not pass through its entire thickness; they serve to fix that part of 
the wall of the stomach which is immediately adjacent to the tube to the parietal peritoneum.

The abdominal incision is then closed, except for a small portion above, just sulficient to allow the catheter to emerge, with several interrupted silk-worm gut sutures, each passing through all the layers of the abdomen, including the parietal peritoneum.

Gastrorrhaphy.-Suture of the wall of the stomach for perforation, stab, or gunshot wound.

An incision is made in the middle line, through the linea alba, and the stomach exposed.

If the wound in the stomach is small, it may be closed with a single row of interrupted Lembert sutures of fine silk. These should take a good, broad bite in the wall of the stomach, and should include the serous and muscular coats only; they should not pierce the entire thickness of the wall of the stomach or enter the mucous membrane layer.

If the wound in the stomach is large, this first row of interrupted Lembert sutures may be reinforced by a second continuous Lembert stitch, which will positively insure the accurate approximation of the serous surfaces and bury the first row of sutures.

Before applying the sutures the surface of the stomach immediately adjacent to the wound should be swabbed with a wet bichloride pad. If the peritoneum has become soiled by escaping stomach contents, it is well to thoroughly wash out the abdominal cavity with salt solution after the opening in the stomach has been closed.

Pylorectomy. - Resection of the pyloric end of the stomach; adapted to early cases of malignant disease of this part of the stomach where the neighboring organs, especially the pancreas and liver, are not involved. The stomach, immediately preceding the operation and after the patient has been anæsthetized, should be washed out through a stomach tube.

The incision is placed in the middle line and should be sufficiently large, 10 to $15 \mathrm{~cm}$., extending from the ensiform process down to the umbilicus or beyond this point. The pyloric end of the stomach is drawn into the wound and well surrounded with gauze pads so arranged as to protect the abdominal cavity during the operation, and the left lobe of the liver is held up out of the way by an assistant.

Billnoth's Metrod.-The first step in the operation is the isolation of the pylorus (diseased part, which is to be excised) from 
the greater omentum (transverse colon) below and from the lesser omentum above. With a blunt-pointed ligature carrier, armed with catgut or fine silk, the greater and lesser omenta, corresponding to the diseased pylorus, are transfixed and tied off in sections; the greater

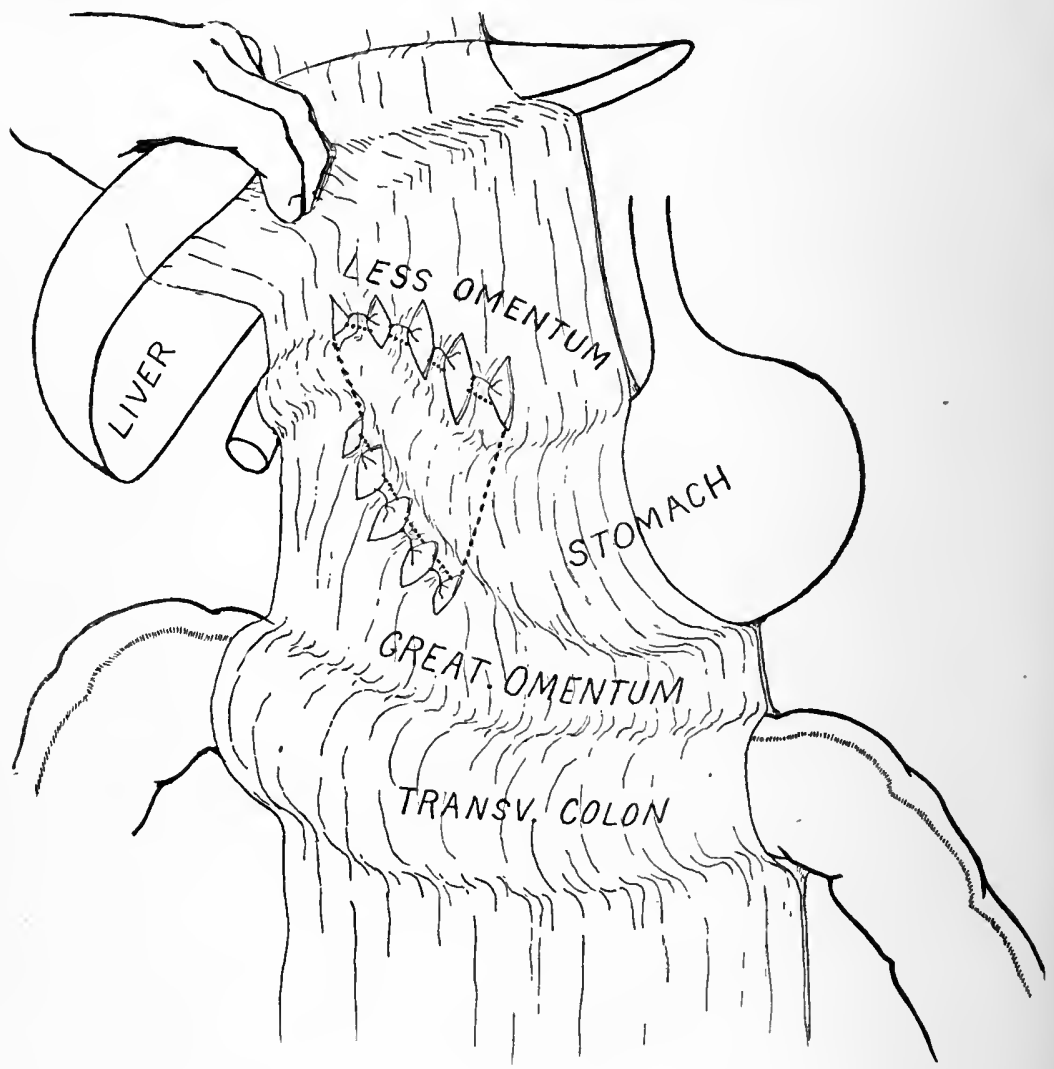

Fig. 101.-Pylorectomy. Anterior edge of the liver is lifted up; the lesser and greater omenta are shown. The lesser and greater omenta, correspondIng to the portion of the stomach that is to be excised, have been ligated in sectlons. The dotted lines indicate the line of section through the stomach and omenta. Instead of being applied as represented in this plcture, the ligatures may be placed double and the line of incision carried between them.

and lesser omenta are then cut away from the pylorus, cutting with the scissors between the ligatures and the pylorus. Each ligature should include one to one and one-half inches of the omentum. Instead of a ligature carrier a sharp-nosed artery forceps may be used 
to pass the ligatures. One should be mindful of the location of the common bile-duct and the portal vein in the free edge of the lesser omentum.

After the pylorus (tumor) has been thus entirely freed from its omental attachment above and below, it may be drawn pretty well out through the abdominal incision, so that the operation may be continued with more ease.

Before excising the pylorus (tumor) compression clamps are applied about the stomach and duodenum close to the tumor; ordinary long-limbed artery forceps will answer for this purpose. Two are applied to the stomach, close to the tumor, one reaching from the lesser currature and one from the greater curvature, and one is placed about the duodenum, also rather close to the tumor. The stornach is then seized by an assistant, who compresses it between the fingers of both hands, grasping it just beyond the place where the clamps are applied in order to prevent the escape of the stomach contents when it is cut, and then the operator, with a sharp scissors, cuts across the stomach from above downward, between the fingers of the assistant and the clamps.

The stump of the pylorus is now enveloped in a compress and turned to one side, the clamps preventing any leakage. The hemorrhage from the cut edge of the stomach is controlled by catching the bleeding points with forceps; any spurting arterial points should be ligated with fine silk; the hemorrhage from the divided veins ceases when the suture is applied.

The opening in the stomach is closed, commencing above and working downward toward the greater curvature, first with a continuous stitch of silk, which includes all the coats of the stomach and which is applied with a long, straight needle. Each loop of the suture is drawn fairly tight. The lower part of the opening in the stomach is left unclosed for a sufficient length to allow the insertion, later, of the stump of the duodenum. A second continuous Lembert stitch is then introduced, which inverts and buries the first line of suture. Through the opening left below some strips of iodoform gauze are packed into the stomach to prevent leakage, and then, enveloped in a compress, it is temporarily laid aside.

The attention of the operator is now directed to the duodenum. Its contents are stripped along with the finger, and a compressor applied, or it may be surrounded by a strip of gauze which is tied moderately tight to prevent the escape of its contents when it is 
cut. If the gauze strip is used, it may be carricd around the duodenum in the mouth of a sharp-nosed artery forceps, which is thrust through its mesentery. Instead of a compressor or strip of gauze the duodenum may be compressed between the fingers of an assistant; but the assistant's fingers occupy considerable space, and may thus interfere with the work of the operator.

The duodenum is divided a short distance beyond the clamp which had been applied to it, and the resection of the pylorus is thus accomplished. Any escaping contents from the end of the duodenum should be caught upon a gauze pad, and the edge of the duodenum wiped off with a wad of wet bichloride gauze.

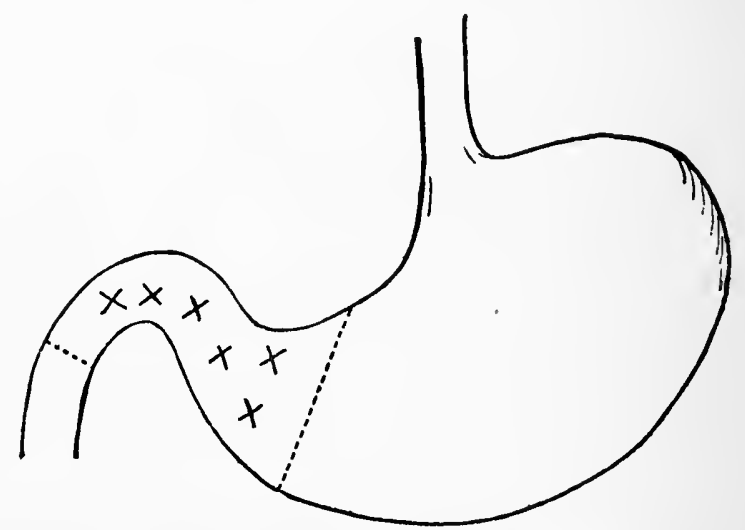

Fig. 102.-Pylorectomy (Billroth). Dotted lines indicate lines of section in excising diseased pyiorus. $\times \times \times$ represent diseased portion that is to be excised.

The protecting gauze pads are now renewed, the gauze removed from the opening remaining in the stomach, and the end of the duodenum sutured into this opening. There is first applied, with a curved surgeon's needle, a continuous silk suture; this should be applied from within the gut so that the raw edges present toward the interior of the gut. This line of suture should include all the coats, take a good bite, and be drawn fairly tight. When this suture is completed, it is reinforced by a second row of outside sutures, uninterrupted and of fine silk, which includes only the serous and muscular coats and buries the first line of suture. Special care should be taken to secure the point where the stomach suture meets the suture that unites the stomach and the duodenum. 
With the Murphy Button.-The junction of the end of the duodenum and the stomach may be accomplished by means of a Murphy button. The opening left in the end of the stomach, after the pylorus has been cut away, is closed throughout its entire length, and a junction then made between the stump of the duodenum and a new opening, which is made in the posterior wall of the stomach about one inch away from its sutured edge.

The end of the stomach and the end of the duodenum may be both closed completely by inversion and suture, and a regular gastrojejunostomy then performed with or without a Murphy button or with McGraw's rubber suture or with the Laplace or O'Hara forceps, etc.

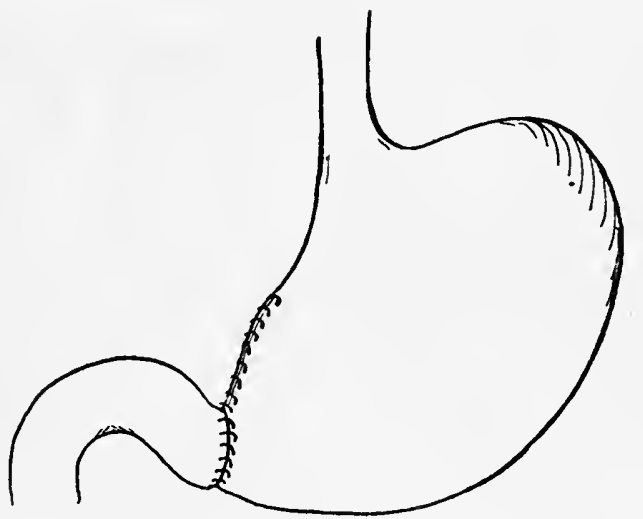

Fig. 103.-Pylorectomy (Billroth). Diseased portion bas been excised and the end of the duodenum sutured to the end of the stomach.

In excising the diseased pylorus one should not go too far along on the duodenum, as the second part of this segment of the gut is only partly invested with peritoneum, and is therefore unfavorable for union with the stomach.

Method of Kocher (Resection of the Prlorus, with GastroDuodenostomy).-Marked success in Kocher's hands.

The pyloric tumor is exposed through a long median incision and then isolated from its omental attachments as described above. After the pyloric tumor has been thus isolated compresses are packed about it to protect the abdominal cavity, and clamps applied: ordinary long-limbed artery clamps. Upon the stomach side of the tumor-in order to include the whole width of the stomach-two clamps are necessary: one reaching from above downward and the 
other from below. Upon the duodenum, to the distal side of the tumor, a clamp is likewise applied; parallel with this, but farther along, a second clamp is applied to the duodenum.

The duodenum is now divided with sharp scissors, between the two clamps, but not too close to the distal clamp, in order to leave room enough for necessary manipulation in suturing, etc. The cut edge of the duodenum, protruding between the limbs of the clamp, is wiped clean of intestinal contents with a wet bichloride pad and then enveloped in sterile gauze, and with the clamp still applied it is turned to the right and held against the right edge of the abdominal incision by an assistant. The clamp which compresses the stump of the duodenum should not be too tight, although Kocher says that it does not damage the gut, that the gut still bleeds from its edge after the clamp is removed.

We then turn our attention to the stomach. The stomach is seized between the fingers of both hands by an assistant, and the pyloric portion cut away in a direction from above downward, between the clamps and the assistant's fingers, and removed. The tumor mass, being tightly clamped at both ends, when cut away does not leak. Bleeding, spurting points in the cut edge of the stomach are clamped and tied with fine silk; oozing and venous hemorrhage stop when the suture is applied.

The margins of the wound in the stomach are now wiped off with a wet bichloride pad and brought together throughout their entire length with a continuous silk stitch in a long, straight needle. This stitch includes all the coats, and takes a good bite; this sutured edge is then again wiped off with a wet bichloride pad and a fine, continuous Lembert stitch is applied, inverting the raw edges of the stomach wound and completely burying the first suture.

The protecting abdominal pads are now again renewed, and we may proceed with the last step of the operation: the union of the stump of the duodenum to the posterior wall of the stomach.

The assistant, still holding the stomach, directs its sutured end forward out of the wound; so that its posterior surface looks toward the duodenum, which is held over against the right edge of the abdominal incision. The cut end of the duodenum, with the compressor clamp still applied, is joined to the posterior wall of the stomach by a continuous stitch of fine silk, which is applied with a cambric needle and which corresponds to the posterior half of the circumference of the duodenum as it protrudes from the clamp. This 
suture catches the duodenum just beyond its cut edge. This stitch does not pass through the entire thickness of the duodenum, but catches only the serous and muscular coats of the duodenum and the corresponding coats of the stomach. It forms the posterior half of the "outside serous ring suture." This needle, still threaded, is then laid aside until it is wanted later to complete this "outside serous ring suture." The end of the duodenum is sutured to the posterior wall of the stomach about one inch distant from the sutured edge of the latter.

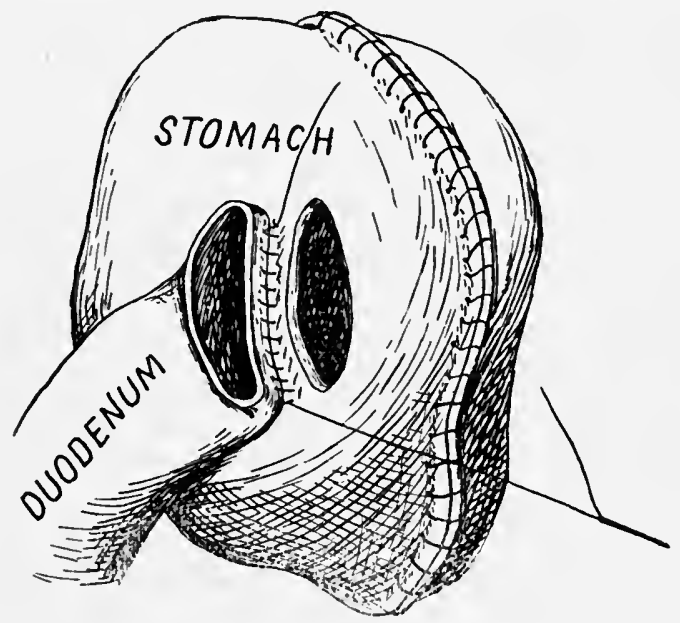

Fig. 104.-Pylorectomy (Kocher). The end of the duodenum has been sutured to the edge of the opening made in the fosterior wall of the stomach with a continuous non-penetrating stitch.

The clamp is now removed from the duodenum, the hemorrhage controlled, and any escaping contents sponged away, finally wiping the margin with a wet bichloride pad. An opening is then made in the stomach of a size to correspond with the end of the duodenum. This should be placed one-fourth inch from the line of suture by which the duodenum is already joined to the wall of the stomach. The edge of the duodenum stump is then sutured all around to the edges of the opening in the stomach, with a curved needle and silk, this being a continuous stitch applied from within, and including the whole thickness of the wall of each organ and taking a good bite, so that the raw edges of the parts look inward toward the lumen of the gut. When the parts have been thus united all around, the original needle is again taken in hand, and the Lembert suture which forms 
the anterior half of the "outside serous ring" applied and the union is complete. Before joining the serous coats the parts should be swabbed with a bichloride pad.

The protecting pads are now removed and the abdominal incision closed. With a continuous catgut stitch the edges of the peritoneum are coapted, and with several interrupted silk-worm gut sutureswhich pass through the skin, muscle, and aponeurosis-the closure of the abdominal wound is completed.

Gastrectomy.-Extirpation of the entire stomach. First case by Schlatter, 189\%. A healthy heart is essential to the success of this operation. The operating room should be kept warm and the patient dressed in flannel garments to prevent as much as possible loss of body-heat by radiation. The stomach should be washed out immediately before the operation is commenced, after the patient has been anæsthetized.

The incision is best made in the linea alba, and must be liberal, -from six to seven inches in length,-reaching from the ensiform process to the umbilicus or even beyond this point.

After the abdomen has been opened the stomach is recognized and examined, and search made for secondary deposits in the liver and adjoining lymphatic glands. In many cases the stomach can be drawn almost entirely out of the abdomen, and thus the performance of the operation is facilitated.

The first step consists in the isolation of the stomach, freeing it from the greater and lesser omenta and from its attachment to the spleen: gastro-splenic omentum.

Commencing at the pyloric end of the stomach, the omenta are tied off in sections,-first the lesser and then the greater omentum,-each ligature being tied double and including about one and one-half inches of the omentum. In ligating the lesser omentum the liver must be drawn up out of the way and the stomach pulled down. The presence of the common bile-duct between the layers of the lesser omentum, near its free right border, should not be forgotten. After the lesser and greater omenta have been ligated as far as the middle of the stomach and divided, the section may be made between the pylorus and duodenum, in order that the stomach may the better be drawn down, so as to make the isolation of its cardiac end less difficult, or else one may wait until the whole length of the lesser and greater omenta has been ligated and cut away from the stomach before the division is made at its duodenal end. The 
division of the omentum should be made betwêen the double ligatures with the scissors, cutting from one ligature hole into the next.

After the stomach has been freed of its omentum, along the lesser and greater curvatures, we are ready for the next step of the operation: the removal of the stomach. The stomach is divided first at its pyloric end, if this has not already been done. An intestinal clamp is placed about the duodenum, about one and one-half inches from the pylorus, and a clamp about the pyloric end of the stomach, and between these the intestine is divided with the scissors. Any escaping contents are caught upon a pad, and the end of the duodenum, sterilized and wrapped in gauze, and with the compressor still applied, is dropped temporarily into the abdomen.

A ligature is then thrown around the gastro-splenic omentum; this is the peritoneal fold that reaches from the fundus of the stomach to the spleen, and through it the vasa brevia pass to the stomach.

This ligature is applied double, that we may divide between them. Special pains should be taken to secure the vessels in the gastro-splenic omentum, leaving the ligature long that the pedicle may be drawn forward, so that, if necessary, the vessels may be secured with additional ligatures. .

To reach the csophagus the stomach must be pulled well downward. An intestinal compressor is placed about the œsophagus a short distance below the diaphragm, and a clamp about the œsophageal end of the stomach, and then between these the cesophagus is divided with the scissors. The stomach is thus removed.

After the stomach has been excised it becomes necessary to restore the continuity of the alimentary canal, either by joining the end of the duodenum to the œsophagus, œsophago-duodenostomy, or else by inserting the end of the œsophagus into the jejunum, œsophago-enterostomy.

In most cases the œsophagus can be drawn down and the duodenum sufficiently mobilized to allow of its being brought up into apposition with the end of the œsophagus without tension. In this case the parts may be joined with a Murphy button No. 3 (see "End-to-End Anastomosis"). After the button has been inserted the compression clamps may be removed from the duodenum and œsophagus, and a row of outside Lembert sutures applied to make the junction still more secure. These sutures include the serous and muscular coats, but do not pass through the mucous membrane. If unable to approximate the parts as described, the end of 
the duodenum may be inverted and closed with a double row of sutures and an œsophago-enterostomy done, the end of the œsoph-

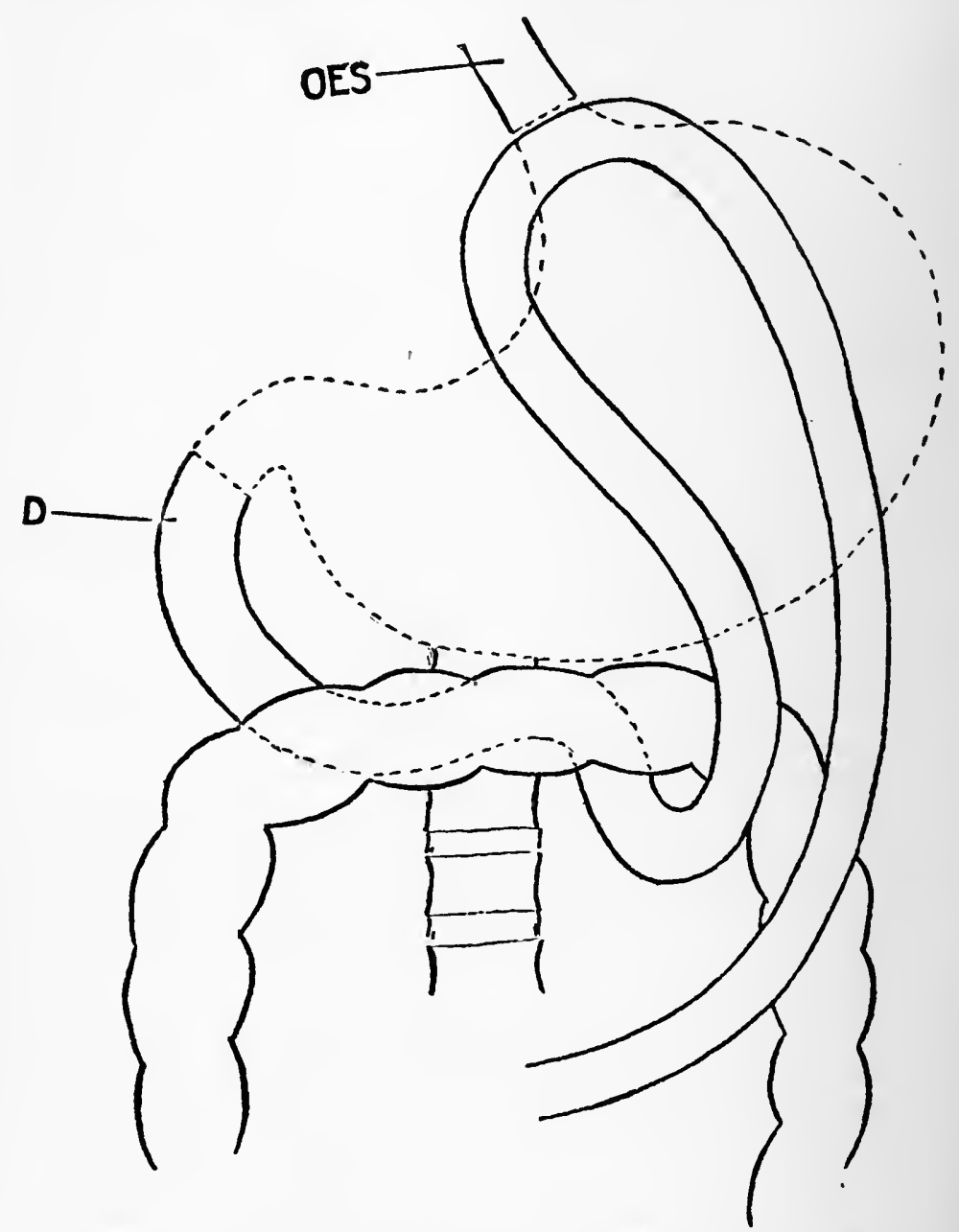

Fig. 105.-Gastrectomy. OES, stump of csophagus; $D$, end of the duodenum. Dotted iines indicate the excised stomach. The smali intestine (jejunum) has been drawn up into apposition with the stump of the cesophagus, as in œsophago-enterostomy.

agus being sutured into an opening which is made in the small intestine. The upper part of the jejunum is sought in the upper back part of the abdominal cavity,- to the left of the body of the second 
lumbar vertebra,-and a coil of gut about eighteen inches beyond this point selected. A segment of this coil of gut about six inches long is tied off with tapes, first one and then the other, after the contents of the segment have been stripped along with the fingers. This segment of gut is then brought up, around the transverse colon, into apposition with the end of the œsophagus.

The posterior half of the circumference of the end of the œsophagus is sutured to the wall of the coil of gut with a row of continuous Lembert sutures. These sutures secure the wall of the œsophagus about one-fourth inch beyond its cut edge, and include the serous and muscular coats, but not the mucous. This needle is then discarded temporarily, and an incision is made in the gut corresponding in length to the size of the opening in the osophagus. The edge of this opening in the gut is sutured to the edge of the œsophagus all around with a continuous silk stitch that includes all the layers. When this suture has been completed and the end of the œosophagus thus securely fixed to the opening in the intestine, the first needle, that with which the posterior half of the end of the oesophagus was joined to the gut, is again taken in hand and the anterior half of the "outside serous ring" suture applied. It is well to use silk exclusively for both sutures and ligatures in this operation. The abdominal wound is closed without drainage.

During the course of the operation the solar plexus may be considerably molested, and at the time that the œsophagus is severed both pneumogastric nerves are also divided. The shock is therefore apt to be marked, and should be counteracted by avoiding as much as possible loss of body-heat and by administering proper stimulation. The division of the pneumogastrics leads to disturbance of the heart's action; it becomes very greatly accelerated, and an attempt should be made to regulate this, probably with proper doses of digitalis hypodermically. For the first few days the patient is nourished per rectum; after forty-eight hours fluids may be given per mouth, first small quantities of water and then broth, milk, etc., may be added. At the end of a weck a moderate amount of solid food may be taken through the mouth.

\section{THE SMALL INTESTINE.}

The Surgical Anatomy of the Small Intestine. The Duodenum is the first part of the small intestine. It is about ten inches long and commences at the pyloric end of the stomach and ends at the 
jejunum. Its wall is moderately thick. It is usually described as consisting of three parts.

The first, or ascending, part is freely movable, continuous with the pylorus, and entirely invested by peritoneum. It passes from the pyloric end of the stomach upward and backward toward the right as high as the level of the twelfth dorsal vertebra; it reaches close to the under surface of the liver, with which it is connected by the so-called ligamentum hepatico-duodenale. This ligament is simply the free, thickened, right edge of the lesser omentum: ligamentum gastro-hepaticum. Between the layers of this lesser omentum are the hepatic artery, portal vein, and common bile-duct, the artery ascending to the liver, and the duct and vein descending behind this first part of the duodenum. Between the layers of the lesser omentum the artery lies to the left, the duct to the right, and the vein between and behind both.

The duodenum then makes a turn downward along the right side of the first and second lumbar vertebræ, lying upon the front of the right kidney, with the head of the pancreas to the left (i.e., internal to this part of the duodenum). This is called the second part of the duodenum. It differs from the first part in being fixed to the posterior wall of the abdomen and in not being completely surrounded by peritoneum, but simply covered by the peritoneum upon its front surface, and therefore when we look for this part of the duodenum, after reflecting the transverse colon and the great omentum upware, it is not to be seen, and is only exposed to view after the peritoneum which covers its anterior surface has been cut through. The common bile-duct and the pancreatic duct open into the second part of the duodenum, between it and the head of the pancreas. These ducts pass obliquely through the wall of the duodenum, and join with each other, before entering the gut, through a single common orifice, which is found upon the inner wall of the duodenum in the center of a papilla. A sound may be passed from this part of the duodenum into the common duct or into the pancreatic duct.

Between the head of the pancreas and this part of the duodenum in the injected cadaver there may be seen the anastomosis between the superior and inferior pancreatico-duodenalis arteries: branches derived from the hepatic and superior mesenteric, respectively.

At the level of the third lumbar vertebra the duodenum makes 
another turn, passing across the body of the third lumbar from the right to the left side of this vertebra, and at the same time ascending to the level of the second lumbar, vertebra. This is known as the third part of the duodenum. The aorta, etc., lie behind this part of the duodenum, and the head of the pancreas lies above it.

Upon the left side of the second lumbar vertebra the duodenum is fixed to the vertebral column by a thickened portion of peritoneum; this fold contains some unstriped muscular fibers, and is called the suspensory ligament of the duodenum, the ligament of Treitz. This third part of the duodenum is also covered upon its anterior surface only by the peritoneum, and is fixed by this layer in the back of the abdomen in common with the pancreas. This portion of the duodenum is not to be seen when we examine this part of the abdomen until after the layer of peritoneum which covers its anterior surface and conceals it from view has been cut.

The whole duodenum, in its curved course, resembles a horseshoe in the hollow of which the head of the pancreas is received.

The second and third parts of the duodenum are rather unfavorable parts for operation, on account of their fixedness and depth and the incompleteness of their peritoneal covering.

The Jejunum and Ileum, about twenty feet long, make up the rest of the tube of small intestine, and are the direct continuation of the duodenum, terminating in the cæcum in the right iliac fossa.

Upon the left side of the second lumbar vertebra, where the duodenum ends and the jejunum begins, the intestinal canal becomes again provided with a complete peritoneal investment and a long mesentery, and is known as the jejunum.

The jejunum forms about two-fifths of the length of the small intestine, and becomes the ileum where the valvulæ conniventes, which characterize its inner surface, cease to exist. It is thick walled and large in caliber, and therefore resembles somewhat the large intestine; still, it is readily distinguished from this part of the gut by the absence of the longitudinal striæ and appendices epiploicæ and in not being sacculated.

At its commencement, upon the left side of the second lumbar vertebra, the jejunum seems to project directly forward, through the parietal peritoneum which lines the back of the abdominal cavity. This appearance is due to the fact that the portion of the gut, duodenum, which immediately precedes the jejunum, is not pro- 
vided with a mesentery, lying behind the peritoneum and covered by it upon its anterior surface only, whereas the commencement of the jejunum and the rest of the small intestine are provided with an investment of peritoneum, which completely surrounds them, and a mesentery, which suspends them to the back of the abdomen, and, therefore, where this arrangement commences, the gut appears to project directly forward through the peritoneum from the posterior wall of the abdomen. The process of peritoneum that incloses the first part of the jejunum marks the commencement of the mesentery.

We can locate this first portion of the jejunum by reflecting the great omentum, and with it the transverse colon, upward out of the way, and then, passing the hand back to the vertebral column, this coil of intestine is found lying just to the left of the body of the second lumbar vertebra. An attempt to draw this coil of gut out of the abdomen will show that it is fixed within the abdomen, and this fact will serve to identify it positively.

The ileum, which is the continuation of the jejunum, constitutes three-fifths of the length of the small intestine. It becomes progressively smaller in caliber and thinner as we trace it toward its termination at the cæcum, where its wall is thinnest and its caliber narrowest.

The jejunum and ileum are suspended free in the abdominal cavity arranged coil upon coil, and are provided with a complete peritoneal envelope and a long mesentery, through which they are attached to the vertebral column in the back of the abdomen.

The Mesentery is a reflection of peritoneum containing some unstriped muscular fiber, fat, etc.; it serves to suspend the gut in the abdomen and at the same time supports the blood-vessels, lymphatics, nerves, etc., in their course to and from the small intestine.

The mesentery is fan-shaped. The distal border is very long, corresponding to the whole length of the small intestine, to which it is attached; the proximal border is short and is fixed to the anterior surface of the vertebral column, reaching from the left side of the second lumbar vertebra, where the duodenum ends and the jejunum commences, downward, to the right side of the fifth lumbar vertebra; its line of attachment is thus oblique from the left side, above, downward and to the right. The vertebral edge of the mesentery is but six inches long, whereas the distal, intestinal edge is about twenty feet long, and in order to accomodate these two borders to 
each other the intestinal end of the mesentery is folded and folded upon itself, making a series of plaits.

Where the two layers of peritoneum of which the mesentery is composed meet the intestine, they diverge and surround the intestine in a sling-like fashion, the intestine being entirely invested except for the small "dead" space which corresponds to the separation of the layers of the mesentery at the so-called mesenteric border of the intestine. Here the mesentery is not applied directly to the surface of the intestine, but is separated from it, leaving a small space- "dead space"-where the serous layer does not form part of the wall of the intestinal tube.

The Blood-supply of the Small Intestine is furnished by the superior mesenteric artery. This vessel is given off from the anterior aspect of the aorta, and passes forward between the lower border of the pancreas and third part of the duodenum; it is located between the layers of the mesentery, and courses, in a curved direction downward and to the right, toward the right iliac fossa. The superior mesenteric is a short, thick trunk. From its convex side it gives off branches to supply the whole length of the small intestine; from its concave side it gives off branches to the large intestine, to the cæcum and vermiform appendix, ascending colon, and transverse colon, finally anastomosing with a branch from the inferior mesenteric (see below). The superior mesenteric vein accompanies the artery and its branches, and behind the pancreas joins with the splenic to form the portal vein. The blood in the portal vein is derived from the intestine; before reaching the general circulation it passes through the liver; it leaves the liver through the hepatic veins, two or three in number, which empty into the inferior vena cava.

The branches of the superior mesenteric, which supply the small intestine, are given off, as already mentioned, from the convex, left, side of the artery. These branches do not pass direct to the intestine, but anastomose with each other, forming a series of arches. From this set of arches another series of branches is given off, and thus this peculiar anastomotic arch formation continues until the intestine is almost reached; finally the individual branches from the ultimate arches are distributed to the wall of the intestine. They pass to the intestine from between the layers of the mesentery, where these separate to envelop the intestinethat is, at the mesenteric border-through the so-called "dead space." After the ultimate vascular branches reach the wall of the 
gut they do not communicate freely with each other, so that each scgment of gut is dependent almost entirely upon one or two definite vessels for its nutrition and integrity. The same arrangement holds good for the ultimate veins. Therefore, if several of these ultimate vascular branches are severed close to the gut or become embolized or thrombosed, we are apt to have, as a result, gangrene of the corresponding segment of the gut. Wounds of the intestine at the mesenteric border are unfavorable for suture on account of the absence of the serous, peritoneal covering, at this part. Wounds at the mesenteric border of the gut almost of necessity include division of the ultimate intestinal arteries and veins; and therefore interfere seriously with the blood-supply to the corresponding part of the gut.

\section{OPERATIONS UPON THE SMALL INTESTINE.}

Enterorrhaphy.-Suture of the intestine for gunshot and stab wounds and for perforations due to ulceration, etc.

These injuries are frequently accompanied by hemorrhage from wounded vessels in the mesentery. These vessels should be ligated with catgut. If large, and especially if divided close to the gut, it is well, after ligating the bleeding vessels, to resect the corresponding segment of the gut, as such injuries are very apt to be followed by gangrene of that part of gut which is dependent for its supply upon the injured vessels.

The incision for injuries of this character is usually made in the middle line, four to five inches long, reaching from the umbilicus downward toward the symphysis. The incision may be prolonged upward toward the ensiform cartilage, passing to the left of the umbilicus. The operator should avoid laying the abdomen open from the ensiform cartilage down to the symphysis pubis in the eagerness of his search for wounds in the gut. If it becomes necessary to increase the length of the incision very much, that portion of it which is not in immediate use may be brought together temporarily with a fow interrupted silk sutures which pierce the whole thickness of the abdominal wall.

Having opened the abdomen, one should make a systematic examination of the intestine from one end to the other, commencing at the lowest part of the ileum, where it enters the cæcum. This part of the gut should be sought and drawn out upon the abdomen, and from this point on the small intestine and mesentery should be 
carefully inspected, coil after coil being drawn out and examined and then replaced until the upper end is reached.

As a rule, penetrating gunshot and stab wounds of the abdomen are accompanied by multiple perforations of the gut and mesentery, - may be as many as fifteen or twenty, -and, when one perforation in the gut is located, usually a second is found in the same segment at a corresponding point opposite. Each time a projectile passes through the gut it makes two wounds-one of entrance and one of exit.

Where we locate a perforation of the gut we usually find the mucous membrane protruding and tending to plug up the opening, nature's effort. Here we pause, replace the mucous membrane, wipe off the margins of the opening with alcohol followed by a weak bichloride solution, and close it with two or three interrupted Lembert sutures of fine silk; these sutures should be placed about one-eighth inch apart, and care should be taken to invert the edges of the wound and to bring the serous surfaces into close apposition.

In suturing these wounds the caliber of the gut should not be reduced more than one-third.

We then continue along in the search for further wounds. Wounds involving the mesenteric border of the gut, especially if the adjoining mesentery is torn, are unfavorable for suture; in the first place, the serous coat on this part of the gut is imperfect, has a "dead space"; and, in the second place, if any of the mesenteric vessels are divided close to the gut, the corresponding segment of the gut is apt to become gangrenous; therefore it is wise, in many cases, to resect such a segment of gut at once.

Bleeding vessels in the mesentery should be clamped and tied with plain catgut.

After the whole length of the small intestine has been explored one should examine the whole length of the large intestine, the stomach, and the bladder for perforations, and look further for hemorrhage, which might indicate wounds of the liver, spleen, kidneys, etc.

Hemorrhagic oozing from the solid viscera is usually readily controlled by the Paquelin cautery or by packing, or the edges of a gaping wound may be brought together with several deep catgut sutures, although these tend to tear through if any tension exists. Any spurting vessels in the solid viscera should be clamped and tied with catgut. 
Having thus completed the examination of the entire length of the alimentary canal, etc., closed all wounds, and controlled the hemorrhage, the whole abdominal cavity may be flushed out with hot saline solution, using a considerable quantity - best poured from a pitcher.

During the search for wounds, etc., one should replace the gut, coil after coil, as fast as it is examined. While the intestine is outside the abdomen it should be carefully protected with hot sterile towels, which may be wet in hot saline solution. After a time the wet cloths, if not repeatedly wet with hot water, become cooled; therefore some surgeons prefer dry sterile compresses for this purpose.

If it is necessary to have a considerable portion of the length of the gut outside upon the abdomen, it should be supported so that it does not drag upon the mesentery; this should be avoided, however, as much as possible, as it adds greatly to the shock and there may be some difficulty experienced in returning the distended coils of gut into the abdomen again.

If, owing to the distension of the guts with gas, it becomes difficult to replace them within the abdomen, it may be necessary to make punctures to allow the gas to escape. In doing this it is probably better to make a few rather large openings with a fairly large aspirating needle or a scalpel to allow gas to escape, closing them afterward with a Lembert stitch; this plan is probably better than numerous small punctures made with a fine instrument.

The abdominal incision should be carefully closed, first sewing the edges of the parietal peritoneum together with a continuous No. 3 catgut suture; then, with a sufficient number of interrupted silkworm sutures, the edges of the skin and aponeurosis are brought together, each stitch including all the layers of the abdominal wall except the parietal peritoneum.

Enterectomy.-Resection of a portion of the gut (small intestine); the length of gut resected may vary from several inches to several feet. The operation is performed for wounds which may not be safely closed by suture; for those associated with division of the mesenteric vessels, especially if they are divided close to the intestine; for malignant growths; for gangrene, strangulation; for fistula, etc.

The incision is usually made in the middle line, four or five inches long, reaching from the umbilicus downward toward the symphysis. The portion of intestine to be resected should be gently 
freed from adhesions, if there are any, and brought out upon the abdomen, together with an adjoining portion of healthy gut, four to six inches to either side of the part which is to be resected; the gut should be supported upon dry, sterile gauze compresses, some of which are also packed into the abdominal incision to protect the peritoneal cavity.

In order to prevent the escape of intestinal contents during the operation, two gauze strips may be tied around the gut, one beyond each extremity of the segment which is to be excised. An assistant may compress the gut between his fingers or temporary intestinal clamps may be applied, but the gauze strips are probably more convenient. In order to carry the gauze strips around the gut, a thin-nosed artery forceps is thrust through the mesentery close to the gut, and with this the end of the gauze strip is seized and pulled through. One strip is tied and the contents of the gut gently stroked along with the fingers beyond the second strip, and then this is tied also. We have thus a fairly empty coil to operate upon, the strips

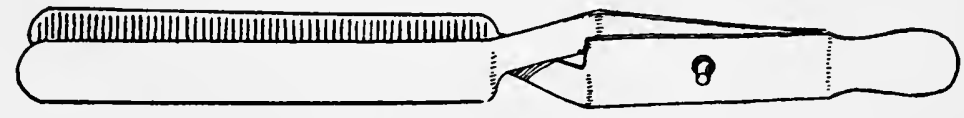

Fig. 106.-Intestine Compressor.

being tied just tight enough to prevent the re-entrance of contents. The strips should be applied to the gut at a sufficient distance beyond the portion which is to be excised to allow convenient working space.

We then proceed to separate the portion of gut that is to be excised from its mesenteric attachment. This is done by tying the mesentery off in segments, each ligature including about one inch of the length of the mesentery; the ligatures should be of thin catgut (No. 1 or 2), and each tied single about one inch away from the mesenteric edge of the gut. These ligatures may be passed either with a narrow-bladed artery forceps or a blunt ligature carrier. One must be careful not to tie off a greater length of mesentery than that which actually corresponds to the segment of gut which is to be excised, because gut which has been deprived of its mesentery is deprived of its blood-supply and is bound to slough. One should rather err in the other direction, tying off a little less mesentery than that which corresponds to the length of gut to be excised. After the mesentery has been thus tied off the segment of gut that 
is to be excised is cut away from its mesenteric attachment, using the straight scissors and cutting between the ligatures and the gut; the point of the scissors should be introduced into the openings made
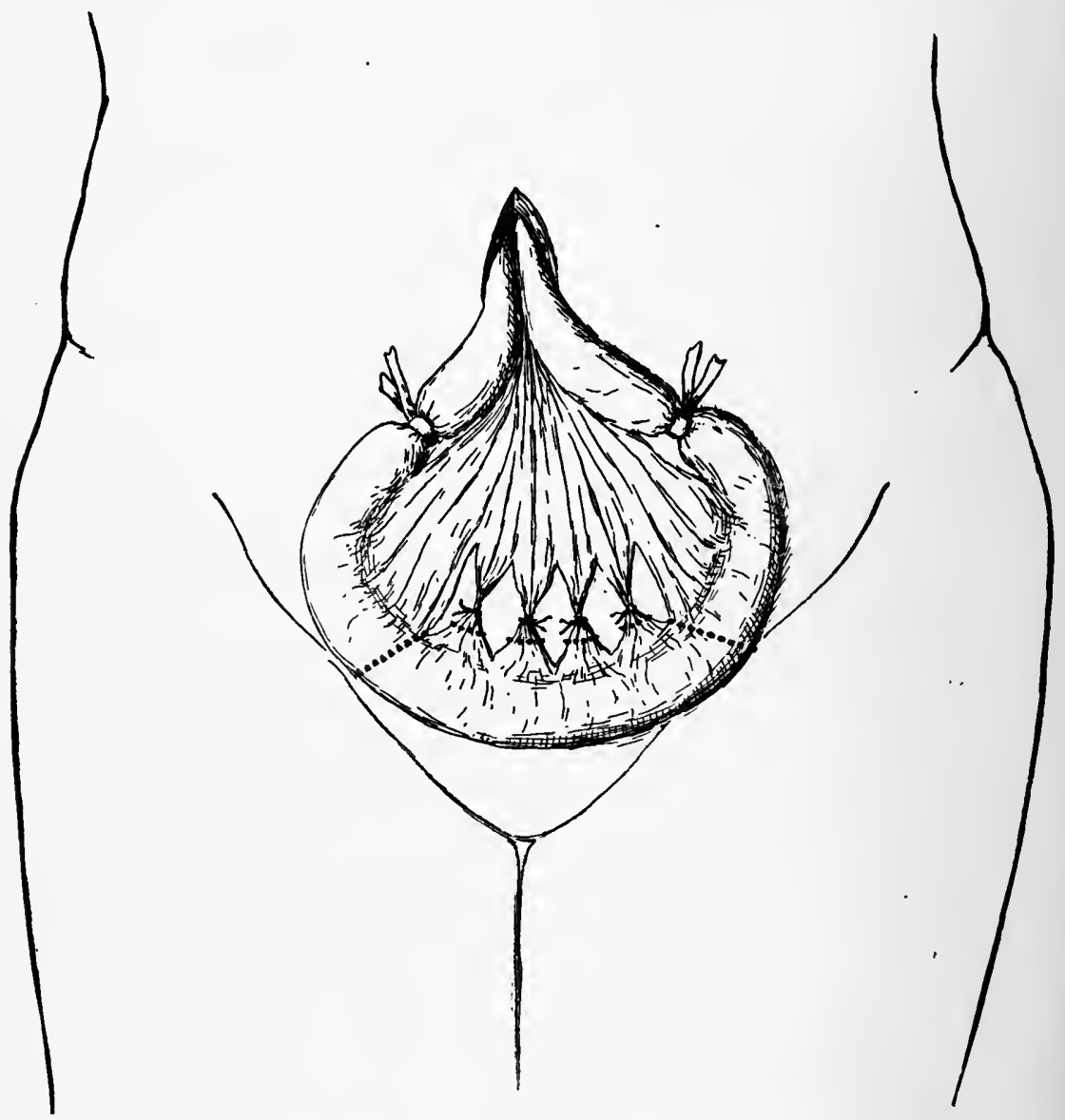

Fig. 107.-Enterectomy. A loop of intestine has been drawn out through the abdominal incision and tied off with tapes. The mesentery corresponding to the portion of gut that is to be excised has been tied off in sections. The dotted lines indicate the lines of section through the mesentery and gut.

by the ligatures, and the mesentery cut from hole to hole, and thus finally through into the last ligature opening. We are now ready to sever the gut, and this is done with long, straight scissors that will divide the gut in one clean sweep. The gut should be divided 
straight across at right angles to its long axis or, better, somewhat obliquely, so that the segment of gut excised measures rather more upon its distal border than upon its mesenteric border. Bleeding points on the cut edges of the intestine should be clamped, but, as a rule, these do not require ligation, since after a few moments' pressure they usually stop, especially after the ends of the gut have been united. Spurting points, however, should be clamped and tied with fine catgut. Contents that escape from the ends of the bowel as the section is made should be sponged away, and care should be taken that the pads of gauze are so arranged as to prevent the entrance of any of this material into the abdominal cavity.

We are now ready to restore the continuity of the intestinal canal. This may be accomplished by:-

1. End-to-end anastomosis, the most desirable.

(a) Suture.

(b) Invagination and suture (Mounsell).

(c) Murphy button.

(d) Laplace anastomosis forceps.

(e) O'Hara anastomosis forceps.

2. Side-to-side, or lateral, anastomosis; applicable to both small and large intestine.

(a) Suture.

(b) Murphy button.

(c) McGraw's rubber ligature.

(d) Laplace anastomosis forceps.

(e) O'Hara anastomosis forceps.

3. End to side; this method is used to join the ileum to the large intestine (see "Resection of Cxcum") and to join the end of the duodenum to the stomach after pylorectomy (see "Pylorectomy, Kocher").

End-to-End Anastomosis. Suture.-The ends of the intestine, after being cleansed and swabbed off with a bichloride pad, are joined together all around with a continuous suture. This suture commences at the mesenteric border of the gut and unites the two segments of the gut end to end all around. This suture is continuous, and may be of fine silk or catgut (No. 2). It is applied with a curved surgeon's needle, sewing from within, so that the resulting suture line presents into the lumen of the gut. This suture includes all the layers of the wall of the gut, should take a good, broad bite, and each loop should be drawn fairly tight. 
In beginning the suture, near the mesenteric border of the gut, special care is needed, in passing the first stitch, to include the serous coat in the bite of the needle, as it is in this situation that the mesentery splits to invest the intestine and is here not applied close down upon the muscular coat of the intestine; therefore, unless special pains are taken to include the serous coat in the stitch, this will be a weak spot, slow to heal, and may allow leakage.

In uniting the two ends of the gut in this manner the last few stitches must be applied interrupted, but they should be tied so that the joined edges of the gut will be inverted and look inward into the lumen of the gut, and the knots present upon the inner aspect of

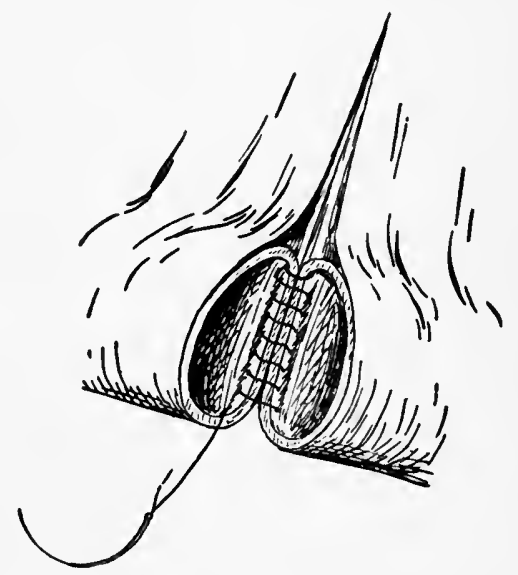

Fig. 108.-End-to-End Anastomosis. Gut joined end to end by a continuous stitch introduced from within the gut and penetrating all the layers (Woelfer). Corresponding to the mesenteric border of the gut there may be observed the "dead space" left by the diverging layers of the mesentery.

the united intestine. The knots of the last one or two stitches will, of necessity, have to be on the outside of the gut, but this will not prevent the edges of the gut being properly inverted. As before noted, especial care is required, in applying this stitch to that part of the gut immediately adjacent to the mesenteric attachment, to include the serous layer of the gut and thus do away with the "dead space" which normally exists in this situation between the serous and muscular coats of the intestine.

Having thus completed the junction of the gut end to end, and having wiped the parts immediately adjacent to the line of suture with a wet bichloride pad, we may proceed to apply a second con- 
tinuous Lembert suture of fine black silk. This Lembert stitch is introduced with a straight cambric needle, and includes only the serous and muscular coats; it should completely bury the preceding penetrating stitch, special caution being used to appose serous surface to serous surface, especially near the mesenteric attachment.

The hole which is left in the mesentery, after the segment of gut has been resected and the ends sutured, should be closed with a continuous catgut stitch, again using special care to bring the edges of the opening close together near the surface of the gut.

The strips which were placed around the gut are now removed and the sutured segment of bowel returned into the abdomen, and placed in the immediate neighborhood of the abdominal incision.

The incision in the abdomen is closed, either with a single series of interrupted silk-worm gut sutures which include the whole thickness of the abdominal wall, and especially the edges of the parietal peritoneum, or, better, the edges of the parietal peritoneum may be brought together, first with a continuous catgut stitch, and then, in addition to this, the other layers of the abdominal wall may be united with a sufficient number of interrupted silk-worm gut sutures.

Mounsell's Method. - After having excised the segment of gut as above described, the cut ends are placed close together, edge to edge, supported upon gauze pads outside the abdomen. With a moderately large, straight needle and fairly thick silk the edges of the cut ends of the gut are fixed to each other at four different points of their circumference equidistant from one another. These sutures are to serve simply as tractors. The first is applied at a point corresponding to the mesenteric attachment, the second at a point directly opposite this, and the other two at points midway between these. Each of these sutures should include all the coats of the gut, special care being taken to catch the mucous membrane and the serous coats; the suture at the mesenteric border, particularly, should take a good hold of the serous coat to insure its inversion at this point. Each suture should be applied from within the gut, so that, when tied, the knot will be upon the inner, mucous membrane aspect of the gut. As each of these four tractor sutures is passed, it is immediately tied and one end cut short, leaving the other end long. In tying, the suture should not be tied right down upon the edges of the gut, but rather loosely, so that afterward they may be readily removed.

In one or the other segment of the gut, a longitudinal incision 


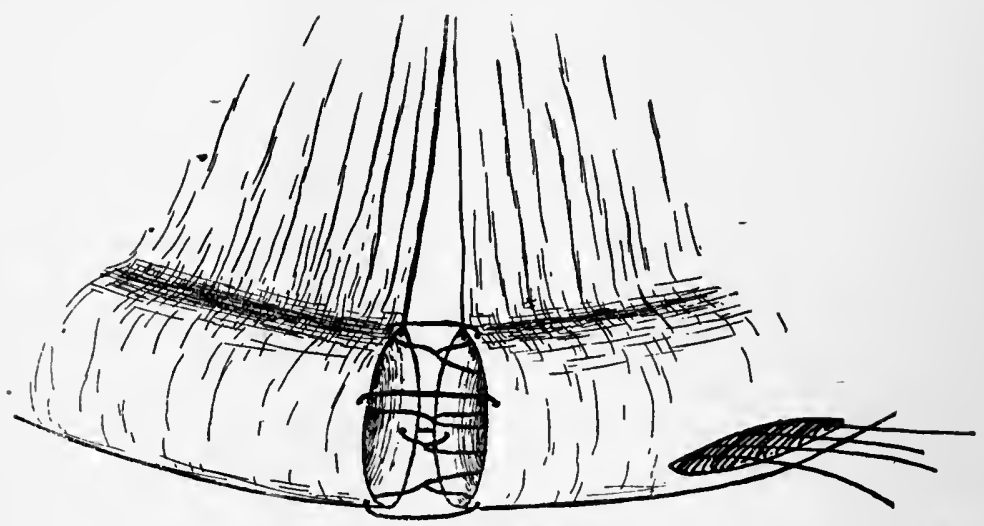

Fig. 109.-End-to-End Anastomosis (Mounsell). The ends of the two segments have been joined by four tractor sutures, the ends of whlch are drawn out of an opening made in the gut.

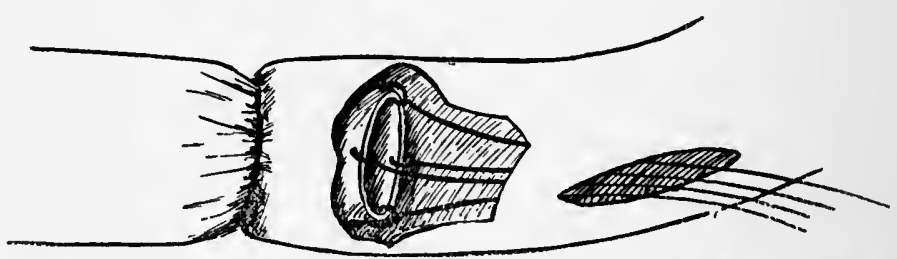

Fig. 110.-Segment of the Wall of the Gut Removed to Show the Invagination of One Coil of Gut into the Other which is Caused by Pulling upon the Tractors.

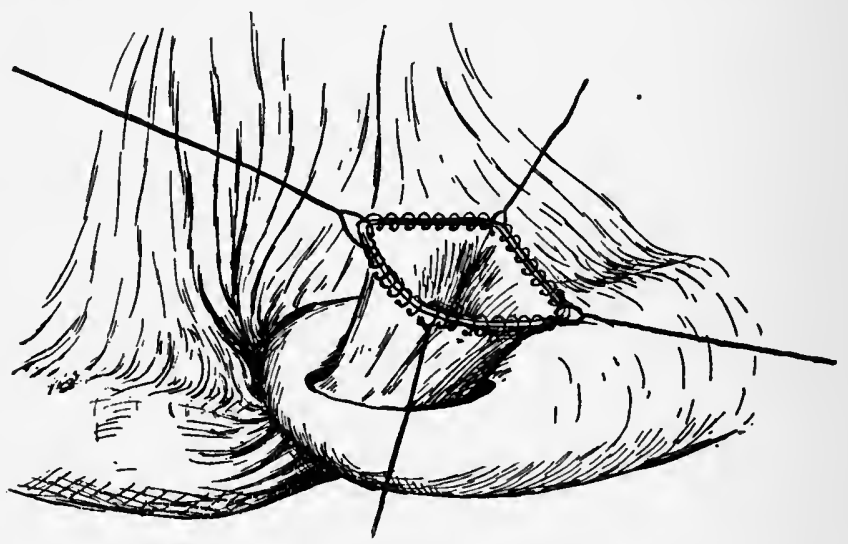

Fig. 111.-The Two Colls of Gut, One Invaginated within the Other, have been Drawn through the Incision in the Gut and their Edges United all Around with a Continuous Penetrating Suture. 
is now made. This incision is placed opposite the mesenteric border, should be about one inch long, and commences about one and one-half inches distant from the cut edge of the gut. It is best made by picking up the wall of the gut with two toothed forceps, and between these, with a sharp, straight scissors, a clean-cut incision is made through the whole thickness of the wall of the gut. Through this incision a narrow artery forceps is passed into the gut and the tails of the four tractor sutures seized and pulled through, thus drawing the ends of the gut after them, with the result that the one segment of gut is invaginated into the other, their serous surfaces lying in contact with each other and their corresponding edges in apposition all around. The four tractor sutures are held by assistants and put somewhat upon the stretch, and then the corresponding edges of both segments of the gut are ready to be joined by suture. The edges are sewed together with an overhand stitch with a straight needle and fine silk. This suture, at each puncture, should take a good bite and include a margin of rather more than one-eighth inch, should be fairly close (intervals of about one-eighth inch between needle punctures), and should be drawn fairly tight. The stitch should include all the coats of the gut, special pains being taken to include the serous coat, particularly at the mesenteric border of the gut. Catgut may be used for this stitch, but silk is probably better.

Having united the edges of the segments of the gut as above described, the temporary tractor sutures are removed and the gut restored to its natural position by reducing the invagination; the incision in the gut is then closed with a continuous Lembert stitch.

All around the circular junction of the segments, after swabbing with a pad moistened with alcohol, followed by one wet with a weak bichloride solution, a continuous Lembert stitch of fine silk is applied; this inverts the edges of the gut and buries completely the penetrating through and through suture.

The opening which is left in the mesentery, after the segment of gut has been excised, is closed with a continuous catgut suture, special care being taken to make the union accurate close to the intestinc. The gut is then returned to the abdominal cavity, being placed near the abdominal incision, and the opening in the abdomen closed.

Murphy Butron.-Having resected the gut as above described, a running string is placed in the edge of each segment of the gut which, when drawn tight and tied, puckers the end of the gut and 
grasps the button about its shank, leaving the flange, or cup, of the button within the gut. This running stitch, or purse-string, is applied in overhand fashion, is of fine silk, and carried upon two long straight needles. This stitch should include all the layers of the gut, especially the serous and the mucous membrane; it should not include too wide a margin of the gut, since the amount of tissue which is grasped between the flanges, or cups, of the button may be too bulky to allow exact coaptation; a margin rather less than one-fourth inch is sufficient. For the running stitch we require a single strand of fine silk with a straight cambric needle at each end. The running stitch is commenced by piercing the mesentery close to the surface of the gut, and then, carrying the same needle back over the edge of the mesentery, it is again thrust through, so that we thus have a loop around the cut edge of the mesentery close to the surface of the gut. Now, with this same needle, the running suture is applied to the corresponding half of the circumference of the cut edge of the gut; each puncture of the needle should be made from within the lumen of the gut from its mucous membrane aspect, and the punctures should be about one-third inch apart. When a point is reached directly opposite the mesenteric border of the gut, this needle is discarded; the second needle is then taken in hand and the second half of the circumference of the gut treated in exactly the same manner.

In this way the whole circumference of the gut is included, leaving the two free tails of the suture, which emerge upon the serous surface of the gut opposite its mesenteric attachment, ready for tying.

The object in catching the edge of the mesentery in the loop of the purse-string suture is to insure the turning in of a serous surface at this point and at the same time to do away with the "dead" mesenteric space; besides, it gives a fixed point to the suture. Before introducing the button the first double loop of a surgeon's knot should be taken with the ends of the purse-string.

One-half of the button, grasped with a thumb forceps by the edge of its tubal part, is now introduced into the end of the gut, turning the button a little on the side to facilitate its introduction, and while it is thus held the purse-string is tied around its shank, leaving the flange within the intestine. The ends of the pursestring are cut short so that they will not protrude between the flanges of the button when this is closed. This procedure is repeated upon 
the other segment of gut. The two halves of the button are then deliberately pressed home, and in doing this one should note that the corresponding mesenteric attachments of either segment of gut are opposite each other.

When the two halves of the button are locked there should be

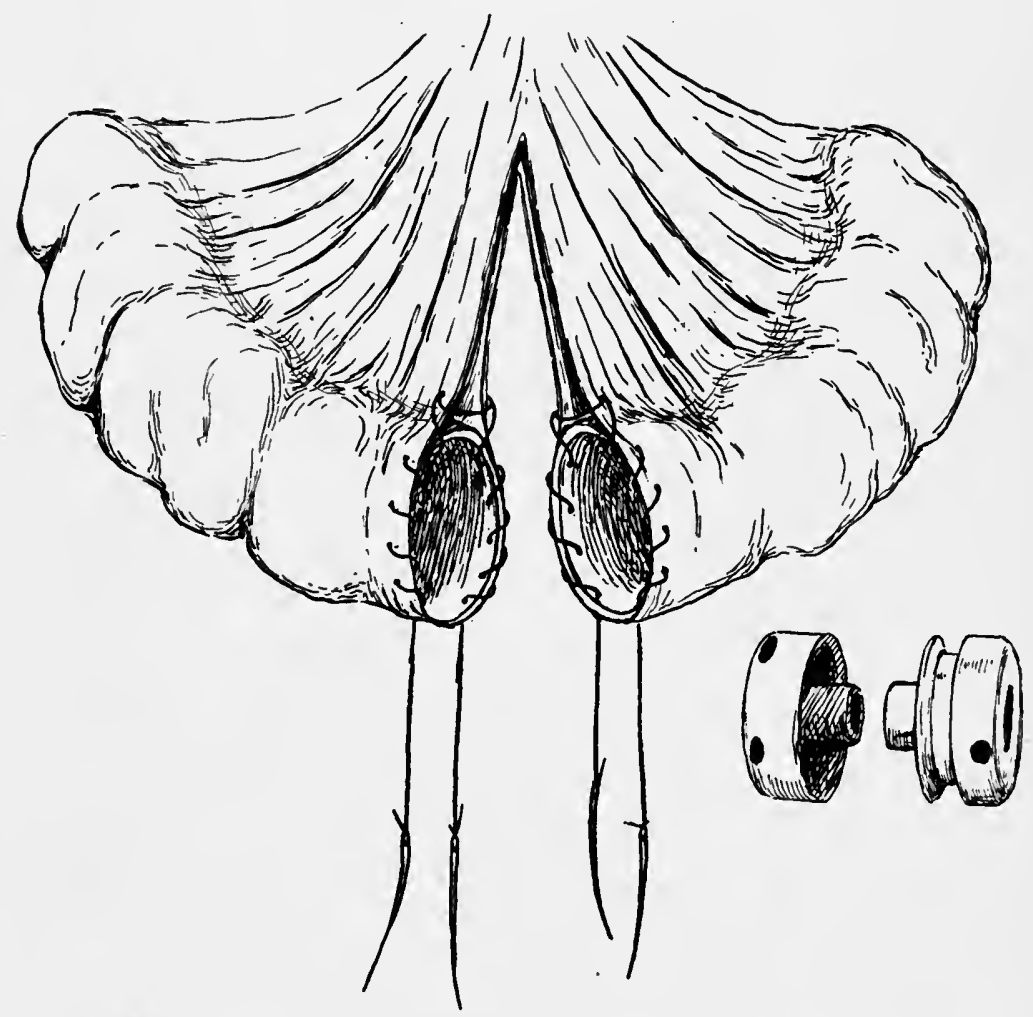

Fig. 112.-End-to-End Anastomosis (Murphy Button). With the purse-string suture a loop is taken through the layers of the mesentery, close to the wall of the gut, in order to obliterate the "dead space."

Fig. 113.-Murphy Button, the Two Haives Separated.

presented between them a clean, smooth line with no raw mucous membrane edge protruding, and at the mesenteric attachment the apposition of serous surfaces should also be assured. Should there be any protruding edge of mucous membrane between the flanges of the button after this has been locked, it may be seized with a thumb 
forceps and trimmed off short with the scissors. Any doubtful points should be made secure by adding several Lembert sutures.

Although it is probably not necessary in all cases to use a layer of Lembert sutures in addition to the Murphy button to secure accurate apposition, nevertheless it is wise in many cases to place a continuous Lembert stitch outside of the button after the halves have been pressed home, especially as the presence of the button makes the application of this stitch rather an easy matter.

After the hole in the mesentery has been closed as described in the preceding operation the abdominal wound is sutured.

The Murphy button causes a pressure atrophy of the edges of the gut which are caught between its flanges. When this atrophy is complete, the button is liberated, taking the atrophied ring of tissue with it, and thus an opening is left, at the site of the anastomosis, which corresponds in size to the full diameter of the flanges of the button.

With Laplace Anastomosis Forceps.-The Laplace anastomosis forceps resembles two pair of hæmostatic forceps, the blades of each being bent to form half a ring. When the two forceps are united side by side, their blades together form a complete ring. The two parts of the instrument when joined are held securely together by means of a clamp that is applied at the rivet. When clamped the two halves of the forceps work in harmony, and may be opened and closed like a single instrument. The handles are provided with a ratchet, like an ordinary hæmostatic forceps, so that when the blades are closed they remain locked. The instruments are supplied in five sizes. The McLean anastomosis forceps is a modification of the Laplace instrument, and is more simple in its construction.

After the diseased portion of the gut has been resected the ends of the bowel are united to each other fairly close with four penetrating sutures of catgut placed equidistant apart, taking care, at the same time, that the mesenteric portions of both segments are placed opposite each other. The ring-blades of the anastomosis forceps are then introduced, closed, between two of the four sutures, and then spread apart so that one ring-blade passes into each end of the gut. In order to facilitate the turning in, inversion, of the edges of the gut so that they may be grasped all around when the blades of the forceps are closed, a strand of silk may be thrown around the four stitches that unite the edges of the gut so as to encircle these stitches. By tightening this thread the edges of both segments are 


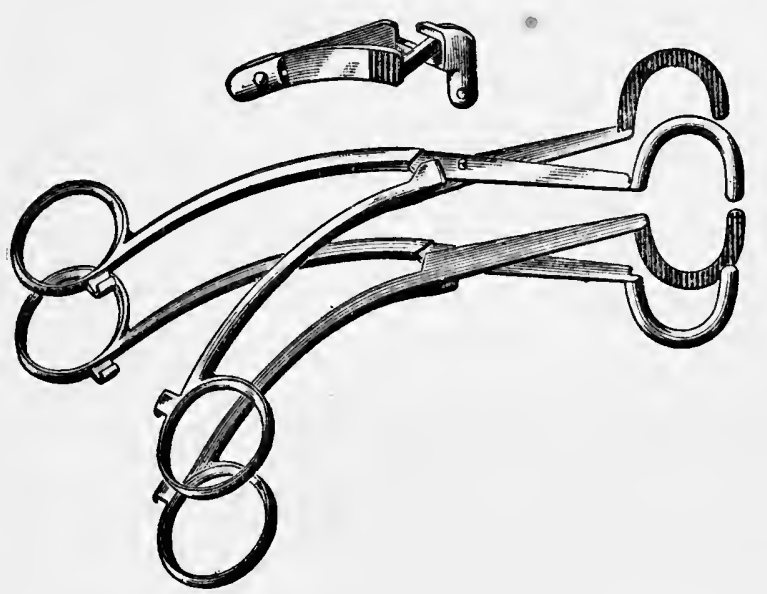

Fig. 114.-Laplace Anastomosis Forceps Separated into its Component Parts.

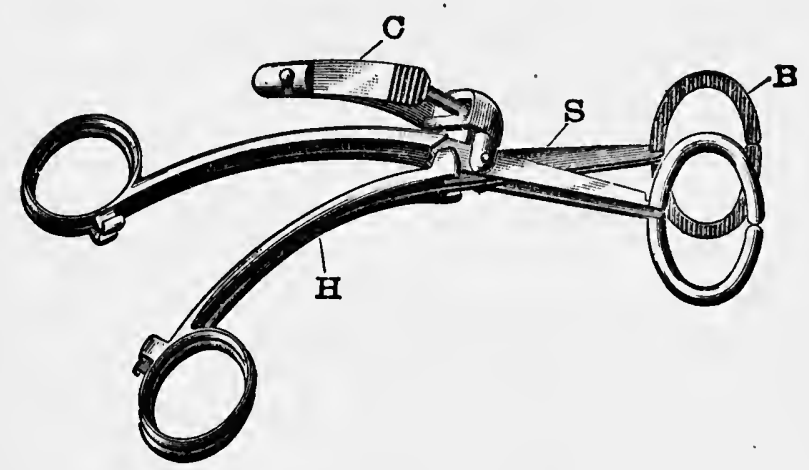

Fig. 115.-Laplace Anastomosis Forceps Jolned Together, its Blades Slightly Open. $B$, rlng blades; $C$, clamp; $H$, handle; $S$, shank.

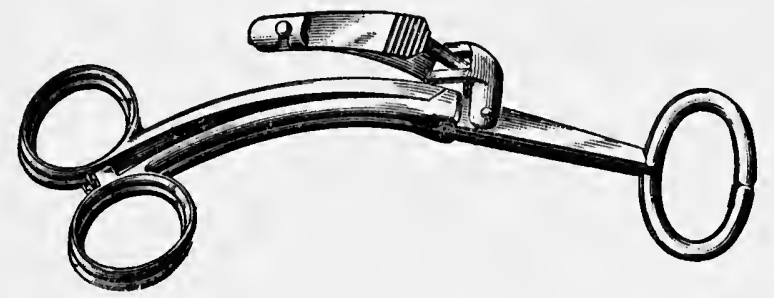

Fig. 116.-Laplace Anastomosis Forceps Joined Together, its Blades Closed. 
turned inward toward the axis of the intestine, with the result that when the ring-blades of the forceps are closed they grasp the margins of each segment of the gut all around, serous surface to serous surface. The margin of each segment, for its entire circumference, should be grasped between the closed ring-blades of the forceps. The strand of silk is then withdrawn.

The two ends of the gut are united to each other, all around the circumference of the ring-blades of the forceps, with a continuous, non-penetrating Lembert suture of silk except at the point where the shank of the forceps emerges. After this suture has been applied the clamp is removed from the forceps, which is then separated into its two component parts; the blades of each portion represent but half a ring, and these are withdrawn from within the intestine, one at a time. The small opening through which the two parts of the forceps were removed is closed with one or two nonpenetrating Lembert sutures. If desired, a second outside row of Lembert sutures may be applied to still further secure the union of the two ends of gut.

With O'Hara ANastomosis Forceps.-0'Hara's anastomosis forceps is composed practically of two long, thin-bladed hæmostatic forceps that may be joined securely to each other, side by side, with a clamp. When thus joined together both forceps work in harmony as one single instrument. The handles of the instrument are provided with a ratchet arrangement like ordinary artery forceps, so that when the blades are closed they remain locked until released. The edges of the blades are serrated, so that the parts within their grasp cannot escape, and they are also graduated with file-marks, so that one may accurately estimate the length of tissue that is grasped between them.

The coil of gut that is to be resected is brought out through the incision in the abdomen and the corresponding portion of the mesentery is tied off. One O'Hara forceps is then applied to the gut below the seat of disease and locked, and the other forceps secures the intestine above the seat of disease and is likewise locked. Each forceps grasps the gut at right angles to its long axis. The tip of each forceps, as it grasps the intestine, should reach just to the mesenteric border. The diseased segment of gut is then excised with long, straight scissors in the usual way, cutting fairly close to the blades of each O'Hara forceps. Before severing the gut ordinary compression clamps may be applied to it-one immediately above and an- 


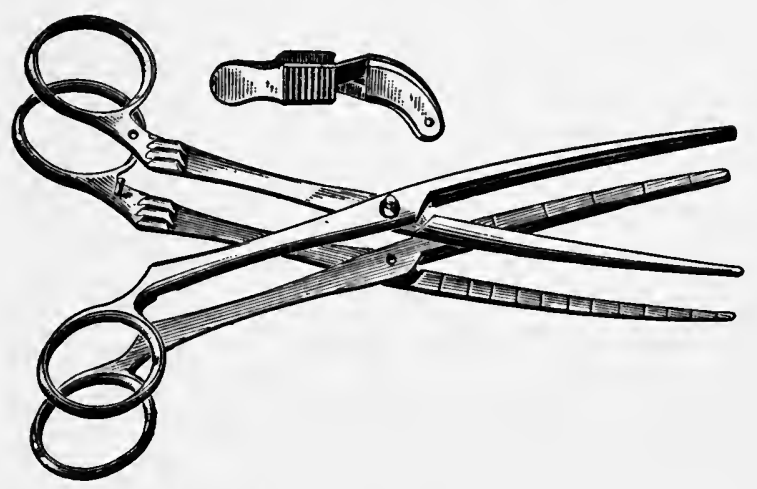

Fig. 117.-O'Hara Anastomosis Forceps Separated into its Component Parts.

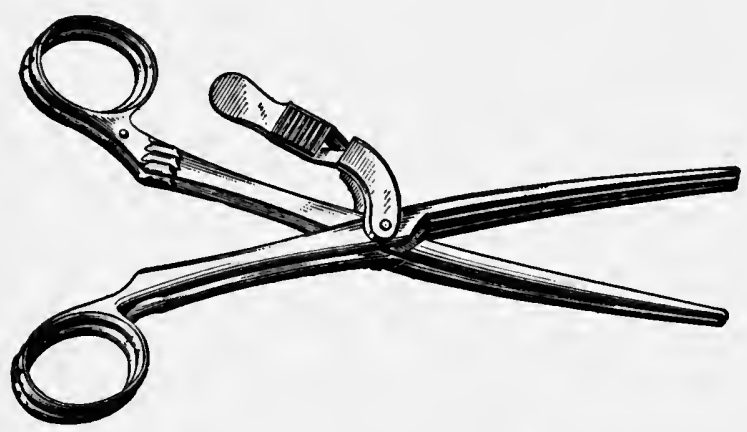

Fig. 118.-O'Hara Anastomosis Forceps Joined Together, its Blades Open.

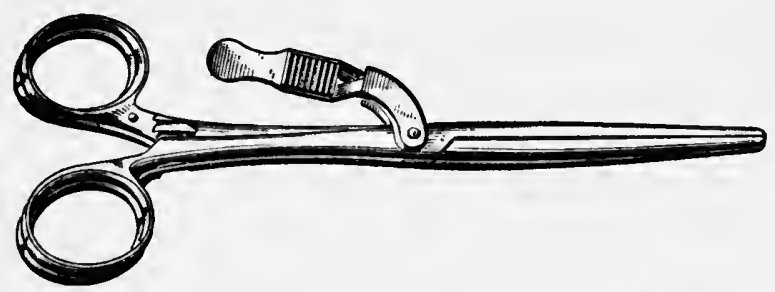

Fig. 119.-O'Hara Anastomosis Forceps Joined Together, its Blades Ciosed. 
other immediately below the diseased portion; so that when the gut is divided there will not be any escape of the contents of the intestinal canal. The mesentery corresponding to the diseased segment of gut may be tied off before or after the gut has been severed. The two O'Hara forceps are now approximated and fixed securely together side by side with the clamp.

Commencing near the rivet and working toward the tip of the forceps, the two segments of gut are united with a continuous, nonpenetrating Lembert suture. This suture catches the wall of each segment of gut just beyond the blades of the forceps; so that the forceps are thus gradually buried, being invaginated into the lumen of the gut as the suture progresses. When the tips of the conjoined forceps are reached, the gut and forceps are turned over, so as to gain access to the other aspect of the gut, and the suture is continued along this side of the gut toward the rivet of the forceps until the point is reached where the suture commenced. In working around the tips of the united forceps at the mesenteric border of the gut care is needed to include the serous coat in the suture. The clamp is now removed, separating the two forceps. First one forceps is unlocked and withdrawn, then the second is unlocked and its blades passed up and down through the line of junction to show that this is patent and that none of the stitches have been carried across the lumen of the gut so as to include the opposite wall, and then this is likewise withdrawn. The small opening that is left in the line of junction after the forceps have been withdrawn is closed with one or two interrupted sutures.

Side-to-Side, or Lateral, Approximation (Lateral Intestinal Anastomosis).- This is the formation of a fistulous opening between two coils of intestine joined side to side.

This operation is indicated when the ends of gut that are to be united differ much in caliber,-for example, to unite the end of the ileum to the cxcum,- or where the intestinal tube is very narrow, as, for example, in children.

Suture.-The parts are brought well up into the wound or, if possible, outside upon the abdomen, and are surrounded with gauze pads to protect the peritoneal cavity. Gauze strips are then tied around the intestine, and after the diseased portion of the gut has been excised the cut end of each segment of the gut for about one inch of its length is inverted and closed with a double row of Lembert sutures, thus converting each end of the gut into a blind pouch. 
Care should be taken to include the invaginated mesentery in the suture. One should commence the invagination of the end of the gut at its mesenteric edge, inverting it about one inch of its length, and then the rest of the gut is very conveniently inverted and sutured.

The next step is the union of the two blind ends of the gut to each other, side to side, so that the intestinal canal, through the new opening that is to be made, will be continued in a direct line, and not reversed in passing from one segment to the other. The ends

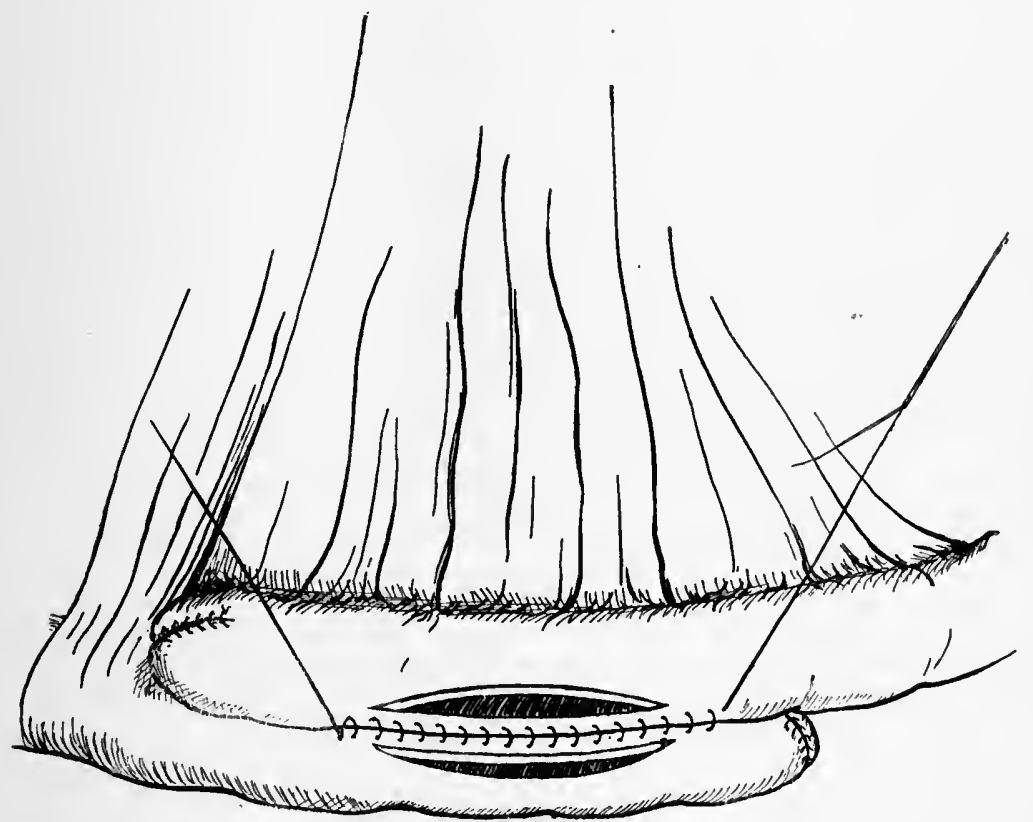

Fig. 120.-Lateral Anastomosis. The end of each coil of gut has been closed by suture. The two coils have been piaced side by side and joined by a continuous non-penetrating suture. An opening has been made in each coll of gut.

of the gut should be so placed that they overlap each other for a distance of four to five inches; their apposed surfaces are then united to each other, for a distance of from three to four inches, by a single row of continuous Lembert sutures of fine silk. After this row of Lembert sutures, which forms the posterior half of the "outside serous ring," has been applied, this needle, still carrying the fine silk, is laid aside until required later to complete this "outside serous ring." This line of Lembert sutures should be one inch longer than 
the proposed openings in the gut, and each stitch should be rather less than one-fourth inch distant from its neighbor and should be drawn tight.

Each segment of the bowel is now opened with the scissors, the incisions being placed about one-fourth inch distant from the line of the Lembert suture; the openings in the bowel should be large so as to allow for subsequent contraction,- -three inches long and at least one inch shorter than the line of the Lembert suture.

Bleeding from the edges of the incisions in the bowel is controlled with clamps, which may be removed after a few minutes' pressure, as the hemorrhage usually ceases. The edges of the open-

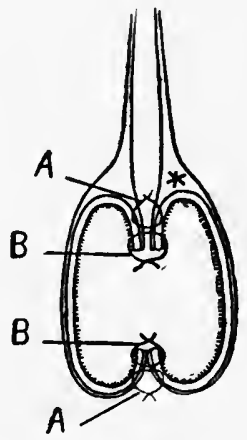

Fig. 121.-A Cross Section of the Apposed Coils of Gut After the Anastomosis has been Completed. $A, A$, the outer, non-penetrating suture, the "outside serous" suture; $B, B$, the buried suture that penetrates all the layers of the wall of the gut, foining the contiguous edges of the opening in each segment all around. *, the "dead space" corresponding to the mesenteric border of the gut where the layers of the mesentery separate to invest the gut.

ings in the gut are wiped with alcohol followed by a weak bichloride solution, and then, with a continuous silk suture, which at the same time controls the hemorrhage, the edges of the opening in each segment of the bowel are united with each other all around.

Having thus united the edges of the openings all around, we again take up the needle carrying the original fine silk suture and complete the anastomosis by making the anterior half of the Lembert suture, the "outside serous ring."

The line of Lembert suture serves to bury the suture by which the margins of the openings in the gut are joined to each other.

In making the lateral anastomosis one should not have the blind ends of the overlapped gut too long, and, further, the blind ends 
should be anchored to the adjoining portion of the intestine by several Lembert stitches.

It may be necessary to tear the mesentery somewhat in order to allow sufficient overlapping of the ends of the bowel. After the anastomosis has been made the adjoining overlapping layers of the mesentery may be united with a continuous catgut stitch. The parts should then be returned to the abdomen and the wound closed up.

MURPHY ButTon. - A lateral intestinal anastomosis may be made with the Murphy button. After the ends of the gut have been inverted and closed with a suture as described in the preceding operation, the two ends are placed side by side and a purse-string suture placed in each segment; the incisions are then made and the buttons inserted in the usual way. This is rather an unsatisfactory method of doing a primary lateral intestinal anastomosis, because the opening left by the Murphy button is too small to allow for the subsequent contraction that always takes place. We may use the Murphy button with satisfactory result in making a lateral anastomosis in connection with a primary gastro-enterostomy (see Fig. 125 ; also "Cholecysto-duodenostomy" for description of the pursestring suture and the method of its introduction).

McGraw's RUbBer SUTuRE.-With the rubber suture a lateral intestinal anastomosis may be made in a manner analogous to that described for the gastro-enterostomy. The surfaces of the two segments of gut that are to be joined are placed side by side and united for a distance of about two and one-half inches with a continuous Lembert stitch of silk as described in the previous operation, and then the needle carrying this stitch is temporarily laid aside. The rubber suture, 2 to $3 \mathrm{~mm}$. thick, is now introduced with a straight needle, so as to include both segments of the gut in its grasp, is drawn tight, and tied. A silk ligature is tied around the knot in the rubber suture so as to secure the latter from slipping. About two inches of the wall of each segment of the gut should be included in the constricting rubber suture; so that, when this cuts through, the opening left between the two coils of gut will be two inches in length (see "Gastro-enterostomy with McGraw's Rubber Suture").

The needle carrying the silk Lembert suture is now again taken up, and with this the two coils of gut are still further united along a line just outside of the rubber suture. This forms the second half of the "outside serous ring" suture, and buries the rubber suture beneath it out of sight. 
Wi'th LAplace Forceps. - Lateral anastomosis with Laplace forceps is accomplished in a manner analogous to that described for gastro-enterostomy.

With O'Hara Anastomosis Forcers.-Lateral anastomosis with the O'Hara forceps is done in a manner similar to that described for gastro-enterostomy.

Gastro-enterostomy.-Gastro-enterostomy is the formation of a fistulous communication between the stomach and the small intestine.

The operation is indicated where the pyloric orifice is constricted, either simple, following ulcer, etc., or malignant. The operation was first performed by Woelfler in 1881. The loop of small intestine may be fixed to either the anterior or the posterior wall of the stomach.

The Anterior Operation (Woelfler). - This consists in bringing a coil of the small intestine-jejunum-up in front of the great omentum and transverse colon and fixing it to the anterior wall of the stomach.

The stomach should be washed out while the patient is anæsthetized, immediately before the operation.

An incision is made in the middle line through the linea alba from a point one inch below the ensiform cartilage down to the umbilicus, or even beyond this point if necessary. The incision is usually about four inches long (10 to $15 \mathrm{~cm}$.).

Through this opening the stomach is sought and examined. A portion of the wall of the stomach which is not involved in the disease should be selected. The stomach is partly covered by the liver, the anterior thin edge of the left lobe of the liver being a good guide to the stomach. Lying below and close to the greater curvature of the stomach is the transverse colon, and from this the great omentum, apron-like, is suspended, hanging down free in the abdominal cavity in front of the small intestine.

After the stomach has been recognized the transverse colon, and with it the great omentum, are drawn upward out of the wound, and search is then made for the commencement of the jejunum. This part of the gut lies in the back of the abdominal cavity, to the left of the vertebral column, upon a level with the second lumbar vertebra, its mesentery being very short and serving to anchor it in this position. To secure this coil of gut the hand is introduced into the abdomen and carried backward, along the under surface of the 
transverse mesocolon, as far as the posterior abdominal wall; just below the attachment of the transverse mesocolon to the vertebral column, at the place indicated upon the left of the column, this coil of gut is found.

This part of the small intestine is readily identified by the fact that it is fixed within, as is shown when an effort is made to draw it out of the abdomen; any other part of the small intestine may be

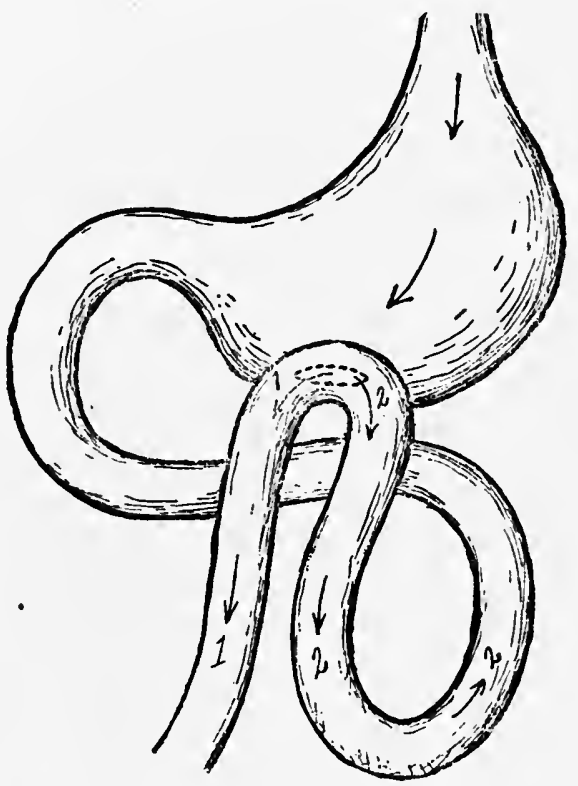

Fig. 122.-Gastro-enterostomy. The jejunum has been fixed to the stomach and an opening made between them. Arrows $(1,1)$ show the proper course of the stomach contents into the long arm of the gut. Arrows $(2,2)$ show the course of stomach contents into the short arm of the gut, through which they may again enter the stomach, "vicious circle."

drawn through the fingers in either direction, and may be readily drawn out upon the abdomen.

We select a loop of gut for attachment to the stomach from eighteen to twenty inches distant from this fixed part of the jejunum. A loop of gut about eight inches long is drawn out upon the abdominal wall and surrounded and constricted by two thin strips of sterile gauze. A sharp-nosed artery forceps is thrust through the mesentery near its attachment to the intestine, and with this the end of each gauze strip is seized and drawn through. The one 
strip is tied and the segment of gut emptied of its contents, to a point beyond the second piece of gauze, by gently stripping it between the fingers, and then the second gauze strip is tied. The gauze strips should be tied sufficiently tight to prevent the reentrance of the intestinal contents into this segment of the gut. The transverse colon and great omentum are now pushed back into the abdomen again, and the anterior surface of the stomach seized and drawn out of the abdomen. Dry, sterile, gauze pads are placed about the stomach and intestine and tucked partly into the incision for the purpose of retaining the parts outside of the abdomen and to prevent the entrance of escaping intestinal contents into the peritoneal cavity. The rest of the operation is done extraperitoneally.

The coil of intestine and the stomach are steadied, side by side, and united by a continuous Lembert suture of fine black silk, using a straight cambric needle. This suture line, which includes the serous and muscular coats, forms the posterior half of the "outside serous ring." It should not penetrate into the cavity of the stomach or intestine. The suture should be applied in a straight line, about two and one-half to three inches long, each puncture of the needle being about one-eighth inch distant from its neighbor, and should take a good, broad bite. Each stitch should be drawn fairly tight. It is probably more convenient for the operator, in applying this suture, to commence at the far end and sew toward himself, steadying the parts with the thumb and index finger of left hand. The tail of the suture should be left long, and may be held by the assistant as a tractor. After this line of suture has been completed, the needle, carrying this thread, is laid aside until needed later to complete the operation by making the anterior half of the "outside serous ring" suture.

The openings in the intestine and stomach are next made. These incisions should be one and one-half to two inches long. They should be shorter than the line of the Lembert suture, and should be placed about one-fourth inch distant from this. The intestine should be incised first, the incision being placed opposite its mesenteric border and any escaping contents carefully swabbed away. These incisions should be straight and clean cut. The wall of the gut is caught up with two toothed forceps, and a small opening made between these with a straight, sharp scissors, and then this opening is sufficiently enlarged. The stomach is then treated in a similar 
manner. Hemorrhage from the edges of these incisions stops after they have been sutured; any spurting points may be clamped and tied with fine silk or catgut.

The adjoining edges of the incisions in the intestine and stomach are now sewed to each other with catgut or silk in a mediumsized, straight needle, each stitch taking a good bite and passing through all the coats, including the mucous membrane, and drawn fairly tight; the needle punctures should be rather less than onefourth inch apart. This suture is continued uninterrupted all around, uniting the corresponding edges of the incisions in the stomach and intestine to each other until these openings are entirely closed in and the anastomosis made. Before beginning this stitch the margins of the openings should be wiped off with alcohol followed by a weak bichloride solution on a swab.

We now again take up the needle with which the posterior half of the Lembert suture-“outside serous ring"-was made, and complete the operation by making the anterior half of the "outside serous ring" suture.

Thus we have the openings in the intestine and stomach, one and one-half to two inches long, united, edge to edge, by a continuous stitch which passes through the entire thickness of the margins of the contiguous openings, and this surrounded, reinforced, by a continuous Lembert suture which passes through the serous and muscular coats only and which serves the purpose of burying the penetrating mucous stitch. Should there be any doubtful points where the mucous penetrating stitch is not certainly buried, one or more supplementary interrupted Lembert stitches may be taken to remedy this.

The coil of intestine should be joined to the stomach near its greater curvature and about three inches from its pyloric opening. The incision in the intestine should be made opposite its mesenteric attachment. The intestine should be joined to the stomach in such a way that the current of food in the stomach and in the loop of intestine will be in the same direction-the distal limb of the loop of gut toward the right or pyloric end of the stomach; this is accomplished by taking care not to twist the loop of intestine upon itself when drawing it up into apposition with the stomach.

The transverse colon and great omentum rolled upon itself lie together behind the junction formed between the jejunum and the stomach. 
The tapes are now removed from the intestine and the parts replaced within the abdomen.

The opening in the abdomen is closed with several silk-worm gut sutures, which pass through the whole thickness of the abdominal wall, care being taken to include the edges of the parietal peritoneum. These stitches should be about one-half inch apart. It is probably better to suture the edges of the peritoneum first with a continuous catgut stitch and after this the silk-worm stitches are applied so as to include the other layers of the abdominal wall.

Posterior Operation (von Hacker). - The jejunum is sutured to the posterior wall of the stomach, which is exposed through an opening torn in the transverse mesocolon.

This operation is preferred by many surgeons to the anterior. The position of the parts is more natural and the transverse colon is not displaced, and cannot drag upon the coil of jejunum that is fixed to the stomach.

The technique of this operation is no more difficult than that of the anterior; so that the choice between the anterior and posterior operation will probably depend, in most cases, upon the location of the disease.

An incision is made in the middle line through the linea alba, as in the preceding operation. The stomach is recognized and examined. The transverse colon and greater omentum are then reflected upward and the stomach drawn out of the incision in the abdomen. An opening at right angles to the long axis of the transverse colon is now cut, or, better, torn, in the transverse mesocolon, penetrating from its inferior aspect, in order to expose a sufficient area of the posterior wall of the stomach.

The posterior wall of the stomach is then drawn partly through the opening torn in the transverse mesocolon, the edges of the opening in the mesocolon being fixed at once to the posterior wall of the stomach by several fine silk sutures (which do not, of course, pierce the entire thickness of the stomach wall). The exposed area of the stomach is then brought up into the incision in the abdomen and out upon the abdominal wall, where it is retained by an assistant.

As in the preceding operation, the commencement of the jejunum is sought for and found in the back of the abdomen to the left of the body of the second lumbar vertebra, just below the vertebral attachment of the transverse mesocolon. A coil of intestine about twenty inches farther along is selected, and this is also brought out 

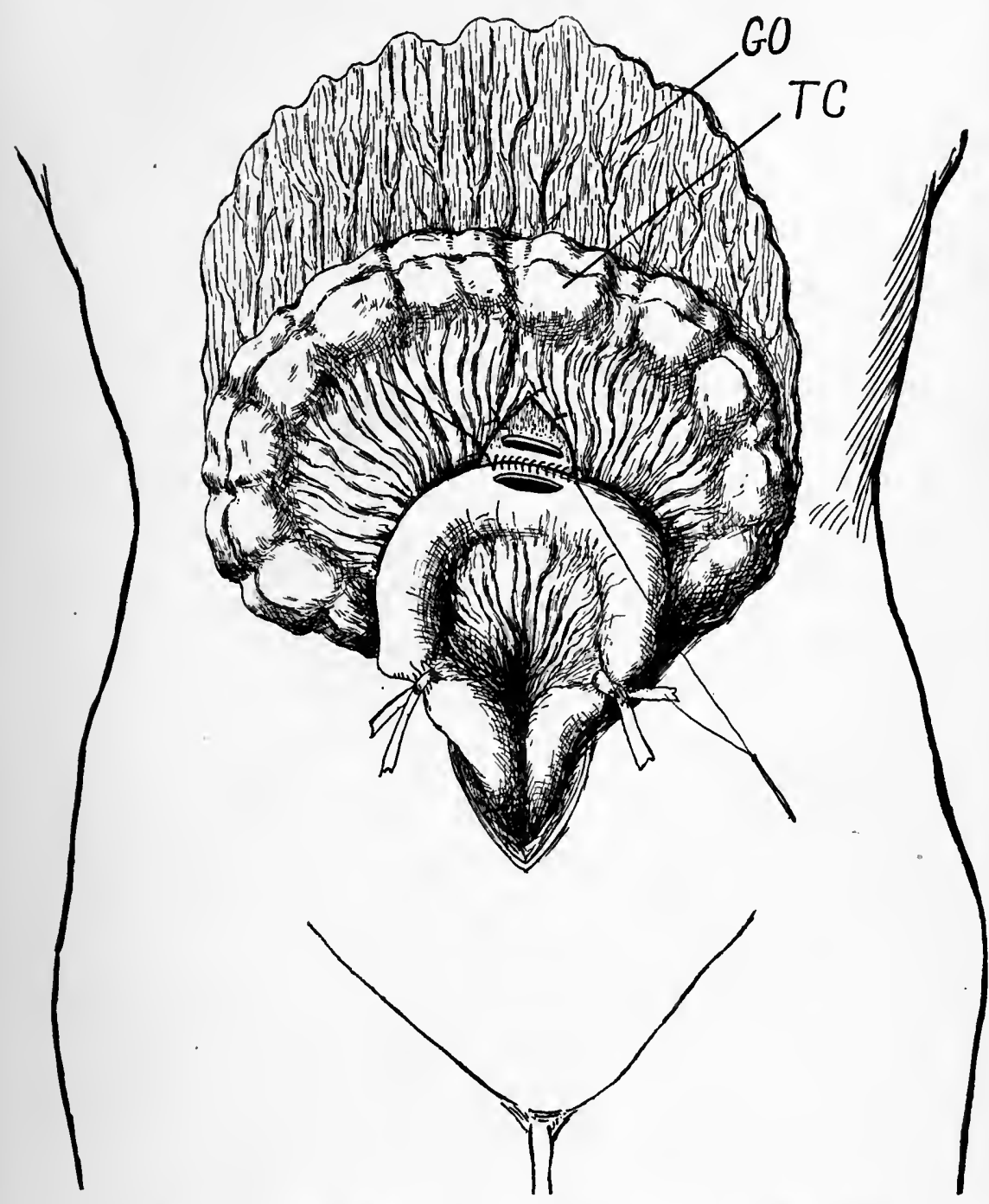

Fig. 123.-Posterior Gastro-enterostomy. Great omentum $(G O)$ and transverse colon $(T C)$ have been drawn out of the incision and turned upward. An opening has been made in the transverse mesocolon in order to expose a portion of the posterior wall of the stomach. A loop of jejunum has been fixed to the wall of the stomach with a continuous, non-penetrating stitch and openings made in the stomach and the attached coil of gut. The loop of gut has been temporarily tied off with tapes. 
of the abdominal wound and placed alongside of that portion of the posterior wall of the stomach which presents through the opening in the transverse mesocolon. Pads of gauze are then tucked about the viscera and partly into the abdominal incision to steady the parts and to prevent the entrance of infectious material into the peritoneal cavity and the gastro-enterostomy is performed as in the preceding operation. The intestine is fixed to the posterior wall of

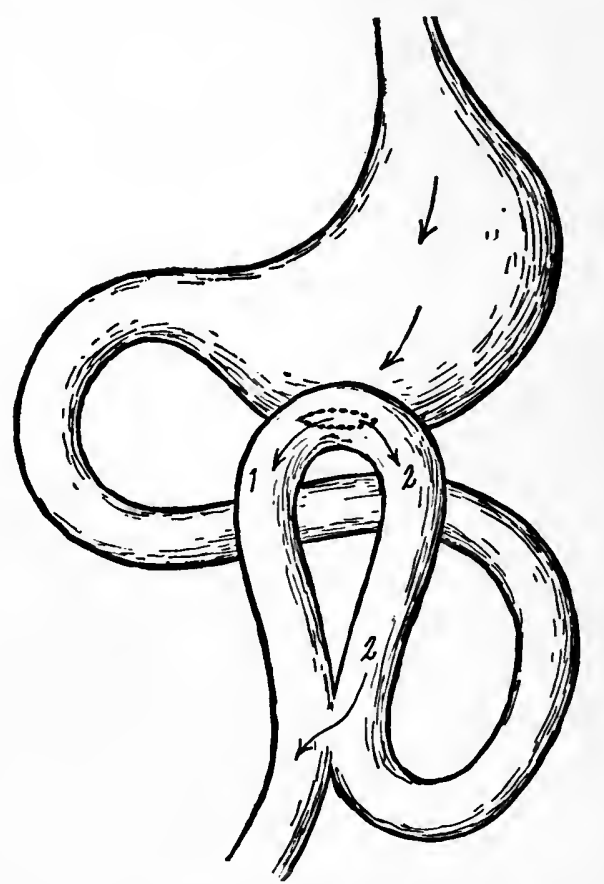

Fig. 124.-Gastro-enterostomy (Jaboulay-Braun). Compare with Fig. 122. Lateral anastomosis has been made between the arms of the attached loop of gut; so that if the stomach contents do enter the short arm of the gut $(2,2)$ they may still escape into the proper long arm. This measure prevents the occurrence of the "vicious circle."

the stomach, near the greater curvature, and from three to three and one-half inches away from the pylorus. The coil should be fixed so that the current of food through the stomach and the intestine are in the same direction.

Jaboulay and Braun Modification.-In some cases, after the anterior or posterior gastro-enterostomy as described above has been performed, there occurs an accumulation of food, bile, and pan- 
creatic juice in the short (proximal) limb of the loop of the intestine that is fixed to the stomach, with a consequent regurgitation into the stomach, and this is characterized by exhausting and fatal vomiting. This regurgitation and vomiting are due to a spur formation in the attached coil of gut which directs the stomach contents into the short or proximal arm of the gut. In order to avoid the occurrence of this vomiting-"vicious circle"-a lateral communication between the two limbs of the coil of intestine which has been attached to the stomach may be made, and this may be done either at the same time that the gastro-enterostomy is performed, or, since this regurgitation, etc., do not occur in all cases, it may be done later as a secondary operation, in this latter case waiting for the appearance of
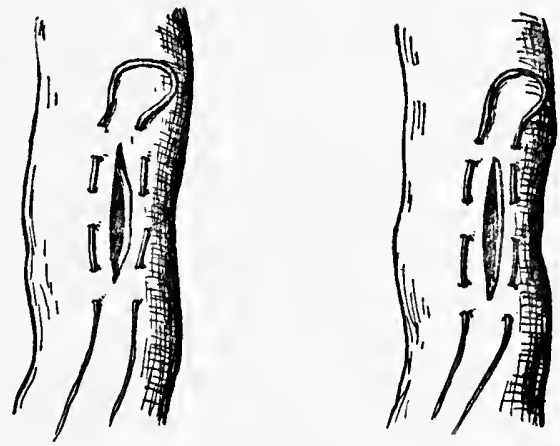

Fig. 125.-Lateral Anastomosis (Murphy Button). Running, or pursestring, sutures applied and incisions made in two coils of gut preparatory to the introduction of the Murphy button.

symptoms indicating the necessity of the additional operation before submitting the patient to the additional risk. It is probably wise, in most cases, to do this entero-anastomosis at the same time as the primary gastro-enterostomy, as it occupies but a few minutes' additional time. This secondary entero-anastomosis may be made with suture, Murphy button, or McGraw's rubber suture or with the Laplace or O'Hara forceps, etc. The communication between the two limbs of the loop of gut should be made at their most dependent part,- -at least five inches distant from their point of attachment to the stomach.

For description of the running, purse-string stitch and the method of its introduction in connection with the Murphy button see "Cholecysto-duodenostomy." 
Gastro-enterostomy with Murphy Button.-This is simple, and much time can be saved by the use of this device.

The use of the Murphy button is no doubt indicated in many cases, especially where a malignant condition exists and the time permitted for the performance of the operation is short. The medium-size button may be used for the gastro-enterostomy, and a smaller one for the entero-anastomosis, if this latter operation is performed in addition.

If a posterior gastro-enterostomy is done, the button, when liberated, is less likely to fall into the stomach than when the anterior gastro-enterostomy is done. According to the suggestion of Weir, the margin of that half of the button which presents into the intestine may be provided with projecting flanges, which should hinder the button from falling into the stomach.

The stomach and intestine are brought out upon the abdomen as in the operations above described. A running stitch is introduced into the wall of the intestine and the wall of the stomach, penetrating through the entire thickness of the wall of each. The space included between the two limbs of this running, or purse-string, suture should be about one-half inch (for description of the running, pursestring suture and the method of its introduction see "Cholecystoduodenostomy"; as applied in lateral anastomosis, between two coils of gut, see Fig. 125). This purse-string suture is applied first to the jejunum, and then between the two limbs of the suture line an incision is made into the gut; this should be barely large enough to permit the introduction of the half button. The half button which is provided with the spring is seized with a thumb forceps and introduced sidewise through the incision into the gut, and, while it is thus steadied, the running string is drawn tight about its shank, tied, and the ends cut short.

In a similar manner, after the purse-string has been applied to the wall of the stomach, this is incised, and the other half of the button is introduced into this incision and the string tied about its neck. The parts adjacent to the openings are then sponged off with a wet bichloride pad and the two halves of the button deliberately pressed home. They should be applied sufficiently tight to cause a gradual pressure necrosis of those parts of the walls of the viscera that are included within their grasp. No raw edge of mucous membrane should protrude between the two halves of the button. If any raw edge of mucous membrane should present itself between 
the flanges of the button, it should be seized with the thumb forceps and trimmed close with sharp scissors, and then be still farther buried with several additional Lembert stitches. Murphy claims that the additional outside Lembert stitch is, as a rule, unnecessary; nevertheless, it is well to use it if time permits, and especially if there are any doubtful points. The button being within the gut makes the application of the Lembert stitch easy. Spurting vessels in the edges of the openings in the intestine and stomach may be clamped and tied with fine catgut or fine silk.

Carle applies the Murphy button without using the purse-string. A simple clean-cut incision is made in the jejunum and in the stomach barely large enough to admit the button. After the button has been introduced the incision is diminished by a single Lembert stitch at each end of the incision; and the two halves of the button then pressed home. It is said to be perfectly safe and to give more perfect apposition than with the purse-string and saves much time. After the button has been introduced and locked several outside Lembert sutures may be introduced, especially if there are any doubtful points and if time permits.

Gastro-enterostomy with McGraw's Rubber Suture.-The gut is brought into apposition with the anterior or posterior surface of the stomach, as described in the preceding operations, and these two portions of the alimentary canal are joined to each other with a continuous silk Lembert stitch for a distance of about two and one-half inches. The needle carrying this suture is then temporarily laid aside.

The stomach is then united to the intestine with a single suture of solid rubber, smooth and round and from 2 to $5 \mathrm{~mm}$. in thickness. This suture is carried in the eye of a long straight needle; a large worsted needle or Hagedorn needle answers well for this purpose. It will be necessary to shave the end of the rubber suture so that it may enter the eye of the needle. The point of the needle is passed into the stomach and then out again, so that about two inches of the wall of the stomach, corresponding to its long diameter, is included between the two punctures, and then, with the same needle and suture, and in a similar manner, the intestine is secured, entering and emerging at points opposite the puncture holes in the stomach.

The rubber suture is then drawn as tight as possible, thus constricting the parts included in its grasp, and tied. In order to secure the knot in the rubber suture a strand of stout silk may be placed 
underneath the rubber at the place where the knot is to be, and after one loop of the knot has been taken in the rubber suture the silk ligature is tied over it and then the second, final loop of the knot is taken in the rubber suture.

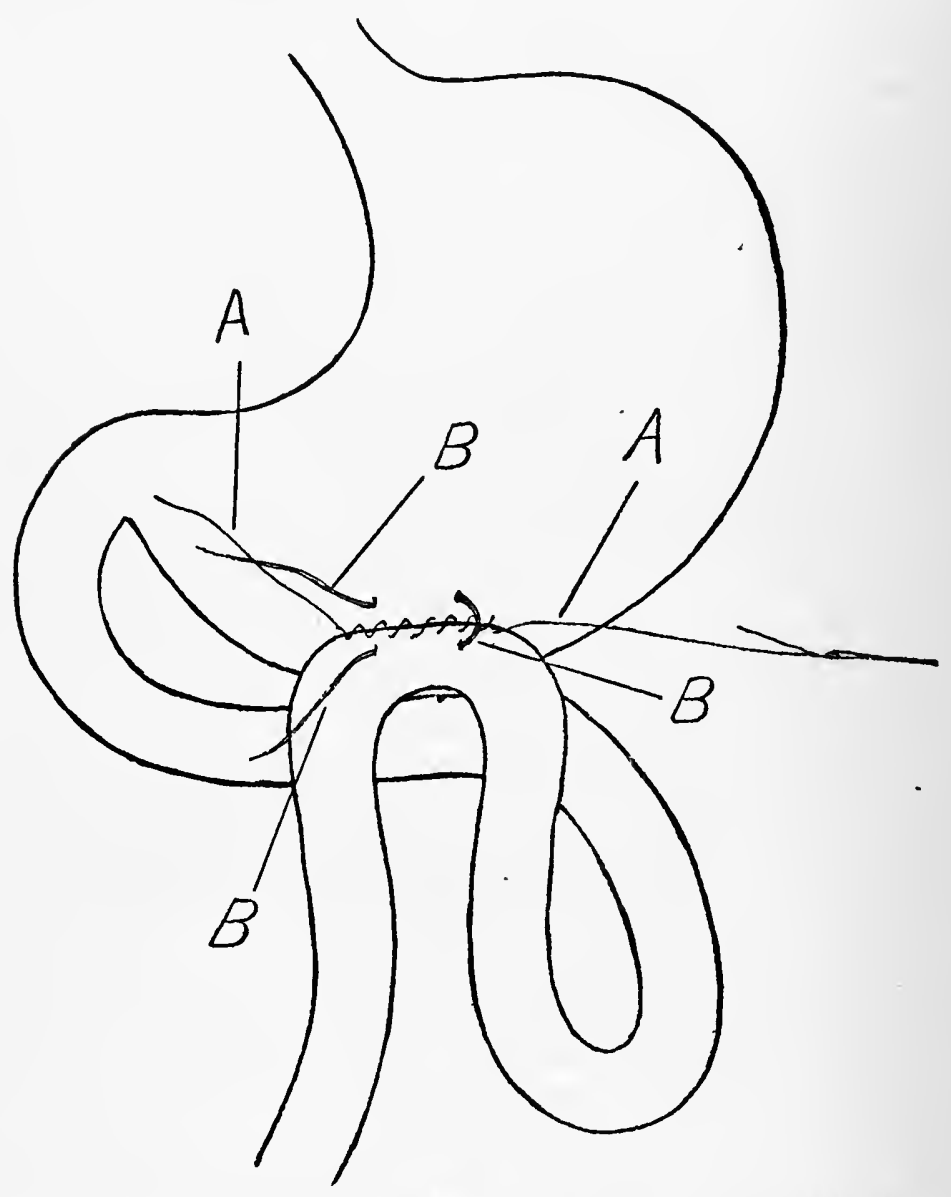

Fig. 126.-Gastro-enterostomy (MCGrav). A loop of intestine has been fixed to the wall of the stomach with a continuous, non-penetrating stitch $(A, A)$. Rubber ligature $(B, B, B)$, which has been passed through the stomach and intestine, ready for tying.

In passing the rubber suture one should make certain that the needle pierces the entire thickness of the wall of the organ and that it does not pick up the mucous membrane on its way; in each viscus 
there should be two punctures only, - one as the needle passes in and one as the needle passes out.

In drawing the rubber suture after the needle, through the wall of the stomach and intestine, it may be stretched so that it becomes thinner, and may thus the more readily follow the needle through the punctures. When the suture is relaxed it becomes increased in diameter so that it more than fills the puncture holes and thus prevents leakage.

Finally, to complete the operation, the needle, carrying the silk thread with which the first half of the Lembert "outside serous ring" suture was applied, is again taken in hand and with it the wall of the stomach and intestine are joined with a continuous stitch, which is applied along a line just outside the location of the rubber ligature, and which buries this latter and completes the "outside serous ring" suture.

By this operation corresponding portions of the opposed stomach and intestine are included in the grasp of a single, tight, elastic rubber suture, which gradually cuts its way through the walls of the opposed viscera; so that after the lapse of two or three days the gastro-enterostomy is established and the liberated rubber suture is passed unobserved through the bowel. This method may be employed in making a lateral anastomosis between two coils of small intestine or between the small and large intestine.

Gastro-enterostomy with Laplace Forceps. - After the stomach and intestine have been brought up into the abdominal incision an opening is made in each viscus, which opening should be rather smaller than the ring of the forceps that is to be used.

The blades of the forceps, joined together and secured with the clamp, are introduced, one into each organ, and then closed, with the result that the margins of each opening are securely grasped between them, serous surface being apposed to serous surface. Care must be exercised to include the entire margin of each opening in the grasp of the closed blades, and this is facilitated by using an instrument whose ring is larger in circumference than the corresponding openings in the stomach and intestine. The wall of the stomach and the wall of the intestine are then united all around the circumference of the ring-blades of the forceps, except for the small space through which the shanks of the forceps emerge. This union should be made with a continuous, non-penetrating Lembert suture of fine silk. The ring-blades of the forceps which are within the stomach 
and intestine retain the parts in accurate apposition, and make the application of the suture a matter of comparative ease.

After the suture has been applied the clamp is removed from the forceps, which is thus separated into its two component parts, the blades of each of which form but half a ring; these are then withdrawn from the stomach and intestine, first one and then the other. Finally the small opening through which the separated forceps were withdrawn is closed with one or two interrupted non-penetrating sutures. A second row of outside non-penetrating Lembert sutures may be applied all around the first row of sutures if desired, in order to make the union of the intestine to the stomach still more secure.

Gastro-enterostomy with O'Hara Forceps. - After the stomach and intestine have been drawn out through the abdominal incision the wall of each is picked up with a mouse-tooth forceps and securely grasped between the blades of one of the forceps, along a line corresponding to the long axis of each organ, in the same manner as one would grasp the prepuce with a circumcision clamp. The length of the wall of the organ thus secured between the blades of the forceps will vary according to the size of the intended opening. The blades of the forceps are graduated so that there should be no difficulty in securing the same amount of each organ, and this is desirable. As the wall of the stomach or intestine is grasped the tip of the blade should reach exactly to the edge of the process thus secured. That part of the wall of the stomach and intestine which protrudes beyond the blades of the forceps is cut away with the knife or scissors fairly close to the blades. The two forceps are then united together and securely locked with the clamp, and we are ready for the next step of the operation, the suturing of the wall of the intestine to the wall of the stomach. This is done with a continuous, non-penetrating Lembert suture of silk. Commencing near the rivet, this suture is carried along the blades of the forceps toward the tip and then around the tip of the forceps and back again upon the other side to a point near the rivet where the suture was commenced. As this suture progresses it serves to bury the blades of the forceps beneath it.

The junction of the stomach and intestine is thus complete except for the small space through which the forceps emerge. In applying the second half of the suture it will be necessary to turn the forceps over and with them the stomach and intestine in order to make the parts upon this under side accessible. The clamp is now 
removed from the forceps, which is thus separated into its two component parts. One forceps is unlocked and withdrawn. The second forceps is unlocked, and, after being passed in and out through the opening between the stomach and the intestine to make certain that this is patent and that none of the stitches have been inadvertently carried across to the opposite margin of the orifice, this is likewise withdrawn. The small opening that still remains and through which the forceps were withdrawn is now closed with one or two non-penetrating Lembert sutures. If desired, an additional row of Lembert sutures may be placed outside the first row in order to strengthen the union.

\section{THE LARGE INTESTINE AND VERMIFORM APPENDIX.}

The Surgical Anatomy of the .Large Intestine, etc.-The large intestine may be distinguished from the small intestine by its large ealiber and by its sacculation; attached along its whole length is the great omentum or the analogues of this structure, the appendices epiploicæ. The large intestine is also marked by three longitudinal bands, which traverse its entire length. These longitudinal bands are made up of an aggregation of the longitudinal muscular fibers; one of them is found along the mesenteric border of the gut, another corresponds to the attachment of the great omentum and the little fatty processes, - the appendices epiploicæ, - and the third is located between these two.

The large intestine may be divided into three parts: the cæcum, colon (ascending, transverse, descending, and sigmoid flexure), and the rectum.

The CAcum is the dilated, pouched commencement of the large intestine. It is found in the right iliac fossa, near the brim of the pelvis, resting upon the psoas or iliacus muscle.

It is provided with a complete peritoneal investment, is movable, and has a mesentery which is short and scrves to anchor it to the posterior abdominal wall. The mesentery is sufficiently long, however, to allow this part of the intestinal canal to be drawn out upon the abdominal wall. The layers of the mesocæcum are but loosely adherent to each other and may be readily separated. The cæcum is continued upward into the ascending colon without any definite line of separation between them.

The Vermiform Appendix is a blind, worm-like process, which is given off from the inner posterior aspect of the cæcum at the 
point where the longitudinal bands meet and from one to one and one-half inches below the junction of the small intestine with the cæcum. It is found lying more or less free in the abdominal cavity or dipping into the pelvis.

The base of the appendix corresponds to a point on the abdominal wall called "McBurney's point," which is located two inches to the inner side of the anterior superior iliac spine, upon a line drawn from the anterior superior iliac spine to the umbilicus.

The appendix varies much in size; it is usually as thick around as a lead pencil and its average length is four inches; it varies from two to six inches and may be longer. Usually it is a hollow tube, its canal extending as far as its tip; at times, however, the canal does not extend to the tip or may be absent entirely. Its inner surface is lined with mucous membrane. The appendix is an intraperitoneal structure, being completely invested by the peritoneum, and in nearly all cases it is provided with a mesentery of its own. This mesentery is a little fold derived from the under layer of the mesentery of the small intestine where the latter enters the cæcum; it incloses the appendix between its folds, and usually extends only part way down to the tip, leaving the lower third or half of the appendix free. This mesentery gives one the impression of being too short, causes the appendix to present its curled-up appearance, serves to limit its range of movement, and holds it in close relation with the cæcum. That part of the appendix which is unprovided with mesentery is freely movable, and in those cases in which the mesentery is nearly or entirely absent the appendix enjoys a considerable range of motion. In most cases the appendix is more or less fixed to the cæcum and to the posterior abdominal wall through its mesentery. Its position, as regards the cæcum, varies in different individuals; most commonly it is found lying upon the inner or left side of the cæcum, with its tip behind the ileum and pointing upward in the direction of the spleen. In other cases it lies upon the outer or right side of the cæcum, rather behind it, its tip pointing upward toward the liver; again, it may be found dipping down into the pelvis or lying across the front of the cæcum. In any of these positions it may be more or less fixed either naturally or by adhesions.

In the female the appendix is connected with the broad ligament by a thin band, the so-called appendiculo-ovarian ligament.

The appendix gets its arterial supply from a single small vessel derived from the ileo-colic, which is a branch of the superior mesen- 
teric. The venous return is through a corresponding single venous channel which empties into the superior mesenteric vein. These vessels run parallel with the appendix in the edge of the mesentery between its two layers; when the mesentery is absent they are found upon the surface of the appendix, beneath its serous coat. In the female the appendix receives an additional vessel through the appendiculo-ovarian ligament. The appendix is dependent for its nutrition upon this very limited blood-supply, and no doubt this arrangement is, at least in part, responsible for the readiness with which the wall of the appendix becomes necrotic when its circulation is disturbed.

Occasionally some difficulty may be experienced in finding the appendix. If the longitudinal bands upon the cæcum are traced downward, they will be found to lead directly to the point where the appendix is given off, and therefore these bands are good guides to the root of the appendix.

Just above the root of the appendix the small intestine terminates by entering the cæcum; it enters the cæcum upon its left side. The opening between the ileum and cæcum is guarded by the ileo-cæcal valve. This valve consists of two folds of mucous membrane containing some circular muscular fibers. These folds, projecting into the lumen of the gut, allow the contents of the ileum to pass freely into the cæcum, but prevent the reverse. Fluids injected through the rectum, into the large intestine, cannot pass into the ileum unless this valve is forced, and that requires enough pressure to threaten the rupture of the large intestine.

The Ascending Colon.-This is the continuation upward of the cæcum. It lies close to the posterior wall of the abdomen. The ascending colon has no mesentery, and is only partly invested by the peritoneum, which is absent upon its posterior surface. The ascending colon ascends along the outer border of the right kidney, lying partly upon the kidney, from which it is separated by some interposed loose connective tissue and fat only. Continued upward as far as the under surface of the liver, it makes a turn-the hepatic flexure-and becomes the transverse colon. The under surface of the liver shows a shallow depression corresponding to the hepatic flexure, and here the colon is attached to the liver by a reflection of peritoneum, the ligamentum hepatico-colicum.

The Transverse Colon stretches from right to left across the upper part of the abdominal cavity, lying below the first part of the 
duodenum and greater curvature of the stomach. Close to the spleen, on the left side, the colon makes a second turn,- the splenic flexure,-and from this point is continued downward as the descending colon. At the splenic flexure the colon is fixed to the diaphragm by a fold of peritoneum, the ligamentum phrenico-colicum.

The transverse colon is completely invested by peritoneum and has a long mesentery which suspends it from the posterior wall of the abdomen. The transverse colon enjoys considerable freedom of movement, but is connected with the greater curvature of the stomach by the peritoneum.

In the very young child the connection of the transverse colon to the greater curvature of the stomach does not exist, because the layers of peritoneum which invest the stomach and unite with each other at the greater curvature to form the great omentum have not become adherent to the peritoneum which envelops the transverse colon; this does not occur until later in life (see Fig. 90).

The Descending Colon passes downward in the left side of the abdominal cavity, lying close to its posterior wall, to which it is partly fixed. It has no mesentery, is only partly invested by the peritoneum, and cannot be drawn out upon the abdomen. The posterior wall of the descending colon, which is devoid of peritoneum, lies close to the outer border of the left kidney, lying partly upon its anterior surface. It is continued below into the sigmoid flexure.

The Sigmoid Flexure is the last part of the colon; it is a redundant loop of gut curved upon itself and lying in the left iliac fossa. Its caliber is rather smaller than that of the other parts of the colon; it is completely invested by the peritoneum, and has a fairly long mesentery, which suspends it to the posterior abdominal wall and permits of much freedom of motion. It may be freely drawn out upon the abdominal wall. At the sacro-iliac synchondrosis it is continued down into the pelvis as the rectum.

The Blood-supply of the Large Intestine. - The cæcum, appendix, and ascending and transverse colon are supplied by branches which are given off from the right, or concave, side of the superior mesenteric artery.

The descending colon and sigmoid flexure are supplied by the inferior mesenteric, which comes off from the front of the aorta just below the origin of the superior mesenteric; after supplying the parts mentioned this vessel dips into the pelvis, between the layers of the mesorectum, to supply the rectum as far as its lower end. 
The arterial branches which are derived from the superior and inferior mesenteric for the supply of the ascending and descending colon, as they pass to their destination, lie upon the posterior abdominal wall covered by the peritoneum which lines the back of the abdomen; those which supply the cæcum, transverse colon, and sigmoid flexure, which parts of the large intestine are provided with a mesentery, reach their destination between the layers of the mesentery corresponding to the part.

The veins have a course similar to the corresponding arteries. The inferior mesenteric joins with the splenic vein, which, in turn, unites with the superior mesenteric to form the portal; hence, blood from the intestinal tract and rectum ${ }^{1}$ must first traverse the portal circulation (through the liver) before entering the general circulation. Poisonous matter from the intestinal tract (colitis, hemorrhoids, etc.) may cause thrombosis in the veins leading from these parts or abscess in the liver, etc.

As is the case with the vessels of the small intestine, the terminals of the arteries that are distributed to the large intestine do not anastomose freely; hence division of a considerable branch will often result in gangrene of the corresponding part of the gut.

\section{OPERATIONS UPON THE LARGE INTESTINE.}

Colostomy.-The formation of a fistulous opening into the large intestine, a so-called artificial anus. It is performed for obstruction in the large intestine or rectum or as a preliminary to extirpation of the rectum. This operation may be done to save life when the danger is imminent and the nature of the lesion or the patient's general condition precludes the probability of doing a radical operation with a reasonable likelihood of success. At times one does not decide upon the formation of an artificial anus until after an exploratory laparotomy shows its necessity.

Unless one can previously locate the seat of the obstruction, the exploratory incision is best placed in the middle line, between the umbilicus and the symphysis, and the artificial anus made by bringing the most accessible portion of the large intestine into this same incision and fixing it there. The bowel above (proximal to) the ob-

1 Some venous blood from the rectum enters the general circulation direct through the middle and inferior hemorrhoidal veins. 
struction is found distended, and that below (distal), diminished in caliber or collapsed.

Descending Colon.-If the obstruction can be located in the sigmoid flexure or rectum, the abdomen is opened in the left iliac region and the lower part of the descending colon is brought up into the wound and fixed to its edges.

An incision about three inches long usually suffices; it is made slightly oblique from above downward and inward, corresponding to the linea semilunaris, the middle of the incision being upon a line drawn from the umbilicus to the anterior superior spinous process.

The incision is carried through the layers of the abdominal wall until the parietal peritoneum is reached. After bleeding points have been clamped the peritoneum is caught up with two mouse-toothed forceps, and between them a small opening, large enough to admit the finger, is made with the knife. This opening is enlarged upon the finger with a blunt-pointed scissors so as to correspond in length with the incision in the skin.

The edge of the peritoneum, upon each side, is then fixed to the corresponding margin of the skin, near the middle, with two or three catgut sutures ( $E$, Fig. 127); this is done to prevent retraction of this layer of peritoneum.

Instead of placing the incision as indicated above it may be made nearer the middle line, so as to pass between the fibers of the rectus muscles, separating between these bluntly with the handle of the knife. This may give an artificial anus which is less difficult to control.

A silk stitch ( $A$, Fig. 12\%) is now passed through the edges of the upper part of the incision, through all the layers, including the skin and the edges of the peritoneum; a second similar suture $(D$, Fig. 127) is passed through the lower part of the incision. These two sutures are not tied. The lower part of the descending colon is now sought and brought up into the wound. In order to secure this two fingers are introduced into the abdomen and carried outward and backward, along the inner aspect of the abdominal wall, as far as the lumbar region, where the colon is found; one may meet with coils of the small intestine, and these may get in the way of the hand, but they may be recognized as being entirely surrounded by peritoneum and easily pushed aside; the fingers are then allowed to glide from the posterior wall of the abdomen on to the colon. The sigmoid flexure should not be brought up into the wound by mistake for the descending colon, because this part of the 
gut has such a long mesentery that, if used to form the artificial anus, it may after a time become very much prolapsed, and this is undesirable. Having secured the descending colon, we select a portion sufficiently high up as to just allow of it being conveniently drawn into the wound. If it can be readily drawn out upon the abdomen we may know that we have the sigmoid flexure, and we should then select a portion of the gut above this.

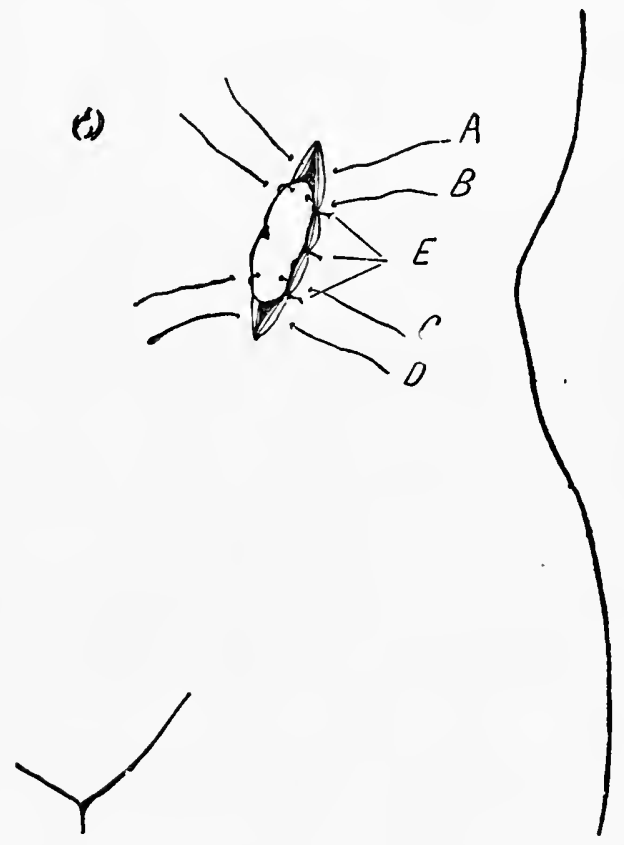

Fig. 127.-Colostomy. The wall of the descending colon drawn Into the incision and fixed. $A, D$, stitches which pass through all the layers of the abdominal wall, including the peritoneum; $B, C$, stitches which pass through all the layers of the abdominal wail, including the peritoneum, but catch up the wall of the gut as well in their course; $E$, stitches that join the parietal peritoneum to the skin.

While that part of the gut which has been selected is steadied in the wound, a silk stitch ( $B$, Fig. 127$)$ in a curved surgeon's needle is passed through the upper part of one edge of the incision, through all the layers, care being taken to include the peritoneum; it then passes superficially through the wall of the gut, picking up its serous and muscular coats and taking a good, broad bite, but not penetrat- 
ing into its lumen, and finally through the opposite edge of the abdominal incision. A second stitch $(C$, Fig. 127) is similarly introduced in the lower part of the abdominal wound, and this also catches the wall of the bowel on the way. These two stitches ( $B$ and $C$ ) should be about two inches apart; they are now tied, likewise the two stitches previously introduced through the edges of the wound, above and below, and the bowel is thus partially fixed in the abdominal incision.

The bowel is now still further fixed to the margins of the abdominal incision by three or four interrupted fine silk sutures on either side; each one of these should secure the serous and muscular coats of the bowel and the edge of the incision in the abdomen, including the parietal peritoneum and skin.

If the condition is not very urgent, the bowel had better not be opened until after the lapse of forty-eight hours, thus allowing time for adhesions to form and shut off the peritoneal cavity. If it is necessary to open the bowel at once a few more extra sutures should be taken in order to secure as accurate a union as possible between the surface of the bowel and edges of the abdominal incision.

The opening in the bowel may be made with a knife, the bowel being held between two forceps, or it may be made with a Paquelin cautery; it should be sufficiently large to allow the introduction of a fairly thick, snugly-fitting tube. Iodoform gauze is packed well about this tube in dressing the wound.

MaYdL MeThod. - An incision is made as above and two or three interrupted catgut sutures are introduced on each side, which serve to fix the parietal peritoneum to the skin ( $E$, Fig. 128). A silk suture ( $A$ and $D$, Fig. 128) is also introduced in the upper and lower ends of the incision. These are left untied until the coil of gut has been secured and are simply for the purpose of diminishing the size of the incision. A convenient portion of the gut is then seized and drawn out through the abdominal incision and a glass cylinder or an artery forceps or a strip of gauze is thrust through its mesentery and placed at right angles to the incision in the abdomen, so as to retain the loop of gut in situ, outside the abdomen, until adhesions have had time to form. The loop of gut may be still further secured by fixing it to the edges of the incision with several catgut sutures. The gut is then, subsequently, divided completely through, down to the glass cylinder or forceps.

If a portion only of the wall of the bowel is fixed to the opening 
in the abdomen, as described in the first operation, there is permitted ready escape of the contents of the bowel, but, at the same time, the possibility of some of the contents passing onward, into the lower segment, is not precluded. This condition is easily restored to the normal, so that this method of operating is preferable unless we

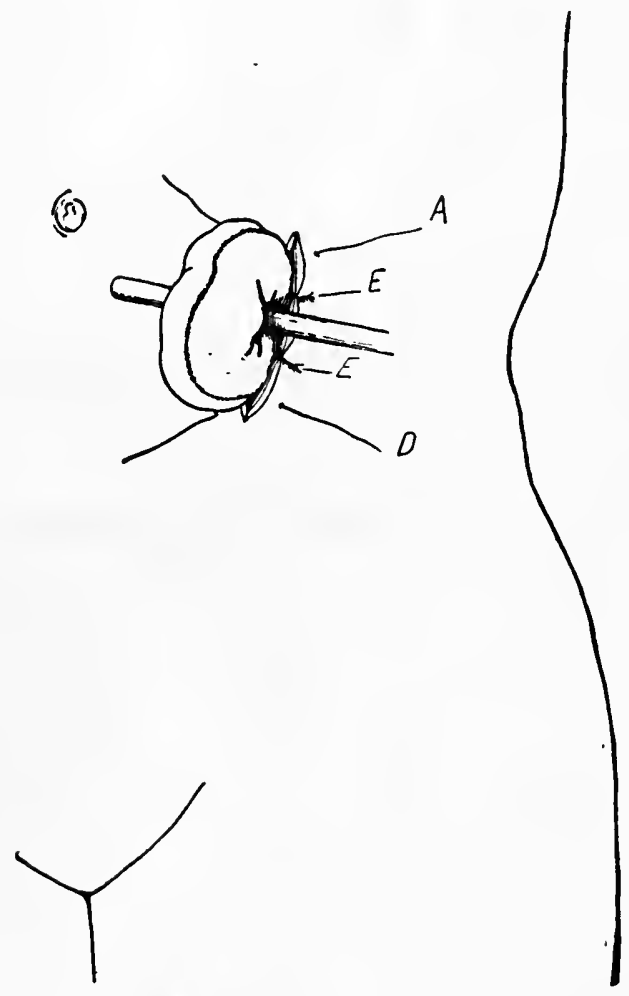

Fig. 128.-Colostomy ( $M / a y d l$ ). A whole loop of large intestine has been drawn out of the abdomen and a glass rod thrust through its mesentery to prevent its returning. $A, D$, sutures that penetrate all the layers of the abdominal wall and serve simply to dimlnish size of the wound. $E, E$, sutures that join the edges of the peritoneum to the skin.

desire the artificial anus to remain permanently. On the other hand, if a whole knuckle of gut is brought out of the wound, as described in the second (Maydl) operation, a "spur" is formed which acts as a valve, directing the bowel contents out through the opening upon the abdomen, and at the same time hindering the passage of any 


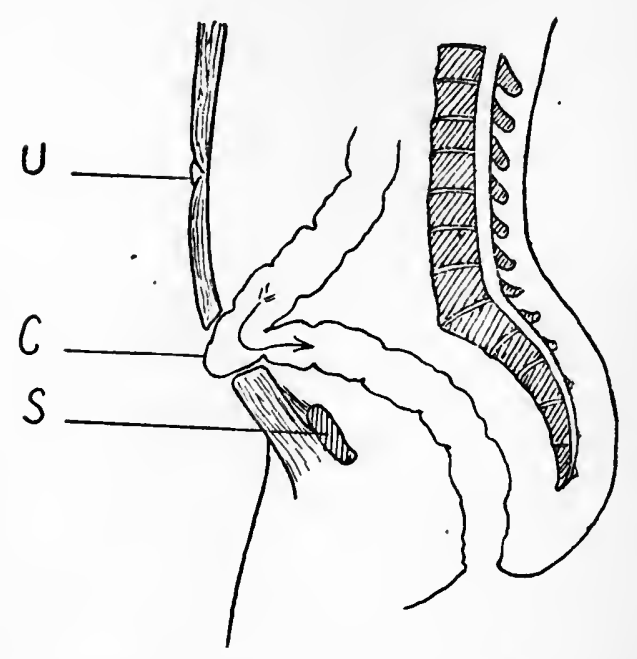

Fig. 129.-Colostomy. Antero-posterior section shows the wall of the colon drawn into the incision in the abdomen. $C$, colon; $S$, symphysis pubis; $O$, umbilicus.

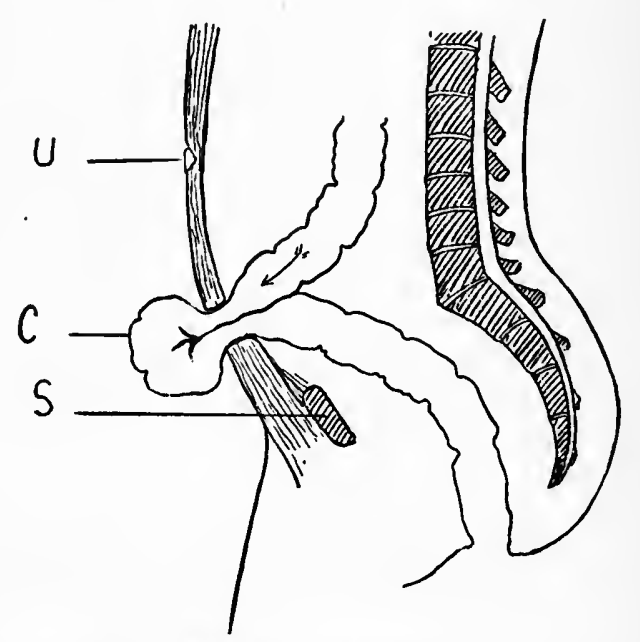

Fig. 130.-Colostomy (Maydl). An antero-posterior section showing a whole loop of intestine drawn out of the incision in the abdomen. The apposed walls of the loop become joined to each other by adhesion, and thus a spur, or partition, is formed which prevents the contents of the upper part of the gut entering the lower portion. C, S, $U$, same as Fig. 129. 
part of the bowel contents onward into the lower segment; it also insures the permanency of the artificial anus.

Ascending CoLon.-If the growth-obstruction-involves the transverse or descending colon, the operation may be performed in a similar manner upon the right side of the body; in this case the lower part of the ascending colon is brought into the wound and fixed.

Resection of the Cæcum.-This may include, in addition to the cæcum, the whole or a part of the ascending colon and part of the ileum. For malignant disease, tuberculosis, and intussusception.

If, before operating, the disease can be located in this part of the gut or a tumor felt, the incision is probably best placed directly over the tumor along the outer border of the right rectus muscle, in the linea semilunaris. If the incision is made for the purpose of exploration, it is usually placed in the middle line, reaching from the umbilicus down, toward the symphysis; through this incision, however, the cæcum may also be excised if found advisable. In either case the incision must be long enough to allow sufficient room for work.

If the incision is made along the outer border of the rectus it should commence about one inch above the middle of Poupart's ligament and is carried in a direction upward to a point located midway between the umbilicus and the anterior superior iliac spine or, if necessary, it may be continued farther upward toward the tip of the tenth rib. It may vary from five to ten inches in length. Having penetrated through the abdominal wall down to the parietal peritoneum, this layer is picked up with two toothed forceps and incised between them. We may then find it necessary to separate some adhesions before the cæcum is exposed. This, together with the adjoining portion of the ileum, is then brought out of the incision upon the abdomen.

If the cæcum is the seat of malignant disease and already so fixed within the abdomen that it cannot be brought out of the wound, it is probably inadvisable to proceed with the extirpation, because, if one is not reasonably certain of removing all the diseased tissue, the risk is probably out of proportion to the best result that can be hoped for.

The cæcum, being steadied outside the abdominal incision, is surrounded by gauze pads to protect the abdominal cavity, and two strips of gauze are tied about the intestine beyond the part which 
is to be excised. Before tying the second piece of gauze the segment of gut may be emptied by stripping it between the fingers. The gauze strips should be placed well beyoud the limits of the part to be excised, and may be carried around the gut in the mouth of a sharp-nosed artery forceps which is thrust through its mesentery.

The mesentery, corresponding to the segment of gut which is to be excised, is tied off in sections with catgut ligatures. The ligatures may be carried in the eye of a blunt ligature carrier or with a pointed-nosed artery forceps. Each ligature should be single and placed some distance away from the gut, so as to leave space to cut between them and the gut. The segment of gut which is to be excised is detached by cutting the mesentery between the ligatures and the gut. One should take care to excise all of the gut whose mesentery has been tied off, because, if an end of the gut which has been deprived of its mesentery is left, it is likely to become gangrenous. It now remains to divide the gut above and below and remove the diseased segment. This is done with a long, straight scissors in one sweep, long clamps having been previously placed upon the gut close to the diseased segment in order to prevent the escape of its contents when it is cut.

Instead of proceeding as above, one may, after the strips of gauze and clamps have been applied about the gut, excise the diseased segment and then tie off the corresponding part of the mesentery in sections as already described.

We are now ready for the final step of the operation, the restoration of the continuity of the alimentary canal by joining the ileum to the colon (ileo-colostomy), and this may be accomplished by:-

1. End-to-end anastomosis (with suture or Murphy button).

2. Lateral anastomosis (with suture or McGraw's rubber ligature or Laplace or O'Hara forceps, etc.).

3. Lateral implantation (with suture or Murphy button).

END-To-End ANastonosis.-This method may be employed if both ends of the gut which are to be united are of the same caliber; this condition at times exists, owing to the fact that the obstruction in the cæcum or at the ileo-cæcal opening may have caused a dilatation and hypertrophy of the ileum, the large intestine at the same time having become more or less diminished in caliber. The anastomosis is made with a double row of silk sutures, the first row passing through all the layers of the wall of the intestine, including the mucous membrane and serous layers, and applied in such a manner 
that the sutured edges are inverted inward into the lumen of the gut; this first row of sutures may be continuous and applied with a medium-sized curved surgeon's needle. The second row of sutures (Lembert) is passed through the serous and muscular coats only, they do not penetrate into the lumen of the bowel, - and serve to bury the first row. This second row may also be continuous. In applying both of these rows great care should be exercised to include the serous coat, especially near the mesenteric attachment. This is the weak spot, especially in suturing the large intestine.

The end-to-end anastomosis may also be made with a Murphy button of largest size.

Lateral Anastomosis.-This is a satisfactory method of restoring the continuity of the intestinal canal, particularly if the ends are of unequal size; for example, in joining the ileum to the cæcum or colon.

The cut edge of each segment of gut is inverted, a margin of three-fourths to one inch being turned in and the opening closed with a continuous silk stitch, which passes through the serous and muscular coats, always taking special care, particularly at the mesenteric border, to appose serous surfaces to each other. A second continuous silk suture is then introduced; this second suture also includes only the serous and muscular coats and serves to bury the first line of suture. The ends of the bowel which have been thus closed up are now placed side to side, overlapping each other for a distance of five or six inches, and they are then united, surface to surface, for a distance of four or five inches with a continuous Lembert suture of fine silk. This forms the first half of the "outside serous ring" suture, and when completed the needle, with the suture left long, is temporarily laid aside.

An incision is then made in each segment of the gut threc to four inches long, but not so long as the line of the Lembert suture (one inch shorter), and at a distance of about one-fourth inch away from the line of the Lembert suture. The corresponding edges of these incisions are then joined together all around with a continuous overhand silk or catgut suture, which includes all the coats of the gut and which may be introduced with a large, straight needle. After the edges of these openings have been thus united, the needle with which the first half of the "outside serous ring" suture was made is again taken up and the second half of the "outside serous ring," Lembert suture, inserted. The gut may be kept free of con- 
tents during the operation, as usual, by strips of gauze passed around each segment of gut beyond the site of the operation. The margins of the gut may be wiped off with a moist bichloride pad as often as they are soiled by escaping intestinal contents.

Having completed the union of the two segments of gut, any rent or opening which is left in the mesentery may be closed by sevcral catgut sutures and the parts returned to the abdomen.

The lateral anastomosis may also be made with MeGraw's rubber ligature in a manner analogous to that described in gastro-enterostomy or with the Laplace or O'Hara forceps.

End-To-Side, Lateral Implantation.-Analogous to Kocher's gastro-duodenostomy (see "Pylorectomy"). This is probably not as satisfactory a procedure as either of the préceding methods. It may be done by suture or with a Murphy button.

After closing the end of the large intestine the end of the ileum is united to the edges of a slit which is made in the large intestine opposite its mesenteric border, the union being accomplished either by suture or with a Murphy button (see "Pylorectomy").

By any of these means the ileum may be joined to any part of the large intestine. Owing to the frequent imperfections of the serous coat at the mesenteric border of the large intestine, the suture in all these operations is always somewhat doubtful, and it may be wise in many cases to leave a strand of gauze, reaching from the sutured gut through the abdominal incision, for drainage in case of leakage.

Ileo-colostomy Without Resection of the Cæcum or Colon.-This operation may be done in cases of obstruction at the ileo-cæcal valve when the advisability of a more radical operation-resection-is doubtful. An anastomosis may thus be made between the ileum and the ascending colon, or, if the obstruction is located in another part of the colon, the anastomosis may be made between the ileum and the sigmoid flexure. Care should be taken to secure a coil of small intestine as low down, near the cæcum, as possible; so that the nutrition of the patient may not be seriously interfered with.

Resection of the Sigmoid Flexure.-This operation is usually performed for malignant obstruction. This part of the large intestine is a favorite seat of malignant disease.

The incision is probably best made analogous to that for excision of the cæcum, but upon the other side of the abdomen, along the outer border of the left rectus, commencing below, about one 
inch above the middle of Poupart's ligament. The sigmoid may also be resected through an incision in the linea alba, extending from the umbilicus downward to the symphysis pubis if such an incision has bcen already made for the purpose of exploration before the growth was definitely located.

The sigmoid, owing to its long mesentery, may be readily drawn out throngh the abdominal incision. It is surrounded by gauze pads to protect the abdominal cavity, and after the mesentery, which is usually quite long, has been tied off in sections, that part of the bowel which is to be resected is clamped off, cut free from its mesenteric attachment, and finally excised. The ends of the bowel are then united, end to end, by suture or with a large Mrurphy button. The same care, etc., should be exercised in suturing near the mesenteris attachment to include the serous coats. After resection of the sigmoid each end of the bowel may be inverted and closed and a lateral anastomosis done as already described in connection with resection of the crecum.

If the sigmoid is fixed and the neighboring parts already infiltrated, it may be better to make an artificial anus above the seat of obstruction and omit the radical operation.

The colon has also becn resected at the hepatic and the splenic flexures, the incision being made above, along the outer border of the corresponding rectus, or in the middle line, from the ensiform cartilage downward to or beyond the umbilicus. The continuity of the canal may be restored by any one of the methods described above.

\section{OPERATIONS UPON THE VERMIFORM APPENDIX.}

Appendicectomy.-Removal of the appendix.

As performed in cases of chronic relapsing catarrhal and recurrent appendicitis, there being no abscess present. In these cases the incision in the abdomen is closed immediately upon completion of the operation and without drainage.

The Incision is oblique from above, downward and inward, and should be about three inches long. Some operators use a much shorter incision: Morris, one and one-half inches. The incision should be sufficiently long to allow proper performance of the operation. It is well to commence with a short ineision, which may be lengthened later should it become necessary.

The incision should be placed about one and one-half inches to 
the inner side of the anterior superior iliac spine, crossing, almost at a right angle, a line drawn from the anterior superior spine to the umbilicus and so arranged that one-third of its length is above the line and two-thirds below it.

All bleeding points should be clamped, but need not be ligated immediately, as they usually stop after the forceps have been applied for a few minutes. The aponeurosis of the external oblique is exposed and divided, separating between its fibers along a line corresponding to the skin incision. The muscular fibers of the internal oblique are then exposed and incised, together with those of the transversalis, which muscle lies beneath the internal oblique; the fibers of these muscles are divided at right angles to their course. Finally the incision is carried through the fascia transversalis and parietal peritoneum, the latter being picked up with two mousetoothed forceps and divided between these. The gut as it lies beneath the peritoneum may be adherent to the latter, and therefore care should be exercised in cutting through the peritoneum not to wound the gut. It may not be necessary to divide the muscles for the whole length of the skin incision. In many cases, if the muscular layer is divided for a distance corresponding to the middle half of the length of the skin incision, this will suffice, and, if necessary, later the incision in the muscles can be lengthened above and below.

In closing this incision each layer should be united separately: first, the parietal peritoneum with a continuous catgut stitch; then the transversalis fascia and the muscles with a second continuous catgut stitch; then the aponeurosis of the external oblique, also with a continuous catgut suture; and finally the skin, with a catgut stitch. The incision in the skin may be closed with an intracuticular suture.

The McBurney Gridiron Incision. - The cut through the skin is the same as that described in the preceding paragraphs; the aponeurosis of the external oblique is split by separating between its fibers, and then two broad, sharp retractors are introduced, and, retracting the skin and aponeurosis, the muscular fibers of the internal oblique are exposed; these are not cut, but are separated.with the handle of the knife in the direction of the fibers, which is nearly at a right angle to the direction of the skin incision. The fibers of the transversalis muscle are next exposed and treated in a similar manner. Two broad, blunt retractors are now introduced and the edges of the muscles drawn apart, when the transversalis fascia is 
exposed and incised, and finally the peritoneum. These last two layers are divided in the same direction as the internal oblique; i.e., at right angles to skin incision.

Two sets of retractors are necessary to hold the wound open: one set, sharp, for the edges of the skin and aponeurosis of the external oblique, retracting from side to side; the other set, blunt, for the muscles and deeper layers, retracting from above and from below. We have thus a four-sided opening in the abdomen which may be enlarged by prolonging the separation and incision in the different layers.

In closing this incision the edges of the peritoneum are first brought together with a continuous catgut stitch. The edges of the muscles of themselves return to place and are secured by two or three interrupted catgut sutures, which include the transversalis fascia also in their bite. The aponeurosis of the external oblique is sewed with a continuous catgut suture from above downward, and the skin then closed with an intracuticular catgut suture.

There being no muscles or aponeurotic fibers cut, the liability to hernia is very much diminished.

The Battle Incision.-A perpendicular incision, four inches in length, which is placed one and one-half inches to the inner side of the linea semilunaris, is made through the skin and fat, down to the aponeurosis of the external oblique. The lower two-thirds of this incision should be below a line which is drawn from the anterior superior spine to the umbilicus. The aponeurosis, which really forms the anterior layer of the sheath of the rectus, is then divided to correspond with the skin incision and the fibers of the rectus thus exposed. The rectus muscle is not cut, but is drawn inward until its outer edge is reached, and, while it is thus held with a blunt retractor, the posterior layer of its sheath is incised for a length corresponding with the incision in its anterior layer. This incision through the posterior layer of the rectus sheath is not placed immediately behind the incision in the anterior layer, but rather external to it. The parts being well retracted, good access is had to the abdominal cavity.

In closing this opening each layer is sutured separately: first, the peritoneum, and then the opening in the posterior layer of the sheath of the rectus. The rectus muscle is allowed to resume its normal position, and a suture is placed in the anterior layer of its sheath. These sutures are all continuous and of simple catgut; 
finally the skin incision is closed. This incision also diminishes the liability to hernia.

After having opened into the abdomen we may proceed with the next step of the operation, the search for the appendix. At times it may be found presenting at once in the wound, more or less changed, thickened, etc., or, occasionally being bound down and fixed within the abdomen by adhesions, it does not come into view, and then it will be necessary to search for it.

The appendix may be directed downward and may dip into the pelvis, or, with its tip pointed upward, it may lie to the outer or to the inner side of the cæcum. It' may be more or less confined in any of these positions by its mesentery or by adhesions.

If difficulty is experienced in finding the appendix, the cæcum may be brought out of the wound to serve as a guide. The cæcum may be identified by its sacculation, by the little fatty processes attached to it, and by its longitudinal, white striæ, two of which can usually be seen: if these striæ are followed they will be found to lead down to the point where the appendix is given off.

The appendix is gently liberated from its adhesions with the fingers,- - there is no danger of hemorrhage in this procedure,--and gradually it is brought out of the wound, the cæcum being at the same time returned into the abdomen.

It is wise, before beginning to free the appendix, to introduce into the wound one or more gauze pads to protect the peritoneal cavity during the removal of the appendix and in the event of unexpectedly meeting a small collection of pus.

After the appendix has been sufficiently freed it is held in the wound or outside the abdominal incision, and after having properly arranged the gauze pads so as to protect the peritoneal cavity, we proceed at once to tie off the mesentery of the appendix. This is done by transfixing the mesentery close to the appendix and near its root with a ligature carrier or with a straight or curved needle carrying a loop of No. 2 simple catgut. This ligature is tied and the appendix then cut away from the mesentery, cutting between the appendix and the ligature with the scissors. The appendix being thus separated from its mesentery, we are ready to proceed with the final step of the operation,--the removal of the appendix. This may be done in one of several ways.

1. Ligature Without Inversion.-After the mesentery has been tied off and cut away from the appendix with the scissors, a 
catgut ligature (No. 2) is tied tightly around the appendix about one-fourth inch distant from its root; the ends of this ligature are left long to serve as a temporary tractor. The appendix is then

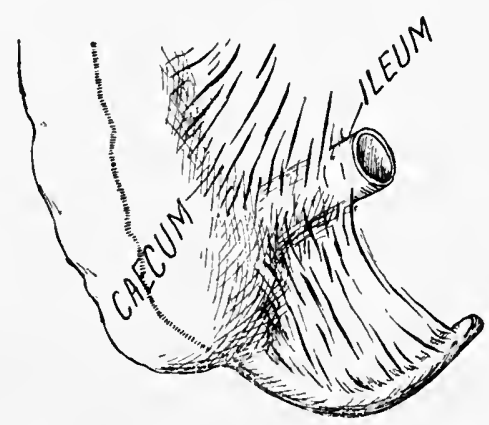

Fig. 131.-Appendix. Meso-appendix shown.

seized with an artery clamp upon the distal side of the ligature to prevent leakage when it is cut, and with the straight scissors it is amputated between the clamp and ligature. While the stump of

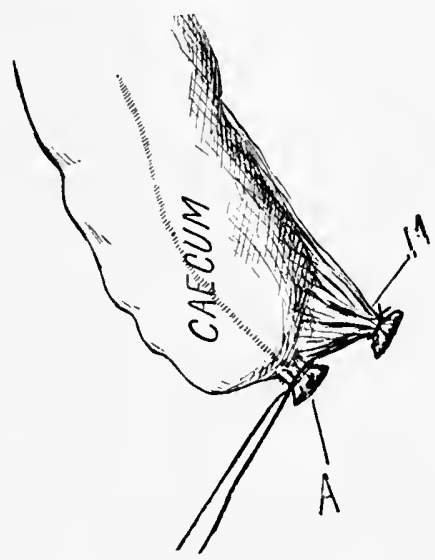

Fig. 132.-Appendicectomy. Appendix (A) ligated without inversion and amputated; $\boldsymbol{H}$, meso-appendix tied off.

the appendix is steadied by making traction with the ligature, which was left long intentionally for that purpose, the raw end of the stump is touched with pure carbolic acid on a very small swab or else it is cauterized with a pointed Paquelin. Some aristol may then be 
rubbed in, the ligature cut short, and the stump of the appendix allowed to drop back into the abdomen.

2. Inversion of the Stump of the Appendix Without LigaTURE.-After the mesentery has been tied off and cut away from the appendix and without applying a ligature around its root, the appendix is seized with an artery clamp and amputated within one-half inch of its junction with the cæcum. The short stump of the appendix that remains is then inverted into the lumen of the cæcum and the opening into the cæcum corresponding to the base of the inverted appendix stump closed with a row of fine silk Lembert sutures. There is no bleeding from the stump of the appendix. The amputation of the appendix may be done with the scissors or the

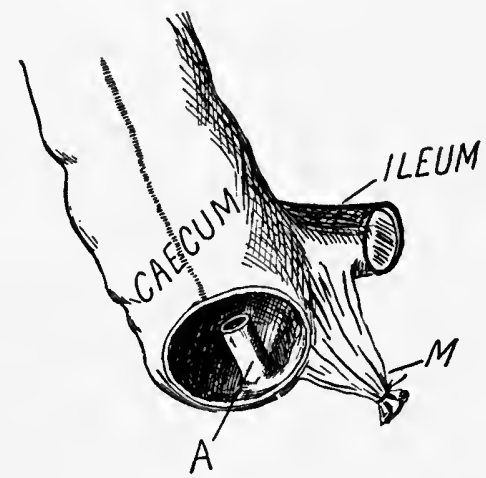

Fig. 133.-Appendicectomy. A portion of the wall of the cæcum removed in order to show the inverted appendix stump $(A)$. $M$, meso-appendix tied off.

Paquelin. This is a very simple and satisfactory way of treating the appendix.

3. Inversion of the Stump of the Appendix with PurseSTRING (DAWBARN).-After the mesentery has been ligated and cut free from the appendix, the latter is steadied and a silk purse-string suture introduced into the wall of the cæcum so as to surround the root of the appendix at a distance of about one-fourth inch all around. This stitch should include only the serous and muscular coats of the cæcum; it should not penetrate into the lumen of the bowel. The purse-string suture is not drawn tight or tied, but the first double loop of a surgeon's knot is taken. Then, without applying any ligature around its root the appendix is seized with an artery 
forceps and cut away with the scissors, leaving a stump about onehalf inch long. The stump does not bleed. One may amputate the appendix with a Paquelin instead of a scissors.

A thin artery forceps or the ends of a thumb forceps are next introduced into the canal of the stump like a glove stretcher, and the stump is stretched. The cut end of the stump is then seized with the thumb forceps or thin artery forceps and inverted into the cæeum; it is turned "outside in" like a reversed glove finger. The foreeps is then withdrawn, and at the same time the pursestring drawn tight, thus leaving the inverted stump presenting into the cæcum and closing the opening in the cæcum. If thought neeessary, one may still further secure the opening in the cxeum by applying two or three Lembert stitches in addition to the pursestring. This is a very satisfactory method of dealing with the stump of the appendix.

4. Inversion of the Appendix (Edebohls).-This procedure is applicable to cases of catarrhal appendicitis that do not demand amputation of the organ. It may also be practiced incidentally during the course of other operations in order to preclude the possibility of the appendix becoming a source of trouble at some future time. The meso-appendix is first tied off close to the root of the appendix and then cut away from the appendix for its whole length. The point of a probe is then applied to the tip of the appendix, and with this the appendix is turned "outside in" into the lumen of the excum as one would reverse the finger of a glove. After the appendix has been inverted into the eæcum and while it is thus held by the probe one or two stitches of silk are taken so as to close the orifice that eorresponds to the root of the turned-in appendix. The probe is then withdrawn and if neeessary another stitch may be taken.

These stitches that unite the margins of the orifice that corresponds to the root of the appendix serve to retain the appendix in its new inverted condition; they are usually of silk and, of course, are non-penetrating.

In connection with any of these methods the stump of the mesentery may, in addition, be sutured over the site of the inverted appendix stump.

During any of these manipulations it is necessary for an assistant to steady the excum, grasping it between the fingers with a gauze pad, which gives a better hold and at the same time protects it from becoming soiled. 
The incision in the abdomen is finally closed, as indicated above, without drainage. Proper apposition and primary healing of the incision are necessary to secure the patient from the liability to subsequent ventral hernia.

Operations for Appendicular Abscess.-Cases that go on to suppuration, resulting in the formation of a localized intraperitoneal abscess which is shut off from the general peritoneal cavity by adhesions between immediately adjacent peritoneal surfaces.

The abscess is opened and drained, the appendix being removed at the same time or left, according to the circumstance of each individual case.

During the opening of the abscess and the removal of the appendix care should be exercised not to break through the barrier of adhesions, which are the result of nature's effort to protect the general peritoneal cavity from infection.

The location of the abscess differs in different cases: it may be located anterior to the cæcum within a mass of matted guts and may be opened as soon as the incision in the abdominal wall is carried through the parietal peritoneum. The abscess may be located behind and to the outer or right side of the cæcum, reaching upward toward the kidney and liver or downward into the pelvis, or it may be located to the inner or left side of the cæcum, or it may lie almost entirely within the pelvis and cause symptoms of pressure upon the bladder. Occasionally there is more than one collection, and care should be exercised that such a condition does not escape our attention at the time of the operation.

The Incision. - The simple incision described above, cutting through the various layers, is usually employed. It should be, as a rule, about four inches long, and may be increased if necessary to allow proper work. The position of the incision may be somewhat changed from that described above in order to better expose the tumor; thus it may be placed farther away from the iliac spine-nearer the middle line, or lower down, nearer Poupart's ligament if the position of the tumor should indicate.

Some surgeons use McBurney's gridiron incision for abscess cases as well as for the simple appendicectomies.

The incision is carried through the abdominal wall, layer by layer, until the peritoneum is reached, and then after the hemorrhage has been controlled the peritoneum is incised carefully in order to avoid wounding the underlying gut, which may be adherent to the 
parietal peritoneum. This is best done by seizing the peritoneum with two mouse-tooth forceps and cutting between them.

Having incised the parietal peritoneum, we may find ourselves at once within the abscess cavity. In such cases the abscess is found to be located in front of the cæcum. When this condition exists, we may often note, in making the incision through the abdominal wall, that the deeper layers-subperitoneal tissue, etc.-are œdematous and infiltrated.

In most cases, however, after the parietal peritoneum has been incised, we come down upon a mass, consisting of the crecum and small intestine adherent and matted together, and within this the appendix and abscess are inclosed. This mass may be still further adherent to the overlying parietal peritoneum, which lines the posterior surface of the anterior abdominal wall, in which case the general peritoneal cavity is excluded from the likelihood of infection from the field of operation; on the other hand, this adhesion between the mass of matted intestine and the immediately adjoining parietal peritoneum may be absent; so that, when the fingers are introduced into the abdominal cavity through the incision, they may be passed freely in all directions, between the matted mass and the overlying parietal peritoneum: inward toward the umbilicus, upward toward the liver, and downward into the pelvis.

Having exposed the mass within which the abscess and appendix are inclosed, we are ready to evacuate the abscess. Before doing this, however, the parts should be properly protected by gauze pads placed around and into the incision in the abdomen, and, if the condition exists as described above,-i.e., if no adhesions have been formed between the mass of intestine which incloses the appendix, etc., and the parietal peritoneum,- - the pads should also be tucked into this space (between the matted mass and the anterior abdominal wall), in order to block it off, so that when the abscess is opened the entrance of pus into the general peritoneal cavity will be prevented. Later, after the abscess has been evacuated, etc., these pads may be removed and replaced by fresh sterile pads or strips of gauze, which are allowed to remain, with their ends protruding from the wound, in order to obstruct this space and to promote the formation of protecting adhesions.

The abdominal incision is held open with retractors and search made for the abscess. The appendix is not to be seen, being adherent and buried within the mass of matted guts. We can locate the point 
where the appendix comes off from the cæcum by following down along the course of the longitudinal striæ and gently, with the fingers, working between the adhesions until the abscess is reached.

As the abscess is opened the patient may be turned on the right side to facilitate the escape of the pus, which is swabbed away as fast as it escapes. The abscess cavity may be gently flushed with peroxide of hydrogen, which may be preceded and followed by irrigation with saline solution.

If the suppurative process involves the connective tissue behind the colon, reaching up toward the kidney, a counter-opening may occasionally, with advantage, be made in the loin.

After the pus has been evacuated and the abscess cavity sterilized we may attempt the removal of the appendix. Too much force, however, especially in the hands of inexperienced operators, should not be used in this effort, and one may wisely in many cases terminate the operation at this stage, being content with packing the abscess cavity and leaving the appendix to take care of itself, or to be removed later after suppuration has ceased, thus giving the patient the best chance for relief from immediate danger.

There is no question as to the desirability of removing the appendix at the time that the abscess is opened if the conditions permit, and one should make an effort to accomplish this.

If the appendix lies to the inner side of the cæcum, there is probably more danger in attempting to remove it than if it is located to the outer side or below. The manipulations required to remove the appendix where an abscess has been present may cause a breaking through of the adhesions and may be followed by peritonitis, under which circumstances the patient's chance of recovery is greatly diminished. On the other hand, to leave the diseased appendix in the wound subjects the patient to the liability of a fæcal fistula, and -of more consequence than this-to a subsequent attack of appendicitis, which may be fatal.

At times, after the pus has been evacuated, the appendix is fairly accessible, and may be felt or seen in the abscess cavity; so that, by farther separating the adhesions with the fingers and with the introduction of deep retractors, it may be reached and removed. It usually suffices to simply tie a catgut ligature around the appendix close to its root-say, one-half inch distant from the cæcum-and amputate it with the scissors. The stump, which is steadied by the ligature, left long for this purpose, may then be sterilized with pure 
carbolic acid or the Paquelin. If conditions permit, instead of treating the stump in this simple manner it may be inverted into the crcum, as described above.

For drainage the most satisfactory material is iodoform gauze, which may be loosely packed in the abscess cavity or else made into separate bundles inclosed in perforated casings of oil-silk or guttapercha tissue; in addition to the iodoform gauze it is often advisable to use one or more glass or rubber tubes perforated on the sides, especially if the abscess cavity reaches down into the pelvis. Some surgeons use lamp-wick instead of the gauze for drainage.

The wound in the abdomen should be closed in part with two or three silk-worm sutures which include all the layers, especially the parietal peritoneum, and which are introduced with a large curved surgeon's needle. Several similar sutures may also be introduced through the edges of that part of the wound which is to be left open, but these are left untied until after the suppuration has ceased and the drains have been removed.

Operations for Appendicitis Accompanied by Progressive or General Peritonitis or Peritoneal Infection due to perforation or sloughing of the appendix before adhesions have been formed or to rupture or leakage of an appendicular abscess after adhesions have been formed. In these cases the appendix should be removed and an attempt made to prevent or check the general peritoneal infection.

The incision should be sufficiently long-four to six inches; if a tumor has existed in the right iliac region it is probably best to place the incision in the right semilunar line, reaching down to Poupart's ligament. The incision in other cases may be better placed in the middle line of the abdomen between the umbilicus and the symphysis pubis, especially if previously there has been no tumor in the right iliac fossa and the onset has been sudden or in cases where the diagnosis is somewhat in doubt and signs of a general peritonitis are present.

After the abdomen has been opened the appendix is at once sought and removed. The fluids in the immediate neighborhood of the appendix are sponged away, and the whole abdominal cavity and intestine thoroughly flushed with normal salt solution. The pelvis, where fluids are especially apt to collect, should be cleared and flushed. This flushing should be thorough, using quarts of water poured from a pitcher or thrown into the abdomen through a thick glass tube. This washing should be done, if possible, without re- 
moving the intestines from the abdominal cavity, as this adds greatly to the shock, and there may be some difficulty in replacing them. One may follow the saline irrigation with peroxide of hydrogen, this in turn being followed up by a final washing with saline solution.

If the intestine is coated with flaky exudate and matted with fresh adhesions, it may be advisable to break these up with the fingers in order to make the flushing satisfactory and thorough.

A glass or rubber drainage tube with a gauze strip passed through it may be introduced into the abdomen, reaching well down into the pelvis, and strips of iodoform gauze and drainage tubes may be introduced into the abdomen down into the region of the appendix, and in other directions also. The incision in the abdomen is closed in part with interrupted sutures of silk-worm gut which pass through all the layers of the abdominal wall, including the parietal peritoneum.

\section{THE LIVER AND GALL-BLADDER.}

The Surgical Anatomy of the Liver.-The liver is a solid glandular organ almost completely invested by the peritoneum, suspended in the upper right portion of the abdomen (right hypochondrium) and extending beyond the middle line into the left side (left hypochondrium). It is situated under cover of and protected by the ribs, except in the epigastric region. Behind and toward the right the liver is thick, gradually becoming thin toward the front and left. From side to side it measures eleven inches; from before backward, eight inches; and its posterior border has a thickness of two and one-half inches.

Above, the diaphragm separates the liver from the pleura and pericardium; below it are the gall-bladder, hepatic flexure of the colon, the first part of the duodenum, the pylorus and stomach (which it overlaps), and the right kidney and suprarenal capsule.

The superior surface of the liver looks forward as well as upward, and is in relation with the diaphragm and with the ribs and costal cartilages from the fifth or sixth to the tenth. The lower limit of this surface corresponds to the free border of the ribs (costal cartilages). This upper surface of the liver is smooth, and presents a fold of peritoneum running from the anterior border backward, the suspensory ligament. This serves to suspend the liver to the diaphragm, and is the continuation of the falciform fold of peritoneum, which is thrown around the round ligament from the anterior 
abdominal wall and which extends from the umbilicus to the anterior cdge of the liver. The suspensory ligament divides the upper surface of the liver into the larger right lobe and the smaller left lobe; the latter overlaps the stomach and reaches to the left beyond the middle line. Toward the posterior border of the liver the folds of the suspensory ligament spread out right and left, and, still passing between the liver and the diaphragm, form the anterior layer of the coronary ligament.

The posterior border of the liver, really a surface, is thick, gradually becoming thin toward the left, and is not covered by peritoneum; the peritoneum which covers the upper surface of the liver upon reaching its posterior border is reflected upward to the diaphragm as the anterior layer of the coronary ligament, and that which covers the under surface upon reaching the posterior border of the liver is reflected on to the posterior abdominal wall (diaphragm), forming the posterior layer of the coronary ligament. The coronary ligament, at either end, forms the right and left lateral ligaments of the liver. The posterior border of the liver, to the left of the middle line, presents a notch which corresponds to the œsophagus and which marks the division of the liver into its right and left lobes. The posterior border of the liver is in relation with the diaphragm and lower ribs, with the vertebral column, tenth and eleventh dorsal, the aorta, vena cava inferior, etc. The œsophagus is received in the notch above mentioned.

The anterior border is thin, reaches just below the free border of the ribs (costal cartilages), and corresponds to a line drawn from the tip of the right tenth to the tip of the left eighth costal cartilage, where this joins the cartilage of the seventh.

The under surface of the liver is irregular and marked by grooves and impressions for the colon, gall-bladder, kidney, etc., and is covered by the peritoneum, which is reflected downward at the transverse fissure, as the lesser omentum, as far as the lesser curvature of the stomach, where its folds separate to include the stomach between them.

Besides the right and left lobes, the under surface of the liver presents three smaller lobes: the quadrate, caudate, and the lobus Spigelii. The large right lobe is marked by the transverse fissure, which passes from right to left and is situated rather more than half-way back from the anterior border.

At this fissure, the ressels, ducts, lymphatics, and nerves pass 
in and out of the liver. These structures descend in the right free border of the lesser omentum, between its two folds, the common bile-duct to the right, the hepatic artery to the left, and the portal vein between and behind these two. The hepatic duct, which is formed by the junction of the right and left bile-ducts, emerges from the right end of the transverse fissure and descends between the folds of the lesser omentum, where it is joined by the cystic duct to form the common bile-duct, ductus choledochus.

If we examine the under surface of the liver as this organ lies in its normal position in the abdomen, through a vertical incision made in the abdomen from the tip of the ninth costal cartilage, we note, in sweeping across the surface from right to left, two wellmarked grooves, or depressions, into which the finger sinks; the first, that toward the right, corresponding to the tip of the ninth costal cartilage, lodges the gall-bladder; the second, nearer the middle line, corresponds to the round ligament (fotal umbilical vein).

The Surgical Anatomy of the Gall-bladder.-The gall-bladder is a pear-shaped, hollow receptacle. Its wall is fairly thick and is conposed of muscle and mucous membrane. The serous coat (peritoneum) invests the under surface of the body and all of the fundus of this organ, binding it to the under surface of the liver. The gallbladder lies in direct relation with the under surface of the liver, in the fossa of the gall-bladder, the apposed surfaces of the gallbladder and liver being joined to each other by loose connective tissue.

The fundus of the gall-bladder is directed downward, forward, and to the right, usually appearing below the anterior thin edge of the liver, opposite the tip of the ninth costal cartilage. Sometimes it does not reach quite as far as the anterior edge of the liver, and is then concealed underneath the liver. The edge of the liver, corresponding to the fundus of the gall-bladder, is sometimes marked by a slight notch.

The gall-bladder is three to four inches long and has a capacity of about one and one-half ounces. The fundus rests upon the transverse colon, and the neck, the posterior narrow part, upon the first part of the duodenum. To the outer side of the gall-bladder-i.e., to the right-is the hepatic flexure of the colon; to the left is the pyloric end of the stomach. The neck of the gall-bladder is continued into the cystic duct. The cystic duct is about one-twelfth 
inch in diameter and one to two inches long. Its interior has an irregular, spiral, twisted shape, which makes difficult the passage of a sound through it. It curves down behind the first part of the duodenum, and joins with the hepatic duct between the folds of the lesser omentum to form the common bile-duct.

The hepatic duct is one-sixth inch in diameter and two inches long; it is formed by the junction of the bile-ducts from the right and left lobes of the liver.

The common bile-duct, ductus communis choledochus, varies

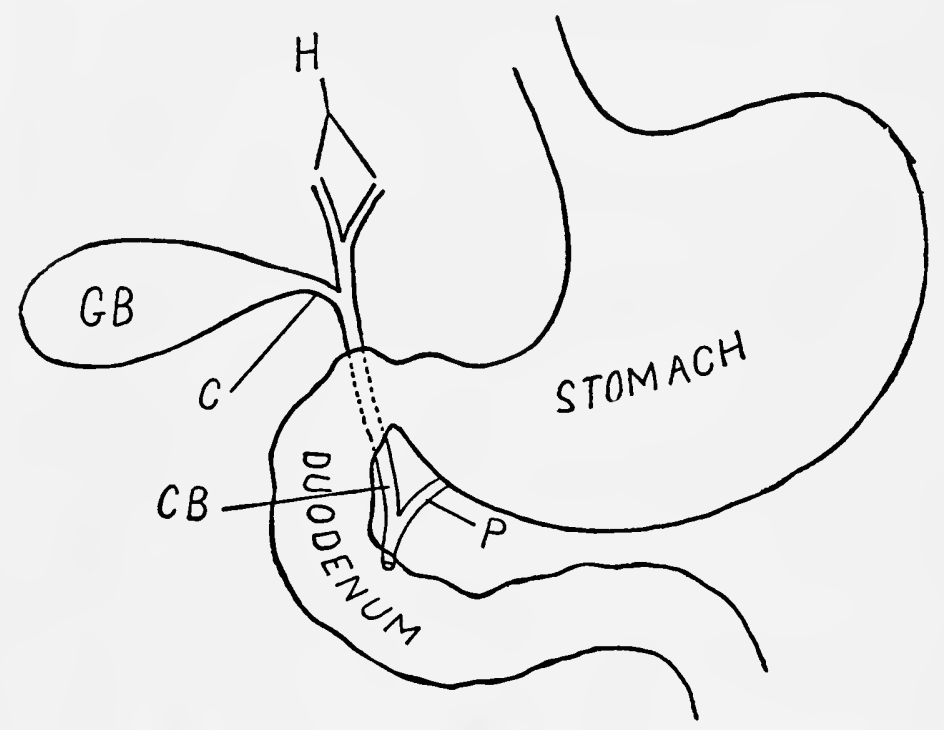

Fig. 134.-Bile-ducts, etc. $C$, cystic duct; $C B$, common duct; $G B$, gall-bladder; $H$, hepatic ducts; $P$, pancreatic duct.

in length: it is usually three inches long and one-fourth inch in diameter; it continues the course of the hepatic duct, descending between the folds of the lesser omentum, lying near its right free edge. In this situation it lies in front of the portal vein with the hepatic artery on its left side; continuing downward it passes behind the first part of the duodenum, and finally behind and to the inner side of the second part of duodenum, between it and the head of the pancreas, where it meets the pancreatic duct; these unite and perforate the wall of the duodenum (second part) upon its inner, posterior aspect, running very obliquely in the wall of this part of the 
gut for one-half to three-fourths inch; the opening of the duct upon the inner surface of the gut is marked by a papilla. This papilla is distant about four inches from the pylorus.

\section{OPERATIONS UPON THE LIVER.}

Hepatotomy.-Incision of the liver for abscess, hydatid cyst, etc.

The incision, when the disease involves the right lobe, is placed along the outer border of the rectus muscle, extending from the tip of the ninth costal cartilage downward for a distance of from three to fire inches.

At times it may be desirable to place the incision elsewhere in order that it may correspond with the prominence of the tumor; for example, if the abscess is located in the left lobe of the liver, then the incision is better placed in the middle line, linea alba. The incision is carried through the integument, fascia, etc., down to the peritoneum, and after the hemorrhage has been controlled the parietal peritoneum is incised between two mouse-tooth forceps. We may find the tumor adherent to the parietal peritoneum, and in this case, after aspirating to discover the nature of its contents, we may incise the tumor and evacuate. The finger is then introduced into the abscess cavity to explore and break up septa, etc. The cavity is finally packed with iodoform gauze. Under these circumstances the operation is very simple and there is no danger whatever of infecting the general peritoneal cavity.

If, however, after incising the peritoneum we find that the tumor is not adherent to the parietal peritoneum,-i.e., if we can pass the hand freely between the tumor and the parietal peritoneum,we must take measures to prevent infection of the general peritoneal cavity while the contents of the cavity are being evacuated, and to accomplish this we do the operation in two sittings.

First, having exposed the tumor, the parietal peritoneum is united to the edges of the skin with two or three catgut sutures on either side, and then strips of gauze are packed into the wound between the tumor and parietal peritoneum for the purpose of shutting off the general peritoneal cavity in case of leakage and to stimulate the formation of adhesions between the tumor and the parietal peritoneum. One may then aspirate with a fine needle in order to discover the nature of the contents and to relieve the tension somewhat.

The incision in the abdomen is left open and packed down to 
the surface of the tumor or the incision may be closed in part by one or two sutures of silk-worm gut which pass through all the layers of the abdomen, including the parietal peritoneum.

After an interval of several days, when adhesions have formed, etc., the tumor, abscess, or hydatid cyst may be incised either with the Paquelin cautery or the knife, and drained.

Hepatectomy (Excision of Diseased Portion of the Liver).-Portions of the liver have, in a few instances, been excised when involved primarily or by extension from growths of the gall-bladder and ducts either by means of the Paquelin cautery or by blunt dissection (enucleation) with the finger. Large individual vessels may be clamped and tied as they are met with during the operation. The space which remains in the liver after the removal of the diseased part may be closed by approximation of its edges with sutures of catgut; but if there is much tension these may tear through.

Injuries of the Liver.-The liver may be lacerated by blows upon the abdomen, by fractured ribs, or by bodies causing penetrating wounds. These injuries may be accompanied by free hemorrhage. On account of the solid structure of the liver large venous channels cannot collapse, and thus hemorrhage is favored. Hemorrhage may be controlled by the cautery or by packing, or by packing combined with suture.

\section{OPERATIONS UPON THE GALL-BLADDER.}

Aspiration of the Gall-bladder.-Drawing off the contents of the gall-bladder, usually for purposes of diagnosis.

Aspiration may be resorted to in order to determine the nature of a tumor which can be felt through the abdominal wall.

The needle is introduced over the most prominent part of the tumor, usually below the tip of the ninth costal cartilage, and some of the contents withdrawn. 'The needle should be of small caliber.

This is a dangerous procedure and one to be condemned, even if the needle and skin are made aseptic, because some of the contents is very apt to escape through the puncture in the wall of the gallbladder upon withdrawing the needle, especially if the needle used is not fine, and if the material is infectious a fatal peritonitis may be thus set up.

Cholecystotomy.-Opening the gall-bladder for the purpose of removing stones, etc.

An incision is made which reaches from the tip of the ninth 
costal cartilage vertically downward, in the linea semilunaris, along the outer side of the rectus or just exposing the outer edge of this muscle, three to four inches long. Having cut through the several layers of the wall of the abdomen, the parietal peritoneum is exposed. Before incising the peritoneum, all blecding points in the abdominal wall are clamped. The parietal peritoneum is then caught up with two mouse-tooth forceps and snipped between these with the knife, whereupon the finger is introduced and the opening enlarged with the scissors, cutting upon the finger as a guide.

Instead of the vertical incision one may make an oblique incision, one finger's breadth distant from and parallel with the free border of the ribs, the middle of the incision corresponding to the tip of the ninth costal cartilage. This incision is usually four to five inches long. The vertical incision is probably the preferable one. After the parietal peritoneum has been incised the sharp anterior edge of the liver is exposed in the upper part of the incision and the transverse colon in the lower part of the incision. The gallbladder may be seen more or less distended, projecting beneath the anterior border of the liver, or it may be small and concealed beneath the edge of the liver.

Occasionally in order to bring the fundus of the gall-bladder into the wound it may be necessary to draw the liver well upward or to incise the thin layer of liver-tissue that overlies the fundus of the gall-bladder, or it may be necessary, with the finger, to break up some adhesions that bind down the gall-bladder.

In the cadaver some difficulty may be experienced in locating the gall-bladder and bringing it up into the wound, as the liver, postmortem, sinks backward and away from the anterior abdominal wall and upward into the thorax, carrying the gall-bladder with it. The gall-bladder is sought in the fossa of the gall-bladder upon the under surface of the liver, its fundus corresponding to the tip of the ninth costal cartilage.

When the fundus of the gall-bladder has been located, two silk tractors may be introduced into its wall to steady it; these should take a good, firm, broad bite, but should not penetrate through the entire thickness of its wall. Gauze pads are then tucked into the incision and around the gall-bladder in order to shut it off from the peritoneal cavity.

If the gall-bladder is much distended, one may aspirate to discover the nature of the contents and to diminish the tension and 
size of the tumor, and then, making an incision in its fundus, it is emptied of its contents, any remaining fluid being withdrawn with sponges on a stick, and the finger introduced for exploration. If any stones are present, these may be removed with a scoop or forceps. With the hand in the abdominal cavity one should thoroughly palpate the cystic, hepatic, and common bile-ducts. Stones impacted in the cystic duct may be forced back into the gall-bladder and removed.

Having emptied the gall-bladder and convinced one's self that . the ducts are patent and if the contents of the gall-bladder were not purulent, one may proceed at once to close the opening in the gallbladder and the incision in the abdomen.

To test the patency of the ducts one may introduce a gum catheter through the incision in the gall-bladder into the cystic duct and onward through this into the common duct. Owing to the twisting and irregularity of the interior of the cystic duct, however, the catheter may catch in its wall and fail to pass even when the duct is pervious. If not successful with the catheter, Abbe has suggested a stream of water introduced into the gall-bladder under pressure; if it flows freely it indicates the patency of the ducts.

The incision in the gall-bladder may be closed with a double row of sutures. The first row, which may be of catgut, includes the whole thickness of the wall and serves to close the opening; it may be continuous or consist of several interrupted stitches, This first line of suture is reinforced by a second row of Lembert sutures, which should include only the serous and muscular coats of the gallbladder; these serve to bury the first row and bring the adjoining serous surfaces into accurate apposition. Unless one is certain that no obstruction exists in the bile-ducts, it is advisable to allow the opening in the gall-bladder to remain; i.e., to sew the edges of the incision in the gall-bladder to the margins of the abdominal incision (cholccystostomy).

If the incision in the abdomen is to be closed, this is effected by uniting, first, the edges of the parietal peritoneum with a continuous catgut suture. The transversalis fascia and muscle (aponeurosis) are then brought together with a second continuous catgut suture, and finally the edges of the skin are united by a catgut suture. After the edges of the peritoneum have been sutured we may approximate the other layers-skin, aponeurosis, fascia, ctc.-with several interrupted sutures of silk-worm gut, each suture including all of these layers, but omitting the peritoneum. 
Cholecystostomy.-Formation of a fistulous opening in the gallbladder; for the removal of calculi from the gall-bladder. The operation may be done in one or two sittings.

An incision as described in the preceding operation, either vertical, passing from the tip of the ninth costal cartilage downward, or oblique, parallel with the free border of the ribs. Probably the vertical incision is preferable in most cases.

The incision may be located nearer to the middle line if the presence of a tumor indicates, so that it may be over the most prominent part of the tumor.

Cholecystostomy in oxe Sitring.-Having opened into the abdomen, the gall-bladder is usually found distended and presenting beneath the free anterior edge of the liver, and may be more or less adherent to neighboring parts-colon, duodenum, etc. These adhesions may be gently broken down by the fingers in the abdomen and the cystic; hepatic, and common bile-ducts palpated for an impacted calculus, etc.

The edge of the parietal peritoneum is fixed to the margin of the skin by two interrupted catgut sutures on either side. The gall-bladder is then drawn into the incision, and, after pads have been arranged so as to protect the peritoneal cavity, two silk tractor sutures are introduced into its fundus, care being taken to avoid passing entirely through the wall into its cavity. While the bladder is steadied by the tractor sutures it is emptied of its contents as completely as possible with the aspirator or a trochar, and an incision then made in the fundus of the bladder and the edges of the incision seized with artery forceps. The contents of the gall-bladder should be entirely evacuated, any remaining fluid being sponged out with pads upon sticks, and stones removed with a scoop or forceps. A stone impacted in the cystic duct may be pushed back into the bladder and removed. If the cystic duct has been obstructed, as soon as the obstruction is relieved there is apt to be a copious flow of bile from the cystic duct into the gall-bladder. This bile should be prevented from entering the peritoneal cavity. Although bile in the peritoneal cavity does not cause a septic peritonitis, still its entrance in any considerable quantity should be avoided.

After having explored the interior of the gall-bladder, removed stones, examined the ducts, and tested their patency, we are ready to sew the edges of the opening in the gall-bladder to the edges of the incision in the abdominal wall. This is done with a number of 
interrupted silk sutures placed fairly close together, the ends being left long to facilitate their removal later. The stitches should be about one-fourth inch apart. Care should be taken to bring the peritoneal surface of the gall-bladder into accurate apposition with the parietal peritoneum, which has already been sutured to the skin. That part of the abdominal incision which is to be closed should be brought together with several silk-worm gut sutures, each passing through all the layers and including especially the parietal peritoneum. The stitches, above and below, immediately adjacent to the fixed gall-bladder, may also include the wall of the gall-bladder in their course.

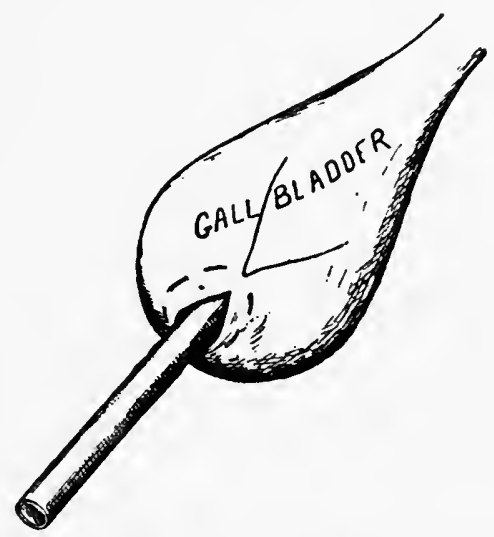

Fig. 135.-A Purse-string Suture has been Introduced Around the incision in the Fundus of the Gall-biadder Close to its Edge. When the pursestring is drawn tight it tends to invert the edges of the opening around the tube, and thus accelerates its closure.

Finally a drainage tube is introduced into the opening in the gall-bladder and the wound loosely packed with iodoform gauze.

McBurney Modification.-In order to facilitate the rapid obliteration of the resulting fistula, the gall-bladder may be united to the edge of the abdominal incision by a row of sutures which do not include the edge of the incision in the gall-bladder, but which catch its wall without passing entirely through it, just external to the opening all around; and then, before the drainage tube is introduced, a purse-string suture which passes through the whole thickness of the wall of the gall-bladder is introduced around and close to the margin of the opening in the gall-bladder, so that when it is drawn tight it will grasp the tube and at the same time invert the 
edges of the opening in the gall-bladder closely around it. The wound is then packed and dressed in the usual way. After the tube is withdrawn the opening in the gall-bladder, with the inverted edges, tends to close more rapidly.

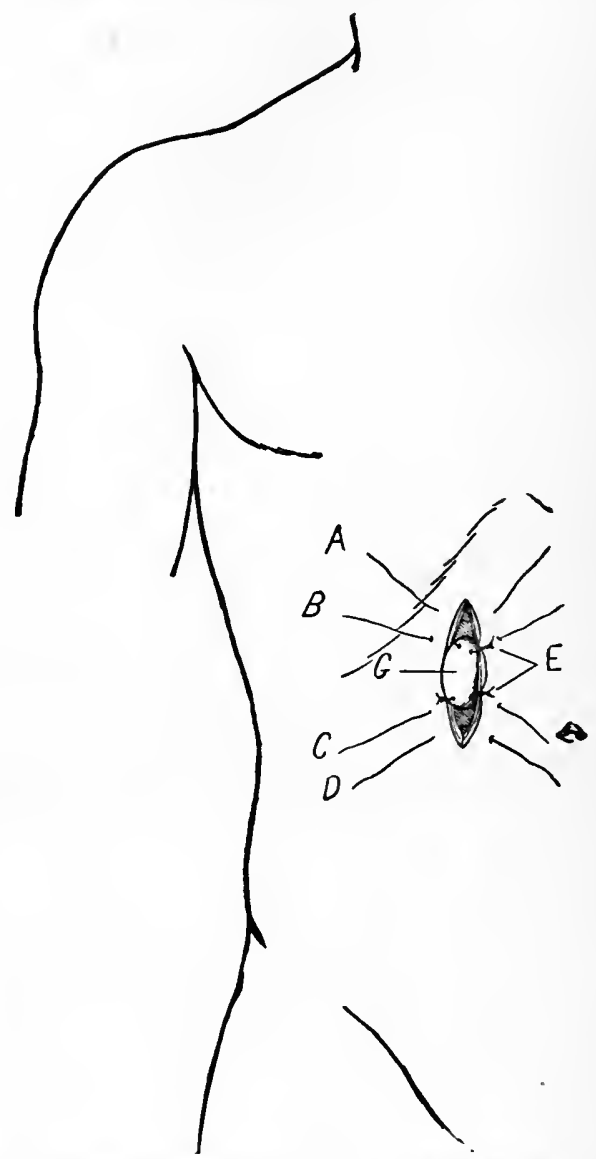

Fig. 136.-Cholecystostomy. Fundus of the gall-bladder (G) drawn into the incision and fixed; $A, D$, sutures through all the layers of the abdominal wall that serve to diminisb size of the incision; $B, C$, sutures that pass through the edges of the incision in the abdomen, but catch up the wall of the gall-bladder as they pass across from one edge of the incision to the other; $E$, sutures that join the edges of the peritoneum to the skin.

Cholecrstostomy in two Sitrings.-This method is especially applicable if the contents of the gall-bladder are purulent.

Through the vertical incision, about four inches long, in the ab- 
dominal wall, the gall-bladder is exposed and the bile-ducts, etc., palpated. After pads have been arranged so as to protect the peritoneal cavity, a fine aspirating needle may be thrust into the bladder and some of the contents drawn off. If the contents are purulent, the operation had better be done in two sittings.

The parietal peritoneum is first fixed to the margins of the abdominal incision with two interrupted catgut sutures ( $E$, Fig. 136) on each side; these join the edge of the peritoneum to the edge of the skin. Two tractor sutures which do not penetrate through the entire thickness of its wall are then introduced into the fundus of the bladder in order to steady it.

Four silk-worm gut sutures $(A, B, C, D$, Fig. 136) are now introduced through the edges of the abdominal incision: two in the upper part of the incision and two in the lower part.

Each of these sutures passes through all the layers of the abdomen; the two middle sutures, marked $B$ and $C$ (Fig. 136), pick up the wall of the gall-bladder in their course, but do not pass through its entire thickness. They serve to partially fix the bladder in the abdominal incision. There should be a space of one and one-half to two inches intervening between these two sutures.

The uppermost and lowermost sutures, marked $A$ and $D$, which are simply for the purpose of closing the abdominal incision for part of its length, are introduced first, but they are not tied until after those which pick up the wall of the gall-bladder have been introduced.

The fundus of the gall-bladder is then still further united to the edges of that part of the abdominal incision which is to be left open -i.e., between the sutures $B$ and $C$-with several additional catgut or silk sutures on either side. These do not pass through the entire thickness of the wall of the gall-bladder, and are best introduced with a narrow, curved surgeon's needle. It may facilitate the introduction of these accessory sutures if the sutures $B$ and $C$ are left untied until these accessory sutures have been introduced. As a result, we have the abdominal wound left open for one and one-half to two inches of its length, and the fundus of the gall-bladder fixed there.

The wound is then packed, leaving the tractor sutures in place, and after two to five days the gall-bladder may be opened between the tractors with a knife or Paquelin cautery and emptied of its contents. 
Cholecystostomy when the Gall-bladder is Adherent to the Parietal Peritoneum, thus Shutting off the Peritoneal CAvitr.-Having made the incision through the abdominal wall as described above, we may find the gall-bladder adherent to the parietal peritoneum, thus shutting it off entirely from the general peritoneal cavity. In this case one may well avoid breaking down the adhesions, and proceed at once to open and empty the gallbladder; a drainage tube is introduced into the bladder and the incision in the abdomen is left open and packed.

Cholecystostomy when the Gall-bladder Cannot be BRoUght UP INTO THE INCISION.-At times it is not possible to draw the gall-bladder up into the abdominal incision for the purpose of fixing it to the edges of the wound, even after adhesions, that may be present, have been broken up.

Under these circumstances it may be better to extirpate the gall-bladder, or else, after emptying it in part with the aspirator, it may be freely incised, emptied, and thoroughly disinfected. A drainage tube may then be introduced into the bladder and fixed with a catgut suture (No. 2) to the edge of the opening in the bladder, and in addition to this a purse-string suture of catgut may be placed in the wall of the gall-bladder, close to the margin of the opening, which, when drawn tight, grasps the tube, the end of which is allowed to project through the incision in the abdomen. Finally, gauze is packed into the abdominal incision around the tube, reaching down to the gall-bladder.

Cholecystectomy.-Extirpation of the gall-bladder.

This operation may be done in cases of cholelithiasis when one is unable to bring the gall-bladder sufficiently well upward, forward, to sew it to the abdominal incision; for rupture of the gall-bladder, due to falls, blows, or a run-over. One must always be certain of the patency of the common bile-duct before extirpating the gallbladder.

The incision is vertical, four to six inches long, corresponding to the outer border of the rectus muscle and commencing above at the tip of the ninth costal cartilage.

After the abdomen has been opened the gall-bladder is sought. It may be distended and present below the edge of the liver, or it may be small and concealed beneath the free edge of the liver.

Adhesions between the gall-bladder and neighboring parts should be broken up with the fingers, and the bile-ducts, especially 
the common, should be thoroughly palpated," since extirpation of the gall-bladder is naturally counter-indicated if the common duct is obstructed. If a stone is found in the common duct it may be crushed with padded forceps or forced back into the bladder or onward into the duodenum, or it may be removed by one of the operations described below.

After having assured one's self of the patency of the common duct one may proceed with the extirpation of the gall-bladder. If the gall-bladder is distended, one may aspirate for the purpose of relieving the tension and to discover the nature of the contents. The liver is drawn upward and the pylorus downward out of the way.

The layer of peritoneum which covers the inferior aspect of the gall-bladder and binds it to the under surface of the liver is incised or torn, and the gall-bladder separated from the under surface of the liver subperitoneally, and, as much as possible, bluntly with the finger, at the same time making traction upon the gall-bladder, which is held in the grasp of a forceps. The separation of the gallbladder is commenced at the fundus, gradually working backward toward the neck of the organ. After freeing the neck one continues along the cystic duct as far as its junction with the common duct; so that the gall-bladder finally hangs free, suspended only by the cystic duct. The hemorrhage from the raw surface of the liver is usually but slight and may be controlled by a few minutes' compression with a hot gauze pad or by the Paquelin, if necessary. A double silk or catgut ligature is thrown around the cystic duct. close to its junction with the common duct and tied, and the cystic duct then divided between the ligatures, and the gall-bladder. thus removed. The stump of the cystic duct is drawn into the incision by the ligature, which is left long for that purpose, and cauterized; the ligature is then cut short and the stump allowed to drop back into the abdomen. The edges of the layer of peritoneum which bound the gall-bladder to the under surface of the liver, and which was torn to allow the enucleation of the gall-bladder, may be brought together with a catgut suture, thus closing in the raw area of the liver and the stump of the cystic duct.

The incision in the abdomen is closed without drainage with several interrupted silk-worm gut sutures, the edges of the opening in the parietal peritoneum being first brought together in the usual way with a continuous catgut suture. 
Cholecyst-enterostomy. - The formation of a fistulous opening between the gall-bladder and the intestine in case of inoperable obstruction of the common duct.

A vertical incision four to six inches long is made, corresponding to the outer border of the right rectus muscle, in the semilunar line, commencing above, just below the free border of the ribs at the tip of the ninth costal cartilage.

Having cut through the abdominal wall, the distended gallbladder usually presents. It is aspirated and emptied as nearly as possible of its contents and then incised, and, if stones are present, these are removed; it is then packed with gauze to prevent further escape of its contents. A loop of the jejunum, sixteen to twenty inches below the duodenum (see "Gastro-enterostomy"), is secured and brought up, in front of the great omentum and transverse colon, into the incision in the abdominal wall.

Gauze pads are then properly placed to prevent soiling of the peritoneal cavity, and with a fine, straight needle and fine silk the gall-bladder, at a convenient point near its fundus, and the gut, opposite its mesenteric border, are united to each other. This stitch takes a good, broad bite, including the serous and muscular coats, but does not pierce the whole thickness of the wall of either organ. The gall-bladder and jejunum are joined together in this way for a distance of about one and one-half inches. This needle is then temporarily laid aside, and an incision one inch long is made in both the gall-bladder and the intestine; these openings are placed opposite each other and are shorter than the line of suture which has already been applied. With a curved surgeon's needle and silk or catgut the contiguous edges of both these openings are joined together all around with a continuous overhand stitch, which penetrates all the coats, and thus the communication between the two organs is effected. After this the first straight needle carrying the fine silk thread, with which the first half of the "outside serous suture" was made, is again taken up and the second half of this "outside serous suture" is introduced. In this way the gall-bladder and the intestine are united by a double line of suture, one joining the edges of the openings to each other all around, and the other, a non-penetrating suture, which surrounds this first suture and buries it.

Having thus completed this part of the operation, the opening which was made in the fundus of the bladder for the purpose of 
emptying it and removing stones, etc., may be closed with a Lembert suture of fine silk, or else the margins of this opening may be fixed to the edges of the abdominal incision in order to insure drainage for a day or two (cholecystostomy). The abdominal wound may be closed in part, that portion which is left open being packed (see "Cholecystostomy"). If the opening in the gall-bladder has been closed, then the abdominal incision may be likewise closed.

The result of this operation is the establishment of a communication which allows the bile to flow from the gall-bladder into the intestine. The fistula (cholecystostomy) closes readily. The disadvantage of this operation is that the bile enters the intestine rather low for digestive purposes. This objection would be obviated if the communication were made between the gall-bladder and the duodenum. This anastomosis may also be effected by the Murphy button or with Laplace forceps, etc.

Cholecysto-duodenostomy with Murphy Button.-The formation of a fistulous opening between the gall-bladder and the duodenum, the upper part that adjoins the gall-bladder, for obstruction in the common duct.

A vertical incision four to six inches long is made from the tip of the ninth costal cartilage, downward, along the outer border of the rectus muscle, in the linea semilunaris, or a vertical incision (Murphy) may be employed which commences above, just below the free border of the ribs and reaches downward for a distance of three or four inches; this incision is placed two inches to the right of and parallel with the middle line, penetrating between the fibers of the rectus muscle.

Having cut through the abdominal wall, the gall-bladder is located and drawn into the wound and steadied there; then the duodenum is secured and drawn into the wound. The duodenum is cleared of its contents by gentle stripping with the fingers, and a compressor applied to prevent the re-entrance of contents. Pads are arranged to protect the peritoneal cavity, and with a fine, straight needle a silk thread is introduced into the free surface of the gut in the fashion of a purse-string. This suture should include about one and one-half inches of the length of the gut and be in a straight line; it should be made with three punctures of the needle, each bite including about one-third inch and passing through the entire thickness of the wall of the gut; a second similar suture line is then made with the same thread in the reverse direction parallel with the first 
and distant from this rather more than one-fourth inch, finally terminating alongside of where the needle first entered in commencing the suture (see Fig. 125). Corresponding to the point where the thread turns back to form the second half of the suture a little slack, or loop, should be left. With the ends of this running stitch the first loop of a surgeon's knot is taken. The gut is then incised between the two rows of suture for a distance corresponding to two-thirds the length of the diameter of the button to be used (No. 1 or 2 preferable), the incision being shorter than the suture line. The male half of the button, grasped with a thumb forceps, is then slipped sideways into the opening in the gut and the running string drawn tight about it and tied. This half of the button is thus fixed in the opening in the intestine and steadied until the female half has been fixed in the gallbladder.

If the gall-bladder is distended, one may first empty it with the aspirator. A similar running suture and incision are made in the gall-bladder at a convenient point near the fundus, and any stones that are present may be extracted. After this the female half of the button is introduced into the opening and the pursestring drawn tight and tied; the two halves of the button are then gently and steadily forced home.

It might be wise in addition to make a fistula by incising the gall-bladder and sewing the edges of the opening thus made into the abdominal wound, as already described (cholecystostomy). As a rule, this is unnecessary, however, and the abdominal wound may be closed without drainage.

The anastomosis between the gall-bladder and duodenum may also be accomplished with suture or with the Laplace forceps.

\section{OPERATIONS UPON THE GALL=DUCTS.}

Choledochotomy.-Incision into the common bile-duct.

Choledocho-lithectomy. - Incision into the common duct and removal of a stone. A stone may be found impacted in the common bile-duct and so fixed that it cannot be forced onward into the duodenum.

The common bile-duct lies fairly deep in the abdominal cavity between the layers of the lesser omentum, passing down behind the first part of the duodenum. If it contains a stone, it is usually found dilated, pouched, and its wall thickened. In order to reach the 
common duct it may be necessary to lengthen the usual gall-bladder incision, which passes downward from the tip of the ninth rib, and introduce deep abdominal retractors; the liver is drawn upward and the pylorus pulled downward out of the way. The hepatic artery lies to the left of the duct and the portal vein behind it. It may be necessary to separate bluntly with the fingers adhesions between the neighboring organs, working in between the pyloric end of the stomach and the under surface of the liver.

After the common bile-duct has been recognized and the stone felt within it, pads are properly placed to isolate the field of operation and protect the rest of the peritoneal cavity. The duct is then incised in its long diameter and the stone extracted whole or after crushing it, if it is very large. Escaping bile is caught and sponged away with gauze pads. After the duct has been sponged out and the adjoining parts disinfected with a pad moistened with alcohol, the opening may be closed with a single continuous suture of silk that includes the whole thickness of the edges of the incision. This will usually suffice, but it may be reinforced by an additional continuous layer of Lembert sutures of fine silk. If in doubt as to the security of the suture, one may introduce a plug of gauze through the incision in the abdomen down to the suture line in the duct for the purpose of drainage, and this may be removed after forty-eight hours.

Choledocho-lithotripsy.-Crushing a stone within the common bile-duct without making an incision into the duct.

This is not advisable unless the calculus is quite soft. It may be done with padded forceps.

Duodenotomy for Removal of Stone Impacted in the Common Duct.-McBurney, and after him others, have removed stones of considerable size, which had become impacted low down in the common duct, through an opening in the duodenum. This operation is especially adapted to the removal of stones impacted low down in the common duct near its duodenal end; they may present into the duodenum through the mouth of the duct.

A vertical incision about five inches in length is made in the abdomen, commencing above at the tip of the right ninth costal cartilage. This incision corresponds to the outer margin of the rectus muscle. With the hand in the abdomen, the diagnosis may be confirmed by feeling the stone in the common duct.

The greater omentum and the transverse colon are drawn up- 
ward out of the way and the second part of the duodenum made accessible. Several gauze pads are arranged in the wound to shut off the rest of the abdomen from the field of operation, and deep retractors then introduced.

A vertical incision one to one and one-half inches long is made in the middle of the descending part of the duodenum, any escaping intestinal contents being wiped away with gauze pads. Within the duodenum search is made for the orifice of the conjoined common and pancreatic ducts. This is found on the inner posterior wall of the second part of the duodenum; i.e., upon that part of the wall of the duodenum which is contiguous to the pancreas and about four inches distant from the pylorus.

In the normal subject this orifice is marked by a papilla. If a stone is present in the common duct, the orifice may be found enlarged, with the stone presenting into it, or a probe may be carried through the orifice into the duct and the stone felt. A forceps may be introduced through the orifice into the duct, and the stone grasped and withdrawn.

In order to extract the stone it may be necessary to dilate the mouth of the duct or to nick it with the knife or scissors. When the stone is withdrawn there follows a free flow of bile, which should be mopped away as fast as it escapes.

The opening in the duodenum is closed with a row of silk Lembert sutures, which are introduced with a small, curved surgeon's needle in a holder.

The incision in the abdomen is closed in the usual way.

\section{THE SPLEEN.}

The Surgical Anatomy of the Spleen. - The spleen is a solid organ located in the upper left part of the abdomen in close relation with the fundus of the stomach, to which it is attached by the gastrosplenic ligament (omentum), being suspended from the diaphragm by the phrenico-splenic ligament, its lower end resting upon the phrenico-colic ligament. The spleen is rather ellipsoidal, although its shape may vary. It measures usually about $12 \mathrm{~cm}$. in its long diameter, $8 \mathrm{~cm}$. in breadth, and $3 \mathrm{~cm}$. in thickness. Its size may vary considerably.

Its outer surface is smooth and rounded, and looks outward, upward, and backward toward the diaphragm, which separates it 
from the pleura and the edge of the lung and the ninth, tenth, and eleventh ribs. Its inner surface consists of two areas: the anterior, the gastric surface, which is the broader, looks inward and forward, and lies close to the posterior surface of the fundus of the stomach; the posterior portion of the inner surface is in contact with the upper and outer part of the left kidney and the tail of the pancreas. Between these two areas the inner surface presents the hilum, where the vessels and nerves pass in and out of the organ.

The lower end of the spleen is in relation with the splenic flexure of the colon, and rests upon the phrenico-colic ligament, which supports it. The anterior border is rather sharp, and marked by a varying number of notches. The posterior border is rounded and thick.

The splenic artery is a branch of the cœliac axis, and in its course to the hilum of the spleen runs along the upper border of the pancreas, lying above the splenic vein.

The splenic vein is as large around as one's finger-twice as large as the splenic artery. It emerges in several branches from the hilum of the spleen, runs along the upper border of the pancreas, and after receiving the inferior mesenteric vein joins with the superior mesenteric to form the portal vein.

The spleen is almost completely invested by the peritoneum, which is intimately blended with the firm capsule proper of the organ. The spleen is fixed to the stomach by the gastro-splenic ligament (omentum) and to the diaphragm by the phrenico-splenic ligament, the suspensory ligament. Its lower end rests upon the phrenico-colic ligament.

The gastro-splenic ligament, or omentum, is the fold of peritoneum which is reflected from the fundus of the stomach over to the spleen, and between its layers the splenic vessels pass to and from the hilum of the spleen and the vasa brevia to the fundus of the stomach. The phrenico-splenic ligament, or suspensory ligament, is the fold of peritoneum which is reflected from the diaphragm to the spleen.

\section{OPERATIONS UPON THE SPLEEN.}

Splenotomy.-Incision of the spleen for the purpose of evacuating an abscess or an hydatid cyst.

The abdominal incision may depend upon the location of the tumor, if any is present. Usually the incision is made along the 
outer edge of the left rectus, in the semilunar line from the lower border of the ribs downward.

After having cut through the abdominal wall and parietal peritoneum, if the spleen is found adherent to the parietal peritoneum, thus shutting off the general peritoneal cavity, one may incise at once with the knife or Paquelin cautery and drain. If adhesions, shutting off the general peritoneal cavity, are not present, one may pack down to the surface of the spleen, leaving the abdominal incision open in part, and only after adhesions which isolate the exposed splenic surface from the general peritoneal cavity have formed, after from two to five days, is the incision into the organ made.

Splenectomy.-Extirpation of the spleen; for wandering spleen, wounds, rupture, prolapse, hemorrhage, or sarcoma.

The incision must be liberal-from 10 to $15 \mathrm{~cm}$. or longer. It may be placed in the middle line, commencing below the ensiform cartilage and passing downward and around the left side of the umbilicus, or it may be conveniently placed along the outer border of the left rectus muscle in the linea semilunaris, or, if there is a tumor present, the incision may be placed to correspond.

After the abdomen has been opened and the hemorrhage from the edges of the incision controlled, the hand is introduced into the abdomen and the spleen seized. If adhesions are present, these may be gently broken down with the fingers, or, if vascular, they may be tied double with catgut and cut. If the capsule of the spleen is not wounded, one should avoid injury to it, as this will save considerable hemorrhage.

After the spleen has been freed it is drawn forward into the wound; it is fixed within the abdomen by the peritoneal folds, which connect it to the stomach and to the diaphragm. Its pedicle, which consists practically of the gastro-splenic omentum (including the splenic vessels), may be transfixed, through its middle, with a curved, blunt-pointed ligature carrier, provided with a long strand of strong silk or catgut. This ligature is then cut so as to make two, and tied, one including the upper half of the pedicle and the other the lower half. One should avoid including the tail of the pancreas in tying these ligatures. These ligatures should be tied tight and left long to serve as tractors in order to pull the stump of the pedicle into the wound for final inspection after the spleen has been cut away. If the phrenico-splenic ligament is not included in the ligatures placed as indicated, this may now be ligated also and in a 
similar manner. The pedicle is then cut through close to the spleen and the organ removed; the stump of the pedicle may be drawn gently forward and an effort made to isolate and ligate the splenic artery and vein, each separately. If the pedicle is properly secured there is little danger of hemorrhage.

The wound in the abdomen is closed without drainage, first bringing the edges of the parietal peritonemm together with a continuous catgut suture and then the other layers with interrupted silk-worm gut.

\section{THE PANCREAS.}

Surgical Anatomy of the Pancreas.-The pancreas is an elongated glandular organ six inches long, its breadth equal to about -one-fourth its length; it is about one-half inch in thickness from before backward.

It is placed transversely in the upper back part of the abdominal cavity, lying behind the stomach across the body of the second lumbar vertebra.

It consists of a head, body, and tail, the tail abutting against the :spleen.

The head lies to the right of the vertebral column, resting upon the inferior vena cava, right crus of the diaphragm, and right renal vessels, and separated from the inner border of the right kidney by the second part of the duodenum. The common bile-duct is located between the second part of the duodenum and the head of the jpancreas.

The body of the pancreas lies opposite the second lumbar vertebra upon the crus (left) of the diaphragm, aorta, thoracic duct, etc. To the left of the vertebral column it is in relation with the renal vessels and left kidney. In front of the pancreas are the peritoneum, stomach, and transverse colon.

The splenic artery and vein run along its upper border. Its lower border is in relation with the third part of the duodenum, and passing forward between this part of the duodenum and the lower border of the pancreas are the superior mesenteric artery and vein.

The tail of the pancreas projects to the left as far as the spleen, to which it is connected by a fold of peritoneum, ligamentum pancreatico-lienale.

The pancreas is covered by the peritoneum upon its anterior :surface only. The transverse mesocolon passes backward, and upon 
reaching the pancreas its layers separate; the upper layer passes. upward, covering the front surface of the pancreas, and lines the back wall of the upper part of the abdomen (lesser sac).

The duct of the pancreas courses through the organ from left to. right, and opens into the second part of the duodenum, through an orifice which it has in common with the common bile-duct (see "Common Bile-duct").

\section{OPERATIONS UPON THE PANCREAS.}

Injuries of the abdomen involving the pancreas are, from their very nature, usually associated with such serious injuries to the neighboring organs that death results without special regard to the pancreas.

Parts of the pancreas may be tied off and excised. Abscess and tumor of the pancreas may cause obstruction of the intestinethrough pressure and adhesions.

Abscess of the pancreas may be opened from in front through an incision in the anterior abdominal wall or it may be incised behind, extraperitoneally. If opened from in front, the contents of the sac may be eracuated with an aspirator in part and the enveloping wall of the abscess or cyst cavity then sewed to the edges of the. abdominal wound, or, without evacuating, the sac may be fixed to the edges of the incision in the abdomen and opened later after adhesions have formed.

A retention cyst may be due to occlusion of the pancreatic duct by stone. Hydatid cysts may also be found in the pancreas. Theseare incised and drained through an incision in the middle line above the umbilicus; the cyst wall may be sewed to the edges of the incision in the abdomen and opened later after adhesions have formed.

\section{OPERATIONS UPON THE SPINAL COLUMN.}

Laminectomy.-Resection of the laminæ of the vertebræ for thepurpose of relieving compression of the cord due to traumatism or disease, depressed or displaced bone, extravasated blood, pus, tuberculous products, Pott's disease, tumors, etc.

The patient is placed prone upon the table with a shallow cushion under the ribs to give the back a slight curve. A long incision is made, in the middle line, through the soft parts down to the tips: 
of the spinous processes. The middle of this incision should correspond to the probable location of the injury or disease.

The soft parts-muscles, etc- - upon either side of the middle line are then freely separated with a periosteum elevator so as to expose the laminæ of from three to five vertebræ.

Hemorrhage should be controlled, oozing, by temporary pressure of a pad, etc., and spurting points by clamps and ligatures. The spinous processes may be snipped off at their bases with the cutting bone forceps, the blades of which may be conveniently bent at an obtuse angle.

While the soft parts, detached muscles, etc., are well retracted, the laminæ, if not already fractured by a traumatism, are divided and then removed.

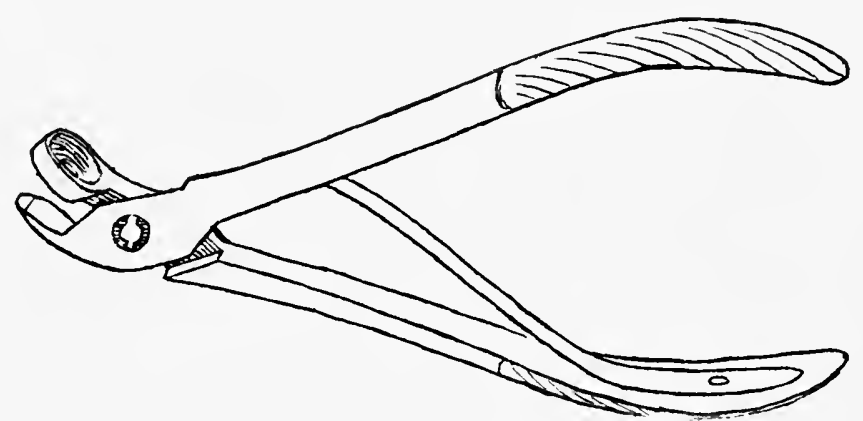

Fig. 137.-Keen Bone Forceps. The end of the upper blade is fenestrated.

The laminæ that are to be resected should first be stripped bare of their periosteum and any remaining soft parts with the sharp-edged periosteum elevator, and then divided as close as possible to the transverse processes, first on one side and then on the other. The laminæ may be divided with a Hays saw, chisel, or rongeur forceps, or they may be gnawed through with a Keen forceps. The laminæ which correspond to the middle of the wound are first resected and then those of the vertebræ above and below. In this way the spinal canal is opened and in some cases of traumatism the compression will have been relieved.

The dura mater proper is exposed by tearing with a blunt director through the loose connective tissue that overlies the dura. In thus exposing the dura mater, there may be considerable hemorrhage from the venous plexus that is located in the posterior part of 
the vertebral canal between the bony wall and the dura, but this is. readily controlled by a few minutes' compression with a gauze pad. As already mentioned, after the spinal canal has been opened, the immediate cause of the symptoms may present itself and the condition may be remedied without opening the dura; for example, a dislocated vertebra, tuberculous granulation tissue, extradural tumor, etc. Prominent angular deformity of the anterior wall of the spinal canal due to fracture, dislocation, Pott's disease, should be corrected by reduction or by chiseling or gouging away the offending process of bone; carious bone may be curetted and sequestra removed.

In order to reach the anterior wall of the canal, it may be necessary to divide several nerve-trunks upon one side and lift the cord partly out of its bed. The severed nerves may be reunited afterward by suture.

If the cause of the symptoms is not apparent the dura should be laid open. Before opening the dura, its color, degree of bulging, pulsation, etc., should be noted. The dura is picked up with a toothed forceps and a small opening made in the middle line, and through this opening the dura is incised upon a grooved director to any requisite length. When the dura is incised there is an escape of cerebro-spinal fluid and may be pus or blood. Adhesions. between the dura mater and the arachnoid should be gently broken up. The edges of the dura may be then well retracted and the cord carefully examined. A bent probe may be used to investigate the sides and anterior aspect of the cord.

In closing the wound the edges of the dura are brought together with interrupted catgut sutures placed about one-eighth inch apart, and the edges of the muscles and skin approximated with interrupted sutures of silk-worm gut. For the purpose of drainage, a narrow strip of gauze is introduced into the bottom of the wound, its extremity emerging through the lower end of the skin incision. The wound usually heals by first intention.

The parts should be immobilized by incasing the patient in plaster or by the use of a proper extension apparatus.

Lumbar Puncture.-J. Leonard Corning, of New York, in 1885 reported experiments of injecting solutions of cocain into the spinal canal through a puncture in the dorsal region for the purpose of inducing analgesia, etc.

Quincke, of Kiel, in 1891, practiced lumbar puncture for the 
purpose of drawing off fluid to diminish intracranial pressure in cases of hydrocephalus. With this object in view he has drawn off as much as 100 c.c. in some cases.

Bier in 1899 reported a number of cases which had been operated upon painlessly under the influence of cocain introduced into the subarachnoid space through a lumbar puncture.

Tuffier in 1899 brought the matter prominently before the general profession, and since then the method has been practiced by many operators with varying degrees of satisfaction.

The necessary instruments consist of a needle and a syringe. The needle should be about $10 \mathrm{~cm}$. long, with a diameter of about $1.1 \mathrm{~mm}$. and with a canal, or bore, of $0.8 \mathrm{~mm}$. The point of the needle should be sharp, but the bevel should be short. The needle throughout may be made of steel or its body may be made of a flexible alloy and its extremity of steel. Such a needle would bend without breaking (Bainbridge). The syringe should have a capacity of 30 minims, and be so constructed as to permit of proper sterilization; a glass barrel with a solid metal piston would answer. The nozzle of the syringe and the cap of the needle should form a smooth bevel joint,-not a screw thread,-in order to permit of their rapid adjustment and to eliminate the use of washers.

The puncture may be made between the laminæ of the fourth and fifth lumbar vertebræ or between the third and fourth or the fifth and first sacral. The puncture between the laminæ of the fourth and fifth seems to be preferred by most surgeons.

The needle is introduced just below and to the right of the tip of the spinous process of the fourth lumbar vertebra and is pushed in a direction forward and inward and slightly upward into the spinal canal.

The patient should be seated upon the side of the table with his back to the operator, his trunk bent forward, and his elbows resting upon the thighs. The tips of the spinous processes should form a straight line from above downward, deviating neither to the right nor left.

To locate the tip of the spinous process of the fourth lumbar vertebra, which is the guide in performing the operation, a line may be drawn across the back from the highest point of one iliac erest to a corresponding point upon the other. The tip of the spinous. process of the fourth lumbar will be found to correspond to this line.

The patient being bent forward causes the space between the 
laminæ of the fourth and fifth lumbar vertebræ to become wider. The index finger of the left hand is placed upon the lower part of the tip of the spinous process of the fourth lumbar vertebra, and with the right hand the needle is introduced; it is entered just below and about $1 \mathrm{~cm}$. to the right of this point (tip of the spine of

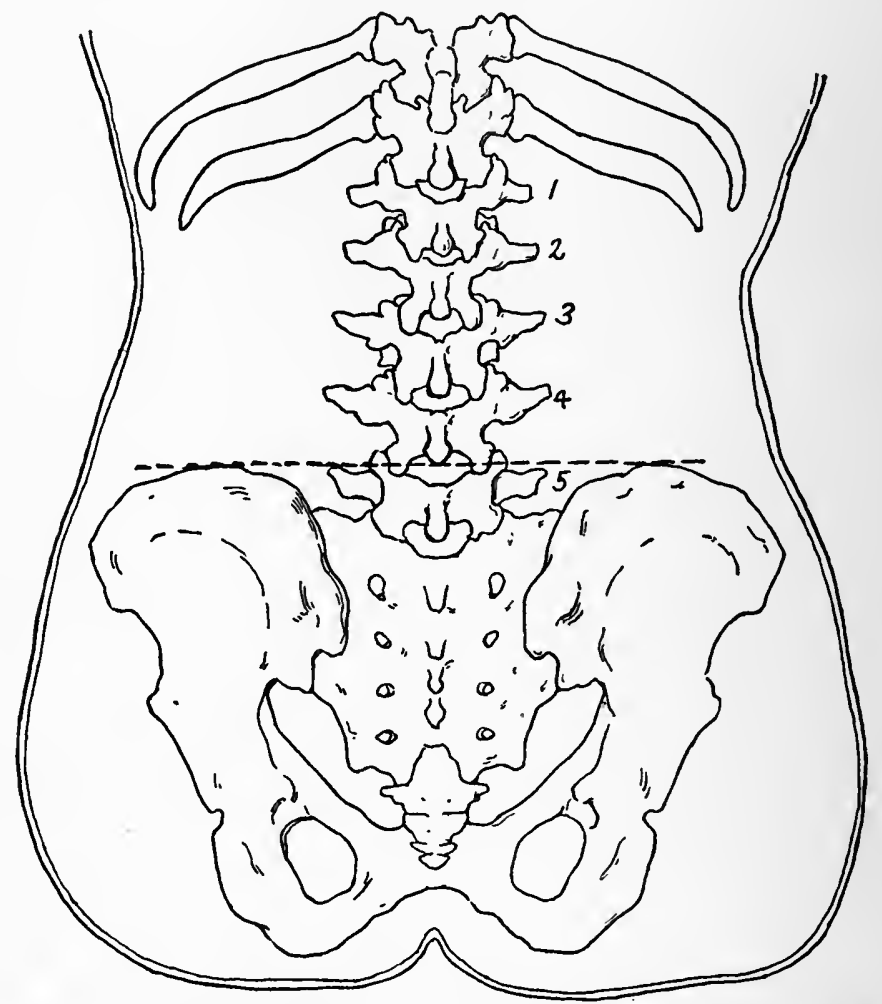

Fig. 138.-Lumbar Puncture. Tip of spinous process of fourth lumbar vertebra corresponds to a line drawn across the back touching the highest point of each iliac crest. The needle is inserted just below and to right of the tip of the spinous process of the fourth lumbar vertebra.

the fourth lumbar). The skin may be anæsthetized and a small incision made with the point of the knife in order to permit the easy passage of the needle through this structure, which is sometimes pretty tough and difficult to penetrate. The needle is then pushed slowly and deliberately forward and inward through the soft parts, entering the spinal canal in the middle line between the 
laminæ of the fourth and fifth lumbar vertebræ. After the needle has passed through the ligament between the laminæ, ligamentum subflavum, and the dura mater into the subarachnoid space there is felt a sense of diminished resistance which is readily appreciated, especially by the experienced. The positive proof that the extremity of the needle is in the subarachnoid space is the escape of the clear cerebro-spinal fluid, which flows from the end of the needle drop by drop. Not more than about ten drops of the cerebro-spinal fluid should be allowed to escape. The syringe containing the cocain solution which is to be introduced is now adjusted to the needle and its contents slowly injected. From 15 to 20 minims of a 2-percent. solution is the quantity usually injected.

Unless the escape of cerebro-spinal fluid occurs to indicate positively that the end of the needle is in the subarachnoid space the injection should not be made.

If the needle strikes an impediment, bone, on the way, it should be partly withdrawn and its direction changed so as to avoid the obstruction. One should not attempt to forcibly change the course of the needle by bending it without withdrawing it at least in part, as it may break off; a sudden movement or jerk on the part of the patient may also break the needle. With the flexible needle of Bainbridge this danger is eliminated. 


\section{PART VI.}

\section{THE RECTUM.}

Surgical Anatomy of the Rectum. - The rectum is the termination of the alimentary canal and is contained within the true pelvis, the posterior wall of which is formed by the sacrum and coccyx.

The SACRUM is an irregular, triangular-shaped bone formed by the coalescence of five vertebræ. With the coccyx it forms the lower part of the vertebral column and the posterior wall of the pelvis, where it is wedged in between the ossa innominata.

It is flattened from before backward and curved upon itself, and is placed very obliquely, so that its anterior surface looks downward as well as forward. Above, it articulates with the fifth lumbar vertebra, forming a prominent angle which projects forward and forms the back part of the inlet into the true pelvis. Its lower end articulates with the base of the coccyx. The lateral borders of the sacrum are broad and irregular above, for articulation with the iliac bones and for the attachment of the posterior sacro-iliac ligaments. The lower part of the lateral border is thin, and gives attachment to the greater and lesser sacro-sciatic ligaments and to a portion of the gluteus maximus muscle. Its anterior surface is smooth, concave, looks downward and forward, and presents on either side, one below the other, the four anterior sacral foramina, through which openings the anterior sacral nerves escape from the sacral canal. The branches which emerge from the first, second, and third anterior sacral foramina are large and go to form the sacral plexus. Through the fourth anterior sacral foramina emerge nerves which are distributed to the rectum and the bladder.

The posterior surface of the sacrum is convex, rough, and irregular. In the middle line from above downward are three or four tubercles, which represent the corresponding spinous processes; usually the fourth and always the fifth are absent. External to the spinous processes, on either side of the middle line, are the four posterior sacral foramina, one below the other. These provide exit to the posterior sacral nerves, which are of no importance surgically. Between the posterior sacral foramina and the spinous processes the 
bone is smooth, and corresponds to the laminæ of the other vertebræ, forming the posterior wall of the sacral canal; the laminæ of the fourth usually and of the fifth always are absent, thus leaving the sacral canal open at its lower part. The margins of the laminæ below, where the canal is open, are prominent, and are called the cornua. They artieulate with the corresponding cornua of the coceyx. The posterior surface of the sacrum is covered by and gives attachment to the ereetor spinæ muscle.

THE CoccYx is formed of four rudimentary vertebræ, and contains no spinal canal. Below, at the tip, the coccyx is pointed and gives attachment to the sphincter ani. Above, it presents a base with a prominent process on each side, the cornu. Its base articulates with the lower end of the sacrum; its cornua articulate with those of the sacrum. Its lateral border gives attachment to the greater and lesser sacro-sciatic ligaments, to the coecygeus muscle, and low down near its tip to a few fibers of the levator ani muscle.

The Rectum is continuous with the sigmoid flexure and terminates at the anus. It is about eight inches long, and is located in the back part of the true pelvis, surrounded by loose connective tissue. It includes that part of the large intestine which reaches from the left saero-iliac synchondrosis to the anus. It is usually described as consisting of three parts.

The first, or upper, part of the rectum extends from the left sacro-iliae synchondrosis toward the middle line, and, dipping into the pelvis in front of the sacrum, becomes continuous, opposite the second sacral vertebra, with the second, or middle part. This upper part of the rectum is narrower than the middle portion, and comprises about one-half its entire length. It is provided with a complete investment of peritoneum, which, as mesoreetum, is attached to the front of the sacrum, and thus serves to suspend the rectum in the pelvis. Dipping down into the pelvis, behind the rectum, between the folds of the mesorectum, is the termination of the inferior mesenteric artery, which is known as the superior hemorrhoidal. This part of the rectum is in relation behind with the left sacroiliac synchondrosis and the front of the saerum. Interposed between it and the sacrum are the pyriformis muscle, the sacral plexus of nerves, and the left internal iliae vessels and their branches. Anteriorly it is covered by the peritoneum, and is in relation with some coils of small intestine.

The second, or middle, part of the rectum is more roomy than 
the first part, and is known as the ampulla; it corresponds to the front of the sacrum and coccyx, reaching from the second sacral vertebra to the tip of the coccyx. It is curved, with its concavity forward. This part of the rectum is covered only upon its anterior aspect by the peritoneum. In the male the peritoneum is reflected from this part of the rectum forward on to the bladder, which it reaches just above the seminal vesicles (see Fig. 177). In the female the peritoneum reaches lower down upon the front surface of the rectum than in the male, and is reflected from this organ forward upon the upper fourth of the posterior wall of the vagina and upon the uterus, forming the pouch of Douglas. This pouch often contains coils of small intestine and in the female may contain a displaced ovary.

In the male the lower part of this middle portion of the rectum is in relation with the base, or trigone, of the bladder, the latter lying directly in front of the rectum. Between the base of the bladder and this part of the rectum are the seminal vesicles and the prostate gland, and here upon either side the ureters enter the bladder. In the female the lower portion of this part of the rectum is in relation with the posterior wall of the vagina.

The third, or lowest, part of the rectum is that portion which extends from the tip of the coccyx to the anus, and is directed downward and backward; it has no relation whatever with the peritoneal cavity. In the male the perineum separates this third portion of the rectum from the urethral canal, and in the female from the lower part of the vagina. This part of the rectum is rather narrow, and corresponds to the location of the sphincters. Upon either side of this part of the rectum, the levator ani, which extends downward and inward from its origin along the lateral wall of the true pelvis, is attached.

Besides the antero-posterior curves already described the rectum presents a lateral curve. The first part of the rectum in dipping into the pelvis from the left sacro-iliac synchondrosis reaches a little to the right of the middle line, while the lower part lies a little to the left of the middle line.

The lumen of the rectum presents three half-moon folds, or plicæ sigmoidea, with corresponding constrictions on its outer surface. These folds contain muscular fibers. The most marked and constant of these folds, plica transversalis recti, is located about half-way up upon the right wall, 5 to $6 \mathrm{~cm}$. from the anal orifice and upon a 
level with Douglas's fold. The two others are upon the left wall, not so constant nor so prominent, and are placed one nearer and the other farther away from the anus than the one first mentioned. These folds may offer considerable obstruction to the passage onward of bougies, etc.

In the lower part of the rectum the mucous membrane is thrown into longitudinal folds,-—columnæ Morgagni,-—so that upon section it would present a star-shaped appearance. About one inch above the anal opening the circular muscular fibers are increased in number and aggregated into a bundle-the sphincter internus; this is composed of unstriped muscular fibers.

Surrounding the anal orifice and attached behind to the tip of the coccyx and in front to the midpoint of the perineum is the collection of muscular fibers which is known as the sphincter externus; this is formed of striped voluntary muscular fibers.

The skin about the anus is thrown into folds, which radiate toward the anus, and often in the form of tags, etc., may become hypertrophied, inflamed, and itch-external, or itching, piles; or they may present cracks and fissures between them, at the edge of the anus-fissure in ano.

The rectum is supplied by the superior hemorrhoidal artery, the termination of the inferior mesenteric. This vessel descends behind the rectum between the folds of the mesorectum, and opposite the middle of the rectum divides into two branches; these distribute branches upon the sides of the rectum almost to the lower end. One may cut into the posterior wall of the rectum (strictures, etc.) for a distance of about three inches above the anal orifice without meeting this vessel. It bifurcates above this point.

The middle hemorrhoidal branches are derived from the internal iliac. The inferior hemorrhoidal, several on each side, are derived from the internal pudic (branch of the internal iliac) as it courses forward upon the inner aspect of the tuber ischii; they pass inward toward the anus and beneath the skin, and supply the integument about the anus and the lower end of the rectum; these branches are divided when incisions are made in this region in the skin or into the ischio-rectal fossæ. The branches from these three sets of vessels anastomose freely with each other up and down the rectum.

The veins of the rectum form a plexus of interanastomosing branches upon the wall of the rectum; they terminate above in the 
superior hemorrhoidal, which empties into the inferior mesenteric, which in turn empties into the portal. The middle and inferior hemorrhoidal veins empty, the middle into the internal iliac and the inferior into the internal pudic. Thus the rectum is liberally supplied with arterial blood from both the inferior mesenteric and the internal iliac arteries, the branches from both freely anastomosing with each other; it is drained by venous branches which carry blood to both the portal and general circulation, these also freely intercommunicating with each other.

The venous plexus situated in the lower part of the rectum, just above the anus and beneath the mucous membrane, is tortuous, and in certain conditions-disturbance of the portal circulation, habitual constipation, pressure of the gravid uterus, etc.-may become enlarged, pouched, and varicose, and give rise to the condition known as "bleeding piles," or internal hemorrhoids. Through the veins which drain the rectum infection may be carried to the liver - abscess of the liver, etc.

The nerves that emerge from the first, second, and third anterior sacral foramina join with each other to form the sacral plexus. The rectum is supplied by nerves that emerge through the fourth anterior sacral foramen. Branches from these nerves are also distributed to the bladder.

\section{OPERATIONS UPON THE RECTUM.}

Dilatation of the Sphincter.-This operation is practiced as a curative measure for fissure in ano and as a preliminary step in other operations upon the anus and rectum.

The patient is placed in the lithotomy position. Under anæsthesia two fingers or the thumb of each hand are introduced through the anus and well up into the rectum beyond the level of the internal sphincter, and a gradually increasing steady force is exerted in a lateral direction toward either tuber ischii until the sphincter is thoroughly relaxed. Considerable force may be employed, but it should be applied gradually, and not abruptly.

Fistula in Ano.- This may be either complete or incomplete. The incomplete may be either blind external or blind internal.

A complete fistula is a tract, or sinus, which opens internally into the rectum and externally upon the skin near the margin of the anus, and may allow the escape of gas and fæces from the bowel. 
The opening into the rectum is usually single, but there may be several openings upon the skin.

If the finger is introduced into the rectum and a probe passed into the fistula through the opening in the skin, its point may be felt beneath the rectal mucous membrane and may be guided through the inner orifice of the fistula into the rectum. This opening will be found a variable distance above the anal orifice and at times may be somewhat difficult to discover; it may be located above the internal sphincter or it may be just above the external sphincter close to the margin of the anus.

An incomplete, or blind, fistula is one which presents an orifice at only one end. If it opens into the rectum, but not externally

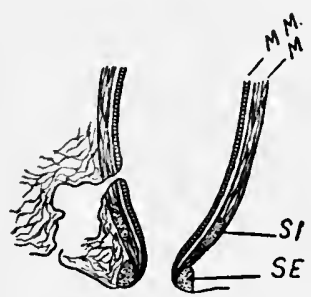

Fig. 139.-Compiete Fistula in Ano. $\boldsymbol{M}$, muscular layer of the rectum; $M . M$. mucous membrane layer of rectum; $S E$, cross section of external sphincter; $S I$, cross section of internal sphincter.

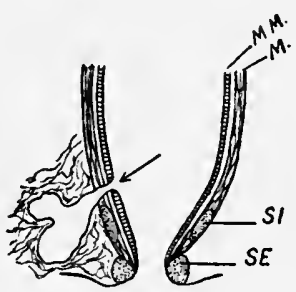

Fig. 140.-Bllnd Internal Fistula. Arrow indicates openlng from rectum. For letters, see Flg. 139.

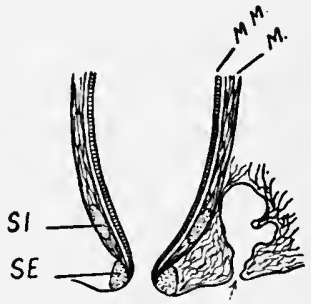

Fig. 141.-Blind External Fistula. Arrow shows openlng upon the skin. For letters, see Fig. 139.

upon the skin, it is called a blind internal fistula; if it opens externally upon the skin, but not internally into the rectum, it is called a blind external fistula.

Operation for CoMplete Fistula.-The anus is first thoroughly stretched. The finger is then introduced into the rectum and a blunt-pointed grooved director passed into the fistula through the opening in the skin. The point of the director, which may be recognized by the finger in the rectum beneath the rectal mucous membrane, is guided into the bowel through the internal orifice of the fistula. It is important to find this opening. The end of the director is then brought out through the anus,- - the director may be bent somewhat in order to do this, - and the bridge of tissue upon the director is divided with the knife, carried along the groove 
of the director; the fistula is thus laid open through its whole length into the rectum. If there is more than one external orifice upon the skin, the intervening tissue between the separate openings should be divided. Any secondary sinuses branching off from the main fistulous tract should also be laid open. As the internal orifice of the fistula is above the external sphincter or may be above the internal sphincter, these muscles are naturally divided when the fistula is laid open. One may curette the tract of the sinus after it has been laid open, but too much force should not be used. The whole wound is finally packed with iodoform gauze. This packing should not be too tight, but should reach well to the bottom of the wound in every direction. The bleeding is usually readily con. trolled by the packing. Any spurting vessels should be clamped and tied or the clamps may be left on until the first dressing.

Operation for Incomplete Fistula is practically the same as the foregoing. If there is no opening into the rectum, - a blind external fistula, - the point of the director, which is passed into the fistula through the external orifice and which is felt beneath the rectal mucous membrane by the finger within the rectum, may be forced into the rectum, the sinus being thus converted into a complete fistula, and the parts then divided as already described.

If there is no external opening,- - a blind internal fistula,-we make one. The skin about the margin of the anus at the point corresponding to the blind external extremity of the fistulous tract is usually marked by redness, induration, etc. After the skin has been incised at this point, thus converting the sinus into a complete fistula, it is treated as above described.

Hemorrhoids. External, or Itching, Piles present themselves about the margin of the anal orifice outside, external to the sphincter; they consist of cutaneous tags, which may be snipped off with the scissors, the edges of the skin being then, if necessary, brought together with a single suture. Occassionally they contain a varicosed vein, which may be thrombosed; this may be laid open, the clot turned out, and the edges of the skin brought together with one or two catgut stitches.

Frequently a fissure is located at the base of one of these external tags, or piles, and it is therefore wise, in all these cases, to stretch the sphincter before removing the pile.

Internal, or Bleeding, Piles.-These are located entirely within the anus, only appearing externally when the patient strains, 
or bears down. They may be caught in the grasp of the sphincter and become strangulated. When the patient strains they may appear as one or more fairly well defined bunches. Each mass consists of a bunch of dilated, pouched, varicose veins covered over by mucous membrane which may be normal in appearance or may be more or less ulcerated.

Ligation axp Excision.-The sphincter is first stretched and the rectum thoronghly irrigated. Each individual hemorrhoidal mass is then seized with a clamp, an ordinary artery forceps, and while it is pulled down the mucous membrane around its base is cut through by snipping with the blunt-pointed scissors. This incision should extend through the mucous membrane into the submucous

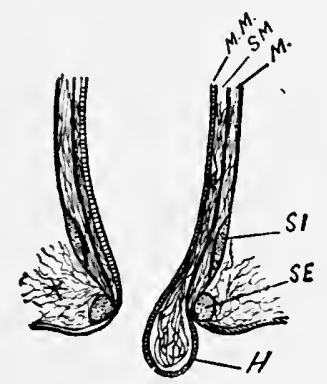

Fig. 142.-Hemorrhoids. $H$, hemorrhoidal mass (internal piles), consisting of a bunch of tortuous veins covered by mucous membrane protruding through the anal orifice; $M$, muscular layer of the rectum; $M . M$., mucous membrane layer of rectum; $S E$, cross section of external sphincter muscle; $S I$, cross section of lnternal sphincter muscle; $S I I$, submucous layer in which the veins ramify; *, loose connective tissue to either side of lower end of rectum in ischio-rectal fossa.

connective tissue layer, but should not cut into the vessels that go to form the hemorrhoidal mass. After this the mucous membrane at the base of the pile may be peeled back with the finger-nail or the end of the scissors, and the base or pedicle of the pile surrounded with a strong catgut ligature; this should be tied very tight so that it cannot slip (No. 2 plain catgut). The pile is cut away close to the ligature and the ligature then cut short. Each hemorrhoidal mass that presents itself is treated in like manner. They usually vary from two to four in number. The edges of each opening may be brought together over the stump of the pile with one or two catgut sutures, but this is probably, in most cases, unnecessary.

After the operation has been completed strip gauze is packed 
into the rectum fairly tight. It should reach to a point above the level of the site of the operation. Instead of the strip gauze one may introduce a tampon in the shape of a square piece of gauze, the center of which is seized with a forceps and, pouch-like, pushed into the rectum beyond the site of the operation, and then into this gauze pouch strips of gauze or a wad of cotton may be packed. The packing is to guard against hemorrhage from the slipping of a faulty ligature. One should bear in mind that the patient may suffer considerable hemorrhage into the bowel without any blood appearing externally; hence the importance of carefully tamponing.

Clamp axd Cautery.-After the anus has been stretched, etc., each pile is seized at its most prominent part with an artery forceps and drawn well down and a special clamp-pile clamp-applied to its base. The end of the clamp as it grasps the pile should be directed upward into the rectum; i.e., it should not grasp the hemorrhoidal mass along a line parallel with the margin of the anus, as this would result in an annular scar, which is not desirable. The pile should be firmly caught between the blades of the clamp and secured by turning the screw down tight. The pile is then cut away with the scissors, rather close to, but not flush with, the surface of the blades of the clamp; a small part of the tissue should be left protruding beyond the surface of the clamp after the pile is cut away. The cautery at a red heat is now applied to the cut edge of the remaining portion of the pile which protrudes beyond the surface of the blades of the clamp and this is slowly burned to a crisp down to the surface of the blades. The clamp is then removed. Each pile is treated in this manner. It is unnecessary to tampon the rectum.

Excision of a Circumscribed Part of the Rectal Wall.-Before proceeding with this operation the bowel should be thoroughly emptied by a course of laxatives and thorough irrigation of the rectum. This preparatory treatment may well occupy several days to a week. The ease with which a limited portion of the rectal wall is excised depends upon the situation of the disease.

If the Disease Ixvolves the Lower Part of the Bowed situated at or near the anus, the operation is comparatively easy. The sphincter is first thoroughly stretched so that it is completely relaxed. The tumor or diseased area is seized with a vulsella forceps or the fingers and is drawn down and out through the anal orifice, and may then be excised, together with that part of the rectal wall which forms its base, with the scissors or a sharp knife. The hem- 
orrhage should be controlled, seizing or tying bleeding points as they are encountered, and the edges of the defect in the rectal wall brought together by suture step by step as the operation progresses. The wound in the rectal wall should be closed in a transverse direction, because if sewed in a vertical line we may get a troublesome diminution of the caliber of the bowel, and this should be avoided in this narrow part. The stitches should be of catgut and interrupted, and should pass through the whole thickness of the wall of the bowel; the ends of the sutures should be left long to serve as tractors to facilitate the placing of the succeeding sutures. After the diseased part has been entirely removed additional sutures may be placed between those already introduced, but these should pass only through the mucous and submucous layers of the bowel, and are for the purpose of giving a more exact union of the edges of the mucous membrane.

If the Disease is Located Higher up, Beyond the Region of THE Avus.-The field of operation must be made accessible to view and touch, if possible; but this is more difficult than is the case when the disease is located lower down, nearer the anus.

The sphincter should be forcibly dilated, so that it is entirely relaxed. The tumor or diseased area may then be seized with a vulsella forceps, and can often be pulled down and out through the anal orifice, under which circumstances the operation may be done practically as described for disease situated lower down, in the anal region. Usually, however, the stretching of the anal orifice does not suffice to allow access to the diseased area, and we may find it desirable to make an incision, from within the bowcl, through the posterior wall of the rectum, including the anus, back to the coccyx. In this way we may make the field of operation accessible. Occasionally, however, even with this posterior incision, we are still unable to bring the disease within easy reach, or we may wish to avoid this posterior incision. Under these circumstances the work must be done within the rectum with the aid of retractors. For this purpose, after the sphincter has been thoroughly dilated, two flat-bladed retractors are introduced well up into the rectum, one on either side, the mucous membrane that tends to prolapse between the blades of the retractors being held back with a pad upon a long sponge holder. One should thus be able to see the ficld of operation, and this is necessary in order to control the hemorrhage and to suture the edges of the wound which is left in the rectal wall after the diseased por- 
tion has been extirpated. The retractors being in position, the diseased area is seized with a vulsella forceps and excised as already described. The portion of the rectum inmmediately above the anal region is roomy, and one may suture the wound left in this part of the rectal wall, after the excision of the disease, in a vertical direction without fear of constriction. The sutures should be of simple catgut, and the ends of each should be left long to serve as tractors to facilitate the introduction of the succeeding sutures; when the operation is complete they are all cut short. The stitches should be interrupted; every second stiteh should pass through the whole thickness of the rectal wall and the intermediate ones through the mucous membrane and submucous layers only.

If an accessory posterior incision has been made through the wall of the rectum back to the eoccyx this part of the wall of the rectum should also be closed in a similar manner, but the back part of this posterior incision which opens through the skin behind the anus, between it and the coccyx, should be left partly open for the purpose of drainage. The drainage is arranged by inserting a strip of gauze, which is packed into the wound behind the rectum and well up as far as the site of the suture line. This packing should not be tight, but should surely reach to the bottom of the wound.

Volkmann strongly advises drainage in all cases of excision of a portion of the wall of the rectum even where the wall of the rectum has not been split by the posterior incision. In those cases where the posterior incision through the rectal wall has been made one may drain as described above. If the posterior incision has not been made, one may make an incision in the skin near the margin of the anus, and through this penetrate sufficiently deep to reach the site of the suture line in the wall of the rectum when a strip of gauze is introduced.

Innocent Rectal Polypi.-After the anus has been dilated these may be seized with a clamp and twisted off the wall of the rectum with great ease or they may be amputated with the cautery. They usually do not bleed, but, if they do, the stump may be clamped and tied.

Extirpation of the Rectum, Amputatio Recti (Volkmann).Special pains should be taken to thoroughly empty the bowel, especially above the point of constriction, with a course of laxatives and copious rectal irrigations. This preparatory treatment may require several days or a week. 
This operation is adapted to those cases in which the disease has already involved the lower part of the rectum, including the anus-where the lower end of the bowel (sphincter) cannot be saved. The diseased portion, including the anal part, is amputated, and after this has been accomplished the upper part of the gut is pulled down and sutured to the margin of the skin about the anus.

The patient is placed in the lithotomy position. A strip of gauze may first be introduced into the rectum to prevent leakage, etc., and then an incision which encircles the anus is made through the skin. This incision is carried down into the loose connective tissue about the lower end of the rectum, and, when this part of the bowel has been liberated all around, it is seized and drawn down. The levatores ani, which are inserted into the sides of the lower part of the rectum, are encountered. These are divided with the knife or scissors close to the wall of the rectum, and then, gradually working deeper and deeper, the rectum is thoroughly separated all around from the loose connective tissue which surrounds it, and pulled down more and more as this step of the operation progresses. The isolation of the rectum is accomplished chiefly by dissecting with the fingers or with bluntpointed scissors.

If more space is required, accessory incisions may be added. A posterior incision which reaches from the circular incision that surrounds the anus backward to the tip of the coccyx may be made. This incision may still farther be extended upward upon the back of the coccyx, and, if necessary, this bone may be enucleated, after the soft parts which cover it have been separated with a periosteum elevator. An anterior incision may also be added; this passes forward from the circular incision which surrounds the anus as far as the bulb of the urethra in the male and the posterior wall of the vagina in the female. This anterior incision not only provides more room, but allows the operator to keep himself informed of the location of the urethra and vagina, and may thus diminish the liability of injuring these parts. A catheter may be introduced into the bladder as an additional caution. These accessory incisions should not extend through the wall of the rectum, as it is advisable to amputate the rectal tube intact, without cutting into it, in order to avoid soiling the wound with its contents.

In liberating the rectum in the female we have to separate it upon its anterior aspect from the posterior wall of the vagina. The vagina may also be involved in the disease, and it will then be nec- 
essary to excise a part of its wall together with the rectum. In this case one should pause and close the opening made in the vaginal wall before proceeding further with the isolation of the rectum. In the male the rectum has to be separated anteriorly from the prostate and from the base of the bladder.

As we continue deeper with the isolation of the rectun upon its anterior aspect, especially if the disease reaches pretty well up, we meet the fold of peritoneum which dips down in front of the rectum: in the female between the rectum and the vagina, in the male between the rectum and the bladder. The depth to which this peritoneal fold is reflected upon the front wall of the rectum varies. Usually it descends to a level which is just above a point that can be reached by the finger introduced into the rectum through the anus; i.e., to a point 5 to $6 \mathrm{~cm}$. above the anus. This fold may, however, extend down to a point within a short distance of the anus. If this fold of peritoneum is not involved in the disease, it may be simply stripped away from the front wall of the rectum without opening into it. At times, however, it is opened, either intentionally when it is diseased or accidentally. This is of no special significance, especially if the rectum itself has not been opened. The opening in the peritoneum may be closed at once. If small, its edges may be caught in an artery clamp and tied with a catgut ligature as one ties a bleeding vessel. If larger, its edge may be sewed to the peritoneum that covers the front wall of the rectum with several catgut stitches, or it may be left unsutured and packed with gauze. In separating the rectum posteriorly there may be considerable hemorrhage. All bleeding vessels should be clamped as they are cut. Diseased glands which lie behind the rectum may also be enucleated.

After having separated the rectum beyond the upper limits of the disease the whole tube is pulled down and steadied with the vulsella forceps, which serve as tractors, grasping the bowel above the level of the disease, and then the lower diseased portion is amputated, making a straight cut transversely across the bowel. After this has been done the edge of the bowel is sewed to the edges of the skin around the anus with alternating superficial and deep stitches of silk. Before the edge of the bowel is sewed to the margins of the skin about the anus it may be twisted on its long axis through a quarter of a circle. This may make the artificial anus somewhat more retentive (Gersuny). If twisted to a greater degree, it may result in gangrene of the lower part of the bowel. 
If there have been made accessory posterior and anterior incisions, these may be closed with several interrupted sutures; but this closure should not be complete, as there should be sufficient space between the sutures to allow free drainage from the parts about the rectum.

Drainage is made with strips of gauze, which are packed loosely into the incision, both in front and behind the rectum.

Resection of the Rectum in Continuity (Dieffenbach).-This operation may be performed for excision of cicatricial stricture (Halsted).

This operation is applicable to those cases where the disease involves the wall of the rectum above the sphincter, the lower portion of the tube being free and healthy.

The diseased portion of the rectum is resected in its continuity and the lower end of the upper healthy segment then sutured to the upper end of the lower healthy anal part, which includes the sphincter. The Kraske method of resecting the rectum is probably preferable to this method, especially if the parts outside the rectum are involved.

After the sphincter has been thoroughly dilated the lower, healthy part of the rectum is divided into two lateral halves by two incisions, one of which, commencing within the rectum, passes backward, dividing the lower part of the rectum, including the anus, back to the coccyx. The second incision divides the front wall of the rectum, passing forward through the perineum as far as the bulb of the urethra in the male and the posterior wall of the vagina in the female. Both these incisions reach upward through the wall of the rectum to a point just below the lower limits of the disease. Two broad, blunt-pronged retractors are then introduced, one on either side, and the wound thus held wide open.

In either lateral half of the rectum which has been thus split and just below the lower limits of the disease a transverse incision is now made. This incision passes through the entire thickness of the rectal wall, and separates the lower healthy part of the rectum from the upper diseased portion. Into these lateral incisions upon either side of the rectum the blunt-pronged retractors are introduced, and, after inserting a strip of gauze into the diseased portion to prevent its contents from soiling the wound, the lower cut edge of the upper diseased portion of the rectum is secured with vulsella forceps or silk tractor sutures, which at the same time close its lower 
end, and its isolation from the loose connective tissue by which it is surrounded upon all sides is commenced. Steadily drawing the diseased portion of the bowel more and more downward, its separation from the adjoining connective tissue is continued until it is entirely free and we are able to reach beyond the upper limits of the disease. This separation of the rectum is accomplished chiefly by blunt dissection with the finger or the end of the blunt-pointed scissors, working all the time fairly close to the rectal wall. Vessels are clamped as they are cut during the course of the operation. All spurting vessels should be ligated.

In liberating the rectum anteriorly we may meet the fold of peritoneum that projects downward upon its front aspect. If this is not involved in the disease it can usually be peeled away from the wall of the rectum with the finger without opening into it. If diseased, or if it cannot be separated from the front wall of the rectum, we may cut through it close to the wall of the rectum, and, introducing the finger into the opening thus made, draw the rectum down. A pad may be temporarily introduced to prevent the prolapse of intestine through the opening and to protect the peritoneal cavity. After the rectum has been drawn down for a sufficient distance the opening in the peritoneum may be closed by suturing its edge with catgut to the peritoneal layer that covers the anterior wall of the rectum, or it may be left unsutured and drained with a strip of gauze, which is left protruding through the wound in the perineum in front of the anus. The part of the rectum above the disease should not be separated from its surrounding parts any more than is absolutely necessary to permit its being drawn down to the edge of the lower segment of the bowel, and furthermore one should not work too close to the wall of the rectum in order not to damage the blood-supply to such a degree that the nutrition of the rectum might be seriously impaired.

After the rectum has been liberated to a point beyond the upper limits of the disease we may then proceed to excise the diseased portion. Before doing this two tractors of silk are passed through the whole thickness of the wall of the rectum above the diseased area in order to steady it and to hold it after the diseased segment has been excised. When this has been accomplished the end of the healthy bowel is drawn down and sutured to the upper edge of the lower segment (anal portion). This is done with fine silk sutures which alternately pass through the whole thickness of the bowel and 
through the mucous membrane only. The edges of the anterior and posterior incisions in the lower segment of the rectum, including the ends of the sphincter, are then brought together in a similar manner, and thus the continuity of the bowel is restored. The incision in the skin in front of the anus and that behind the anus are only partly closed, and a strip of gauze is packed to the bottom of each incision, as thorough drainage is imperative. Before commencing the suture of the bowel the parts may be irrigated and a soft rubber tube surrounded by gauze introduced well up into the upper part of the rectum beyond the proposed site of suture. This is to prevent soiling of the suture line, and also to allow the passage of gas and possibly fluid frees during the few days immediately following the operation.

If the peritoneal pouch has been opened and packed the end of the gauze packing emerges through the incision in the perineum in front of the anus.

Resection and Amputation of Rectum through the Sacral Route (Kraske).- This method is well adapted for resectio recti for disease. situated high up, but with the lower end of the rectum and the anus still healthy. It also furnishes the best route for amputatio recti in those cases where the anal portion is also involved.

The bowel should be thoroughly emptied before the operation by a course of laxatives and repeated rectal irrigations. This preparatory treatment should be thorough, and may require one or two weeks. If the stricture of the rectum is so tight that the bowel above the site of the constriction cannot be emptied before the operation, one may do a preliminary colostomy. This should be done one or two weeks before the rectal operation. The transverse colon should be used for this purpose, because if the sigmoid or the descending colon is used there may be some difficulty in drawing down the bowel at the time of the rectal operation.

For Resectio Recti (the anal portion being healthy).

The operation is described in three steps:-

1. Sacral "Vor operation": resection of the coccyx and part of the sacrum.

2. Resection of the diseased portion of the bowel.

3. Apposition of the ends of the bowel and treatment of the incision, etc.

Sacral "Vor Operation."-The patient lies upon the left side (Hochenegg), with the belly inclined somewhat toward the table, the 
lower limbs strongly flexed at the knees and hips, and supported thus by an assistant, or he nay rest upon the abdomen with the lower limbs hanging over the end of the table. A slightly curved incision with the concavity toward the left is made. It begins above on a level with the middle of the sacrum and from two to three fingers' breadth (about two inches) to the left of the middle line; it is carried down to the middle of the upper border of the coccyx, and from this point it is continued down in the middle line upon the coccyx, ending at its tip. This incision divides the skin, subcutaneous fat, and superficial fascia, and exposes in the upper part of the wound the lower portion of the gluteus maximus muscle, the fibers of which run at right angles to the line of the incision.
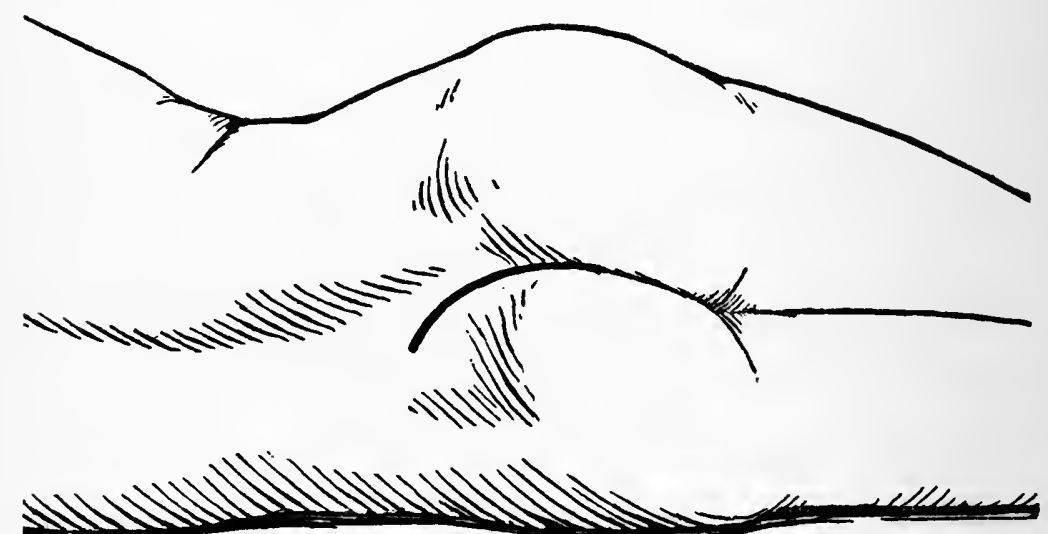

Fig. 143.-Incision for Resection of the Rectum (Kraske).

That part of the gluteus maximus muscle which presents itself in the wound is incised and retracted, and there are then exposed, lying underneath, the attachment to the sacrum of the greater and lesser sacro-sciatic ligaments. These structures are also divided close to the edge of the sacrum.

In dividing the gluteus maximus muscle branches of the gluteal artery are cut; these may be clamped and tied. Penetrating through the fat in the ischio-rectal fossa the coccygeus, which is attached to the border of the coccyx and sacrum, and the levator ani, which is attached to the coccyx near its tip, are exposed. These muscles are covered over by a thin fascia-the anal; they are divided with the knife close to the edge of the sacrum and coccyx. The soft parts are 
then separated with a periosteum elevator from the posterior surface and right border of the coccyx, and while it is forced forward the sacro-coccygeal joint is opened from behind and the bone seized with the bone forceps and extirpated. The sphincter ani is cut away from the tip of the coccyx close to the bone. If the arteria sacra media, which descends in front of the sacrum, is injured, it may be clamped and tied.

The levator ani and coccygeus muscles having been already divided, the operator now penetrates through the loose, fatty tissue which lies behind the rectum with the fingers so as to expose the posterior surface of the rectum. The rectum moves with respiration, and shows an impulse if the patient coughs or strains.

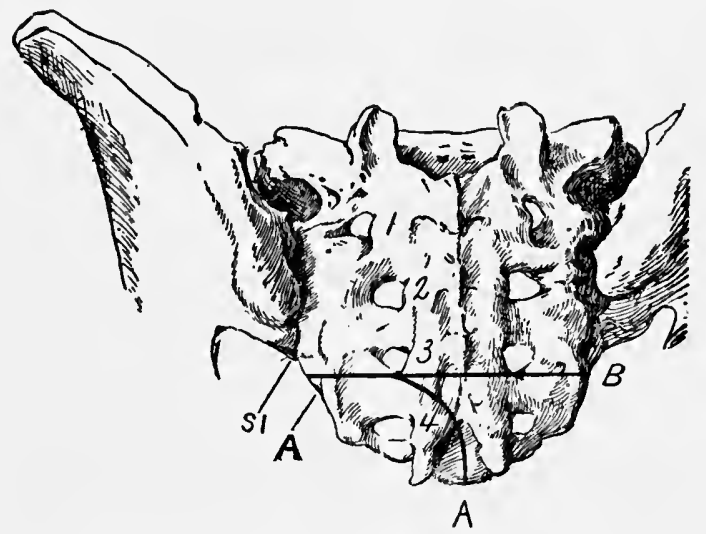

Fig. 144.-Back Part of Ilium and Sacrum. Coccyx removed. A, A, usual line of section through sacrum; $A, B$, line of section to remove all of lower part of sacrum; $S I$, lower end of sacro-iliac articulation; $1,2,3$, 4, posterior sacral foramina.

In many cases one may proceed at once with the second step of the operation: the extirpation of the diseased part of the rectum. At times, however, the space is not sufficiently ample, especially if the tumor is adherent and cannot be readily drawn down into the wound, or if the space between the border of the sacrum and the ascending ramus of the ischium (spatium sacro-ischiadicum, Kraske) is unusually narrow. In these cases in order to obtain more room it will be necessary to resect a portion of the sacrum. This may be done with the chisel, bone forceps, or saw. The soft parts are separated from the lower part of the left half of the posterior surface of the sacrum with the periosteum elevator, and that portion of the 
sacrum then resected which lies below a line that commences at the left border of the bone, just below the level of the third posterior sacral foramen; curving downward and inward toward the middle line and passing between the third and fourth posterior sacral foramina, this line terminates at the middle of the lower border of the sacrum. If necessary to get still more room the line of section through the sacrum may be carried straight across the sacrum, just below the third posterior sacral foramina from the left to the right border of the bone, thus removing all of the sacrum below the third sacral foramina. The line of section through the sacrum may terminate at any point between those described above. The guide to the location of the third sacral foramen is the lower end of the sacroiliac articulation. The lower end of the sacro-iliac articulation lies just above the lower margin of the third posterior sacral foramen.

In making the resection of the sacrum it is unwise to go above the lower border of the third posterior sacral foramen on account of the important structures which emerge from the first, second, and third anterior sacral foramina (sacral plexus). Through the fourth anterior sacral foramen branches emerge which are distributed to the bladder and the rectum. If these branches are damaged some disturbance of the function of these organs will follow, but this is only temporary, control being rapidly regained. If the left half only of the lower portion of the sacrum is removed, this disturbance will be much less marked.

Resection of the Diseased Pontion of the Bowel (the Anal Portion being Free from Disease). - With the fingers the diseased portion of the rectum is freed upon its posterior aspect and upon the sides from the loose fat and connective tissue that surround it. It is then likewise freed upon its anterior aspect. As we proceed with the isolation of the reetum, it may be necessary to cut some connective tissue bands with the scissors. All blood-vessels are clamped and tied as they are divided. During this step of the operation one should take care not to open into the rectum. When the diseased part of the rectum has been thus freed all around, a heavy silk ligature or strip of gauze is tied tightly around it, just below the lower limits of the disease, and through the anus, the lower part of the bowel, after being again thoroughly irrigated, is packed with gauze. The bowel is then divided transversely below the ligature with the seissors or knife, thus cutting the diseased portion away from the lower healthy (anal) segment of the bowel. The wound is 
not sailed, because the diseased segment is shut off by the ligature which has been applied about it, and the lower anal segment, besides having been thoroughly sterilized, is packed with gauze.

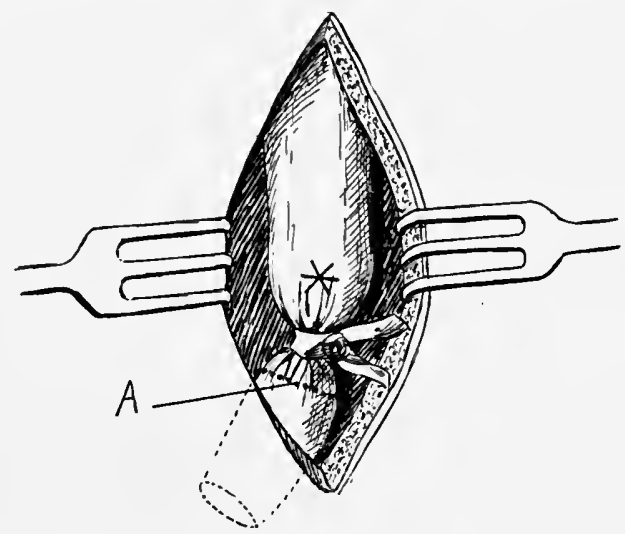

Fig. 145.-Resection of Rectum (Kraske). Rectum exposed and ligature passed around it just below the diseased portion $\left(^{*}\right)$ that is to be excised. $\boldsymbol{A}$, line of incision through rectum.

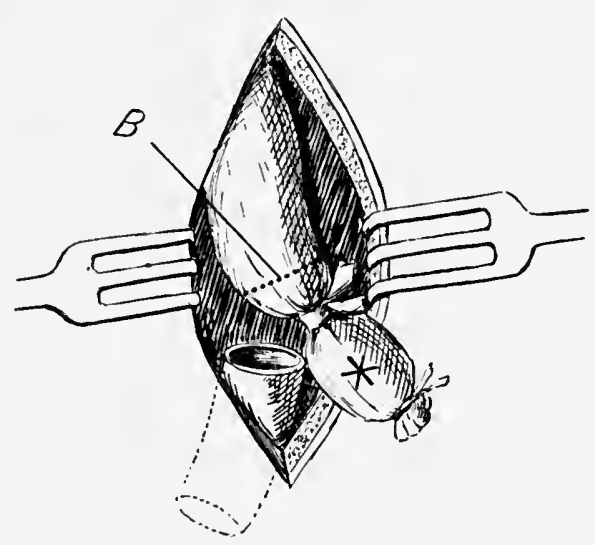

Fig. 146.-Resection of Rectum (Kraske). Diseased portion (*) cut away from the healthy lower, anal portion. A ligature has been placed about the rectum just above the diseased portion. $B$, line of section that separates the diseased portion from the healthy upper portion.

The diseased portion of the gut is now seized, and, while traction is made, it is gradually dissected out of its bed of fat and connective tissue, being thoroughly isolated upon all sides, so that it 
can be pulled down as far as necessary. This is accomplished largely by blunt dissection with the fingers. Just beyond the upper limits of the disease, when this becomes feasible, a second ligature is thrown around the rectum and tied, and thus the contents of the diseased segment are imprisoned within that portion of the bowel which is to be resected. The rectum is now drawn out of the wound as far as possible, and placed upon sterile, gauze pads, and the diseased part cut away from the upper healthy portion of the bowel. Before this is done an assistant grasps and compresses the lower part of the upper healthy segment of the bowel, beyond the intended line of section, between the fingers, so that, when the diseased portion is cut away, the end of the bowel cannot escape, and also to prevent the escape of its contents. Should there, however, accidentally be any leakage, the wound is protected by the compress which has been arranged beneath the bowel before the section is made.

The diseased portion having been thus excised, the proximal, or upper, segment of the bowel is immediately packed with gauze. Bleeding from the edge of the bowel may be checked by clamps and, if necessary, ligatures.

In freeing the rectum upon its anterior aspect, one has to deal with the pouch of peritoneum which dips down upon its front wall, between it and the uterus and vagina in the female and the bladder in the male. One should recognize this pouch, as it may be necessary to open it, and, indeed, this is probably desirable in all cases, as it enables one to bring down the upper part of the bowel with more ease. After an opening has been made into this pouch, it may be enlarged by cutting with the scissors, upon either side, close to the wall of the rectum; through the opening thus made two fingers may be introduced and the bowel drawn down; after it has been pulled down sufficiently, one may sew the edge of the opening in the peritoneum to the peritoneal layer that covers the bowel, upon either side, with several catgut sutures; the anterior portion, however, should be left open for drainage. Kraske advises against closing this opening in the peritoneal pouch even in part. He says that it should be loosely packed with strip gauze, surrounding the rectum in front, upon the sides, and behind, and reaching well up into the peritoneal cavity; the extremities of the gauze strips are allowed to emerge through the upper part of the skin incision, and should be marked for identification, so that they may be removed after five or six days. If this fold of peritoneum is involved in the disease, it may be oblit- 
erated by its opposing surfaces having become agglutinated, or the growth may have extended still farther so as to involve the uterus or bladder. This will add to the difficulty of the operation; but some surgeons do not consider it a counter-indication to the continuance of the operation, because, if necessary, the parts of these organs that are involved may be resected.

If the peritoneal fold is not involved in the disease it can usually be peeled away from the front wall of the rectum with the finger, and in this case one may be able to complete the operation without opening into the peritoneal cavity.

Diseased lymphatic nodes located behind the rectum, between it and the sacrum, should also be enucleated. There may be considerable bleeding caused by separating the rectum upon its posterior aspect and sides from branches of the superior hemorrhoidal; they should be clamped and ligated.

Suture of the ENds of the Bowel.-The upper segment should be sutured to the lower (anal portion), and this union may be either complete or partial.

Complete Union, the Ideal Method.-During the application of the sutures care should be taken that no fæces soil the suture line; a wad of gauze packed into the upper, central, segment of the bowel prevents this. There should be no tension on the upper segmentno tendency for it to draw up into the abdomen away from the anal portion. Proper isolation of the rectum and the opening of the peritoneal pouch will obviate this.

One may further fix the upper, central, segment of the bowel in the wound by several non-perforating sutures. For uniting the ends of the bowel fine silk sutures should be used. One may commence the suture in the middle line anteriorly and work around upon either side toward the back. The sutures should be introduced from the inner surface of the bowel and tied so that the knots are within the lumen of the bowel-they should be interrupted, and each should include the whole thickness of the wall of the gut, and be placed about $1 / 2 \mathrm{~cm}$. distant from each other. Those sutures which are introduced last, and which join the two segments of the bowel posteriorly, must be introduced from the outer surface, and do not penetrate the whole thickness of the wall of the bowel, but simply include the outer coats. When these latter sutures are tied, the knots will be found upon the outer aspect of the bowel. Before closing this posterior portion of the wound Hochenegg advises the introduction 
of a rubber tube surrounded by gauze from the anus well up into the bowel beyond the suture line. This prevents soiling of the suture line and also permits the passage of gas and fluid fæces during the few days immediately following the operation. A strip of gauze should be introduced into the wound, so as to lead from the suture line upon each side of the bowel out through the incision, for the purpose of providing drainage for this part, in the event of the sutures giving way. There is some danger in complete closure of the bowel. The sutures may tear through and allow the contents of the bowel to escape into the wound, especially if the obstruction

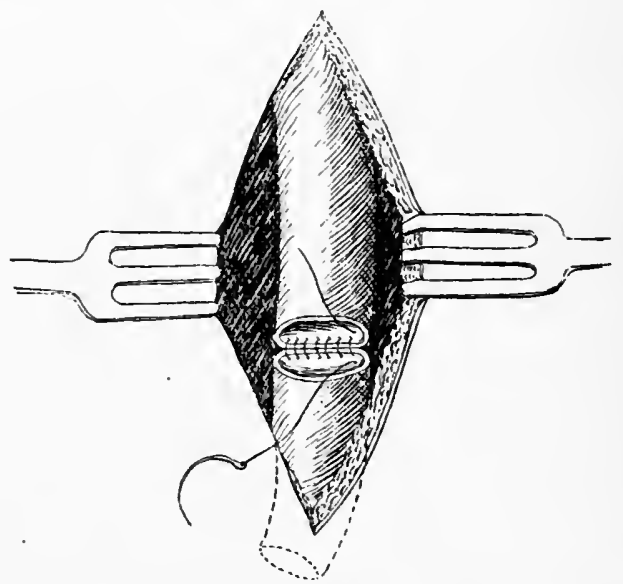

Fig. 147.-Resection of Rectum (Kraske). Diseased portion has been excised and the healthy upper and lower portions have been partially unlted with interrupted sutures that penetrate the entire thickness of the wall of the bowel.

offered by the disease had prevented the complete evacuation of the bowel before the operation. Masses of fæces come down and put a strain upon the stitches; if this accident occurs, the wound becomes infected, and we may get, as a result, a fatal peritonitis.

Union most often fails in the posterior part of the suture line in the bowel; this is due probably to the damage done to the vessels which supply the bowel, in isolating it. Such a break of the suture line, however, usually does no harm if proper drainage of the wound has been provided, and usually the resulting fæcal fistnla closes spontaneously, or may be closed by use of adhesive plaster strips in dressing or by a subsequent operation. 
Partial Union.-Instead of making a complete union we may join the ends of the segment of the bowel only anteriorly and upon the sides, leaving the posterior part of the wound open. The. upper segment of the bowel is then fixed in the wound to prevent its retraction. In this case we wait for the fæcal fistula that results to close spontaneously, or else we accomplish this by a subsequent operation.

However the ends of the bowel are treated, the wound should be well packed with iodoform gauze-not too tight, but reaching well down to the bottom of all parts of the wound. This packing is allowed to remain until it becomes loosened,-usually for about one week,-when the wound is again dressed and repacked. The incision in the skin is partly closed.

For Ajiputatio Recti (the Anal Portion of the Bowel being Involved in the Disease).-If it is desired to remove the lower (anal) portion of the bowel, together with the rest of the rectum, the skin incision should be prolonged from the tip of the coccyx, so as to encircle the anus. After the coccyx and part of the sacrum have been resected as described above, the whole length of the bowel, including the anal portion, is isolated, beginning below at the anus and working upward. Upon either side near the anus the attachment of the levator ani is separated from the rectim with the scissors, working close to the wall of the rectum. At times, some difficulty in separating the rectum from the prostate or the vagina is experienced. A sound may be introduced into the bladder, and this part of the operation done in the perineal position. This change of position, however, is probably unnecessary. When the bowel has been isolated to a point beyond the upper limits of the disease, a ligature may be thrown around the rectum and the diseased portion cut away. The end of the proximal (upper) part of the bowel into which a strip of gauze has been packed is then sewed to the margins of the skin in the upper part of the incision close to the edge of the sacrum with interrupted silk sutures. The wound is then packed carefully about the bowel, above and below, and the skin incision partly closed with several silk sutures. The bowel may be twisted through a quarter of a circle before uniting it to the margin of the skin, with the idea of making the artificial anus more retentive. 


\section{PART VII.}

\section{HERNIA, SPERMATIC CORD, TESTES, ETC.}

The Surgical Anatomy of the Groin.--The groin may be divided into the inguinal and femoral regions. These parts may be considered more or less together, on account of the close relationship that exists between them.

The inguinal region corresponds to that part of the anterior abdominal wall which lies just above Poupart's ligament, and is traversed by a canal for the passage of the spermatic cord, in the male, and the round ligament, in the female. By invaginating the integument of the scrotum, the finger may be introduced into this canal.

The femoral region corresponds to the upper anterior part of the thigh-the area immediately below Poupart's ligament. Underneath Poupart's ligament, between it and the pubic bone, there is a space through which the ilio-psoas muscle and anterior crural nerve, and the femoral vessels, etc., pass from the abdomen into the thigh.

The Superficial Layer of the Superficial Fascia. - Beneath the skin of the groin there is a loose connective tissue layer which contains a varying amount of fat, and in which the bloodvessels, nerves, lymphatic glands, etc., are located. This layer is called the superficial layer of the superficial fascia. In some subjects it is very thick. It is continuous with the general fatty layer of the body. In the male it is continued on to the penis, where it is thin and loose, forming one of the coats of that organ, and in the scrotum is continued into the dartos. From the scrotum it may be traced back into the perineum, where it is known as the superficial layer of the superficial perineal fascia. In the female it is continuous with the fatty layer of the labia majora, each one of which corresponds to one-half of the scrotum. The vessels which are found in this layer, and which may be cut in making the skin incisions in operating upon these parts, are the superficial epigastric, superficial circumflex iliac, and superficial external pudic arteries, together with their corresponding veins. 
The Lymphatic GLands. - The lymphatic glands of this region are arranged in two groups: one group, the inguinal, is spread along Poupart's ligament, and drains the external genitals, scrotum, penis, etc.; the other group lies along the saphenous vein, and in and about the saphenous opening. These drain the lower limb. In extirpating the inguinal group of glands there is but little hemorrhage, but it is necessary to avoid the spermatic cord. In extirpating the lower, femoral, group there may be considerable hemorrhage, and one must avoid injury to the internal saphenous vein and to the femoral vein, especially when excising those glands that are lodged in the saphenous opening.

The Deep Layer of the Superficial Fascia.-After the fatty layer has been removed from this region the deep layer of the superficial fascia is exposed. This fascia is thin, and covers the aponeurosis of the external oblique muscle in the inguinal region, and the fascia lata in the femoral region. It is adherent, in the middle line, to the linea alba, and, just below Poupart's ligament, to the fascia lata. In the male it forms one of the corerings of the penis, and is continued into the scrotum, where it forms the dartos, and backward beyond the scrotum, into the perineum, where it forms the deep layer of the superficial perineal fascia. In the perineum it is attached laterally to the rami of the pubes, and behind to the transverse perineal raphé. In the female this layer is continued into the labia majora. This fascia is firmly attached to the margins, or pillars, of the external ring, and is known as the external spermatic fascia. Entrance into the inguinal canal cannot be effeeted until this layer of fascia has been incised. From the margins of the ring this layer of fascia is continued downward, surrounding the cord and forming one of its investments, and below, as already mentioned, it is found in the scrotum as the dartos. Below Poupart's ligament, in the femoral region, this layer of fascia is firmly adherent to the margins of the saphenous opening in the fascia lata, where it is perforated by numerous vessels and lymphatics, and is called the cribriform fascia. From this point on, the inguinal and femoral regions may be studied separately.

The Inguinal Regiox.-The inguinal region is the site of inguinal hernia. After removing the deep layer of the superficial fascia from the inguinal region (including the margins of the external ring), we expose the aponeurosis of the external oblique and the external inguinal ring, into which the finger may be introduced, and 
from which the spermatic cord (the round ligament in the female) is seen to emerge.

The aponeurosis of the external oblique is the strong, smooth, glistening, bluish-white, fibrous expansion of the external oblique muscle. Its fibers have an oblique direction downward and inward toward the middle line, and join with each other in the linea alba. The lower fibers of the aponeurosis of the external oblique are collected into a thick bundle to form Poupart's ligament.

Poupart's ligament is a strong, fibrous band which extends from the anterior superior spinous process of the ilium downward and inward to the spine of the pubes. Both these bony processes are easily made out; the latter, the spine of the pubes, is readily felt beneath the soft parts upon the upper border of the pubic bone, about three-fourths inch from the symphysis. The fibers of the aponeurosis of the external oblique immediately above Poupari's ligament pass inward toward the middle line, interlacing with those from the opposite side, and are attached to the symphysis, and there is thus left a triangular opening in the aponeurosis, which is called the external inguinal ring. This so-called ring is simply a split in the aponeurosis of the external oblique. Its outer, or lower, border, or pillar, is formed by Poupart's ligament; its inner, or upper, border, or pillar, is formed by those fibers of the aponeurosis of the external oblique which are attached in the middle line to the symphysis, interlacing with those of the opposite side. The apex of this opening is directed upward and outward; its base corresponds to the crest, or upper surface, of the body of the pubic bone, that portion of the bone which is included between the pubic spine, to which Poupart's ligament is attached, and the symphysis. Various stay fibers are seen in the aponeurosis, passing from below upward and inward, near the apex of the external ring. These serve to bind the pillars of the ring firmly together, and are called the intercolumnar fibers.

The spermatic cord (round ligament in the female) is seen emerging from the external ring, and a director may be introduced through the ring upward and outward into the inguinal canal. From the inner end of Poupart's ligament-i.e., from the external pillar of the ring-a triangular sheet of fibers is given off, which is reflected upward and inward toward the middle line, and is continued into the anterior layer of the sheath of the rectus muscle. This is called the triangular ligament, or Colles's ligament, and is situated behind the inner end of the external ring, and in front of the conjoined tendon, 


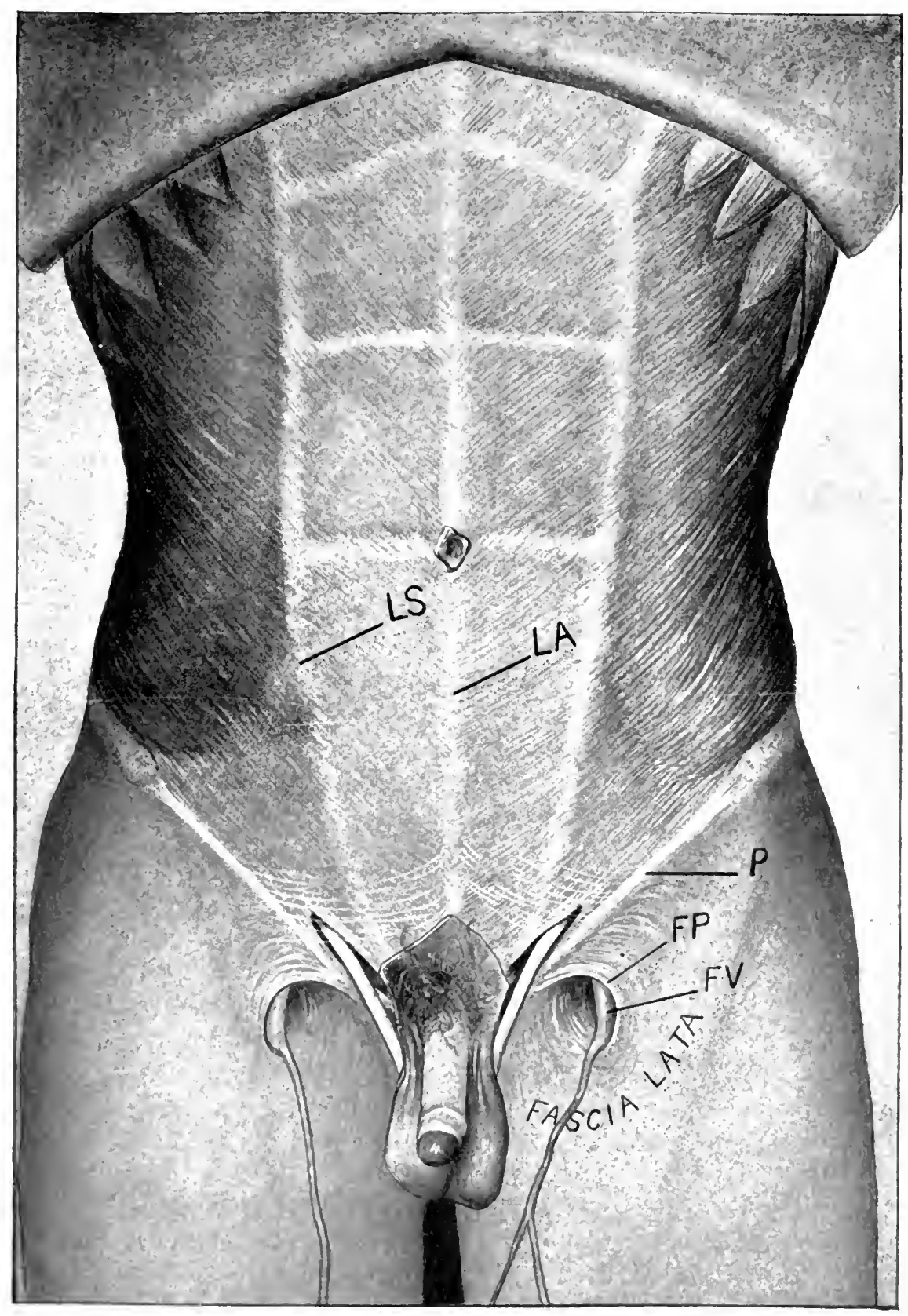

Fig. 14R, - Inguinal and'Femoral Regions. FP, whe of falcitorm procen: Fl, femeral vein : L.A. linea alha; $L S$, linea semilunatis; $P$, Poupart - ligament. The external inguinal ring is shown with the spermatic cord emerging. The fibers croming the upper outer angle of the ring are known as the intercolumnar fiters. 

and serves to strengthen this part. If we examine still further this inner end of Poupart's ligament,-i.e., the external pillar of the ring,-we find given off from its lower border, just before its attachment to the pubic spine, a strong triangular band, which is attached to the ilio-pectineal line, a prominent ridge upon the upper surface of the pubic bone, which is continued outward and backward from the pubic spine to the edge, or brim, of the true pelvis. This band in known as Gimbernat's ligament. It presents an outer, sharp, curved edge, and is of much anatomical interest in the study of femoral hernia.

The Inguinal Canal.-The inguinal canal is an oblique slit in the abdominal wall, and, under ordinary circumstances, the greater the intra-abdominal pressure, the tighter its closure. It is from 4 to $5 \mathrm{~cm}$. (one and one-half inches) long, and lies above and parallel with Poupart's ligament. It terminates beneath the integument at the external inguinal ring, a triangular opening in the aponeurosis of the external oblique, which is located just above the crest of the pubes.

If we introduce a director through the external ring into the inguinal canal, and pass it in a direction upward and outward underneath the aponeurosis of the external oblique, to a point about half an inch above the middle of Poupart's ligament,-i.e., the location of the internal ring, - and then split the aponeurosis upon this, we open up the inguinal canal and expose its contents: the spermatic cord, in the male; the round ligament, in the female. The cut edges of the aponeurosis should be seized with artery forceps and separated freely from the underlying parts with the finger. The spermatic cord is a structure as big around as the little finger. It is made up of the vas deferens, which is the efferent duct of the testicle; the artery of the vas deferens and the cremasteric artery, and their corresponding veins; the spermatic artery, and the pampiniform venous plexus. As these structures traverse the inguinal canal they are all bound together into a single rounded cord by a strong sheath of fascia, the infundibular process of the transversalis fascia. Descending upon the cord are also seen the fibers of the cremaster. muscle, which are derived from the lower edge of the internal oblique in the descent of the testes. The cord is also accompanied, in its course through the inguinal canal, by the genital branch of the genito-crural nerve and the inguinal branch of the ilio-inguinal nerve. 
After the inguinal canal has been opened by splitting the aponeurosis of the external oblique, the free, curved, fleshy edge. of the internal oblique is exposed to view. This muscle, the part seen here, arises from the outer half of Poupart's ligament. If the edge of this muscle is raised and drawn upward and outward for a short distance, or incised, we expose the transversalis muscle, which lies beneath the internal oblique. That portion of the transversalis which is thus exposed arises from the outer third of Poupart's ligament, and is covered by the internal oblique, and is not seen until the edge of this latter muscle has been drawn aside.

Toward the outer part of the inguinal canal these two muscles, where they arise from Poupart's ligament, are situated for a short distance in front of the spermatic cord. They then arch inward above the cord, and, joining with each other, become tendinous, and, as the conjoined tendon, descend behind the cord, to be attached to the upper surface of the pubic bone; i.e., the crest and the pectineal line. The conjoined tendon, at its attachment to the pubic bone, is placed behind the external ring, and participates in the formation of the inner part of the posterior wall of the inguinal canal. It is important to note that that portion of the posterior wall of the inguinal canal which is included between the arching free edge of the internal oblique muscle above and Poupart's ligament below is formed by the transversalis fascia only. This fascia is a fibrous layer which lines the whole inner surface of the abdomen, including the posterior surface of the anterior abdominal.wall, and it is here exposed to view where the muscle is deficient; i.e., between the edge of the internal oblique muscle above and Poupart's ligament below. Through the outer part of the posterior wall of the inguinal canal the several structures which go to make up the spermatic cord (round ligament in the female) pass forward into the inguinal canal, being provided with a strong, fibrous sheath, which is known as the infundibular process, by the fascia transversalis. This sheath incloses the several elements of which the cord is composed, and serves to bind them together into a single bundle, which traverses the inguinal canal and emerges at the external inguinal ring. The point at which the structures which constitute the spermatic cord pass forward into the inguinal canal is the site of the internal inguinal ring. The internal ring is an opening in the transversalis fascia, which is located half an inch above the middle of Poupart's ligament. The inguinal canal proper has no internal opening; i.e., it 


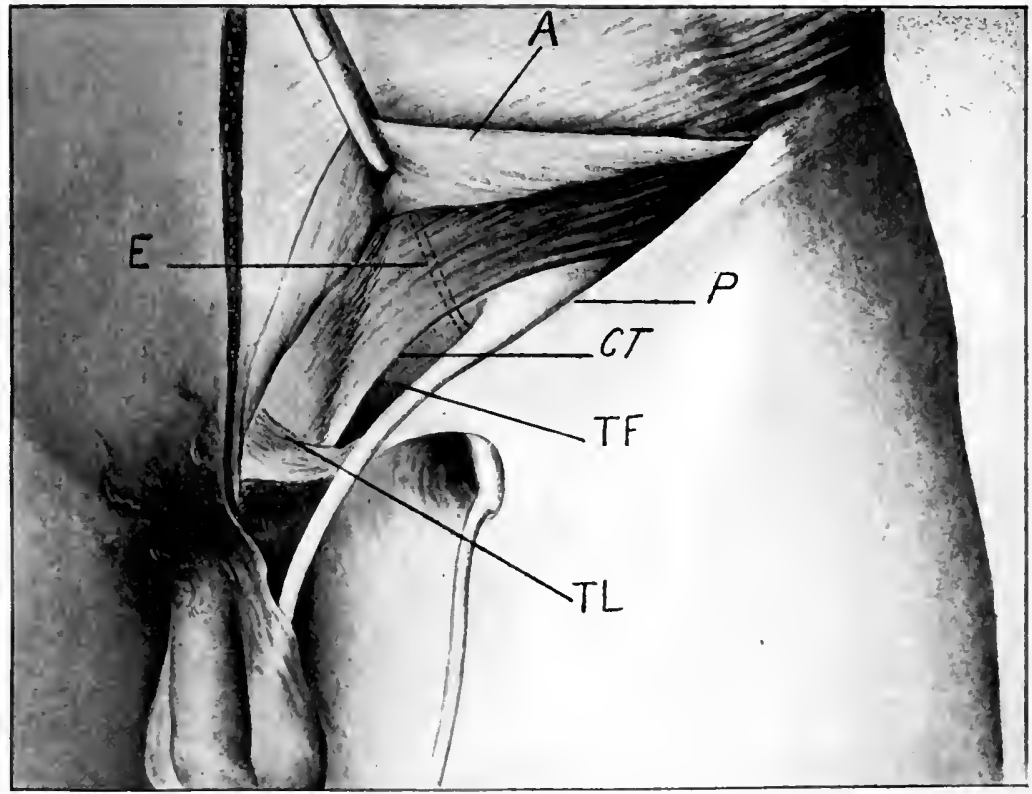

Fig. 149.-The Inguinal Canal. The canal has been laid open by splitting the aponeurosis of the external oblique $(A)$, which is grasped with the artery forceps and drawn upward; $C T$, edge of the internal oblique muscle (conjoined tendon); $E$, dotted line represents the course of the deep epigastric artery, which is located heneath the transversalis fascia; $P$. Poupart's ligament; $T F$, transversalis fascia, which forms the posterior wall of the inguinal canal: $T L$, triangular ligament, which is given off from the inner end of Poupart's. 

does not communicate with the abdominal cavity. The internal inguinal ring is really the mouth of the infundibular process, which is, in reality, the sheath that is provided to the spermatic cord from the transversalis fascia.

The infundibular process is a glove-finger-like diverticulum, or pocket, which is derived from the fascia transversalis, being prolonged downward into the bottom of the scrotal sac, and, through this, the testicle, drawing the vas deferens, etc., after it, descends in its journey from the abdomen into the scrotum. After the testis has reached the bottom of the scrotal sac, the upper part of this infundibular process-i.e., the part which corresponds to the cord -contracts and shrinks so closely around the structures which make up the cord, and which are contained within it, that its cavity is, in this way, entirely obliterated, and the shrunken infundibular process remains permanently as the proper fibrous sheath of the spermatic cord.

The lower part, however, of the infundibular process remains permanently unchanged as one of the layers of the scrotum.

The contraction of the infundibular process about the upper part of the cord may be incomplete, and there may be thus left a space within the sheath of the cord (infundibular process), into which the point of the finger may be insinuated from within the abdomen. The finger under these circumstances does not enter the inguinal canal, but passes through the internal ring into the proper sheath of the spermatic cord. The mouth of the infundibular process, the "internal ring," may be best studied from within the abdomen, after the peritoneum, which lines this portion of the $a b$ dominal wall, has been stripped away.

Beneath the transversalis fascia-i.e., the posterior wall of the inguinal canal-is found the parietal layer of the peritoneum, with an intervening stratum of loose connective tissue, containing fat, between it and the transversalis fascia; this is the so-called subperitoneal connective tissue layer. The layer of peritoneum which lies behind, or rather beneath, the posterior wall of the inguinal canal presents no opening whatever. Within the abdomen, about the mouth of the infundibular process, "internal ring," the parietal peritoneum is adherent to the transversalis fascia, and may show a slight bulging into the neck of the infundibular process (sheath of the cord).

In the study of these parts the deep epigastric artery plays 
an important rôle. This artery may be seen, or its pulsation felt, as it lies beneath the transversalis fascia in the subperitoneal connective tissue between the transversalis fascia and the peritoneum. The artery is accompanied by one or two veins. It arises from the external iliac (femoral) just before this vessel passes out of the abdomen under Poupart's ligament, and ascends obliquely upward and inward toward the umbilicus to reach the outer border of the rectus muscle. It passes across the posterior wall of the inguinal canal about the middle, and so divides it into two parts, an outer and an inner. The outer part of the posterior wall of the inguinal canal, that part which lies external to the deep epigastric artery, is formed by the transversalis fascia and the underlying peritoneum, and presents the opening through which the structures that form the spermatic cord (round ligament) leave the abdomen, the internal ring. The presence of this orifice tends to weaken this outer part of the posterior wall of the inguinal canal. The inner portion of the posterior wall of the inguinal canal, that part which lies internal to the deep epigastric artery, is strengthened, in part, by several additional layers. From before backward this part of the posterior wall of the inguinal canal is formed of the triangular ligament, conjoined tendon, transversalis fascia, and parietal peritoneum. This inner portion of the posterior wall of the inguinal canal is, therefore, much more secure than the outer part.

A hernia that protrudes through the posterior wall of the inguinal canal external to the deep epigastric-i.e., one which passes through the "internal ring" and works its way downward along the cord-is an oblique, or external, inguinal hernia, the common rariety. In those cases in which the upper part, or neck, of the infundibular process has failed to become tightly contracted around the elements of the cord right up to the point at which they emerge from the abdomen, the predisposition to hernia is, without doubt, more pronounced, and this is especially the case if, in addition, the peritoneum, which is normally adherent about the site of the "internal ring," shows a certain degree of bulging into the mouth of the patent infundibular process.

A hernia that bulges forward through the posterior wall of the inguinal canal to the inner side of the deep epigastric artery is a direct, or internal, inguinal hernia. Such a hernia does not pass through the "internal ring" and descend along the course of the cord, within its sheath (infundibular process), but bulges directly 


\section{At Sixth Month.}

Testls located in the back part of the abdominal cavity, covered by the peritoneum upon its anterior aspect.

$G$, gubernaculum of Hunter.

$I P$, iniundlbular process of the transversalis fascia.

$P$, peritoneum lining the interlor of abdominal cavity.

$S$, scrotum.

$T$, testis.

$T F$, transversalis fascia.

$V D$, vas deferens.

\section{At the Seventh Month.}

The testis has descended into the inguinal region toward the mouth of the infundibular process-future internal Ingulnal ring.

\section{At the Eighth MONTh.}

The testis has entered the Infundibular process, carrying a process of the peritoneum with it.

$V P$, vaginal process of peritoneum.

\section{At Ninth Month.}

Testis has reached the bottom of the Infundibular process,-scrotum,-carrying process of peritoneum with it.

\section{THIRD to FOURTH WeEk AFter BirTh.}

Testis is located in the bottom of the infundibular process-scrotum. Obliteration has begun in the vaginal process.

\section{Several Months after Birth.}

Normal adult condition.

Testis rests in bottom of infundibular process-scrotum. The vaglnal process which accompanied the testis in its descent has become obliterated except for that portion of its extent which corresponds to the testis. This remains as the tunica vaginalis testls.

$C T$, cavity of tunica vaginalls testis. 

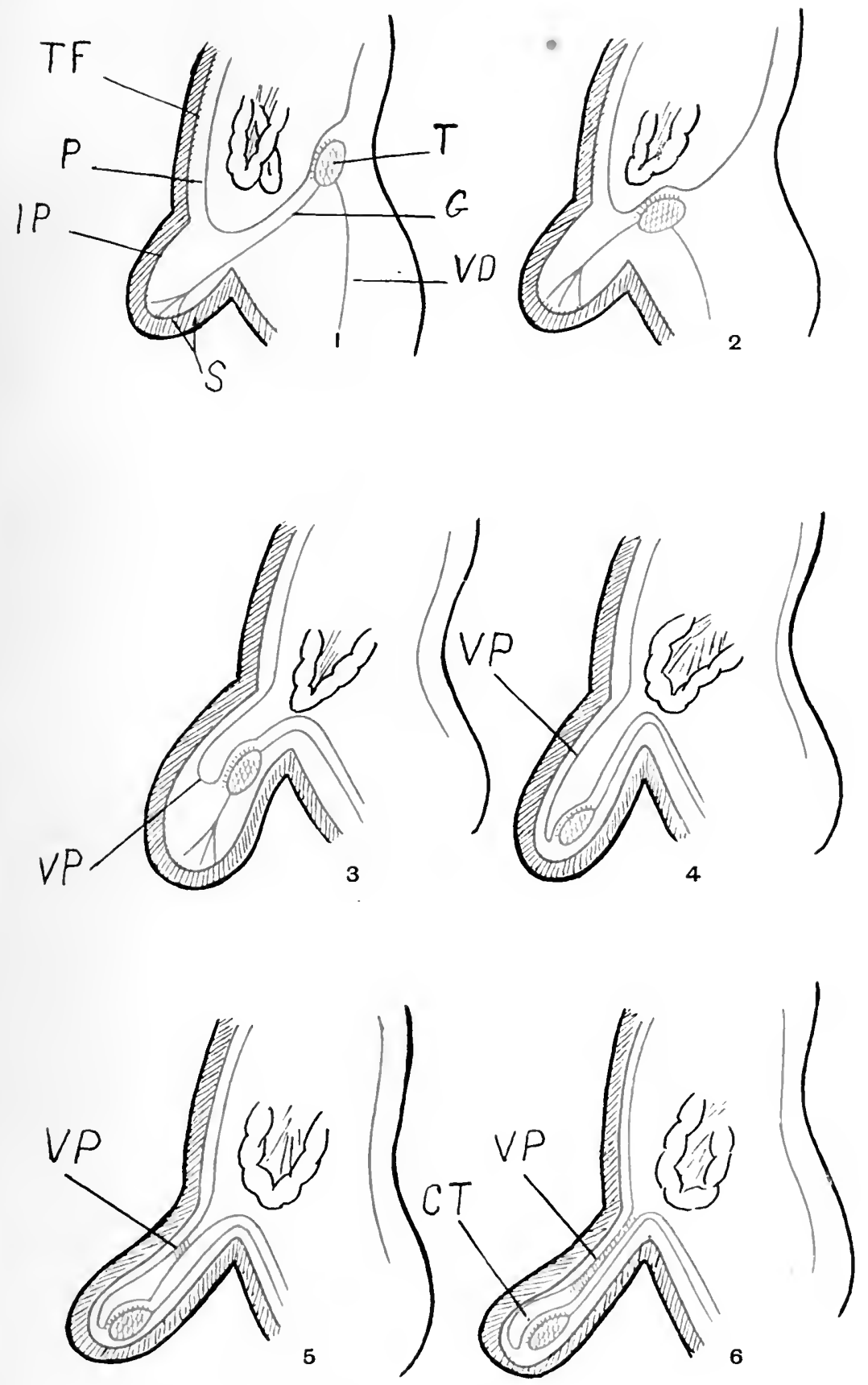

Fig. 1.)1.-Descent of the Testis. 

forward into the inguinal canal, to the inner side of the cord, and, besides the transversalis fascia, it may have to push the conjoined tendon, etc., before it, or else force its way between the fibers of this structure. These accessory structures form a strong barrier against the formation of a direct hernia, which variety is much less common than the oblique.

In the female the inguinal canal and rings are all less well developed than in the male. The round ligament is a thin structure, often difficult to find. After passing through the inguinal canal it emerges from the external ring, and is then lost in the connective tissue about the external ring and in the labia majora.

Inguinal hernia is comparatively infrequent in the female. When it occurs, it is analogous to that in the male, and may descend into the labia majora.

The Descent of the Testes. - The testes (ovaries in the female) are developed within the abdomen from the Wolffian body, and in early fotal life they are situated in the back part of the abdominal cavity near the kidneys. They lie not within the peritoneal cavity, but, like the kidney, behind the peritoneum, which is adherent to their front surface. From this position, the testes, during the later months of fœtal life, gradually descend. They descend behind the peritoneum and enter the infundibular process through its mouth, the "internal ring." Finally, during the last month of intra-uterine life they arrive at their normal destination, the bottom of the scrotal pouch.

The ovaries descend in an analogous manner, but do not pass out of the abdominal cavity.

Preparatory to the descent of the testis there is a pouch-like bulging of the lower part of the anterior abdominal wall in either inguinal region. A shallow pouch is thus formed on either side, which gradually becomes deeper, and finally the two join together in the middle line to form the scrotum. Each of these pouches is lined on its internal aspect by a sac-like prolongation from the transversalis fascia (infundibular process). These pouches are empty and ready to receive the testes.

Reaching from the testis as it lies within the abdomen, downward into the bottom of the infundibular process (scrotum), there is a musculo-fibrous structure, the gubernaculum of Hunter. It serves to lead the testis down into the scrotal sac.

About the sixth month of fœtal life the descent of the testis 
begins. The gubernaculum contracts and draws the testis downward toward the inguinal region. About the seventh month the testis arrives at the "internal ring," the wide-open mouth of the infundibular process. The testis then passes into the infundibular process, and, as it does so, it brings a bag-like process of the peritoneum, which is adherent to it, with it. This is called the vaginal process of the peritoneum. At the eighth month the testis is found in the infundibular pouch, together with the vaginal process of the peritoneum, which accompanies it, and during the last month of intrauterine life it is found at the bottom of the infundibular pouch, the scrotum, together with its vaginal peritoneal process.

The testis may be interrupted in its journey into the scrotum at any point, and may remain stationary either in the abdomen or in the inguinal canal. This condition occasionally complicates congenital hernia. After the testis has reached the bottom of the scrotal sac, the peritoneal pouch, which accompanied it, becomes, for that part of its extent which corresponds to the vas deferens, gradually obliterated. This process of obliteration commences in the middle of the tube and extends upward toward its abdominal orifice, and downward toward the testis, and, in the adult, this obliterated portion of the vaginal process is represented only by a fibrous strand that is found, together with the vas deferens, etc., inclosed within the proper sheath of the cord.

The lower part of the vaginal process, that portion which corresponds to the testis, remains permanently as the tunica vaginalis testis. At birth the canal of the vaginal process is still pervious, but very much shrunken, and becomes rapidly obliterated during the first few weeks of extra-uterine life.

If the peritoneal pouch, the vaginal process, which accompanies the testis in its descent, remains pervious after birth throughout its whole extent, and, if its orifice is large enough to permit, a coil of intestine may enter; and we shall then have a congenital hernia.

In the female the round ligament is the remains of the gubernaculum. The ovary descends like the testis, but does not leave the abdominal cavity; it remains in the pelvis. It does, however, exceptionally leave the abdominal cavity, and may then be found in the labia majora. Congenital hernia is uncommon in the female.

To recapitulate: There are two varieties of inguinal hernia, the direct, or internal, and the oblique, or external. The direct is always acquired, and is less common than the indirect. In this 


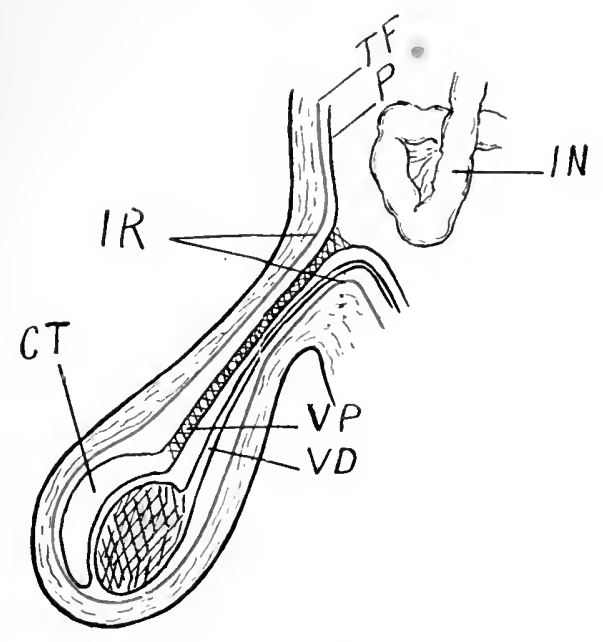

Fig. 151.-Normal Condition of Inguinal Region, Scrotum, etc. Testis in bottom of scrotum and vaginal process obliterated. $C T$, cavity of tunica vaginalis testis; $I N$, intestine within abdominal cavity; $I R$, internal inguinal ring-the mouth of the original infundibular process of the transversalis fascia; $P$, peritoneum lining abdominal cavity; $T F$, transversalis fascia ; $V D$, vas deferens; $V P$, vaginal process of peritoneum-obliterated.

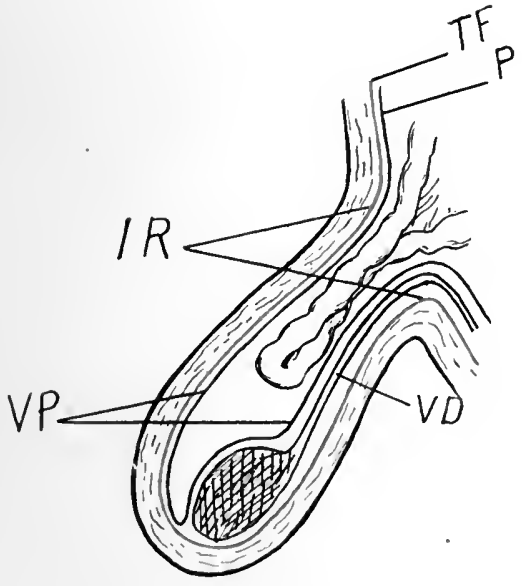

Fig. 152.-Condition of Parts in Presence of a Congenital (Oblique Inguinal) Hernia. Note that the vaginal process is patent, unobliterated, and that a coil of intestine has entered.

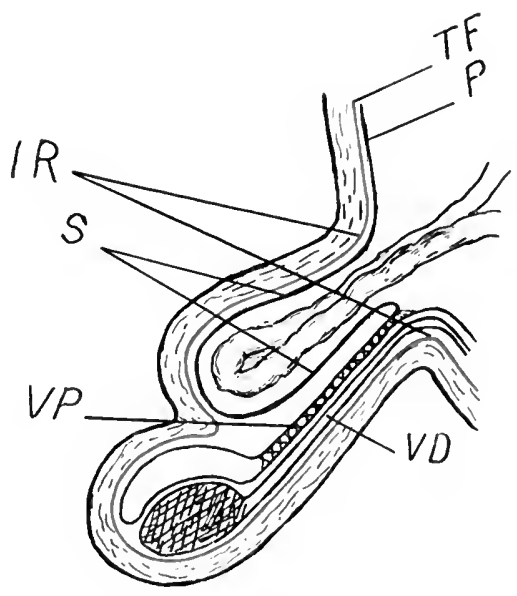

Fig. 15\%,-Condition of Parts in Presence of an Acquired Oblique Inguinal Hernia. Note that the vaginal process $(V P)$ is obliterated and that a coil of intestine has pushed its way down into the original infundibular process (sheath of the spermatic cord), driving a new process of peritoneum (S) before it. This peritoneal process forms the sac of the hernia. 

variety a pouch of peritoneum (the hernial sac)-containing, for example, a loop of gut-simply forces that part of the posterior wall of the inguinal canal that lies to the inner side of the deep epigastric artery before it into the inguinal canal, and finally down through the external ring.

The oblique variety may be either congenital or acquired.

A congenital hernia is due to the absence of obliteration in the vaginal peritoneal process. If this process remains patent throughout its entire length, the hernial contents-for example, a coil of gut - simply drop into the open pouch, and we have the usual form of congenital hernia.

An acquired oblique hernia is produced after the vaginal process has become completely and permanently obliterated. In this variety the contents-for example, a coil of gut-must force an entirely new pouch of peritoneum, which constitutes the hernial sac, before it. This peritoneal sac enters the mouth of the infundibular process ("internal ring") like a wedge, and works its way downward along the spermatic cord, inclosed within the sheath of the cord (infundibular process), which it simply distends; or else, after passing through the internal ring into the infundibular process (sheath of the cord), it causes a bulging of a circumscribed portion of the sheath of the cord, with the result that a pocket, or pouch, is formed, which is really an offshoot from the proper sheath of the cord, and in this pouch the hernial peritoneal sac is found, together with the hernial contents.

An acquired hernia may traverse the whole length of the inguinal canal and enter the scrotum, but its sac is always entirely distinct from the original vaginal peritoneal process, and its contents are never to be found in the same cavity with the testis, as is the case in the congenital variety.

A partial obliteration of the vaginal process of the peritoneum may occur, and we may then have an infantile, or encysted, hernia. In this case the vaginal process is occluded at or near its mouth, but remains open throughout a part of its extent below. We then have a hernia, with its own newly acquired peritoncal sac, like an ordinary acquired hernia, passing through the internal ring and downward within the sheath of the cord, pushing the closed, but unobliterated, vaginal peritoneal process in front of it. When such a hernia is operated upon, it looks as though there were two separate and distinct sacs. The unobliterated vaginal process, within which the testis is found, is entered first, and then a second serous sac, the 
true hernial sac, is met with and incised, and within this the hernial contents are encountered.

The Femoral Region.-The area immediately below Poupart's ligament is known as the femoral region.

The Fascia Lata is exposed after the skin and superficial fascia have been removed. This is a strong, aponeurotic layer which entirely surrounds the muscles of the thigh, and serves to bind them into a compact mass. It is attached above, in front, to the whole length of Poupart's ligament, from the pubic spine to the anterior superior iliac spine; externally, to the crest of the ilium; behind, to the sacrum; and, internally, to the rami of the pubes and ischium.

Just below Poupart's ligament, where the internal saphenous vein enters the femoral vein, the fascia lata presents an oval opening, the saphenous opening. It is only exposed after the cribriform fascia (that part of the deep layer of the superficial fascia which is attached to the margins of the saphenous opening) has been removed. The outer margin of the saphenous opening is sharp and curved, and was called by Allan Burns the falciform process. If the falciform process is traced upward and inward, it is found to be continuous with the inner end of Poupart's ligament and with Gimbernat's ligament, some of its fibers being attached, with this latter ligament, to the pubic bone. Below, the falciform process is seen to curve inward underneath the internal saphenous vein, becoming continuous here with that part of the fascia lata which covers the pectineus muscle (pubic portion of the fascia lata). The free edge of the falciform process, and that part of the fascia lata external to it, cover the femoral sheath upon its anterior aspect, and are known as the "iliac portion" of the fascia lata. It is attached above to the whole length of Poupart's ligament, and externally is continuous with the sheath of the sartorius muscle.

That portion of the fascia lata upon which the internal saphenous vein rests, and which covers the pectineus muscle, may be traced upward, under Poupart's ligament, as far as the ilio-pectineal line, to which it is attached, and from which the pectineus muscle arises. This is known as the "pubic portion" of the fascia lata. Beneath the femoral vessels this pubic portion of the fascia lata is continuous, externally, with the fascia which covers the ilio-psoas muscle (fascia iliaca). Above, under Poupart's ligament, this fascia, which covers the pectineus muscle, is thickened, and is known as the pubic ligament of Cooper. These two portions of the fascia lata, the iliac and 

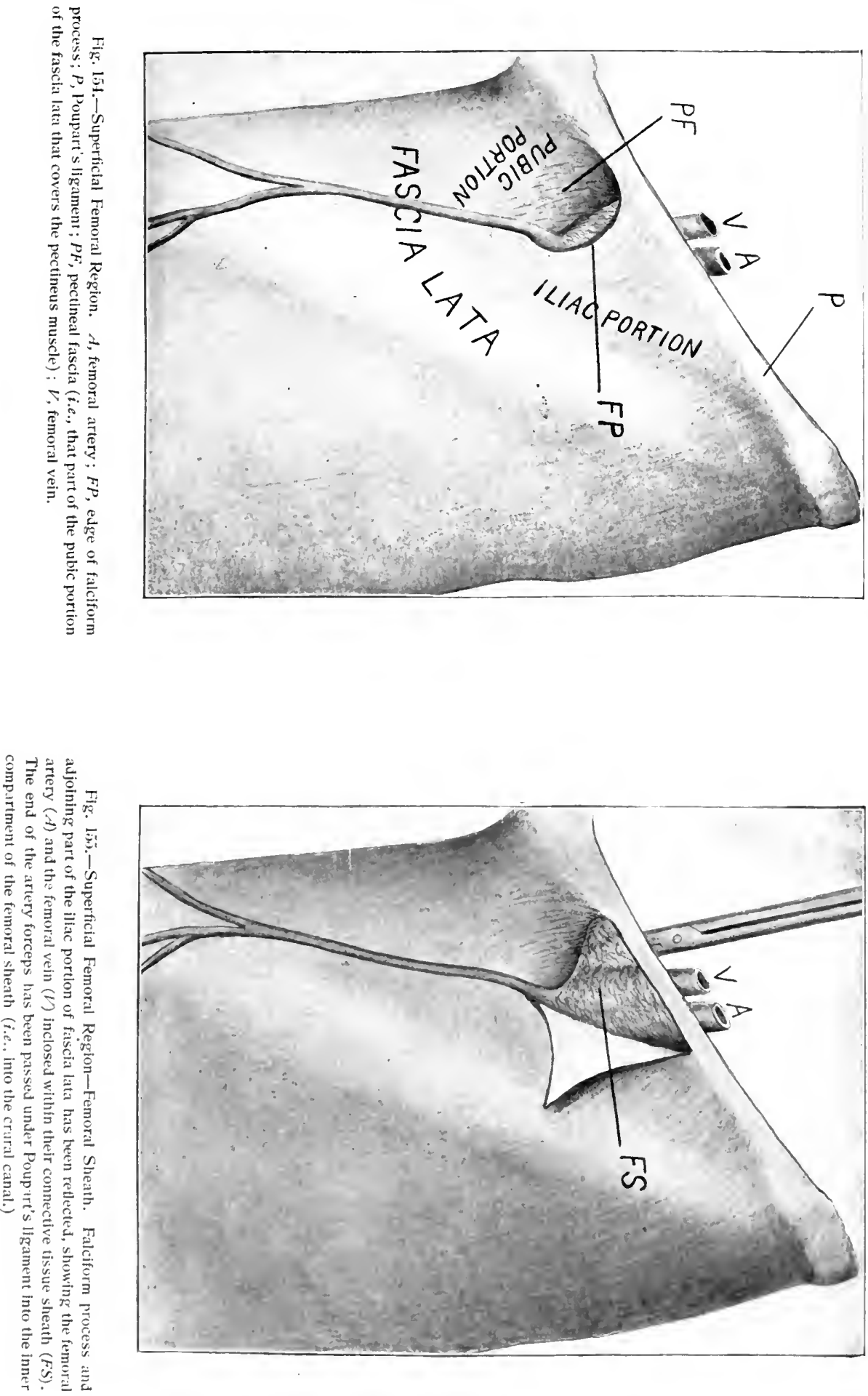

pubic portions, are so arranged that a slit-like opening, the saphenous opening, exists between them, and through this the internal saphenous vein joins the femoral vein.

The femoral vessels, inclosed within their sheath, are sandwiched in between these two portions of the fascia lata, resting behind upon the fascia which covers the pectineus and ilio-psoas muscles, and covered in front by the iliac portion of the fascia lata. The two portions of the fascia lata, which have just been described, the iliac and pubic portions, are simply parts of one and the same fascia, and are seen to be directly continuous with each other, below the saphenous opening upon the front of the thigh. The pubic portion of the fascia lata, which corresponds to the pectineus muscle, is, as already said, continuous externally, behind the sheath of the femoral vessels, with the iliac fascia, which invests the ilio-psoas muscle. One should not confuse the names "iliac portion of the fascia lata" with "iliac fascia."

The Space Beneath Poupart's Ligament. - Through this space the ilio-psoas muscle and the anterior crural nerve and the femoral vessels pass out of the abdomen into the thigh.

The ilio-psoas muscle, with the anterior crural nerve, occupies the outer part of the space. The ilio-psoas muscle is a thick mass of muscle which has its origin within the abdomen from the iliac fossa, bodies of the lumbar vertebræ, etc. It consists of the psoas and iliacus muscles, and passes downward under Poupart's ligament into the thigh, where it is attached to the lesser trochanter of the femur and to the surface of the bone immediately below this.

Within the abdomen the ilio-psoas muscle is covered by a thick fascia, the fascia iliaca, which is attached to the bodies of the lumbar vertebræ and to the sacrum, to the crest of the ilium, and to the brim of the pelvis.

At Poupart's ligament, that part of the iliac fascia which covers the outer portion of the ilio-psoas muscle-i.e., corresponding to the outer third of Poupart's ligament-does not pass down into the thigh with the muscle, but is attached to Poupart's ligament, whence it is reflected upward, becoming continuous with the transversalis fascia, which lines the whole posterior surface of the anterior abdominal wall. Internal to this, however, corresponding to the inner portion of the ilio-psoas muscle, the fascia which covers the muscle passes with the muscle, underneath Poupart's ligament, down into the thigh, and in the upper part of the thigh is continuous, behind the 
sheath of the femoral vessels, with the fascia which covers the pectineus muscle (pubic portion of the fascia lata). Immediately beneath Poupart's ligament the iliac fascia is thickened, and this thickened portion is called the ilio-pectineal ligament. This is not an isolated ligamentous band of fibers, but simply a thickened portion of the fascia iliaca as it passes with the ilio-psoas muscle under Poupart's ligament into the thigh. It extends from the junction of the outer and middle thirds of Poupart's ligament downward and inward to the ilio-pectineal eminence, and serves thus to divide the space underneath Poupart's ligament into two portions: an outer, the iliopsoas space, which contains the ilio-psoas muscle and the anterior crural nerve, and an inner and upper, the femoral space, through which the femoral vessels pass from the abdomen into the thigh.

The femoral space is bounded above by Poupart's ligament; below, it is bounded externally by the ilio-pectineal ligament, and, internally, by the pubic ligament of Cooper. The so-called pubic ligament of Cooper is simply the thickened upper portion of the fascia which covers the pectineus muscle. Internally, the space is bounded by the sharp, curved edge of Gimbernat's ligament. The space is limited externally by the junction of Poupart's ligament and the ilio-pectineal ligament.

The Femoral Sheath.-As the femoral vessels pass into the thigh, through the femoral space, they are inclosed in a special connective tissue sheath, and rest upon the ilio-psoas and pectineus muscles. The femoral sheath is a funnel-shaped connective tissue envelope which is prolonged downward from the margins of the femoral space, inclosing the vessels as they pass into the thigh. Corresponding to its commencement at Poupart's ligament, the femoral sheath is widemouthed, and attached all around to the margins of the femoral space. Above, it is attached to Poupart's ligament; below, to the ilio-pectineal ligament (thickened portion of the fascia covering the ilio-psoas muscle) and to the ligament of Cooper (thickened upper portion of the fascia that covers the pectineus muscle). Internally, it is attached to the edge of Gimbernat's ligament. The femoral sheath is continued but a short distance downward upon the femoral ressels, becoming narrow and contracted below, and closely applied to the walls of the vessels.

The femoral sheath is divided into three compartments, which are entirely separate and distinct from each other, by connective tissue septa. In the outer compartment the femoral artery is 


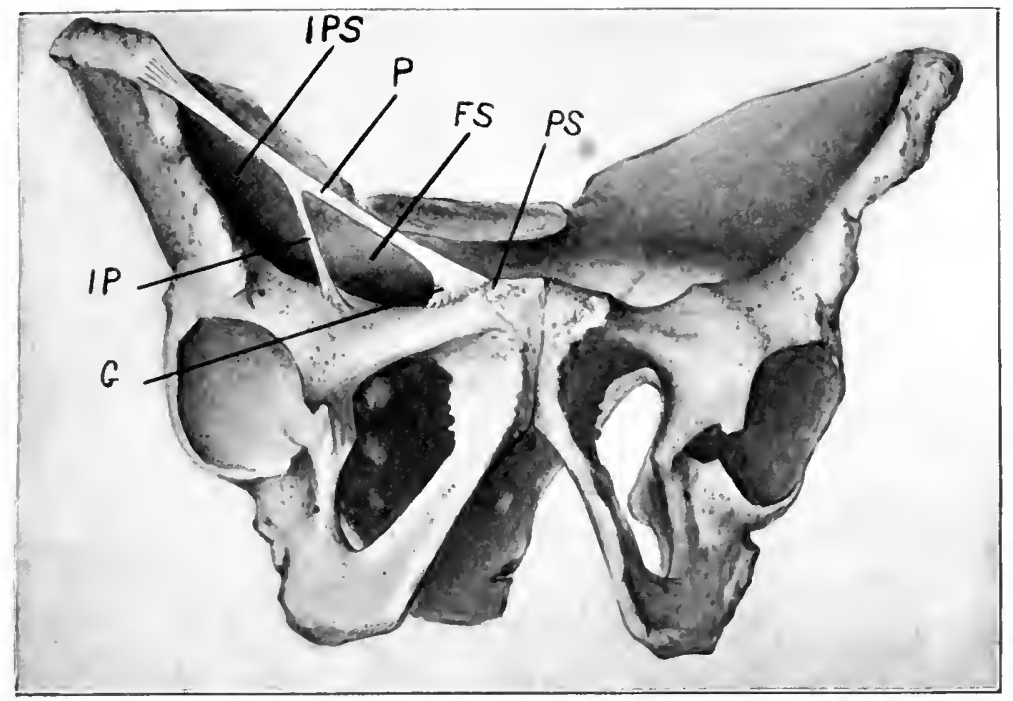

Fig. 156.-The Pelvis and Ligaments of the llio-pubic Region. FS, femoral space; $G$, Gimbernat's ligament; $I P$, ilio-pectineal ligament; $I P S$, ilio-psoas space; $P$, Poupart's ligament; $P S$, pubic spine.

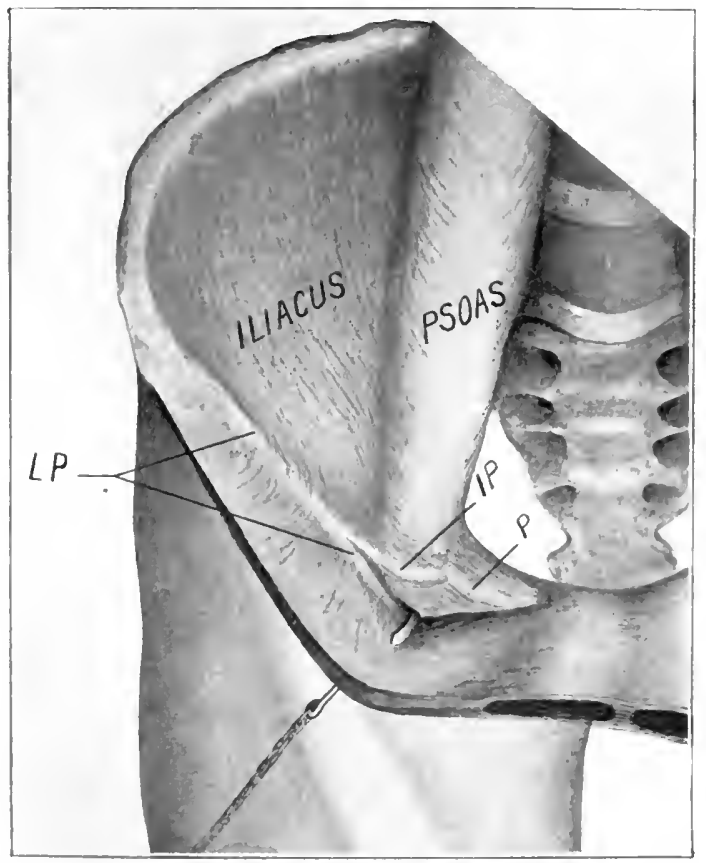

Fig. 15i.-Femoral Space. Femoral vessels and sheath as they pass under Poupart's ligament have been cleared away. Poupart's ligament lifted upon hook. The iliacus and psoas muscles are covered by their fascia, the fasci iliaca; $I P$, ilio-pectineal IIgament-thickened portion of the fascia that invests the iljo-psoas muscle; LP, Poupart's Ilgament; $P$, pubic ligament of Cooper-upper thickened part of the fascia that covers the pectineus muscle. 




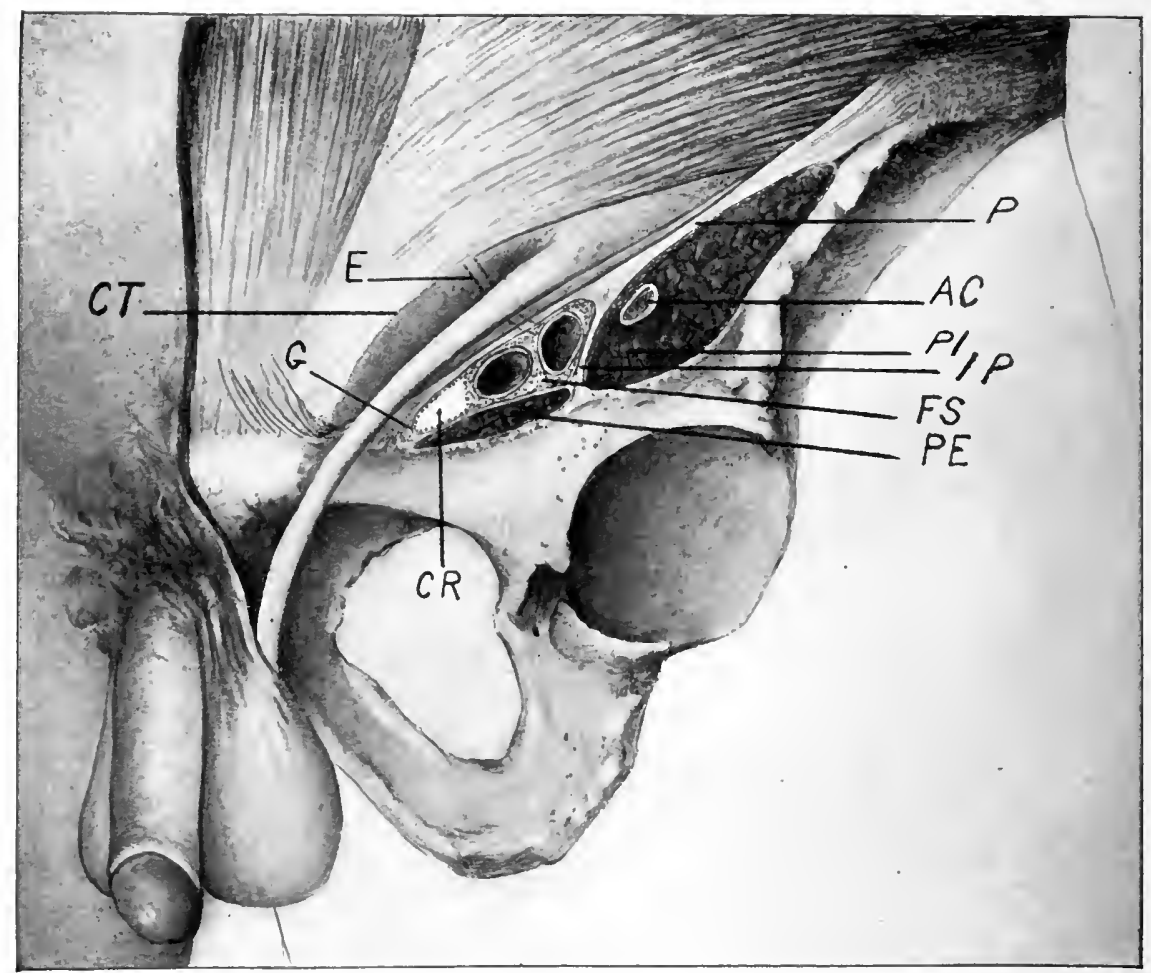

Fig. 158.-Deep Femoral Region-the Femoral Vessels, etc., Cut Across as they Emerge Under Poupart's Ligament. $A C$, anterior crural nerve ; $C T$, edgre of the conjoined tendon; $C R$, crural ring ; $E$, dotted line indicates the course of the deep epigastric artery; $F S$, femoral sheath; $G$, Gimbernat's ligament; $I P$, ilio-pectineal ligament; $P$, Poupart's ligament; $P E$, pectineus muscle. This muscle rests upon the pubic bone and is covered by its fascia, - the pectineal fascia, $\rightarrow$ which is somewhat thickened immediately beneath Poupart's ligament, where it Is known as the pubic ligament of Cooper. It will be noticed that the femoral sheath is divided into three compartments : the outer for the femoral artery; the middle for the femoral vein; the inner $(C R)$ is the crural ring, the mouth of the crural canal. 
lodged; in the middle, the femoral vein; the inner compartment contains a lymphatic gland and some loose connective tissue, and gives passage to the lymphatic vessels that enter the abdomen from the lower extremity. This space, the inner, is called the crural canal. It is inclosed within the femoral sheath, and reaches from Gimbernat's ligament downward upon the inner side of the femoral vein as far as the junction of the internal saphenous vein with the femoral, at which point the crural canal ceases to exist, because here the femoral sheath is applied directly to the wall of the femoral vein.

The orifice of this crural space, or canal, is called the crural ring. The crural ring is bounded above by Poupart's ligament; below, by the pectineus muscle and the fascia which covers it, and which is here thickened and called the pubic ligament of Cooper; internally, by Gimbernat's ligament; and, externally, by the femoral vein. A femoral hernia, as it descends into the thigh, usually occupies this crural canal, lying to the inner side of the femoral vein, and, just above the junction of the internal saphenous vein with the femoral vein, where the crural canal terminates, it presents in the saphenous opening.

Study of the Inguinal and Femoral Regions from Within the Abdomen.-To examine these regions from within the abdomen, an incision is made through the anterior abdominal wall, on either side, passing from the umbilicus outward and then downward to a point just external to the anterior superior spine of the ilium.

The Inguinal Region.-The bladder is seen to occupy the anterior median portion of the true pelvis, and when moderately full reaches as high as the symphysis. It will be observed that the peritoneum which covers the bladder is continued forward from the fundus of that organ over on to the posterior surface of the anterior wall of the abdomen, where it presents several folds, or ridges, which are caused by the projection of prominent underlying structures. These several ridges, or plicæ, converge in a direction upward, toward the umbilicus, and include between them areas which are more or less depressed, and which are called foveæ. In the middle line, reaching from the summit of the bladder upward to the umbilicus, the peritoneum is raised in the shape of a fold by the superior ligament of the bladder, the remains of the fœtal urachus. External to this, passing from either side of the body of the bladder upward to the umbilicus, there is a fold, beneath which the obliterated hypogastric artery runs. Still more externally there is another fold, which corre- 


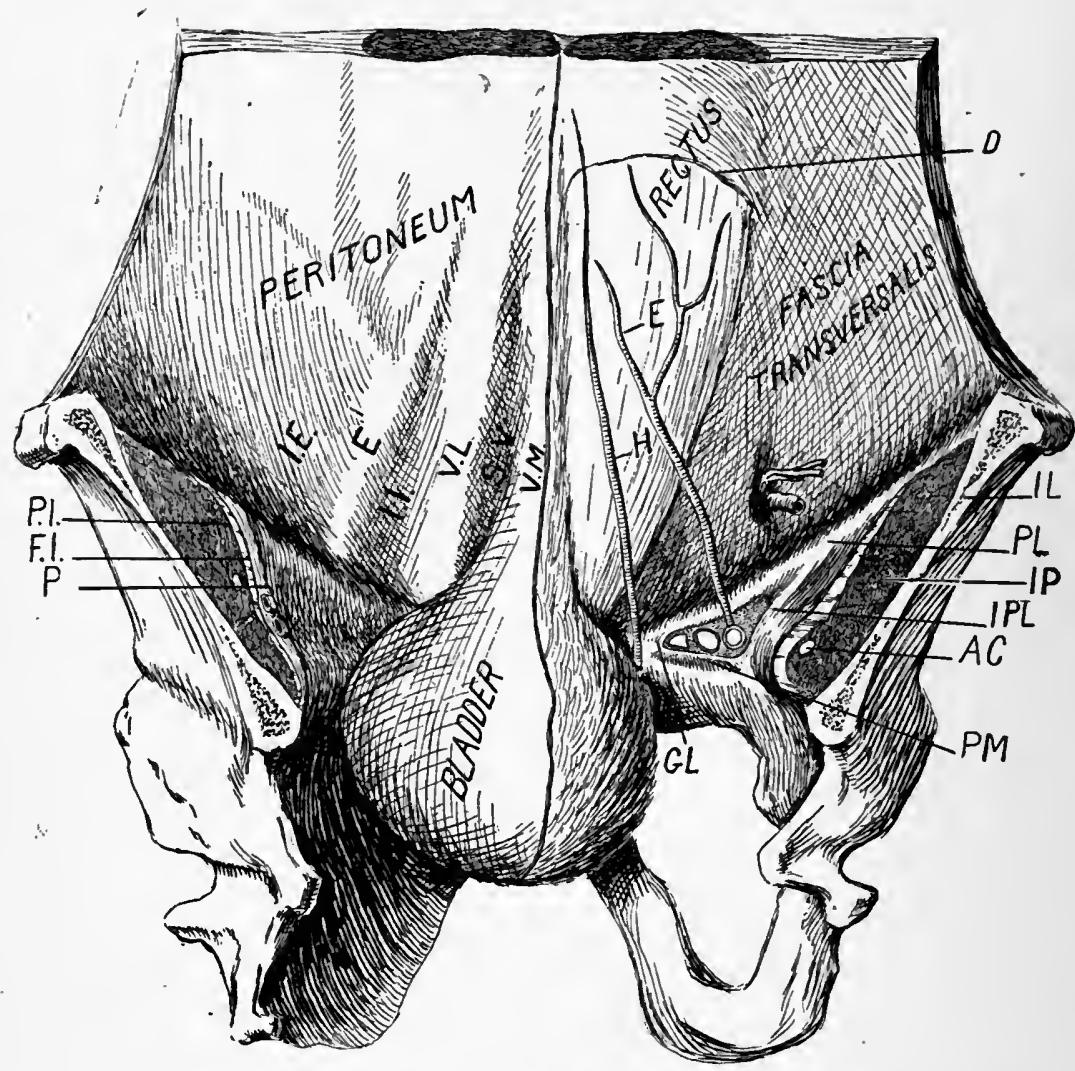

Fig. 159.-The Inguinal and Femoral Regions from Within the Abdomen. Upon the right side the peritoneum has been stripped off, exposing the transversalis fascia. $A C$, anterior crural nerve imbedded in the ilio-psoas muscle; $D$, semilunar fold of Douglas-the lower edge of the posterior layer of the sheath of rectus; $E$, deep epigastric artery; $E^{1}$, plica epigastrica (the deep epigastric vessels are situated beneath this fold); F.I., cut edge of the fascia illaca, which invests the illo-psoas muscle; $G L$, Gimbernat's ligament; $H$, obliterated hypogastric artery; $\boldsymbol{I . E .}$., fovea inguinalis externa; I.I., fovea lngulnalis Interna; $I L$, sawn surface of the llium; $I P L$, llio-pectlneal ligament, a thickened portion of the lliac fascia; $P$, cut edge of the peritoneum; P.I., cut edge of the llio-psoas muscle; $P L$, Poupart's ligament; $P M$, pectineus muscle covered by its fascia, which is here somewhat thickened and is known as the pubic ligament of Cooper; $S \nabla$, fovea supravesicalis; $\nabla . L$., plica vesico-umbilicalis lateralis (the obliterated hypogastric artery lies beneath this fold); V.M., plica vesico-umbilicalis media (the urachus, which reaches from the fundus of the bladder to the umbilicus, is situated beneath the fold). Above the mlddle of Poupart's ligament there is an openlng in the transversalis fascia-internal inguinal ring-mouth of the infundibular process. The vas deferens and other component parts of the spermatic cord which pass in and out of the abdomen through this orlfice have been cut short in the plcture; this opening is the exit for indirect inguinal hernia. Beneath Poupart's ligament the femoral vessels, inclosed with their sheath, are seen. These structures have been divided close to Poupart's ligament. The femoral sheath occuples the space described as the femoral space, and is divided into three compartments-the outer for the artery and the middle for the vein; the orifice of the inner compartment is called the crural ring. 
sponds to the course of the deep epigastric artery; this is a large vessel given off from the external iliac (femoral) just before it passes out of the abdomen under Poupart's ligament, and is accompanied by one or two veins. The peritoneal folds are named, respectively, the plica vesico-umbilicalis media, corresponding to the urachus, in the middle line; the plica vesico-umbilicus lateralis, corresponding to the obliterated hypogastric artery; and the plica epigastrica, corresponding to the epigastric artery and vein. Between these peritoneal folds, or plicæ, are the foveæ, already mentioned, which are deeper in some subjects than in others. External to the plica epigastrica is the fovea inguinalis externa. Between the plica epigastrica and the plica vesicoumbilicalis lateralis is the fovea inguinalis interna. Between the plica vesico-umbilicalis lateralis and the plica vesico-umbilicalis media is the fovea supravesicalis.

The Fovea Inguinalis Externa.-After the peritoneum has been stripped off from this area, and some loose connective tissue (subperitoneal connective tissue) which lies beneath it has been removed, we expose the transversalis fascia. This fascia presents the opening into the infundibular process, the so-called "internal ring," which is located about half an inch above the middle of Poupart's ligament. The vas deferens, spermatic artery, veins, etc., structures of which the spermatic cord is formed (in the female, the round ligament), pass into this opening. The lower, inner, margin of the internal ring presents a distinct, sharp, crescentic edge. A probe or the finger can be introduced into the internal ring, and may be insinuated for a greater or less distance into the sheath of the spermatic cord, infundibular process. About the internal ring the peritoneum is more or less plaited upon itself, and is adherent to the margins of the ring, and may bulge for a certain distance into it. A fibrous cord passes from the peritoneum into the internal ring, and may be traced downward into the infundibular process along with the other constituents of the spermatic cord. This fibrous band, or string, represents the shrunken, obliterated vaginal process of peritoneum which accompanies the testis in its descent into the scrotum. Directed upward and inward toward the umbilicus, and passing to the inner side of the internal ring is the deep epigastric artery, with its accompanying vein. If a hernial protrusion occurs in this location, the process of peritoneum which forms the sac of the liernia forces its way through the internal ring (to the outer side of the deep epigastric), and gradually works its way downward within the fibrous 
sheath of the cord, which is the remains of the original infundibular process, and we then have a typical external, or oblique, inguinal hernia. The coverings of this variety of hernia, from within outward, are, besides its peritoneal sac, the infundibular fascia (pouch derived from fascia transversalis), cremaster muscle and fascia, deep layer of the superficial fascia (spermatic fascia), superficial layer of the superficial fascia (fat), and the skin.

If a congenital hernia is present, the vaginal peritoneal process which accompanied the testis in its descent into the scrotum is found patent, unobliterated, reaching downward through the internal ring and along the cord within its sheath (infundibular process) to the bottom of the scrotum.

The coverings of a congenital hernia are the same as those given for the oblique, or external, acquired variety. The difference between the oblique acquired and the congenital is that the acquired must form a peritoneal sac for itself, whereas the congenital finds its sac already present; i.e., the unobliterated vaginal peritoneal process.

The Fovea Inguinalis Interna.-This is the space between the plica epigastrica and the plica vesico-umbilicalis lateralis. After the peritoneum has been stripped away from this part we expose the transversalis fascia. The fovea inguinalis interna is the part which is involved in direct inguinal hernia. It presents no opening. In the event of a direct inguinal hernia, a bulging or pouching of this part of the posterior wall of the inguinal canal occurs, and the hernial sac, composed of the parietal peritoneum, will have as coverings, from within outward, the various layers that form this part of the posterior wall of the inguinal canal, viz.: the fascia transversalis, the conjoined tendon, and the triangular ligament, and, in addition, the deep layer of the superficial fascia (spermatic fascia), the superficial layer of the superficial fascia (fat), and the skin.

The neck of the sac in a direct inguinal hernia lies to the inner side of the deep epigastric vessels.

Fovea Supravesicalis. - This is the space between the plica vesico-umbilicalis lateralis and media. Its floor is formed by the rectus muscle. This region is of but little surgical interest, and is not the site of hernial protrusions.

The Femoral Region.-Below Poupart's ligament we have the femoral region. This part is, at times, depressed, and is called the fossa cruralis. If we dissect away the peritoneum, we expose Pou- 
part's ligament, passing from the anterior superior spinous process of the ilium inward and downward, to be attached to the spine of the pubes. From the lower border of Poupart's ligament, just before its attachment to the pubic spine, a triangular band of fibers, which is attached to the ilio-pectineal line, is given off. This is called Gimbernat's ligament. Its sharp outer edge may be readily felt.

Between Poupart's ligament and the pubic bones there is a large space through which the ilio-psoas muscle and anterior crural nerve and the femoral vessels pass into the thigh. The ilio-psoas muscle arises within the abdomen and passes down toward Poupart's ligament in one mass, which is invested by a strong fascia, the iliac. At Poupart's ligament, the fascia that covers the outer part of the psoas-iliacus-i.e., that part of it which corresponds to the outer third of Poupart's ligament-is attached to Poupart's ligament, and is thence reflected upward, becoming continuous with the transversalis fascia, which lines the whole posterior surface of the anterior abdominal wall. Internal to this, however, where the femoral vessels pass out under Poupart's ligament, the fascia is continued downward with the muscle underneath Poupart's ligament, into the thigh. As the femoral vessels descend into the thigh they rest upon the pectineus and ilio-psoas muscles, separated from them, however, by the fascia which covers them, the pectineal fascia ${ }^{1}$ covering the pectineus muscle, and the iliac fascia covering the ilio-psoas muscle.

The fascia iliaca, immediately beneath Poupart's ligament, is thickened, and is called the ilio-pectineal ligament. It reaches from the junction of the outer and middle thirds of Poupart's ligament to the ilio-pectineal eminence, and serves to divide the space under Poupart's ligament into two portions: that for the ilio-psoas muscle and anterior crural nerve, below and externally, and that through which the femoral vessels pass, above and internally. This latter is called the femoral space. The boundaries of the femoral space are, above, Poupart's ligament; below and externally, the ilio-pectineal ligament (thickened portion of the iliac fascia); below and internally, the pubic ligament of Cooper (the upper thickened portion of the fascia that covers the pectineus muscle); internally, the edge of Gimbernat's ligament.

As the femoral vessels pass down through the femoral space

1 That part of the pubic portion of the fascia lata that covers the pectineus muscle. 
into the thigh, they are inclosed in a connective tissue sheath, which is prolonged downward from the margins of this space. It is called the femoral sheath. The femoral sheath is divided into three compartments by septa: the outer contains the artery; the middle one, the vein; the innermost, that between the vein and the edge of Gimbernat's ligament, is the so-called crural canal, and gives passage to lymphatics from the thigh to the abdomen. The abdominal orifice of the crural canal is called the crural ring.

In the event of a femoral hernia, a process of peritoneum (hernial sac) is forced into the crural ring and down through the crural canal, appearing below in the upper part of the thigh in the saphenous opening.

The coverings of a femoral hernia, from within outward, are, besides its peritoneal sac, the femoral sheath, the deep layer of the superficial fascia (the cribriform fascia), the superficial layer of the superficial fascia (fat), and the skin.

The Obturator Foramen.-This foramen is located below the brim of the pelvis. It is an opening in the upper part of the obturator membrane, between its upper edge and the lower border of the ramus of the pubes. This foramen gives exit to the obturator artery, vein, and nerve, and is sometimes the site of a hernial protrusion. The obturator artery usually arises from the external iliac, passes forward just below the brim of the pelvis, and out through the obturator foramen into the thigh. Occasionally, however, this artery is derived from the deep epigastric, close to the origin of this vessel from the external iliac (femoral), and in its course to reach the obturator foramen it is found in close proximity to the margin of the crural ring. After its origin from the deep epigastric, in its course to reach the obturator foramen, it either passes around the upper and inner margins of the crural ring or else it descends close to the inner wall of the femoral vein and behind the outer border of the crural ring.

\section{OPERATIONS FOR HERNIA.}

Herniotomy. - Incision of the coverings of a hernia, opening into the sac, and the division of constricting rings or bands constitute the operation of herniotomy. The operation is done for the purpose of liberating a strangulated hernia. The constriction may be caused by bands in the body of the sac or by the neck of the sac itself, but in 
most cases it is probably caused by the firm, unyielding ring by which the neck of the sac is encircled.

At one of the usual sites of a hernial protrusion there is found a tense, elastic tumor. The incision is placed over the most prominent part of the tumor, cutting carefully through the skin and the deeper layers until the sac proper is reached. The sac may then be pinched up with two forceps and incised between them, when there is an escape of serous fluid, and the contents of the sac are exposed.

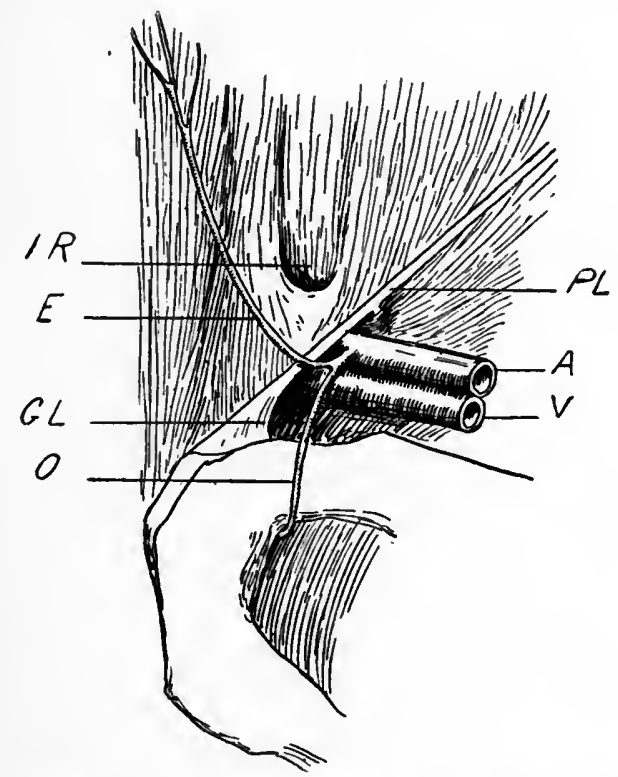

Fig. 160.-Irregular Origin of Obturator Artery. In its course into the pelvls it lies close to the inner side of the femoral vein. $A$, femoral artery: $E$, deep eplgastric artery; $G L$, Gimbernat's ligament; $I R$, Internal inguinal ring; $O$, obturator artery; $P L$, Poupart's ligament; $\nabla$, femoral vein. The space between the femoral veln and Gimbernat's ligament is known as the crural ring, and through this femoral hernia leaves the abdomen.

The contents vary; they may consist of intestine, large or small; of omentum, or of both; and occasionally there may be other organs, such as the bladder, ovary, etc. After the sac has been freely opened, its contents should be examined. Any constricting bands in the body of the sac should be divided, and an attempt then made to pull the gut down so as to ease it at the point of constriction; but in this effort much force should not be used. An effort is made to 
insert the finger into the neck of the sac, and, if this is successful, a probe-pointed, curved knife may be introduced upon the finger and the constricting ring incised. If one is unable to insert the finger into the neck of the sac, a director may be carried through, and upon this the ring may be divided. In freeing the constriction, a succession of nicks should be made rather than a single free cut, and these may be repeated until the parts are liberated.

For the relief of an indirect inguinal hernia the incision in the

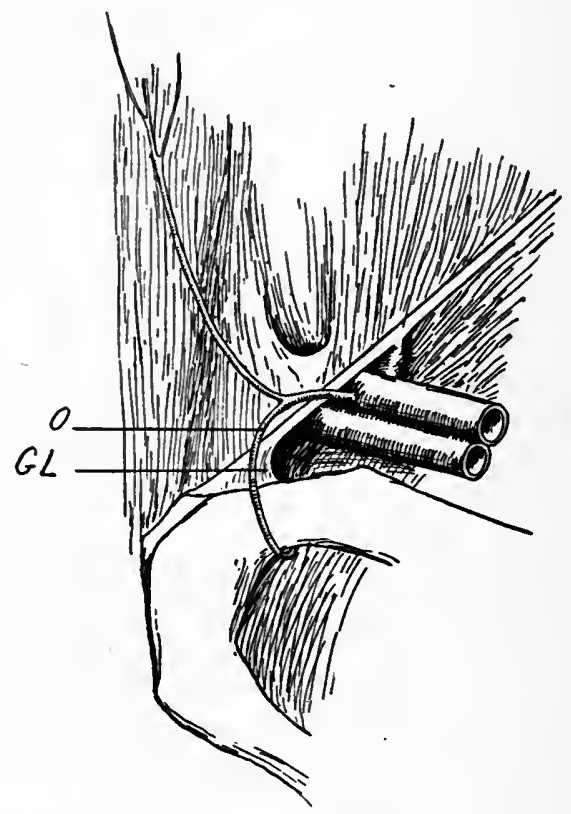

Fig. 161.-Irregular Origin of Obturator Artery. In its course into the pelvis it curves around the upper and inner edge of the crural ring. Letters same as 160 .

constricting ring should be directed upward. For a direct inguinal hernia the incision should be directed upward and inward, toward the umbilicus. For a femoral hernia the incision should be directed inward toward Gimbernat's ligament and somewhat upward.

For practical purposes, if in doubt as to the exact variety of the hernia, the direction of the liberating incision for both varieties of inguinal and for femoral hernia may be upward and inward, toward the umbilicus. By cutting in this direction, upward and inward, 
toward the umbilicus, we work in a line which is parallel with the course of the deep epigastric vessels, and the danger of wounding these is thus obviated.

Occasionally the obturator artery, as described above, is given off from the deep epigastric, and in its course to reach the obturator foramen this vessel would then have a close relationship to the neck of the sac of a femoral hernia. From its origin, at the deep epigastric, the obturator artery either descends close to the inner wall of the femoral vein, and therefore behind the outer margin of the crural ring, and would thus lie to the outer side of the neck of a femoral hernial sac, or else it curves inward and then downward, behind the upper and inner borders of the crural ring, and would then lie above and to the inner side of a femoral hernial sac. In the first case, this vessel would be out of the way in making the liberating incisions at the crural ring, whereas in the second instance the vessel would be jeoparded in making the liberating incisions if caution were not exercised.

If the constriction at the neck of the sac is relieved by a succession of nicks, rather than by a single free incision, we will be very much less liable to divide an abnormally placed obturator artery. After the contents of the sac have been liberated they may be drawn down for examination, especially at the points of constriction. If omentum is present, this may be ligated and amputated. As to the treatment of the gut, careful deliberation must be used. If the gut is healthy, it may be returned at once into the abdomen. If doubtful, one may wait for a short time to note if it tends to clear up. After the gut has been reduced the finger should be introduced through the neck of the sac in order to make certain that there are no adhesions about the neck which might continue to constrict the gut.

If the gut is gangrenous, or too doubtful to return into the abdomen, the incision in the abdomen at the neck of the sac should be enlarged and the gut drawn down and resected; or else the gut may be allowed to remain without disturbing the adhesions about the neck of the sac, and an artificial anus made by incising the strangulated coil of gut, if it has not already slonghed through. The wound, under these circumstances, should be left open and packed.

Radical Operation for Inguinal Hernia (Bassini Method). For an Oblique Acquired Hernia.-An incision is made through the skin, commencing at a point half an inch above and somewhat ex- 
ternal to the middle of Poupart's ligament, carrying it downward and inward as far as the spine of the pubes; or it may be prolonged for a short distance downward upon the scrotum, if necessary. This incision penetrates into the subcutaneous fatty layer. In its upper part the incision should be deepened until the fibers of the aponeurosis of the external oblique are plainly visible. The fingers are then introduced into this upper, deeper part of the incision, and it
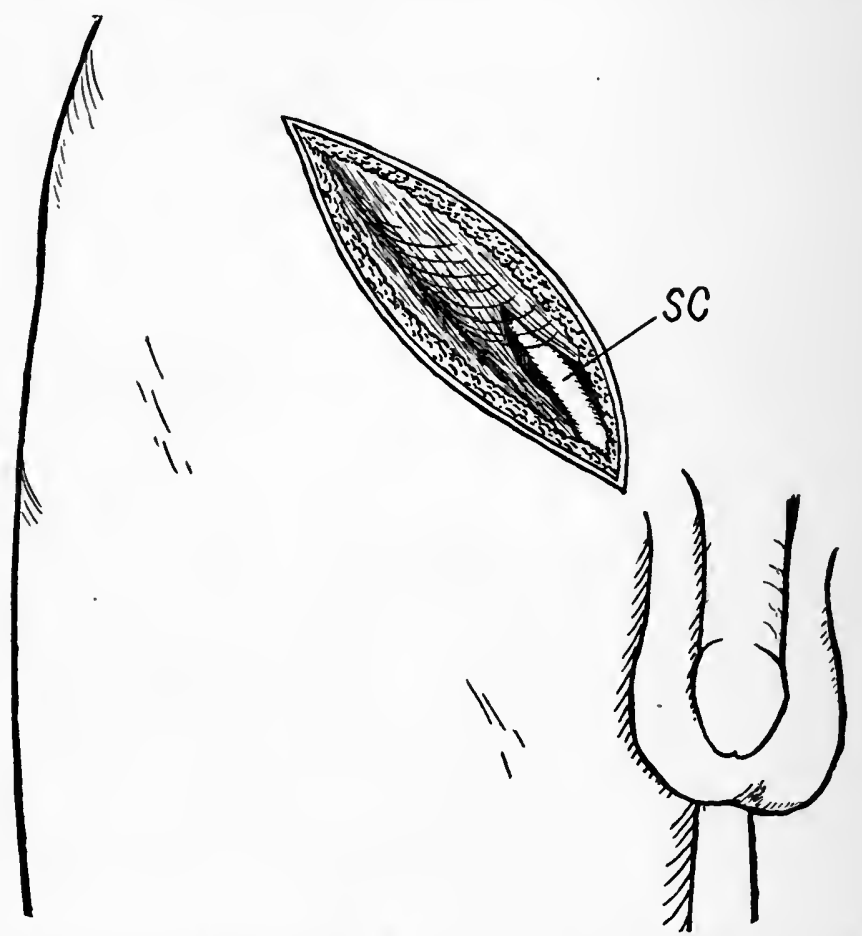

Fig. 162.-Operation for Inguinal Hernia. Incision penetrates through the skin and fat, exposing the aponeurosis of the external oblique. SC, spermatic cord emerging from the external inguinal ring.

is torn open down to its lower end. After this has been done the aponeurosis of the external oblique and the pillars of the external ring, through which the cord emerges, are exposed.

Any bleeding points are caught in artery forceps; but it is not necessary to ligate them immediately, as the hemorrhage usually ceases after a few minutes' compression.

A blunt director is now passed into the external ring, and car- 
ried upward and outward beneath the aponeurosis of the external oblique to a point beyond the middle of Poupart's ligament, the location of the "internal ring," and upon this the aponeurosis is divided. Some obstruction to the introduction of the director through the external ring will be experienced if the deep layer of the superficial fascia, which is attached to the margins of the ring, has not been incised.

The edges of the split aponeurosis of the external oblique are seized with artery forceps and separated with the finger from the structures which lie immediately beneath. The inguinal canal is thus laid open, and the spermatic cord, together with the hernial sac, is exposed. The lower, free fleshy edge of the internal oblique muscle is seen arching inward over the cord and hernial sac. It is blended with the tendon of the transversalis muscle to form the conjoined tendon, which descends behind the cord, and which can be felt as a strong, resistant band attached to the crest of the pubic bone.

The spermatic cord, together with the hernial sac, which is usually found empty, unless its contents are irreducible or the patient is straining, is now hooked up, upon the finger, and we proceed to separate the sac from the cord. At times it is difficult to recognize the sac. It is formed of the pouch of peritoneum, with some loose connective tissue (subperitoneal connective tissue layer) and is situated within the proper sheath of the spermatic cord (infundibular process of transversalis fascia), which must be incised or torn in order to expose it (the sac). The sac has a peculiar, white, aponeurotic appearance, and may be very thin or of moderate thickness. The isolation of the sac from the cord is accomplished chiefly by tearing and separating with the fingers, occasionally cutting a resisting band with the scissors. At times the sac is very intimately united with the cord, and much patience is required to separate it. One should recognize the vas deferens, and constantly be familiar with its location, in order to avoid injuring it. In isolating the sac, one may have considerable hemorrhage from the pampiniform plexus of veins, which runs along with the vas deferens, etc., in the cord. It usually ceases, however, after clamps have been applied to the bleeding points for a few minutes. If one of the arterial branches which run in the cord is torn, it will be necessary to apply a ligature. One may begin the separation of the sac from the cord above at the neck of the sac and work downward, toward its lower part (fundus), or commence at the fundus and work upward, toward the neck. The op- 
erator may assist himself in separating the sac from the cord by incising it in order to introduce the finger into it, and thus inform himself of its limits.

After the sac has been completely separated from the cord, especially above, about the neck at the location of the "internal ring," it is raised, and (if not already incised) is seized by an assistant with the fingers of both hands, or with two artery forceps, and incised between them with the knife. In incising the sac, especially if the contents are adherent, or if operating upon a strangulated hernia when there is much distension, one should use caution not to wound the parts within. After the sac has been opened the contents may be reduced, and, if there are no adhesions, this is very readily done. If there is a considerable amount of prolapsed omentum in the saic, this may be tied off with stout catgut and amputated in preference to returning it to the abdomen. If the contents are adherent to the sac, they must be gently separated before they can be reduced. This can usually be accomplished with the finger, taking care to avoid tearing the gut, and ligating any points that bleed freely. Dense adhesion bands may be first tied double and then divided between the ligatures. If omentum is adherent within the sac, it may be ligated and amputated. The contents should be free, especially at the neck of the sac, in order that they may be properly reduced.

After the sac has been emptied we may tie it off. The finger is introduced into the sac and carried well within its mouth, and a catgut ligature (No. 2) thrown around its neck. As this ligature is drawn tight and tied, one should feel it slip over the end of the finger,. which is within the mouth of the sac. It should be applied about the neck of the sac as high up as possible, in order to avoid leaving any pouched portion of the sac to invite the recurrence of the hernia. The ligature is left long for use as a tractor, and the sac is cut away, about one-fourth inch distal from the ligature. Then, after a final examination of the stump of the sac, the ends of the ligature are cut short, and the stump of the sac allowed to retract into the abdomen. If the sac is rather wide-mouthed, instead of simply surrounding it with a ligature one may transfix it with a ligature carried in a curved needle and tie double.

The next step in the operation is the strengthening of the posterior wall of the inguinal canal, and this is done by approximating the free edge of the internal oblique and transversalis muscles (conjoined tendon) above to Poupart's ligament below. While this is 


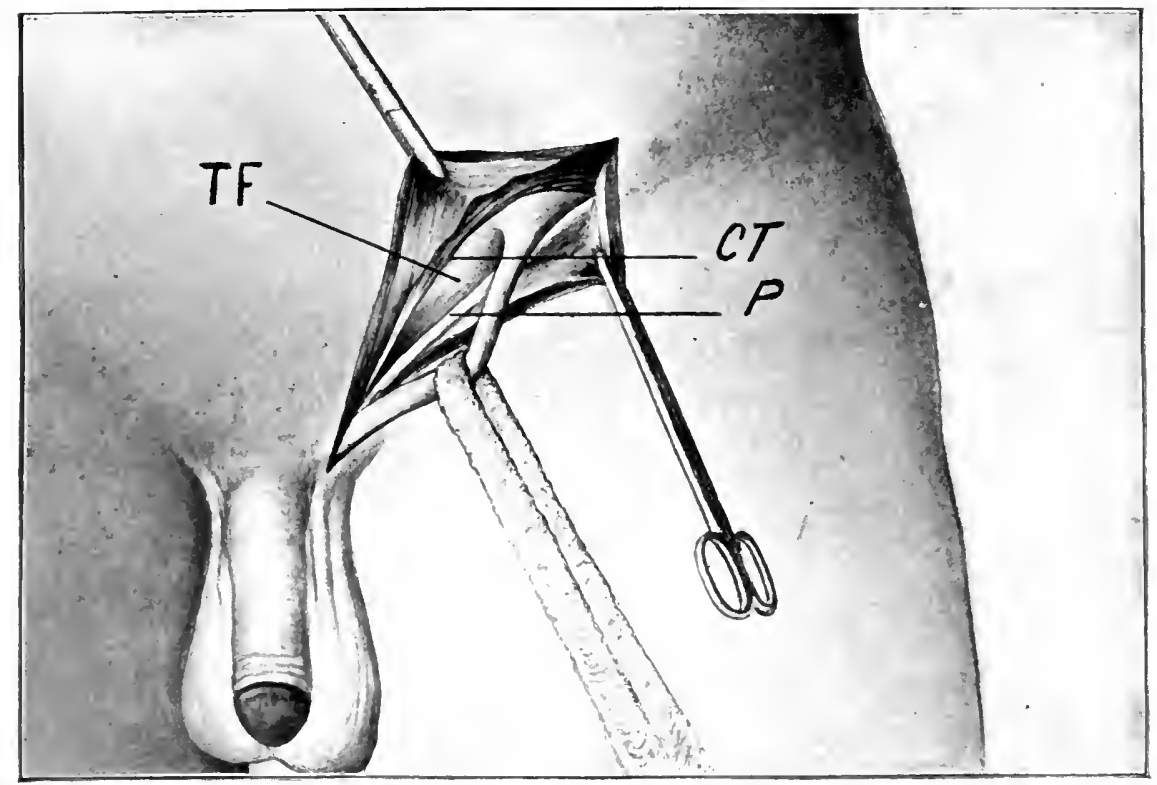

Fig. 163.-Bassini Operation for Inguinal Hernia. The inguinal canal lais open by splitting the aponeurosis of the external oblique. The edges of the split ap.meurosis are seized with artery forceps and drawn aside. Spermatic cord pulled aside with a loop of gauze preparatory to suturing the edge of the conjoined tendon to Poupart's ligament; $C T$, edge of conjoined tendon; $P$, edge of Poupart's ligament; $T F$, transversalis fascia, which forms the posterior wall of the inguinal canal.

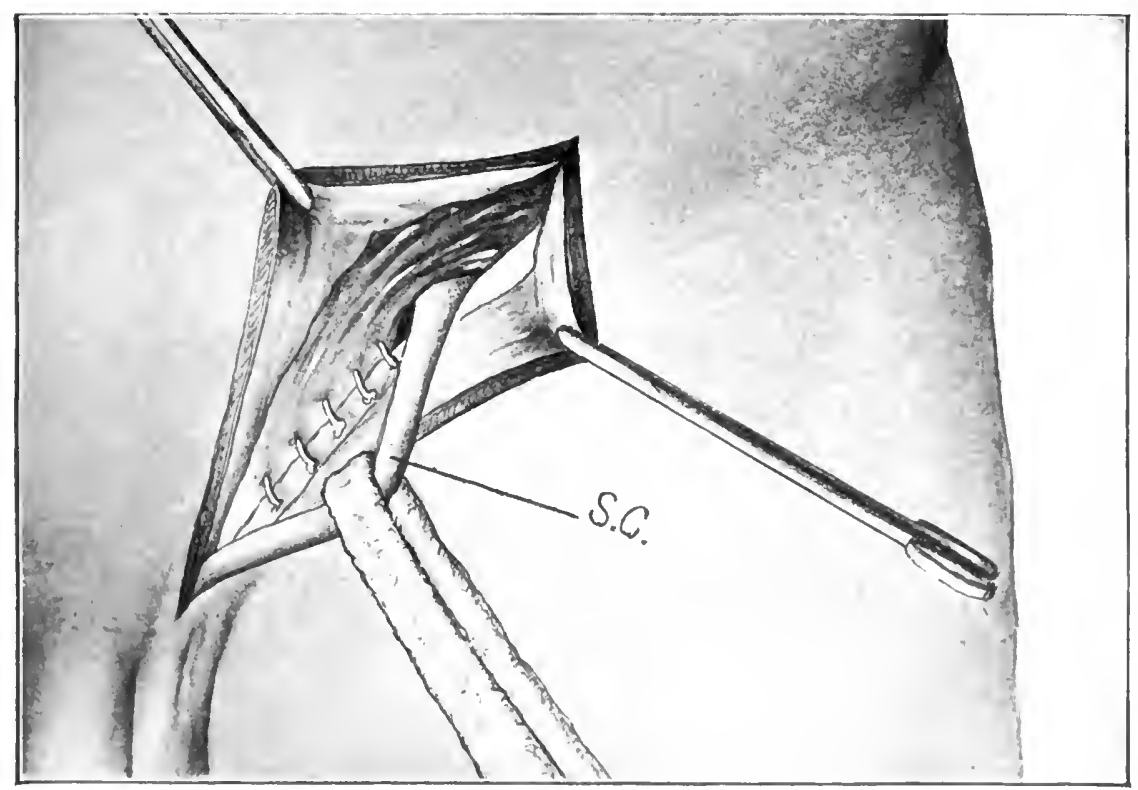

Fig. 16il.-The Bassini Operation. The edges of the split aponeurosis held aslde with artery forceps. Conjoined tendon sutured to the edge of Poupart's. Spermatic cord (S.C.) drawn aside with gauze loop. 

being done the spermatic cord is held out of the way of the operator upon a strip of gauze, and the upper edge of the divided aponeurosis of the external oblique, which is held in an artery forceps, is retracted, in order that the edge of the internal oblique and transversalis (conjoined tendon) may be made out. These parts can be readily seen and may be plainly felt by the finger in the wound. Poupart's ligament is likewise freely exposed, when the lower edge of the aponeurosis of the external oblique is strongly retracted. This structure may be recognized as a sharp, white band. These parts, the conjoined tendon above and Poupart's ligament below, are now brought together with three to five interrupted sutures of some permanent material, such as silk-worm gut, silk, silver wire, kangaroo tendon, or chromicized gut. These sutures are introduced with a large, curved needle grasped in a needle holder. The first suture is placed externally, just to the inner side of where the cord emerges from the abdomen; the last one or two sutures, those nearest the middle line, should take a sufficiently broad bite to include, together with the conjoined tendon, the edge of the rectus muscle. Ea $h$ suture should take a good, broad bite. In introducing the sutures through Poupart's ligament there is said to be some danger, especially with the middle sutures, of piercing the femoral vein with the needle. This might happen if the needle were inserted too deeply, but this is not necessary, as a good, broad bite of the ligament is easily sccured without introducing the needle deep enough to reach the vein. The sutures are left long, and are not tied until all are introduced. Usually three or four sutures suffice; sometimes five are necessary. The most external suture should be placed so as to leave just space enough for the cord to emerge comfortably without constriction between the edge of the internal oblique and transversalis above and Poupart's ligament below. When the sutures are tied, the edge of the internal oblique and transversalis muscles (conjoined tendon) and Poupart's ligament are seen to be closely approximated, and in this way there is formed a solid posterior wall to the inguinal canal, upon which the cord rests when it is dropped back into the wound. The edges of the split aponeurosis of the external oblique are now brought together over the cord with a continuous suture of catgut, simple or chromicized, No. 2. This suture is commenced above and externally, and terminates below at the site of the former external abdominal ring. In this way the anterior wall of the inguinal canal is restored, and beneath this the cord is situ- 
ated. One should take care that the cord is not gripped too tightly between the posterior and anterior walls of the new canal, and that, at the site of the external ring, sufficient space is left for the cord to emerge without danger of its becoming strangulated.

The wound should be dry-free from oozing. No drainage is necessary. The incision in the skin may be closed with a continuous intracuticular catgut suture.

In the female this operation is simplified in that we have no spermatic cord to consider; the round ligament, its analogue, is simply cut away, and the deep sutures which strengthen the posterior wall of the inguinal canal introduced in the manner described above.

For a Congenital Hernia.- In this variety of hernia the sac is formed of the unobliterated vaginal process of the peritoneum, at the bottom of which the testis usually lies. In some cases the testis does not reach the bottom of the scrotum in its descent, and may remain stationary, in any part of the inguinal canal or within the abdomen, when it may be wise to remove it. The incision in the skin and aponeurosis of the external oblique are made as in the foregoing operation. After the inguinal canal has been laid open, the cord, together with the sac, is picked up, upon the finger. The hernial sac is really included within the proper sheath of the cord, infundibular process, and its isolation from the elements of the cord may be somewhat difficult. The sheath of the cord (infundibular process of the transversalis fascia) must be incised or torn through in order to reach the sac. In separating the sac we may commence above at the neck of the sac, and work downward, toward the testis. After the sac has been separated from the cord, vas deferens, etc., to a point which is just above the testis, it is opened and its contents reduced. The sac is then cut across, allowing the lower part, that which corresponds to the testis, to remain to form the tunica vaginalis. The upper part of the sac, after having been thoroughly isolated, is then tied off at the point where it emerges from the abdomen, and the edge of the internal oblique and transversalis (conjoined tendon) sutured to Poupart's ligament, as already described in the preceding operation. The lower part of the vaginal process (hernial sac) which remains, and which corresponds to the tunica vaginalis testis, is then closed with a continuous catgut suture, so that the testis is shut up within its tunica vaginalis. The edges of the split aponeurosis of the external oblique are then brought together over the cord, and the incision in the skin closed. If the 
testis has been much handled, it may be wise to introduce a thin strip of gauze into the cavity of the tunica vaginalis, through the bottom of the scrotum, for the purpose of drainage; usually, however, this is not necessary.

For a Direct Inguinal Hernia.-In this variety of hernia the peritoneal pouch (hernial sac) does not enter the "internal ring," mouth of the infundibular process, and work its way down along the cord, within the sheath of the cord, but bulges directly forward, into the inguinal canal, to the inner side of the deep epigastric artery, pushing the transversalis fascia, conjoined tendon, and triangular ligament before it, and is found upon the inner side of the spermatic cord as this descends through the inguinal canal. The sac consists of a wide-mouthed pouch of peritoneum and subperitoneal connective tissue, and, as it presents into the inguinal canal, is covered by the transversalis fascia, the conjoined tendon, and the triangular ligament. It is also covered by the aponeurosis of the external oblique, superficial and deep layers of the superficial fascia, and the skin. The mouth of the sac is wide, and may reach from the external edge of the rectus as far outward as the deep epigastric artery, or even beyond this, pushing the artery in front of it, in which case the artery may form a deep groove upon the sac, and thus divide it into two pouches. Under these circumstances it may be necessary to tie the artery double and divide it. There may be no well-formed sac present, but simply a wide, conical bulging of the posterior wall of the inguinal canal. In direct hernia the sac is readily separated from the cord, after which it is opened and its contents reduced. If the sac is very wide-mouthed, it may be necessary to approximate the margins of the opening with a catgut suture, and then cut away what remains of the sac. The operation is completed as described above for the oblique variety. While the cord is held aside, the edge of the conjoined tendon (internal oblique and transversalis muscles) is sutured to Poupart's ligament. The cord is then replaced and the edges of the aponeurosis of the external oblique sutured over it, and finally the incision in the skin closed.

Halsted's Operation for Inguinal Hernia.-The incision reaches from a point $5 \mathrm{~cm}$. above and external to the site of the internal ring, which is located half an inch above the middle of Poupart's ligament. It is carried downward and inward as far as the spine of the pubes (site of the external ring). This incision extends through the skin and superficial fascia, freely exposing the aponeurosis of 
the external oblique muscle and the external inguinal ring. All bleeding points are clamped. As a rule, it is not necessary to tie them, as the hemorrhage ceases after a few minutes' compression.

The next step in the operation consists in the division of the aponeurosis of the external oblique, the internal oblique and transversalis muscles, and the transversalis fascia. These structures are incised from the external ring below to a point about $2 \mathrm{~cm}$. above and external to the location of the internal ring, or farther if necessary, in order that the upper and outer part of the incision may extend into the fleshy part of the internal oblique and transversalis muscles. The vas deferens is now sought, and, together with its vessels, isolated, and then all the veins which accompany the vas deferens except two or three, after being tied off above and below, are excised. In this way the size of the cord is markedly. diminished. The remains of the cord are now held to one side, and the isolation of the hernial sac is begun. After this has been completed, the sac is incised and its contents returned into the abdomen. When the transversalis fascia is incised the constriction about the neck of the sac disappears, and its mouth, from a narrow orifice, becomes a wide-open space, through which one may easily introduce several fingers or the whole hand into the peritoneal cavity. The margins of the mouth of the sac are now brought together with a continuous or interrupted suture of catgut, and the sac below this suture line resected. This step of the operation is really like closing any ordinary opening in the parietal peritoneum. During the application of this suture a gauze pad may be introduced, through the opening into the peritoneal cavity, to prevent the intestine from prolapsing into the wound. After the mouth of the sac, peritoneum, has been thus sutured and closed, and the sac cut away, we proceed with the next step of the operation, the approximation of the cut edges of the several layers of the abdominal wall. While this is being accomplished the cord is raised upon a hook and held out of the way, well toward the outer part of the incision. To unite these parts from six to eight mattress sutures of silk are required. The layers which are approximated consist above of the aponeurosis of the external oblique, the internal. oblique and the transversalis muscles (conjoined tendon), and the transversalis fascia. Below they consist of Poupart's ligament and the aponeurosis of the external oblique and the transversalis fascia, and in part, externally, of the cut edges of the internal oblique and transversalis muscles. The sutures pass 
through all these layers. Between the two most external of these sutures the cord emerges through the abdominal wall, between the cut edges of the internal oblique and transversalis muscles. The cord should be firmly grasped by these muscles, but not tightly enough

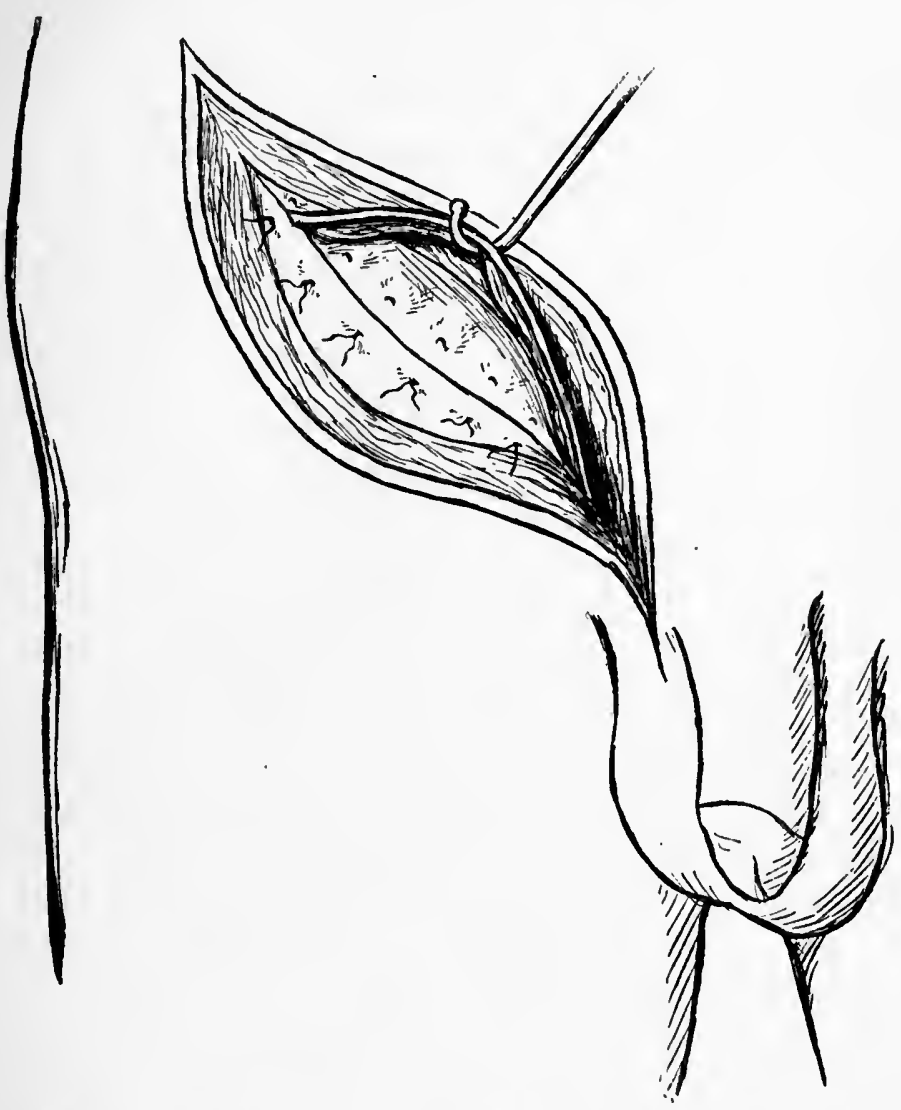

Fig. 165.-Halsted's Operation. The vas deferens, with a few remaining vessels of the cord, drawn aside with a hook. Mattress sutures have been applied, uniting the different layers that have been cut, inciuding the aponeurosis of the external oblique.

to strangle it. The cord, as it emerges through the abdominal wall, in its new position, should be surrounded by the fleshy fibers of these muscles; it should not emerge between the tendinous portions of the muscles. If the incision through the internal oblique and trans- 
versalis muscles and the transversalis fascia has not been carried sufficiently far, in a direction upward and outward, to accomplish this, it should be extended farther, so as to reach well into the fleshy portion of these muscles.

After the mattress sutures have been applied and the parts already mentioned approximated, the cord is dropped back into the wound and rests upon the aponeurosis of the external oblique. The edges of the skin are then sutured over the cord with a continuous intracuticular suture, thus completing the operation. The cord is transplanted so that it emerges through the abdominal wall above and external to the site of the "internal ring," where it is surrounded by muscular fibers and lies just beneath the skin, instead of beneath the aponeurosis of the external oblique.

Operation for the Radical Cure of Femoral Hernia. - Femoral hernia descends through the crural canal upon the inner side of the femoral vein, and presents in the thigh, just below Poupart's ligament. In order to expose the sac of the hernia an incision is made below and parallel with Poupart's ligament, the middle of the incision being over the center of the tumor. This incision is carried through the skin and subcutaneous fatty tissue and the deep layer of the superficial fascia (cribriform) down to the sac. Instead of being placed parallel with Poupart's ligament, the incision may be made in an oblique direction from above downward.

The sac is now isolated, and separated from the adjoining parts up to and beyond the level of Poupart's ligament. Special care is required in separating the sac on the side which adjoins the femoral vein. After the sac has been thoroughly isolated it is opened and the contents reduced. The sac is then twisted and tied off as high up as possible. It may be surrounded with a simple catgut ligature, or it may be transfixed and tied double. The portion of the sac below the ligature is then cut away, the ends of the ligature cut short, and the stump of the sac pushed back beyond Poupart's ligament into the abdomen.

We are now ready to close the orifice through which the hernia descended into the thigh. We should first recognize the margins of this orifice, the crural ring. This is bounded above by Poupart's ligament; internally by the edge of Gimbernat's ligament; below by the fascia that covers the pectineus muscle, the upper, thickened portion of which is called the pubic ligament of Cooper, and which extends from Gimbernat's ligament to the pectineal eminence; externally 


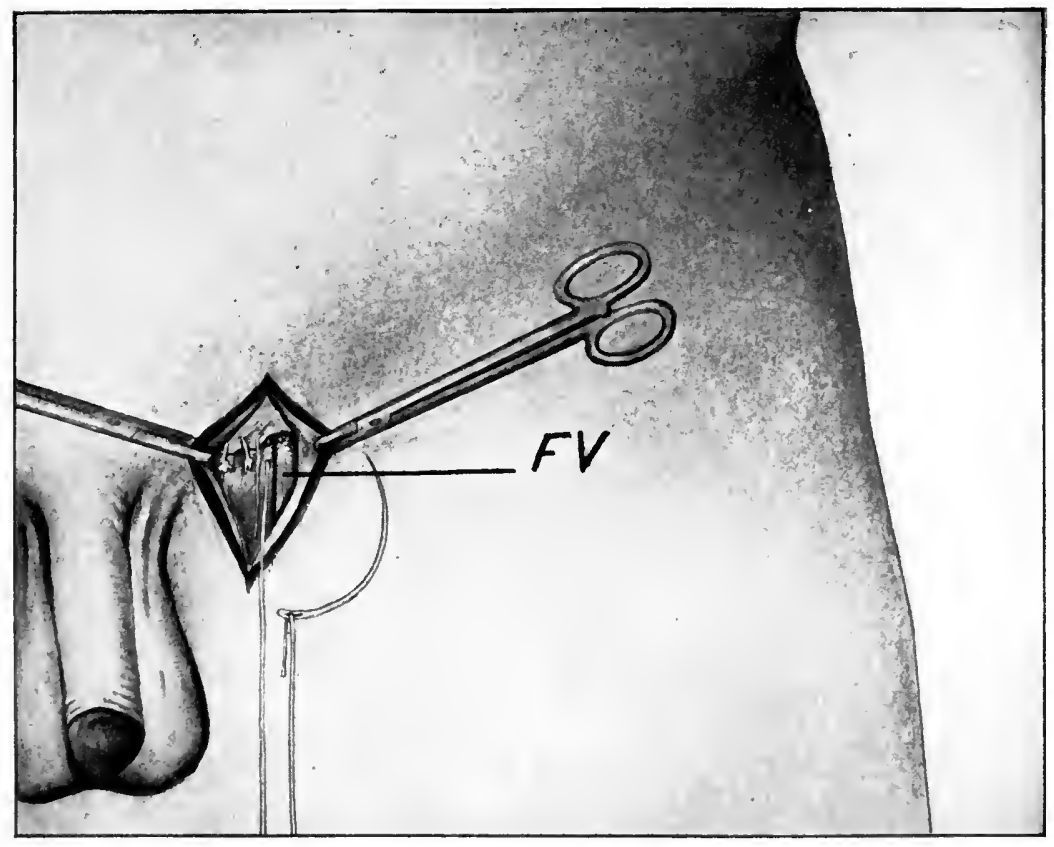

Fig. 166.-Operation for Femoral Hernia. FV, femoral vein. Poupart's ligament has been sutured to the upper part of the fascia that covers the pectineus muscle. 

it is bounded by the femoral vein. The edge of the falciform process should also be recognized, and likewise the internal saphenous vein, where it joins the femoral. The crural ring is obliterated by suturing the lower edge of Poupart's ligament to the fascia which covers the pectineus muscle; i.e., to that part of it which covers the upper part of the pectineus - the pubic ligament of Cooper. The stitches should be of silk, and should be introduced with a short, full-curred needle. The first suture catches Poupart's ligament just external to its attachment to the pubic spine, and should take a good bite. After the needle is drawn through Poupart's ligament is pulled upward and backward with a blunt hook in order to permit the needle to catch the pectineal fascia as high up under Poupart's ligament as possible; i.e., near the ilio-pectineal line, from which the pectineus muscle arises. Half a centimeter external to this suture a second suture is introduced in a similar manner, and then, at a distance of another half-centimeter, a third suture. These three sutures suffice to close the opening. The third and last suture is located about $1 \mathrm{~cm}$. to the inner side of the femoral vein. When these sutures are tied, the lower edge of Poupart's ligament and the pectineal fascia (the thickened portion, high up near the origin of the pectineus muscle from the ilio-pectineal line) are approximated, and the crural ring is thus obliterated. The opening in the skin is closed in the usual way. No drainage is required.

THE SPERMATIC CORD, SCROTUM, ETC.

The Spermatic Cord.-The spermatic cord descends through the inguinal canal, emerging at the external inguinal ring. As it emerges from the external ring it lies just beneath the integument in the subcutaneous fat, and descends into the scrotum, where it is joined to the posterior border of the testis. It is about as thick around as the little finger, and is made up of a bundle of structures, the vas deferens, the artery of the vas deferens, and the cremasteric artery, their corresponding veins, the spermatic artery, and a tortuous venous plexus, the pampiniform. The vas deferens, the efferent duct of the testis, occupies the posterior part of the cord. The vas deferens is about as big around as a goose quill, has a firm feel, and may be readily recognized as it is rolled between the fingers. The artery of the vas deferens ramifies upon the vas deferens, supplies it, and anastomoses below with the spermatic artery. The cremasteric artery is distributed 
to the constituents of the cord, and supplies its sheath. The spermatic artery is given off from the aorta; it supplies the testis and has a strong current of blood. The pampiniform plexus is a tortuous, intercommunicating plexus of venous channels that accompanies the other elements of the cord. Through this plexus the blood is returned from the testis. The vessels of the pampiniform plexus join together above to form the spermatic vein. This vein upon the right side enters the vena cava directly; upon the left side it empties into the renal vein, so that the venous return on the left side is less direct than upon the right side. Varicocele is usually found upon the left side.

As these structures traverse the inguinal canal they are all contained within the infundibular process, which serves to bind them together into a single bundle and which forms the real fibrous sheath of the cord, the fascia propria. Descending upon the cord is a series of looped, muscular fibers, each joined to the other by an intervening thin fascia. These are the cremaster muscle and fascia. These fibers, that form the cremaster muscle, are derived from the lower border of the internal oblique.

As the cord emerges from the external inguinal ring, the deep layer of the superficial fascia (spermatic fascia), which is attached to the pillars or margins of the ring, is continued down upon the cord, inclosing it and forming one of its investments.

The Scrotum.-The scrotum is a pouch with two compartments, one on each side, separated by a median septum. It consists of several layers, from without inward. The skin is redundant, corrugated, and wrinkled. Beneath the skin is the dartos. The dartos is a loose, reddish, contractile layer, which is found immediately beneath the skin. It contains some muscular fibers, and is continuous behind with the two layers of the superficial perineal fascia, and laterally with the same layers in the groin. It sends a septum into the scrotum, which divides it into its two halves. Beneath the dartos is the cremaster muscle and fascia, and beneath this the infundibular fascia, and, finally, most internal, the parietal layer of the tunica vaginalis.

The Testes.-The testes are situated in the scrotum, each suspended by its spermatic cord. They are partially invested by a closed, serous sac, the tunica vaginalis. This is the unobliterated part of the vaginal process of the peritoneum, the peritoneal pouch that accompanies the testis in its descent from the abdomen into the infundibular process, the scrotum, before birth. 
$C T$, cavity of the tunica vaginalis testis.

$\mathrm{Cl}$, cremaster artery and artery of the vas deferens and their corresponding veins, all in close proximity to the vas deferens.

$I R$, internal inguinal ringthe mouth of the original infundibular processt)rough which the structures that constitute the cord escape (the infundibular process becomes contracted around the elements of the cord and forms their proper sheath-the fascia propria [red line]).

$P$, peritoneum that lines the interior of the abdomen.

$S$, symphysis pubis.

$S A V$, spermatic artery and veins (below, along the course of the cord, the spermatic veins consist of a plexus of intercommunicating branches-the pampiniform plexus).

$T F$, transversalis fascial.

$Y D$, vas deferens.

$1 P$, remains of the obliterated vaginal process of peritoneum that accompanies the testis in its descent into the scrotum (the arrow indicates the site of the former opening or mouth of this process).

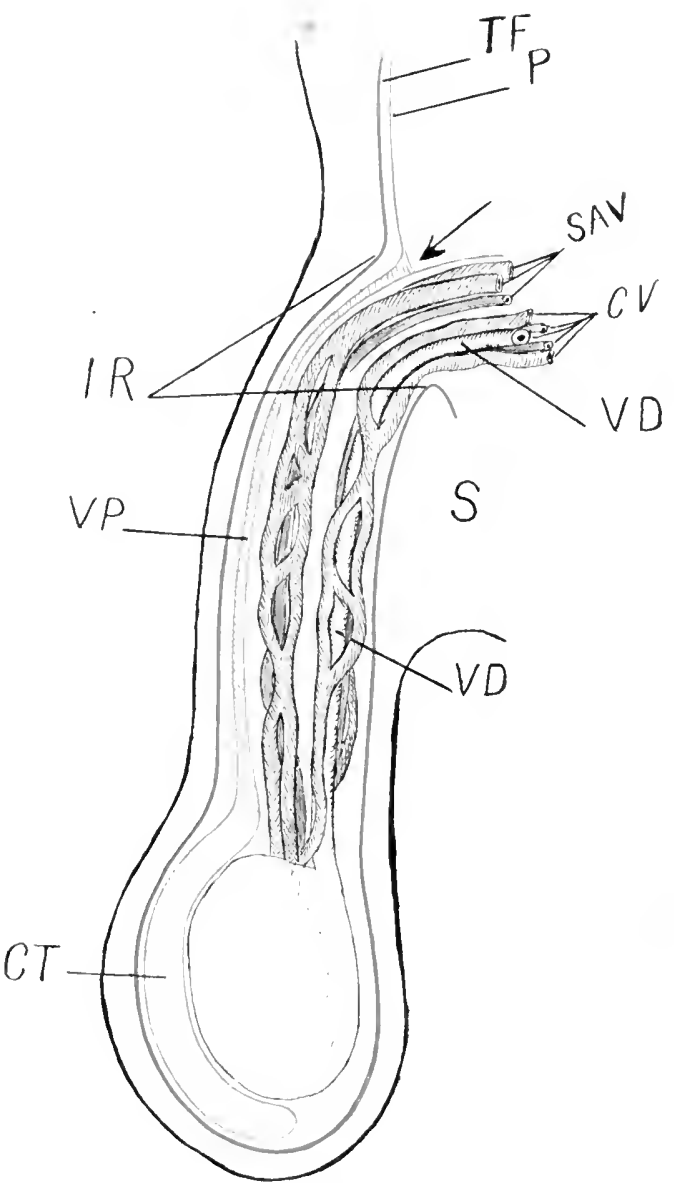

Fig. 14i.--Spermitic Cord.

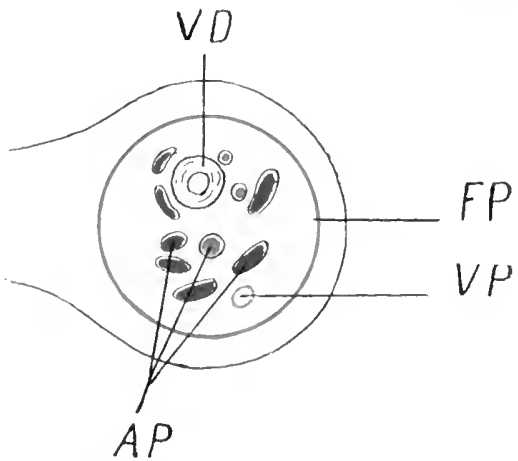

AP, spermatic artery and pampiniform plexus.

FP. fascia proprial (sheath of the cord and original infundibular process).

$F D$, vas deferens surrounded closely by the cremaster artery and artery of the vas deferens and their corresponding veins.

$I P$, remains of the oblitesated vaginal process.

Fig. 168,-Cross Section of Spermatic Cord. 



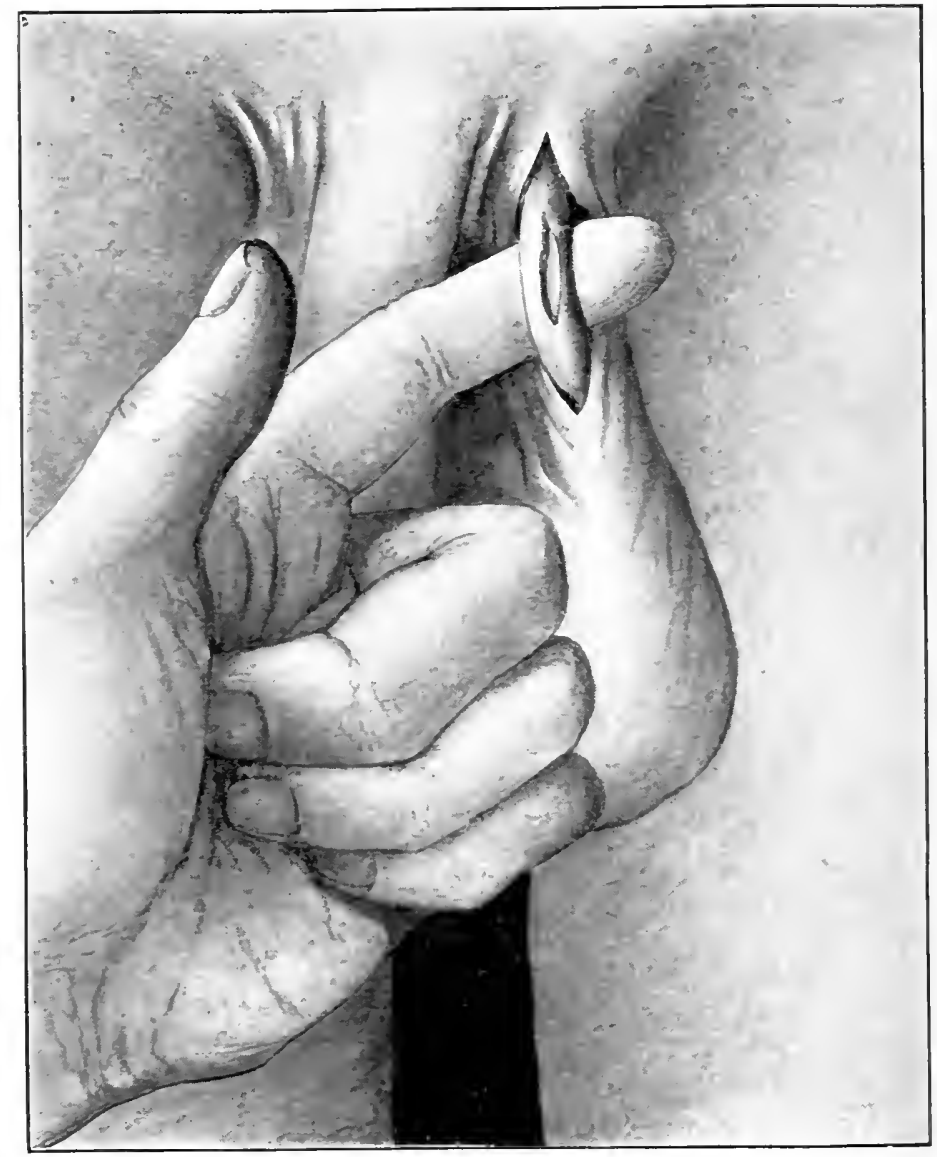

Fig. 169.-Exposure of Spermatic Cord. The spermatic cord has been hooked up out of the incision upon the finger, and its sheath incised preparatory to separating the vas deferens and adjoining vesseis from the other structures of the cord. 
If we cut through the anterior wall of the scrotum, through these various layers, we enter the cavity of the tunica vaginalis, which contains normally a small quantity of serous fluid. The testis presents into this cavity, being partially invested by the visceral layer of the tunica vaginalis. The posterior border of the testis is not covered by the tunica vaginalis, and is excluded from the cavity of the tunica raginalis.

Along the posterior border of the testis is the epididymis. It surmounts the testis above like a cap. It has a body, an upper, larger portion, the globus major; and a lower, smaller portion, the globus minor. The vas deferens is the continuation of the epididymis. It commences at the lower end of the globus minor, and, passing upward along the posterior, inner border of the testis, is found in the posterior part of the spermatic cord, passing through the "internal ring" into the abdomen. Within the abdomen it dips down into the pelvis, to terminate between the base of the bladder and the rectum, where it joins with the duct of the seminal vesicle of the corresponding side to form the ejaculatory duct.

The Ejaculatory Ducts.-The ejaculatory ducts are two in number, one on each side. They are about three-fourths inch long, pass forward through the prostate gland, one on either side of the middle line, between the middle and lateral lobes of the prostate, and open upon the floor of the prostatic urethra.

\section{OPERATIONS UPON THE SPERMATIC CORD, SCROTUM, ETC.}

For Varicocele. OPEN OPERation.-An incision is made, about one and one-half inches long, into the upper part of the front of the scrotum, commencing just below the spine of the pubes, and passing through the skin into the subcutaneous fatty layer. This incision can be made by pinching up the skin and transfixing it with a sharppointed knife or by cutting it with the scissors. The cord is then hooked up, upon the finger, out of the loose, fatty layer in which it lies, and with one or two strokes of the knife its sheath (the spermatic fascia and the fascia propria) is opened. The vas deferens is sought and recognized, and together with the immediately adjacent veins is separated from the other parts of the cord. This is done with the fingers, holding the vas deferens and the several adjacent veins, which are to be allowed to remain securely between the finger and thumb of the left hand, while the work of separating the other structures of 
the cord, veins of the pampiniform plexus and the spermatic artery, from the vas deferens, may be accomplished with the fingers of the right hand.

After the vas deferens, together with the several immediately adjacent veins, has been isolated for a distance varying from one to two inches, depending upon the laxness of the scrotum and the length of the cord, etc., a double catgut ligature is passed with an artery forceps and then cut so that we have two ligatures. These ligatures, which surround all those structures of the cord that have been separated from the vas deferens, etc., are tied, one above and the other

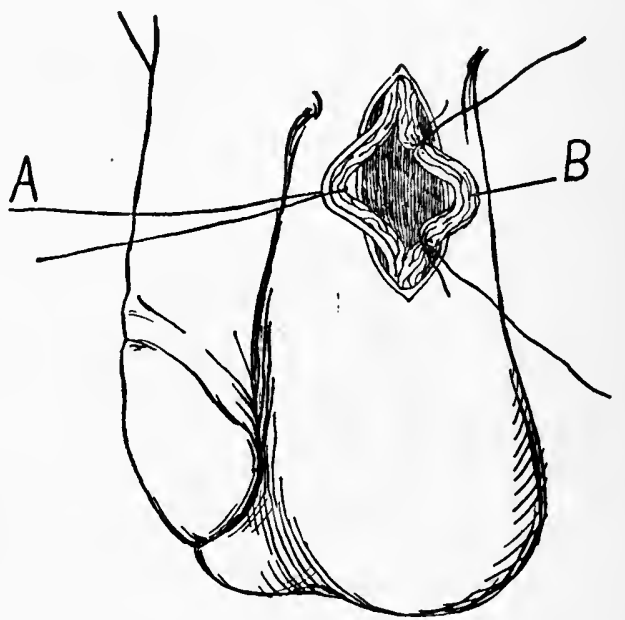

Fig. 170.-Varicocele. The vas deferens and adjoining vessels $(A)$ have been separated from the other structures of the cord-from the spermatic artery and pampiniform plexus $(B)$. Ligatures have been tied about $B$ above and below preparatory to excising the intervening portion.

below. The portion intervening is excised with the scissors, not too close to the ligatures, and the ends of the ligatures, which have been purposely left long, are then tied together, in this way bringing the ends of both stumps into apposition. The ends of these two portions may be still further secured by one or two catgut sutures, which should take a good bite through the whole thickness of each stump.

The portion of the cord which is stripped away from the vas deferens, and which is ligated and excised, is composed of all the veins of the pampiniform plexus and the spermatic artery. When the vas is isolated, the artery of the vas deferens, which anastomoses 
below with the spermatic artery, and the cremasteric artery, together with their corresponding veins, go with it; these vessels are therefore not interfered with, and they are sufficient to provide for the nutrition of the testis after the pampiniform plexus and the spermatic artery have been ligated.

For the ligatures, plain catgut, not too thick (No. 1 or 2) may be used, and special care should be taken to apply the upper ligature securely that it may not slip, as this would result in a very free hemorrhage from the end of the spermatic artery.

In this operation one not only ties off the veins of the pampiniform plexus, but also shortens the cord, and thus draws the testis up, a result which is much to be desired. Before closing the incision in the skin all bleeding points should be clamped and ligated or twisted, and the wound should be dry. The edges of the incision in the skin are brought together with a continuous stitch of catgut, which may be intracuticular.

For Hydrocele.-A condition in which the tunica vaginalis is distended with serous fluid. The testis is usually found in the lower, back part of the sac, the fluid being collected above and in front of it.

Puncture and Injection.-This is suitable for simple cases, and for those where tapping has not been previously resorted to. The scrotum is grasped in the left hand, in order to make it tense and to steady it. A fine needle, attached to a hypodermic syringe, is introduced through the anterior wall of the scrotum, and a small quantity of the fluid drawn off, both for the purpose of confirming the diagnosis and to demonstrate the fact that the needle is in the cavity of the tunica vaginalis. The hypodermic needle is left in situ, its end free in the cavity of the tunica vaginalis. A fairly large trochar is then thrust through the bottom of the scrotum rather toward the front, and in an upward direction into the cavity of the tunica vaginalis. In doing this one should remember that the testis occupies the lower back part of the sac. With the trochar in the cavity of the tunica vaginalis one should be able with it to touch the hypodermic needle previously introduced into the sac above. The sac is allowed to empty itself through the cannula, and this is then withdrawn.

The barrel of the hypodermic syringe is now filled with the fluid to be injected. Twenty minims of a 95-per-cent. carbolic-acid solution may be used, with satisfactory results, for this purpose. 
This is thrown into the cavity of the tunica vaginalis through the hypodermic needle, and then this needle is also withdrawn. The fluid that has been thus introduced into the cavity of the tunica vaginalis is distributed over the whole cavity by manipulating the scrotum. The punctures made by the instruments are covered over with a thin coat of collodion, and a very thin film of absorbent cotton.

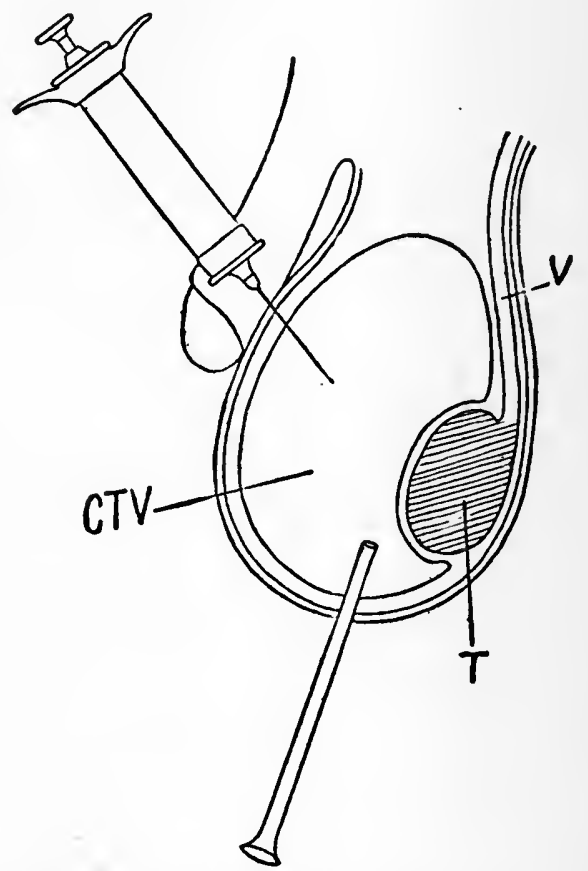

Fig. 171.-Hydrocele, Tapplng. CTV, cavity of the tunica vaginalis testis: $T$, testis; $V$, vas deferens. Hypodermic needle introduced into the upper part of the sac; trochar cannula into the lower part.

This operation is usually followed by some effusion into the sac, and with but little or no pain. After a few days' rest in bed with the scrotum supported, these symptoms subside. The operation is not painful, but the part where the trochar is to be introduced may be anæsthetized with ethyl chloride if desired.

OpEn Operation (VolKMANN).-This operation is suitable for those cases that have already been tapped many times or where the operation previously described has been tried and has failed. 


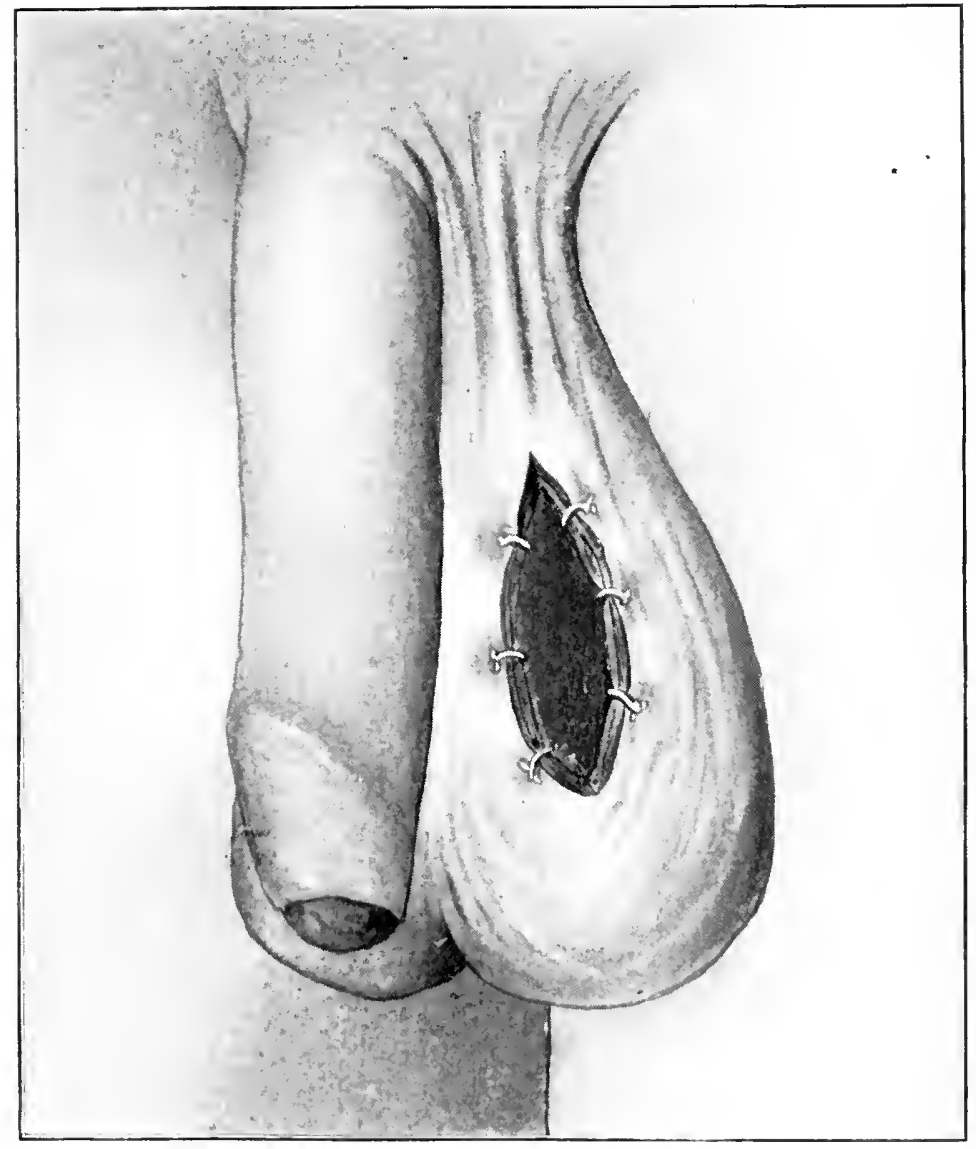

Fig. 172.-Volkmann Operation for Hydrocele. Edge of tunica vaginalis sutured to the edges of the skin incision. 

The scrotum is grasped by an assistant in order to make it tense and to steady it. An incision is made through the anterior wall of the scrotum, opening into the cavity of the tunica vaginalis. The length of the incision depends upon the size of the tumor, but is usually two or three inches. When the tunica vaginalis has been opened, and while the fluid is escaping, the edge of the parietal layer of the tunica vaginalis-i.e., the inner lining of the scrotal sac-is seized on either side with an artery forceps, and with the finger this is torn away from its attachment to the inner aspect of the scrotum, and excised in part with the scissors. If the tumor has been very large, it will be necessary to excise more of the tunica vaginalis than if the tumor is smaller. The tunica vaginalis may be much thickened. In trimming away this redundant portion of the tunica vaginalis one must take care to leave enough to conveniently cover the testis and also avoid cutting into the epididymis. It is rather better to excise too little than too much of the tunica vaginalis. After this part of the operation has been done the edge of that portion of the tunica vaginalis. which remains is fixed to the corresponding edge of the skin incision all around with a continuous or with several interrupted fine catgut sutures. Then, with a wad of cotton on a stick, the whole interior of what remains of the tunica vaginalis, including that covering the testis, is swabbed out with 95-per-cent. carbolic acid. The cavity is then loosely packed with sterile gauze. The strips should reach well down into the deepest recesses of the cavity, but the packing should not be tight. A loose dressing is applied, which may be held in place by a T-bandage. The packing should be removed at the end of forty-eight hours, simply retaining a strip in the opening in the skin, and the parts allowed to granulate. If too much of the tunica has been removed, there will be too much inversion of the skin, and this will delay the healing process.

Excision of the Tunica (von BergmanN).-After the tunica vaginalis sac has been opened and its contents evacuated, the parietal layer of the tunica vaginalis is seized and stripped away from its attachment bluntly with the fingers as far back as the posterior border of the testis, or rather epididymis, and then excised in its entirety with the scissors. After all bleeding has been controlled with forceps and ligatures, the wound in the skin is closed with sutures, without any drainage whatever. As a rule, the skin incision heals by first intention, and the patient is able to be around in about twelve days. 
This method is very satisfactory, and is especially applicable to those cases where the tunica vaginalis is excessively redundant after the evacuation of a large hydrocele, or when the tunica is markedly thickened.

Retroversion of the Tunica Vaginalis.-This method has been variously ascribed to Jaboulay, Doyen, Garampozzi, and Winkelmann. An incision is made in the front of the scrotum, usually about two inches in length, into the cavity of the tunica. Through this opening the fluid contents of the distended tunica vaginalis escape, and the testis is then drawn forward out of the scrotum.

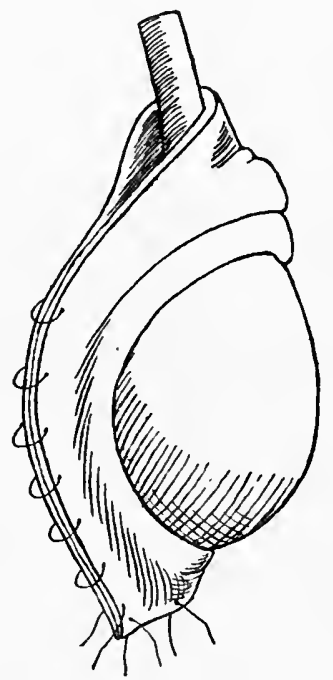

Fig. 173.-Hydrocele. Retroversion of the tunica vaginalis. The tunica has been turned back beyond the epididymis and fixed there by sutures.

As the testis is drawn forward out of the scrotum, the vaginal layer of the tunica is reflected backward,-turned inside out, as it were,-so that the opening in the parietal layer of the tunica, through which the testis has been drawn, gets to lie behind the testis, encircling the cord and covering over the epididymis, and in this position it is fixed by joining its edges together with several catgut sutures so that it may not again slip forward over the testis. The edges of the incision in the scrotum are now sufficiently detached to allow the integument of the scrotum to be drawn forward and cover 
.

$\checkmark$ 


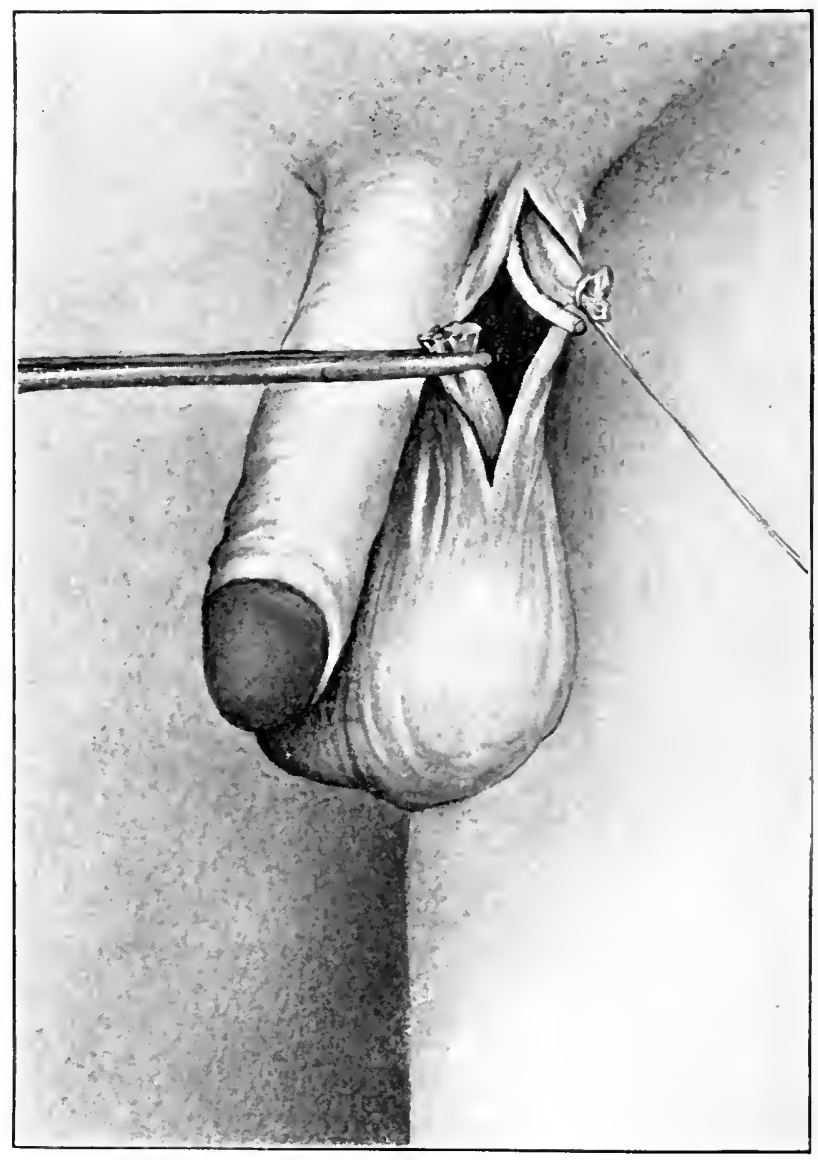

Fig. 17.,-Castration. Cord has been divided. The end of the lower portion grasped with an artery forceps. A ligature has been tied around the end of the upper stump. It will be noticed that the vas deferens is not included in the ligature. 
over the testis and reflected tunica vaginalis, and they are thus united to each other without drainage, in this way completing the operation.

The result of this operation is that the free secreting surface of the tunica vaginalis which has been turned inside out is brought into contact with the raw internal wound surface of the scrotum, to which it becomes united, effecting the cure.

If the tunica vaginalis is very redundant after evacuating a large hydrocele, a part of the tunica may be excised with the scissors, leaving just enough to complete the operation as described above; but for those very large hydroceles, and those with a markedly thickened tunica, the von Bergmann is probably the more satisfactory operation.

Castration (Extirpation of the Testis).-An incision, about two inches long, is made upon the front of the upper part of the scrotum through the skin and fat, commencing at a point just below the external ring-the spine of the pubes. If operating for malignant disease, and if the skin is involved, the incision may be arranged so as to circumscribe that part of the skin which is involved. In the upper part of the incision the cord is found, and hooked up, upon the finger, and just below the point where it emerges from the external ring its sheath is incised with the point of the knife. The vas deferens is then recognized, and should be separated from the rest of the cord. A catgut ligature is then passed about those parts of the cord which have been separated from the vas deferens, and tied so tightly that it cannot slip off. This ligature should include all the elements of the cord except the vas deferens. The ends of this ligature are left long, to serve as a tractor; the cord, including the vas deferens, is then divided with the scissors, at least half an inch below, distal to the ligature. Before dividing the cord it is grasped, below the point at which it is to be divided, with an artery clamp. The cord having been divided, the lower end, that which is held in the grasp of the artery forceps, together with the testis, and including the tunica vaginalis, is enucleated from the scrotum, usually without opening into the cavity of the tunica vaginalis, and almost entirely by blunt dissection. Where the knife or scissors is used to assist in this enucleation one should take care not to cut through the septum into the other half of the scrotum, and one should also avoid button-holing the skin.

After the testis has been enucleated we return to the stump of the cord. This may be brought into view by drawing upon the liga- 
ture, which was left long to serve as a tractor, and if there is no bleeding this ligature may be cut short and the stump of the cord allowed to retract up into the inguinal canal. Should there be any bleeding points, these may be clamped and ligated. One should avoid including the stump of the vas deferens in the ligature, as it may result in disagreeable symptoms; e.g., colicky pain, etc.

The wound is large, and may be closed with catgut sutures; in most cases, however, it is well to place a drain in the lower end of the wound. If operating for tuberculosis, the cord should be divided as high up as one can reach. 


\section{PART VIII.}

\section{THE URINARY SYSTEM.}

\section{THE KIDNEYS.}

The Surgical Anatomy of the Kidney.-One kidney may be absent in apparently normal subjects, the left more frequently than the right. This is said to occur once in about two thousand four hundred subjects. Absence of one kidney has been met with twice in five hundred subjects in the writer's experience. When one kidney is absent that which is present is usually larger and assumes the function of both kidneys.

There may be two kidneys present, joined together below or above, horseshoe kidney, or both above and below, either with connective tissue or kidney tissue. This condition is met with about once in one thousand subjects.

At times the kidney becomes loosened in its bed, and may become dislodged,-movable kidney,-or it may be provided with a nearly complete peritoneal covering and mesentery and enjoy a considerable range of motion, when it is called "floating, or wandering," kidney.

The kidneys lie in the upper back part of the abdomen, one on each side of the vertebral column, from the twelfth dorsal to the third lumbar vertebra. They are extraperitoneal organs, being covered by peritoneum upon their anterior surface only.

The kidneys are provided with a fibrous capsule, which is usually very thin and closely adherent to the organ. They are lodged within a bed of loose fat and connective tissue, out of which they may be readily enucleated. The anterior surface of the kidney is directed forward and outward, and is covered by the peritoneum. The descending part of the duodenum lies in front of the right kidney, the pancreas in front of the left kidney.

The upper part of the posterior surface of the kidney is separated from the eleventh and twelfth ribs by the diaphragm and pleura; the lower part of the posterior surface of the kidney rests upon the quadratus lumborum muscle, which is covered by the anterior layer of the lumbar fascia. Normally the upper half of the kidney lies above the twelfth rib, and the lower half below the twelfth rib. 
The inner border of the kidney is concave, and is directed toward the psoas muscle and the vertebral column; the inner border of the kidney really rests upon the edge of the psoas muscle, and the kidney is thus tilted somewhat outward. Corresponding to the inner border of the kidney are the artery and vein and the ureter. At the hilum the relation of these structures from before backward is vein, artery, and ureter; upon the left side the artery lies above the vein, and upon the right side the vein lies above the artery; upon both sides the ureter is the lowest of the three structures.

The outer border of the kidney is rounded and convex, and is related, the right, with the ascending colon, and the left with the descending colon. The colon really lies a little in front of the kidney, as well as to its outer side.

The upper end of the kidney is covered by the suprarenal capsule, which sits upon it like a cap. The upper end of the right kidney is in close relation with the under surface of the liver. The upper end of the left kidney lies close to the spleen. The lower end of the kidney reaches to within one or two inches of the crest of the ilium.

The right kidney is located about one inch lower than the left, and this is due to the presence of the liver upon the right side; the right kidney is, therefore, more accessible than the left, and this is also the one which is more frequently movable and the object of operative measures.

\section{OPERATIONS UPON THE KIDNEY.}

Nephropexy.-Suture or fixation of a movable or floating kidney.

A movable kidney is one that enjoys a limited range of motion in the posterior part of the abdomen, but which does not leave the lumbar region. A floating kidney is one that is more or less completely invested with a peritoneal coat and provided with a more or less complete mesonephron, and therefore enjoys a considerable range of motion, and is capable of leaving the lumbar region entirely.

In operating upon the kidney through the lumbar incision the patient may be placed prone upon the table with an Edebohls cushion under the abdomen, or he may rest upon the side of the body corresponding to the kidney which is not the object of operation, with the knees and thighs somewhat flexed and the front of the body turned toward the table. In this latter position the patient is steadied with sand bags placed against the eliest and abdomen; an- 
other sand bag or cushion may be placed between the lower part of the side of the chest and the table, with the idea of increasing the space between the lower border of the twelfth rib and the crest of the ilium upon the side of operation.

It is important that the abdomen be relaxed in order that the assistant during the operation may be able through the abdominal wall to push the kidney upward toward the incision in the loin. Probably the most satisfactory position is with the patient lying prone upon the
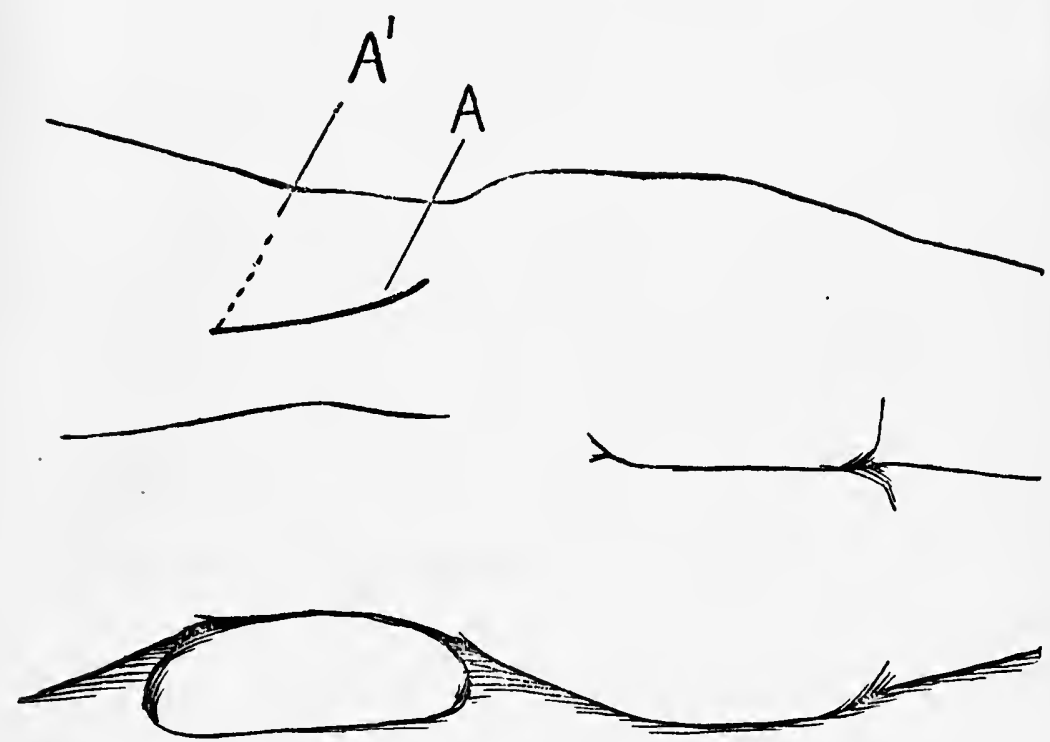

Fig. 175.--lncision to Expose Kidney. $A$, along the edge of the erector spinæ. $A^{\prime}$, additional incision along the edge of the last rib.

table with the Edebohls cushion under the abdomen, especially if both kidneys are to be exposed during the operation.

The incision corresponds to the outer border of the erector spinæ muscle, commencing, above, just below the twelfth rib, about two and a half inches from the middle line (spinous processes); it passes downward, curving somewhat outward, and terminates just above the crest of the ilium. This incision should extend through the skin and subcutaneous fat down to the surface of the latissimus dorsi, the fibers of which muscle are exposed. The incision is then carried through the fibers of the muscle, when the outer border of 
the erector spinæ may be recognized; without opening its sheath, this muscle is drawn toward the middle line with retractors. The quadratus lumborum, covered by its layer of lumbar fascia, is then exposed in the bottom of the wound. The fascia that covers the quadratus lumborum is incised along the outer border of the muscle, which is then also drawn toward the spine. It is important to recognize the edge of this muscle. One should be on the lookout for the ilio-hypogastric nerve, which is derived from the lumbar plexus and passes downward and outward across the front of the quadratus lumborum; this nerve is usually seen after the edge of the quadratus lumborum has been exposed, and should not be cut, but rather drawn aside, out of the way.

There remains now only the deepest and last layer of the lumbar fascia to ineise, and this should be split the full length of the skin incision. In cutting through the various layers of the back, if the patient rests upon the side, there is a tendency to work inward, toward the spinal column, instead of directly downward, through the different layers, toward the kidney, and this should be avoided. The incision should reach above to the lower border of the last rib, but should not be carried beyond this level by carelessly passing the point of the knife, within the wound, upward underneath the last rib, as the pleural cavity may be thus accidentally opened.

After having cut through the deepest and last layer of lumbar fascia, the fatty capsule, in which the kidney is imbedded, is encountered. This is separated from the kidney bluntly, with the fingers in the wound, in order to bring the kidney into the incision for fixation.

During this step of the operation one should be careful not to penetrate through the proper fibrous capsule of the kidney, since, if this accident oceurs, one may detach the true capsule of the kidney from the kidney substance proper, instead of isolating the kidney with its proper eapsule intact from the loose mass of fat in which it is lodged. If the kidney is displaced, movable, it is easy to reach it, as it then lies lower in the abdomen. The right kidney normally is situated lower than the left. When the kidney is sufficiently free, its outer, rounded border is brought up into the wound; this is greatly facilitated by the assistant forcing it up by pressure from the front of the abdomen if the patient lies upon the side. If the patient lies prone, with the Edebohls cushion under the abdomen, 
the kidney may be brought into the wound or entirely out upon the back without any counter-pressure being made upon the abdomen from in front.

The proper fibrous capsule is incised from above downward along the whole length of the outer, rounded border of the kidney, and each edge seized and separated from the kidney substance-it peels off easily-for a distance of about one inch on each side.

Either edge of the detached capsule is then sutured above and below with chromicized catgut to the corresponding edge of the muscles deep in the wound. Two additional sutures of No. 2 chromicized catgut are passed through the edges of the muscles and carried deep through the kidney tissue proper: one of these sutures passes through the upper part of the kidney and one through the lower part. It is probably more convenient to pass these two deep kidney sutures first, leaving them untied, to be used as tractors to hold the kidney in position until the stitches through the capsule have been introduced and tied. The two deep stitches are then tied also, but not too tight, as they might cut through the kidney tissue. When all the sutures have been tied, it will be seen that the external, rounded border of the kidney, denuded of its capsule, is firmly fixed to the edges of the muscles deep in the wound, and in this position it remains fixed as the wound heals. Before tying the two sutures that pass through the kidney the edges of the muscles in the incision may be united by several deep catgut stitches.

The incision is closed with interrupted silk-worm gut stitches, which pass deep through both the skin and muscles, or the muscles may be united separately by several deep, interrupted, catgut sutures. It is unnecessary to drain the wound.

There are usually no large vessels encountered during the operation, but any spurting points may be clamped and ligated.

Nephropexy (Edebohls). - An incision is made which reaches from the twelfth rib to the crest of the ilium along the outer border of the erector spinæ; it passes through the skin and fat down to the latissimus dorsi. The fibers of the latissimus dorsi are not cut, but are separated bluntly, in the direction of their course, with the handle of the knife.

Corresponding to the outer edge of the quadratus lumborum muscle, which lies beneath the erector spinæ, the transversalis fascia is incised, thus entering the abdomen and exposing the mass of fat (fatty capsule) within which the kidney, enveloped in its proper 
fibrous capsule, is imbedded. One should avoid division of the iliohypogastric nerve, a moderately large branch of the lumbar plexus which passes obliquely downward and outward across the front surface of the quadratus lumborum; it should be sought for at the outer edge of the quadratus lumborum, and drawn to one side.

Upon its anterior aspect and near its outer edge the sheath of the quadratus lumborum is now incised from the twelfth rib to the crest of the ilium; this exposes a considerable area of the raw anterior surface of the muscle. With the fingers in the wound the fatty capsule is peeled off the kidney, and the organ, enveloped in its proper fibrous capsule, is delivered through the wound out upon the back. At times the kidney, still enveloped in its fatty capsule, may be delivered through the wound, and under these circumstances the fatty capsule may be separated from the kidney almost as far as the pelvis, and excised with the scissors.

The position of the patient, prone, and with the Edebohls cushion underneath the abdomen, makes the delivery of the kidney comparatively easy. If the opening in the loin is not sufficiently roomy, it may be enlarged by nicking the outer edge of the quadratus lumborum below, near its attachment to the ilium.

A small incision is made in the capsule of the kidney, near the middle of its outer, rounded border, and through this opening a director is introduced upon which the capsule is divided along the entire length of the outer border of the kidney. The capsule is then peeled back, about half-way toward the pelvis upon either surface, thus laying bare about one-half of the entire kidney surface. The detached part of the capsule is not excised unless it is quite redundant; it is simply folded back toward the pelvis of the kidney upon the non-detached portion.

Four fixation sutures of forty-day chromicized catgut are now introduced through the capsule; these pass through the capsule only, two on each side, one above and the other below. Each of these fixation sutures should take a good, broad bite, and passes through both the detached and the non-detached portions of the capsule, parallel with and close to the margin that corresponds to the line of its reflection. After these four fixation sutures have been introduced the kidney is returned into the abdomen.

The ends (eight in number) of the fixation sutures are then, in succession, one after the other, threaded in a large curved Hagedorn needle, and carried through the muscles and fascia that correspond 
to the edges of the incision, from within outward. The sutures are not tied until later. Those sutures that pass through the inner edge of the incision penetrate the retracted edge of the incised sheath of the quadratus, the quadratus itself, and the erector spinæ and latissimus dorsi; the sutures that pass through the outer edge of the incision pierce the edge of the transversalis fascia and the latissimus dorsi musele.

Now, before tying the fixation sutures the edges of the wound in the back are approximated with from four to six ehromicized eat-

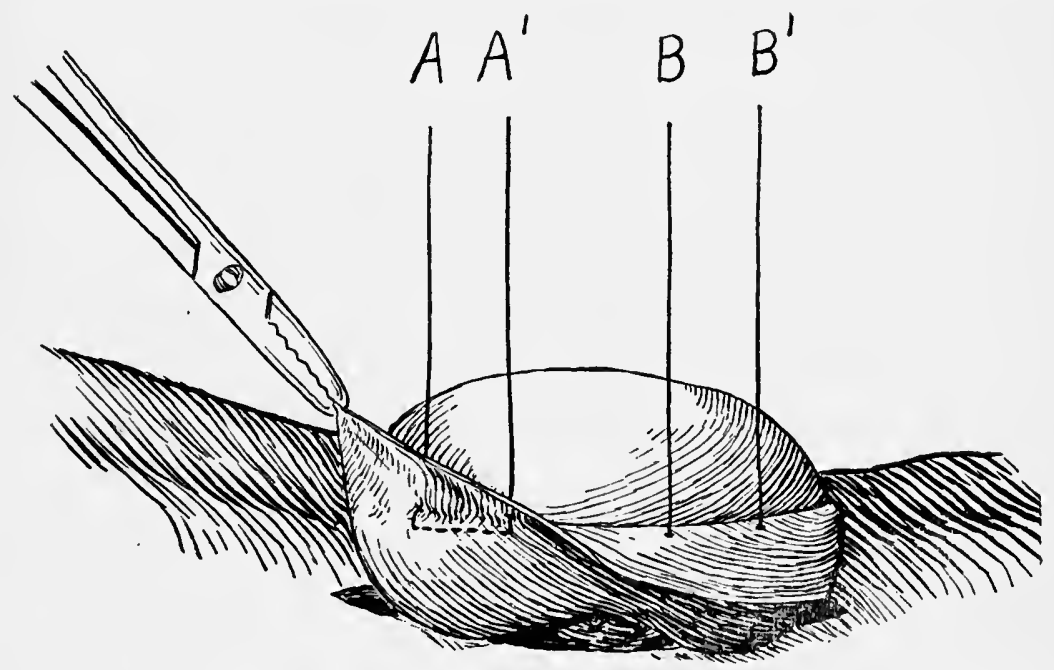

Flg. 176.-Nephropexy (Edehohls). Kidney delivered through an incision in the back. Projer fibrous capsule reflected and two flxation sutures introduced, one above $\left(A, A^{\prime}\right)$ and one below $\left(B, B^{\prime}\right)$. These sutures pass through the reflected and attached portions of the capsule close to the line of reflection. The two sutures that secure the capsule upon the opposite side of kidney are not seen.

gut sutures; these are interrupted, and pass through all the fasciæ and muscles in the edges of the wound. The fixation sutures are then tied; they emerge upon the posterior surface of the latissimus dorsi, four upon each side of the line of division in the muscle. They are not tied across the wound, but the adjoining ones of each side are tied to each other upon the same side of the wound.

The edges of the skin are finally united without drainage with an intracuticular suture.

The result of this operation is to fix the denuded, raw surface 
of the kidney, corresponding to the whole length of its convex border, and extending half way to the pelvis upon either surface, to the denuded anterior surface of the quadratus lumborum, the upper extremity of the kidney projecting upward, beneath the last ribs.

Nephrotomy.-Cutting into the kidney for the purpose of evacuating an abscess or to explore the pelvis of the kidney.

The position of the patient and the incision are as described for nephropexy (page 404). The patient is placed either prone, with the Edebohls cushion underneath the abdomen, or else he rests upon the well side. The incision is carried down, step by step, until the last layer of the lumbar fascia has been cut and the kidney is reached. If operating for nephritic abscess, we may find, as soon as the kidney is exposed, that the indications of the abscess immediately present themselves, or it may be necessary to search with an exploring needle. When pus is located, the cavity containing it is incised with the point of the scalpel and enlarged with dressing forceps, which are introduced closed and expanded as they are withdrawn. At times the entire kidney substance is destroyed, and simply a bag of pus remains. We may or may not find a stone. The abscess cavity is irrigated and packed loosely with a strip of iodoform gauze, the end of which emerges through the lower part of the wound in the loin.

In closing the incision in the back the stitches should be carried deep in order to include the muscles, together with the skin; the lower part is left open for drainage.

At times, in order to explore the pelvis of the kidney or to drain it, it may be necessary to bisect or split the kidney from its posterior rounded border right through into its pelvis. In doing this care should be exercised to divide the kidney midway between its two surfaces, as this is attended with less hemorrhage. The kidney must be brought up into the wound, and may be steadied there by an assistant exercising pressure from in front. It is usually sufficient if the incision in the kidney extends through only a part of its length. In this way a stone which may escape the exploring needle may be detected and removed, or, if there is no stone present, and the symptoms are due to an inflammatory condition of the pelvis, this may be drained through the kidney by leaving a small tube or a strip of iodoform gauze, which reaches from the pelvis of the kidney and emerges through the incision in the loin. A resulting urinary fistnla usually closes spontaneously, provided the ureter is not obstructed. 
The cut surfaces of the kidney may be brought together, thus controlling hemorrhage from the renal vessels, by passing several deep sutures through the substance of the kidney. If an individual spurting artery of some size is seen, it should be ligated separately. For these sutures catgut should be used, and they should be passed in a curved surgeon's needle.

The wound in the loin is closed in part by interrupted, silkworm gut sutures, which penetrate deep through the edges of the muscles, or the edges of the muscles may be united separately by several interrupted catgut sutures.

Nephrolithotomy.-Cutting into the kidney for stone.

The steps of this operation are like those already described in the preceding operation. After the kidney has been reached and brought up into the wound it may be palpated and punctured with a fine needle, here and there, in order to locate the stone. It may be found in the pelvis of the kidney or in the kidney tissue proper. With the point of the knife, which is passed along the needle as a guide, an incision is made in the kidney, this opening being enlarged with an artery forceps, or the pelvis of the kidney may be incised, and the stone extracted.

If one is unable to locate the stone with the exploring needle, and the symptoms warrant it, the kidney may be laid open, as in the preceding operation.

As a rule, pus is associated with stone, and it is, therefore, usually necessary to drain these cases.

If there is no pus, or if small in quantity and if the ureter is not obstructed, one may omit drainage and allow the wound in the kidney to close; if the opening is large, a suture may be introduced. If the pelvis of the kidney has been opened, it may be closed with several catgut sutures introduced with a small, curved needle in a holder. It is well to provide drainage for the incision in the back, a strand of gauze being packed into the wound down to the site of the incision in the kidney or pelvis of the kidney.

Nephrectomy.-Extirpation of the kidney.

The position of the patient is the same as that already described for nephropexy. The steps of the operation are as above indicated down to the point of exposing the kidney. The incision is the same as that described for nephropexy (page 404) and should reach from the last rib to the crest of the ilium. If necessary, we may obtain more room by curving the lower end of the incision forward, above the 
upper border of the crest of the ilium, or we may make a cut from the upper end of the lumbar incision outward along the lower border of the last rib (see Fig. 175).

The isolation of the kidney must be thorough, and this is accomplished with the hand in the wound, working patiently, with the fingers, around the kidney, eare being taken not to tug upon the kidney, as one may tear the vessels at the hilum. The suprarenal capsule may be left behind, although, if diseased, it may be removed also. After the kidney has been isolated, its outer, rounded border is brought well into the wound, or, as may be done in nearly all cases, the kidney is bronght entirely out of the wound, so that a ligature may be thrown around it and worked down about the structures at the hilum - the vein, artery, and ureter-and tied, or one may pass the ligature with a large, curved, blunt ligature carrier, the ligature being carried about the artery and vein, without including the ureter, which lies below the vessels and on a plane posterior to them.

The ligature should be of strong catgut; after the ligature has been tied its ends should not be cut short, as it is desirable to use the ligature as a tractor to bring the stump of the kidney into view for final inspection.

In cutting away the kidney the division should not pass through the pedicle, which is made of the vessels, but, if possible, should pass through the kidney tissue near the hilum, in order to leave a little mass of kidney tissue as a cap, or knob, to prevent the slipping of the ligature.

The wound is treated as in the foregoing operations; it is probably better to introduce a drain, which is left for seventy-two hours.

When the kidney is the seat of a very large tumor, it may be difficult to remove it through this posterior incision.

We should be positive that a second kidney, which is capable of carrying on the work, is present, and, if necessary at the time of the operation, an incision may be made down upon the other kidney in order to satisfy ourselves of its presence.

Decortication of the Kidney (Edebohls). - This operation was. first suggested for the cure of chronic Bright's disease, by Edebohls. The operation is recent, and its real valne still sub judice. The beneficial effect of the operation is, no doubt, due to the increased supply of blood that is brought to the kidney through the new vascular connections that are formed between it and the adjacent parts.

Edebohls says that one may use the anæsthetic, ether or chloro- 
form, with which he is most familiar. Mixed nitrous oxide and oxygen is very well adapted to certain cases. It would seem that chloroform would be more satisfactory in most cases. Spinal analgesia would, no doubt, be appropriate in some of these cases, where the patient's condition counter-indicates the use of a general anæsthetic.

The patient lies prone upon the table, with the Edebohls cushion under the abdomen. The incision, the same as that described for nephropexy (page 40\%), corresponds to the edge of the erector spina, and penetrates the transversalis fascia along the outer edge of the quadratus lumborum. The kidney is recognized in the mass of fat, fatty capsule, that incloses it.

With the fingers in the wound the fatty capsule is separated bluntly from the surface of the kidney as far as the pelvis. The kidney, inclosed within its unbroken fibrous capsule, is then drawn into the wound, or, if possible, lifted out of the wound upon the back.

Corresponding to the middle of the outer, rounded border of the kidney, the capsule proper is incised, and divided upon a director along the entire length of the outer, rounded border of the organ, and around its extremities, above and below. Each half of the capsule is then stripped away from the surface of the kidney toward the pelvis, taking care not to break or tear the kidney substance proper, which may be friable and firmly adherent to the capsule.

The stripped off capsule is finally cut away near the pelvis of the kidney, and removed. If the kidney cannot be brought out through the incision in the back, the capsule must be peeled off the kidney, with the fingers in the wound, and excised, as far as possible.

Any portion of the capsule that still remains may be rolled back toward the pelvis of the kidney, where it remains coiled up, upon itself.

The kidney is finally replaced in the abdomen, and the incision closed without drainage. At the time of operation it may appear that but one kidney is the seat of chronic Bright's disease, but it is probably wise in all cases to decapsulate both kidneys at the same sitting.

\section{THE BLADDER.}

Surgical Anatomy of the Bladder.-The bladder is a hollow muscular organ whose function is to receive and hold the urine during the intervals of micturition. It has a capacity ordinarily of about sixteen ounces. 
In the infant the bladder is rather conical, and projects into the abdomen above the level of the symphysis.

In the adult the bladder, when empty, lies deep within the pelvis behind the symphysis, its cavity obliterated and its walls collapsed and in contact with each other. When distended moderately, it reaches as high as the symphysis, farther distension causing it to rise up, out of the pelvis, into the abdominal cavity a varying distance toward the umbilicus. When it is distended with about a pint of fluid, the bladder is pear-shaped, and reaches for a distance of about four inches above the symphysis.

The body of the bladder is free, and, when the organ is distended, rises out of the pelvis into the abdomen, toward the umbilicus.

The base of the bladder in the male is in close relation with the anterior surface of the second part of the rectum, and upon its inner aspect, on either side, shows the openings of the ureters.

The neck of the bladder is continuous with the commencement of the urethra, and in the male is surrounded by the prostate, like a collar.

Relations of the Bladder. In the Male the bladder is in relation, behind, with the rectum, the base of the bladder lying directly in front of the second portion of this part of the bowel, the two being joined together more or less intimately by connective tissue.

The seminal vesicles and vas deferens are located on either side of the middle line, in the space between the contiguous walls of the rectum and the bladder; they converge anteriorly toward the prostate, which surrounds the neck of the bladder, and which is readily felt through the rectum.

In the Female the uterus and vagina are located behind the bladder.

In both sexes the bladder lies immediately behind the symphysis pubis, from which it is separated by a space, which is filled with loose connective tissue more or less firmly connected with the anterior wall of the bladder, and which is called the space of Retzius. When the bladder is distended, it reaches above the symphysis, and is then in relation, in front, with the anterior abdominal wall.

Passing from the summit of the bladder to the umbilicus is the urachus, which occasionally remains patent after birth.

The peritoneum covers the sides, part of the posterior surface, and the summit of the bladder, but does not cover its anterior surface, being reflected from the summit of the bladder over on to the poste- 
rior surface of the anterior abdominal wall." When the bladder is well distended, it rises upward into the abdomen; its summit, as it approaches the umbilicus, carries the peritoneum with it, and its anterior surface, which is devoid of peritoneum, then comes into relation with the abdominal wall; so that under these circumstances the bladder may be entered through an incision in the anterior abdominal wall, low down, close to the symphysis, without molesting the peritoneum or entering the peritoneal cavity.

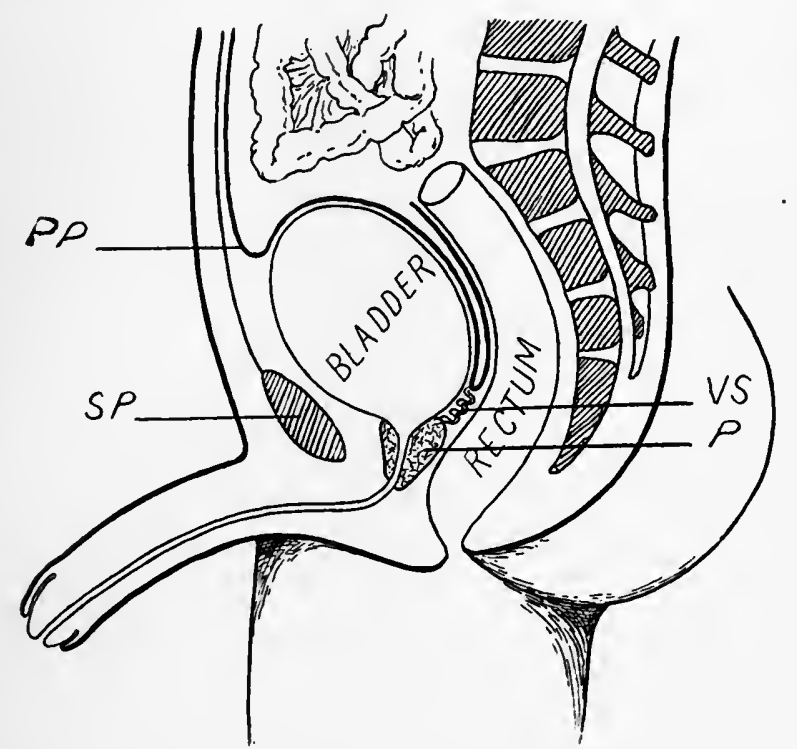

Fig. 177.-An Antero-posterlor Section Showing Relations of the Peritoneum to the Bladder, etc. Bladder moderately distended. $\boldsymbol{P}$, prostate gland surrounding commencement of the urethra (neck of the bladder); $P P$, anterior fold of peritoneum reflected from the posterior aspect of the anterior abdominal wall over on to the fundus of the bladder; $S P$, symphysis pubis; VS, vesiculæ seminales.

The higher the bladder ascends into the abdomen, the larger the area of its anterior, non-peritoneal surface which is presented for operation.

\section{OPERATIONS UPON THE BLADDER.}

Suprapubic Cystotomy. - The patient is placed in the usual position upon the back and fully anæsthetized, so as to relax the abdominal muscles. If one is unable to distend the bladder, owing to 
the existence of an impassable stricture of the urethra, etc., much advantage is gained by placing the patient in the Trendelenburg position.

A soft rubber catheter is introduced into the bladder, and, through this, the bladder is washed out with boric-acid solution, 10 to 12 ounces being allowed to remain; the catheter is then withdrawn, and a band tied about the penis to prevent the escape of the fluid. The fluid which is thrown into the bladder causes it to ascend into the abdomen, carrying the peritoneum with it; so that its anterior surface, uncovered by peritoneum, is exposed for several inches for operation. It is well not to introduce more than 10 to 12 ounces, as oftentimes the capacity of the bladder is diminished, and a quantity above 12 ounces might do harm.

In order to throw the distended bladder farther forward toward the anterior abdominal wall, a bag may be introduced into the rectum and distended with about 6 ounces of water. Most operators dispense with the rectal bag as unnecessary. The incision, which is placed in the middle line, linea alba, commences below, at the symphysis pubis, and reaches upward, toward the umbilicus, for a distance of about three inches, and extends through the skin and fat down to the deep fascia. Bleeding vessels in the skin are clamped.

The incision is carried down through the linea alba, between the edges of the recti and pyramidales, until the layer of connective tissue, which is located in front of the bladder, dipping down between it and the symphysis pubis, is reached.

The edges of the wound are then drawn apart with retractors, and this layer of connective tissue, which covers the anterior wall of the bladder, is scraped upward, toward the umbilicus, with the fingernail; so that, in case the fold of peritoneum reaches abnormally low, or the bladder has not been sufficiently distended, we may thus still separate it and carry it upward toward the umbilicus. The muscular wall of the bladder is then easily recognized, especially if the organ is distended. A plexus of veins, more or less visible, which ascends upon the anterior wall of the bladder from below, may help to identify it.

With a curved surgeon's needle two rather stout silk stitches are introduced, one on either side of the middle line, through the whole thickness of the bladder wall, and these are used as tractors to steady the bladder while it is being incised.

In cutting into the bladder the point of the knife is introduced 
between the two silk tractor stitches about one inch above the symphysis, and the bladder incised in a direction downward, toward the symphysis. When the bladder is opened the fluid contained within it escapes in part. The incision should be large enough to permit the introduction of one or two fingers for the purpose of exploration, etc.

The incision in the bladder may be enlarged sufficiently to allow necessary manipulation; caution should be exercised in extending the opening in the bladder, for any considerable distance, in an upward direction, toward the umbilicus (fold of peritoneum).

If a stone is present, it may be removed with the forceps, guided by the finger; if the stone is very large, it may first be crushed. One should search the bladder carefully for stones which have become almost completely encysted in pockets in the bladder wall. If the operation is done for ulcer of the bladder, the diseased area may be scraped or cauterized, etc. With the patient in the Trendelenburg position and the edges of the wound drawn asunder with broad retractors, the interior of the bladder may be illuminated and made visible; an hypertrophied prostate may be enucleated through the suprapubic opening.

After the work within the bladder has been completed, the opening may be closed with a line of sutures. They should bring the edges of the opening in the bladder into accurate apposition, and should pass through all the layers of the wall of the bladder down to, but not including, the mucous membrane. None of the sutures should pass through the whole thickness of the wall of the bladder. Fine silk or catgut may be used.

If the opening in the bladder wall is closed, the incision in the wall of the abdomen should be left open, at least in part, and packed, in order to provide drainage; it will also be necessary to leave a catheter in the urethra for several days. In most cases it is probably wise to leave the incision in the bladder unclosed, stitching the margins of the opening in the bladder to the edges of the muscles in the abdominal wound with two or three interrupted silk sutures on either side, their ends being left long to facilitate their removal later. There are introduced through the suprapubic opening into the bladder two drainage tubes. One of the tubes is long, and reaches from the bottom of the bladder over the side of the bed into a bottle partly filled with an antiseptic solution and suspended from the side of the bed. The second tube, which is short, is for the purpose of assisting siphonage 
of the bladder and to facilitate irrigation. These tubes are fixed in the bladder by passing the silk tractor stitches (which were introduced in the early stage of the operation) through the tubes. The wound is packed loosely about the tubes with iodoform gauze.

The fistula that remains after the tubes are removed rapidly diminishes in size, and finally closes spontaneously, provided the urethral canal is unobstructed. In many cases it will suffice to fix the tubes in the bladder with the silk tractor sutures, omitting the suturing of the edges of the opening in the bladder to the abdominal incision.

Puncture of the Bladder may be made in the middle line just above the symphysis, or through the rectum. It is done for the purpose of drawing off the urine when the patient is unable to empty the bladder through the urethra. One should first satisfy himself by percussion, etc., that the bladder is actually distended.

A medium-sized curved trochar is introduced above the symphysis; it should be thrust through the anterior abdominal wall in the middle line just above the symphysis, and in a direction backward and downward, toward the sacrum, for a distance of two or three inches.

If introduced through the rectum, the trochar should be guided upon the finger into the rectum beyond the base of the prostate, at which point it is thrust into the bladder in a direction upward and forward, toward the symphysis. The suprapubic route is probably preferable.

\section{THE PENIS.}

Surgical Anatomy of the Penis.-The penis when erect is prismoid in shape. It is composed of the corpora cavernosa and the corpus spongiosum.

The corpora cavernosa are two cylinders of erectile tissue which run parallel with each other and occupy the upper part of the organ. They consist of a mesh-work of vascular spaces, which may readily become distended with blood, thus bringing the penis into a condition of erection. They are each provided with a strong, fibrous envelope, the tunica albuginea, and behind diverge, to be attached to the rami of the pubes.

The corpus spongiosum is situated below the corpora cavernosa, and contains the urethral canal, which is also surrounded by cavernous, or erectile, tissue.

The end of the penis is enlarged, rather bulbous, and is known 
as the glans; this is really the enlarged extremity of the corpus spongiosum. Behind, in the perineum, the corpus spongiosum is enlarged and forms the bulb. The penis at its root is firmly connected to the symphysis by a fibrous band, the suspensory ligament.

The three cylinders which together form the penis are bound together by a fibrous sheath, and covered with a soft, loose, movable envelope of skin, which, at the extremity, is reflected over the glans for a greater or less distance, forming the prepuce. The constriction behind the glans is called the corona.

Passing forward along the dorsal surface of the penis, in the groove between the corpora cavernosa, are two arteries, one on each side, the dorsal arteries of the penis, branches of the internal pudic, and lying between the two arteries is the single dorsal vein.

\section{OPERATIONS UPON THE PENIS.}

Forcible Dilatation of the Prepuce for Phimosis.-This may be practiced in many cases, especially in newborn and young children, instead of a dorsal section or circumcision. An anæsthetic is unnecessary. The skin of the prepuce is seized and peeled forcibly backward over the glans as far as the corona. This is readily done in most cases, even when the orifice of the prepuce is quite narrow. The margin of the prepuce stretches and suffers slight tears here and there about its circumference; it should be drawn back and forth several times, and again repeated daily for several days. When the prepuce is drawn back, any hardened smegma that has accumulated should be removed, and the glans washed and smeared with oil or vaselin; the skin is then again drawn forward over the glans, since the constriction of the narrow prepuce might cause some inconvenience if allowed to remain back behind the glans. After the foreskin has been drawn back and forth over the glans a dilator may be introduced into its orifice, and it may then be forcibly and thoroughly dilated. In most cases this is unnecessary.

Dorsal Section.-This operation is done for phimosis in the young, when one is unable to retract the skin and when it is not desirable to do a complete circumcision, and in adults in all cases where it is necessary to expose the glans for treatment.

The skin of the penis is rolled slightly back toward the root of the organ with the finger and thumb, and one blade of a bluntpointed scissors introduced beneath the prepuce, between it and the 
glans, as far back as the corona, and the foreskin then divided along the middle line, steadying it so that it will not roll or slip. The scissors should be sharp, especially toward the ends.

The prepuce should not be divided for its whole length, but only to within a short distance of the corona.

One should be careful not to introduce the blade of the scissors into the urethral canal instead of between the glans and prepuce; this might happen if the prepuce were intimately adherent to the surface of the glans, as is sometimes the case.

Instead of using the scissors the section may be made with a sharp-pointed, curved bistoury, guided upon a grooved director,

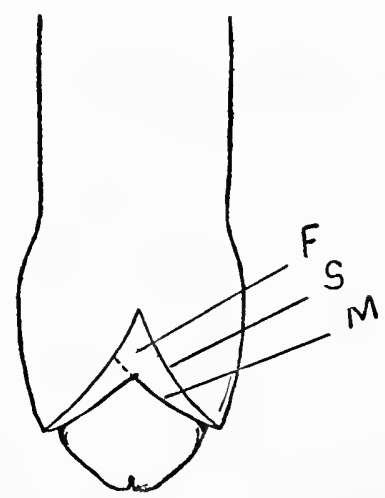

Fig. 178.-Dorsal Section (Roser). Prepuce has been divided upon the dorsal aspect. $M$, edge of incised mucous membrane; $S$, edge of skin. Dotted lines indicate little triangular flap $(F)$ of mucous membrane that is cut from the mucous to the skin edge of the divided prepuce. The flap is turned back and sutured into the angle of the wound; the edge of mucous membrane and skin may also be joined on each side with one or two stitches.

which is introduced underneath the prepuce, between it and the glans. As a rule, there is but little hemorrhage.

If the parts are not infected, one or two catgut stitches may be introduced on either side. Usually no suture is necessary in the child.

Roser's Method of Dorsal Section.-After the dorsal section has been made, the mucous membrane not being cut as far back as the skin, an oblique incision is made, on either side, from the corner of the mucous membrane backward and outward as far as the edge of the skin. The little triangular mucous membrane flap which is thus formed is then turned up into the angle in the skin, to insure rapid 
healing in the corner of the incision; it may be held in place with one stitch in the angle of the incision. One or two stitches may also be introduced on either side of the incision proper.

Circumcision.-In children an anæsthetic is necessary; in adults the operation may be done under the influence of cocain, which is injected into the prepuce after a strip of gauze has been tied fairly tight about the body of the penis near its root to prevent diffusion of the cocain. One should avoid cutting the skin too short. After the parts have healed there should be a little redundancy of the skin marking the previous reflection of the prepuce, and this is best accomplished by drawing the skin a little backward, toward the root of the penis, before applying the constricting band. The first step

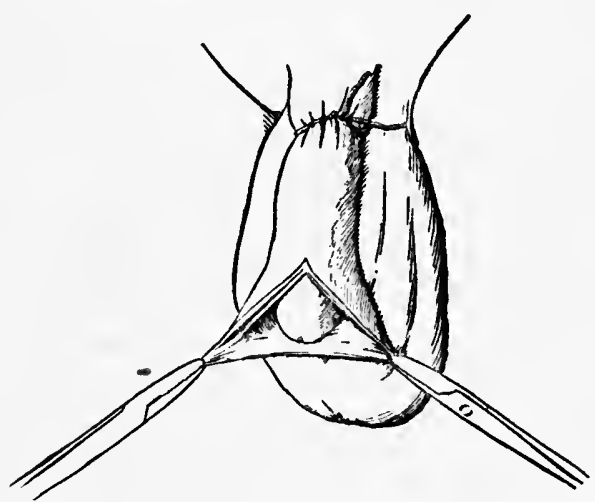

Fig. 179.-Circumcision. Dorsal section has been made. The corners of the divided prepuce are grasped with artery forceps preparatory to trimming it away with the scissors.

in the operation is the dorsal section of the prepuce. One blade of a scissors is introduced underneath the prepuce to a point just in front of the corona, and the prepuce then divided to within a short distance of the corona. Either corner of the divided prepuce is seized with an artery clamp close to the edge of the incision, and with a straight, blunt-pointed scissors the redundant portion of the prepuce is trimmed off, first around one side and then around the other as far as the attachment of the frænum, and finally cut through in this situation, just in front of the frænum and without dividing the frænum.

The entire length of the prepuce should not be amputated; about one-fourth its length should remain. 
As a rule, the bleeding stops when the ligature around the penis is removed and after a few minutes' compression. Bleeding arterial points, however, should be seized with a clamp and twisted. All bleeding should be checked before suturing, if necessary applying fine catgut ligatures.

The edges of the skin and mucous membrane are united with interrupted catgut sutures, the first being applied in the middle line above, the next in the middle line below, then one on each side, and finally in the intervals between these, making eight sutures in all.

In the child, as a rule, the four sutures are sufficient.

Circumcision with the Clamp.-After the parts have been anæsthetized, etc., the edge of the prepuce is seized above in the middle line and below in the middle line with artery forceps, and drawn forcibly forward over the glans. That part of the prepuce which is thus pulled beyond the glans is grasped between the blades of a long, straight clamp, which is applied obliquely from above downward and forward; the clamp should seize the foreskin firmly, and care should be observed that the glans is not included; this accident, however, is not likely to occur.

That part of the prepuce which protrudes beyond the blades of the clamp is trimmed off with a sharp knife or with the scissors plane with the surface of the clamp, and the clamp then removed. The hemorrhage is controlled and the sutures applied as above.

Amputation of the Penis.-This operation is done for malignant disease. A sound is passed into the urethra, and, supported upon this, the penis is lifted away from the body. An elastic ligature is placed about the organ close to its root.

A circular incision is made through the integument and a flap reflected sufficiently long to cover over the stump of the penis; it should be equal in length to half the diameter of the penis plus onethird for shrinkage. After the flap has been turned back like a cuff the portion of the penis that is to be amputated is cut away. The urethral portion of the penis should be cut about one-fourth inch longer than the part that corresponds to the corpora cavernosa.

The blade of the scalpel is thrust flatwise through the penis between the urethral portion, which may be recognized by the sound within, and the corpora cavernosa, and carried a good one-fourth inch forward toward the glans, when the urethral portion is cut through with a circular sweep of the knife down upon the sound contained within. The corpora cavernosa are then divided upon a 
plane farther back, corresponding to the base of the skin flap, so that the urethral portion will project about one-fourth inch beyond the cut surface of the corpora cavernosa.

The tourniquet is now removed from the root of the penis. The dorsal arteries bleed, and require to be clamped and ligated. The arteries of the corpora cavernosa usually require no ligatures; if they

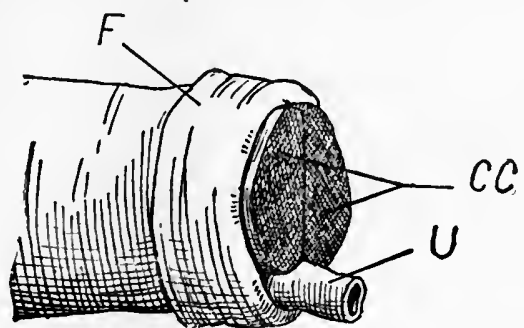

Fig. 180.-Amputation of the Penis. $C C$, corpora cavernosa; $F$, skin flap turned back; $U$, urethral portion cut long.

spurt, they may be clamped or touched with the Paquelin. A few minutes' compression usually suffices to check bleeding from any remaining sources.

The edges of the urethra are seized with two artery clamps, and the urethra then split upon its under aspect for a distance of about one-fourth inch. The skin flaps are turned over the end of the

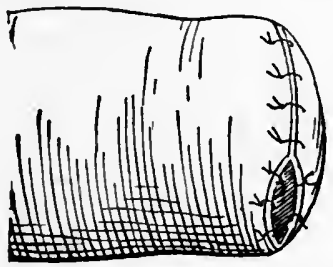

Fig. 181.-Amputation of the Penis. Edges of skin flap united to each other over the ends of the corpora cavernosa and to the edges of the spilt urethral portion.

stump, and are united from before backward with several interrupted sutures, and the edges of the split urethral orifice are sewed to the adjoining edges of the skin flaps.

The object of cutting the urethra long and splitting it is to provide a larger orifice to allow for subsequent contraction.

A soft rubber catheter is introduced into the bladder and al- 
lowed to remain for several days, its end emerging through the dressings. It may be fixed with a silk stitch to the edge of the urethral orifice.

\section{THE PERINEUM AND ISCHIO-RECTAL REGION.}

The Floor of the Pelvis from Without Inward. - This space is lozenge-shaped; its front portion is limited on either side by the rami of the pubes and ischium; its posterior part is limited on either side by the edges of the great sacro-sciatic ligaments. The anterior angle corresponds to the symphysis pubis, the posterior angle to the tip of the coccyx, and on either side the tuber ischii may be felt. There is a more or less complete fibrous raphé running from before backward in the middle line, and also one from side to side where all the layers of the perineal fascia are blended together. Where these lines intersect there is a point where muscles are attached and take origin and where all the fasciæ are joined. This is known as the central tendinous point of the perineum. The space in front of the transverse raphe is the perineum proper; the space behind it is occupied by the anus and upon either side by the ischio-rectal fossa, and is known as the ischio-rectal region.

The Superficial Layer of the Superficial Perineal Fascia.-Beneath the skin there is a layer of loose fascia which is continuous with the superficial fascia of the thighs and buttocks. This is the superficial layer of the superficial fascia of the perineu:n and ischio-rectal regions; it corresponds to the subcutaneous fat, and is continuous in front with the dartos layer of the scrotum, and behind, upon either side of the anus, it is packed into the ischio-rectal fossa as a pyramidal plug of fat and loose connective tissue.

The Deep Layer of Superficial Perineal Fascia.-If we remove this superficial layer of fascia and fat, including the mass from the ischio-rectal fossa, we come down upon a second layer of fascia, the deep layer of the superficial fascia of the perineum. Corresponding to the perineal region proper, the fascia is attached upon each side to the edge of the pubic arch and behind to the transverse raphé; in front it is continuous with the dartos of the scrotum; behind, in the ischio-rectal region, it is continuous with the anal fascia, which covers the perineal surface of the levator ani muscles.

Anteriorly this fascia is dense, and serves to close in the structures proper to the perineum. If fluid is injected underneath this layer of fascia, it will not spread backward beyond the transverse 
raphé, because this layer of fascia is attached along this raphé with the next underlying fascial layer; it will not escape laterally, owing to the attachment of the fascia to the margins of the bony pelvic arch; but anteriorly it will escape, passing into the dartos tissue of the scrotum and thence upward upon the front of the pubes.

The Ischio-rectal Region.-This is the region which lies behind the transverse raphé-that part which corresponds to the anus and the ischio-rectal fossa.

In the middle is the anus, surrounded by its external sphincter muscle. This muscle arises from the tip of the coccyx behind, and, passing forward, is attached, in front of the anus, to the middle tendinous point of the perineum, which corresponds to the junction of the sphincter from behind, the transversus perinei from each side, and the bulbo-cavernosus from in front.

On either side of the anus there is a pyramidal space, the ischiorectal fossa; this space is occupied by a mass of fat and loose connective tissue, the base of which corresponds to the superficial layer of superficial perineal fascia, and reaches from the tuberosity of the ischium to the anus. This space is about two inches deep. Its outer wall is formed by the tuber ischii and the obturator internus muscle, which muscle is covered over by a layer of fascia, the obturator fascia. Passing forward upon this outer wall of the ischio-rectal fossa, beneath the obturator fascia and about one and one-half inches above the tuberosity of the ischium, are the internal pudic ressels and nerve.

The inner wall of the ischio-rectal space is formed by the levator ani (to be described later). The superficial surface of this muscle, which looks into the ischio-rectal space, is covered by the anal fascia, which is derived from the obturator fascia along the line of the origin of the levator ani from the side of the pelvis. This anal fascia is attached in front to the transverse fibrous raphe and is continuous there with the deep layer of the superficial perineal fascia.

The ischio-rectal space is thus walled off from the perineal space proper and from the rectum. It is the seat of the so-called ischiorectal abscess, and when this breaks through into the rectum it forms the fistula in.ano.

Some small vessels and nerve branches cross this space transversely just beneath the skin, passing from the tuberosity of the ischium toward the anus, and these are cut when incisions are made into the space. 
The Perineum.- Upon removing the deep layer of superficial perineal fascia we open into the proper perineal space.

Occupving the middle of the space is a thin muscle, the bulbocavernosus; it arises from the middle tendinous point of the perineum, and, passing forward, covers the bulb of the urethra, which is the posterior enlarged portion of the corpus spongiosum, joining,

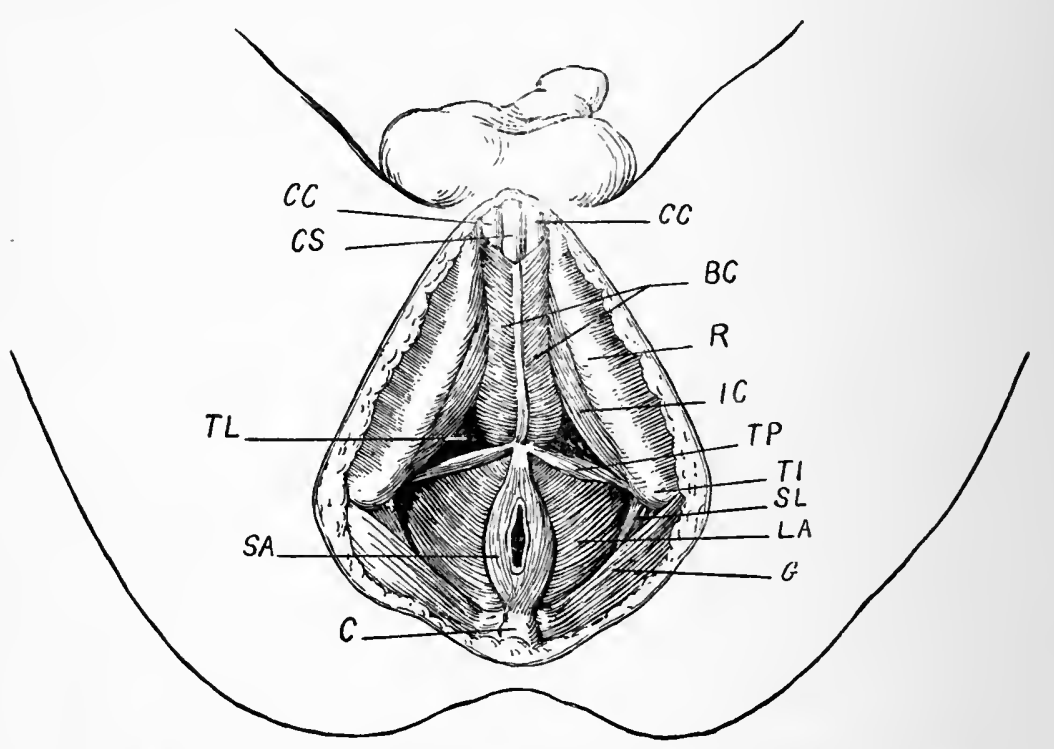

Fig. 182.-The Perineum and Ischio-rectal Region. The superficial and deep layers of the superficlal perineal fascia have been removed. The space in front of the transversus perinel $(T P)$ corresponds to the perineum; that behind the transversus perinei to the ischio-rectal region. The floor of the space $(T L)$ corresponds to the anterlor layer of the triangular ligament. $B C$, bulbo-cavernosus muscle; $C$, tip of coccyx; $C C$, corpus cavernosum (crus penis); $C S$, corpus spongiosum (the posterior part of the corpus spongiosum is called the bulb of the urethra); $G$, edge of gluteus maximus muscle; $I C$, ischio-cavernosus muscle; $L A$, levator ani muscle; $R$, ramus of the pubes and ischlum; $S A$, sphincter anl; $S L$, edge of great sacro-sciatic ligament; $T I$, tuberosity of the ischlum; $T L$, superficial or anterior layer of the trlangular ligament; $\boldsymbol{T P}$, transversus perinei muscle.

with fibers from the muscle of the opposite side upon its upper surface, in a strong aponeurosis. The most anterior fibers of the bulbocavernosus muscle are attached on either side to the crus penis, some entirely encircling these bodies and joining upon the upper surface of the root of the penis in such a way as to bind down the dorsal vessels of the penis, obstructing the return flow through the vein. 
This muscle shows a median fibrous raphé. "Upon either side, arising from the ascending ramus of the ischium, is the ischio-cavernosus. The fibers of this muscle partly cover the crus penis, and are attached to its sheath. The crus penis is the posterior portion of the corpus cavernosum, and is attached to the ramus of the ischium and pubes.

Forming the posterior border of this space on either side is the transversus perinei muscle. This muscle arises from the inner surface of the tuberosity of the ischium; it passes inward and forward to the central tendinous point of the perineum, where it is attached, joining with the muscle of the opposite side and the other muscles already described.

Passing forward through this space are the superficial perineal vessels and nerve, and directed inward along the border of the transversus perinei is the transverse perineal artery.

The floor of this space is formed by a dense layer of fascia, the superficial layer of the deep perineal fascia, or, better, of the triangular ligament. This layer of fascia is perforated by the urethral canal about one and one-half inches below the symphysis. Beneath this layer of fascia there is a second layer, similar in structure, the deep layer of the deep perineal fascia or triangular ligament.

Behind, corresponding to the transverse perineal raphé, these two layers of deep fascia are blended with each other and with the deep layer of the superficial perineal fascia. They are attached laterally to the inner surface of the rami of the pubes and ischium; above, in front, they do not reach to the symphysis, but terminate in the ligamentum transversum pelvis, a ligamentous band passing between both pubic rami, leaving a space above, between it and the symphysis, for the passage of the vena dorsalis penis.

Between the two layers of the triangular ligament the deep transverse perineal muscle, the compressor urethræ, is located; this muscle is made up chiefly of striped muscular fibers passing across from one pubic ramus to the other above and below the urethra, and also of unstriped fibers which pass in various directions, some encircling the membranous part of the urethra.

The two layers of the triangular ligament, together with the muscle contained between them, form the uro-genital diaphragm. In the space between the two layers of the triangular ligament, besides the muscle, are contained the urethra, its membranous portion, and behind, on either side, Cowper's gland, the duct of which is seen passing forward to enter the bulbous portion of the urethra. Poste- 
riorly, close to the lateral border of the space, is seen the internal pudic artery. It gives off the artery of the bulb, and passing forward divides into the artery of the crus penis, which enters the crus, and the dorsal artery of the penis, which perforates the suspensory ligament and runs forward along the upper surface of the penis.

As the urethra perforates the superficial layer of the triangular ligament it is provided with a fibrous prolongation, which is continued forward upon the bulb of the urethra.

The posterior or deep layer of the triangular ligament is continous (within the pelvis) with the fascia which covers the obturator internus muscle and the upper or pelvic surface of the levator ani muscle, and at the side of the prostate it is reflected upward upon this gland.

The prostate gland, which encircles the neck of the bladder and contains the prostatic portion of the urethra, rests upon the upper, or pelvic, surface of the triangular ligament and the levator ani.

The levator ani serves to close in that part of the pelvic outlet which lies posterior to the triangular ligament. The anterior fibers of the muscle unite in the middle line with those of the opposite side in a sling-like fashion to support the prostate. The fibers more posteriorly are continued into either side of the rectum and to the tip of the coccyx.

The seminal vesicles and the vas deferens lie within the pelvis, between the second part of the rectum and the base, or trigone, of the bladder, above the upper border, or base, of the prostate. They may be brought into view by separating the rectum from the base of the bladder and drawing it backward toward the coccyx.

The Pelvic Cavity from Within. - Examining the pelvic cavity from within, after removal of the bladder and rectum, we find it bounded in front by the pubic bones, behind by the coccyx and sacrum, laterally by the pubes and ischinm and the sacro-sciatic ligaments. The lateral wall of the pelvic cavity is partly covered by the obturator internus muscle, which arises from the inner surface of the pubes and ischium around the margin of the obturator foramen.

The obturator internus is covered by a thick fascia, which is attached above to the margin of the brim of the pelvis, being continuous above with the fascia that covers the psoas and iliacus muscles (the fascia iliaca). In front this obturator fascia is continued into the posterior or deep layer of the triangular ligament.

The obturator fascia is marked by a thick, white, fibrous band, 
which extends along the lateral wall of the pelvis from before backward, from the posterior surface of the pubic bone in front to the spine of the ischium behind, and is known as the tendo arcuatum. Along this line upon either side of the pelvis the levator ani takes its origin. The muscles pass in a general direction obliquely downward and inward, joining with each other in the middle line. The anterior fibers pass downward, inward, and backward, and unite in the middle line underneath the prostate, which they support in a sling-like manner. The more posterior fibers pass downward and inward, and are inserted into the sides of the rectum just above the anus; the fibers behind these are attached to the tip of the coccyx.

Still more posteriorly lies the coccygeus. This muscle looks like a continuation of the levator ani, and serves to close in the outlet of the pelvis behind the levator ani. It is fan-shaped, and is attached by its apex to the spine of the ischium and by its broad base to the lateral margin of the coccyx.

Lying upon the same plane, but still farther above and behind, and corresponding to the upper border of the coccygeus muscle, is the pyriformis. This muscle arises from the sides and from the anterior surface of the sacrum, and passing outward leaves the pelvis through the great sacro-sciatic notch, and closes the pelvic cavity behind.

Thus, taking part in the formation of the floor of the pelvis, there is a muscular layer which is formed in front by the levatores ani, behind this by the coccygei, and still farther behind and above by the pyriformi.

In the front part of the floor of the pelvis, between the margins of the levatores ani, there is a space which corresponds to the posterior, or deep, layer of the triangular ligament.

The fascia that covers the obturator muscle, the obturator fascia, is continuous in front with the postcrior, or deep, layer of the triangular ligament; corresponding to the line, the tendo arcuatum, which marks the origin of the levator ani, this obturator fascia, which is simply a portion of the general pelvic fascia, gives off a layer that covers the pelvic surface of the levator ani; farther back the pelvic surface of the coccygeus and the pyriformis and the front of the sacrum are also covered by a continuation of this same fascia.

Where this fascia, after covering the pelvic surface of the levator ani, strikes the prostate and the rectum, it is reflected upward upon the sides of these organs. 
A process of this fascia is reflected inward between the rectum and the base of the bladder, and serves to bind the seminal vesicles and vas deferens to the base of the bladder.

The under surface of the levator ani, which is directed toward the perineum and ischio-rectal fossa, is also covered by a thin layer of fascia, which is derived from the obturator fascia along the line of the origin of the levator ani. This is called the anal fascia.

The anal fascia is continued backward upon the under surface of the coccygeus muscle, and anteriorly is continued forward into the deep layer of the superficial perineal fascia, joining along the transverse septum, or raphé, with all the other fasciæ of the perineum.

\section{OPERATIONS UPON THE PERINEUM, ETC.}

Perineal Section (External Urethrotomy) With a Guide.-This operation is performed for stricture of the deep urethra or for the purpose of draining the bladder. The patient is placed in the lithotomy position and a tunneled sound introduced through the urethra into the bladder.

An assistant steadies the sound with the right hand, throwing the groove as much as possible toward the surface of the perineum, and at the same time drawing the whole urethra upward, away from the rectum toward the symphysis. The scrotum is drawn up toward the symphysis, out of the way of the operator.

An incision is made in the middle line from the base of the scrotum backward to within a short distance of the anus. This incision reaches through the skin and fat down to the deep layer of the superficial perineal fascia.

The edges of the wound are drawn asunder with small, sharp retractors, and with another stroke of the knife the deep layer of the superficial perineal fascia is incised and the bulb of the urethra exposed in the forward part of the wound. Then, with the finger in the wound, the groove in the tunneled guide within the urethra is recognized and the point of the knife, guided upon the finger-nail, is placed in the groove of the sound, piercing the membranous part of the urethra just behind the bulb. The knife is then shoved backward, carrying the point of the blade along the groove of the sound toward the neck of the bladder and raising the handle, at the same time, toward the symphysis. Having carried the point of the knife beyond the location of the stricture into the prostatic portion of the 
urethra, the handle is depressed, the knife at the same time being withdrawn and cutting as it is withdrawn; in this way the membranous portion of the urethra is laid open and the stricture divided.

While the urethra is being incised upon the grooved sound the sound should be lifted straight up toward the symphysis, carrying the urethra with it, and thus drawing it farther away from the rectum. If some urine or fluid is in the bladder, its escape will demonstrate the fact that the bladder has been entered.

A director gorget may now be introduced into the bladder along the groove of the sound and the latter withdrawn. A soft rubber catheter of large caliber is introduced through the opening into the bladder, and fixed in place to the edge of the incision in the skin with a silk stitch, and the wound then packed.

Usually there are no vessels to tie, although spurting arterial branches should be clamped and twisted and, if necessary, ligated. One should avoid wounding the bulb of the urethra if possible, and, for a certainty, the rectum and anus.

Before dismissing the patient, a large metal sound, at least a No. $30 \mathrm{~F}$., should be passed through the anterior urethra and into the bladder to make certain that no remaining obstruction exists in any part of the canal.

Perineal Section Without a Guide.-This is a difficult procedure.

All attempts to introduce a guide through the constricted part of the urethra into the bladder fail. One should not be satisfied with a single attempt, but should try, if possible, to at least get a small whalebone or rubber guide through. After having made the attempt and found it impossible to get any guide whatever past the stricture, a tunneled sound may be introduced as far as the obstruction.

As described in the preceding operation, an incision is made in the perineum and the urethral canal opened upon the guide just in front of the stricture. After all the bleeding has been arrested, the edges of the wound, including the edges of the incised urethra, are retracted with small, sharp hooks, and an effort then made to find the opening through the stricture into the posterior part of the urethra by inspection or by attempting to pass a fine probe-pointed director or a fine whalebone guide. At times pressure upon the bladder will force a few drops of urine through the orifice of the stricture, and this may assist us in locating it (Koenig).

If we do not succeed in getting through the stricture by these means an effort may be made to open into the urethra behind the 
stricture, and then, if this is successful, the stricture may be divided from behind. It is difficult, however, to locate the deep urethra (membranous portion) without a guide. It lies between the layers of the triangular ligament, reaching from the bulbous portion of the urethra to the apex of the prostate gland. Occasionally the urethra is diverted from the middle line or a false passage may be encountered which will still further confuse us.

At times, especially if the bladder contains fluid and pressure be made above the pubes, the urethra may be felt as a rounded, compressible tube, occupying the middle line and perforating the triangular ligament about one and one-half inches below the symphysis.

The prostatic urethra, which is the continuation of the membranous urethra, is surrounded by the prostate gland, and, if one finger is introduced into the rectum and the thumb placed in the incision in the perineum the operator may get the prostate between them, and the apex of the prostate may thus serve as a clue to the location of the membranous urethra. One should refrain from blindly jabbing in the wound in the hope of accidentally striking the urethra.

If all these measures fail, a suprapubic cystotomy may be performed and a guide passed from within the bladder into the urethral canal, in this way locating the posterior part of the deep urethra for the purpose of incision.

If it becomes necessary to do a suprapubic cystotomy, this may be more conveniently done with the patient in the Trendelenburg position. A suprapubic cystotomy under these circumstances is also a difficult procedure, as the bladder may contain little or no urine and may therefore lie very low in the pelvis behind the symphysis.

Median Lithotomy.-This operation is performed for small calculi. The bladder should be washed out with boric-acid solution, 5 or 6 ounces being allowed to remain in the bladder. The operation is practically the same as the preceding perineal section (with a guide) except that the incision into the urethra is made rather more extensive, cutting through the anterior part of the prostatic as well as through the membranous portion of the urethra. The incision should not extend entirely through the prostate. Oftentimes after the bladder has been opened a small stone will of itself drop out of the wound, or it can be removed with forceps, scoop, etc. It may be necessary to enlarge the internal urethral orifice somewhat with a dilator or with the finger. If necessary, a larger stone may be crushed before removal. 
The finger should be introduced into the bladder to search for partially encysted stones, etc. Finally the bladder is washed out and a large, rubber catheter introduced through the perineal wound and fixed to the edge of the skin with a silk stitch. The wound is packed about the catheter and left open.

Lateral Lithotomy.-The bladder is washed out with boric-acid solution, 4 or 5 ounces being left remaining in the bladder. A tunneled sound is introduced through the urethra into the bladder and steadied by an assistant. An incision is made through the skin and fat, commencing in front at the base of the scrotum and passing backward and outward to a point midway between the tuberosity of the ischium and the anus. A second sweep of the knife incises the deep layer of the superficial perineal fascia. The index finger of the left hand is then introduced into the wound, and the finger-nail placed in the groove of the sound in the front part of the wound, just behind the bulb of the urethra. The sound is then drawn upward toward the symphysis, thus lifting the whole urethra away from the rectum, and the point of the knife placed in the groove of the sound, cutting through the membranous urethra. The handle of the knife is then elevated and the point shoved backward along the groove of the guide into the prostatic urethra. The handle of the linife is then depressed, at the same time withdrawing the blade and cutting as it is withdrawn. In this way the membranous urethra, together with the side of the prostate itself, are incised, the division of these deep structures being made along the line of the skin incision.

In making this last incision upon the sound the superficial transverse perineal muscle, and the artery of the bulb, together with the membranous urethra, the prostate gland, and the triangular ligament, are cut. It is usually necessary to clamp and tie the artery of the bulb, and sometimes, if the incision extends too far backward and outward, the internal pudic may be divided; this branch bleeds profusely, and must be tied. After the bleeding has been controlled and the stone removed, a catheter is introduced into the bladder and fixed to the edge of the incision. The wound is packed about the catheter and left unsutured.

Prostatectomy (McGill-Fuller).-The bladder is washed out with boric-acid solution, and 8 or 10 ounces of this allowed to remain in the organ. A suprapubic cystotomy is then done, as already described, with the patient in the ordinary position, lying upon the back. The incision in the abdomen and bladder may be held open with long, 
rather broad retractors, and the interior of the bladder explored. The retractors are then removed and the fingers of the left hand introduced into the bladder, and the wall of the bladder incised over the enlarged, prominent prostate, which is readily recognized by the fingers in the bladder.

This incision is made with long scissors, which are guided by the fingers in the bladder, and extends through the whole thickness of the bladder wall into the substance of the hypertrophied prostate; it is placed transversely and just behind the urethral orifice, and is made sufficiently large to admit the finger. The finger is then introduced, and, working between the wall of the bladder and the prostate, the entire hypertrophied mass is enucleated. While this is being accomplished the whole perineum is forced up from below by counter-pressure made in the crotch by the closed fist of the operator enveloped in a sterile towel. Guiteras makes this counter-pressure with two fingers in the rectum. Cutting forceps or a sharp spoon may be used to assist in the extirpation of the mass, although this is usually unnecessary and undesirable.

The hypertrophied middle or lateral lobes, or practically the whole prostate, may be removed in this way. The hemorrhage is considerable, chiefly oozing, but this is, as a rule, easily controlled by irrigating the bladder with hot saline solution.

The bladder may be drained through the suprapubic opening, introducing two tubes, as already described for suprapubic cystotomy. It is probably well, in addition, to do a perineal section and introduce a third tube through the opening thus made in order to drain the bladder from below (Fuller). The perineal opening is readily made upon a tunneled sound. The tubes may be left in the suprapubic opening for several days and fixed by the two silk tractor sutures which are passed through the wall of the bladder; if the tubes are thus secured, one may usually omit fixing the edges of the opening in the bladder to the margins of the abdominal incision.

In enucleating the hypertrophied prostate one should work with the fingers close to the surface of the mass and avoid any such force as might tear into the rectum.

Prostatectomy (Alexander). - A suprapubic cystotomy is performed, and then placing the patient in the lithotomy position a perineal section is made and the membranous urethra opened back to the apex of the prostate (see "Perineal Section").

The fingers of the left hand are introduced into the bladder 
through the suprapubic opening, and the enlarged prostate pressed down into the perineal wound and steadied.

According to Guiteras, the prostatic mass may be pressed down into the perineum, without opening the bladder, through an incision in the lower part of the linea alba as for suprapubic cystotomy, with two fingers in the prevesical space of Retzius.

The forefinger of the right hand is introduced into the perineal wound, and, pushing the rectum backward away from the prostate, we tear or poke through the capsule of the prostate upon its posterior inferior surface (that which presents toward the rectum), and then with the finger the whole of the hypertrophied prostate may be shelled out, working close to the surface of the mass so as to avoid tearing through the mucous membrane of the neck of the bladder and prostatic urethra. In this way the lateral lobes are first enucleated, and then the middle lobe, if this is also enlarged, may be removed in a similar manner.

The hypertrophied prostatic mass may be seized with vulsella forceps and traction made first toward one side and then toward the other during its enucleation. The bladder is drained through the perineal wound, but in addition two tubes may be introduced through the suprapubic opening and fixed to the edges of the opening in the bladder; these are allowed to remain for several days.

Prostatotomy (Bottini's Operation).-The apparatus consists of an incisore prostatico and a rheostat to regulate the current accurately.

One should have previously made an examination with the cystoscope for stone, etc. The patient lies upon the back with his legs hanging over the end of the table and the thighs spread apart. The bladder should contain about 6 ounces of boric-acid solution.

Usually sufficient local anæsthesia is obtained by the use of a solution of cocain which is thrown into the urethra and stripped backward into the posterior urethra with the finger, or a general anæsthetic may be employed. With the finger in the rectum the size and the shape of the prostatic tumor may be determined.

The incisore is introduced into the bladder beyond the enlarged prostate and its nose turned downward toward the base of the bladder, so that, as it is slowly withdrawn, it catches or hooks upon the prostatic mass. The extremity of the instrument may be felt with the finger in the rectum through the bladder wall above the prostatic tumor. The instrument is now held firm and steady in the whole of 
the left hand and the current closed and regulated by the rheostat until sufficiently strong to give a red heat, which usually requires fifteen seconds. Now, slowly turning the screw in the handle of the instrument, the heated blade is gradually withdrawn, thus burning a furrow through the prostatic mass. If the ear is held near the symphysis, a sizzling sound can be heard. If, in withdrawing the blade, we note increased resistance in the mass, the current is augmented; if too little resistance to the blade-if it cuts too easilythe current is correspondingly diminished. After the incision has been made sufficiently long the blade is shoved back with a little increase of the current.

Several such channels or incisions, usually three, should be made through the prostatic mass: one through the middle line, toward the rectum, with the nose of the instrument directed downward; one with the nose of the instrument directed upward toward the symphysis; and one upon one or both sides of the middle line with the nose of the instrument again pointed downward toward the rectum. The operation should occupy from five to ten minutes.

The incisore resembles a lithotrite, having a male and a female blade, the male blade fitting into the female and consisting of platinum iridium, which may be heated to any degree by the electric current, whose strength is regulated by the rheostat.

By turning the screw at the handle the male blade is withdrawn from the groove in the female blade, and is thus made to cut or burn its way through the hypertrophied prostatic mass.

The shaft of the instrument is hollow, so that it may be supplied with a current of cold water, which flows in through one tube and out through another; these tubes are both placed near the handle. The cold water current is for the purpose of keeping that part of the instrument cool which rests in the anterior part of the urethra.

Immediately before using the instrument it should be tested with the current, and an observation made upon the rheostat to determine just what degree of current is necessary to bring the blade to the proper heat; usually about 45 ampères are required. The screw in the handle permits of an incision up to $4 \mathrm{~cm}$. in length being made. 


\section{PART IX.}

\section{THE UPPER EXTREMITY.}

\section{THE AXILLA.}

The Axilla is a four-sided pyramidal space. Its apex is above, and corresponds to the depression upon the upper surface of the first rib, external to the attachment of the tendon of the scalenus anticus muscle, where the subclavian artery enters the axillary space to become the axillary. The base of the axilla corresponds to the fold of skin and fascia which is stretched between the edge of the pectoralis major in front and that of the latissimus dorsi behind.

The anterior wall of the axilla is made up of the pectoralis major and pectoralis minor; the posterior wall is formed by the subscapularis and the tendon of the latissimus dorsi and the teres major. The inner wall corresponds to the side of the chest, and is made up of the first, second, third, and fourth ribs and corresponding intercostal muscles and the upper serrations of the serratus magnus. The outer wall of the axilla is a narrow space, which is included between the anterior and posterior walls and corresponds to the floor of the bicipital groove. In the bicipital groove is lodged the long tendon of the biceps. The coraco-brachialis muscle, which arises from the coracoid process, descends in the outer part of the axillary space, lying close to the humerus.

To the anterior lip of the bicipital groove is attached the tendon of the pectoralis major, and to its posterior lip are attached the tendons of the latissimus dorsi and teres major.

The contents of the axilla consist of the axillary artery and vein, the large nerve-trunks which are derived from the brachial plexus, lymphatic vessels and nodes, and a mass of loose connective tissue and fat which is continuous with the connective tissue and fat of the root of the neck and the mediastinum.

The Axillary Artery.-The axillary artery is the continuation of the subclavian, and passes through the axillary space from its apex to its base, where it is prolonged downward into the arm as the brachial. The vessel passes through the upper part of the axillary space, 
lying close to its anterior wall. The lower, or outer, portion of the artery lies close to the humerus, beneath the edge of the coraco-brachialis, resting upon the tendon of the latissimus dorsi, and covered by the pectoralis major. The axillary vein, which is sometimes double, accompanies the artery, lying below it, and both artery and vein are in close relation with the nerve-trunks which traverse the axillary space. With the arm extended to a right angle, the course of the artery is nearly straight, and corresponds to an imaginary line which is drawn from the junction of the inner and middle thirds of the clav-

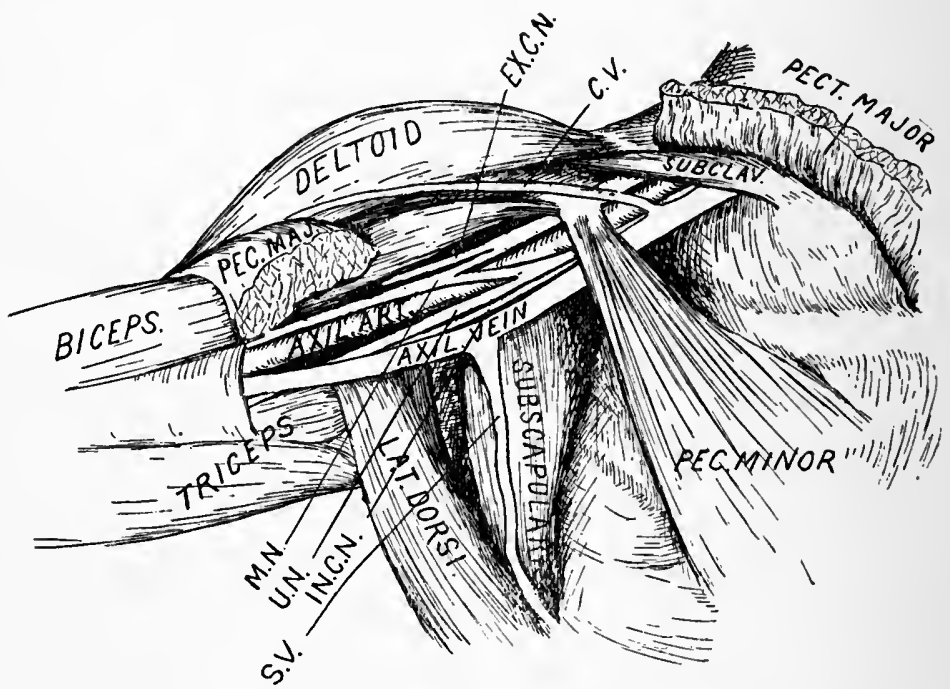

Fig. 183.-Axillary Region. Costo-coracoid membrane has been cleared away to show upper part of the axillary vessels, etc. C.V., cephalic vein; EX.C.N., external cutaneous nerve; IN.C.N., internal cutaneous nerve; $M . N$. , medlan nerve; S.V., subscapular vein; U.N., ulnar nerve.

icle to a point upon the front of the elbow midway between the two condyles; with the arm hanging by the side, the artery describes a curve which is convex upward and outward.

After the pectoralis major has been separated from its attachment to the clavicle and reflected downward, the pectoralis minor, together with the costo-coracoid membrane, will be exposed. The costo-coracoid membrane is a rather thickened sheath of fascia which reaches from the inner border of the pectoralis minor upward, to be attached to the under surface of the clavicle and to the first rib; it is simply a reflection of the deep fascia which invests the pectoralis 
minor, and serves to cover in the upper, or first, part of the axillary vessels and adjoining structures.

The axillary artery is crossed about its middle by the pectoralis minor muscle, and may be conveniently considered in three parts. The upper, or first, part of the artery reaches from its commencement at the first rib to the inner border of the pectoralis minor, and is not exposed until after the costo-coracoid membrane has been cleared away; the second part of the artery is that portion which lies behind the pectoralis minor muscle, and the third is that part which reaches from the outer border of pectoralis minor to the point below where it becomes the brachial.

In the first part of its course the three trunks of the brachial plexus lie above the axillary artery. In the second part of its course one trunk lies above, one behind, and one below it. In the third part the cords of the brachial plexus communicate with each other, surrounding the axillary artery, and divide into a number of branches to supply the upper extremity. The median nerve lies external to the artery, taking one root from the external cord of the plexus and a second root from the internal cord, the latter root passing across the front of the artery. The external cutaneous nerve also lies to the outer side of the vessel, being derived from the outer cord of the plexus. To the inner side of the artery, and derived from the inner cord, are the uInar, internal cutaneous, and lesser internal cutaneous nerves. Derived from the posterior cord of the brachial plexus and situated behind the artery are the posterior circumflex and the musculo-spiral nerves. Immediately after its origin the circumflex passes directly backward between the subscapularis and latissimus dorsi (and teres major) muscles, and is distributed to the deep surface of the deltoid.

The cephalic vein pierces the costo-coracoid membrane and passes across the first part of the axillary artery to empty into the axillary vein.

The lymphatic vessels and nodes are intimately related to the axillary vessels along their whole course within the axilla.

From the upper, or first, part of artery are given off the superior thoracic and acromial thoracic branches, which are distributed to the anterior wall of the axilla and to the axillary contents. A branch from the acromial thoracic is found in company with the cephalic vein in the groove between the deltoid and pectoralis major muscles (Mohrenheim's fossa). 
At the lower border of the pectoralis minor the long thoracic is given off; this branch passes downward close to the lower border of this muscle, lying beneath the edge of the pectoralis major, and ramifies upon the side of the chest.

Still lower, and close to the posterior wall of the axilla, the artery gives off the subscapular, a large branch which descends upon the posterior wall of the axilla, along the outer border of the subscapularis muscle; it is accompanied by the large subscapular nerve, and enters and supplies the latissimus dorsi. External to this branch is given off the posterior circumflex, which passes backward between the latissimus dorsi and subscapularis muscles together with the circumflex nerve; they wind around the surgical neck of the humerus beneath the deltoid, which they supply. The axillary vessels and adjoining nerves, etc., in the upper, or inner, part of the axillary space, are located close to the anterior wall, and in the lower, or outer, part of the axilla they are found close to the humerus, resting upon the tendon of the latissimus dorsi and beneath the edge of the coracobrachialis. Branches of the axillary artery ramify upon the anterior and posterior walls of the axillary space, and, descending upon the inner wall, side of the chest, posteriorly, is the long thoracic nerve, which supplies the serratus magnus; the middle of the axilla is, therefore, free for incisions for abscess, etc.; if it is desired to extirpate completely the axillary contents, it is well to commence by making a clean dissection of the main vessels and nerves.

\section{THE ARM.}

Upon the front of the arm there is seen a prominent spindleshaped mass, which consists of the belly of the biceps and, joined to its inner side, the coraco-brachialis muscle. Occupying the inner side and back of the arm is a thick mass of muscle, the triceps. Upon the outer side, above, covering over the shoulder-joint, is a large mass of muscle, the deltoid. Beneath the deltoid, between it and the surgical neck of the humerus, the circumflex nerve and the circumflex arteries are found. The circumflex nerve, although well protected by the mass of deltoid muscle, on account of its relation with the neck of the humerus is often injured by blows and falls upon the shoulder, with a resulting disability of the deltoid.

Vessels of Arm. The Brachial Artery.-In the depression corresponding to the inner margin of the biceps and coraco-brachialis, 
beneath the deep fascia, lies the brachial artery. The brachial artery is the continuation of the axillary; it passes down along the inner side of the arm in the space between the anterior muscular mass, biceps, etc., and the inner muscular mass, triceps; externally and behind, the artery rests against the humerus, and below the bend of the elbow it divides into the radial and ulnar.

The linear guide to the artery with the arm abducted is a line drawn from the coracoid process to a point upon the front of the elbow, midway between the condyles; the muscular guide is the inner edge of the biceps and the coraco-brachialis muscles.

The brachial artery is covered by the integument and deep fascia,

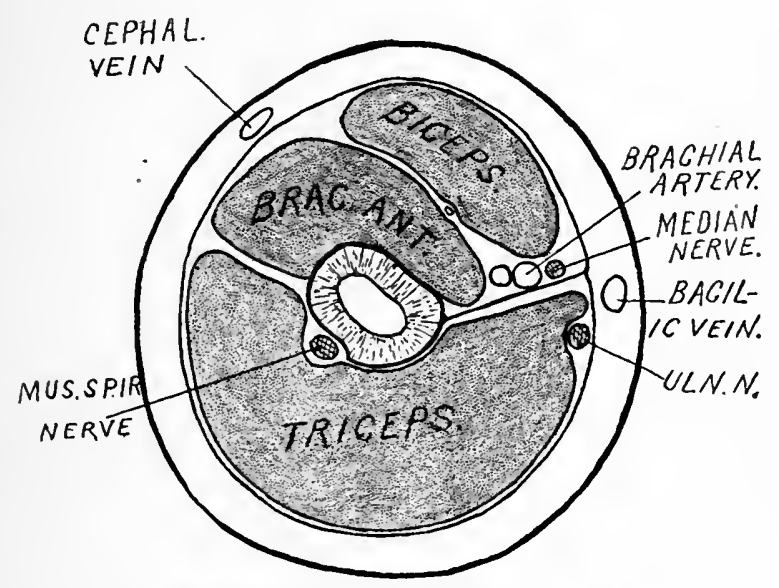

Fig. 184.-Section through Middle of Right Arm.

and is accompanied by two veins, venæ comites, which lie directly upon the vessel and anastomose with each other by numerous transverse branches. Above the median nerve lies to the outer side of the artery, crosses the artery about its middle, and below lies to its inner side; the ulnar and internal cutaneous nerves lie to the inner side of the artery, the ulnar resting upon the inner head of the triceps and gradually getting farther away from the artery as it descends to reach the back of the internal condyle. Behind the artery, in the upper part of the arm, lies the musculo-spiral nerve.

The basilic vein runs parallel with the brachial artery, lying superficial to it and rather to its inner side. One may meet this vein 
in making the incision to expose the brachial artery. In the lower half of the arm this vein is separated from the artery by the deep fascia, but about the middle of the arm it pierces the deep fascia, and thus gets into closer relation with the artery. In the upper part of the arm the basilic vein joins the venæ comites to form the axillary vein. Along the outer side of the arm, superficial to the deep fascia, runs the cephalic vein; above this vein is found in the groove between the pectoralis major and the deltoid, and, after piercing the costocoracoid membrane, passes across the first part of the axillary artery . to empty into the axillary vein.

At the Elbow, upon the front aspect of the arm, there is a triangular space with its apex directed downward toward the hand; the inner border of the space is formed by the pronator radii teres, passing obliquely downward and outward from the internal condyle; the outer border is formed by the spinator longus, and the floor of the space by the brachialis anticus and supinator brevis.

In this space are found the tendon of the biceps, the brachial artery and its accompanying veins, the median and the musculo-spiral nerves, and the bifurcation of the brachial artery into the radial and ulnar, which occurs about one inch below the bend of the elbow. In this space the median nerve is about half an inch to the inner side of the brachial artery, owing to the latter's verging outward, away from the nerve, toward the middle line.

The musculo-spiral nerve lies in the outer part of the space upon the supinator brevis, and is covered by the overlapping edge of the supinator longus. This region is corered by the skin, superficial and deep fascia. The skin of this region has a marked tendency to retract when cut, and this should be remembered in marking out the flaps for exarticulation at the elbow-joint. Lying just beneath the skin upon the deep fascia is the median cephalic vein externally, and the median basilic internally. The latter, the median basilic, is separated from the brachial artery, not only by the deep fascia, but also by a fibrous expansion which is given off from the biceps tendon to the deep fascia of the forearm. The median cephalic is the vein selected by preference for intravenous infusion.

The Radial Artery. - From its origin below the bend of the elbow the radial passes somewhat outward and then downward upon the outer side of the anterior aspect of the forearm; it lies superficial, though partly covered by the overlapping edge of the supinator longus. In its course it rests upon the tendon of the biceps, the supinator 
brevis, the radial origin of the flexor sublimis digitorum, the pronator radii teres, the flexor longus pollicis, and the pronator quadratus. The lower part of the artery, above the wrist, lies beneath the integument and the deep fascia, to the outer side of the tendon of the flexor carpi radialis, between it and the tendon of the supinator longus.

In the upper part of the forearm the artery is accompanied by the radial branch of the musculo-spiral nerve, which lies to its outer side. Usually two venæ comites accompany the artery.

At the wrist the radial artery curves around the outer side of the joint, lying beneath the extensor tendons of the thumb and resting upon the external lateral ligament; it then passes across the posterior surface of the scaphoid and trapezium, and then forward, through the opening in the first dorsal interosseous muscle, into the palm of the hand.

In the hand the radial artery is situated deep and passes from without inward, resting upon the bases of the metacarpal bones and the anterior interosseous muscles, covered by all the structures of the hand: tendons, nerves, superficial arch, etc. Upon reaching the inner side of the hand it anastomoses with the communicating branch from the ulnar, and in this way completes the deep palmar arch. The deep palmar arch is located one finger's breadth nearer the wrist-joint than the superficial palmar arch. The deep arch is accompanied by the deep branch of the ulnar nerve. From the deep arch are given off the palmar interosseous branches; these descend upon the interosseous muscles between the metacarpal bones, and at the clefts of the fingers anastomose with the branches from the superficial arch.

The Ulsar ARTERY. - Immediately after its origin the ulnar artery approaches the inner side of the forearm, passing deep beneath the superficial flexors, and lying upon the flexor profundus digitorum; the upper half of the artery is thus covered by the superficial flexors (pronator radii teres, flexor carpi radialis, palmaris longus, and flexor sublimis digitorum); in the lower half of its course the ulnar artery is still found resting upon the flexor profundus digitorum, but it is rather more superficial and lies between the tendon of the flexor carpi ulnaris internally and those of the flexor sublimis digitorum externally. In the forearm the artery is accompanied by the ulnar nerve, which lies to its inner side; above, at its origin, the median nerve lies to its inner side, but soon this nerve crosses the artery, and thus gets to lie to its outer side. The artery is accompanied by venæ comites. Just below its origin the ulnar gires off the interosseous, 
which divides into an anterior and a posterior interosseous branch. The anterior passes down the front of the forearm, resting upon the interosseous membrane; the posterior passes through an opening in the upper part of the interosseous membrane, and runs down the back of the forearm between the superficial and the deep layers of muscles.

At the wrist the ulnar artery lies superficial, passing across the anterior annular ligament, on the ulnar side of the hand, just to the radial side of the pisiform bone, with the ulnar nerve lying to its inner side; here it turns outward toward the radial side of the hand

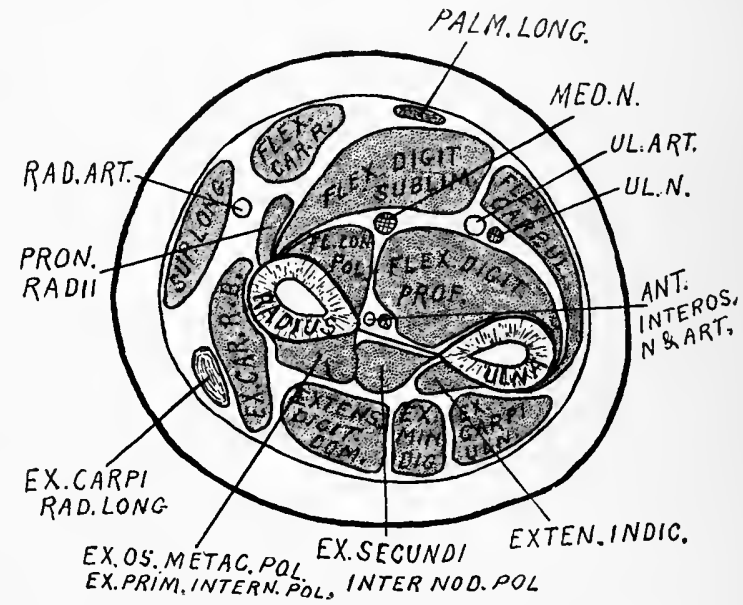

Fig. 185.-Section through Micdle of Right Forearm.

and anastomoses with a branch (superficial) from the radial, thus forming the superficial palmar arch.

In the hand the superficial palmar arch is about on a level with the palmar surface of the thumb, when it is abducted and is covered by the skin and palmar fascia, resting upon the flexor tendons, etc.; it gives off digital branches, four in number, which pass downward and after anastomosing with the palmar interosseous branches, from the deep palmar arch, at the clefts of the fingers, divide into two. branches to supply the contiguous sides of the fingers.

The superficial palmar arch supplies all the fingers except the thumb and the radial side of the index finger, which are supplied. through branches from the radial. 
The Musculo-spiral Nerve.-The musculo-spiral nerve passes down the back of the arm. It is lodged in the musculo-spiral groove upon the posterior surface of the humerus between the inner and outer heads of the triceps muscle and covered by the long head of this muscle. In its course it crosses the posterior surface of the humerus obliquely from above downward, and from within outward, and at the elbow-joint is found in front of the external condyle beneath the supinator longus. The nerve is accompanied by the superior profunda branch of the brachial artery.

The Mediax Nerve.-In the upper arm the median nerve is closely related with the brachial artery. In the forearm it lies beneath the flexor sublimis muscle, resting upon the flexor profundus digitorum. Just above the annular ligament this nerve becomes more superficial, lying to the inner side of the tendon of the flexor carpi radialis.

The Ulxar Nerve.-In the upper arm the ulnar nerve lies some little distance to the inner side of the brachial artery, resting upon the inner head of the triceps, beneath the deep fascia. At the elbow the ulnar nerve lies behind the joint in the groove between the internal condyle and the olecranon process; it then swings forward, and is continued down the anterior aspect of the forearm, resting upon the flexor profundus digitorum beneath the flexor carpi ulnaris and lying close to the inner side of the ulnar artery.

\section{THE HAND.}

Beneath the integument in the palm of the hand is the palmar fascia. This is a dense, aponeurotic layer intimately joined to the integument.

Beneath the palmar fascia are the flexor tendons, superficial and deep palmar arches, nerves, etc. As the flexor tendons pass across the wrist-joint into the palm of the hand they are bound down by the anterior annular ligament. The extensor tendons, as they pass over the back of the wrist-joint into the hand, are bound down by the posterior annular ligament.

Beneath the anterior annular ligament the flexor tendons are inclosed within a synovial sheath, which extends for a short distance upward into the forearm and downward into the palm of the hand. From this common sheath there are given off two processes, one of which accompanies and envelops the tendon of the flexor longus 
pollicis into the thumb; the other accompanies the flexor tendons of the little finger to their destination.

The sheaths which surround the tendons of the other fingersi.e., the index, middle, and ring-do not, as a rule, reach beyond the metacarpo-phalangeal articulation, and do not communicate with this common flexor sheath. This fact is important in determining the extension of inflammatory processes which involve the tendon sheaths of the fingers up into the hand and forearm. Inflanmatory processes which involve the thumb and little finger are found more apt to extend into the hand and forearm than those of the other fingers.

The hand gets its arterial supply from the radial and ulnar arteries (see description of these vessels).

The Nerve-supply of the Hand.-The nerve-supply of the hand is derived from the median and ulnar and musculo-spiral nerves. The median nerve passes into the palm of the hand beneath the annular ligament; the ulnar nerve passes into the palm of the hand across the annular ligament: i.e., in company with the ulnar artery. In the hand, in close relation to the superficial arch, the median and ulnar nerves give off their digital branches, which supply the integument of the palmar aspect of the fingers with sensation, the ulnar supplying the little finger and half the ring finger, the median supplying the other fingers.

The dorsal aspect of the hand and the fifth, fourth, and part of the third fingers are supplied by the ulnar nerve; the thumb and the second and part of the third fingers are supplied by the radial nerve, which is a branch of the musculo-spiral.

All the interossei, both anterior and posterior, and the two inner lumbricales are supplied by the deep branch of the ulnar nerve which accompanies the deep palmar arch; the two outer lumbricales are supplied by the median.

A collection of pus in the palm of the hand may be situated superficially beneath the skin, between it and the palmar fascia, or deep, beneath the palmar fascia or within the proper synovial sheaths of the flexor tendons.

Incisions into the palm of the hand may be freely made without troublesome hemorrhage, if placed over the metacarpal bones and below the line of the superficial palmar arch.

Ligations. The Axillary Artery.-The axillary artery is not cften exposed for the purpose of ligation, but frequently the artery and 
vein and adjoining structures are laid bare during the course of operations which require a thorough cleaning out of the axilla.

A ligature may be applied to the third part of the axillary artery as it lies upon the tendon of the latissimus dorsi close to the humerus. The arm should be abducted from the side of the chest to a right angle and slightly flexed at the elbow-joint, in order that the structures may not be placed too much upon the stretch; with the arm in this position the course of the artery corresponds to a line drawn from the junction of the middle and inner thirds of the clavicle to the middle of the elbow. An incision two and one-half inches long is made through the integument down to the deep fascia; this incision is placed midway between the anterior and posterior borders of the axilla, along the edge of the coraco-brachialis muscle. This incision penetrates through the skin and fat. With a second stroke of the knife the deep fascia is incised, and one may then, with the handle of the knife, seek the white, shiny tendon of the latissimus dorsi, which is the guide to the axillary vessels in this part of their course. When this tendon is recognized, it is followed up toward its attachment to the humerus, diminishing the tension of the parts by flexing the arm somewhat at the elbow, and then the vessels and nerves are readily located, the vein, which lies below and internal to the artery, being the first structure encountered.

The artery is carefully isolated for a short distance, using blunt hooks to retract the adjacent structures, and the loose connective tissue which immediately surrounds the ressel is picked up with a toothed forceps and nicked with the point of a knife; through the small opening which is thus made in the connective tissue sheath a director may be introduced between the vein and the artery and gradually worked around the vessel, taking care to keep close to the wall of the vessel, so as not to include any of the adjoining structures -one should avoid, especially, the musculo-spiral nerve, which is located behind the artery, upon the tendon of the latissimus dorsi. A small aneurism needle is then carried around the vessel, a ligature passed through its eye, and the needle withdrawn, thus leaving the ressel surrounded by the ligature, which is tied with a single square knot.

The Brachial Artery.-The linear guide to the brachial artery is a line drawn from the coracoid process to a point upon the front of the elbow, midway between the condyles, the arm being abducted to a right angle with the trunk. The muscular guide to the artery is 
the inner edge of the mass of muscle, composed of the biceps and coraco-brachialis.

The incision, two inches in length, is made along the inner border of the coraco-brachialis, penetrating through the skin and subcutaneous fat and exposing the deep fascia. At this stage, below the middle of the arm, the basilic vein, lying superficial to the deep fascia and to the inner side of the brachial artery, is met. In the upper part of the arm we would not encounter the basilic vein until after we had cut through the deep fascia.

The deep fascia is now incised in a direction corresponding to the skin incision, and the bundle of structures-which consists of

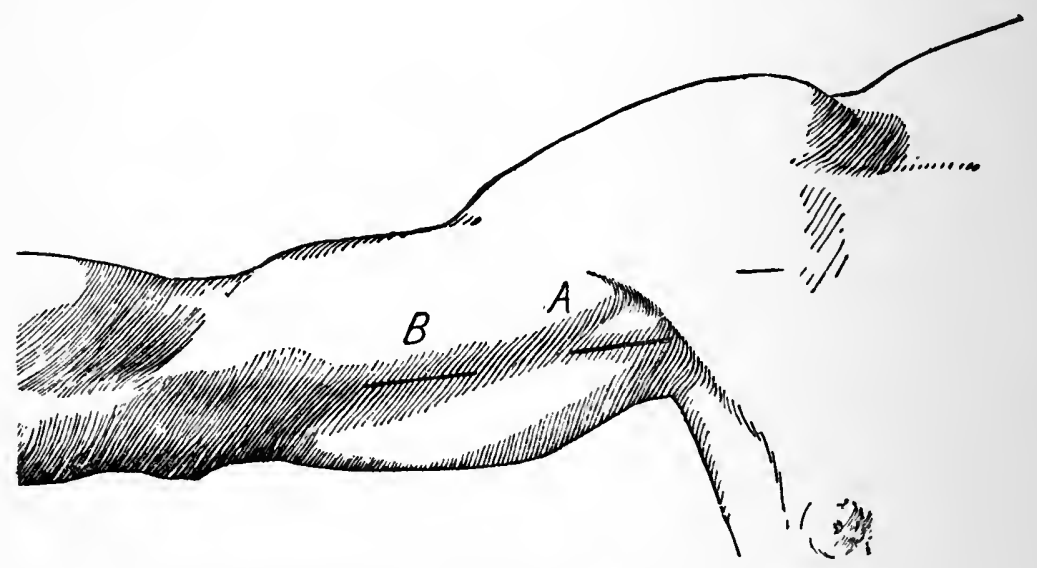

Fig. 186.-Right Arm. A, incision for ligation of axillary artery; $B$, incision for ligation of brachial artery.

the artery, venæ comites, and adjoining nerves and which is readily felt beneath the deep fascia-is exposed.

In the middle of the arm we find the median nerve lying upon and crossing the artery from without inward; above the middle of the arm the median nerve lies close to the outer side of the artery; below the middle it lies along its inner side. The ulnar nerve is situated upon the inner side of the artery, getting farther away from it as it descends toward the elbow-joint. The loose connective tissue that surrounds the brachial artery may be now picked up with a mouse-toothed forceps and nicked with the point of the knife; through the small opening thus made a director is introduced and 
gradually worked around the artery, which is thus isolated from the adjoining structures, avoiding the venæ comites, which lie direetly upon it. A small aneurism needle is then passed around the artery through the path made by the director, and after a ligature is carried through its eye the needle is withdrawn, leaving the artery surrounded by the ligature, which is tied.

The Radial Antery. In the Middle of the Forearm. - An incision one and one-half inches long is made between the middle and inner thirds of the forearm, reaching through the skin and fat down to the deep fascia; the deep fascia is then incised and the artery found lying partly concealed by the overlapping edge of the supinator longus, which is drawn aside with a retractor. The artery is accompanied by venæ comites, which lie close upon it, and also to its outer side by the radial nerve, which is a branch of the musculo-spiral.

Just Above the Wrist.-Here the artery is found beneath the deep fascia, lying between the tendons of the supinator longus externally and the flexor carpi radialis internally. The radial nerve quits the artery three inches above the wrist-joint, and is not met with here.

The Ulnar Artery. In the Middle of the Forearm.-An incision one and one-half inches long is made between the middle and inner thirds of the forearm, through the skin and fat down to the deep fascia; the deep fascia is then incised, and the artery is found lying beneath the edge of the flexor carpi ulnaris, which must be drawn inward to expose the vessel. The artery rests upon the flexor profundus digitorum; to the outer side of the artery is the edge of the flexor sublimis digitorum. The artery is accompanied by venæ comites, which lie in close relation with it. The ulnar nerve is found upon the inner side of the artery.

Just Above the Wrist.-The ulnar artery lies beneath the deep fascia, with the tendon of the flexor carpi ulnaris to its inner side and the tendons of the flexor sublimis to its outer side; the ulnar nerve lies elose to the inner side of the vessel in this part of its course.

\section{AMPUTATIONS, RESECTIONS, ETC.}

Surgical Anatomy of the Hand.-The hand is composed of the carpus, metacarpus, and phalanges. Each finger is made up of three phalanges, the thumb of two (sce Fig. 189).

Phalango-phalangeal Joints. - The fingers are formed by the phalanges, which articulate with each other, end to end. Upon the 
anterior aspect are found the flexor tendons; upon the posterior are the extensor tendons.

Each phalango-phalangeal joint has an anterior ligament and two lateral ligaments, the posterior ligament being formed by the spreadout extensor tendon.

Flexion and extension are permitted in these joints. Flexion occurs by the gliding of the distal phalanx around the head of the proximal, and therefore when the finger is flexed the joint is found below the angle of the knuckle at a distance which corresponds to the thickness of the end of the proximal bone.

Metacarpo-PHalangeal Joints.-These joints are quite similar to the phalango-phalangeal; they are formed by the articulation of the heads of the metacarpal bones with the proximal ends of the phalanges. They are provided with an anterior ligament and two lateral ligaments; the extensor tendon spreads out in the form of a broad, fibrous sheath as it passes across the posterior aspect of the joint, and thus serves as a posterior ligament, completely covering the joint upon its posterior aspect. The anterior ligaments are firmly united with each other (except that of the thumb), so as to bind the heads of the metacarpal bones firmly together into one strong, solid row, which lends a great element of strength to the hand.

The lateral ligaments are attached to the bones, excentrically, in such a manner that, although a considerable range of adduction and abduction is allowed when the fingers are extended, this is not permitted when they are flexed; when flexion takes place, the lateral ligaments become relatively short, since the points to which they are fixed become more widely separated. When flexion takes place between the phalanx and the head of the metacarpal bone, it is accomplished by the proximal end of the phalanx gliding around the head of the metacarpal bone, and, therefore, in this position, the level of the joint will be found at a distance below the angle of the knuckle which corresponds to the thickness of the head of the metacarpal bone.

Exarticulation of the Finger at the Phalango-phalanGEAL JOINT.- - In amputating a portion of a finger an effort should be made to use what integument may be available, with a view to preserving as much of the length of the finger as possible. No doubt, where one may choose, the best amputation is through a joint and with a long anterior flap; this brings the suture line upon the posterior aspect of the stump and out of the way of pressure.

The end of the finger which is to be amputated is seized by the 
operator with the left hand and strongly flexed, and a transverse incision, reaching down to the bone, is then made across its dorsal surface, about one-half inch below the point of the knuckle; this incision should not include more than one-half of the circumference of the finger. An additional incision is then made upon either side, extending from the end of the transverse incision, along the side of the finger, for a distance corresponding to the length of the proposed flap, and this should also penetrate to the bone.

With the finger still strongly flexed, the joint is now opened upon its dorsal aspect, remembering that the line of the joint lies below the point of the knuckle. After the joint has been opened the point of the knife should be passed in on each side and the lateral ligaments freely divided, when the joint surfaces may be separated

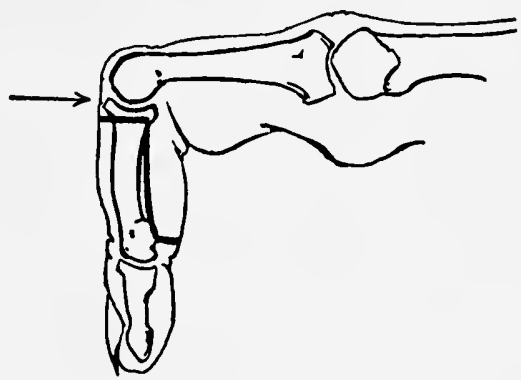

Fig. 187.-Exarticulation of the Finger at the Phalango-phalangeal Jolnt. The arrow indicates level of the joint when the finger is fixed. Heavy line Indicates the long anterior flap.

from each other. The blade of the knife is then introduced between the joint surfaces and behind the bone, between the bone and the anterior flap, and with a sawing motion the anterior flap is cut, with the edge of the knife directed toward the bone, down to the level of the next joint, or until a flap of sufficient length is obtained, when it is cut from within outward by turning the edge of the knife toward the skin. The digital arterial branch on either side should be tied with catgut. The corners of the flap may be rounded off, although this is probably unnecessary. The anterior flexor tendons may be united by two catgut sutures to the edge of the extensor tendons, as this increases the probability of a movable, useful finger stump. The edges of the skin are approximated with two to four catgut sutures, and the operation is complete. 
Exarticulation of the Finger at the Metacarpo-phalanGEAL JoINT.-Amputation through the metacarpo-phalangeal joint may be done with or without the removal of the head of the metacarpal bone. Removal of the head of the metacarpal bone allows the adjoining fingers to be approximated, thus diminishing, somewhat, the apparent deformity, but this is accomplished at the expense of the solidity and strength of the hand; so that, in most cases, especially in laboring people, the end of the metacarpal bone is better not removed.

The finger is seized and flexed as in the previous operation, and an incision made upon the dorsal aspect of the hand, commencing

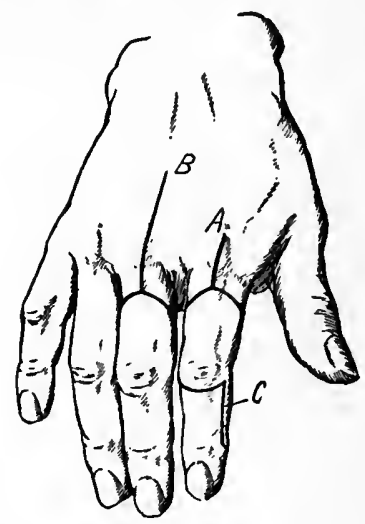

Fig. 188.-Exarticulation of the Finger. $A$, incision for exarticulation at the metacarpo-phalangeal joint; $B$, incision for amputation of finger with excision of the head of the metacarpai bone; $C$ indicates long anterior flap in exarticulation through the phalango-phalangeal joint.

one-half inch above the point of the knuckle and carried down as far as the level of the web of the finger. This incision should penetrate to the bone, dividing the skin and also the aponeurotic expansion of the extensor tendon. At the lower end of this incision, upon a level with the web of the finger, a second incision is carried around the finger, cutting all the structures, including the anterior and posterior tendons, down to the bone.

A corner of the flap is now seized, the finger being drawn toward the opposite side, and the flap, including the tendinous expansion, is stripped away from the bone with the knife; this is then done, in a similar manner, with the other remaining half of the flap.

Now strongly flexing the finger, the joint, which is located a 
good one-half inch below the angle of the knuckle, is opened by inserting the point of the knife, and the lateral ligament on either side is then completely divided. In opening the joint and dividing the lateral ligaments the knife may be grasped by the blade, being thus held short and firm. The bone is readily dissected out of the flap, care being taken not to perforate the integument with the point of the knife.

The vessels are caught and tied, usually one on each side of the flap; the corners of the flap may be rounded off and the end of the bone covered by uniting the edges of the flap with several interrupted catgut sutures.

If, in addition, the distal end of the metacarpal bone is to be removed, the dorsal incision should be extended somewhat farther upward, toward the wrist, and through all the structures down to the bone. With the point of the knife the soft parts are then separated from the bone, and with a strong cutting forceps the bone is divided about one inch above its lower end, taking care to cut the bone straight across. The loose lower end of the bone is then seized with a toothed bone forceps and enucleated, cutting with the edge of the knife applied close to the bone. After the vessels have been ligated, the edges of the flap are united with interrupted catgut sutures. If the head of the metacarpal bone is taken away it is not necessary to make the flap so long.

Exarticulation of the Hand at the Carpo-metacarpal Articulation.-Applicable to cases of traumatism where the thumb can be saved.

The hand which is to be amputated is seized by the operator and an incision made which crosses the palm of the hand, somewhat curved, with the convexity downward toward the fingers; it commences on the radial border of the hand near the head of the metacarpal bone of the index finger and ends on the ulnar border of the hand near the base of the fifth metacarpal bone. The incision extends through the soft parts, including the integument and palmar fascia, down to the flexor tendons. This anterior flap is reflected upward to the level of the carpo-metacarpal articulation.

Upon the back of the hand the incision extends through the skin only, and passes across the hand somewhat curved, with the concavity downward toward the fingers. If the anterior flap is scant, the posterior may be made correspondingly longer. The extremities of this posterior incision join with those of the anterior. The flexor tendons 
on the front of the hand and the extensors on the back of the hand are now divided transversely down to the bone with a sharp knife. The hand is again supinated and the carpo-metacarpal articulation opened, working from the ulnar side of the hand toward the thumb. Care should be taken in exarticulating the metacarpal bone of the index finger from the trapezoid not to injure the joint between the metacarpal bone of the thumb and the trapezium.

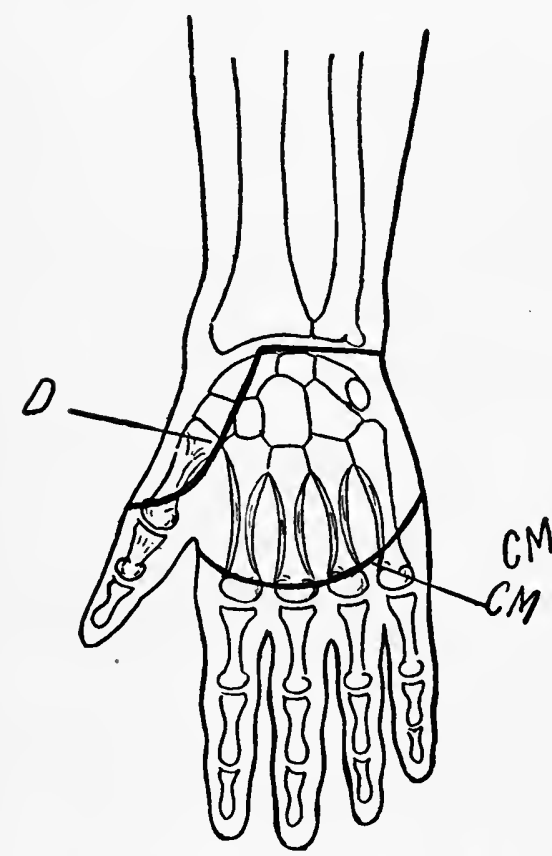

Fig. 189. - Palmar Aspect of Right Hand. $C M$, outline of the palmar flap in exarticulation of the hand through the carpo-metacarpal joint; $D$, incision for exartlculation of hand at the wristjolnt (Dubrueil), front view.

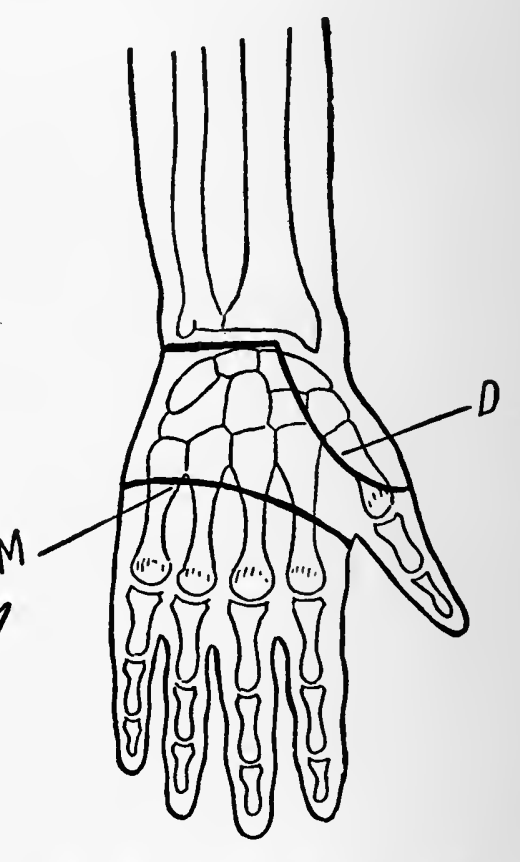

Fig. 190. - Dorsal Aspect of Rlght Hand. $C M$, dorsal Incision for exarticulation of the hand at the carpometacarpal joint; $D$, lnclslon for exarticulation at the wrist-joint (Dubrueil), back view.

The branches of the radial and ulnar arteries must be clamped and ligated before the tourniquet is removed.

The edges of the flaps are brought together with interrupted catgut sutures, the stump being thus covered by the strong palmar integument, and the suture line upon the posterior edge of the stump free from pressure, etc. 
If the condition of the integument of the palm of the hand is such that the longer flap cannot be taken from this part of the hand, then one may get a sufficiently long flap from the posterior surface, or two flaps of equal length, one from the anterior and one from the posterior surface of the hand may be made.

Surgical Anatomy of the Wrist-joint.-The wrist-joint is formed of the first row of the carpal bones in order, from without inward, scaphoid, semilunar, and cuneiform, and of the lower extremities of the radius and ulna.

The three carpal bones are united to each other, and present

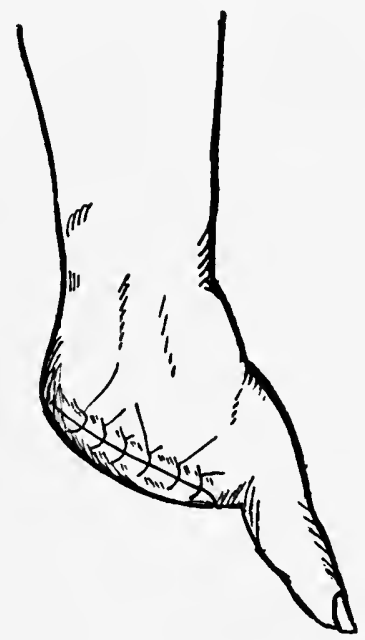

Fig. 191.-Stump Result of Exarticulation of the Hand at the Carpo-metacarpal Joint.

one continuous surface, smooth, "covered with articular cartilage, and convex from side to side and from before backward. The outer extremity of this surface slopes downward to a much lower level than the inner extremity.

The articular surface presented by the lower ends of the radius and ulna is concave in order to accommodate the convex articular surface of the upper row of carpal bones. This radio-ulnar articular surface is directed obliquely downward externally, so that the outer, or radial, end is a considerable distance below the level of the inner, or ulnar, end, and is continued into the external styloid proces:, to the tip of which the external lateral ligament is attached; the inner, 
or ulnar, side of this radio-ulnar articular surface presents the inner styloid process, prolonged from the lower end of the ulna. The tip of this process gives attachment to the internal lateral ligament of the wrist-joint.

Of the three carpal bones, the outer two, the scaphoid and semilunar, correspond to and articulate with the radial articular surface; the inner, the cuneiform, corresponds to the ulnar articular surface, an interarticular fibro-cartilage being interposed betwcen them.

There is a broad anterior and a broad posterior ligament, and these, together with the lateral ligament on either side, practically form a capsular ligament, which is lined upon its inner aspect by a thin, serous layer, the synovial membrane of the joint.

Exarticulation of the HaNd at the Wrist-Joint (DUBRUEIL).-An assistant steadies the forearm, drawing the integument rather toward the elbow. The hand which is to be amputated is seized by the operator, and commencing upon the front of the wrist, between the middle and outer thirds, an incision is made, which is carried inward around the inner border of the wrist, below the level of the styloid process, and across the back of the wrist, terminating at a point between the middle and outer thirds and directly opposite the point where the incision commenced. This incision should extend through the skin and subcutaneous fatty tissue and should be placed well below the level of the wrist-joint; otherwise, after the integument has retracted, the cut edge will be found to be above the level of the wrist-joint.

A tongue-shaped flap, with its base corresponding to the radial third of the circumference of the wrist, is now marked out by an incision reaching from either end of the circular incision described above. This flap of integument is taken from over the metacarpal bone of the thumb, its lower extremity corresponding to the metacarpo-phalangeal articulation of the thumb (see Fig. 189). This flap, including the superficial fascia and fat, is dissected back to the level of the wrist-joint. The wrist-joint is then entered by introducing the blade of the knife into the joint on its radial side, below the styloid process, and the hand severed from the forearm, thus completing the exarticulation.

The radial and ulnar arteries are picked up and ligated, the median and ulnar nerves seized and cut short, and the ends of the bones covered over with the flap, which is fixed with interrupted 
catgut sutures. The first stitch should unite the apex of the tongueshaped flap to the skin at a point corresponding to the tip of the styloid process of the ulna, and the other stitches are then placed so as to distribute the flap evenly, should it be found to be a little redundant.

An analogous operation may be done at the wrist-joint, taking the tongue-shaped flap of integument from the ulnar side of the hand.

One may also exarticulate at the wrist-joint, using two flaps, an anterior and a posterior flap, of equal length; or else one long, preferably the anterior, and one short; or the circular method may be used.

Amputation through the Forearm.-The forearm is a good place at which to practice the old musculo-tegumentary flap method. Practically, this method is now almost entirely discarded in favor of the skin flap or circular method. The arm overhangs the edge of the table. The hand, which is supinated, is supported by an assistant. A long, sharp amputating knife is introduced through the skin upon the outer side of the forcarm, at the level where it is intended to divide the bones, until its point touches the outer surface of the radius; it is then pushed through the soft parts upon the front of the forearm, keeping close to the anterior surface of the bones, and emerging at a corresponding point upon the inner, or ulnar, side of the forearm. Now, with a sawing motion and with the edge of the knife directed toward the radius and ulna, the anterior flap which includes the integument and all the muscular tissuc, is cut away from the bones. If the anterior and posterior flaps are to be of equal length, each flap should correspond in length to onehalf the diameter of the limb, at the point where the bones are to be divided, plus one-third extra, which is allowed for retraction. When the flap has been cut to a sufficient length, the edge of the knife is turned toward the integument and the flap cut square from within outward.

The posterior flap is formed in a similar manner. The point of the knife is again introduced upon the outer, or radial, side of the forearm in the upper angle of the incision which marks the anterior flap, and thrust through the forearm behind the bones, between them and the soft parts, emerging at the upper part of the incision upon the inner, or ulnar, side of the forearm, and then, with the edge of the knife closely applied to the bones, the posterior flap is cut equal 
in length to the anterior. The flaps should be square, and not tongue-shaped.

The flaps are turned back and held thus by the hands of an assistant or with sharp retractors, or by the operator. With a scalpel the interosseous membrane is cut through and the bones cleaned of any remaining soft parts, in order to make way for the saw.

The heel of the saw is placed upon one of the bones, and by drawing it backward firmly and steadily a groove is made, after which the bones can be rapidly severed, engaging the second bone after the first has been partly sawn through, and completing the section of both simultaneously. No cloth retractor is necessary, the flaps being held back by the operator's hand while he saws through the bones.

In the dead subject it will be seen that the muscles in the flap protrude beyond the edge of the integument; this is due to the unequal retraction of skin and muscle, and does not occur to the same degree in the living subject. Should the ends of the muscles or tendons protrude, they may be trimmed off with the scissors.

The radial and ulnar arteries are sought for and ligated; also the anterior and posterior interosseous. These latter are found close to the anterior and posterior surfaces, respectively, of the interosseous membrane. The median and ulnar nerves should be drawn down and cut short. The edges of the flap are joined all around with interrupted catgut sutures.

We may amputate through the forearm, using skin flaps, anterior and posterior, of equal length, or one long and the other short; or we may reflect a circular tegumentary cuff, in all of these operations, dividing the muscles on a level with or just below the point at which the bones are to be divided.

Surgical Anatomy of the Elbow-joint. - The elbow-joint is an irregular, rather complicated joint, formed by the lower end of the humerus and the upper end of the radius and ulna.

The lower end of the humerus is broad from side to side and flattened from before backward, and presents below two partially separated, smooth, rounded, articular surfaces, the smaller, outer one being for articulation with the radius, and the broader, inner one for articulation with the ulna. The plane of this double articular surface is oblique from without downward and inward, its inner end being on a much lower level than its outer.

The surface for articulation with the radius, the external, is a 
portion of a sphere, and occupies the lower and anterior aspect of the humerus.

The surface with which the ulna articulates, the inner, is broad, spool-shaped, and occupies not only the anterior and inferior, but also the posterior, aspect of the bone. This portion articulates with the greater sigmoid cavity of the ulna.

Below, the joint is formed by the upper end of the radius externally and the upper end of the ulna internally. The upper end or head of the radius presents a shallow cup-shaped surface, covered with cartilage for articulation with the radial part of the articular surface of the humerus; this surface is surrounded by a smooth, narrow margin, which rotates within the ring formed by the lesser sigmoid cavity of the upper end of the ulna and the orbicular ligament.

The head of the radius lies just below the external condyle, and may be recognized even when the joint is considerably swollen; by supinating and pronating the hand it may be felt to rotate beneath the skin. The elbow-joint is readily entered between the head of the radius and the external condyle.

The upper extremity of the ulna is irregular, and presents an articular surface, the greater sigmoid cavity, which is made up of the superior surface of the upper end of the ulna and the anterior surface of the olecranon process. The olecranon is a strong, squareshaped process of bone which projects upward from the posterior part of the upper end of the ulna. The greater sigmoid cavity is covered by articular cartilage and articulates with the trochlear surface of the lower end of the humerus. The upper end of the ulna further presents, upon its outer edge, a smooth depression, the lesser sigmoid cavity, to either end of which the orbicular ligament is attached. Within the ring formed by the orbicular ligament and the lesser sigmoid cavity the upper end of the radius rotates in pronation and supination.

Besides the parts entering directly into the formation of the elbow-joint there may be felt, internally, the internal epicondyle, very prominent and giving attachment, upon its anterior aspect, to the common tendon of origin of the flexor muscles of the forearm, and, externally, the less prominent external epicondyle, giving attachment, upon its posterior aspect, to the common tendon of the extensor muscles of the forearm. Behind may be felt the prominent olecranon process. At its junction with the ulna the olecranon proc- 
ess is somewhat constricted, and is here often the site of fracture. Its anterior surface enters into the formation of the elbow-joint, forming the upper part of the greater sigmoid cavity. Its posterior surface is subcutaneous and triangular in shape, with its apex below, where it is continuous with the posterior border of the shaft of the ulna. To the broad, upper border of the olecranon process is attached the triceps tendon, and around its margin the posterior and lateral ligaments of the joint. The upper border, or surface, of the olecranon process, when the arm is extended, is on a line drawn between the two epicondyles.

The elbow-joint is provided practically with a capsular ligament, which is lined, upon its inner surface, by a synovial membrane which also dips into that part of the joint between the head of the radius and the lesser sigmoid cavity of the ulna and orbicular ligament.

The ulnar nerve lies in close rclation with the elbow-joint, posteriorly, in a groove between the internal epicondyle and the olecranon process.

Exarticulation of the Forearm at the Elbow-Joint (Double Circular Method).-The arm overhangs the side of the table, and is steadied by an assistant, who draws the integument somewhat toward the shoulder-joint. The operator grasps the limb with the left hand, and with a long amputating knife a circular incision is made around the forearm, through the skin and fat down to the deep fascia. This incision should be placed below the level of the elbow-joint a distance corresponding to one-half the diameter of the arm at the elbow-joint, plus one-third extra, which is allowed for shrinkage of the skin. The upper surface of the head of the radius marks the level of the elbow-joint. This tegumentary flap is dissected away from the deep fascia and reflected upward like a turned-up cuff as far as the level of the elbow-joint. At this level the muscles are divided with the long knife down to the bone, and the elbow-joint then entered externally above the head of the radius, finally passing in between the ulna and the humerus, cutting the anterior and lateral ligaments. The forearm then hangs suspended by the attachment of the triceps tendon, and, this being cut close to the olecranon, the exarticulation is complete.

In this operation a common fault is that the muscles, being cut on a level with the elbow-joint, retract and leave the end of the humerus projecting into the wound. Even if the muscles are divided 
a considerable distance below the level of the joint and stripped away from the bone from a point below the level of the joint, it helps but little, as, upon the posterior aspect, there are no muscles, and even the tendon of the triceps, when cut close to the olecranon, lies well above the level of the joint; therefore, in most cases. it is desirable to supplement this operation by resecting the lower articular end of the humerus, which may be readily done. As regards the usefulness of the resulting stump, it matters little if we make the section just above, instead of through, the elbow-joint.

It is necessary to ligate the brachial artery and its accompanying vein separately. The median, ulnar, and musculo-spiral nerves are drawn down and cut short. The edges of the skin are united from side to side with interrupted catgut sutures, and a small drain introduced, which may be removed after forty-cight hours.

Amputation of the Arm.- Here the double circular method is preferable. The arm, hanging over the side of the table, is grasped above, near the shoulder, by an assistant, who, at the same time that he steadies the arm, draws the integument somewhat toward the shoulder. With a long amputating knife a circular incision is made, which reaches through the skin and superficial fascia down to the deep fascia. This incision should be placed below the level at which the bone is to be divided a distance equal to onc-half the diameter of the arm, plus one-third, which is allowed for retraction of the skin.

The circular flap, which includes all the fatty tissue, but not the deep fascia, is now dissected back like a cuff to a point one inch below the level at which the bone is to be divided; at this point the muscles are severed down to the bone with one circular sweep of the long knife.

With a blunt elevator or the back of the scalpel the muscles, but not the periosteum, are separated from the humerus for another inch, and thus the level is reached at which the bone is to be divided. After the periosteum has been cut by drawing the knife around the bone, the heel of the saw is applied and with a firm backward movement a groove is made in which the saw may work, and then the bone is rapidly severed.

While sawing the bone it is unnecessary to use a cloth retractor, as the soft parts may be held back so as to give the saw freedom, by the hands of an assistant, or with two sharp retractors.

Having completed the amputation, it will be seen that the mus- 
cles slightly overhang the end of the bone without covering it, and that the skin flap is sufficiently long to cover the whole stump.

The brachial artery and accompanying veins are found lying anterior and internal to the bone, and should be clamped and tied;

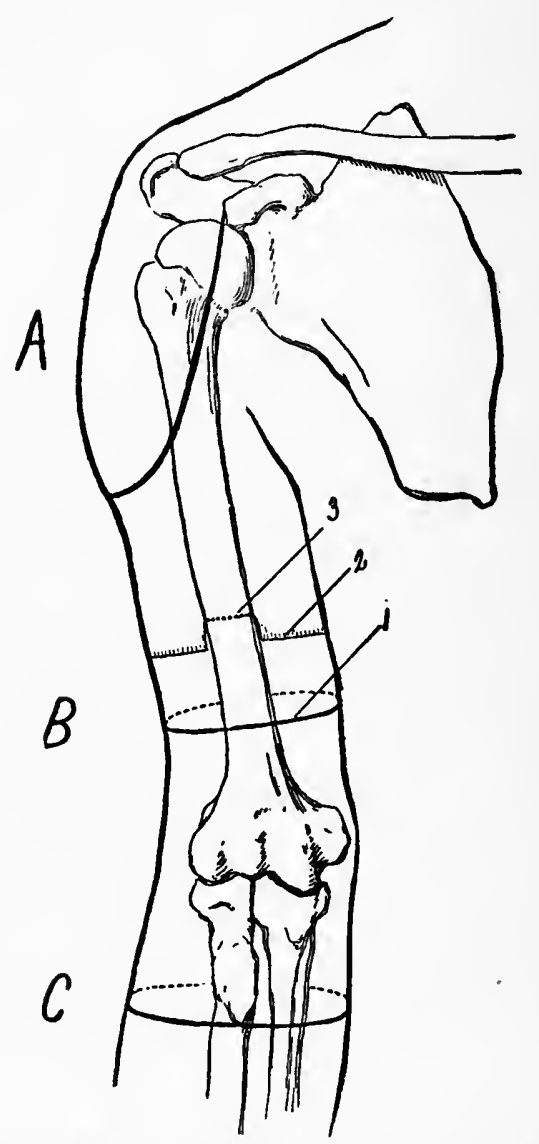

Fig. 192.-Right Arm, Anterior Aspect. A, outline of the lateral deltold flap in exarticulation at the shoulder-joint; $B$, amputation through the arm; 1 , incision through the skin; 2 , incision through the muscle; 3 , line of division through the bone; $C$, incision for exarticulation through the elbow-joint (circular method).

the median and ulnar nerves, which are in close proximity to the brachial artery, should be cut short; likewise the musculo-spiral, which is found upon the posterior surface of the humerus. The superior profunda, a branch of the brachial artery, which accompanies the 
musculo-spiral nerve, is also seized and ligated. The tourniquet is then removed and any remaining bleeding vessels clamped and ligated.

The edges of the skin are united from side to side, making a transverse line, by several interrupted catgut sutures; if the wound is clean, one may omit drainage, or a temporary drain may be introduced, and removed after forty-eight hours.

The arm may also be amputated with the formation of musculotegumentary flaps, as described for the forearm, or, instead of a cuff skin flap, one may use lateral or antero-posterior skin flaps of equal length, or one long and the other short.

Surgical Anatomy of the Shoulder-joint. - The shoulder-joint consists of the articulation of the upper end of the humerus and the glenoid cavity of the scapula. The articular surface of the upper end of the humerus looks inward and backward and is hemispheroidal in shape; it presents the arc of a smaller sphere from before backward, and of a larger sphere from above downward; that is, the diameter from before backward is shorter than that from above downward.

The articular surface is limited by the anatomical neck, which is narrow and well marked above, but broad and less well marked below; the anatomical neck marks the junction of the head of the bone with the shaft.

Externally may be observed the broad, large tuberosity major; internally and below the head is the smaller tuberosity, the tuberosity minor. To the tuberosity minor is attached the tendon of one muscle, the subscapularis; to the tuberosity major-i.e., to its upper and posterior borders-are attached the tendons of threc muscles: the supraspinatus, the infraspinatus, and the teres minor, in that order from above downward.

The anterior border of the greater tuberosity forms the extcrnal border of the bicipital groove, the external, or anterior, bicipital ridge; the lesser tuberosity and the ridge that is prolonged downward from it forms the inner border of the bicipital groove, the internal, or posterior, bicipital ridge. Between the two is the bicipital groove.

To the external bicipital ridge is attached the tendon of the pectoralis major; to the internal bicipital ridge are attached the tendons of the latissimus dorsi and teres major. Lying in the groove itself, held in place by a process of fibrous tissue, is the long tendon 
of the biceps muscle. Close to the humerus, between the tendon of the pectoralis major in front and the tendons of the latissimus dorsi and teres major behind, are the brachial vessels and accompanying nerves, which descend in a bunch from the axilla, partially overlapped by the coraco-brachialis muscle. The bicipital groove really forms the outer wall of the axilla when the arm hangs by the side.

Below the tuberosities is the surgical neck, so called because it is a rather common site of fracture.

The glenoid cavity, a depressed area upon the head of the scapula, is much less extensive in area than the articular surface presented by the humerus; it is shallow, longer from above downward than from before backward, and is connected with the body of the scapula by the neck.

The glenoid cavity presents a slightly raised margin, to which margin is attached the glenoid ligament, which serves to deepen the cavity.

Overhanging the shoulder-joint is the acromion process, the extreme outer end of the spine of the scapula; this process articulates with the outer end of the clavicle, and forms the prominent outer part of the shoulder-girdle and a protecting ledge over the shoulderjoint.

In front and internal to the shoulder-joint the coracoid process may be felt, and in thin subjects seen; it projects forward from the upper border of the scapula, lying below the outer end of the clavicle, to the under surface of which it is connected by strong ligaments. Passing from the coracoid to the acromion process is a strong ligamentous band, the coraco-acromial ligament. This ligament passes over the head of the humerus, across the upper part of the shoulderjoint, deepening the cavity in which the head of the humerus plays and serving to add strength to the joint.

The shoulder-joint is provided with a capsular ligament, which is attached above to the neck of the scapula around the glenoid cavity, and below to the anatomical neck of the humerus. A separate fibrous band, called the coraco-humeral ligament, extends from the coracoid process down to the neck of the humerus, where it is attached in common with the capsular ligament, of which it is really a part.

The long tendon of the biceps is attached to the upper margin of the glenoid cavity; it passes across the upper surface of the head of the humerus, through the shoulder-joint, and emerges through 
the anterior part of the capsule, and then passes down the arm, being lodged in the bicipital groove. In its course through the shoulderjoint the long tendon of the biceps is entirely enveloped in a tubular process of the synovial membrane, and thus, although it passes through the shoulder-joint, the tendon is at the same time excluded from it.

Like a hood or cushion, the deltoid muscle covers and serves to protect the shoulder-joint; beneath the deltoid there is a bursa, which sometimes becomes diseased.

Below the acromion and beneath the deltoid muscle the head of the humerus may be readily recognized. It may be felt to rotate underneath the soft parts upon manipulation. It is responsible for the rounded contour of the shoulder; if the head of the humerus leaves the glenoid cavity, the shoulder presents a peculiar flattened appearance, which is very striking, and the sharp outer end of the acromion process becomes especially prominent and tends to direct attention to the fact that the head of the humerus has been dislocated.

Exarticulation at the Shoulder-Joint (Spence). - The shoulder should overhang the edge of the table and the arm should be abducted a little from the side of the thorax and at the same time rotated somewhat outward, so that the great tuberosity is directed outward.

The incision is about six inches long, and commences above, at the clavicle, between the acromion and coracoid processes, and passes down the front of the arm as far as the point where the deltoid is attached to the humerus. This incision is deep, penetrating through the skin, fascia, and muscle down into the bicipital groove. With the long knife a circular incision is then made around the arm, on a level with the lower end of the longitudinal incision; this incision, upon the inner aspect of the arm, should pass through the integument and superficial fascia (subcutaneous fat) only, care being taken not to sever the brachial vessels; for the rest of the circumference of the arm, however, this circular incision penetrates through all the soft parts to the bone.

The outer edge of the wound is seized, and with a scalpel the soft parts are dissected away from the outer surface of the humerus, the arm being rotated inward by the assistant, to facilitate this step of the operation.

The capsular ligament being now exposed, the joint should be 
opened; this is done, not by passing the blade of the knife flatwise between the head of the humerus and the acromion process, but by cutting directly down upon the upper surface of the head of the humerus, from behind forward, as though one would cut into the head of the bone. During this step of the operation the assistant may help by rotating the arm first inward and then outward. In this way the joint is freely opened, the long tendon of the biceps being cut at the

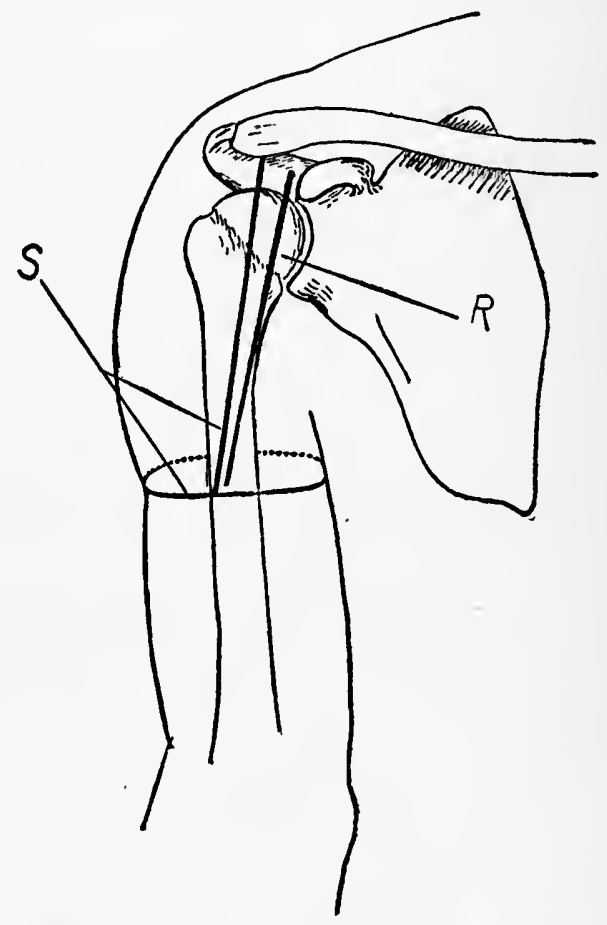

Fig. 193.-Right Shoulder, Anterior View. $R$, line of incision for resection of shoulder-joint; $\delta$, incision for exarticuiation at the shouider-joint (Spence).

same time. The head of the bone is now turned out of its socket and drawn forcibly outward, away from the glenoid cavity; the long knife is introduced into the wound, behind the head of the humerus, and the soft parts, with the edge of the knife applied close to the surface of the bone, are separated from the inner aspect of the humerus to a point a little below the level of the circular incision, care being taken not to injure the brachial vessels, which run parallel 
with the inner surface of the humerus, and which have not, as yet, been divided.

Now, with a final stroke of the knife, the operation is completed by cutting through the soft parts upon the inner aspect of the arm down to the surface of the bone, thus severing the vessels and nerves. Just before this final cut which divides the vessels is made an assistant grasps the mass of soft parts which have been separated from the inner side of the humerus and which include the brachial vessels, and thus compresses them while they are being cut, and continues to hold them until the operator can secure the divided vessels with artery forceps, after which they are tied. Other vessels which spurt are clamped and tied as the operation progresses.

The edges of the skin may be brought together with interrupted catgut sutures, a drain emerging from the lower end of the wound and left in place for forty-eight to seventy-two hours; or the edges of the wound may be closed throughout and an opening made through the posterior part of the flap, near the glenoid cavity, and the wound thus drained. This latter plan is very satisfactory.

The above is a good method for exarticulation at the shoulderjoint, which may thus be accomplished with the loss of but little blood. Through the longitudinal incision, which is first made, the joint may be opened and freely explored and drained, or the joint may be excised; this is a great advantage, as we are often in doubt as to the necessity of exarticulation until after the joint has been opened and inspected.

Exarticulation at the Shoulder-Joint with aN Esmarch BANDAge APPLIED.-The shoulder overhanging the side of the table and the arm somewhat abducted, an Esmarch bandage or rubber tube is applied tightly about the axilla, passing around the shoulder over the outer part of the clavicle. With a long knife a circular incision is then made through the integument and fat down to the deep fascia. This incision should be placed just above the insertion of the deltoid muscle. The integument, which retracts at once, is drawn toward the shoulder by an assistant, and the muscles, vessels, etc., divided by a second circular sweep of the long knife down to the bone as high up as the retracted integument permits; the bone is then sawn through at this level. The brachial artery and accompanying veins are now clamped and tied; also the superior profunda, which is found upon the back side of the humerus in company with the musculospiral nerve. 
After these vessels have been tied the Esmarch bandage is removed and any further spurting vessels ligated.

A second incision is now made from the acromion process down upon the front of the stump of the humerus and penetrating to the bone. The soft parts are then cut away from the outer surface of

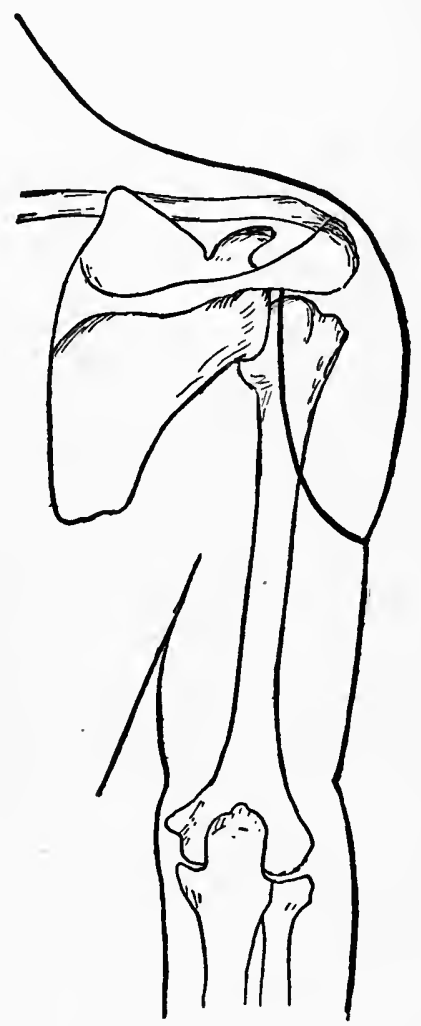

Fig. 194.-Right Shoulder, Posterior View. Outline of the lateral deltold flap for exarticulation at the shouider-joint.

the stump of the humerus, tying vessels as they are cut, and the joint opened by incising the capsule from behind forward, including the tendon of the biceps; the head of the bone is then turned out of its socket, and while it is drawn forcibly outward, away from the glenoid cavity, the soft parts upon its inner side are stripped away from the bone and the operation thus completed. But little 
blood is lost. The wound may be treated as in the preceding operation.

After the circular incision has been made through the soft parts, including the muscles, brachial vessels, etc., down to the bone, and while the tourniquet is still applied and without sawing through the bone, one may ligate the vessels and then, after removing the tourniquet, proceed to complete the operation by turning the head of the

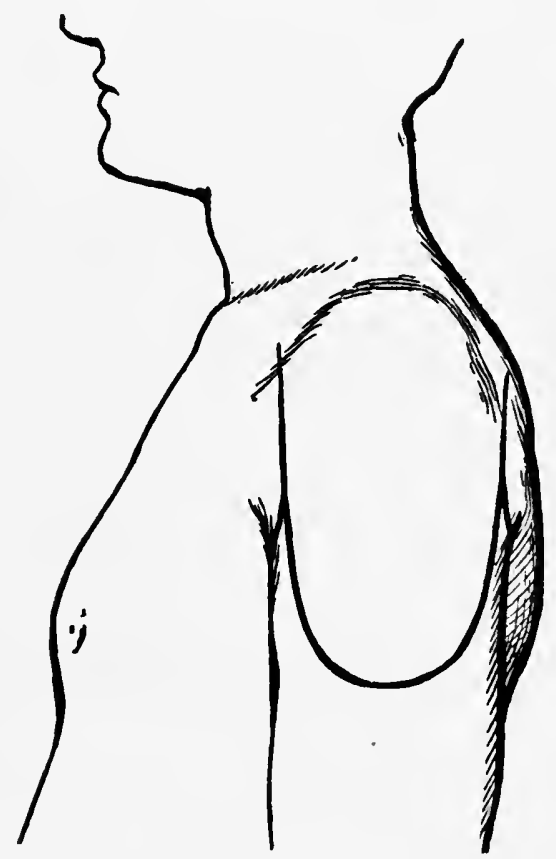

Fig. 195.-Left Shoulder, Side View. Outlíne of the lateral deltoid flap for exarticulation at the shoulder-joint.

bone out of its socket and stripping the soft parts away from the upper part of the bone through the longitudinal incision as described above. This would save sawing through the shaft of the humerus.

Exarticulation at the Shoulder-Joint with the Formatron of a Lateral Deltord Flap.-The position of the patient is the same as in the previous operation, the shoulder overhanging the edge of the table. A large musculo-tegumentary flap, U-shaped and corre- 
sponding to the deltoid muscle, is taken from the outer aspect of the arm. The incision commences anteriorly, just external to the coracoid process, and passes down upon the front of the arm to a point a short distance above the insertion of the deitoid muscle, whence the incision is carried backward across the outer aspect of the arm and then upward as far as the spine of the scapula to a point just posterior to the acromion process; this incision reaches to the bone throughout its whole course. In dividing the muscles the knife should be directed rather obliquely, in order that the edge of the musculo-tegumentary flap may be beveled at the expense of the deeper structures so that the muscles will not protrude beyond the edges of the skin, which retracts considerably when it is cut. Care should be taken that this flap is not tongue-shaped.

This outer deltoid flap is seized with the fingers and dissected away from the surface of the bone and reflected up over the shoulder. The spurting branches of the circumflex artery are seized with forceps and tied. The capsule of the joint being now exposed, the joint may be opened by cutting through the capsule, from before backward, with the edge of the knife applied directly against the upper surface of the head of the bone, the long tendon of the biceps being cut at the same time. The arm is rotated outward, and the attachment of the subscapularis cut from the lesser trochanter; then rotating inward, the tendons which are attached to the upper and posterior border of the greater trochanter are divided, when the head of the bone drops away from the glenoid cavity.

The joint being thus widely open, the upper end of the humerus is dragged outward away from the glenoid cavity, and with a long knife the soft parts, attached to its inner aspect, are cut away from the bone, the edge of the knife being held close against the surface of the bone, in order to avoid injuring the brachial vessels, which run parallel with and close to the inner surface of the humerus. After the soft parts have been thus separated from the inner aspect of the humerus to a point about one inch below the anterior fold of the axilla, the edge of the knife is turned inward, and with a final stroke a short inner flap is cut, dividing the vessels at the same time. Just before this final cut, which divides the vessels, is made, an assistant grasps the mass of soft parts, which includes the brachial vessels, and compresses them until the operator can secure the ends of the severed artery and accompanying veins; these are then ligated and the nerves drawn down and cut short. 


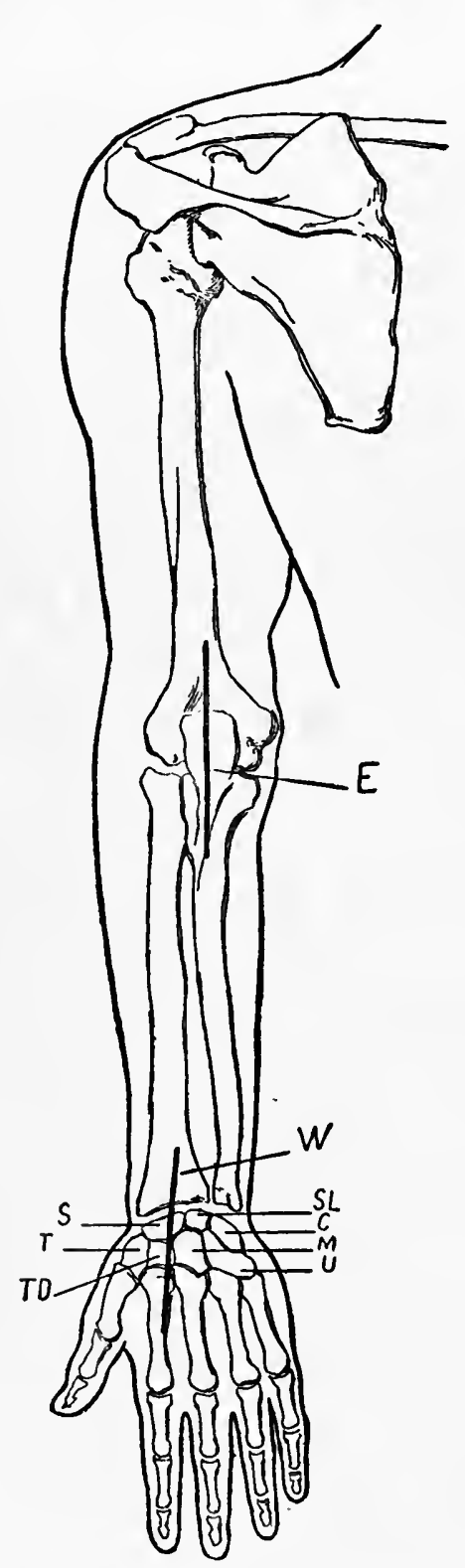

Fig. 196.-Left Arm, Posterior Vlew. $E$, incision for resection of elbowfoint; $C$, cunelform; $M$, os magnum; $S$, scaphold; $S L$, semilunar; $T$, trapezium; $T D$, trapezoid; $U$, unciform; $W$, incis:on for resection of wrist-joint. 
The wound is closed with interrupted catgut sutures, a drainage tube which reaches to the glenoid cavity being left protruding through the posterior part of the wound.

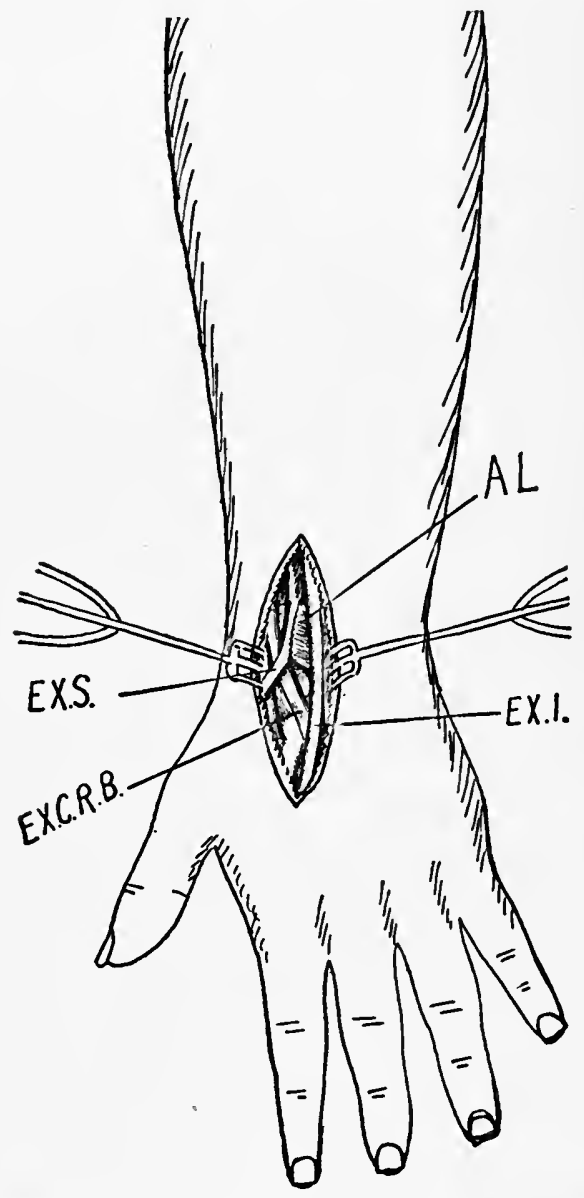

Fig. 197.-Resection of Wrist-joint. $A L$, annular ligament split to show the tendons of extensor secundi $(E X . S)$ and extensor carpi radialis brevior $(E X . C . R . B) ; E X . I .$, tendon of extensor indicis.

Resections. WRIST-JoINT.-A tourniquet is applied above the elbow, in order that the operation may be bloodless. A dorsal incision is made, commencing below, at the middle of the ulnar border of the metacarpal bone of the index finger, and this is continued upward, 
over the middle of the posterior surface of the radius, to a point one inch above the level of the wrist-joint. This incision passes through the skin and fat and runs parallel with the outer border of the extensor tendon of the index finger, the extensor indicis.

This incision is then gradually deepened step by step, and in its lower part one should avoid opening the sheath of the extensor indicis; in the upper part of the incision, nearer to the wrist-joint, the tendon of the extensor carpi radialis brevior, which is attached to the base of the third metacarpal (that of middle finger), and the tendon of the extensor secundi are exposed. We keep to the inner, ulnar, side of these tendons, drawing them toward the outer, radial, side of the wound with a blunt hook, and thus avoid cutting them. The wrist-joint is then entered by cutting through its posterior ligament, between the tendons of the extensor indicis and the extensor secundi. With blunt retractors the tendons of the extensor indicis and extensor communis are drawn toward the ulnar border of the hand, and the tendons of the extensor secundi and extensor carpi radialis brevior toward the radial border. Above the joint the incision penetrates to the surface of the radius between the bunch of tendons (extensor communis digitorum and extensor indicis), to the ulnar side, and the extensor secundi, to the radial side. The edges of the wound, including the tendons, being well retracted, an elevator is introduced and all the soft parts separated from the bones, working as close as possible to the surface of the bone. It may be necessary to partially separate the attachment of the tendon of the extensor carpi radialis brevior from the base of the third metacarpal. This is accomplished with the elevator or by chipping away a thin sliver of the bone with a chisel; the tendon should not be divided with the knife.

After the carpal bones have been freely exposed the wrist is flexed and the first row is removed, commencing with the scaphoid, then the semilunar, - which adjoins it, -and finally the cuneiform. The pisiform, which articulates with the anterior surface of the cuneiform and to which the tendon of the flexor carpi ulnaris is attached, is allowed to remain unless it is diseased.

With the wrist still flexed, thus giving better access to the carpus, the second row of carpal bones is now excised, commencing with the os magnum, which is easily recognized by its prominent rounded head. This bone is seized with a small lion-tooth forceps, isolated, and removed. Then the trapezoid lying to the outer side of the os magnum 
and articulating with the metacarpal bone of the index finger; after this, the unciform is seized with the forceps and removed; the trapezium, which articulates with the metacarpal bone of the thumb, is allowed to remain, if its condition permits, as its removal interferes much with the function of the thumb.

It.is not always necessary to remove all the bones of the carpus; when diseased, they may often be readily enucleated with a sharp spoon; at other times the ligamentous bands which join the bones to each other and to the bases of the metacarpal bones must be cut before they can be enucleated, and in doing this one should be careful that the point of the knife does not wound the structures in the palm of the hand. There may be some difficulty in removing the scaphoid. In excising this bone, and also the trapezium, one should remember that the radial artery lies in close proximity to their posterior surfaces. Although this vessel is usually separated from the bones when the soft parts are detached with the elevator, and is therefore not endangered, yet one should look out for the point of his knife.

The removal of the unciform is rather difficult, owing to the irregularity of its hook-like process and its muscular attachments. It may be seized with a toothed bone forceps, and, by twisting it and at the same time cutting with the edge of the knife close to the bone, it may be removed.

If the ends of the radius and ulna are to be removed, the soft parts, including the tendons, are separated from the posterior surface of the bones with the periosteum elevator; the lateral ligaments are also detached from the bones, preferably with the periosteum elevator rather than with the knife, taking care to avoid the radial artery as it winds around the outer side of the wrist. The lower ends of the bones are then forced well out of the wound and the soft parts separated from their anterior surfaces, working close to the bone or subperiosteally, and finally the ends of the bones are sawn off. One should avoid the ulnar artery and nerve, anteriorly, toward the ulnar side. The tourniquet may now be removed.

There are usually no vessels to tie, none of importance being cut. The hand is placed upon a straight anterior splint and the wound partly closed by interrupted sutures and packed with iodoform gauze.

ELBOW-JOINT (LANGENBECK).-A tourniquet is placed about the upper part of the arm. The operation should be done subperiosteally.

The arm, with the elbow flexed, is thrown across the patient's chest and steadied by an assistant; the operator usually stands on 
same side as the diseased joint, although at times it is convenient to change to the other side. An incision, about four inches long, is made upon the posterior aspect of the joint. This incision commences about two inches above the upper border of the olecranon process and is continued downward upon the posterior triangular surface of the olecranon and ulna, passing, not through the middle of this surface, but a little to the inner side of the middle line and ending on its inner border (see Fig. 196). This incision should be made with a heary resection knife, and throughout its whole length should penetrate through all the soft parts, including the periosteum, down to the bone. The upper part of this incision splits the tendon of the triceps lengthwise right down to its attachment to the upper border of the olecranon, and passes through the posterior ligament of the joint to the surface of the humerus. The lower part of the incision, corresponding to the posterior surface of the olecranon, passes through the periosteum to the bone.

Sharp retractors are introduced into the upper part of the wound, and the attachment of the triceps tendon is chiseled away from the upper border, surface, of the olecranon process on either side, taking a very thin shell of bone with it; this separation may also be accomplished with the knife, cutting close to the bone, but the subcortical separation with the chisel is preferable.

The periosteum elevator is now used to separate the soft parts, including the periosteum, from the posterior surface and sides of the olecranon process and the adjoining upper part of the ulna and lower end of the humerus, working first inward toward the inner condyle and keeping close to the bone, as this mass of soft parts includes the ulnar nerve, which is lodged in the groove between the inner condyle and the olecranon; if we work subperiosteally, or very close to the surface of the bone, the ulnar nerve is not seen and not endangered. To retract this mass of soft parts as it is detached from the bone, one should use a blunt-pronged retractor. The separation of the soft parts is continued inward and around the inner epicondyle. In separating the soft parts from the inner epicondyle one should use the chisel rather than the knife, since the tendon common to the superficial flexor muscles is attached here, and it would be disadvantageous to cut it. In a similar nianner the soft parts, including the periosteum, are detached from the outer side of the olecranon, continuing outward until the external epicondyle is denuded. To the external epicondyle is attached the tendon common 
to the superficial extensors, and therefore one should avoid using the knife here.

The separation of the soft parts can be accomplished almost entirely with the elevator, if necessary using considerable force with the sharp edge of the elevator applied directly upon the bare surface of the bone; but it may be necessary here and there to help one's self with the chisel and occasional snips with the knife. Upon the posterior surface of the olecranon the knife may be used a little more freely, as here the periosteum is thick and fibrous, being reinforced by the triceps tendon, and is almost impossible to separate with the elevator.

After having denuded the whole of the olecranon process and the contiguous portions of the humerus, ulna, and radius out beyond the epicondyles, the elbow is flexed and the lower end of the humerus forced out of the wound, cutting away any remaining restricting bands. The soft parts about the anterior aspect of the lower end of the humerus are then quickly separated with the elevator and the articular end of the bone sawn off. The section should be made through a plane parallel with the articular surface. Then, in a like manner, the upper end of the radius and ulna are stripped of soft parts and sawn off. The diseased synovial membrane may now be completely excised with toothed forceps and blunt-pointed, curved scissors. One should avoid injuring the structures in front of the joint, brachial artery, etc., with the point of the knife. After the resection has been completed the tourniquet may be removed. As a rule, there are no vessels to tie. The incision is closed, except for a part of its length, which is left open for drainage, and the arm put up in a position of almost complete extension in a splint or plaster of Paris with a big wad of dressings.

After two weeks the arm may be gradually or at once flexed to nearly a right angle, which is the best position for ankylosis. Occasionally we get some motion.

Shoulder-JoINT (Subperiosteal Method of Ollier and Hueter).-The arm lies at the side, slightly abducted and rotated outward, so that the greater tuberosity looks outward. An incision is made which commences above, to the outer side of the coracoid process, and passes downward, upon the front of the arm, for a distance of five inches; this incision, throughout its whole length, is carried deep to the bone (see Fig. 193). When the edges of the wound are held apart with blunt-pronged retractors, the long tendon of the 
biceps, as it lies in the bicipital groove between the two tuberosities, is exposed. This tendon emerges from within the joint beneath the lower border of the capsule.

This incision above, to the outer side of the coracoid process, should extend as high as the clavicle, in order to allow easy access to the capsule and to the head of the humerus.

A director is now introduced alongside of the long biceps tendon, beneath the lower border of the capsule, and well up into the joint, and upon this the capsule is divided as far upward as the upper border of the glenoid cavity; in this way the capsule is split longitudinally throughout its entire length (the coraco-humeral ligament, which is a part of the capsule, is also divided in this cut), and the joint is thus freely opened upon its anterior aspect.

The tendon of the biceps is now lifted out of its groove and drawn outward with a blunt hook, and the periosteum incised in the upper part of the floor of the bicipital groove, between the two tuberosities; an elevator, with a sharp edge, is then introduced into the incision in the periosteum, and this, together with the attachment of the capsule, is separated from the inner side of the neck of the bone. The tendon of the subscapularis is very intimately attached to the lesser tuberosity, and in order to separate this it may be necessary to use the knife to some extent, cutting close upon the surface of the bone, or, what is preferable, one may, with the chisel, chip off a thin layer of the cortex, carrying the attached tendon with it.

In separating the capsule from its attachment around the neck of the bone it will be necessary, here and there, to use the knife, cutting with its edge applied close to the surface of the bone.

After the parts on the inner aspect of the bone have been thus separated, and while the arm is rotated inward and the long tendon of the biceps hooked over toward the inner side, the periosteum, together with the attachment of the capsule, is separated from the outer side of the bone; this is accomplished chiefly with the periosteum elevator, with occasional snips with the knife. The tendons attached to the upper and posterior borders of the greater tuberosity are intimately united with the bone, and, if they cannot be separated with the periosteum elevator, one may use the chisel, as on the inner side, removing a thin shell of the cortex along with the tendon attachments. During this part of the operation the arm is rotated more and more inward. 
After the upper end of the bone has been thoroughly isolated we find it lying in a sac, formed above by the detached capsule, which is continuous below with the periosteum and tendons that have been separated from the bone.

The head of the bone is now thrown out of this sac and out of the incision, and may be sawn off with the flat saw, protecting the neighboring soft parts, or the chain or Gigli saw may be used, or it may be knocked off with a broad chisel.

After the head of the bone has been removed, the interior of the joint becomes accessible, and one may dissect away all the synovial membrane lining the joint with toothed forceps and strong, blunt-pointed scissors, curved on the flat.

The glenoid surface of the scapula, if diseased, may be thoroughly curetted with the sharp spoon, or chiseled or gouged out with the rongeur bone forceps. Usually no vessels of importance are cut; any spurting points may be caught and tied as the operation progresses.

The cavity of the joint should be freely drained through the lower part of the incision, using a good-sized tube. An additional opening may be made posteriorly to provide still better drainage. This opening is made by pushing an artery forceps through the mass of deltoid muscle from within and then incising the skin with the knife upon this. We avoid making the opening through the deltoid with the knife in order not to wound the circumflex nerve and vessels. The opening through the muscle may be made as large as. desired by spreading the blades of the forceps. In closing the incision interrupted silk-worm gut sutures, which pass through all the structures, including the edges of the split capsule, should be employed.

If it is intended to remove the head of the bone only, it is not necessary to separate the periosteum for more than a short distance upon the shaft. Usually separation of the capsule around the anatomical neck and the tendons partially from the greater and lesser tuberosities will give sufficient room to permit of the excision of the head of the bone. Only when the head of the bone is to be excised below the trochanters is it necessary to separate the periosteum and tendons for a greater distance below the anatomical neck.

The operation as described above differs from Langenbeck's only as regards the incision. The incision of Langenbeck commences above at the acromion process, and is therefore more external; pass- 
ing through the body of the deltoid, it divides the circumflex nerve, and is therefore likely to be followed by impairment of the function of the deltoid.

Tendon Suture.-Tendons may be found divided as a complication of a wound, or they may be accidentally cut by the surgeon during the course of an operation about a joint; one or several may be severed. The proximal portion of the tendon, that which is joined to the muscle, may be separated a considerable distance from the distal portion, owing to the contraction of the muscle, and at times considerable search may be necessary to secure it, or it may be necessary to lay the sheath of the tendon open for this purpose.

The ends should be approximated and joined by a catgut suture,

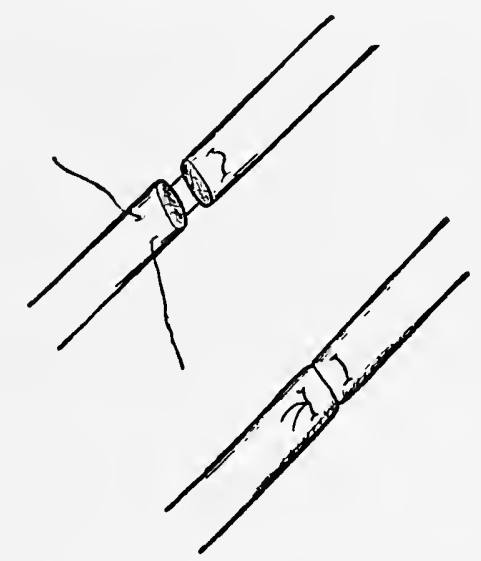

Fig. 198.-Divided Tendon Reunited by a Single Mattress Suture.

one passing through the tendon proper being probably the most satisfactory. If the flexor tendons are divided, in order to coapt the ends and retain them in position with the minimum degree of tension, the joint must be placed in a position of flexion, and the reverse when the extensor tendons are severed. Asepsis is a necessary condition to healing; if the parts are infected, an effort should be made to render them sterile, and under these circumstances drainage in addition is probably advisable.

Nerve Suture.-A nerve-trunk may be severed, cither accidentally by the surgeon during the course of an operation or the condition may be encountered as a complication of an accidental wound.

The ends should be approximated and united with one or more 
plain catgut sutures, which may be passed through the body of the nerve proper. The union may be effected immediately after the occurrence of the accident or after the lapse of considerable time. If immediate, it is simply necessary to coapt the ends and retain them in position with one or two sutures; if after the lapse of a considerable period, it will be necessary to search for the ends of the divided nerve, and, after they are found, freshen them, before uniting them, end to end, by suture. Plain catgut is preferable for the suture material.

Intravenous Saline Infusion. - Any prominent superficial vein may be used for this purpose; the median cephalic at the bend of the elbow is the one usually selected. A tourniquet is first applied about the arm, high up near the axilla and just sufficiently tight to constrict the superficial veins, but not tight enough to shut off the arterial current; this causes the superficial veins to become swollen and more con-

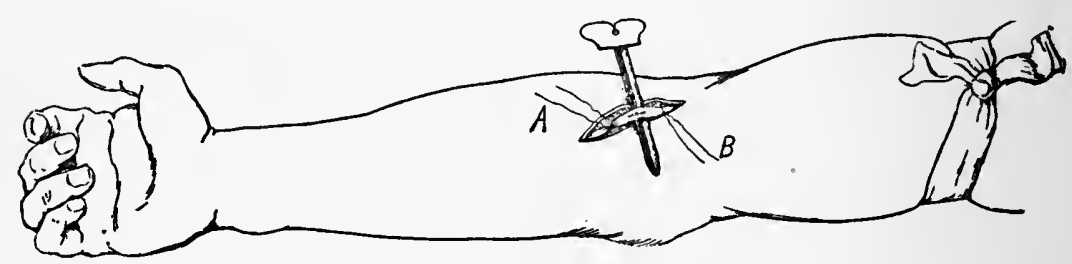

Fig. 199.-Superficiai Vein Exposed for Saine Infusion. The vein, which is raised upon the director, has been opened ready to introduce the cannula. Suture $(A)$ has been tied. Suture $(B)$ surrounds the vein, but has not been tied; one loop of the knot has been taken, but not drawn tight.

spicuous. The skin is then pinched up over the vein and may be incised by transfixion with the knife or with the scissors, care being taken not to injure the vein itself. The vein is then thoroughly isolated for about one inch and raised well out of its bed upon a director, after which a double catgut ligature is passed around the vein. This ligature is then cut, so as to leave the vein surrounded by two ligatures, one above and the other below. A single loop of a knot is taken loosely in the upper ligature, the ends of which are left long. The vein is now freely opened with a narrow-bladed knife and the lower ligature then tied tight around the vein. Through the opening made in the vein the end of the cannula is slipped up into the vein beyond the upper ligature, which is then tied fast about the cannula, in order to retain it securely in place within the vein.

Care should be taken to introduce the cannula into the lumen of the vein, and not into the loose connective tissue that surrounds 
the vein. This is an accident which may readily occur, and is to be avoided by thoroughly isolating the vein and lifting it well out of its bed before incising it. Before the cannula is introduced into the vein the solution should be allowed to flow in order to fill the cannula and thus avoid carrying air into the vein; although it is of no consequence if a small quantity of air does enter the rein, nevertheless this should be avoided if possible.

After the ligature has been tied and the cannula thus secured in the vein, the tourniquet is remored from the arm and the fluid allowed to flow; from 1 to 2 quarts at a temperature of about $115^{\circ} \mathrm{F}$. may be introduced. ${ }^{1}$ The reservoir should be held at an elevation of two feet.

1 A degree of heat that the hand can conveniently endure if no thermometer is at hand. 


\section{PART $X$.}

\section{THE LOWER EXTREMITY.}

\section{THE THIGH.}

THE muscles and other structures of the thigh are enveloped by the skin and the superficial fascia, which is areolar in structure and includes the subcutaneous fat. These layers are loose, and movable upon the deeper parts. Beneath the fat (superficial fascia) there is a strong, tense, fibrous envelope, thicker in some parts than in others, - the proper, or deep, fascia,-which, in the region of the thigh, is called the fascia lata. This layer is attached above to Poupart's ligament, the crest of the pubis, sacrum, and rami of the pubis, and below-about the knee-joint, to all the prominent bony points; it confines the muscles and furnishes septa, which pass in between the different groups of muscles to be attached to the ridges on the femur. Beneath the skin, in the fatty layer, ramify the various subcutaneous veins and nerves, and in the region of the groin the subcutaneous arterial branches that are derived from the femoral.

The Gluteal Region. - The gluteal region corresponds to the upper back part of the thigh, and presents the prominence of the buttock. This is more developed in some persons than in others, especially in females, and is due chiefly to the cushion of fat beneath the skin.

After the skin and fat have been reflected, the deep fascia, fascia lata, is exposed. This fascia is rather thin in this region, and through it the fasciculi of the gluteus maximus muscle may be recognized. The fascia lata is attached above to the crest of the ilium; below it is continuous with the same layer of fascia upon the back of the thigh; internally it is attached to the sacrum and coccyx.

The gluteus maximus is a broad, thick muscle; it arises from the upper, posterior portion of the external surface of the ilium, from the side of the sacrum and coccyx, from the lumbo-sacral aponeurosis, and from the great sacro-sciatic ligament. In coarse bundles its fibers pass downward and outward; the upper fibers become tendons, and pass across the great trochanter and are inserted into the fascia lata upon the outer aspect of the thigh; the lower fibers are attached 
to the femur along the line which passes from the great trochanter downward to the linea aspera.

The muscle should be cut through at right angles to the course of its fibers and reflected, when the bursæ beneath it, one corresponding to the trochanter major and the other to the tuber ischii, may be examined and the parts which lie beneath the muscle exposed to view. Above and in front is the posterior portion of the gluteus medius, and below this, but upon the same plane, the pyriformis; these two muscles are separated from each other by a cellular interval, through which the gluteal vessels and nerves are seen to emerge from within the pelvis. Below the pyriformis, but still upon the same plane, are the two gemelli and the tendon of the obturator internus. Still lower is found the quadratus femoris, which is really the upper part of the adductor magnus muscle. These muscles are all attached to the femur at or near the great trochanter. Passing downward from the tuberosity of the ischium are the semimembranosus and the semitendinosus and biceps muscles.

The space which exists in the skeleton between the lateral border of the sacrum and coccyx and the margin of the ischium is converted into two foramina, the greater and lesser sacro-sciatic foramina, by the greater and lesser sacro-sciatic ligaments. The greater sacro-sciatic ligament is attached by its broad base to the margin of the sacrum and coccyx and by its other end to the tuberosity of the ischium; the posterior surface of the great sacro-sciatic ligament gives attachment to some fibers of the gluteus maximus muscle. The lesser sacro-sciatic ligament is attached to the margin of the sacrum and coccyx and to the spine of the ischium; the lesser is situated upon a plane anterior to the greater.

Through the greater sacro-sciatic foramen emerge the pyriformis muscle; the gluteal vessels and nerve which appear above the pyriformis, between it and the gluteus medius; the sciatic artery and great sciatic nerve, which appear below the pyriformis, and the internal pudic vessels and nerve. The internal pudic vessels and nerve, after emerging from the pelvis through the great sacro-sciatic foramen, curve around the lesser sacro-sciatic ligament, close to the ischium, and pass forward into the deep part of the perineum.

Stretching the Sciatic Nerve.-The patient lies upon the abdomen with a sand bag under the lower part of the trunk. An incision three inches long is made upon the back of the thigh, the upper end of the incision corresponding to the middle of a line drawn from the 
tuberosity of the ischium to a point a hand's breadth below the great trochanter; this incision passes through the skin and fat down to the deep fascia; the lower edge of the gluteus maximus is now recognized, and at this point the deep fascia, fascia lata, is incised; through the opening thus made in the deep fascia two fingers are introduced and passed under the edge of the gluteus

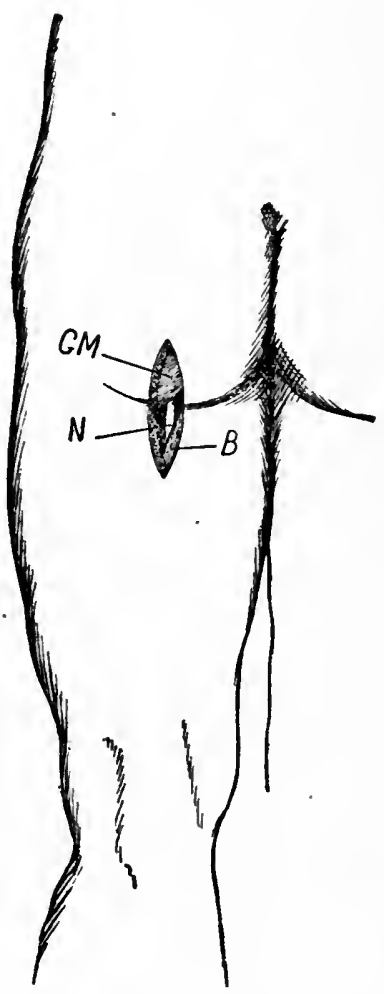

Fig. 200.-Stretching Sciatic Nerve. $B$, tendon of biceps; $G M$, lower edge of gluteus maximus; $\boldsymbol{N}$, sciatic nerve.

maximus, and the sciatic nerve hooked up and drawn out of the wound. Three or four fingers being now passed under the nerve, it may be stretched to the desired degree, pulling with a gradually increasing force up to one hundred pounds; this may be repeated once or twice; in order to regulate the force one may use a scale and hook. No vessels are met with, and it will but rarely be neces- 
sary to apply any ligatures; the wound in the skin is closed without drainage.

The Anterior Femoral Region. - Upon the anterior part of the thigh just below the inner end of Poupart's ligament is the saphenous opening; this is a slit-like opening in the deep fascia, fascia lata, through which the internal saphenous vein passes to join the femoral. Its outer margin presents a prominent, curved, overhanging edge, the falciform process. The femoral vessels are situated beneath the iliac portion of the fascia lata, external and adjacent to this falciform margin, resting upon the pectineus and ilio-psoas muscles (see Femoral Region, Hernia).

This falciform process, or margin, is continuous above with Poupart's ligament, and may be traced farther inward into Gimbernat's ligament; below it curves inward and upward beneath the saphenous vein, and is here continuous with that portion of the fascia lata, pubic portion, which covers the surface of the pectineus muscle, being continued upward upon the surface of this muscle and under Poupart's ligament as far as the pectineal line, where it is attached (see Figs. 148 and 154). In the upper part of the thigh, behind the femoral vessels, this fascia that covers the pectineus muscle is continuous with that which covers the ilio-psoas muscle, the fascia iliaca.

The saphenous opening is partly closed by a wad of fascia, which is adherent around the margin of the opening and which is called the fascia cribrosa. The fascia cribrosa is pierced by the internal saphenous vein, which passes through the saphenous opening and joins the femoral vein on its inner side.

The Ixternal Saphenous Vein lies beneath the fatty layer of the skin; it commences upon the dorsum of the foot, and passes upward in front of the internal malleolus, along the inner side of the leg, and across the knee-joint behind the internal condyle, immediately above which it often presents a pouch-like dilatation; it is continued upward upon the inner, front aspect of the thigh, and just below Poupart's ligament passes through the saphenous opening to join the femoral. It receives many branches all along its course. That part of the vein and its tributaries which correspond to the leg and to the neighborhood of the knee-joint are apt to become very tortuous, dilated, and pouched, exhibiting the common condition known as "varicose veins." Just before it enters the saphenous opening the vein receives many branches from the front and inner side of the 
thigh, all radiating toward the saphenous opening, and here also it receives the veins which correspond to the subcutaneous branches of the femoral artery. The saphenous vein is accompanied by a chain of lymphatics which terminate in nodes located about the saphenous opening, and these may become enlarged and tender when infectious processes are present below in the integument of the leg or thigh.

Those lymphatics which are situated along Poupart's ligament in the groin are usually enlarged when the external genitals are the seat of disease.

In this anterior femoral region also, lying beneath the skin, are found the superficial branches from the femoral artery. The superficial epigastric passes through the saphenous opening and upward across Poupart's ligament to ramify upon the lower abdomen. The superficial external pudic passes through the saphenous opening and inward to supply the skin, etc., of the external genitals. The superfieial circumflex-iliac passes upward and outward, piercing the deep fascia external to the saphenous opening and runs parallel with, and below Poupart's ligament, supplying the skin and glands in this region.

These vessels are usually cut in making the incision for hernia and in extirpating diseased glands in this region.

The Femoral Artery. Scarpa's Triangle.-Upon removing the integument and deep fascia from the upper anterior part of the thigh we expose a triangular space, Scarpa's triangle. This triangle corresponds to the upper third of the thigh; its base, which is above, is formed by Poupart's ligament; its outer border by the sartorius musele, and its inner border by the adductor longus. The apex of the triangle is below where these museles meet. The floor of the triangle is formed, from within outward, by the adduetor longus, the pectineus, and the ilio-psoas.

Passing downward through this space, from the middle of its base-i.e., midway between the anterior superior spine of the ilium and the spine of the pubic bone-to its apex, is the femoral artery accompanied by the femoral vein. The femoral artery is the continuation downward into the thigh of the external iliac, and emerges from the abdomen underneath Poupart's ligament at the point already described. Toward the lower part of Scarpa's triangle the femoral artery is overlapped by the inner edge of the sartorius muscle. 
After traversing Scarpa's triangle the femoral vessels are continued downward along the inner side of the thigh, lying beneath the sartorius muscle, quite close to the femur, within Hunter's canal.

Hunter's Canal is a musculo-fibrous space corresponding to the middle third of the thigh, lying close to the inner side of the femur; its outer wall is formed by the vastus internus, which separates the vessels from the bone; its inner wall by the adductor longus, and

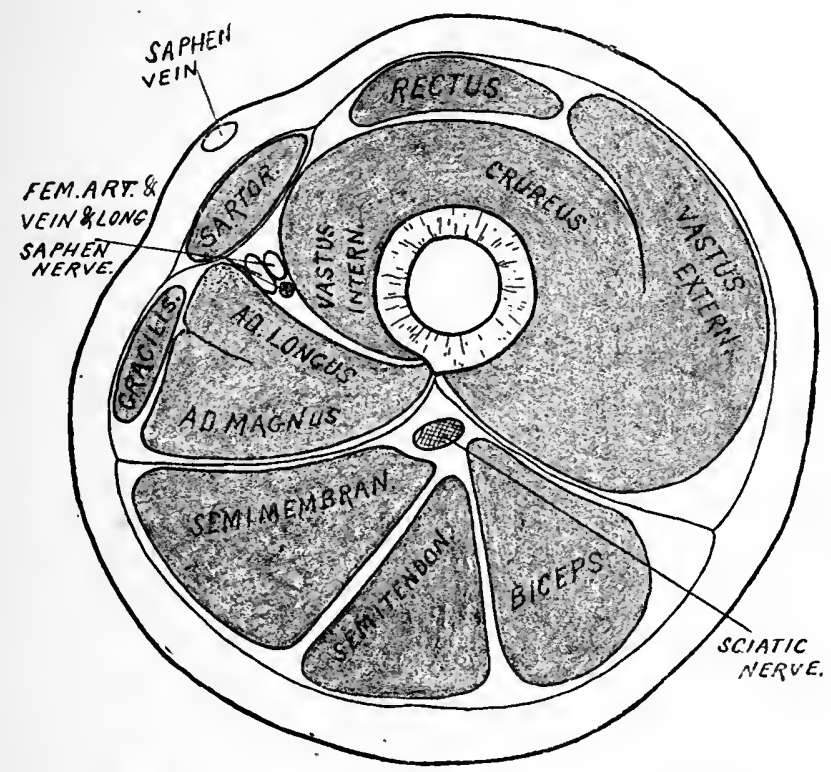

Fig. 201.-Section through the Middle of the Left Thigh.

in the lower part of the thigh by the adductor magnus; the space between the muscles is roofed over by a fibrous sheet, which is derived from the deep fascia. Hunter's canal ends below, above the internal condyle, at the foramen in the adductor magnus muscle, through which the femoral vessels pass into the popliteal space.

About two inches below Poupart's ligament the femoral artery gives off a large branch, the profunda femoris. This vessel arises from the outer and posterior aspect of the femoral artery; at its origin it curves slightly outward and then passes behind the femoral artery and vein, and dips into the floor of Scarpa's triangle, passing 
through the space between the adductor longus and the pectineus; it then descends in the thigh, resting upon the adductor magnus along the inner side of the femur and giving off branches which perforate the adductor magnus to anastomose with branches upon the back of that muscle.

The femoral artery gives off other small branches in Scarpa's triangle, but they are of little surgical importance.

As the femoral artery emerges from underneath Poupart's ligament it is accompanied by the femoral vein, which lies to its inner side. During the course of the artery through Scarpa's triangle the vein gradually gets to lie behind the artery, and in Hunter's canal it is located behind and a little to its outer side.

As the femoral vessels pass out through the femoral space, beneath Poupart's ligament, they are inclosed in a connective tissue sheath, which is continuous with the subperitoneal connective tissue of the abdomen and which is closely adherent all around the margin of the femoral space: above to Poupart's ligament, below to the fascia which covers the ilio-psoas and pectineus muscles, and internally to the margin of Gimbernat's ligament. This femoral sheath is divided into three distinct compartments by fibrous septa; the outer compartment contains the artery, the middle one the vein; the inner compartment contains a small amount of connective tissue and fat, and through it the lymphatics from the thigh pass into the abdomen. This inner compartment is continued but a short distance downward upon the inner side of the femoral vein; it corresponds to the space between the femoral vein and the outer edge of Gimbernat's ligament, and forms the crural canal, into which the gut descends in femoral hernia.

As the vessels emerge from the abdomen under Poupart's ligament they are contained within their sheath, which is, in turn, partially covered anteriorly by that portion of the fascia lata which lies external to the falciform edge of the saphenous opening; underneath Poupart's ligament the vessels within their sheath rest upon the iliopsoas and pectineus muscles.

- The ilio-psoas muscle is covered over by a layer of fascia, the iliac, which is continuous internally with the fascia that covers the pectineus muscle (the pubic portion of the fascia lata). This layer of fascia, which covers the ilio-psoas muscle, is simply the continuation downward, under Poupart's ligament into the thigh, of the fascia iliaca, which covers these muscles within the abdomen. 
The Anterior Croral. Nerve.-At Poupart's ligament, lying to the outer side of the femoral artery and imbedded in the substance of the ilio-psoas muscle, is the anterior crural nerve. This nerve is separated from the femoral artery by the iliac fascia, which invests the ilio-psoas muscle and is not seen in the thigh until this layer of fascia has been incised.

Below Poupart's ligament the anterior crural nerve divides into cutaneous and muscular branches. The internal or long saphenous nerve, the largest of the cutaneous branches, approaches the femoral artery as it lies in Scarpa's triangle, and accompanies it down along the inner side of the thigh, through Hunter's canal. At the lower end of the canal, where the femoral vessels pass through the adductor foramen into the popliteal space and just above the internal condyle, the nerve becomes more superficial, lying beneath the sartorius; below the knee-joint it becomes subcutaneous, and runs down the inner side of the leg in company with the internal saphenous vein, and supplies the skin of the leg.

Ligation of the Femoral Artery. The Common Femoral.The common femoral is sometimes ligated as a preliminary to exarticulation of the thigh at the hip-joint. The vessel is ligated immediately below Poupart's ligament, above the origin of the profunda femoris branch, where it is quite superficial.

An incision about two inches long is made, commencing above, at the middle of Poupart's ligament; i.e., at a point midway between the anterior superior iliac spine and the spine of the pubes. This incision passes through the skin and fat down to the deep fascia, the fascia lata. The pulsation of the artery may be readily felt with the finger in the wound.

The deep fascia is incised and the artery exposed by stripping away its connective tissue sheath. An aneurism needle, carrying a catgut ligature, is passed around the vessel from within outward,i.e., between the vein and artery,-and then withdrawn, thus leaving the artery surrounded by the ligature, which is tied. The femoral vein, which lies to the inner side of the artery, may be tied at the same time, through the same incision. The wound is closed with several interrupted sutures. This procedure makes the exarticulation at the hip-joint practically a bloodless operation.

The Femoral in Scarpa's Triangle.-The femoral artery is occasionally ligated for aneurism involving its lower portion or its continuation; the popliteal. 
For this purpose the ligature is usually applied in the lower part of Scarpa's triangle, about five inches below Poupart's ligament, and therefore below the origin of its profunda femoris branch. The

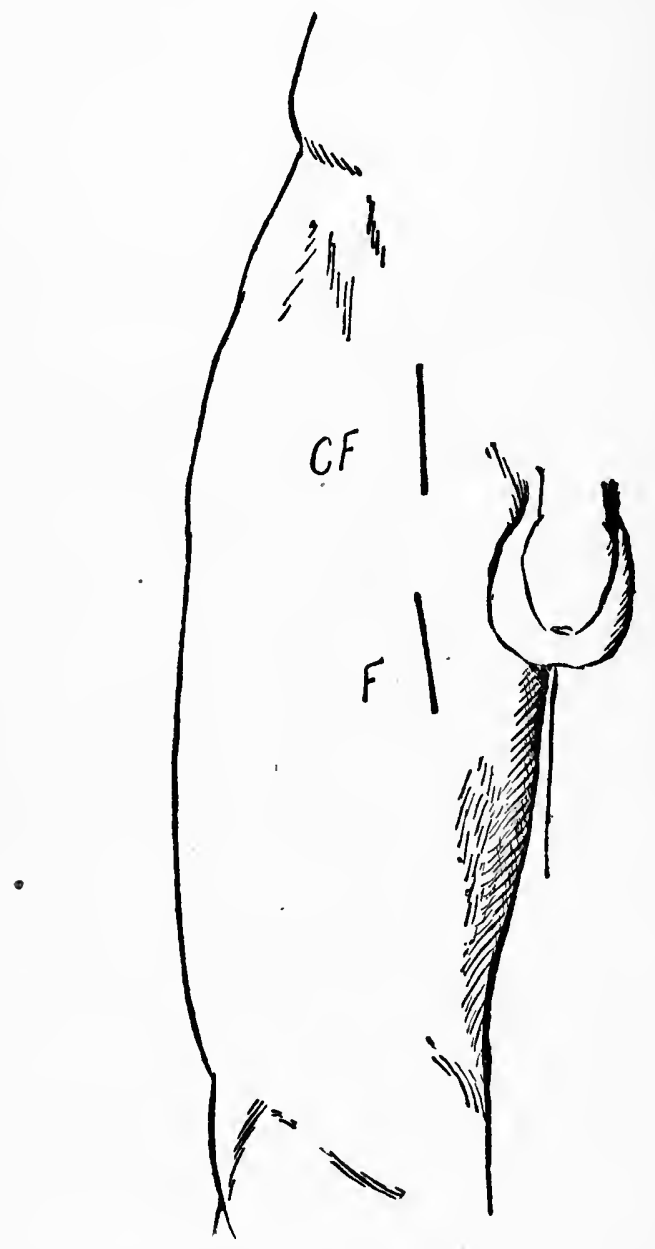

Fig. 202.-Ligation of Femoral Artery. $O F$, incision for ligation of common femoral; $F$, incision for ligation of temoral in Scarpa's triangle.

course of the artery is indicated by a line drawn from a point above, midway between the anterior superior spine of the ilium and the spine of the pubes, to the internal condyle below. The muscular 
guide to the artery, in this part of its course, is the inner border of the sartorius muscle, which slightly overlaps the vessel.

The patient is placed upon the back, with the leg rotated slightly outward. The incision is made about three inches long, corresponding to the inner border of the sartorius muscle; it commences above, about four inches below Poupart's ligament. This incision passes through the skin and subcutaneous fat and through the sheath of the sartorius, exposing the inner edge of this muscle; the muscle is readily recognized by the oblique course of its fibers. In this incision some tributaries of the long saphenous vein are cut and clamped. Having fully recognized the edge of the sartorius muscle, this is drawn outward, and the vessel may then be located by its pulsation beneath the deep fascia; this layer of deep fascia is incised along the course of the artery and the vessel thus exposed. In this situation the vein is found lying behind the artery and still slightly to its inner side; the long saphenous nerve lies a short distance to the outer side of the artery. We may see the internal cutaneous nerve passing obliquely inward across the sheath of the artery.

The loose connective tissue, which forms the sheath of the artery, is now picked up with a thumb forceps and nicked with the point of the knife, and through the opening thus made a director is introduced between the artery and the vein, working around the artery, close to its wall, from within outward. After the artery has been thus isolated a catgut ligature is carried around it, also from within outward, in an aneurism needle. Before tying the ligature one should again investigate to make certain that the artery alone is included, and then tie a single square knot. The incision is closed with several catgut sutures.

The Popliteal Space.-The femoral artery and vein, having passed through the opening in the lower part of the adductor magnus muscle, enter the popliteal space, and are known here as the popliteal artery and vein.

The popliteal space is lozenge-shaped and situated behind the knee. It is bounded above and externally by the biceps; above and internally by the semimembranosus, semitendinosus, gracilis, and -sartorius, the tendons of these muscles being known as the outer and inner hamstrings, respectively. Below and externally the space is bounded by the outer head of the gastrocnemius, and below and internally by the inner head of the same muscle. The floor of the space is formed, from above downward, by the posterior surface of 
the lower end of the femur, the posterior ligament of the knee-joint, and the popliteus muscle.

Passing from the upper angle, through the space, to the lower angle, where it becomes the posterior tibial, is the internal popliteal nerve. In the upper part of the space, emerging from beneath the biceps muscle, is the external popliteal nerve; this nerve passes downward and outward along the inner edge of the biceps tendon.

The popliteal artery, with its accompanying rein, enters the popliteal space above, emerging from beneath the semimembranosus, near the upper angle of the space; therefore in the upper part of the space the artery lies to the inner side of the internal popliteal nerve; about the middle of the space, however, the artery passes underneath the nerve; and in the lower part of the space it is found to the outer side of the nerve.

The popliteal artery lies close to the floor of the popliteal space, separated from the posterior ligament of the knee-joint by a little connective tissue; the vein is placed superficial to the artery and rather to its outer side; the internal popliteal nerve lies superficial to the vessels, crossing them from above downward. The popliteal artery gives off several branches, but they are of no surgical importance.

The popliteal space is covered by the skin and superficial fascia (fat) and by the deep fascia, which is stretched between the hamstring tendons. When the popliteal artery reaches the lower part of the popliteal space it divides into two branches, the anterior and posterior tibial.

It is seldom or never necessary to tie the popliteal; for popliteal aneurism the ligation of the femoral is preferred.

\section{THE LEG.}

The Anterior Tibial Artery.-Just below the lower border of the popliteus muscle the anterior tibial artery passes forward, through an opening in the interosseous membrane between the tibia and the fibula, to the front of the leg; it then passes downward, lying upon the front surface of the interosseous membrane, accompanied by two venæ comites, one on either side. In the upper third of the leg the vessel lies between the tibialis anticus on its inner side and the extensor longus pollicis on its outer side. Upon the front of the ankle the artery lies beneath the anterior annular ligament, having 
the tendon of the extensor longus pollicis on its inner side and the tendons of the extensor longus digitorum on its outer side. Upon the front of the ankle the tendon of the tibialis anticus lies to the inner side of the tendon of the extensor longus pollicis, and the perineus tertius lies to the outer side of the tendons of the extensor longus digitorum. After the anterior tibial artery emerges from beneath the lower border of the anterior annular ligament, it is continued downward as the dorsalis pedis, lying in the first interosseous space, and giving off a branch which passes outward across the tarsus, and, lower down, one which passes outward across the heads of the metatarsal bones. This latter branch, which is

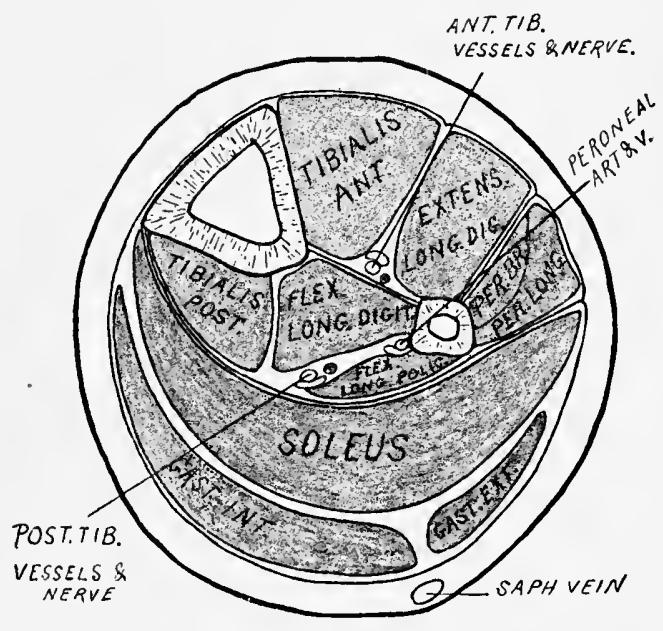

Fig. 203.-Section through the Middle of the Left Leg.

known as the metatarsal, gives off three descending branches, which pass downward upon the second, third, and fourth interosseous muscles as far as the webs of the toes, where they each divide into two lateral branches, which are distributed to the contiguous halves of the adjoining toes. These interosseous branches are for the supply of the adjoining sides of the fifth and fourth, fourth and third, and third and second toes. The dorsalis pedis itself descends upon the first interosseous, this part of the artery-i.e., between the first and second metatarsal bones-being called the dorsalis hallueis; it divides to supply the contiguous sides of the first (big toe) and second toes, supplying also the inner side of the big toe. 
The first dorsal interosseous muscle is perforated above by a large branch of the dorsalis pedis, which passes through to the deep part of the sole of the foot, to anastomose with the external branch of the posterior tibial to form the plantar arch.

The Anterior Tibial Nerve, which is derived from the external popliteal, reaches the anterior tibial artery at the junction of the upper and middle thirds of the leg, and then accompanies it throughout the rest of its course. The nerve reaches the anterior tibial artery, as this ressel lies upon the interosseous membrane, by curving around the upper part of the fibula beneath the extensor longus digitorum. Corresponding to the middle third of the leg, the nerve lies upon the front of the artery, but in the lower part of the leg it lies to the outer side of the artery, and beneath the anterior annular ligament divides into an internal and an external branch.

Ligation of the Anterior Trbial Artery.-The patient lies upon the back, with the knee somewhat flexed and a sand bag placed beneath it. The linear guide to the artery corresponds to a line drawn from the inner side of the head of the fibula to a point below, midway between the internal and external malleoli.

The ressel may be tied in the middle third of the leg, as it lies upon the anterior surface of the interosseous membrane between the tibialis anticus on its inner side and the extensor proprius pollicis on its outer side.

An incision, about two fingers' breadth external to the prominent edge of the shin bone and two or three inches long, is made through the skin and fat down to the deep fascia. The deep fascia is then incised, and working down, between the tibialis anticus on the inner side and the extensor proprius pollicis on the outer side, with the handle of the scalpel, the interosseous membrane is reached. The foot is then somewhat flexed at the ankle-dorsal flexion-to relax the muscles, and retractors are introduced deep into the wound, and the artery, with its venæ comites lying upon it, is exposed. The anterior tibial nerve lies in front of the anterior tibial vessels in this part of their course. After the nerve has been separated from the artery a ligature is carried around the vessel from without inward and tied.

The Posterior Tibial Artery.-This vessel passes down the back of the leg, and below, between the internal malleolus and the tuberosity of the os calcis, it divides into the internal and external plantar. The posterior tibial is larger than the anterior, and at its origin lies deep beneath the muscles of the calf,-_gastrocnemius and soleus,-- 
resting upon the tibialis posticus; from its origin, as it descends, it gradually approaches the tibial side of the leg.

In the lower third of the leg the artery is more superficial, running parallel with the inner border of the tendo Achillis and being covered only by the deep fascia and the integument. The posterior tibial artery is accompanied by two large venæ conites, one on either side of it.

Between the os calcis and the inner malleolus, and beneath the origin of the adductor pollicis, the posterior tibial artery divides into its terminal branches, the internal and exterual plantar. The internal plantar, the smaller, runs along the inner side of the sole of the foot. The external plantar passes outward, beneath the flexor brevis digitorum, lying upon the flexor accessorius as far as the base of the fifth metatarsal bone; it then turns and runs inward to the interval between the bases of the first and second metatarsal bones, where it anastomoses with the large perforating branch from the dorsalis pedis, and thus forms the plantar arch.

From the plantar arch four digital branches descend in the corresponding interosseons spaces as far as the webs of the toes, where they divide for the supply of the adjacent sides of the toes. The contiguous sides of the big toe and second toe and the inner side of the big toe are supplied by the continuation of the perforating branch of the dorsalis pedis, which divides, at the cleft between the big and second toes, into two branches. One passes inward to supply the inner border of the great toe and the other bifurcates to supply the contiguous sides of the great and second toes.

As the posterior tibial artery descends in the middle of the space between the os calcis and the internal malleolus, the venæ comites lie one on each side of it; the posterior tibial nerve, already divided into the internal and external plantar, lies to its outer side; still more externally, close to the os calcis, is the tendon of the flexor longus pollicis, and to the inner side of the artery, lodged in the groove upon the posterior border of the internal malleolus, are the tendons of the tibialis posticus and flexor longus digitorum; of these two, the tibialis posticus being the more internal and the closer to the bone.

Just below its origin the posterior tibial artery gives off a large branch, the peroneal; this branch descends along the fibular side of the back of the leg, covered by the soleus and gastrocnemius and lying upon and partly covered by the flexor longus pollicis. 
The Posterior Tibial Nerve accompanies the posterior tibial artery; it is the continuation of the internal popliteal, and is a large nerve. At its commencement the nerve lies to the inner side of the artery, but, a short distance from its origin the artery passing obliquely inward toward the tibial side of the leg and the course of the nerve being straight; the nerve thereby gets to lie to the outer side of the artery. The posterior tibial nerve continues down the back of the leg upon the outer side of the artery, and divides, in the space between the os calcis and the internal malleolus, into the internal and external plantar.

Ligation of the Posterior Trbial.-This ressel may be exposed and tied just above the ankle-joint and to the inner side of the tendo Achillis. An incision is made about two inches long midway between the posterior border of the inner malleolus and the inner border of the tendo Achillis. This incision reaches through the integument and fat down to the deep fascia. The deep fascia is then incised and the posterior tibial artery exposed; it is found quite superficial, together with its venæ comites, one on either side. To the outer side of the ressels, nearer the tendo Achillis, is the posterior tibial nerve. The veins are separated from the artery, and a ligature then carried around the artery in an aneurism needle, from within outward in order to aroid the nerve, and tied.

Tenotomy.-This operation is done with a narrow-bladed knife through a very small incision in the skin.

Texdo Achillis.- The foot is strongly flexed so as to put the tendon upon the stretch, and a narrow tenotomy knife entered close to the inner border of the tendon and about one and one-half inches above its attachment to the os calcis; the knife is entered upon the flat and pushed through the soft parts in front of the tendon as far as its outer border; the blade of the knife is then turned so that its cutting edge is directed toward the tendon, and with several strokes the tendon is divided. The division of the tendon is really accomplished by strongly flexing the foot and thus making the tendon very tense upon the sharp edge of the knife.

There is no danger of wounding the posterior tibial ressels and nerre if the blade of the knife is introduced close to the inner border of the tendon (see "Posterior Tibial Artery," etc.).

Tendons of the Tibialis Posticus and Flexor Longus DigiToRUM.-These tendons are divided as they descend in the groove upon the posterior border of the internal malleolus. 
The inner edge of this groove, which marks the posterior border of the internal malleolus, should be recognized and the tenotomy knife introduced upon the flat, so that it enters in front of the tendons, between the tendons and the floor of the groove upon the posterior border of the internal malleolus. The knife is then turned so that its cutting edge is directed toward the tendons, and by forcibly flexing (dorsal flexion) the foot and everting it, thus making the tendon tense, their division is accomplished (see "Posterior Tibial Artery," etc.).

Multiple Ligature of the Subcutaneous Veins of the Leg.-This operation is performed for varicose veins of the leg with or without ulcer, and with very satisfactory results.

The operation may, in many cases, be done under local cocain anæsthesia, a few drops being injected into each region just before the incision is made; after several such injections have been made one may often dispense with further injections.

An elastic band is first applied about the thigh, half way between the knee and the hip, and sufficiently tight to obstruct the venous return, but not tight enough to interfere with the arterial current; this serves to make the subcutaneous veins stand out more prominently.

A small incision, usually about one-half inch long, is made with a sharp knife just alongside the vein selected for ligation, care being taken not to wound the vein.

After the vein has been exposed it is separated from its connective tissue bed with a director, and a fine catgut ligature carried around it in the eye of a small blunt-pointed ligature carrier. After the ligature has been tied the small wound in the skin is closed with a single catgut stitch.

The first ligature should be applied to the internal saphena upon the inner aspect of the thigh, several inches above the level of the knee-joint, and the successive ligatures placed below this point, thus gradually working down toward the foot, or, if an ulcer is present, toward the ulcer. Each prominent vein is thus treated, using from ten to twenty separate ligatures, according to the number of veins that are involved.

If an ulcer is prescnt, all the enlarged veins radiating from the ulcer should be ligated, and then the ulcer may be scraped, and, after it has been thoroughly disinfected, covered with skin grafts. Any veins that are cut during the operation should be caught with clamps and tied with catgut. 
AMPUTATIONS, RESECTIONS, ETC.

Surgical Anatomy of the Skeleton of the Foot.-A knowledge of the composition and articulations of the skeleton of the foot is of much practical value in performing the various amputations upon this part.

The tarsus is made up of two rows-or, better, two groups-of irregular-shaped bones. The first row consists of the os calcis and astragalus, the os calcis occupying the outer side of the foot and forming the heel, the astragalus being on the inner side of the foot, par-

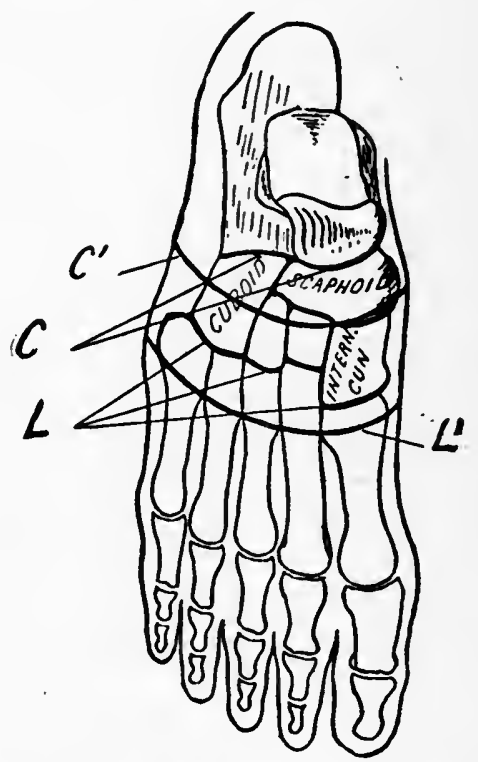

Fig. 204.-Right Foot. $C$, Chopart articulation; $C^{\prime}$, incision for Chopart amputation; $L$, Lisfranc articulation; $L^{\prime}$, incision for Lisfranc amputation.

tially resting upon the os calcis and entering into the formation of the ankle-joint. The anterior, articular surfaces of these bones are on about the same plane, and form an uninterrupted line from the outer to the inner side of the foot. The anterior, articular surface of the astragalus is convex, and is located above and to the inner side of that of the os calcis, which is rather concave.

The second group consists of the cuboid, which is on the outer side of the foot, articulating with the os calcis; the scaphoid, which is on the inner side of the foot, articulating behind with the astragalus; 
and the three cuneiforms. This second group presents anteriorly an irregular row of articular surfaces which is convex toward the toes, its outer end being about one inch nearer the ankle-joint than its inner end.

We next come to the metatarsal bones, five in number, which articulate as follows: The two outer, those of the little toe and the fourth, with the cuboid; the third, middle one, with the external cuneiform; the second with the middle cuneiform; and the first, that of the big toe, with the internal cuneiform. The base of the fifth metatarsal bone presents a prominent tuberosity, which projects outward and is easily felt underneath the skin; this is an important surgical guide. The second metatarsal bone is characterized by its base projecting backward, into the tarsus, beyond the bases of the adjoining metatarsal bones; so that the tarso-metatarsal articular line is interrupted at this point.

We therefore have an articular junction between the os calcis and astragalus behind and the cuboid and scaphoid in front, which we might call the Chopart joint. Through this we do the Chopart amputation. The inner end of the scaphoid presents a prominent tuberosity, which is readily felt beneath the skin just below and in front of the tip of the inner malleolus; this tubercle is the guide to the inner end of the Chopart joint, the outer end of the joint being located one thumb's breadth behind the tuberosity which marks the base of the fifth metatarsal bone.

The articular line between the tarsus behind and the metatarsus in front might be called the Lisfranc junction. This line is curved, with its convexity forward toward the toes. The outer end of the junction corresponds to the base of the metatarsal bone of the little toe, which presents a prominent tuberosity that may be readily felt and which is the guide to the joint. The inner end of the Lisfranc junction is lower than the outer, being about one inch nearer the toes, and may be located two fingers' breadth in front of the tuberosity of the scaphoid.

The line of the Lisfranc articulation is interrupted by the projection of the base of the second metatarsal bone rather less than onefourth inch farther into the tarsus than the third metatarsal, and again by the fact that the articulation between the first metatarsal (big toe) and the internal cuneiform is about half an inch lower, nearer the toe, than that between the second metatarsal and the middle cuneiform. 
Exarticulation of the Big Toe. Oval Method.-The toe is seized with the left hand and a dorsal incision made upon the head (lower extremity) of the metatarsal bone, commencing about one-half inch above the metatarso-phalangeal joint; this incision is carried straight down to a point about one-half inch beyond the web of the toe and then around the toe, cutting everything to the bone.

One should remember that the head of the metatarsal bone of the big toe is large and requires a considerable flap to cover. it. The corners of the flap are seized first on one side and then on the other, and the flap dissected away from the bone. Flexing the toe, the joint is opened upon its dorsal aspect, the lateral ligaments being divided, while the toe is pulled first to one side and then to the other, and finally the remaining attached soft parts are separated, cutting close to the bone and from within outward. Spurting vessels are clamped and tied and the wound closed with four or five interrupted catgut sutures. A small drain may be left in situ for two days. Amputation of the other toes is done in a manner analogous to the above.

Exarticulation of the Big Toe, with Removal of the First Metatarsal Bone.-An incision is made which begins just above the tarso-metatarsal joint, articulation of the metatarsal with the internal cuneiform, which is located about one finger's breadth below the tuberosity of the scaphoid, and this is carried down, upon the dorsal surface of the foot, to the web of the toe, at which point it is carried, in the form of an oval, around the toe (see Fig. 219). This incision, throughout its whole extent, reaches to the bone. The edges of the incision are drawn apart with retractors, and the soft parts separated from the metatarsal bone, after which the joint above, between the metatarsal and internal cuneiform bones, is opened and the metatarsal enucleated out of its bed of soft parts, cutting with the edge of the knife close to the surface of the bone.

The tendons of the big toe are cut short above at the level of the tarso-metatarsal joint. It is unnecessary to use a tourniquet in this amputation. Spurting vessels are caught and tied, and after the bleeding has been checked the wound is closed with several interrupted catgut sutures. The incision may be placed upon the side of the foot instead of upon the dorsum; this is better for drainage, but the scar is not so well located.

Exarticulation of the Little Toe.-Amputation of the little toe and its metatarsal bone may be done in a manner similar to the preceding. 
For Ingrowing Tok-NaIl. Removal of the Offending Half of the Nail. - This operation is done under local cocain anæsthesia. A rubber band is tied tight around the root of the toe for the purpose of confining the cocain to this part and in order to control the hemorrhage. The end of a sharp-pointed scissors is pushed under the nail and down the middle, as far as the root, and with this the nail is split. The half of the nail which is to be removed is then grasped with an artery forceps and torn away from the matrix.

Cotting Operation.-Cocain anæsthesia. A rubber band is tied around the root of the toe. The soft parts, corresponding to the affected side of the toe, are transfixed with a long, narrow-bladed knife and excised. The incision should extend backward well beyond the

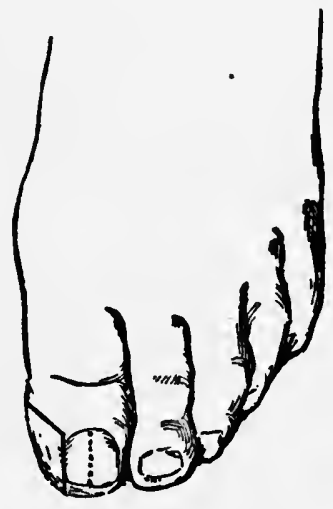

Fig. 205.-Operations for Ingrowing Toe-nail. Solid line Indicates Cotting operation. Dotted line shows line of incision for removal of half of the nail.

root of the nail. In addition, the corresponding half of the nail may be removed as described above. The bleeding digital branch upon the outer side of the toe may be clamped and tied. Although a snug bandage and elevation of the limb usually suffice to control the hemorrhage, still it is wise to ligate the bleeding point. The raw surfaces are disinfected and covered with a wad of gauze and a bandage applied.

amputation through the Tarso-metatarsal articulation (Lisfranc). - A tourniquet is applied just above the knee. The right foot, for example. The foot should extend over the end of the table. The guides to the Lisfranc joint are, on the outer side of the foot, the prominent base of the fifth metatarsal bone (little toe) and, on the inner side, the base of the first metatarsal (big toe) which is located 
a finger's breadth in front of the tuberosity of the scaphoid. The lower part of the foot is grasped in the left hand (the palm of the hand applied to the sole of the foot), with the thumb upon the outer guide and the index finger upon the inner guide, and a curved incision, with its convexity downward toward the toes, is then made; this incision extends across the dorsum of the foot, from its outer to its inner border, commencing and ending a little below the level of the joint, so that when the skin retracts it will not leave the ends of the bones protruding beyond the edge of the flap (see Fig. 204). An incision is then carried down, along the outer and inner borders of the foot, from either end of the dorsal incision, as far as the web of the toes.

The short flap which has been marked out upon the dorsum of the foot is dissected back to the level of the articulation and should include only the integument and the subcutaneous fat.

Now, forcibly flexing the foot, the extensor tendons on the dorsum are divided to the bone and the point of the knife inserted into the joint behind the base of the metatarsal bone of the little toe, and this joint thus opened. The knife is then carried inward across the foot, remembering that the line of the joint is not straight, but convex, the convexity being directed forward toward the toes.

When we reach the point where the base of the metatarsal bone of the second toe projects into the tarsus, the edge of the knife is turned backward toward the ankle for about one-fourth inch, and then, again turning it inward, the joint between the base of the second metatarsal and the middle cuneiform is opened. The edge of the knife is then turned forward toward the toes, and carried in this direction for about one-half inch, in order to reach the level of the joint between the first metatarsal (big toe) and the internal cuneiform, which is then also opened.

Flexing the foot still more forcibly, thus causing the joint to gape widely, the metatarsus, the portion of the foot which is to be amputated, is freed with the point of the scalpel upon its deep plantar aspect, and then, with the long knife, and cutting close to the bone, all the soft parts are separated upon the plantar aspect of the foot down to the webs of the toes, at which point the long plantar flap is cut from within outward and the amputation is complete.

It will be necessary to clamp and tie the dorsalis pedis upon the dorsal surface of the foot, near the inner border, and in the large plantar flap the branches of the plantar arch. 
We have upon the dorsum a short, semilunar flap which is composed of skin and fat only, and upon the plantar aspect a long flap

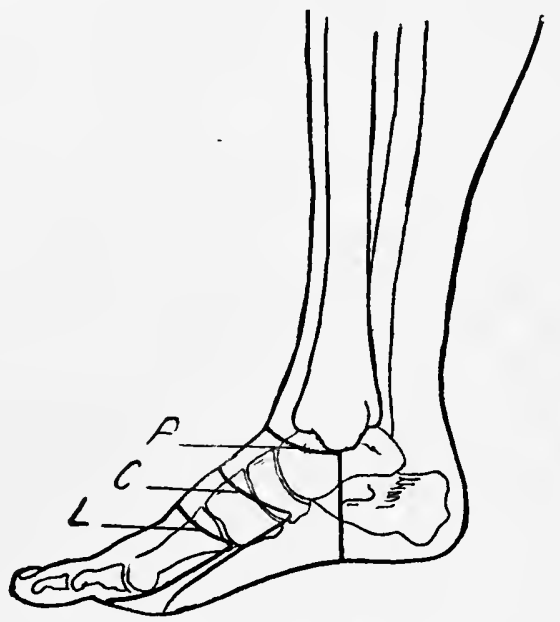

Fig. 206.-Right Foot, Inner Side. $C$, Incision for Chopart; $L$, incision for Lisfranc; $P$, incision for Pirogott.

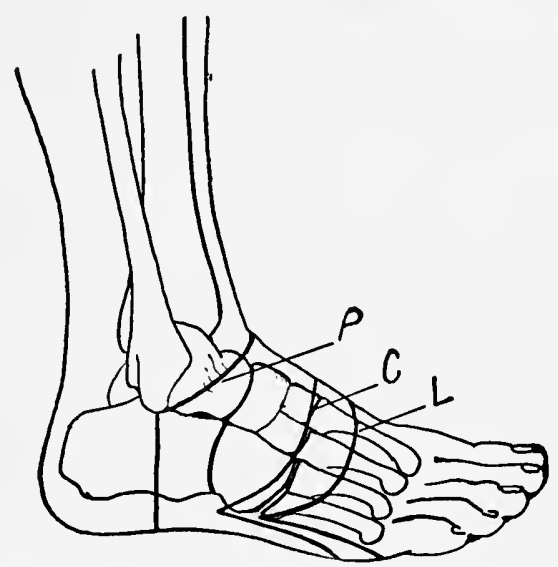

Fig. 207.-Right Foot, Outer Side. $O$, incision for Chopart; $L$, incision for Lisfranc; $P$, incision for Pirogotf.

composed of all the structures of the sole of the foot. The edges of these flaps are brought together with interrupted catgut sutures.

In amputating the left foot it is grasped in the same way by the 
operator, indicating the bony guides with his finger and thumb, the incision being made from the inner toward the outer border of the foot.

Amputation through the Medio-tarsal Joint (Chopart).The tourniquet is placed around the limb above the knee-joint. The right foot, for example. The foot extends over the end of the table. The guide to the Chopart joint, on the inner side of the foot, is the tubercle of the scaphoid; on the outer side of the foot we measure a thumb's breadth behind the tuberosity which marks the base of the fifth metatarsal bone, in order to locate the outer end of the joint. The foot is grasped with the left hand, as described in the Lisfranc, the index finger on the inner guide, tubercle of scaphoid, and the thumb marking the level of the joint externally.

As in the Lisfranc, a short anterior flap is marked out by making a dorsal incision, curved, with the convexity forward toward the toes. This incision commences at the outer border of the foot rather in front of the line of the joint (nearer the toes) and ends on the inner side of the foot, likewise in front of the line of the joint (see Fig. 204). From either end of this dorsal incision a lateral incision is carried forward, along either border of the foot, toward the toes.

The short anterior flap is now seized and, including only the skin and fat, is reflected back a little beyond the line of the joint. Forcibly flexing the foot, the medio-tarsal joint is then opened, from within outward, by inserting the point of the knife into the joint immediately behind the tubercle of the scaphoid so as to enter between this bone and the head of the astragalus; then, continuing outward toward the outer border of the foot, the joint between the cuboid and the os calcis is opened, care being taken not to enter, by mistake, the joint between the astragalus and the os calcis.

Flexing the foot still more forcibly, and thus causing the opened joint to gape, the plantar ligaments, which bind the bones together, are divided with the scalpel, and then a long knife is introduced into the joint and the long plantar flap cut with a sawing motion, the edge of the knife being applied close to the bones, thus separating all the plantar soft parts from the bones as far down as the heads of the metatarsal bones, where, with a cut from within outward, the long plantar flap is completed.

It is necessary to catch the stump of the dorsalis pedis near the inner side of the foot, upon the dorsal surface, and the branches of the plantar arch in the long posterior flap. The dorsal flap is 
short, and consists of skin and fat; the plantar flap is long, and includes all the soft parts of the sole of the foot. The edges of the flaps are united with several interrupted catgut or silk-worm gut sutures.

In operating upon the left foot it is grasped by the surgeon in the same way, the incision marking out the dorsal flap being made from the inner toward the outer border of the foot.

Owing to the action of the tendo Achillis, the stump which results is very apt, after a time, to become extended at the ankle-joint; in order to avoid this the division of the tendo Achillis has been recommended. This, however, helps but little, and many surgeons have discarded this method of amputation entirely.

Surgical Anatomy of the Ankle-joint.-The ankle-joint is formed by the lower ends of the tibia and fibula and the astragalus. The lower ends of the tibia and fibula are bound together by the so-called interosseous ligament, thus forming an arched concavity into which the articular surface of the astragalus is received. The outer portion of the tibio-fibular arch is formed by the external malleolus (lower end of fibula), which extends a finger's breadth lower than the inner malleolus; the vault and inner buttress of the arch are formed by the lower articular surface of the tibia and the inner malleolus. The articular surface of the tibia is broader in front than behind.

The articular surface of the astragalus presents an upper, smooth surface, which slopes downward and backward and which is also wider in front than behind, and is continuous, on each side, with a lateral, smooth facet for articulation with the inner and outer malleoli.

The joint is provided with a capsular ligament, which is described as consisting of several separate portions. Behind, it is very thin and membranous, but is thicker in front and upon the sides.

The capsule is attached above, anteriorly and posteriorly, to the margin of the tibia and fibula, and on the sides to the margins of the inner and outer malleoli; below it is attached to the adjacent rough surface of the astragalus and the os calcis, some of the fibers on the inner side extending forward to the scaphoid.

The joint is provided with a synovial membrane, which is applicd to the inner aspect of the capsular ligament. 
Exarticulation of the Foot at the Ankle-joint (Syme).The right foot, for example. The foot should extend over the end of the table, and is grasped by the operator with the left hand. An incision is made which commences upon the external malleolus, just above its tip, and which is carried straight downward and around the sole of the foot and thence upward as far as the tip of the internal malleolus; this incision reaches to the bone throughout its course. A second incision is made which passes across the front of the anklejoint through the skin, joining the ends of the first incision.

Having incised the integument upon the front of the ankle, the extensor tendons, etc., are exposed; these are divided and the ankle-joint entered by cutting through the anterior ligament. In doing this one should not, by mistake, enter the joint between the head of the astragalus and the scaphoid.

After the anterior ligament has been freely divided the foot is strongly flexed, and then the lateral ligament, upon each side, is divided close to the bone. The joint now gapes, and while a constantly increasing traction is made upon the foot the tendons of the peronei are cut on the outer side and the tendons of the tibialis posticus, etc., on the inner side.

Cutting with the edge of the knife close to the bone, the os calcis is then dissected out of its bed, drawing the foot first to one side and then to the other as this dissection progresses, and occasionally searching with the finger for resisting bands, etc., that interfere with the enucleation of the bone. One should avoid buttonholing the flap, especially as the back part of the os calcis is reached and as the attachment of the tendo Achillis is being separated from the bone; the posterior tibial vessels in the inner side of the flap may also be avoided by keeping the edge of the knife close to the bone.

After the os calcis has been thus enucleated from the soft parts of the heel and the foot removed, the flap is turned up and dissected away from the lower margin of the tibia and fibula for a short distance, in order to make way for the application of the saw. A thin slice of the lower end of the tibia and the malleoli are then removed. The anterior tibial and the internal and external plantar vessels are ligated and the anterior and posterior tibial nerves drawn down and cut short, as are also the ends of any divided tendons that present themselves, and the wound then closed with interrupted catgut sutures. 
If a drain is used, this may emerge through a small longitudinal incision, which is made in the posterior part of the flap upon the outer side of the tendo Achillis. Koenig recommends suture of the divided anterior tendons to the edge of the lower, turned-up flap.

Upon the left foot the incision would be made from the tip of the internal malleolus around the sole of the foot, terminating just above the tip of the external malleolus.

Exarticulation of the Foot at the Ankle-Joint (Pirogoff). -The incisions are the same as in the preceding operation-the Syme. After the ankle-joint has been freely opened, the soft parts are separated from the astragalus and the os calcis backward, beyond the incision that passes through the sole of the foot, as far as the posterior border of the upper articular surface of the astragalus. The soft parts being then retracted, the saw is applied to the upper surface of the os calcis and the bone cut square through upon a plane at right angles to its long axis, and corresponding to the incision that passes through the soft parts around the sole of the foot.

This hooded tegumentary flap, which contains the posterior portion of the os calcis, is now separated from the lower margin of the tibia and fibula, working close to the surface of the bones, and a thin slice of the lower end of the tibia, together with both malleoli, then sawn off. This section is made upon a plane at right angles to the long axis of these bones.

The anterior tibial and the internal and external plantar arteries are ligated and the corresponding nerves are drawn down and cut short.

When the flap is brought into position, the sawn surface of the os calcis and the sawn surface of the tibia are apposed; the edges of the wound are united with interrupted catgut sutures.

If drainage is desired, it may be provided by making a small longitudinal opening in the posterior part of the flap along the outer side of the tendo Achillis. If the traction of the tendo Achillis upon the segment of the os calcis which is left in the flap is considerable, the tendon may be divided subcutaneously.

Koenig advises suture of the ends of the cut anterior tendons to the edge of the turned-up flap to prevent these tendons retracting up the leg, and also to hold the flap in position.

The sawn surfaces of the bones are usually easily retained in apposition by the bandage and dressings, especially if the tendo 


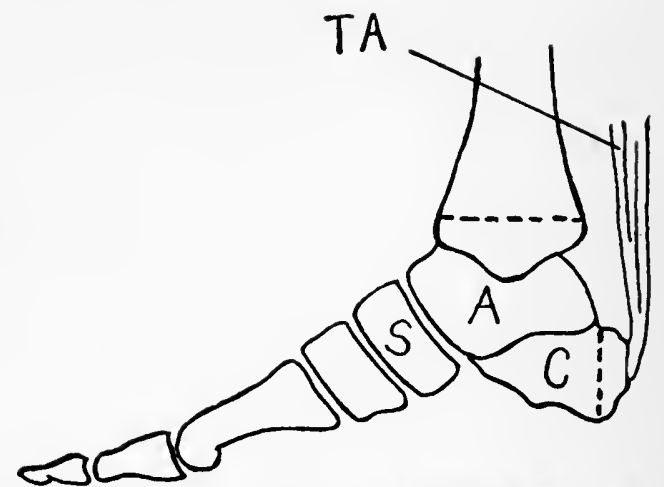

Fig. 208.-Right Foot, Inner Side. $A$, astragalus; $C$, os calcis; $S$, scaphoid; TA, tendo Achillis. Dotted lines show lines of section through the bones in Pirogoff's amputation.

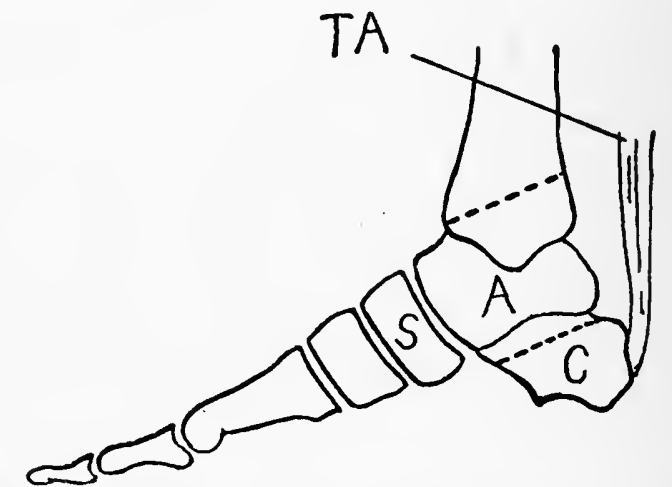

Fig. 209.-Right Foot, Inner Side. Dotted lines show section through bones. Günther's modification.

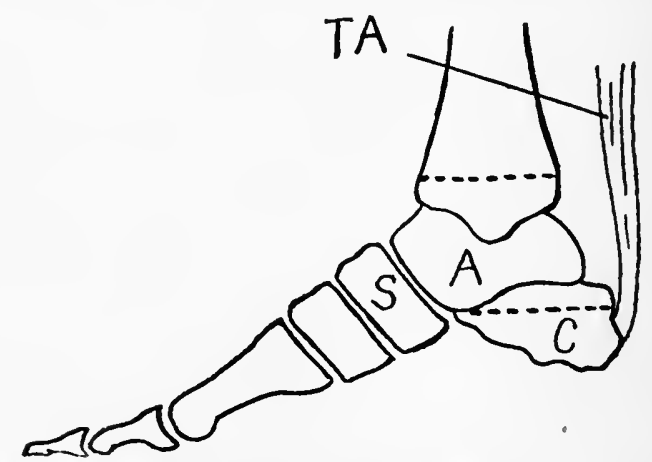

Fig. 210.-Right Foot, Inner Side. Dotted lines show section through bones. Le Fort's modification. 
Achillis has been divided. Some surgeons prefer to fix the segment of the os calcis to the lower end of the tibia by driving a nail through the os calcis into the lower end of the tibia.

Günther's Modification of Pirogoff's Operation. - The incision across the front of the ankle is the same as in the previous operation; the lower incision, which passes through the sole of the foot, instead of passing vertically downward is directed obliquely downward and forward; upon the inner side of the foot this incision passes just behind the tubercle of the scaphoid, and a similar obliquity is also observed upon the outer side of the foot, the incision striking just behind the tuberosity of the base of the fifth metatarsal. The soft parts are dissected back, away from the bones, for a short distance, and, as in the previous operation, the ankle-joint is freely opened and the saw applied to the upper surface of the os calcis behind the astragalus and the os calcis sawn through, not straight down as in the Pirogoff, but obliquely downward and forward so as to end just behind the anterior edge of the lower surface of the os calcis.

The soft parts are then separated from the lower ends of the tibia and fibula, and, being well retracted, the lower ends of these bones are sawn off obliquely from behind forward and downward.

The sawn surface of the os calcis is now applied to the sawn surface of the tibia without any rotation, and thus division of the tendo Achillis is avoided, and, further, that part of the stump which supports the weight and is applied to the ground corresponds to the under surface of the os calcis and the integument covering it.

After the vessels have been ligated the edges of the wound are brought together with interrupted catgut sutures. It may be wise to fix the stump of the os calcis to the lower surface of the tibia with a nail, which is driven through the os calcis into the lower end of the tibia, previously making a small incision in the skin to allow the nail to be introduced. Drainage may be provided as in the preceding operations.

Le Fort's Modification of Pirogoff's Amputation.-A slightly curved dorsal incision is made across the foot, corresponding to the Chopart joint, commencing on the outer side of the foot one inch below and in front of the tip of the external malleolus and ending on the inner side of the foot at the tubercle of the scaphoid. A second incision, passing obliquely forward, is made through the sole of the foot as in Günther's operation, uniting the ends of the dorsal in- 
cision. The integument is then dissected back, and the ankle-joint, under forcible flexion, widely opened as in the Pirogoff.

The upper third of the os calcis, through a plane parallel with the long axis of the bone, is sawn off; this section through the os calcis commences at the posterior end of the bone, after first separating the soft parts and the tendo Achillis sufficiently to apply the saw, and passes forward through the bone as far as the Chopart joint (articulation between the os calcis and cuboid). The foot is then removed, leaving the remains of the os calcis, with the tendo Achillis attached, in the flap. The lower ends of the tibia and fibula, after proper separation and retraction of the soft parts, are then sawn off. The sawn surfaces are apposed and the wound closed. This is a rather difficult operation to perform.

Amputation of the Leg.-The leg may be amputated at any point up to the level of the tuberosity of the tibia. With a view to the use of an artificial limb, one should make an effort to save the knee-joint and as much of the length of the leg as possible.

In amputating the leg we may use flaps of different length, a long anterior and a short posterior, or the reverse, and the flaps may consist of the integument only or may include the muscular tissue as well. The circular method may also be used here, a flap of integument being turned back like a cuff to the point where the muscle and bone are to be divided, and if necessary, owing to the bulging of the muscles of the calf, the circular tegumentary flap may be split, on one or both sides, in order to facilitate its reflection.

It seems to me that lateral skin flaps of equal length, cut in such fashion as to bring the suture line behind the end of the bone, is the preferable operation,- the so-called lateral hooded flap,-yet we should not commit ourselves to any particular method, but take the flaps as best we can when, thereby, more of the length of the limb can be saved.

Amputation of the Leg with Lateral Hooded Flaps.-The tourniquet is placed above the knee. The patient lies with the leg projecting over the end of the table and steadied by an assistant, who grasps it by the foot and elevates it. We must first decide upon the point at which the bones are to be divided, and then make our flaps accordingly (see Fig. 212). The incision is commenced on the front of the limb, one and one-half inches below the level at which the bones are to be divided and just to the outer side of the sharp anterior border of the tibia; from this point the incision curves downward and back- 
ward around either side of the leg, approaching the middle line on the posterior aspect of the limb, where it is carried upward, in the middle line, to a point opposite the level at which the bones are to be divided. This incision extends through the skin and subcutaneous fat down to, but not including, the deep fascia.

Each of the lateral flaps thus marked out should correspond in length to half the thickness of the limb, adding one-third to allow

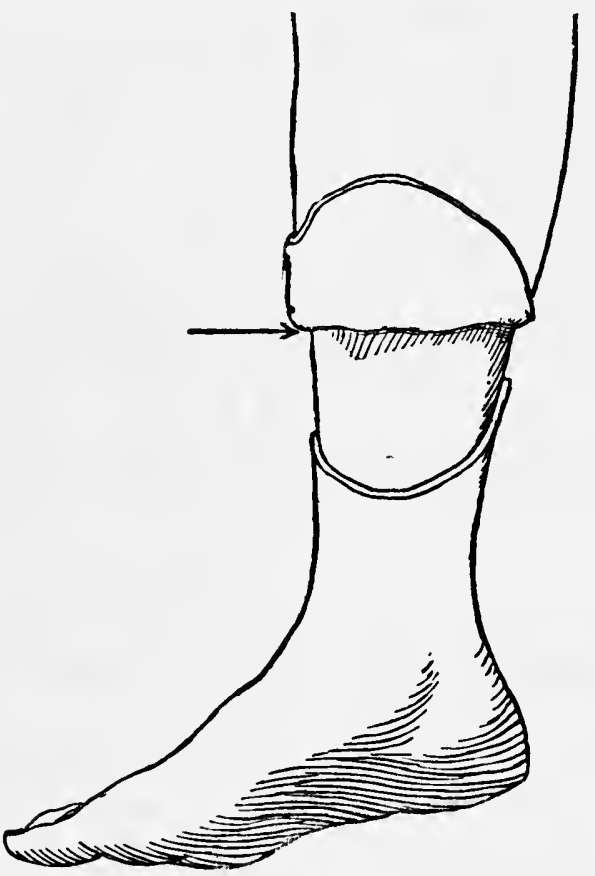

Fig. 211.-Amputation of Leg. Hooded flap of skin and fat turned back. Arrow shows level at which bones are to be divided.

for retraction. The length of the flap is measured from the level at which the bones are to be divided.

The edge of the flap is seized with the fingers, and, making strong traction, it is separated from the deep fascia, taking all the subcutaneous fat with it and eutting with long sweeps of the knife, its edge being always direeted toward the deep fascia in order to avoid cutting the small vessels that ramify in the fat and supply the integument. In reflecting the flap we should work evenly around the whole circumference of the limb. 
After the flaps have been turned back as far as the level at which the bones are to be sawn through, and while they are thus held by an assistant, the muscles are divided with a long knife, down to the bone, with one clean, circular sweep. The muscular tissue between the bones may be divided with a narrow, double edged knife or with a scalpel and the periosteum then incised to make way for the saw.

The heel of the saw is firmly placed upon the edge of the tibia and, drawing back, a groove is made in which the saw works easily. When the tibia is partly sawn through the fibula may be engaged in order to complete the division of both bones simultaneously.

The use of the three-tailed cloth retractor may be dispensed with, as the assistant can better, with his hands or with sharp retractors, hold the divided muscles out of the way of the saw.

While the bones are being sawn the limb is supported below, that its weight may not prematurely break the bones before their section with the saw has been completed.

The prominent anterior angle of the tibia may be sawn off or chiseled away, although this is probably an unnecessary step, especially if the flaps are sufficiently long. The end of the fibula may be cut a little shorter with the bone forceps. In shortening the fibula one should not use the straight bone forceps, as they rather crush and splinter the shaft of the bone; it is better to do this by taking several bites with a sharp rongeur.

Before removing the tourniquet the anterior and posterior tibial vessels are clamped and tied. The anterior tibial is found upon the front of the interosseous membrane between the bones; the anterior tibial nerve may be pulled down and eut short at the same time. The posterior tibial vessels are located in the back of the stump, on the tibial side of the leg, beneath the gastrocnemius and soleus muscles; the large nerve which accompanies these vessels may be pulled down and cut short. The peroneal branch of the posterior tibial artery, which is found just behind the fibula, should also be tied. After the tourniquet has been removed any remaining vessels that bleed may be caught and tied. The edges of the flaps are joined with interrupted catgut sutures, leaving a drain which emerges posteriorly. When the suture is complete, it will be seen that the suture line is located behind the end of the tibia and thus out of the way of pressure. 
Surgical Anatomy of the Knee-joint.-The knee-joint is made up of the lower end of the femur and the upper end of the tibia and the patella. The lower end of the femur is expanded and rather cuboidal in form, having two prominent condyles which project backward beyond the posterior surface of the shaft of the bone.

The inner condyle, when the femur is held perpendicularly, is seen to extend lower than the outer and is also rather narrower than the outer. The inferior and posterior surfaces of the condyles are smooth, rounded, and covered with cartilage; this smooth articular surface is also continued upward upon the anterior surface of the lower end of the femur, extending rather higher externally than internally, and is limited externally by a prominent ridge.

Behind, between the projecting condyles, there is a space large enough to accommodate the thumb, known as the intercondyloid notch; to the contiguous surfaces of this notch the crucial ligaments are attached.

The inner condyle presents upon its inner surface a broad prominence, the inner tuberosity, and to this the internal lateral ligament is attached.

The outer condyle presents upon its outer surface a prominent tubercle, which is located a little behind the center, and to this is attached the external lateral ligament. Immediately below this tubercle there is a smooth groove in which the tendon of the popliteus muscle is lodged.

The lower and posterior portions of the articular surface of the condyles articulate with the articular surface of the tibia; the anterior portion articulates with the patella. The relation of these articular surfaces varies according to the position of the knee-joint.

The upper end of the tibia presents a superior surface, which is divided into two lateral concave, rather ovoidal portions, which articulate with the condyles of the femur, and an intermediate rough area which is marked by a prominence, the spinous process, the summit of which presents two prominent tubercles for the attachment of the extremities of the semilunar interarticular fibro-cartilages. This intermediate space, in front and behind the spinous process, is rough for the attachment of the semilunar cartilages and the crucial ligaments.

The anterior surface of the upper end of the tibia presents a triangular surface, its base corresponding to the anterior border of the upper surface of the tibia and its apex to the tuberosity of the 
tibia. The tuberosity of the tibia gives attachment to the ligamentum patellæ.

The patella presents a smooth posterior surface, covered with cartilage, which articulates with different parts of the articular surface of the condyles in different positions of the knee-joint.

The upper and lateral borders of the patella give attachment to the expanded tendon of the quadriceps; the lower part of the posterior surface, which is rough, gives attachment to the ligamentum patellæ. This ligament, which is attached below to the tubercle of the tibia, fixes the patella to this bone.

The anterior surface of the patella is smooth and is covered by a fibrous expansion from the quadriceps extensor, and is separated from the integument by a bursa which, at times, becomes inflamedhousemaid's knee.

The knee is provided with a capsular ligament which is thin or wanting in places, and is strongly reinforced by expansions derived from the deep fascia (lata) and from the quadriceps and by various accessory ligaments.

In front is the ligamentum patellæ. Behind is the ligament of Winslow, which forms the posterior part of the capsule; this ligament is strong, and extends between the femur and the tibia and is strengthened by bands from the tendon of the semimembranosus, which pass upward and outward from the inner tuberosity of the tibia to the external condyle of the femur; it forms part of the floor of the popliteal space, and the popliteal vessels lie close to it.

The origins of the gastrocnemius, plantaris, and popliteus muscles are intimately connected with the posterior ligament.

Laterally, upon the inner side of the joint, we have the internal lateral ligament, which extends from the tuberosity of the internal condyle to the upper part of the internal border of the tibia, and upon the outer side the external lateral ligament, which is attached above to the tubercle on the external condyle and below to the head of the fibula. These lateral ligaments are attached behind the center of the condyles, and are therefore put upon the stretch by any attempt at overextension of the knee-joint. The capsule is further reinforced, on the sides, by the broad expansions that are derived from the quadriceps extensor and the fascia lata; these are attached to the sides of the patella.

Within the joint are the ligamenta alaria, which are simply redundant folds of the synovial membrane that are reflected from 
the sides of the patella; these are prolonged downward and backward as the ligamentum mucosum, which is attached behind to the femur in the intercondyloid notch between the condyles.

The crucial ligaments, two in number, pass between the lower end of the femur and upper surface of the tibia, crossing one another, and help to fix the bones. The internal passes from the outer side of the internal condyle downward, backward, and outward, and is attached to the rough portion of the upper surface of the tibia behind the spine. The external extends from the inner side of the external condyle downward, forward, and inward and is attached to the rough space in front of the spine of the tibia.

Within the joint, interposed between the articular surfaces of the femur and tibia, are the two semilunar fibro-cartilages, the internal and the external. Placed upon the upper surface of the tibia, they serve to deepen the concavity which receives the articular surface of the femur. They are semilunar in form, and are attached by their borders to the margin of the upper surface of the tibia and to the inner contiguous surface of the capsule; by their extremities they are attached to the rough middle portion of the upper surface of the tibia between the two articular surfaces.

The synovial membrane of the knee-joint is very extensive; it lines the inner surface of the capsule and gives off a large pouch, which extends upward upon the front of the femur beneath the quadriceps extensor; as the ligamenta alaria, the synovial membrane is reflected from the sides of the patella and is continued backward as a process, the ligamentum mucosum, to the back of the femur, between the two condyles, where it is attached. The synovial membrane lines both surfaces of the semilunar cartilages and invests the crucial ligaments, and often communicates with the synovial lining of the tibio-fibular joint and with the bursæ adjacent to the kneejoint. It gives a process externally which is found between the margin of the external semilunar cartilage and tendon of the popliteus muscle, forming a bursa for this tendon. A pad of fat is wedged into the joint below the patella, being covered by the synovial membrane of the joint and prolonged into the ligamentum mucosum.

The Burs $x$ Adjacent to the Knee-Joint.-The arrangement of the bursæ about the knee-joint is somewhat irregular.

Posteriorly. On the outer side: First. Between the posterior 
part of the capsule and the outer head of the gastrocnemius there is a bursa which sometimes communicates with the joint.

Second. Beneath the tendon of the popliteus there is a bursa which always communicates with the joint.

Third. Occasionally there is a bursa between the tendon of the popliteus and the external lateral ligament.

Inner side: First. Between the inner head of the gastrocnemius and the posterior part of the capsule there is a bursa which often communicates with the joint and sends a process between the gastrocnemius and the semimembranosus.

Second. Between the semimembranosus and the head of the tibia.

Third. Occasionally between the tendons of the semitendinosus and semimembranosus.

Anteriorly. First. Between the anterior surface of the patella and the integument.

Second. Between the ligamentum patellæ and anterior surface of the tibia (tubercle tibiæ).

Exarticulation of the Leg at the Knee-Joint (Stephen Smith Hooded FlaP).-The patient lies upon his back, with the leg overhanging the end of the table. One should remember that the end of the femur is large and that a considerable flap is required to cover it. The tourniquet is placed above the knee, high up.

The incision, which passes through the integument and fat down to the deep fascia, commences in front, one inch below the tubercle of the tibia; from this point it curves downward and backward across either side of the leg, and behind, near the middle line, is carried upward into the popliteal space as high as the level of the kneejoint. Two lateral flaps with rounded corners are thus marked out. One should avoid making the flap scant by getting well upon the posterior aspect of the leg before turning the incision upward into the popliteal space.

This tegumentary flap, which includes the subcutaneous fat, is now seized with the fingers and dissected away from the deep fascia with long sweeps of the knife, its edge being directed toward the deep fascia so as not to cut into the flap. Considerable traction should be applied to the flap as it is being reflected, in order to facilitate its separation from the deep fascia. The flap should be dissected up to the level of the joint all around. While the flap is retracted the knee-joint is sharply flexed and entered, cutting first 
through the lower part of the ligamentum patellæ; the blade of the knife is then introduced, flatwise, between the semilunar fibro-cartilages and the upper surface of the tibia, and the cartilages separated

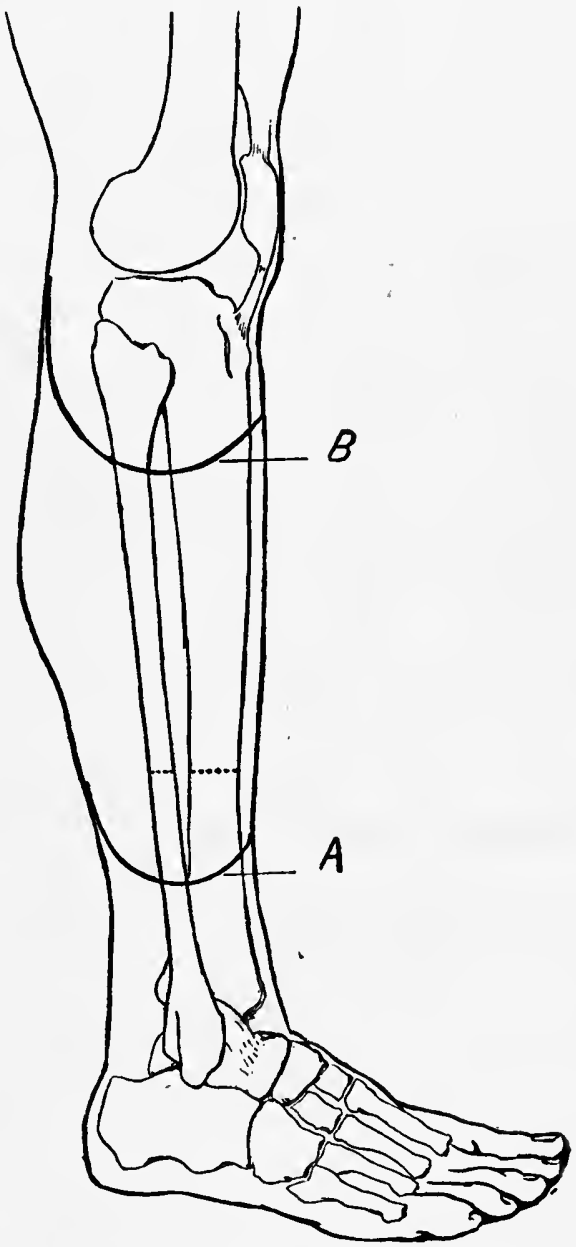

Fig. 212.-Right Leg, Outer Side. A, outline of hooded skin flap in amputation of the leg. Dotted line shows line of division through bones. $B$, outline of skin flap in Stephen Smith hooded flap for exarticulation at the knee-joint.

all around from the edge of the upper surface of the tibia, so that they may be left attached in the stump after the leg has been amputated. 
The lateral ligaments are cut on each side, and with the limb still strongly flexed the attached ends of the fibro-cartilages and the crucial ligaments are cut away from the upper surface of the tibia, and then, with a long knife, the soft parts behind the joint, the posterior ligament, popliteal vessels, etc., and tendons and muscle, are cut square through from within the joint. The amputation is thus complete.

The popliteal artery and its vein, which lies upon (superficial to) it, are each seized and tied. They lie close to the posterior surface of the femur. The popliteal nerves are pulled down and cut short. The edges of the flap are united with interrupted catgut sutures, a space being left posteriorly for drainage.

This operation gives us a good, broad, fairly flat stump, with the suture line behind the extremity of the bone. The reason for leaving the fibro-cartilages in the stump is that they tend to make a better base to the end of the femur.

Transcondylar Amputation at the Knee-Joint (Carden).A long anterior and a short posterior flap are made, the femur being divided through the condyles. Both legs hang over the end of the table, the one to be amputated being extended and supported by an assistant, who grasps the foot. In amputating the right limb the operator stands on the outer side of the leg and with the thumb and forefinger indicates the points at which the incision commences and ends.

A long anterior flap is marked out by an incision which passes through the skin and subcutaneous fat down to the deep fascia. This incision commences at a point a little behind the middle of the internal condyle and upon a level with the knee-joint; it passes down the inner side of the leg as far as the tubercle of the tibia, swings outward across the front of the leg, passing below the tubercle of the tibia, and is then carried upward upon the outer side of the leg to a point upon the outer condyle opposite that at which the incision began upon the inner condyle.

In operating upon the left leg the operator may stand upon the inner side of the limb, making the incision from the outer condyle around to the inner. The corners of the flap should be rounded, but the flap should not be tongue-shaped.

The edge of the anterior flap is seized with the fingers, and the flap, consisting of the skin and subcutaneous fat, is dissected away from the deep fascia and reflected as far as the lower border of the 
patella; in thus detaching the tegumentary flap the edge of the knife should always be directed toward the deep fascia. The knee is then

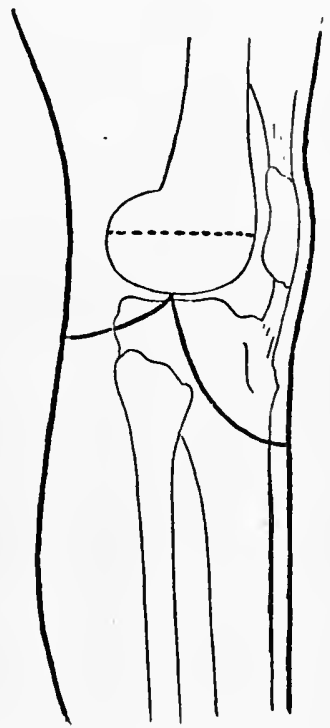

Fig. 213.-Right Leg. Carden's Amputation. Solid line indicates flaps.

Dotted line shows line of division through the condyle.

flexed and the joint opened from in front with the long knife, which first divides the ligamentum patellæ and then passes straight through

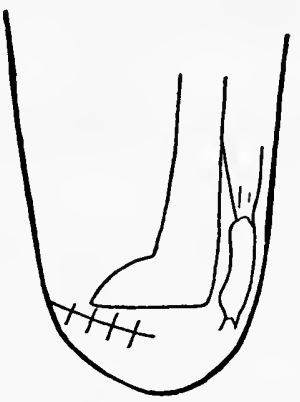

Fig. 214.-Stump After Carden's Amputation.

the joint, cutting capsule, lateral ligaments, and crucial ligaments, and emerging through the structures in the popliteal space; as the knife passes through the integument in the popliteal space the assist- 
ant should draw the soft parts upward toward the hip, and the knife may be turned somewhat downward in order that the posterior flap may not be cut too short, as the integument in this region tends to retract very much.

The soft parts are then separated about the circumference of the condyles and retracted, and the saw applied, the section being made, not above, but directly through, the condyles proper. The sharp edge of the sawn surface of the condyles may be rounded off somewhat with a file or with a rongeur bone forceps. The popliteal artery and vein are found posterior to the bone, and should be tied separately and the popliteal nerves drawn down and cut short.

The stump is covered over by joining the edges of the long anterior skin flap and the short posterior flap with interrupted catgut sutures. It is wise to drain the synovial pouch, which is located in front of the lower end of the femur, under the quadriceps extensor, by introducing two tubes, which reach well up into the pouch, emerging through the incision on either side.

Ampdtation at the Knee-Joint (Gritti-Stokes).-The position of the patient is the same as described in Carden's amputation. A long anterior flap is marked out by an incision commencing upon the internal condyle just behind its middle, and passing down the side and then across the front of the leg just below the tubercle of the tibia, and thence upward to a point on the outer condyle a little behind its center. The flap thus outlined is like the Carden, but somewhat shorter. The edge of this anterior flap is seized with the fingers and, including all the subcutaneous fat, is separated from the deep fascia, cutting with the edge of the knife directed toward the deep fascia and constantly making considerable traction upon the flap. At the lower border of the patella, the flap being retracted and the leg flexed, the knee-joint is opened from before backward, cutting with the long knife through the ligamentum patellæ, capsule, and lateral and crucial ligaments, and finally through the posterior ligaments and the parts in the popliteal space. While cutting through the integument in the popliteal space the skin should be drawn well upward toward the hip-joint so that the posterior flap may not be eut too short. There should be a short posterior flap, one-half to one inch long.

The soft parts are separated from the lower end of the femur, working with the edge of the knife close to the bone, to a point beyond the upper limits of the articular surface; here a circular cut is made around the bone, and with the saw the end of the femur is removed 
parallel with the plane of its inferior articular surface. After the articular end of the femur has been removed, the patella, being surrounded by a towel to give a good, firm grip, is seized with the left hand and the whole of its articular surface sawn off. The sawn surface of the patella is then apposed to that of the lower end of the femur, to which it is fixed by two chromicized catgut sutures, which are passed through drill holes in the posterior edge of the femur and the lower border of the patella. The patella may also be fixed to the femur by a nail driven through it into the femur. The popliteal vessels require ligation. A tube may be introduced on each side

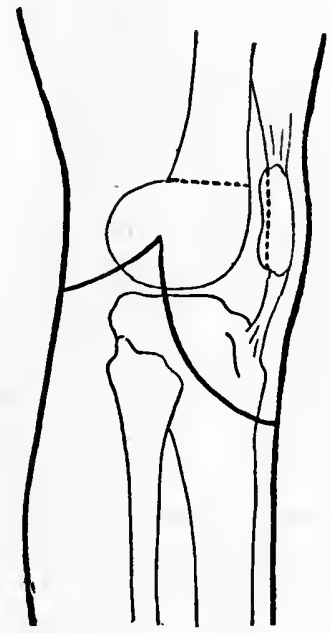

Fig. 215.-Gritti-Stokes Amputation. Solid lines indicate flaps. Dotted lines show section through femur and patella.

to drain the large synovial space under the quadriceps extensor tendon. The edges of the wound are sutured with interrupted stitches of catgut.

Amputation of the Thigh.-As a rule, this is accomplished by a modified circular in two-or, better, three-steps, the skin being divided upon one level, the muscles upon another, and the bone upon a third. A tourniquet is placed about the limb, high up, near the hip-joint.

The thigh should hang over the end of the table. For either the right or the left thigh it is probably more convenient for the operator to stand upon its outer side. An assistant steadies the thigh by grasp- 
ing it above and drawing the integument a little toward the hip. A second assistant may support the limb below.

The point at which the bone is to be divided is first located, and then, with a sweep of the long amputating knife, a circular incision is made around the limb through the skin and fat down to the deep fascia, thus marking the lower limits of the skin flap. This circular incision in the skin should be placed below the point at which the bone is to be divided a distance equal to half the diameter of the limb at that point (where the bone is to be divided), adding one-third more to allow for retraction.

The edge of the skin fiap is seized with the fingers and the flap reflected like a cuff, separating it from the underlying deep fascia with long sweeps of the scalpel, its edge being always directed toward the deep fascia in order to avoid cutting into the flap. While the flap is being dissected away from the deep fascia, upon the posterior aspect of the thigh, the limb may be elevated by the assistant.

After the flap has been dissected back to within one inch of the point at which the bone is to be divided, the long knife is again taken and the muscles are cut, with a circular sweep, down to the bone. The muscular tissue is then scraped back away from the bone with a blunt instrument as far as the point at which the bone is to be divided. While the assistant retracts the skin and muscles with his hands or sharp retractors, a circular incision is made through the periosteum around the bone, and then, planting the heel of the saw upon the bone, it is drawn firmly backward, thus making a groove for itself, and the bone is then quickly severed; the assistant supports the limb lightly below in order that the bone may not be broken before it is sawn completely through. The limb should not be so held by the assistant as to jam the saw.

The femoral and profunda femoris arteries and veins, which are located close to the inner side of the femur, are tied separately, and the tourniquet then removed, after which any remaining bleeding points may be clamped and tied.

While seeking these bleeding points only a limited part of the surface of the stump need be exposed at one time, the rest being covered and compressed with a hot gauze pad. The chief bleeding points are sought between the muscles. The sciatic nerve, which is found between the muscles on the back of the thigh, is pulled down and cut short.

The edges of the flap are brought together from side to side, 
making a transverse line, with interrupted catgut sutures. It is usually wise to leave a drain for several days. If the subject is very muscular and the limb very thick, it may be necessary to incise the flap on one side in order to facilitate its reflection.

This is probably the preferable method of amputating the thigh. Instead of the above described method, one may use a long anterior and a correspondingly shorter posterior tegumentary flap, or flaps which include all the muscle down to the bone as well as the skin may be used.

Surgical Anatomy of the Hip-joint.-The hip-joint is composed of the upper end of the femur and the acetabular cavity of the os innominatum.

The upper end of the femur presents a rounded head which represents about two-thirds of a sphere; it is smooth, covered with cartilage, and is marked in the apex of its posterior, inferior quadrant by a depression in which is attached the ligamentum teres. The head of the femur is directed upward, inward, and forward.

The head of the femur is joined to the shaft by the neck, which passes from the head downward and outward to the shaft; the neck is somewhat flattened from before backward, and is broader at its junction with the shaft than with the head, and is narrowest midway between these points.

The upper end of the shaft presents upon its outer aspect the great trochanter, a prominent, square-shaped mass of bone. The external surface of the great trochanter is continuous with the external surface of the shaft, and is marked by a rough line that passes obliquely from above downward and forward; to this line is attached the gluteus medius muscle; the smooth surface below and behind this line is covered by the gluteus maximus, a bursa being interposed.

The inner surface of the trochanter is applied to the shaft of the bone, except for its upper, posterior part, which is free and hollowed out to form the digital fossa; here the tendon of the obturator externus is attached, and this attachment must be separated before one can dislocate the head of the femur backward in doing a resection of the hip-joint.

The prominent upper border of the great trochanter is free, and gives attachment to the tendons of the obturator internus and gemelli in front and to the tendon of the pyriformis behind. The anterior border of the trochanter major gives attachment to the 
gluteus minimus; its posterior border is thick and rounded and limits the digital fossa behind.

On the inner side of the shaft, at its junction with the neck, is the trochanter minor; it is smaller than the trochanter major, prominent, and pyramidal; to it and to the shaft of the bone immediately below it is attached the ilio-psoas muscle.

Upon the front of the bone, commencing above and externally at the great trochanter and curving obliquely downward and inward and passing around the inner side of the shaft, just below the lesser tuberosity, is the so-called spiral line. This line, on the back of the bone, runs into the linea aspera, forming one of the arms of this prominent ridge. This spiral line is well marked, and upon the front of the bone gives attachment to the capsular ligament.

Upon the posterior aspect of the bone, a prominent, rounded line is presented, which runs from the posterior border of the great trochanter downward and inward to the lesser trochanter; this is known as the posterior intertrochanteric line.

The acetabulum is a large cup-shaped depression corresponding to the junction of the three portions (pubes, ilium, ischium) of which the os innominatum is formed. This cavity extends downward and inward as far as the edge of the obturator foramen, and its floor looks downward, outward, and forward; it is surrounded by a sharp, prominent ridge whose summit gives attachment to the ring-like cotyloid fibro-cartilage which serves to deepen the cavity, constricting its orifice and gripping the head of the femur, thus assisting in retaining it within the socket of the joint. In order to dislocate the head of the bone, in resecting the hip-joint, it is necessary to nick this cotyloid ligament.

The lower portion of the margin or rim of the acetabulum, that part which is adjacent to the obturator foramen, is interrupted by a wide, deep notch, the cotyloid notch. In the recent state this notch is bridged over by a ligamentous band, the transverse ligament; that part of the ring-like cotyloid fibro-cartilage which corresponds to the notch is applied to the upper surface of the transverse ligament. The transverse ligament converts the cotyloid notch into a foramen, through which vessels, nerves, etc., pass into the hipjoint.

The floor of the acetabulum is partly articular and partly nonarticular; the articular part is the smooth, horseshoe-shaped surface which occupies the periphery of the cavity; the non-articular portion 
is the rough, depressed area which occupies the middle of the cavity and is prolonged down along the floor to the site of cotyloid notch; this non-articular, depressed surface lodges a mass of fat and its margins give attachment to the ligamentum teres.

The hip-joint is provided with a capsular ligament, which is attached above around the margin of the acetabulum and transverse ligament (which completes the circumference of the acetabulum below); below it is attached to the femur; in front, to the spiral line as far as the lesser trochanter; behind it is attached to the surface of the neck proper, one-half to two-thirds inch above, away from, the posterior intertrochanteric line. The capsule is materially strengthened by the circular fibers that are woven into it (ligament of Webber).

The capsule is reinforced by three auxiliary bands of fibers. The most important is the ilio-femoral band, which is thickest, widest, and longest; it is attached above to the ilium just below and behind the anterior inferior spinous process and below spreads out and is attached along the spiral line, from the greater to the lesser trochanter; it is known as the " $Y$ " ligament of Bigelow.

The ischio-femoral band is attached to the ischium behind and below the acetabulum (to the upper part of the groove for the tendon of the obturator externus), and to the femur it is attached at the upper part of the trochanter major and spreads out and encircles the capsule.

The pectineo- or pubo- femoral band is thin, and attached to the pectineal eminence on the os innominatum and to the neck of the femur behind the ilio-femoral band, being incorporated with the lowermost fibers of the ilio-femoral band.

The transverse ligament is a fibrous band that bridges across the notch in the lower part of the rim of the acetabulum, thus converting the cotyloid notch into a foramen.

The cotyloid ligament is a complete fibro-cartilaginous ring which is attached to the edge of the bony rim and the transverse ligament, encircling the acetabulum and deepening the cavity and constricting its orifice.

The ligamentum teres is an interarticular fibrous band which passes between the head of the femur and the bottom of the acetabulum. It is attached in the bottom of the acetabulum to the margins of the rough space and to the transverse ligament; its narrow end is attached to a dimple which marks the apex of the posterior 
inferior quadrant of the head of the femur. It is usually a strong band.

The rough depression in the bottom of the acetabular cavity is filled in with a cushion of fat in which the vessels that pass along the ligamentum teres to supply the head of the bone are lodged.

The synovial membrane of the hip-joint lines the inner surface of the capsule, covers the mass of fat in the floor of the acetabular cavity, and is thence reflected upon the ligamentum teres as far as the head of the femur as a tubular prolongation, and thus practically shuts the teres ligament out of the cavity of the joint.

A large bursa lies beneath the ilio-psoas muscle upon the front of the capsule; this often communicates with the joint. Smaller bursæ are located between the various tendons and adjoining bony parts, etc.

The hip-joint is covered in front by the ilio-psoas and the pectineus muscles; on the outer side by the glutei; behind by the gluteus maximus, pyriformis, obturator internus and gemelli, and quadratus femoris; internally and below by the obturator externus.

Exarticulation of the Thigh at the Hip-Joint (Wyeth).The patient lies upon the back with the thigh extended over the end of the table. In order to prevent slipping of the tourniquet, which is placed about the thigh for the purpose of compressing the femoral vessels and thus controlling the hemorrhage, two long pins are introduced through the soft parts, the ligature being applied above these. The pins are about ten inches long and are introduced as follows:-

One, transfixing the soft parts on the outer side of the thigh, is introduced one inch below the anterior superior spine of the ilium, and, passing backward through the soft parts for a distance of about three inches, emerges about one inch below the crest of the ilium; this pin transfixes the upper part of the tensor vaginæ femoris muscle.

A second pin is introduced through the soft parts on the inner side of the thigh, one inch below the pubic bone; it passes through the adductor muscles, and emerges posteriorly one inch below the tuberosity of the ischium; in introducing this inner pin one must avoid injuring the femoral vein. The femoral artery passes into the thigh underneath Poupart's ligament at a point which corresponds to the middle of a line drawn from the anterior superior iliac spine to the pubic spine. The femoral vein lies just to the inner side of the 
artery. Corks are applied to the sharp points of the pins after they have been introduced to prevent one's pricking one's self.

The tourniquet is placed around the thigh above the pins, which prevent its slipping down. A pad may be placed beneath the tourni-

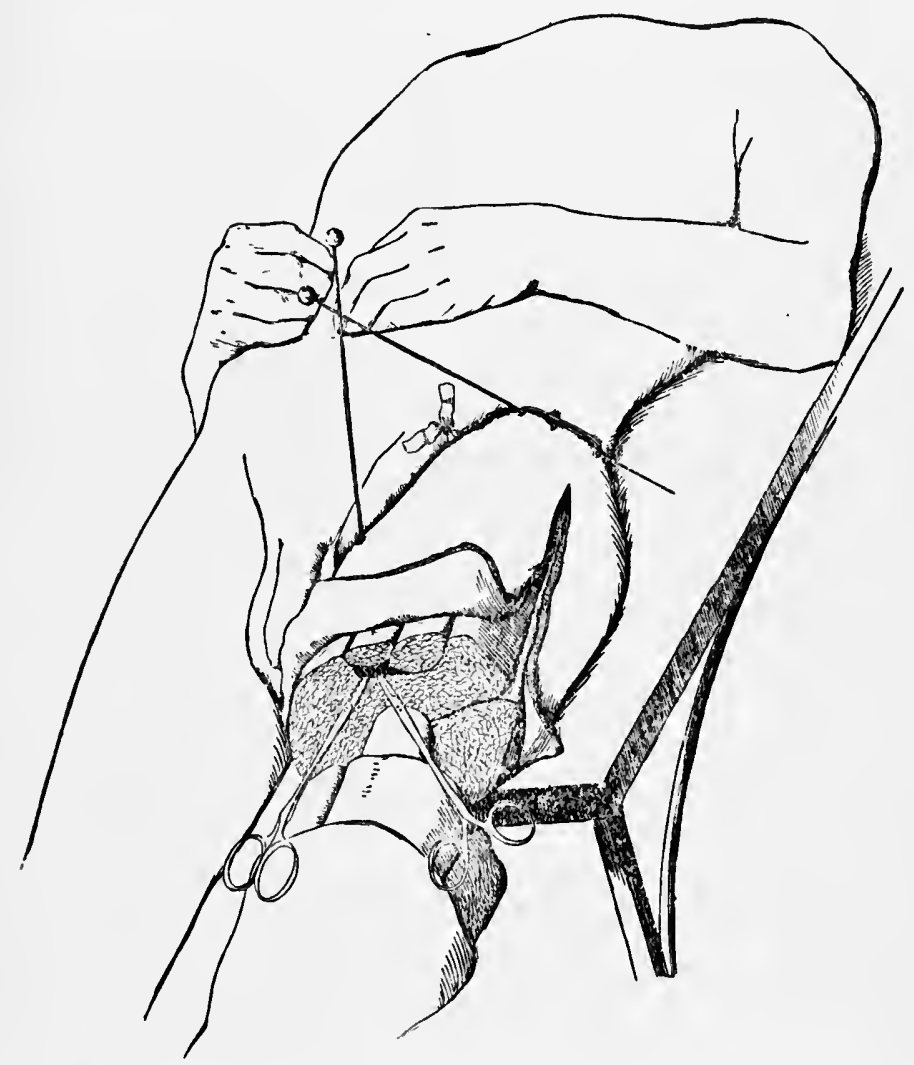

Fig. 216.-Exarticulation at Hip-joint. Wyeth pins in place to prevent ligature from slipping. Upon the outer side of thigh the incision reaches to the bone. A circular skin flap has been turned back and the muscles and blood-vessels divided down to the bone. Clamps applied to femoral artery and veln.

quet, upon the front of the thigh, corresponding to the location of the femoral vessels, to still further secure their compression.

The operator stands on the outer side of the limb, which is supported by an assistant. With a long knife a circular incision is made through the skin and fat down to the deep fascia; this in- 
cision should encircle the thigh a hand's breadth (five inches) below the perineum.

With a stout scalpel a second incision is made along the outer side of the thigh. Commencing above the great trochanter, this incision is carried downward, upon the surface of the trochanter and along the outer side of the thigh, as far as the circular incision, where it terminates. This second incision should reach to the bone throughout its entire extent.

The edge of the skin flap which is marked out by the circular incision is seized and dissected away from the deep fascia for a distance of about three inches. At this point, the skin flap being retracted, a circular cut is made with the long knife, through the muscles, down to the bone, dividing the vessels, the femoral and the profunda femoris, which lie in front and internal to the bone. These vessels are now sought, clamped, and tied. In order to get better access to the vessels the muscles may be scraped downward away from the shaft of the bone for a short distance. We should make sure of the femoral artery and vein and the profunda femoris and its vein; these latter lie in a deeper plane than the femoral vessels. Any other vessels which may be visible, searching in the spaces between the bundles of muscle, are also ligated.

The tourniquet may now be removed, gradually loosening it and catching additional vessels as they bleed, and then the pins are withdrawn or the tourniquet and pins may be left until after the bone has been enucleated and the amputation is complete, but in all cases the main vessels should always be secured immediately after the circular cut through the muscles has been made.

The next step in the operation is the separation of the soft parts from the shaft of the bone and the dislocation of the head of the bone from its socket. The soft parts are retracted and stripped away from the bone, working with the edge of the knife close to the bone and rotating the limb first inward and then outward to facilitate this part of the operation. After the shaft of the bone has been denuded of its soft parts up as far as the capsule of the joint, the joint is opened by incising the capsule and the cotyloid fibro-cartilage, and the head of the bone is then thrown out of its socket, cutting or tearing the ligamentum teres, and any remaining soft parts, and thus completing the exarticulation.

After ligating any bleeding points that show themselves and having cut the nerves short, the edges of the skin are united with in- 
terrupted catgut or silk-worm gut sutures, taking, besides, a few deep eatgut sutures through the muscle. A large drainage tube is introduced; this reaches into the deepest part of the wound, into the acetabular cavity, and emerges through the lower end of the incision.

Exarticulation at the Hip-Joint, with Preliminary Ligation of the Common Femoral.-Amputation at the hip-joint may be accomplished with the loss of very little blood if, as a preliminary step, the common femoral artery and vein have been ligated high up within two inches of Poupart's ligament; i.e., above the origin of the profunda femoris branch. After the common femoral artery and vein have been tied a circular incision is made around the thigh, five inches below the perineum, and in addition to this a longitudinal incision, which commences above the trochantar major and is carried down the outer side of the thigh just the same as in the preceding operation. The integument is then reflected, in the shape of a tegumentary cuff, for a distance of about three inches, at which level the muscles are divided layer by layer, ligating any vessels that bleed as they are met with. In cutting through the muscles on the back of the thigh we meet several large branches, but these are readily secured with clamps as they spurt and are then ligated. Having cut through the muscles down to the bone, the soft parts are separated from this in the usual manner, and the head of the bone turned out of the acetabulum and the amputation thus completed. We may use this method where tumor, etc., prevent the use of the Wyeth pins.

Resections. ANKLE-JoINT (LANGENBECK-HUETER).-This operation is done subperiosteally, and is especially applicable to cases of traumatism. The foot rests with its inner side upon a thin sand bag, the knee being slightly flexed.

An incision about three inches long is made along the posterior border of the fibula just in front of the sheath of the peronei tendons; this is carried downward as far as the tip of the malleolus, where it is turned upward for a short distance along the front border of this malleolus. This incision reaches through the soft parts and periosteum to the bone. The tissues which cover the bone are raised subperiosteally with an elevator, laying bare all of the lower end of the fibula and taking care not to injure the peronei tendons, which are lodged in the groove upon the posterior border of the external malleolus. There is considerable difficulty in separating the periosteum from the surface of the malleolus betow, and in order to accomplish this it may be neces- 
sary to resort to the knife, cutting with its edge close upon the surface of the bone or else one may chisel away a thin shell of the cortex of the bone.

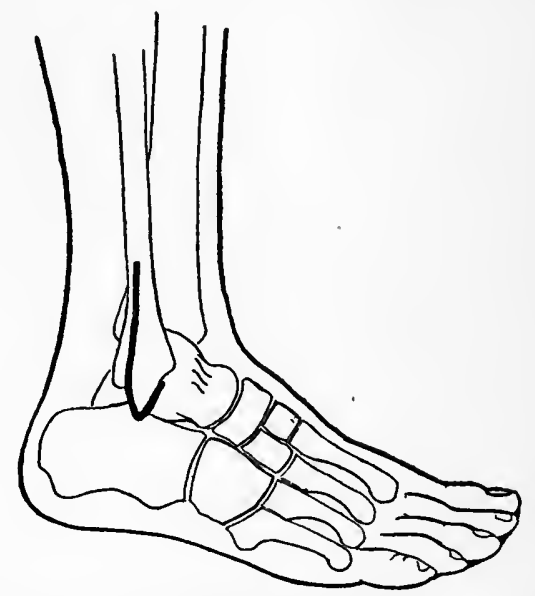

Fig. 217.-Right Foot, Outer Side. External incision for resection of ankle (Langenbeck-Hueter).

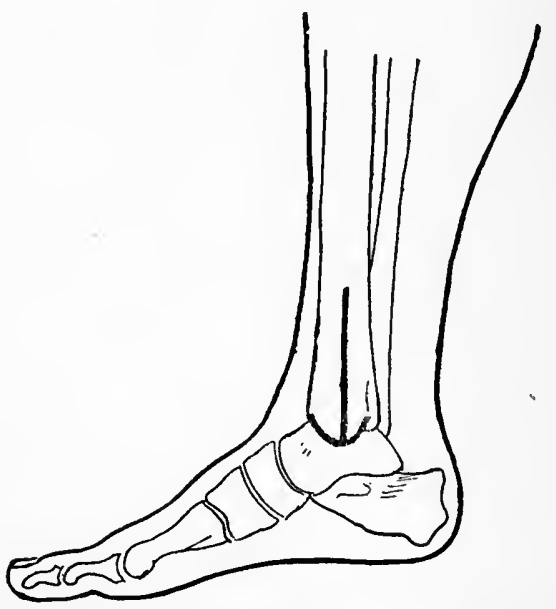

Fig. 218.-Right Foot, Inner Side. Anchor-shaped incision upon inner side of ankle for resection (Langenbeck-Hueter).

In isolating the lower end of the fibula on its inner aspect, corresponding to the attachment of the interosseous ligament which binds 
the lower ends of the tibia and fibula together, care should be taken to stick close to the surface of the bone, so as to leave the periosteum connected with the interosseous ligament.

Now, corresponding to the upper part of the wound, the fibula is encircled with a chain or wire saw and divided, or it may be cut through with a chisel. The upper end of the detached fragment is then seized with the bone forceps and wrenched free from the remaining ligaments (external lateral) which still hold it. This gives access to the interior of the joint, and through this opening the upper articular surface of the astragalus may be removed with the chisel or sharp spoon and the joint irrigated and drained.

One may stop with this partial operation, or else proceed to do a complete resection. In this latter case the foot is turned so that it rests upon its outer side, and an anchor-shaped incision then made which consists of a cut two and one-half or three inches long, down the middle of the inner subcutaneous surface of the tibia as far as the tip of the malleolus, and from this point additional incisions, which are carried upward along the anterior and posterior borders of the malleolus for a distance of about one inch. These incisions all reach through the periosteum to the bone. In many cases the single longitudinal incision will suffice. Through this incision the periosteum and soft parts are separated from the lower end of the tibia in one mass, working first upon the anterior surface and then upon the posterior surface of the bone, and avoiding injury to the tendons; upon the outer surface of the lower end of the tibia, corresponding to the attachment of the tibio-fibular interosseous ligament, one should work as close as possible to the surface of the bone.

During this part of the operation the edges of the wound are held well apart with blunt retractors. The soft parts should be separated from the lower end of the bone as much as possible with the sharp-edged periosteum elevator, but, if necessary, one may resort to the use of the knife, keeping close to the surface of the bone, or may chisel away a thin layer of the cortex of the bone. Finally, the internal lateral (deltoid) ligaments are cut close to the edge of the malleolus, - it is better to separate these also with the elevator or the chisel,-and the ankle-joint is now open upon its inner side. The lower end of the tibia may be cut through with the chain or wire saw or chisel upon the same level as the fibula was divided; it is then seized with a bone forceps and detached from any remaining bands that hold it. 
The upper articular surface of the astragalus, if desirable, may now be sawn off from behind forward with a thin, flat saw, taking care of the tendons on the back and front of the joint, or, better, it may be cut away with the chisel. This section should be made through such a plane that, when the sawn surface of the astragalus is apposed to the sawn surface of the tibia, the foot will be at right angles to the leg. There is a tendency to make the section through the astragalus upon a plane which would place the foot in a position of extension (plantar flexion), and this is to be avoided.

When this operation is performed for traumatism, the result is good. Much of the bone is reproduced and the parts regain almost their former contour; any excess of bone that is produced from the detached periosteum is nsually absorbed. Portions of the tibia, even as much as 8 to $10 \mathrm{~cm}$., have been removed and reproduced. An ankylosed ankle is the preferable result after this operation; the joints between the bones of the tarsus eventualiy give considerable spring to the foot. When the operation is performed for tuberculosis, frequently no bone is reproduced, healing fails, and we have, as a result, a wabbly joint, with sinuses.

It may not be necessary in all cases to do a complete resection, since all of the parts-for example, the articular surface of the astragalus-may not be diseased, etc. Care should be exercised in applying the dressings to place the foot at a right angle with the leg and turned somewhat outward. It is probably wise in all cases to drain, at least for a few days. The edges of the wound are approximated with interrupted catgut sutures.

With Extirpation of the Entire Astragalus.-The long middle incision on the inner side of the ankle is prolonged downward about one inch farther than described in the foregoing operation, so as to reach to the sustentaculum tali, and at its lower end an antero-posterior incision is added which is about two inches long and which penetrates to the bone (see Fig. 222). The soft parts are separated forcibly with the elevator and the whole of the astragalus thus brought into view. The joint between the head of the astragalus and the scaphoid is opened (tuberosity of the scaphoid is the guide), and also the joint between the astragalus and the os calcis (sustentaculum tali); after this the astragalus is seized with a bone forceps, and, twisting and at the same time cutting close to the bone, it is removed. In resecting the ankle-joint for tuberculosis, if the astragalus is diseased, it is well to remove this bone entire. 
Ankle-Joint (Koenig).-This is a satisfactory operation, especially for tuberculous joints. The lower part of the leg rests upon a sand bag, the foot being elevated and turned outward. An incision is made upon the inner side of the ankle, commencing an inch or an inch and one-half above the level of the joint, and passing down along the anterior border of the tibia and inner malleolus parallel with and just internal to the extensor tendons which lie upon the front of the joint.

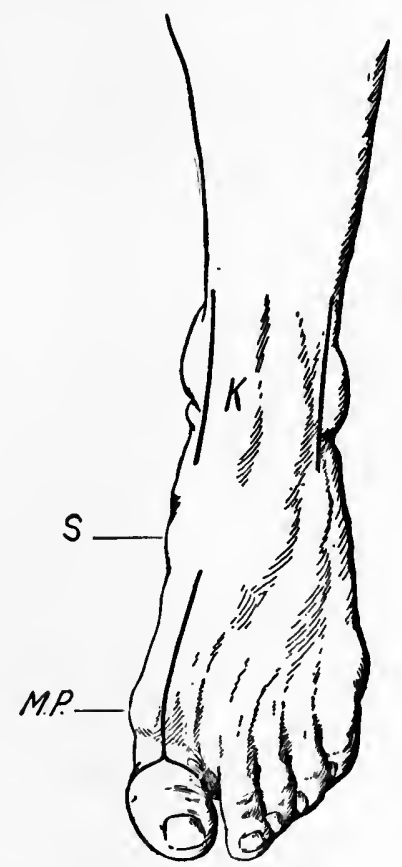

Fig. 219. $-K$, incisions for resection of ankle (Koenig); M.P., articuiation between metacarpal bone of the big toe and first phalanx; $S$, iocation of tubercie of scaphoid. Incision for amputation of big toe with removal of the first metatarsal.

This incision penetrates through the integument and periosteum to the tibia, and is continued downward across the ankle-joint, into which it opens, and then curves forward upon the neck of the astragalus as far as the tubercle of the scaphoid.

A similar incision is made upon the outer side of the joint, commencing above at the same level as the internal incision and passing downward along the anterior edge of the outer malleolus, across the ankle-joint, into which it opens, and ending at a point 
opposite the lower end of the inner incision. This incision runs parallel with the outer margin of the extensor group of tendons.

Between these two incisions there is a bridge of tissues consisting of integument, anterior tibial vessels and nerve, extensor tendons, anterior ligament, and synovial membrane. This mass of soft parts is freely separated from the front of the tibia above and from the astragalus below, as much as possible subperiosteally with the elevator, and when necessary with occasional snips with the scissors or knife.

Access to the ankle-joint is now fairly free, and one may commence the excision of the diseased synovial membrane with mousetoothed forceps and scissors; the ends of the tibia and fibula and the articular surface of the astragalus may also be reached with the sharp spoon.

If it is desirable to resect the ends of the bones and it becomes necessary to gain still better access to the interior of the joint, a thin shell of the cortex, carrying the periosteum and the attachments of the ligaments, may be chiseled away from the surface of the inner and also from the surface of the outer malleolus, leaving them bare and free. Drawing the soft parts widely asunder with blunt hooks, a broad chisel may be applied, through the inner incision, to the lower end of the tibia, and this may then be divided; the fragment which is thus detached is seized with bone forceps and removed, cutting the remaining attachments close to the bone and taking care not to injure the tendons which lie close to the back of the bone nor the posterior tibial vessels and nerve. The lower, bare end of the fibula may be treated in a similar manner, avoiding the peroneal tendons in the groove upon its posterior surface. In laying bare the malleoli one should try to separate the lateral ligaments with the chisel subperiosteally in preference to cutting them.

The articular surface of the astragalus may be removed with the broad chisel or with a narrow, thin-bladed saw, the section being made through a plane which will allow the foot to be placed at a right angle with the leg.

In most cases of tuberculous joints when the astragalus is involved, it is probably better to remove this bone entire; this will also permit treatment of the joints between the astragalus and os calcis and the astragalus and scaphoid if these are involved, and this is frequently the case. The astragalus is readily removed through the inner incision, first opening the joint between the head of the astrag- 
alus and the scaphoid, and then the joint between the head of the astragalus and the sustentaculum tali of the os calcis. The astragalus is seized with a lion-tooth forceps, and, cutting its attachments close to the bone, it is twisted free.

Whether the entire astragalus is removed or not in cases of tuberculosis the whole synovial membrane lining of the ankle-joint should be removed with toothed forceps and scissors; that part of the membrane which lines the posterior portion of the capsule is difficult to reach, but its removal may be facilitated by drawing the foot strongly downward away from the tibia and at the same time strongly reflecting the anterior flap or bridge of soft parts.

Usually there are no vessels to tie. Drainage tubes may be introduced on each side and the wound packed with iodoform gauze. The edges of the wounds are brought together with interrupted catgut sutures, being left partly open to allow for the drainage tubes and gauze. The foot is dressed at a right angle to the leg.

ANKLE-JoINT (LaUENSTEIN).-A very satisfactory method, especially for tuberculous joints. The knee is slightly flexed, and the foot rests with its inner surface upon a thin sand bag. The incision is placed upon the outer side of the joint, passing through the skin and subcutaneous fat and exposing the external surface of the outer malleolus and the lower end of the fibula for a distance of about three inches. The surface of the fibula thus exposed is subcutaneous, and is included between the tendon of the peroneus tertius in front and the tendon of the peroneus brevis behind; from the tip of the outer malleolus the incision curves forward and inward across the dorsum of the foot, terminating just external to the tendon of the peroneus tertius, which should not be cut.

The joint is now opened in front of the external malleolus by cutting the anterior fasciculus of the external lateral ligament, and then the integument, together with the extensor tendons and other soft parts, including the anterior portion of the capsular ligament, are separated from the front of the tibia with the periosteum elevator, these soft parts being meanwhile drawn forcibly forward, away from the front surface of the tibia, with a blunt hook.

The posterior margin of the incision is next seized and retracted and the sheath of the peroneal tendons opened; these tendons, together with the integument, are drawn well back out of the way with a blunt hook and the remaining fasciculi of the external lateral ligament (middle and posterior) then divided. 
The foot, being somewhat extended in order to relieve the tension of the peronei tendons, may now, with moderate force, be completely dislocated by rotating it inward upon its long axis in a hingelike fashion around the internal malleolus.

All parts of the joint are now accessible; the synovial membrane may be dissected away with a thumb forceps and scissors, and the upper articular surface of the astragalus, if desired, may be chiseled away or resected with a thin, flat saw, or, by extending the incision somewhat, the entire bone may be removed. If the tibia and fibula are diseased, the soft parts about the lower ends of these bones may be detached, preferably subperiosteally, with the elevator, and the diseased portion of the bones then resected with the saw. If the articular

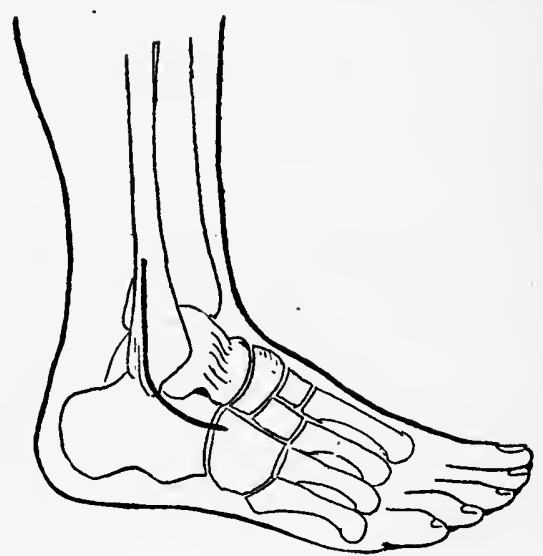

Fig. 220.-Resection of Ankle-joint. Lauenstein's incision.

surface only of the astragalus, and not the whole bone, is to be removed, one should take care to make the section through the bone in such a plane that, when the foot is replaced, the cut surfaces of the astragalus and tibia will permit of the foot being placed at a right angle with the leg. There is a marked tendency, in resecting the articular surface of the astragalus, to carry the section through a plane which would result in the foot's being joined to the leg at an obtuse angle, in a position of extension, and this is to be avoided.

Ankle-Joint, Osteoplastic (Mrkulicz-Wladimirow). — The patient lies upon the abdomen. A transverse incision is made across the sole of the foot. This incision commences on the outer border of the foot a finger's breadth behind the tuberosity which 
marks the base of the fifth metatarsal bone (little toe), and ends on the inner side of the foot at the tubercle of the scaphoid. From either end of this incision, upon either side of the foot an additional incision is carried obliquely upward and backward across the lower end of each malleolus to their posterior borders, and then still another incision is made transversely, just above the heel, uniting the ends of the two lateral incisions and dividing the tendo Achillis and the posterior tibial vessels. All these incisions penetrate to the bone. The foot is now forcibly flexed (dorsal flexion) and the ankle-joint is opened from behind and the lateral ligaments are cut. The astragalus and

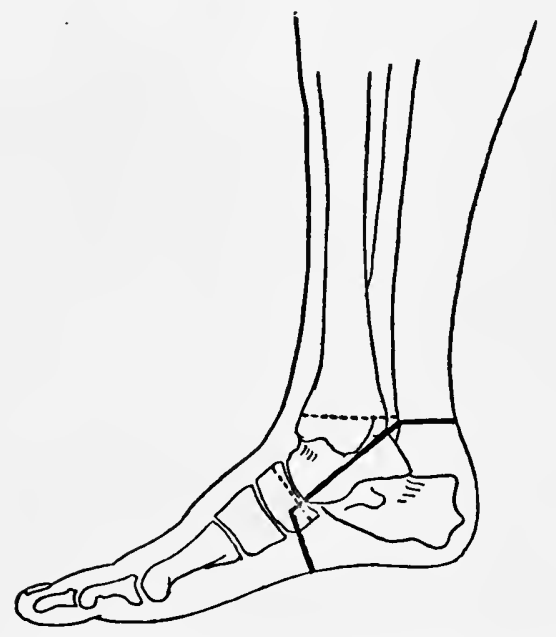

Fig. 221.-Right Foot, Inner Side. Line of incision for Mikulicz-Wladimirow osteoplastic resection of the ankle-joint. Dotted lines indicate section through the bones.

the os calcis are then dissected out of the mass of soft parts in which they are located, working with the edge of the knife close to the surface of the bones; during this step of the operation the bones are forcibly drawn, first to one side and then to the other, in order to facilitate their enucleation, and, working forward, the joint between the astragalus and the os calcis behind and the scaphoid and cuboid in front is finally opened. The remaining ligaments and bands are then severed and the bones removed.

The soft parts around the lower end of the tibia and fibula are now separated, cutting with the edge of the knife close to the surface 
of the bones, and a thin slice of the lower end of the tibia, including both malleoli, is sawn off.

A thin slice, including the articular surfaces, is likewise sawn off from the scaphoid and cuboid, so that when the foot is extended (plantar flexion) the sawn surfaces of the cuboid and scaphoid may be apposed to the sawn surfaces of the tibia and fibula, the long axis of the leg being thus prolonged into the foot, as a direct line, the patient walking upon the heads of the metatarsal bones and the phalanges.

The posterior tibial vessels which lie behind the ankle-joint, to the inner side of the tendo Achillis, are cut, and must be ligated. The bones may be retained in contact with sutures of chromicized catgut carried through drill holes, but this is, as a rule, unnecessary, especially if the foot is put up in plaster. The skin wound is closed with interrupted catgut sutures.

One should be careful that the integument on the front of the ankle, which is redundant after excision of these bones, does not interfere through its bulk, "bunching," with the correct apposition of the bones. One may overcome this tendency by passing several quilting sutures through this mass of soft parts.

KNEE-JOINT.-A tourniquet is applied about the upper part of the thigh. The patient lies upon the back with the leg extended, the operator standing upon the side of the table corresponding to the joint which is to be resected.

The usual incision (Textor) and probably the best for most cases is convex downward, passing across the front of the joint, below the patella, and extending from the middle of one condyle to a similar point upon the other. This incision should reach deep to the bone, and below the patella divides the ligamentum patellæ.

The knee-joint having been thus opened, the limb is strongly flexed at the hip and knee, with the sole of the foot resting upon the table, and it is thus supported by an assistant. The lateral ligaments and the lateral portions of the capsule are now divided, cutting them close to the surface of the femur.

The knee being still more markedly flexed, the crucial ligaments are divided close to their attachment to the upper surface of the tibia, cutting with the edge of the knife directed downward, as if one would cut into the articular surface of the upper end of the tibia; if the ligaments are divided with the edge of the knife directed backward, one may accidentally cut the popliteal vessels. 
The anterior flap, which includes the patella, should be dissected back and retracted sufficiently to allow free access into the synovial pouch, which is located above the patella, between the quadriceps tendon and the front of the femur.

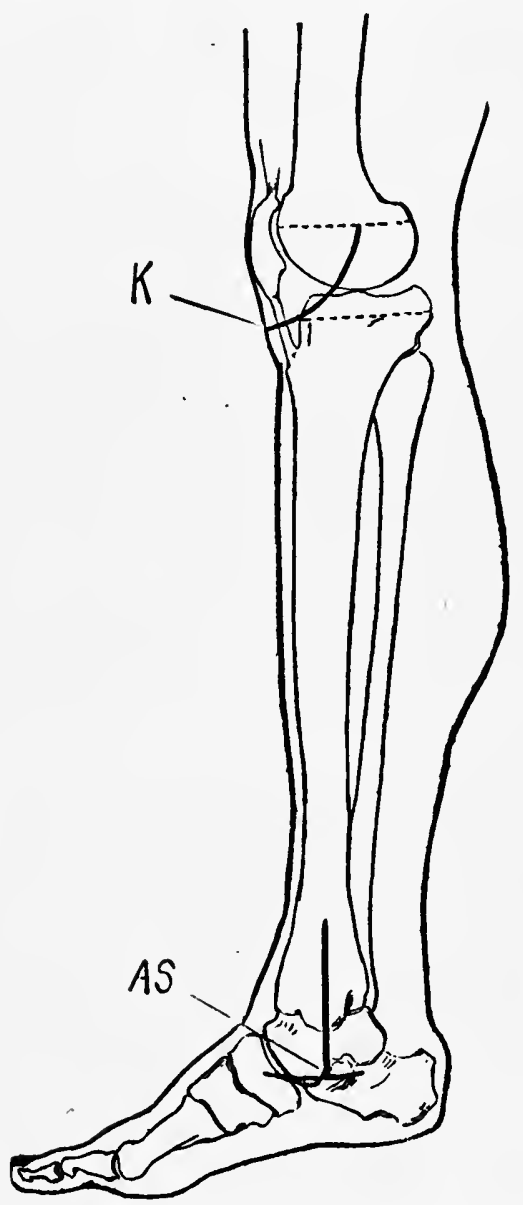

Fig. 222.-Right Leg, Inner Side. AS, incisicn upon the inner aspect of the ankle for resection of the astragaius; $K$, Textor incision for resection of the knee-joint. Dotted lines indicate planes of section through the bones.

With mouse-tooth forceps and blunt-pointed scissors, curved on the flat, the synovial membrane which lines the joint may now be entirely resected. If the bones are healthy, one may stop at this 
stage of the operation and close the wound, after irrigating thoroughly and providing for suitable drainage (arthrectomy).

In resecting that part of the synovial membrane which lines the posterior part of the capsule one should aroid cutting deeply, on account of the liability to injure the popliteal vessels, which lie adjacent to this part of the capsule. There is rather less danger of doing this if the posterior ligament is put upon the stretch by drawing the tibia away from the femur while this part of the synovial sac is being excised. This portion of the synovial membrane is also more accessible after the ends of the bones have been resected.

If the disease in the bones is limited to one or more foci, these may be thoroughly scooped out with a sharp spoon, thus avoiding the resection of the ends of the bones. Especially in children one should avoid, wherever possible, the resection of the ends of the bones, since interference with the epiphyseal line may retard very much the subsequent growth of the limb.

The patella is usually extirpated if the ends of the femur and tibia are resected, even if it is apparently not diseased. It is grasped with double sharp hooks and excised, cutting with the edge of the knife close to the surface of the bone.

If one decides to resect the ends of the bones, the lower end of the femur is first removed, separating the soft parts back as far as necessary, and working with the scalpel close to the bone. The end of the femur, stripped of its soft parts, is forced upward, out of the wound, above the level of the tibia (hip flexed) and with a sharp, broad saw the section is made through the end of the bone from before backward, commencing by placing the heel of the saw upon the bone and making a groove by drawing the instrument firmly backward. The femur should be steadied with both hands of an assistant who supports himself by resting his elbows upon the table. The end of the bone should be forced sufficiently far upward out of the wound so as to make the use of a towel to protect the soft parts during its section unnecessary. The piece of bone resected must be of the same thickness anteriorly, posteriorly, and upon either side, otherwise, when the operation is completed, the limb will be found to be in a position of knock-knee or bow-leg, or there will be too much or too little extension.

The section should pass through a plane which is parallel with the articular surface of the bone, and not at right angles with the long 
axis of the bone, and therefore in making the section one should disregard the long axis of the bone, and rather keep his eye on the plane of the articular surface.

The end of the tibia is now likewise stripped of its soft parts and projected upward out of the wound well beyond the, sawn surface of

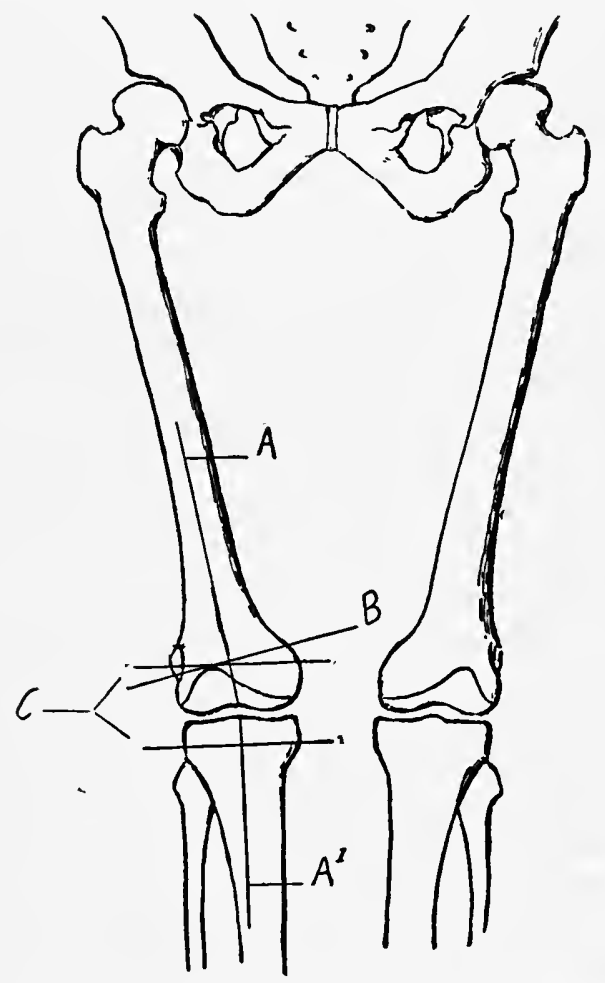

Fig. 223.-Resectlon of Knee-joint. $A, A^{1}$, long axes of the femur and tibia; $B$, line drawn at right angles to the long axis of the femur. $C$, lines through the lower end of the femur and upper end of the tibia parallel with the plane of the articular surfaces. Through these planes the section should be made in resecting the knee-joint.

the femur, and a section of the bone removed, as in the case of the femur, parallel with the plane of its articular surface.

At times it is necessary to excise two or three inches of the bones (femur and tibia together), but one should remove as little as the conditions present will permit, especially in children. The sharp spoon may be used to extirpate foci which extend into the substance of the 
bone beyond the surface exposed by the section, and, if the cortex is healthy, one may remove much of the medullary portion of a bone rather than sacrifice more of the length of the limb by removing a thicker segment of bone.

The limb should be now extended and the position of the joint noted; when the ends of the bones are brought together there should be the normal slight bowing inward and a slight degree of flexion (five degrees).

If the position of the limb is not satisfactory, one may remove a further section from one of the bones to correct it; but the necessity for this second section should be avoided.

The position of the bones being satisfactory, all loose, ragged tissue is cut away and any remaining portion of the synovial membrane that has been overlooked excised.

In most cases it is well to insert tubes for drainage, one on each side. These should reach well up into the recess beneath the quadriceps muscle, between it and the front of the femur; in addition, strips of iodoform gauze may be packed into the wound, the ends emerging through the incision on each side; if tubes are used, they should be fixed to the edge of the skin with a silk-worm gut stitch to prevent their slipping out. The front part of the skin incision is closed with interrupted silk-worm gut stitches.

If the limb is placed in a good, firm, plaster-of-Paris splint, the ends of the bones, as a rule, remain in good position. A sharp edge of either bone should not be left projecting into the popliteal space (popliteal vessels). If it is desired to fix the ends of the bones they may be joined together with two stout chromicized catgut sutures which pass through drill holes placed near the anterior margin of the sawn surfaces of the bones, one on each side of the middle line, or, after the skin has been sutured, the bones may be joined by two nails, one driven through the front of the upper end of the tibia and reaching obliquely upward into the sawn surface of the femur, and the other passing through the front surface of the femur and reaching down into the upper end of the tibia. Small incisions may be made in the skin to allow the introduction of the nails.

These accessory measures, for the purpose of holding the bones in apposition, are, as a rule, unnecessary if the ends of the bones have been sawn square, and fit well, and a good plaster splint is applied. In adjusting the plaster splint one should see that the foot is slightly everted, so that the patient will not "toe in." 
Before suturing the wound the tourniquet may be removed and any spurting vessels secured; usually there are few or none, and any slight oozing may be controlled by the pressure of the dressing.

Instead of the incision described above the knee-joint may be opened by an incision, with the convexity directed upward, passing across the limb above the upper border of the patella. This is the reverse of the incision described above, and gives very free access to the synovial pouch under the quadriceps tendon.

Volkmann makes a transverse incision across the front of the knee, through the skin down to the surface of the patella, the knee being slightly flexed and resting upon a sand bag; corresponding to this incision through the skin, the patella is sawn through transversely. To this may be added two lateral incisions, one on each side of the joint running up and down. We then have an $\mathrm{H}$-shape incision. This incision is especially adapted to those cases where the disease is limited to the synovial membrane alone; so that after its excision the segments of the patella may be sutured with chromicized catgut. This incision is often desirable in children.

Hip-Jornt (LANGENBECK). - This operation is done as much as possible subperiosteally. The patient lies upon the well side of the body, with the hip and knee slightly flexed. A longitudinal incision is made over the outer side of the hip four to five inches long; it commences two or three inches above the upper border of the great trochanter, upon a line corresponding to the long axis of the femur, and from this point is continued straight down upon the outer surface of the trochanter and ends upon the upper part of the shaft of the femur; the incision throughout its whole length penetrates to the bone; that portion of the incision which lies above the trochanter passes through the fibers of the glutei muscles down to the surface of the ilium, which it reaches just above the margin of the acetabulum, exposing the capsule of the joint.

Sharp retractors are inserted deep in the incision and the soft parts drawn forcibly asunder. The capsule is incised from the rim of the acetabulum downward toward the great trochanter, the cotyloid ligament, also, being nicked, or may be separated from the bony rim of the acetabulum for a short distance on either side of the incision in the capsule.

With the periosteum elevator, or, where necessary, with the knife or chisel, the tendons, together with the periosteum and the capsule, are separated from the greater trochanter and the neck of the femur; 
this should be accomplished as much as possible subperiosteally with the sharp-edged periosteum elevator; but, where the attachment of the parts to the bones is very intimate, it may be necessary to resort to the knife, cutting with its edge close to the surface of the bone, or the chisel may be used, chipping off a thin shell of the cortex, which carries the attached tendons with it. An assistant rotates the limb inward or outward as may be necessary to facilitate this part of the operation.

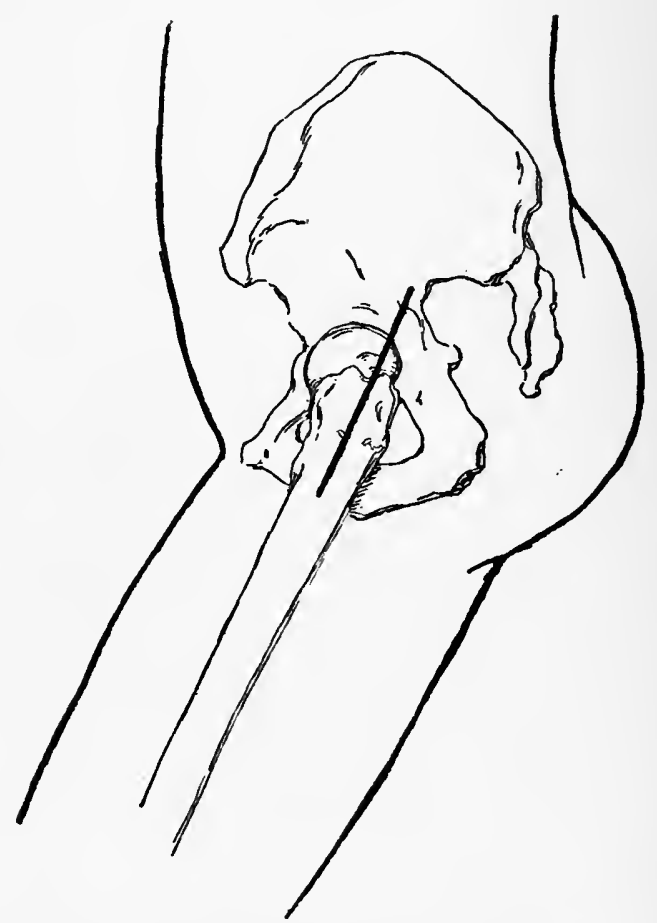

Fig. 224.-Resection of Hip. Langenbeck's incision.

In this way the upper end of the femur is denuded. Pains should be taken to separate the tendon of the obturator externus, which is attached, in the digital fossa, upon the inner aspect of the great trochanter, and also the tendons that are attached to the upper border of the great trochanter.

In order to cut the ligamentum teres the thigh is flexed, rotated inward, and adducted, in this way partly luxating the head of the bone; a long, narrow knife is then introduced into the joint above 
and behind, and sweeping downward and forward across the head of the bone, the ligament is usually cut. In operating on diseased joints it is, as a rule, not necessary to cut the ligamentum teres, as it is, in most cases, already destroyed, or, at any rate, readily ruptures upon forcibly manipulating the joint (adduction and rotation inward). The head of the femur is then completely luxated backward by

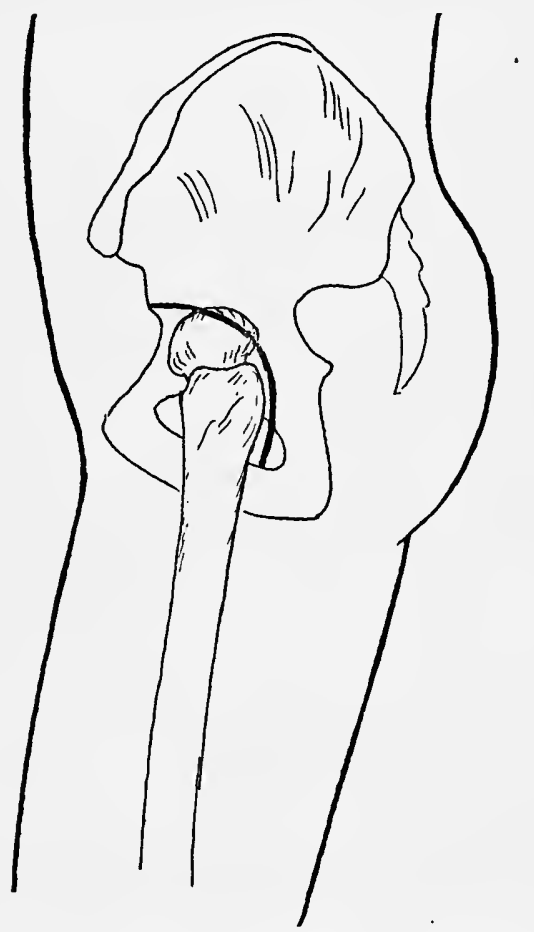

Fig. 225.-Resection of the Hip. Anthony White's incision. Commences anteriorly midway between the anterior superior spine of the ilium and the upper border of the trochanter major and curves backward above the trochanter major and then downward behind the trochanter for a distance of about two inches.

manipulation (flexion, adduction, and rotation inward) and forced out of the wound, when the head and neck may be readily removed with a Gigli or chain saw, with a flat saw, or with a chisel.

Some surgeons make it a practice to remove the trochanter as well as the head and neck of the femur, making the line of section through the shaft of the bone just below the great trochanter. In 
order to do this it is necessary to separate the periosteum, etc., correspondingly lower down upon the shaft of the femur. If the trochanter is healthy, it is unnecessary to remove it; yet, if at all suspicious, it is better to make the section through the bone below the great trochanter, removing the great trochanter as well as the head and neck, because the result is just as good, and many surgeons claim better, than when it is left.

Now, rotating inward and outward, but chiefly by extension of the limb, pulling strongly upon the femur and holding the edges of the wound widely apart to give us room, we may proceed to excise the synovial membrane, using long, sharp scissors, curved upon the flat, and mouse-tooth forceps.

If the acetabulum is diseased, it may be curetted with a sharp spoon or even resected with the chisel and mallet. A sinus may be found leading through the acetabulum to a focus within the pelvis, in which case drainage of the joint may be combined with counter-drainage through an incision made anteriorly just below Poupart's ligament. There is but little hemorrhage during the operation; bleeding vessels may be seized as they are cut during the progress of the operation.

The soft parts are brought together with interrupted silk-worm gut sutures, which should pass deep through the integument, muscles, and periosteum, closing the wound, except for a space below sufficient to allow the passage of a thick tube, which should reach upward as far as the acetabulum for drainage, or the wound may be packed with iodoform gauze, or the gauze packing may be combined with the use of a tube. Before closing the wound it should be washed out with hot bichloride solution.

During the operation one should work as much as possible with the periosteum elevator and chisel, cutting as few tendons as possible with the knife. We should strive to keep the capsule and the periosteum or shell of cortex that is separated from the bone, along with their attached tendons, hanging together in one continuous layer; so that, when we are ready to resect, the denuded upper end of the femur lies in a sort of sac which is made of the above named structures, and which all hang together, continuous with one another, and it is out of this hood or sac that we deliver the upper end of the bone for resection. In closing the wound the upper edges of the hood should be included in the sutures, except the part that is left open for drainage. 
0steotomy of the Femur for Genu Valgum-Knock-knee (Macewen).-The knee is somewhat flexed, its outer side resting upon a sand bag. A short longitudinal incision is made upon the inner side of the thigh just above the knee-joint. It is placed one finger's breadth in front of the tendon of the adductor magnus, its lower

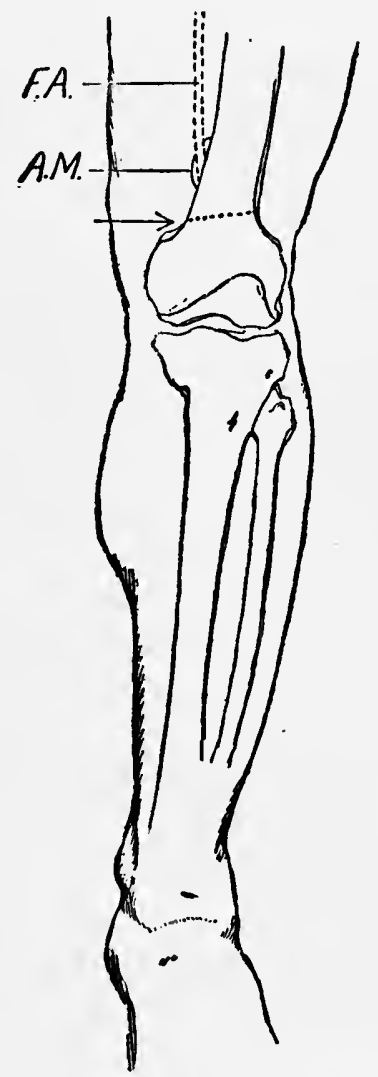

Fig. 226.-Osteotomy (Maceven). A.M., opening in the adductor magnus through which the femoral artery (F.A.) passes into the popiiteal space. Arrow indicates point at which the chisel is applied and the dotted line the plane of section for bow-legs and knock-knee.

end upon a line which is drawn around the lower part of the thigh one finger's breadth above the upper border of the external condyle; or we may locate the lower end of the incision two fingers' breadth above the inner condyle and one finger's breadth in front of the tendon of the adductor magnus. The incision is prolonged upward 
for a distance of $4 \mathrm{~cm}$. and reaches to the bone through the integument, vastus internus muscle, and periosteum.

The periosteum is separated from the surface of the bone over an area sufficient to allow the application of a broad chisel, with which the bone is divided, in a direction outward and slightly upward. The line of fracture does not pass through, but just above, the joint. The deformity is then corrected, and the limb, including the foot, placed in a plaster splint.

It is not necessary to chisel through the entire thickness of the shaft of the femur, but only far enough to allow one to gradually bend the bone into position-it should not be forcibly fractured or bent into position with a sudden jerk. The line of fracture is placed above the epiphyseal line, and therefore this operation may be done upon children and young people without interfering with the natural subsequent growth of the femur. This operation may also be done through an incision upon the outer aspect of the limb upon the same level.

Suture of the Patella.-Approximation and fixation of the fragments of the patella in case of fracture with marked separation, etc.

The failure to obtain bony union in fracture of the patella is due to the interposition of strings of torn periosteum, etc., and blood-clot between the fragments, and to distension of the capsule of the knee-joint with blood and serum. These conditions interfere with immediate close coaptation of the fragments. The object of an operation is to approximate and fix the fragments and to evacuate the knee-joint.

A vertical incision is made sufficiently long to expose the fragments, the edges of the wound being drawn asunder with sharppronged retractors. Each fragment is then, in turn, lifted up into the wound with a sharp hook and its edge freed from shreds of periosteum and blood-clot. The blood is then turned out of the joint and the joint irrigated with a warm saline solution. The fragments are then brought together with sharp hooks and fixed in position with two or more chromicized catgut sutures. These sutures should include the periosteum corresponding to the edges of the fragments and should take a good, firm bite. It is, in most cases, unnecessary to carry these sutures through the bone. If this should be desirable, however, the holes must be drilled through the edges of the fragments. These drill holes should not go through the entire thickness of the bone, but only partly through, emerging upon the edges of the fragments. 
After the fragments have been united the rents in the capsule, fibrous expansion of the quadriceps extensor tendon upon either side, corresponding to the lateral margins of the patella, may also be closed with several catgut sutures. The incision in the skin is sutured with catgut without drainage, an aseptic dressing is applied,

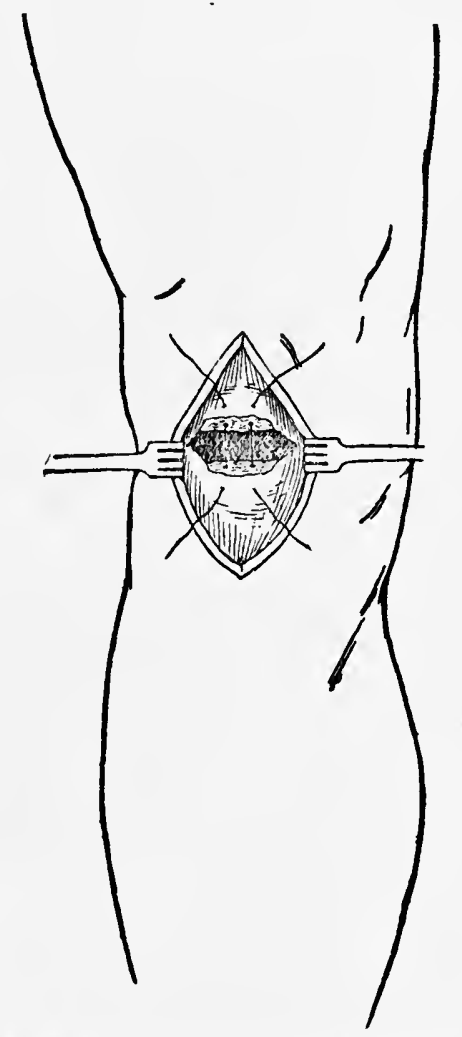

Fig. 227.-Wiring Patella for Fracture. Shows tear in capsule upon either side of fractured patella. Sutures emerge upon the edge of each fragment: i.e., they do not pass entirely through the whole thickness of the bone.

and the limb placed upon a long posterior splint with the foot elevated and the limb flexed at the hip-joint so as to relax the quadriceps. After one week the limb may be placed in plaster, still kept flexed at the hip, and at the end of four weeks passive motion may be commenced. 



\section{INDEX.}

[The names of arteries, muscles, nerres, and veins are placed in the index nnder the heading*

"Artery," " Muecle," etc.]

Abbe, operation for stricture of œsophagus, 230.

Abdomen, 205.

antero-lateral wall of, 207.

muscles of, 208.

aponeuroses of, 210 .

posterior wall of, 206.

Abdominal cavity, 205.

wall, deep vessels of, 212.

superficial vessels of, 208 .

Abscess, extradural, in middle fossa, 50.

of breast, incisions for, 194.

of cerebellum, 50 .

temporo-sphenoidal, 51.

Alexander, prostatectomy, 434.

Alimentary tube, 80.

Alveolar process, cleft of, 92.

Amputation of arm, 461.

of breast, 195 .

of finger at phalango-phalangeal joint, 450 .

at metacarpo-phatangeal joint, 452.

of toot through medio-tarsal joint, Chopart, 504

Pirogoff method, 507.

Günther modification. 509.

le Fort modification, 509 .

Syme method, 506 .

through tarso-metatarsal joint, Lisfranc, 501.

of forearm, 457.

of $\mathrm{leg}, 510$.

of penis, 422

of rectum, Kraske, 361.

Volkmann, 348 .

of thigh, 521

of toe, 500 .

of tongue, 159 .

Anæsthesia, cocaln, 2 .

general, 1.

Incomplete, 1.

local, 2.

Schlelch method, 3.

Anal fascia, 425-430.

Analgesia by subarachnoid Infection of cocain, 3.

Anastomosis, Intestinal (see "Intestinal Anastomosis").

Anglotribe, 11.

Ankle-joint, 505.

exarticulation of foot at, 506 .

PIrogoff method, 507.

Günther modification, 509 .

le Fort modification, 509.

Syme, 506.

resection of, Koenlg, 533.

Langenbeck-Hueter, 529.

Lauenstein, 535 .

Mikulicz-Wladimlrow, 536.

with extirpation of astragalus, 532 .

Antrum of Highmore, 53.

to drain, 69

mastold, 40.

drilling Into, 46.

to open and draln, 46.

Anus, artiflclal, 289.

Aorta, arch of, 182.

thoracle, 187.
Aortlc valve (orifice), 181

Aponeurosis of abdominal muscles, 210.

of external oblique, $209,364$.

Appendicectomy, 299 .

incision, 299

Battle, 301.

McBurney, 300.

Appendicitis accompanied by general per1toneal infection, operation, 309 .

Appendicular abscess, operation, 306 .

Appendix vermiformis, amputation of, 299 .

inversion of, 305 .

stump of, after amputation, 304.

ligation of stump of, without inversion, 302 .

surgical anatomy of, 285 .

Aqueductus Fallopil, 45 .

Arch, mandibular, 82.

of aorta, 182.

Arches, visceral, 82

Arm, amputation of, 461.

Arteries of scalp, 19.

Artery, anterior tibial, 492. ligation of, 494.

axillary, 437 .

ligation of, 447.

brachial, 440 .

ligation of, 448.

common carotid, 136

ligation of, 153 .

common carotid, left, 188.

deep epigastric, 367 .

dorsalis pedis, 493.

external carotid, 138

ligation ot, 155.

facial, 52 .

femoral, 486.

ligation of, 489.

Inferior thyroid, 141

innominate, 188 .

intercostal, ligation of, 200.

Internal carotid, 33, 137. ligation of, 156.

Internal mammary, 170. ligation of, 200 .

internal maxillary, 60 .

lingual, 58, 127.

ligation of, 158.

mlddle meningeal, 25.

ligation of, 25 .

musculo-phrenic, 170.

obturator, 380 .

posterior tibial, 494.

ligation of, 496.

radial, 443 .

ligation of, 449 .

subclavian, 140.

ligation of, 156.

subclavian, left, 100

superior epigastric, 170

temporal, 60 .

ulnar, 443.

ligation of, 449.

vertebral, 141.

Ascending colon, 287

Atresla of facial orifices, 90.

Auditory canal, external, 42-87. process, 42. 
Auricle, 42-87.

Auriculo-ventricular valves (orifices), 182.

Axlla, surgical anatomy of, 437 .

Axillary abscess, incision for, 440. line, 171.

vein (see "Artery, axlllary").

Azygos vein, 187.

Back, 214.

muscles of, 215.

of neck, 120 .

Bardeleben operatlon for harelip, 106.

Bassinl operation for inguinal hernia, 383.

Bier, subarachnoid injection, 335 .

$\mathrm{Big}$ toe, exarticulation of, 500 .

with removal of first metatarsal bone, 500 .

Billroth, extiryation of tongue, 164.

pylorectomy, 237.

Bladder, drainage of (see "Suprapuble cystotomy").

operation for stone in (see "Suprapubic cystotomy").

puncture, 418 .

relations of, 414 .

surgical anatomy of, 413.

suture of wounds of, 17.

Blandin's method, harelip, 106.

Bone, division of, 5 .

- suture of, 15.

Bottini, prostatotomy, 435.

Bowel, suture of wounds of, 16 (see also "Intestine," etc.).

Branchial arches (see “Visceral arches"). clefts (see "Visceral clefts").

Breast, 175.

amputation of, 195 .

Halsted-Meyer method, 197.

extirpation of fibroid from, 195.

flbroid of, 195 .

incisions for abscess of, 194.

lymphatics of, 176.

Bruns method, formation of lower llp, 113.

Bursæ adjacent to knee-joint, 515.

Cæcum, resectlon of, 295.

surgical anatomy of, 285 .

Canal, crural, $375-380,488$.

external auditory, 87 .

Hunter's, 487.

inguinal, 365.

Carden amputation of leg, 518 .

Carotid artery, common, 136. ligation of, 153.

left, 188.

external, 138.

ligation of, 155 .

internal, 137 .

ligation of, 156.

trlangle, inferior, 123.

superior, 125.

Casserian ganglion, 34

extirpation of, Hartley-Krause, 36. Rose-Andrews method, 76.

Castration, 401.

Cavernous sinus, 22-33.

Cavum Meckelil, 35 .

Cerebcllar abscess, 50.

Cerebro-spinal fiuid, 23.

Cervical fascla, deep, 118.

Cheeks, 54

Chest, fascla of, 169.

aspiration (see "Thoracentesis").

tapping (see "Thoracentesis").

Chest wall, muscle of, 169 .

resection of part of, 202.

Cholecystectomy, 322 .

Cholecyst-enterostomy, 324.

Cholecysto-duodenostomy with Murphy button, 325.
Cholecystostomy, 318.

in one sitting, 318 .

in two sittings, 320 .

McBurney modification, 319 .

when bladder cannot be drawn into inclsion for flxation, 322 .

when bladder is already fixed by adhesion: to abdominal wall, 322 .

Cholecystotomy, 315 .

Choledocho-lithectomy, 326.

Choledocho-lithotripsy, 327.

Choledochotomy, 326.

Chopart amputation, 504.

joint, 499

Circumcision, 421.

with clamp, 422 .

Clavicular region, 173.

Cleft of alveolar process, 92.

palate, 93.

operation for, 107.

Clefts, lateral nasal, 96.

median. of upper lip, 95 .

oblique facial clefts, 86,96

of lower lip, lower jaw, and tongue, 98.

orbito-nasal (see "Oblique facial clefts").

transverse facial, 87, 98.

visceral, 82.

Cocain anæsthesla, 2.

sterilization, 4.

subarachnoid injection of, 3 (see also "Lum. bar puncture," 334).

Coccyx, 339.

Colles's ligament, 364.

Colon, resection of (see "Resection of cæcum" and "Sigmoid flexure").

Colostomy, 289.

Maydl method, 292.

Common bile-ducts, 313 .

Congenital deformities of face, 80, 90. classification of, 90 .

hernla, inguinal, 370, 371, Fig. 152.

operation for, 388.

Conjoined tendon, 366 .

Conus terminalis, 220 .

Cooper, puble ligament of, 372, 374, 379.

Corning, spinal injection, 334 .

Corpora cavernosa, 418.

Corpus spongiosum, 418.

Costal cartilages, 168 .

Costo-coracoid membrane, 175.

Cotting operation, 501 .

Cowper's gland, 427.

Craniectomy, 29.

Craniotomy, linear, 29.

Cribriform fascia, 363 .

Crico-thyroid membrane, 132

Crico-tracheotomy, 143.

Crural canal, 375-380, 488.

nerve, anterior, 489 .

ring, 375-380.

Cushing suture, 17.

Cystic duct, 312

Cystotomy, suprapublc, 415 .

Dawbarn, inversion of stump of appendix, 304 . Dartos, 394.

Deformities of face, congenital, $80,89$.

classification of, 90 .

in which first visceral arch is concerned, 98 .

in which frontal palate is concerned, 91.

Deltold flap, exarticulation of arm at shoulderjoint, 469.

Depressed fracture of skull, trephinlng for, 23.

Descending colon, anatomy of, 288.

Development of ear, 40.

Diaphragm, 171-205.

uro-genital, 427 
Dleffenbach Wellenschnitt, 103, 116.

resection of rectum, 351 .

Dieffenbach-Jaesche operation for lower llp, 112.

Dllatation of sphincter, 342.

Diploë, 20, 21.

Division of bone, 5 .

of aoft parts, 4.

Dorsal section, 419.

Roser method, 420.

Drum of ear, $43,87$.

Dubrueil, exarticulation of hand, 456.

Duct, common bile-, 313 .

cystic, 312.

ejaculatory, 395.

hepatic, 313 .

Stenson's, 55, 59.

tear-, 86.

thoracic, 188

Wharton' $8,57$.

Ductus communis choledochus, 313.

Duodenotomy for impacted gall-stone, 327.

Duodenum, 247.

Dura mater, 21

of spinal cord, 220 .

sinusea of, 22 .

Ear, development of, 41. middle, 43.

surgical anatomy of, 41.

Ear-drum, 43, 87.

Edebohls, decortication of kidney, 412. Inversion of appendix, 305 . nephropexy, 407.

Ejaculatory ducts, 395 .

Elbow, apace in front of, 442

Elbow-joint, exarticulation of forearm at, 460 . resection of, 474 .

surgical anatomy of, 458.

End-to-end anastomosia, small lntestine, 257. Mounsell method, 259

with Laplace forceps, 264.

with Murphy button, 261.

with O'Hara forceps, 266.

with suture, 257.

large intestine, 296

End-to-side, lateral Implantation (Intestine), 298.

Enterectomy, 254

Entero-anastomosis (see "Intestinal anastomosis," etc.).

Enterorrhaphy, 252.

Epididymis, 395.

Esmarch, exarticulation of arm at shoulderjoint, 467.

Esmarch's bandage and constrictor, 6.

Estlander, thoracectomy, 202.

Estlander's method, restoration of llp, 115.

Ethyl chloride, 2.

Eustachian tube, 45-87.

Exarticulation of arm at shoulder-joint, deltoid flap, 469 .

Spence method, 465 .

with Esmarch constrictor, 467.

of big toe, 500 .

with removal of metatarsal bone, 500 .

of finger, 450 .

at metacarpo-phalangeal joint, 452

of foot at ankle-joint Pirogoff method, 507.

Günther modification, 509 .

le Fort modification, 509 .

Syme method, 506 .

of forearm at elbow-joint, 460 .

of hand, Dubruell, 456.

at carpo-metacarpal articulation, 453.

of leg at knee-joint, Carden, 518.

Gritti-Stokes, 520 .

Stephen Smith, 516.

of little toe, 500 .
Exarticulation of thigh at bip-joint (Wyeth's pins), 526.

with preliminary ligation of common femoral, 529 .

of toes, 500 .

Excision of internal jugular vein, 49.

Excision of joints (see "Resection").

Face, classiflcation of deformities of, 90 . congenital deformities of, 80,89 .

in which first visceral arch is concerned, 98.

tn which frontal plate is concerned, 91. development of, 80 .

side of, 58 .

skeleton of, 52 .

surgical anatomy of 51 .

Facial cleft, oblique, 86,96 .

transverse, 87,98

vein, 126.

Falciform process, 372-485.

Faltopii, aqueductus, 45 .

Fascia, anal, 425, 430 .

deep cervical, 118.

connective tissue spaces beneath, 119.

deep perineal (see "Triangular ligament"), $427-429$.

endothoracica, 169.

lliaca, $218,373,379$.

lata, 372,482 .

iliac portion of, 372 .

pubic portion of, 372 .

lumbar, 217.

pectineal, 379.

perineal, deep, 427, 429.

superficial, 424.

spermatic, 394 .

superficial of groln, 362.

temporal, 19.

transversalis, 211.

Fasciæ of chest, 169.

of thorax, 169 .

Fauces, isthmus of, 55 .

Femoral artery, 486.

ligation of, 489.

hernia. 380 .

operation for, 392.

region, 372.

anterior, 485 .

from within abdomen, 378 .

sheath, 374-380, 488.

space, $374-379$.

vein (see "Femoral artery").

Fenger's incision, 224.

Fifth (trifacial) nerve, 33.

division of branches at base of skull, Krönlein-Lücke, 79.

Inferior maxillary branch, 63 .

superior maxillary branch, 63

Filum terminale, 220.

Finger, exarticulation of, 450 . at metacarpo-phalangeal joint, 452.

Fistula in ano, 342 .

operation for complete, 343.

for incomplete, 344 .

Foot, amputation of, Chopart, 504. Lisfrane, 501.

exarticulation of, Günther, 509.

le Fort, 509.

Pirogoff, 507.

Syme, 506.

surgical anatomy of, 498.

Foramen lacerum medium, 30.

obturator, 380 .

ovale, 31 .'

rotundum, 31, 63.

spinosum, 31 .

Forceps, Lapiace, 264.

McLean, 264. 
Forceps, O'Hara, 266.

Forcipressure, 11.

Forearm, amputation through, 457.

Forebrain, vesicle of, 81 .

Fossa cruralis, 378 .

ischio-rectal, 425 .

spheno-maxillary, 62 .

zygomatic, 61 .

Fovea inguinalis externa, 377.

inguinalis interna, 378 .

supravesicalis, 378 .

Fowler, pleurectomy, 203.

Frontal plate or process, $83,84,87$. sinus, 21.

Gall-bladder, aspiration of, 315 .

excision of ("Cholecystectomy").

incision into (see "Cholecystotomy"). operations upon, 315 .

surgical anatomy of, 312.

Gall-ducts, operations upon, 326 (see also "Gall-bladder").

Ganglion, Meckel's, 64 .

Casserian (see "Casserian ganglion").

Gastrectomy, 244.

Gastro-enterostomy, 272.

anterior, 272.

Carle, 281.

Jaboulay and Braun, 278.

posterior, 276.

vicious circle after, 279.

von Hacker, 276.

with Laplace forceps, 283.

with McGraw's rubber suture, 281.

with Murphy button, 280 .

with O'Hara forceps, 284.

Woelfler, 272.

Gastroplication, 226.

Gastrorrhaphy, 237 .

Gastrostomy, 232

Ssabanajew and Franck, 233.

von Hacker, 232.

Witzel, 235.

Gastrotomy, 228.

Gimbernat's ligament, 365-379.

Gland, Cowper's, 427 .

parotld, 59.

prostate, 428.

sublingual, 57 .

thyroid, 134.

Gluteal region, 482.

Gritti-Stokes amputation of leg, 520 .

Groin, lymphatic glands of, 362 .

superficial fascia of, 362 .

surgical anatomy of, 362

Günther modification of Pirogoff amputation, 509.

Hagedorn operation for barelip, 103.

Halsted operation for Inguinal hernia, 389.

Halsted-Meyer amputation of breast, 197.

Hand, exarticulation of, at carpo-metacarpal joint, 453.

exarticulation of, Dubrueil, 456.

incision into, 446 .

nerve-supply of, 446.

surgical anatomy of, 445 .

Harellp, 86-91.

Bardeleben's operation for, 106.

Blandin's operation for, 106.

Hagedorn's operation for, 103.

Malgaigne operation for, 101 .

Mirault operation for, 102 .

Nélaton operation for, 100.

operations for, 98 .

complete, 102.

double, with advanced intermaxillary process, 105.
Harelip, double, without advanced intermaxillary process, 104.

incomplete, 100 .

single complete, with cleft of alveolar process and advancement of intermax-

illary bone, 104.

von Graefe operation for, 100.

Hartley chisel, 26.

Head, 19.

surgical anatomy of, 19.

Heart, 179.

outlines of, upon chest wall, 180.

Hemiazygos vein, 188.

Hemorrhage, 5 .

artificial arrest of, 6.

control of, by chemical agents, 10 .

by digital compression in the wound, 10.

by digital compression of main vessels proximal to wound, 8 .

by direct means, 9 .

by forcipressure, 11.

by heat and cold, 9 .

by indirect means, 6 .

by ligature, 11.

by mechanical means, 10 .

by position, 8 .

by preliminary ligation, 8 .

by suture, 11 .

by tamponade, 10.

by torsion, 11.

through nervous system, 9 .

with Esmarch bandage, etc., 6.

intracranial, trephining for, 25 .

from middle meningeal, 25,28 .

natural arrest of, 6.

Hemorrhoids, 342-344.

operations for, 344 .

clamp and cautery, 346.

ligation and excision, 345 .

Henle's spine (see "Spina supra meatum").

Hepatectomy, 315.

Hepatle duct, 313.

Hepatotomy, 314 .

Hernia, 362

femoral, 375,380 .

operations for, 392 .

lngulnal, 368.

acqulred, 371

Bassini operation for, 383.

congenital, 370, 371, 378, Fig. 152.

operation for, Bassini, 3ð১.

direct (see "H., internal").

external or oblique, 368,370 (see also

"Fovea inguinalis externa," 377).

operation for, Bassini, 383 (see also Halsted's, below).

Halsted's operation for, 389.

indirect (see "H., external").

infantile or encysted, 371 .

internal or direct, 368, 370 (see also "Fovea inguinalis interna," 378).

operation for Bassinl, 389 (see also Halsted's, above)

oblique (see "H., external").

strangulated, operation for, 380 .

Herniotomy, 380 .

Highmore, antrum of, 53.

operation to drain, 69 .

Hip-joint, relations of parts behind, 483.

resection of, Langenbeck, 544 .

Anthony White inclsion, 545, Fig. 225.

surgical anatomy of, 523 .

Hunter's canal, 487.

Hydrocele, open operation for, 398.

puncture and injection, 397.

retroversion of tunica vaginalis, 400 .

Volkmann operation, 398 .

von Bergmann operation, 399. 
Hydrocele, Winkelmann operation, 400.

Hyoid bone, 130 .

Idtocy, craniectomy for, 29.

Ileo-colostomy, 296, 298.

end-to-end, 296.

end-to-side, 298 .

lateral (side-to-slde) anastomosls, 297.

without resection of cæcum, 298.

Ileum, 249 .

Iliac fascia (see "Fascla lliaca").

portion of fascia lata, 372 .

Ilio-pectineal llgament, 374-379.

Ilio-psoas space, 374 .

Inferlor maxilla (see "Lower jaw").

Inferior maxillary branch of fifth nerve (see "Fifth nerve").

Infraclavicular region, 174.

Infrahyold region, 130 .

Infundibular process, 367.

Infusion, intravenous, 480

Ingrowing toe-nail, operations for, 501.

Inguinal canal, 365 .

hernia (see "Hernia").

region, 363 .

from within abdomen, 375 .

rlng, Internal, 366, 377 .

external, 364.

Innominate artery, 188

Intermaxillary bone, 87 .

Internal jugular vein, ligation and excision of, 49.

Intestinai anastomosis, large lntestine, endto-end, 296.

lateral, 297.

slde-to-side, 297.

small intestine, end-to-end, 257.

Mounsell method, 259 .

with Laplace forceps, 264.

with Murphy button, 261 .

with O'Hara forceps, 266.

with suture, 257.

lateral, with Laplace forceps, 272.

wlth McGraw rubber suture, 271

wlth Murphy button, 271 .

wlth O'Hara forceps, 272

with suture, 268 .

slde-to-side (see lateral, above).

Intestinal clamp or compressor, 255, Fig. 106.

Intestlne, large, blood-supply of, 288.

operations upon, 289.

surgical anatomy of, 285.

resection of part of, 254 .

small, blood-supply of, 251.

operations upon, 252.

surgical anatomy of, 247.

suture of wounds of (see "Enterorrhaphy").

Intracranial bemorrhage, 25. trephinlng for, 25.

Intravenous lnfusion, 480 .

Ischlo-rectal fossa, 425 .

region, 425

Isthmus of fauces, 55.

Jaboulay and Braun, gastro-enterostomy, 278.

Jaw-bone, lower, 53 (see "Lower Jaw"). upper, 53 (see "Upper jaw-bone").

Jejunum, 249.

Keen bone forceps, 333

Kldney, abscess of, operation for, 410 .

absence of one, 403.

capsule of, 403 .

decortication of, 412.

extirpation of, 411 .

fixation of (see "Nephropexy").

floating, 403 .

incision into, 410.

movable, 403 .
Kldney, stone in, operation for, 411.

surgical anatomy of, 403.

Knee-jolnt, amputation of leg at, Carden, 518. Gritti-Stokes, 520.

Stephen Smith, 516.

bursæ adjacent to, 515 .

resection of, Textor incision, 538.

Volkmann incision, 543.

surgical anatomy of, 513 .

Knot, slip, 12.

square, 12.

surgeon's, 12

Kocher, amputation of tongue, 159. pyiorectomy, 241.

Kraske, amputation of rectum, 360 . resection of rectum, 353 .

sacral route to reach rectum, 353.

Krönlein's modification of Lücke's operation, 79.

Laminectomy, 332.

Langenbeck, extirpation of tongue, 163. formation of lower lip, 114.

incision for resection of upper jaw, 65 .

Laplace forceps, 264. anastomosis, end-to-end, with, 264. lateral, with, 272 .

gastro-enterostomy with, 283

Large intestine (see "Intestine").

anastomosis (see "Intestinal anastomosis").

Laryngeal region, 134 .

Laryngectomy, 148.

Laryngotomy, transverse, 146 (see also "Thyrotomy").

Larynx, extirpation of, 148. of half, 152

Lata, fascia, 482 (see "Fascia").

Lateral anastomosis (see "Intestinal anastomosis").

implantation after resection of cæcum, 298.

Lateral lithotomy, 433.

nasal clefts, 96 .

process, 84

pectoral region, 177.

sinus, 22.

sternal line, 171.

Le Fort, exartlculation of foot, 509 .

Leg, 492.

amputation of, 510

with lateral hooded flaps, 510.

exarticulation at knee-joint, Carden, 518

Gritti-Stokes, 520.

Stephen Smith, 516.

varicose veins of, ligation, 497.

Lembert suture, 16 .

Ligament, Colles's (triangular), 364.

Gimbernat's, 365-379.

ilio-pectineal, 374-379.

Poupart's, 364, 379.

pubic, of Cooper, 372, 374, 379.

sacro-sciatic, greater and lesser, 483

triangular (Colles's), 364

triangular (perineum), 427, 429.

Ligamentum dentatum, 220.

nuchæ, 120.

Ligation of arteries (see "Artery"). preliminary, to control hemorrhage, 8 . of varicose veins of leg, 497 .

Ligature, control of hemorrhage by, 11.

Line, axillary, 171.

lateral sternal, 171.

mammary, 171 .

midsternai, 171.

parasternal. 171

scapular, 172.

Linea alba, 209

Lingual triangle, 127.

Lip, lower, clefts of, 98 . excision of, 111. 
Lip, restoration of, 112.

Bruns method, 113.

Dieffenbach-Jaesche, 112.

Estlander, 115.

Langenbeck, 114

upper, median clefts and notches of, 95 .

restoration of, 116 .

Llps, operations upon, 111.

surgical anatomy of, 54 .

Lisfranc amputation of foot, 501.

joint, 499 .

Lithotomy, lateral, 433.

median, 432.

Little toe, exartlculation of, 500 .

Liver, incision Into (see "Hepatotomy")

injuries of, 315 .

resection of part of (see "Hepatectomy").

surgical anatomy of, 310 .

Longitudinal sinus, 22

Lower anterior pectoral reglon, 177.

Lower jaw, 53.

median clefts of, 98.

resection of entire body ot, 75 .

of half of body of, 73 .

of part of body in continulty, 75 . not in continuity, 76 .

Lücke's operation, Krönlein's modification, 79.

Lumbar fascia, 217.

puncture, 334

Lung, 193.

decortication of (see "Pleurectomy").

limits of, 193 .

root of, 192.

Macewen, osteotomy, 547.

Malgaigne operation for harelip, 101.

Mammary line, 171.

region, 175.

Mandibular arch, 83.

Mastold antrum, 40.

drilling into, 46.

to open and drain, 46

pneumatic, 40 .

region, surgical anatomy, 39 .

Maxillary bone, inferior (see "Lower jaw"). superior (see "Upper jaw-bone").

process, inferior, 83. superior, 83.

Maydl, colostomy, 292.

McBurney, cholecystostomy, 319.

incision, 225, Fig. 91.

McBurney's point, 286.

McGill-Fuller prostatectomy, 433.

McGraw's intestinal anastomosis, 271.

rubber suture gastro-enterostomy, 281.

McLean anastomosis forceps, 264.

Meckelii, cavum, 35.

Meckel's ganglion, 64 .

Median lithotomy, 432.

nerve, 445.

Mediastinum, 177.

contents of, 177

Mesentery, 250.

wounds of, 252 .

Metacarpo-phalangeal joints, 450 exarticulation of finger at, 452 .

Microcephalia, cranlectomy for, 29.

Middle ear, 43 .

fossa of skull, 30 .

extradural abscess in, 50 .

meningeal artery, hemorrhage from, 25.

nasal process, 84 .

Midsternal line, 171 .

Mirault operatlon for harelip, 102.

Mitral valve, 182 .

Mohrenheim fossa, 174.

Mounsell, end-to-end intestinal anastomosis, 259

Mouth, $\mathbf{5 4}$.
Mouth, floor of, 56.

roof of, 55 .

Mouth-gag, Whitehead, 108

Murphy button, cholecysto-duodenostomy with, 325 .

gastro-enterostomy with, 280.

intestinal anastomosis with, 261-271.

Muscle, bulbo-cavernosus, 426 .

compressor urethræ (see "M. transversus perinei").

erector spina, 216

external oblique, 208.

aponeurosis of, 209,364 .

externat pterygoid, 60.

gluteus maximus, $48 \%$

iliacus, 218.

ilio-psoas, 373 .

internal oblique, 209.

internal pterygoid, 64.

latissimus dorsi, 215.

levator anguli scapulæ, 216.

levator ani, 428-429.

masseter, 59 .

occipito-frontalis, 19.

pectoralis major, 173

pectoralis minor, 173 .

psoas, 218.

quadratus lumborum, 217.

rectus, 210 .

rhomboideus, 216.

sphincter ani (internal and external), 341. dilatation of, 342 .

splenius, 216 .

sterno-hyoid, 132.

sterno-mastoid, 120 .

sterno-thyroid, 132.

subclavius, 173.

subcostales, 169 .

temporal, 20.

transversalis abdomlnls, 210.

transversus perinei, 427.

trapezius, 215 .

triangularis sternl, 169

Muscles, Intercostal, 169 .

of back, 215 .

of chest wall, 169.

Musculo-spiral nerve, 445.

Nasal clefts, lateral, 96. process, lateral, 84 . middle, 84.

Neck, back of, 120

blood-vessels of, 136.

front of, 129

side of, 120.

surgical anatomy of, 118.

Nélaton operation for harellp, 100.

Nephrectomy, 411.

Nephrolithotomy, 411.

Nephropexy, 404.

Edebohls, 407.

Nephrotomy, 410

Nerve, anterior crural, 489.

anterior tibial, 494

aurlculo-temporal, 59, 63.

facial, 45, 52, 59 .

fifth (trifacial), 33

division of branches at base of skull, Krönlein-Lücke, 79

inferic $r$ maxillary branch, 63.

superior maxillary branch, 63 .

glosso-pharyngeal, 58.

gustatory, 58 .

hypoglossal, 58.

lingual, 58 .

median, 445.

musculo-spiral, 445.

posterior tiblal, 495

sacro-sciatic, stretchlng, 483 . 
Nerve, suture of, 15, 479. trifacial (see fifth, above).

ulnar, 445 .

vagus (see "Nerves, pneumogastrlc").

Nerves, inferior recurrent laryngeal, 134, 184. of neck, superficial, 122. of tongue, 58 .

phrenic, 184.

pneumogastric, 183.

Neural tube, 80 .

Oblique facial clefts, 86-96.

Obturator fascia, 429.

foramen, 380 .

Occipital triangle, 127.

Asophago-duodenostomy, 245.

Gisophago-enterostomy, 245.

Usophagostomy, 153.

Csophagotomy, external, 152.

Csophagus, 133, 185.

relations of, 186 .

stricture of, operation for (see "Gastrotomy," 228 .

O'Hara forceps, 266.

anastomosis, end-to-end, with, 266.

lateral, with, 272.

gastro-enterostomy wlth, 284.

Olfactory groove, 84.

Ophthalmic nerve (see "Fifth nerve").

Oral pit, 82 .

plate, 80 .

Orbito-nasal cleft, 86-96.

Osteoclast, 5.

Osteotomy (Macewen), 547.

Palate, 55 .

cleft, 93.

operation for, 107.

formation of, 88 .

Pampiniform plexus, 394.

Pancreas, operations upon, 332.

surgical anatomy of, 331 .

Parasternal line, 171.

Parotid gland, 59.

Patelia, suture of, 548.

Pectineal fascia (pubic portion of fascla lata), 379.

Pectoral region, lateral, 177. lower anterior, 177. upper anterior, 172 .

Pelvic cavity (from within), 428.

floor (from within), 429.

Pelvis, floor of (from without inward), 424.

Penis, amputation of, 422.

surgical anatomy of, 418 .

Pericardium, 178

Pericranium, 20.

Perineal fascia, deep, 427. superficlal, 424.

section with guide, 430 .

without guide, 431 .

Perineum, surgical anatomy of, 426.

Peritoneum, parietal, 211.

Phalango-phalangeal joints, 450. exarticulation of fingers at, 450 .

Pharyngeal membrane, 85 .

Phimosis, operations for, 419.

Phrenic nerves, 184

Pia mater, 23,220

Piles (see "Hemorrhoids").

Pirogoff exarticulation of foot, 507.

Plate, frontal, 83, 84, 87.

Pleura, 188.

anterior edge of, 189.

dome of, 191

excision of, 203.

lower edge of, 190.

Pleurectomy, Fowler, 203.

Popliteal space, 491.
Posterior trlangle oi neck, 122.

Poupart's ligament. 364, 379 .

space beneath, 373 .

Prævisceral space, 119.

Prepuce, operations upon, 419.

Process, frontal, 83, 84, 87.

inferior maxiliary, 83.

infundibular, 367 .

lateral nasai, 84-87.

mastoid (see "Mastoid reglon").

middle nasal, 84-87.

superior maxillary, 83.

Prostate gland, 428.

Prostatectomy, Alexander, 434.

McGill-Fuller, 433.

Prostatotomy, Bottini, 435.

Pterygo-maxillary region, 58.

Pubic ligament of Cooper, 372, 374-379.

portion of fascia lata, 372 .

Pulmonary valve (orifice), 181.

Purse-string suture, Murphy button, 279, 325. Pylorectomy, 237.

Billroth, 237.

Kocher, 241

use of Murphy button, 241.

Pyloroplasty, Heinecke and Mikulicz, 231.

Pylorus, resection of (see "Pylorectomy").

Rectal wall, excision of part of, 346 .

polypi, 348 .

Rectum, amputation, Kraske, 361 .

Volkmann, 348

blood-suppiy of, 341 .

resection of, Dieffenbach, 351 .

Kraske, 353 .

sacral route to expose, Kraske, 353.

surgical anatomy of, 338-339.

Regio abdominis lateralis dextra and sinistra, 213.

epigastrica, 213.

hypochondriaca, dextra and sinistra, 213.

inguinalis, 213.

mesogastrica, 213.

pubica, 213

umbilicus, 213.

Region, anterior femoral, 485 .

clavicular, 173

femoral, 372 .

from within abdomen, 378.

gluteal, 482.

infraclavicular, 174.

in frahyoid, 130 .

inguinal, 363 .

from within abdomen, $\mathbf{3 7 5}$.

ischio-rectal, 425.

laryngeai, 134.

lateral pectoral, 177.

lower anterior pectoral, 177.

mammary, 175.

mastoid, 39.

pterygo-maxillary, 58.

sternai, 172.

sterno-mastoid, 123.

suprahyoid, 130.

suprasternal, 135.

upper anterior pectoral, 172.

Regnoli-Billroth amputation of tongue, 161.

Remak, rachenhaut of, 85 .

Resection of ankle-joint, Koenig, 533 .

Langenbeck-Hueter, 529.

Lauenstein, 535 .

Mikulicz-Wladimirow, 536.

with extirpation of astragalus, 532.

of cæcum, 295.

of elbow-joint, 474 .

of hip-joint, Langenbeck incision, 543.

Anthony White incision, 545, Fig. 225.

of intestine (see "Enterectomy").

of knee-joint, Textor incision, 538. 
Resection of knee-joint, Volkmann incislon, 543.

of lower jaw, balf, 70 .

entire body of, 75 .

half of body of, 73 .

part of body in continuity, 75 .

not in continulty, 76 .

of pylorus (see "Pylorectomy").

of rectum (see "'Rectum").

of $\mathrm{rib}, 201$.

of skull, temporary, 26.

of shoulder-joint, 476.

of upper jaw, 64 .

of botb, 69 .

of wrist-joint, 472 .

Retrovisceral space, 119

Rib, first, 167

resection of (thoracotomy), 201.

Ribs, 167.

Riug, crural, 375-380

inguinal, external, 364.

internal, 366, 377 .

Rose position, 9

Rose-Andrews, extirpation of Casserian ganglion, 76 .

Roser, dorsal section, 420.

Sacral route, rectum, 353

Sacro-sciatic llgaments, 483.

Sacrum, 338.

Saphenous opening, $372,485$.

vein, internal, 485.

Scalp, 19

Scapular line, 172.

Scarpa's triangle, 486.

Schlatter, gastrectomy, 244

Schleich infiltration method, 3 .

Sclatic nerve, stretching. 483.

Scrotum, 394.

Sédillot, extirpation of tongue, 162.

Seminal vesicles (see "Vesiculæ seminales").

Serous surfaces, suture of, 16 .

Shoulder-joint, exarticulation at, deltold flap, 469.

Spence method, 465.

with Esmarch constrictor, 467.

resection of, 474

surgical anatomy of, 463 .

Side of neck, 120.

Side-to-side anastomosis (see "Intestinal anastomosis").

Sigmoid flexure, anatomy of, 288 .

resection of, 298 .

sinus, 39 .

thrombosis of, 48

Sinus, cavernous, 22,33 .

frontal, 21

lateral, 22

longitudinal, 22

slgmoid, 39.

thrombosis of, 48

Skin, suture of, 13 .

intracuticular, 14.

Skull, 20.

middle fossa, 30 .

temporary resection of, 26 .

trephining for depressed fracture, 23

Slip knot, 12.

Small intestine, 247 (see also "Intestlne").

anastomosis (see "Intestinal anastomosis")

blood-supply of, 251 .

surgical anatomy of, 247.

Smith (Stephen), amputation of leg at kneejoint, 516 .

Soft parts, division of, 4.

Space, femoral, 374-379.

prævisceral, 119.

retroviscerat, 119

subarachnoid, brain, 23
Space, subarachnoid, spinal cord, 220.

subdural, cranium, 23.

vertebral, 220 .

vascular, 119.

Spence, exarticulation of arm at shoulderjolnt, 465.

Spermatic cord, 365-393.

fascia, 394.

Sphincter ani muscles, 341.

dilatation of, 342 .

Spina supra meatum, 40 .

Spinal column, 218.

cord, 219.

dura mater of, 220.

pia mater of, 220 .

Spleen, excision of, 330 .

incision Into, 329 .

surgical anatomy, 328.

Splenectomy, 330 .

Spleno-maxillary fossa, 62.

Splenotomy, 329 .

square knot, 12

Ssabanajew-Franck gastrostomy, 233.

Staphylorrhaphy, 108.

Stenson's duct, 5u, 59.

Sternal region, 172.

Sterno-mastoid reglon, 123.

Sternum, 168.

Stomach, 221

closure of wounds of (gastrorrhaphy), 237.

excision of (see "Gastrectomy").

foreign bodies in, operation for, 228 .

incision into, 228 .

incisions to expose, 224.

operatlons upon, 226.

surgical anatomy of, 221.

Stricture, urethral, perlneal section for, 430431.

suprapubic cystotomy for lmpassable, 432. Styptics, 10.

Subarachnoid space, brain, 23.

spinal cord, 220 .

injection of cocain, 3 (see also "Lumbar puncture"').

Subclavian triangle, 128.

Subdural space, crantum, 23.

vertebral, 220 .

Sublingual glands, 57.

Submaxillary triangle, 126.

Submental triangle, 130.

Superficial fascia of groin, 362 .

Superior maxilla (see "Upper jaw-bone").

Superior maxillary branch of fifth nerve, 63. process, 83.

Suprahyoid region, 130 .

Suprapubic cystotomy, 415 .

Suprasternal region, 135.

Surgeon's knot, 12.

Suture, Cushing, 17.

Lembert, 16.

material, 13.

of bladder, 18 .

of bone, 15.

of bowel, 16 .

of cartilage, 15 .

of muscle, 14

of nerve, 15,480 .

of patella, 548 .

of serous surfaces, 16.

of skin, 13.

intracuticular, 14

of tendon, 15,479 .

of tissues, 13 .

Syme, exartlculation of foot, 506 .

Tampon cannula, Trendelenburg, 142.

Tamponade to control hemorrhage, 10.

Tarsus, 498.

Tear-duct, 86 
Teeth, development of, 89 .

Temporal faseia, 19.

Temporary resection of skull, 26.

Temporo-sphenoidal abscess, 51 .

Tendon, suture of, 15,479 .

Tenotomy, 496.

of flexor longus dlgitorum, 496.

of tendo Achillis, 496.

of tibialis posticus, 496 .

Testes, descent of, 369 .

Testis, 394.

extirpation of, 401.

Thigh, 482.

amputation of, 521 .

exarticulation of, at hip-jolnt (Wyeth), 526. with preliminary ligation of common femoral, 489.

Thoracectomy (Estlander), 202.

Thoracentesis, 201.

Thoracle aorta, 187.

duct, 188.

wall, muscles of, 169.

Thoracotomy, 201.

Thorax, aspiration (see "Thoracentesls").

tasclæ of, 169.

resectlon of wall of, 202.

gkeleton of, 166.

tapplng (see "Thoracentesis").

Thrombosis of sigmoid sinus, 48 .

Thymus body, 182

Thyro-hyoid membrane, 132.

Thyroid gland, 134.

Thyrotomy, 146.

Toe, amputation, 500

Toe-nail, ingrowing, operation, 501.

Tongue, 57.

amputation, with division of lower jaw, 162. Kocher, with preliminary ligatlon of llngual, 159.

Regnoli-Billroth, 161.

Whitehead, 164.

clefts of, 98 .

development of, 89 .

extirpation of, with divislon of lower faw, 162. Billroth, 164 .

Langenbeck, 163.

of half, Whitehead, 164 .

Sédillot, 162.

nerves of, 58 .

Torsion, 11.

Trachea, 184.

tampon of, 142.

Tracheotomy, 142

high, 143.

low, 144.

median, 145 .

Transversalia fascla, 211.

Transverse colon, anatomy of, 287.

facial cleft, 87.

Trendelenourg position, 8.

tampon cannula, 142 .

Trephine, removal of button of bone, 28 .

Trephining, 23.

for depressed fracture of skull, 23.

for hemorrhage from middle meningeal, 25.

for Intracranial hemorrhage, 25.

Triangle of neck, anterior, 122 .

inferior carotld, 123

lingual, 127.

oceipltal, 127.

posterior, 122

Scarpa's, 486.

subelavian, 128.

submaxlllary. 126

submental, 130

superior carotid, 125.

Triangular ligament (groin), 364. (perineum), 427-428.

Tricuspld valve, 182.
Trlfacial nerve (see "Fifth nerve").

Tympanum, 43.

Upper anterlor pectoral region, 172.

Upper jaw-bone, 53.

resection of, 64

Upper jaw-bones, resection of both, 69 .

Upper lip, median ciefts and notches of, 95. restoration of, 116.

Uranoplasty, 109.

Urethra, perineal section for stricture of, 430 .

suprapublc cystotomy for impassable atricture of, 432 .

Urethrotomy, external, with guide, 430. without guide, 431 .

Vaginal process of peritoneum, 370 .

Valve, aortic, 181 .

mitral, 182.

pulmonary, 181

tricuspid, 182.

Varicocele, open operation, 395.

Varicose velns, ligation of, 497.

Vas deferens, 393-395.

Vascular space, deep cervical fascla, 119.

Vein, anterior jugular, 121

axillary (8ee "Artery, axillary").

azygos, 187.

basilic, 442.

external jugular, 121.

facial, 126.

hemjazygos. 188.

Inferior thyroid, 135.

Internal jugular, 139

ligation and excision of, 49.

Internal saphenous, 485.

aubclavian, 141

temporal, 60 .

temporo-facial, 60

Velpeau incision for resection of upper jaw, 65, Fig. 19.

Vermiform appendix (see "Appendix vermiformis").

Vertebræ, dorsal, 167.

Vertebral column, 218.

Vesicle of forebrain, 81.

Vesiculæ semlnalis, 428.

Vlcious circle (gastro-enterostomy), 279.

Vlsceral arches, 82. clefts, 82.

Vogt's lines, 26.

Volkmann, amputation of rectum, 348 . operation for bydrocele, 398.

Von Bergmann operation for hydrocele, 399.

Von GräPe operation for harelip, 100.

Von Hacker, gastro-enterostomy, 276.

gastrostomy, 232

Incision, 224.

Wagner, temporary resection of skull, 26.

Weber's Incision for resection of upper jaw, 64.

Wellenschnitt, Dieffenbach's, 103, 105, 116.

Wharton's duct, 57 .

Whitehead amputation of tongue, 164.

mouth-gag, 108, Fig. 59.

Wilde's lnclsion, 46.

Witzel, gastrostomy, 235.

Woelfier. gastro-enterostomy, 272.

suture of gut, 258, Fig. 108 .

Wrist-joint, exartlculation of hand, Dubruell, 456.

resection of, 472 .

aurgical anatomy of, 455 .

wyeth, exarticulation of thigh at hip-joint, 526.

Wyeth's pins, 526.

Zygomatic fossa, 61 . 



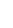







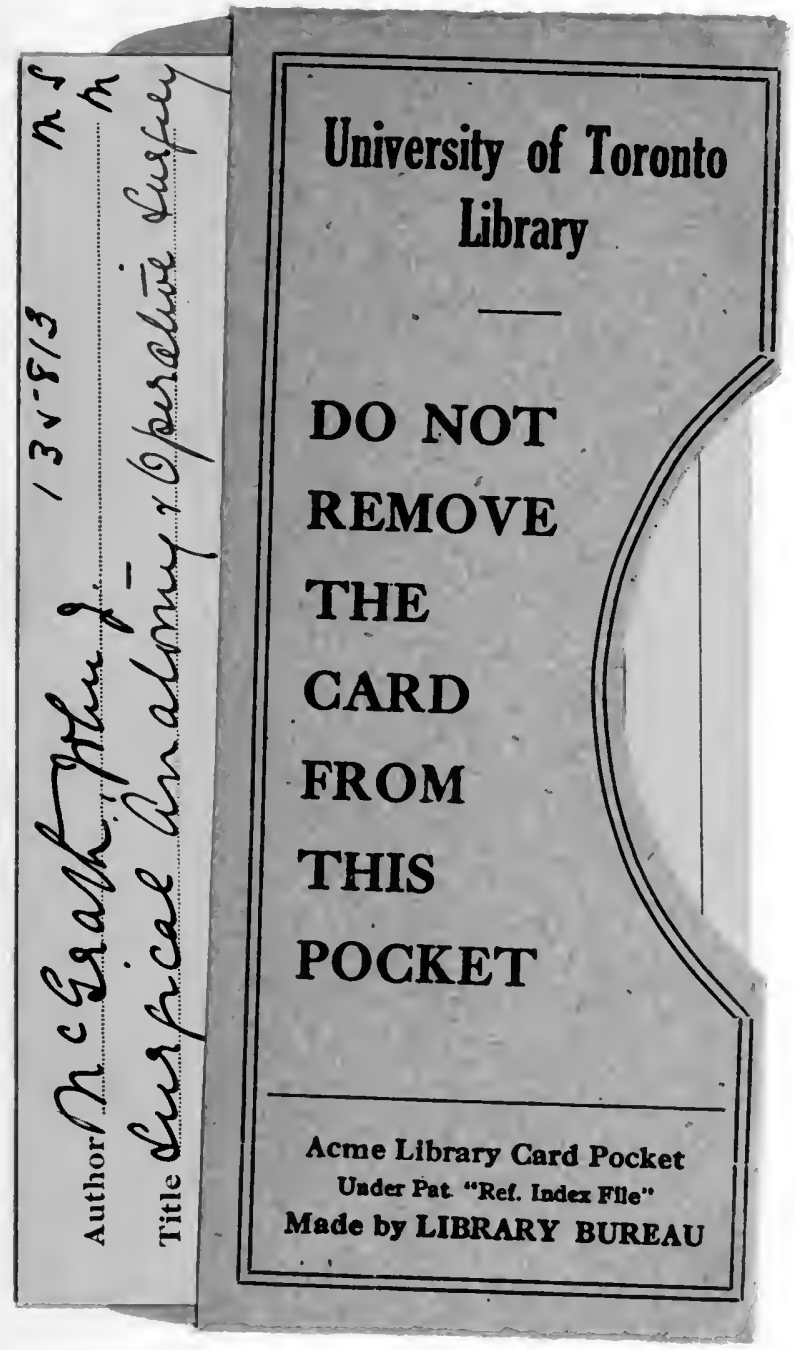


\%

\% 